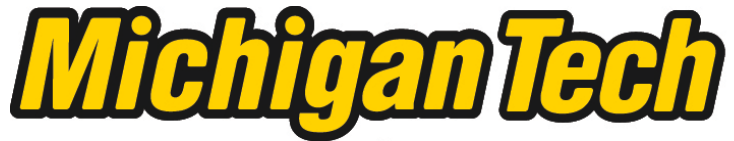 \\ Michigan Technological University Create the Future Digital Commons @ Michigan Tech
}

\section{Translation Studies on an Annular Field Reversed Configuration Device for Space Propulsion}

Carrie Suzanne Hill

Michigan Technological University

Follow this and additional works at: https://digitalcommons.mtu.edu/etds

Part of the Mechanical Engineering Commons, and the Physics Commons Copyright 2012 Carrie Suzanne Hill

\section{Recommended Citation}

Hill, Carrie Suzanne, "Translation Studies on an Annular Field Reversed Configuration Device for Space Propulsion", Dissertation, Michigan Technological University, 2012.

https://doi.org/10.37099/mtu.dc.etds/476

Follow this and additional works at: https://digitalcommons.mtu.edu/etds

3 Part of the Mechanical Engineering Commons, and the Physics Commons 
TRANSLATION STUDIES ON AN ANNULAR FIELD REVERSED CONFIGURATION DEVICE FOR SPACE PROPULSION

By

Carrie S. Hill

\begin{abstract}
A DISSERTATION
Submitted in partial fulfillment of the requirements for the degree of DOCTOR OF PHILOSOPHY
\end{abstract}

In Mechanical Engineering-Engineering Mechanics

MICHIGAN TECHNOLOGICAL UNIVERSITY

2012

(c) 2012 Carrie S. Hill

Distribution A: Approved for public release; distribution unlimited. 

This dissertation has been approved in partial fulfillment of the requirements for the Degree of DOCTOR OF PHILOSOPHY in Mechanical Engineering-Engineering Mechanics.

Department of Mechanical Engineering-Engineering Mechanics

Dissertation Advisor: Dr. Lyon B. King

Committee Member: Dr. Brian Beal

Committee Member: Dr. Jeffrey Allen

Committee Member: Dr. David Kirtley

Department Chair: Dr. William Predebon 



\section{Contents}

List of Figures $\ldots \ldots \ldots \ldots \ldots \ldots \ldots \ldots \ldots \ldots$ ix

List of Tables $\ldots \ldots \ldots \ldots \ldots \ldots \ldots \ldots \ldots \ldots \ldots \ldots \ldots \ldots \ldots$

Acknowledgments $\ldots \ldots \ldots \ldots \ldots \ldots \ldots \ldots \ldots \ldots \ldots \ldots \ldots$

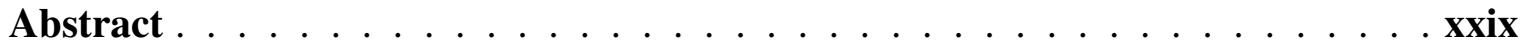

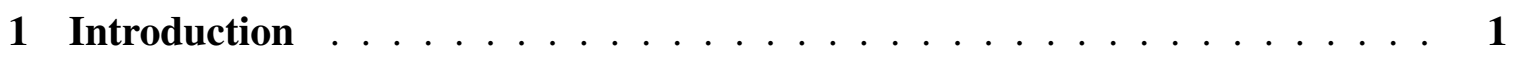

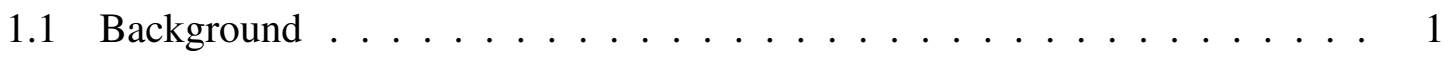

1.2 Aim and Scope $\ldots \ldots \ldots \ldots \ldots \ldots \ldots \ldots$

1.3 Structure of Dissertation $\ldots \ldots \ldots \ldots$

2 Overview of Plasmoid Propulsion . . . . . . . . . . . . . . . . . . . . 7

2.1 Principles of Pulsed Propulsion . . . . . . . . . . . . . . 7

2.2 Plasmoid Propulsion . . . . . . . . . . . . . . . . . . 11

2.3 Plasmoid Thruster Technology $\ldots \ldots \ldots \ldots \ldots$

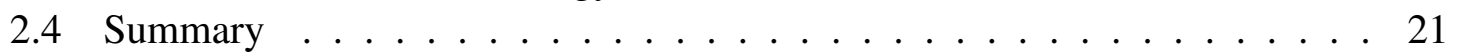

3 Annular Field Reversed Configurations $\ldots \ldots \ldots \ldots$

3.1 AFRC Basics . . . . . . . . . . . . . . . . 23

3.2 AFRC Formation Techniques . . . . . . . . . . . . . . . 25

3.2.1 Parallel Coil Mode: Synchronous _. . . . . . . . . . . . 27

3.2.2 Independent Coil Mode: Synchronous . . . . . . . . . . . . 28

3.2.3 Parallel Coil Mode: Asynchronous . . . . . . . . . . . . . . . . . 29

3.2.4 Independent Coil Mode: Asynchronous . . . . . . . . . . . 32

3.3 AFRC Physics . . . . . . . . . . . . . . . . . . . . . . 34

3.3.1 Radial Balance . . . . . . . . . . . . . . . . . . 35

3.3 .2 Equilibrium Profiles . . . . . . . . . . . . . . . . . . . . . 38

3.3.3 Magnetic Field Diffusion . . . . . . . . . . . . . . . . . 40

3.3.4 Plasma Drifts . . . . . . . . . . . . . . . . . . 42

3.3.5 Termination . . . . . . . . . . . . . . . 43

3.4 Literature Review of AFRCs $\ldots \ldots \ldots \ldots$

3.5 Propulsion Considerations $\ldots \ldots \ldots \ldots \ldots$ 
4 Translation Model of an Annular Field Reversed Configuration . . . . . . . 57

4.1 Annular Electromagnetic Launcher Model . . . . . . . . . . . . . . 58

4.1 .1 Numerical Approach . . . . . . . . . . . . . . . . . 63

4.1.2 Model Validation . . . . . . . . . . . . . . . . . . 67

4.2 Design Studies for the XOCOT-T3 Experiment $\ldots \ldots \ldots \ldots$

4.2.1 Results for Nominal Case . . . . . . . . . . . . . . . . . 75

4.2 .2 Cone Angle Study . . . . . . . . . . . . . . . . . . . . 78

4.2 .3 Inner Coil Radius Study $\ldots \ldots \ldots$. . . . . . . . . . . 81

4.2 .4 Initial Energy Study . . . . . . . . . . . . . . . . . . . . . . 83

4.2 .5 Coil Inductance Study . . . . . . . . . . . . . . . 86

4.2.6 Parasitic Inductance and Resistance Study . . . . . . . . . . . . 88

4.2.7 Final XOCOT-T3 Experiment Design . . . . . . . . . . . . 92

4.2.8 Plasmoid Sensitivity Studies ～. . . . . . . . . . . . . . . . 96

4.3 Magnetic Field Modeling of XOCOT-T3 . . . . . . . . . . . . . . . . . 104

4.4 Summary and Discussion of Results . . . . . . . . . . . . . . . 112

5 Experiment, Facilities, and Diagnostics $\ldots \ldots \ldots \ldots \ldots$

5.1 Experimental Apparatus . . . . . . . . . . . . . . . . . . . 118

5.1 .1 Electromagnetic Coils . . . . . . . . . . . . . . . 119

5.1 .2 Quartz Insulators . . . . . . . . . . . . . . . . . . 122

5.1 .3 Main Bank Discharge Circuit . . . . . . . . . . . . . . . . 123

5.1 .4 Pre-Ionization Source . . . . . . . . . . . . . . . . . . . . 127

5.1 .5 Safety Considerations . . . . . . . . . . . . . . . . . . . 129

5.1.6 Vacuum Facilities and Gas Injection . . . . . . . . . . . 130

5.1 .7 Experiment Operation . . . . . . . . . . . . . . . 132

5.2 Diagnostics . . . . . . . . . . . . . . . . . 1333

5.2.1 Current and Voltage Monitors . . . . . . . . . . . . . 135

5.2 .2 Magnetic Field Probes . . . . . . . . . . . . . . . . . 137

5.2.2.1 B-dot Probe Theory . . . . . . . . . . . . . . . 138

5.2.2.2 Differential B-dot Probe Theory . . . . . . . . . . . . 147

5.2.2.3 Calibration of B-dot Probes . . . . . . . . . . . . . 149

5.2.2.4 Analysis of B-dot Data . . . . . . . . . . . . . 153

5.2.2.5 Error Analysis for B-dot Probes . . . . . . . . . . . 156

5.2.2.6 XOCOT-T3 External B-dot Probes . . . . . . . . . . . 160

5.2.2.7 XOCOT-T3 Internal Magnetic Field Probes . . . . . . . . 170

5.2.2.8 XOCOT-T3 TOF Magnetic Field Probes . . . . . . . . . 173

5.2 .3 Plasma Probes . . . . . . . . . . . . . . . . . . . . . . . . 181

5.2.4 Single Frame Digital Camera . . . . . . . . . . . . . . . . . . . 194

5.3 Translation Measurements . . . . . . . . . . . . . . . . . . . . 194

5.3.1 Velocity Measurements: Time-of-Flight Array . . . . . . . . . . . 195

5.3.2 Momentum Measurements . . . . . . . . . . . . . . . . 197

5.3 .3 Efficiency Estimates . . . . . . . . . . . . . . . . . 199 
5.3 .4 Energy Analysis . . . . . . . . . . . . . . . 200

6 Experimental Data: Translation Study . . . . . . . . . . . . . . . . . 203

6.1 Test Conditions . . . . . . . . . . . . . . . . . . . . . . 204

6.2 Vacuum Characterization . . . . . . . . . . . . . . . . 206

6.2 .1 Vacuum Data: $10 \mathrm{kHz} \ldots \ldots . \ldots 206$

6.2 .2 Vacuum Data: $20 \mathrm{kHz}$. . . . . . . . . . . . . 213

6.3 Plasmoid Formation Characterization . . . . . . . . . . . . . . . . . . 219

6.3.1 Formation Results . . . . . . . . . . . . . . . . . . . . . . 219

6.3 .2 Pre-Ionization . . . . . . . . . . . . . . . . 223

6.3 .3 Main Bank Timing . . . . . . . . . . . . . . . . 226

6.3 .3 .1 Timing: $10 \mathrm{kHz} \ldots \ldots \ldots \ldots 26 . \ldots \ldots$

6.3.3.2 Timing: $20 \mathrm{kHz} \ldots \ldots \ldots . \ldots \ldots 231$

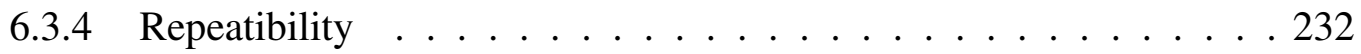

6.3.4.1 Repeatibility: $10 \mathrm{kHz} \ldots \ldots . \ldots 233$

6.3.4.2 Repeatibility: $20 \mathrm{kHz} \ldots \ldots \ldots . \ldots . \ldots 235$

6.4 Translation Study Data . . . . . . . . . . . . . . . . 241

6.4.1 Translation Data: $10 \mathrm{kHz}$. . . . . . . . . . . . . . . 243

6.4.2 Translation Data: $20 \mathrm{kHz}$. . . . . . . . . . . . . 257

7 Translation Results $\ldots \ldots \ldots \ldots \ldots$

7.1 Translation Data Reduction . . . . . . . . . . . . . . . . . . . . . . . . . . . . . .

7.2 Velocity Results . . . . . . . . . . . . . . . . . . . 264

7.2.1 Velocity Results: $10 \mathrm{kHz}$. . . . . . . . . . . . . 267

7.2.2 Velocity Results: $20 \mathrm{kHz}$. . . . . . . . . . . . . . . . 288

7.2.3 Downstream Plasma Flux Measurements . . . . . . . . . . . . . . . 295

7.3 Discussion of Translation Results . . . . . . . . . . . . . . . 303

7.4 Possible Failure Modes . . . . . . . . . . . . . . . . . . . . . 305

7.4.1 Failure Mode: Current Induction Limit . . . . . . . . . . . . . . . . 305

7.4.2 Failure Mode: Low Conductivity . . . . . . . . . . . . . . . . 306

7.4.3 Failure Mode: Instabilities and Imbalances . . . . . . . . . . . . . . . . . . . . . . . . . . . . . . .

7.5 Summary . . . . . . . . . . . . . . . . . . . 309

8 Instability Study Data and Results . . . . . . . . . . . . . . . . 311

8.1 Instability Study Diagnostics . . . . . . . . . . . . . . . . . 311

8.2 Instability Study Data . . . . . . . . . . . . . . . . . 312

8.2 .1 Image Data . . . . . . . . . . . . . . . . 313

8.2.2 Asymmetric Double Langmuir Probe Data . . . . . . . . . . . . . . 316

8.3 Discussion . . . . . . . . . . . . . . . . . . . . 321

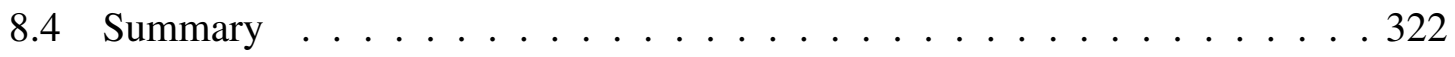

9 Radial Balance Study Data and Results . . . . . . . . . . . . . . . . . 323

9.1 Radial Balance in AFRCs . . . . . . . . . . . . . . . . . . . 323 
9.2 Radial Balance Study Data . . . . . . . . . . . . . . . . . . . . . . . . . . . . . . . . . . . . . . .

9.3 Radial Balance Study Results . . . . . . . . . . . . . . . . . . . . . . . . 342

9.4 Discussion of Radial Balance Results . . . . . . . . . . . . . . . . . . . . . 344

10 Conclusion and Future Work . . . . . . . . . . . . . . . . . . . . . 347

10.1 Contributions of This Work . . . . . . . . . . . . . . . 347

10.2 Future Work . . . . . . . . . . . . . . . . . 350

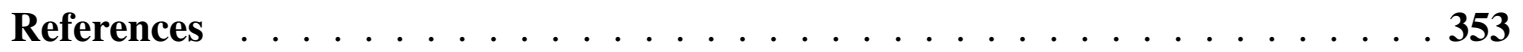

A XOCOT-T3 Supplemental Data . . . . . . . . . . . . . . . . . . 361 


\section{List of Figures}

1.1 Annular field reversed configuration schematic. The poloidal B-field is supported by a toroidal (azimuthal) plasma current. . . . . . . . 2 2

2.1 Family of pulsed indutive plasmoid thrusters, including (a) planar thruster, (b) conical $\theta$-pinch FRC thruster, (c) RMF-FRC thruster, (d) AFRC thruster. 11

3.1 Principle dimensions of an (a) FRC and an (b) AFRC. . . . . . . . . . . . 24

3.2 Force diagram for an AFRC translating from a conical coil. . . . . . . . . 25

3.3 Formation schematic for an AFRC formed with the coils connected in parallel, operating in phase. . . . . . . . . . . . . . 30

3.4 Formation schematic for an AFRC formed with the coils connected in parallel, operating asynchronously. . . . . . . . . . . . 31

3.5 Formation schematic for an AFRC formed with the coils operating indepedently and asynchronously. . . . . . . . . . . . . 33

3.6 Representative internal magnetic field structure of AFRCs showing a reversed field on the inner coil as compared to the outer coil. . . . . . . . . 39

3.7 Representative internal electron density structure of AFRCs, showing a peak density at $\mathrm{B}=0 \ldots \ldots \ldots \ldots \ldots$

4.1 Schematic of the annular electromagnetic launcher model. The plasmoid is depicted with circuit elements $L_{p}$ and $R_{p}$, magnetically coupled to the coils through $M_{o p}$ and $M_{i p} \ldots \ldots \ldots \ldots \ldots$. . . . . . . . . . . . . . . . .

4.2 Geometry and mesh used for the inductance calculations in COMSOL. The coil meshes were refined to resolve the skin depth of the current in the coils. 65

4.3 Results from the modified annular electromagnetic launcher model, using a test case presented by Novac, et al [1]. . . . . . . . . . . . . . . . . 69

4.4 Effective inductance of a slug-coil geometry calculated using COMSOL. Experimental data from Reference [2] are shown with the dotted line. . . . . 70

4.5 Definition of the plasmoid lifetime limit, as determined by the peak in the coil-plasma coupling case where the plasmoid is not allowed to translate. . . 72

4.6 Results from the launcher model, including coil currents, plasma current, capacitor voltage, plasmoid velocity, and axial plasmoid position as a function of time for nominal inputs.

4.7 Positional dependence of the mutual inductance gradient for the inner and outer coil and the combined Lorentz force. . . . . . . . . . . . . . . . . . 77 
4.8 Results from the cone angle study, including the final plasmoid position, final velocity, and energy efficiency. Results are shown for 2 different capacitor banks. . . . . . . . . . . . . . . . . . . . 79

4.9 Mutual inductance gradient and Lorentz force as a function of slug position. Several different cone angles are shown, increasing in the arrow direction. . 80

4.10 Results from the inner coil radius study, including the final plasmoid position, final velocity, and energy efficiency. Results are shown for 2 different capacitor banks. . . . . . . . . . . . . . . . 8 82

4.11 Coupling coefficients and Lorentz force computations for several inner coil radii. . . . . . . . . . . . . . . . . . . 83

4.12 Results from the initial energy study, including the final plasmoid position, final velocity, and energy efficiency. Results are shown for 2 different capacitor banks. . . . . . . . . . . . . . . . . . . 85

4.13 Results from the coil inductance study, showing energy efficiency and minimum required input energy as a function of coil turns. . . . . . . . 87

4.14 Results from the stray inductance study, including the final plasmoid position, final velocity, and energy efficiency as a function of stray inductance. 89

4.15 Results from the stray study, including the final plasmoid position, final velocity, and energy efficiency as a function of stray resistance. . . . . . . 90

4.16 Plasmoid lifetime (related to rise time of circuit) as a function of stray

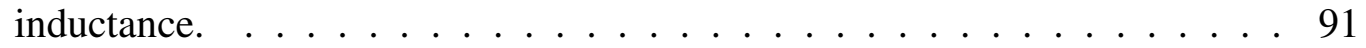

4.17 Lorentz force as a function of slug position for two stray outer coil inductances. The larger stray inductance results in a larger Lorentz force. . . 91

4.18 Results from the launcher model for the XOCOT-T3 design inputs, including coil currents, plasma current, capacitor voltage, plasmoid velocity, and axial plasmoid position as a function of time for nominal inputs. 95

4.19 Results from the XOCOT-T3 design study on initial energy, including the final plasmoid position, final velocity, and energy efficiency. Results are shown for the primary bank $(225 \mu \mathrm{F})$ and the alternate bank $(43.5 \mu \mathrm{F}) . .97$

4.20 Sensitivity results from the XOCOT-T3 design study on plasmoid width, including the final plasmoid position, final velocity, and energy efficiency. Results are shown for the primary bank $(225 \mu \mathrm{F})$ and the alternate bank $(43.5 \mu \mathrm{F}) \ldots \ldots \ldots \ldots \ldots \ldots \ldots \ldots$

4.21 Sensitivity results from the XOCOT-T3 design study on plasmoid length, including the final plasmoid position, final velocity, and energy efficiency. Results are shown for the primary bank $(225 \mu \mathrm{F})$ and the alternate bank

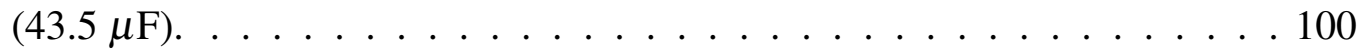

4.22 Sensitivity results from the XOCOT-T3 design study on plasma conductivity, including the final plasmoid position, final velocity, and energy efficiency. Results are shown for the primary bank $(225 \mu \mathrm{F})$ and the alternate bank $(43.5 \mu \mathrm{F}) . \ldots \ldots \ldots$ 
4.23 Sensitivity results from the XOCOT-T3 design study on plasmoid radius, including the final plasmoid position, final velocity, and energy efficiency. Results are shown for the primary bank $(225 \mu \mathrm{F})$ and the alternate bank $(43.5 \mu \mathrm{F}) \ldots \ldots \ldots \ldots$

4.24 Coil connections, circuit connections, and coil geometry used to calculate the vacuum fields. The rectangles in (a) represent FEM objects in COMSOL. The geometry used in COMSOL to represent the multi-turn coils is drawn in (b) . . . . . . . . . . . . . . . . . . 105

4.25 COMSOL results for the vacuum case using the XOCOT-T3 design, including (a) coil currents, (b) time history of midplane axial fields, (c) axial magnetic fields as the length of the coil, and (d) midplane axial field across the radial cross section. . . . . . . . . . . . . . . . . 107

4.26 Magnetic field map from the XOCOT-T3 design, computed in COMSOL. Arrows denote field direction. Detail views of the magnetic field between coil turns is displayed in the smaller figures. . . . . . . . . . . . . 108

4.27 COMSOL results for the plasmoid-coupling case using the XOCOT-T3 design, including (a) coil currents, (b) time history of midplane axial fields, and (c) midplane axial field across the radial cross section. . . . . . . . . 109

4.28 Coil connections, circuit connections, and coil geometry used to calculate the translating plasmoid fields. The rectangles in (a) represent FEM objects in COMSOL. The moving plasmoid in COMSOL is specified by the sliding region of conductivity in (b). . . . . . . . . . . . . . . . . . . . . . . 110

4.29 Coil currents and magnetic field predicted by COMSOL for a plasmoid translating at $50 \mathrm{~km} / \mathrm{s} \ldots \ldots \ldots \ldots 111 \ldots \ldots \ldots$

5.1 The XOCOT-T3 experiment, connected to Chamber 5B. Image is a long exposure photograph of a single pulse discharge in argon. The pre-ionization antenna is shown as the thinner coil upstream (left) of the main 4-turn coils. . . . . . . . . . . . . . . . . . . . . . . . . . 118

5.2 A dimensional drawing of the XOCOT-T3 experiment from several perspectives, connected to Chamber 5B. The coils were concentric, separating an annular space with quartz insulators. . . . . . . . . . . . . . 119

5.3 A system view of the XOCOT-T3 experiment. . . . . . . . . . . . . . . . . 120

5.4 XOCOT-T3 discharge coils, showing (a) a single turn of the inner coil and (b) the outer coil assembly. . . . . . . . . . . . . . . . . . . 121

5.5 Main Bank Discharge Circuit Schematic. . . . . . . . . . . . . . . 123

5.6 Photographs of the XOCOT-T3 main bank circuit. . . . . . . . . . . . . . 125

5.7 A schematic of the pre-ionization circuit. . . . . . . . . . . . . . 128

5.8 Photographs of the pre-ionization circuit. The capacitor bank (far left) is connected to the antenna (far right) through a thyristor stack (middle). The current in the circuit is measured with the Rogowski coil directly in front of the capacitor bank. . . . . . . . . . . . . . . . . . . . . . . 129 
5.9 A schematic of the vacuum facilities used in the XOCOT-T3 testing. This diagram is not drawn to scale. . . . . . . . . . . . . . . . . 131

5.10 Layout of the diagnostics used in the XOCOT-T3. The single frame camera is located outside the chamber, capturing images from an end-on view. Current and voltage monitors are not shown in this figure and are located on the external circuit. . . . . . . . . . . . . . . . . . . . . . . . 134

5.11 Capacitor Voltage Divider Circuit. The capacitor voltage is measured across the $10 \mathrm{k} \Omega$ resistor with an isolated Fluke multimeter. . . . . . . . . 137

5.12 Magnetic field probe locations in the XOCOT-T3 experiment. External b-dot probes run the length of the discharge coils. The placement of the internal b-dot probes shown here is typical for most of the testing. Probe-to-probe spacing is $2.5 \mathrm{~cm}$ apart on the external and internal arrays. . 138

5.13 Illustration of a magnetic field probe. The probe head is connected to a transmission line and $V_{p}$ is measured at the end of the transmission line. . . 139

5.14 The circuit schematic for a b-dot probe connected to transmission lines. The complete circuit (a) can be simplifed to equivalent lumped impedances for low frequency (b) as probe impedances $Z_{P}$, line impedances $Z_{0}$, termination impedance $Z_{L}$, and high voltage capacitive coupling impedance

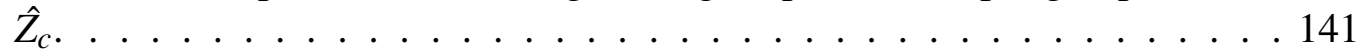

5.15 The frequency response (gain and phase) of a simulated probe assembly with negligible capacitance, $10 \mu \mathrm{H}$ of inductance, and $10 \Omega$ of resistance from $10 \mathrm{kHz}$ to $1 \mathrm{MHz}$. . . . . . . . . . . . . . . . . . . . 143

5.16 A SPICE circuit representation of a magnetic field probe used for examining the effect of the high voltage capacitive coupling on the probe's transfer function $\beta(\omega) \ldots \ldots \ldots \ldots \ldots \ldots$

5.17 Gain and phase changes of a b-dot probes transfer function $\beta(\omega)$ due to high voltage capacitive coupling. The solid lines are from including $\hat{Z}_{c}$ in the transfer function and the dashed lines are from neglecting $\hat{Z}_{c}$. Results from a SPICE simulation are shown as hollow (gain) dots and solid (phase) dots. . . . . . . . . . . . . . . . . . . . . 146

5.18 Illustration of a differential magnetic field probe. Each probe head is wound in a separate direction. . . . . . . . . . . . . . . 148

5.19 Circuit diagram of a differential $b$-dot probe. Mutual inductance between probe heads is indicated using the dot convention. . . . . . . . . . . . 149

5.20 Integrated digital signals sampled from an analog waveform using an 8-bit digitizer and a 12-bit digitizer. . . . . . . . . . . . . 156

5.21 External magnetic field probes and their calibration probes, secured to the quartz insulator. Two arrays of external probes and calibration probes span the length of the electromagnetic coils. . . . . . . . . . . . . . 161

5.22 External magnetic field probe wiring diagram. . . . . . . . . . . 162 
5.23 The complex impedance of an external probe assembly (B007) from $1 \mathrm{kHz}$ to $5 \mathrm{MHz}$, measured with an impedance analyzer. An inset graph shows the detail from 1 to $500 \mathrm{kHz}$. . . . . . . . . . . . . . . . . . . . . . 163

5.24 Sample calibration waveforms for external probe array, including (a) raw b-dot probe signals, (b) corresponding FFTs, (c) intervals of data used for calibration, (d) NA waveform for three intervals, and (e) error in NA. . . . . 165

5.25 Measured phase delay at $9.1 \mathrm{kHz}$ of external probes, compared to their predicted delay. . . . . . . . . . . . . . . . . . 166

5.26 Photograph of internal magnetic field probes. . . . . . . . . . . . . . 171

5.27 Internal magnetic field probes wiring diagram. . . . . . . . . . . . . 171

5.28 Photograph of TOF magnetic field probes. The downstream probe is the larger probe (top) and the upstream probe is the smaller probe (bottom). . . 174

5.29 TOF magnetic field probes wiring diagram. . . . . . . . . . . . . . . 175

5.30 TOF magnetic field probes' impedance as a function of frequency for the (a) upstream probe and (b) downstream probe. . . . . . . . . . . . . . 176

5.31 Measured frequency response of preamplifier \#1 from $10 \mathrm{kHz}$ to $1 \mathrm{MHz}$ for (a) 10x gain and (b) 100x gain. . . . . . . . . . . . . . . . . . 179

5.32 Measured frequency response of preamplifier \#2 from $10 \mathrm{kHz}$ to $1 \mathrm{MHz}$ for (a) 10x gain and (b) 100x gain. . . . . . . . . . . . . . . . . . 180

5.33 Sample I-V characteristic for single Langmuir probe. . . . . . . . . . . . 182

5.34 Ion flux probes, including (a) a photograph of the probes and (b) circuit diagram . . . . . . . . . . . . . . . 185

5.35 Current density measured by the ion flux probes for a $-6 \mathrm{~V}$ bias and $-24 \mathrm{~V}$ bias. . . . . . . . . . . . . . . . . . 186

5.36 Illustration of an asymmetric double Langmuir probe. . . . . . . . . . . . . 188

5.37 Asymmetric double Langmuir probe I-V characteristics for several different electron temperatures. . . . . . . . . . . . . . . . . 191

5.38 Asymmetric double Langmuir probe, including (a) a photograph of the probe and (b) circuit diagram. . . . . . . . . . . . . . . . . . 193

5.39 Plasma probe data from a time-of-flight array in a PIPT experiment [3]. . . 196

6.1 Coil currents as a function of time for the vacuum shot using the $10 \mathrm{kHz}$ bank. . . . . . . . . . . . . . . . . 207

6.2 Vacuum coil current data for the $10 \mathrm{kHz}$ bank, including (a) average waveforms for the $500 \mathrm{~J}$ setting, (b) peak currents versus energy, and (c) peak currents versus discharge voltage. . . . . . . . . . . . . . . 208

6.3 XOCOT-T3 vacuum magnetic fields as a function of discharge energy for the $10 \mathrm{kHz}$ circuit. . . . . . . . . . . . . . . . . . . . . 210

6.4 Downstream magnetic field signals measured with and without a differential amplfier. . . . . . . . . . . . . . . . . . . . . 212 
6.5 TOF peak-normalized vacuum magnetic field traces for the $10 \mathrm{kHz}$ circuit, including average magnetic field waveforms for the (A) upstream probe and the (B) downstream probe. The coil current waveform is shown for comparison. . . . . . . . . . . . . . . . . . . . . . . 214

6.6 Vacuum coil currents for the $20 \mathrm{kHz}$ circuit, including (A) the time history of the coil currents, (B) peak coil currents compared to input energy, and (C) peak currents versus discharge voltage. Uncertainty for all measurements is $1 \% \ldots \ldots \ldots \ldots$. . . . . . . . . . . . . . 216

6.7 Vacuum magnetic fields for the $20 \mathrm{kHz}$ circuit, including average magnetic field waveforms at the coil midplane on the outer wall (A), the inner wall (B), and the average magnetic field at peak coil current along the coil length (C). . . . . . . . . . . . . . . . . . . . . . 217

6.8 TOF vacuum magnetic fields for the $20 \mathrm{kHz}$ circuit, including average magnetic field waveforms for the (A) upstream probe and the (B) downstream probe. The coil current waveform is also shown. . . . . . . . 218

6.9 Typical plasmoid formation data for the $10 \mathrm{kHz}$ circuit at $500 \mathrm{~J}$ with a 4 mTorr fill. A full time-history is shown with vacuum and plasma data for the main bank current, midplane magnetic fields, and light output measured by a photometer. . . . . . . . . . . . . . . . . . . . 221

6.10 Typical plasmoid formation data for the $20 \mathrm{kHz}$ circuit at $100 \mathrm{~J}$ with a 4 mTorr fill. A full time-history is shown with vacuum and plasma data for the (A) main bank current, (B), midplane magnetic fields, and (C) light output measured by a photometer. . . . . . . . . . . . . . . . . 222

6.11 Pre-ionization study data, including current waveforms for the vacuum and plasma case, coil current compared to charge voltage, and energy absorbed by the plasma versus charge energy. . . . . . . . . . . . . . 225

6.12 Timing results for the $20 \mathrm{kHz}, 100 \mathrm{~J}$ tests, showing energy absorbtion into the plasma (top) and midplane magnetic fields for various delay intervals. . 228

6.13 Timing results for the $20 \mathrm{kHz}, 1000 \mathrm{~J}$ tests, showing energy absorbtion into the plasma (top) and midplane magnetic fields for various delay intervals. . .229

6.14 Timing results for the $20 \mathrm{kHz}, 1000 \mathrm{~J}$ tests, showing energy absorbtion into the plasma (top) and midplane magnetic fields for various delay intervals. . 230

6.15 Timing results for the $20 \mathrm{kHz}, 100 \mathrm{~J}$ tests, showing energy absorbtion into the plasma (top) and midplane magnetic fields for various delay intervals. . .232

6.16 Repeatibility study data for the $10 \mathrm{kHz}$ circuit at $100 \mathrm{~J}$, including coil currents and upstream magnetic field measurements. The standard deviation of repeated data sets for each diagnostics is shown on the right. . . 234

6.17 Downstream results from the repeatibility study for the $10 \mathrm{kHz}$ at 100 $\mathrm{J}$, including the measurements recorded from the TOF probes and the standard deviations among repeated shots. . . . . . . . . . . . . 235 
6.18 Repeatibility study data for the $10 \mathrm{kHz}$ circuit at $500 \mathrm{~J}$, including coil currents and upstream magnetic field measurements. The standard deviation of repeated data sets for each diagnostics is shown on the right. . . 236

6.19 Downstream results from the repeatibility study for the $10 \mathrm{kHz}$ at 500 $\mathrm{J}$, including the measurements recorded from the TOF probes and the standard deviations among repeated shots. . . . . . . . . . . . . 237

6.20 Repeatibility study data for the $10 \mathrm{kHz}$ circuit at $1000 \mathrm{~J}$, including coil currents and upstream magnetic field measurements. The standard deviation of repeated data sets for each diagnostics is shown on the right. . . 238

6.21 Downstream results from the repeatibility study for the $10 \mathrm{kHz}$ at 1000 $\mathrm{J}$, including the measurements recorded from the TOF probes and the standard deviations among repeated shots. . . . . . . . . . . . . . . . 239

6.22 Repeatibility study data for the $20 \mathrm{kHz}$ circuit at $100 \mathrm{~J}$, including coil currents and upstream magnetic field measurements. The standard deviation of repeated data sets for each diagnostics is shown on the right. . . 240

6.23 Downstream results from the repeatibility study for the $20 \mathrm{kHz}$ at 100 $\mathrm{J}$, including the measurements recorded from the TOF probes and the standard deviations among repeated shots. . . . . . . . . . . . . . . . . . 241

6.24 Average currents and magnetic fields at $7 \mu$ s compared to number of samples included in average. . . . . . . . . . . . . . . . . 242

6.25 Total current and midplane magnetic field data at various fill pressures for the $100 \mathrm{~J}, 10 \mathrm{kHz}$ circuit. Vacuum traces are shown at each diagnostic by dotted lines. . . . . . . . . . . . . . . . . . . . 245

6.26 Magnetic field data at various fill pressures and locations for the $100 \mathrm{~J}, 10$ $\mathrm{kHz}$ circuit. Vacuum traces are shown at each diagnostic by dotted lines. . . 246

6.27 Peak normalized downstream magnetic field data at various fill pressures for the $100 \mathrm{~J}, 10 \mathrm{kHz}$ circuit. Vacuum traces are shown at each diagnostic by dotted lines. . . . . . . . . . . . . . . . . . . . . . . . 247

6.28 Total current and midplane magnetic field data at various fill pressures for the $500 \mathrm{~J}, 10 \mathrm{kHz}$ circuit. Vacuum traces are shown at each diagnostic by dotted lines. . . . . . . . . . . . . . . . . . . . . . . . . 249

6.29 Magnetic field data at various fill pressures and locations for the $500 \mathrm{~J}, 10$ $\mathrm{kHz}$ circuit. Vacuum traces are shown at each diagnostic by dotted lines. . . 252

6.30 TOF probe data at various fill pressures for the $500 \mathrm{~J}, 10 \mathrm{kHz}$ circuit, including peak normalized downstream magnetic field data and plasma flux measurements. Vacuum traces are shown at each diagnostic by dotted lines. 253

6.31 Total current and midplane magnetic field data at various fill pressures for the $1000 \mathrm{~J}, 10 \mathrm{kHz}$ circuit. Vacuum traces are shown at each diagnostic by dotted lines. . . . . . . . . . . . . . . . . . . . . 254

6.32 Magnetic field data at various fill pressures and locations for the $1000 \mathrm{~J}, 10$ $\mathrm{kHz}$ circuit. Vacuum traces are shown at each diagnostic by dotted lines. . . 255 
6.33 Peak normalized downstream magnetic field data at various fill pressures for the $1000 \mathrm{~J}, 10 \mathrm{kHz}$ circuit. Vacuum traces are shown at each diagnostic by dotted lines. . . . . . . . . . . . . . . . . . . . 256

6.34 Total current and magnetic field data at various fill pressures for the $100 \mathrm{~J}$, $20 \mathrm{kHz}$ circuit. Vacuum traces are shown at each diagnostic by dotted lines. 258

6.35 Magnetic field data at various fill pressures for the $100 \mathrm{~J}, 20 \mathrm{kHz}$ circuit. Vacuum traces are shown for each diagnostic by a dotted line. . . . . . . . 259

6.36 TOF probe data at various fill pressures for the $100 \mathrm{~J}, 20 \mathrm{kHz}$ circuit, including peak normalized downstream magnetic field data and plasma flux measurements. Vacuum traces are shown at each diagnostic by dotted lines. 260

7.1 Full data set from a single shot at $100 \mathrm{~J}$ with the $20 \mathrm{kHz}$ bank and a 4 mTorr gas fill. . . . . . . . . . . . . . . . . 265

7.2 Translation results for the $10 \mathrm{kHz}$ circuit at $100 \mathrm{~J}$, with a 1 mTorr fill pressure.268

7.3 Translation results for the $10 \mathrm{kHz}$ circuit at $100 \mathrm{~J}$, with a 4 mTorr fill pressure.269

7.4 Translation results for the $10 \mathrm{kHz}$ circuit at $100 \mathrm{~J}$, with a 10 mTorr fill pressure. . . . . . . . . . . . . . . 270

7.5 Translation results for the $10 \mathrm{kHz}$ circuit at $100 \mathrm{~J}$, with a 14 mTorr fill

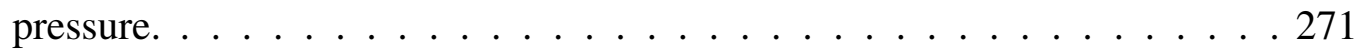

7.6 Translation results for the $10 \mathrm{kHz}$ circuit at $100 \mathrm{~J}$, with a $20 \mathrm{mTorr}$ fill pressure. . . . . . . . . . . . . . . . . 272

7.7 Translation results for the $10 \mathrm{kHz}$ circuit at $100 \mathrm{~J}$, with a 50 mTorr fill pressure.......................... 2727

7.8 Translation results for the $10 \mathrm{kHz}$ circuit at $500 \mathrm{~J}$, with a 1 mTorr fill pressure.275

7.9 Translation results for the $10 \mathrm{kHz}$ circuit at $500 \mathrm{~J}$, with a 4 mTorr fill pressure.276

7.10 Translation results for the $10 \mathrm{kHz}$ circuit at $500 \mathrm{~J}$, with a 10 mTorr fill pressure. . . . . . . . . . . . . . . . . . 277

7.11 Translation results for the $10 \mathrm{kHz}$ circuit at $500 \mathrm{~J}$, with a 14 mTorr fill

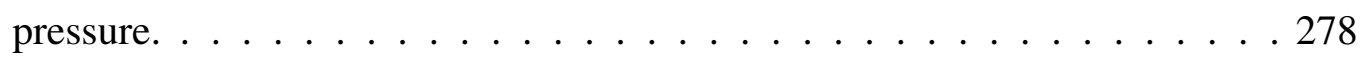

7.12 Translation results for the $10 \mathrm{kHz}$ circuit at $500 \mathrm{~J}$, with a 20 mTorr fill pressure. . . . . . . . . . . . . . . . 279

7.13 Translation results for the $10 \mathrm{kHz}$ circuit at $500 \mathrm{~J}$, with a 50 mTorr fill pressure. . . . . . . . . . . . . . . . . . . 280

7.14 Translation results for the $10 \mathrm{kHz}$ circuit at $1000 \mathrm{~J}$, with a 1 mTorr fill pressure. . . . . . . . . . . . . . . . 282

7.15 Translation results for the $10 \mathrm{kHz}$ circuit at $1000 \mathrm{~J}$, with a 4 mTorr fill pressure. . . . . . . . . . . . . . . . . . . 283

7.16 Translation results for the $10 \mathrm{kHz}$ circuit at $1000 \mathrm{~J}$, with a 10 mTorr fill pressure. . . . . . . . . . . . . . . . . . . 284

7.17 Translation results for the $10 \mathrm{kHz}$ circuit at $1000 \mathrm{~J}$, with a 14 mTorr fill pressure. . . . . . . . . . . . . . . . 285

7.18 Translation results for the $10 \mathrm{kHz}$ circuit at $1000 \mathrm{~J}$, with a 20 mTorr fill pressure. . . . . . . . . . . . . . . . . 286 
7.19 Translation results for the $10 \mathrm{kHz}$ circuit at $1000 \mathrm{~J}$, with a 50 mTorr fill pressure. . . . . . . . . . . . . . . 287

7.20 Translation results for the $20 \mathrm{kHz}$ circuit at $100 \mathrm{~J}$, with a $1 \mathrm{mTorr}$ fill pressure.289

7.21 Translation results for the $20 \mathrm{kHz}$ circuit at $100 \mathrm{~J}$, with a 4 mTorr fill pressure.290

7.22 Translation results for the $20 \mathrm{kHz}$ circuit at $100 \mathrm{~J}$, with a $10 \mathrm{mTorr}$ fill pressure. . . . . . . . . . . . . . . . . . . 291

7.23 Translation results for the $20 \mathrm{kHz}$ circuit at $100 \mathrm{~J}$, with a 14 mTorr fill pressure. . . . . . . . . . . . . . . . . 292

7.24 Translation results for the $20 \mathrm{kHz}$ circuit at $100 \mathrm{~J}$, with a 20 mTorr fill pressure. . . . . . . . . . . . . . . . 293

7.25 Translation results for the $20 \mathrm{kHz}$ circuit at $100 \mathrm{~J}$, with a 50 mTorr fill pressure. . . . . . . . . . . . . . . . . . 294

7.26 ADLP current measured $3.8 \mathrm{~cm}$ downstream of the coil exit plane for several bias voltages. . . . . . . . . . . . . . . . . . 296

7.27 Plasma density and electron temperature measured by an ADLP at $\mathrm{z}=34.3$ $\mathrm{cm}$. Figure (c) shows that thin-sheath approximations are valid for this probe for the time-interval of interest. . . . . . . . . . . . . . . 297

7.28 Translation results for the $100 \mathrm{~J}, 20 \mathrm{kHz}$ circuit including peak-normalized density from an ADLP. . . . . . . . . . . . . . . . . . . . . 298

7.29 Collision cross sections for electron elastic scattering from argon and for electron ionization from argon for various electron energies (a) and mean free path for elastic and ionization scattering as a function of energy energy for various neutral fill pressures (b). . . . . . . . . . . . . . . . 300

7.30 Estimated plasma density for ion saturation currents measured by downstream plasma probes at several pressures. . . . . . . . . . . . . 301

7.31 Time derivative of current compared to observed plasmoid current lifetime for all XOCOT-T3 test conditions, showing the scaling for the outer coil (left) and inner coil (right). . . . . . . . . . . . . . . 306

8.1 Background images for the $10 \mathrm{kHz}$ data (left) and $20 \mathrm{kHz}$ data (right), showing the location of the insulator walls and tank entrance. . . . . . . . . 314

8.2 Images of the plasma from an end view for a $500 \mathrm{~J}, 4$ mTorr plasma test with the $10 \mathrm{kHz}$ circuit. . . . . . . . . . . . . . . . 315

8.3 Images of the plasma from an end view for a $100 \mathrm{~J}, 4$ mTorr plasma test with the $20 \mathrm{kHz}$ circuit. . . . . . . . . . . . . . . 316

8.4 ADLP data at $\mathrm{z}=33.8 \mathrm{~cm}$, showing the temporal data at fixed bias voltages and the reconstructed $\mathrm{I}-\mathrm{V}$ characteristic. . . . . . . . . . . . . . . 318

8.5 Theoretical I-V characteristics for a 4-mTorr backfill with $100 \%$ ionization and various temperatures. . . . . . . . . . . . . . . . . . . 319

8.6 ADLP measurements at the axial midplane, including (A) the temporal probe current, (B) radial distribution of plasma current at various times, and $(\mathrm{C})$ midplane magnetic fields. . . . . . . . . . . . . . . . . . 320 
9.1 Final plasmoid radius, calculated using Equation 9.11. The radial trajectory using XOCOT-T3 circuit parameters is shown in (a) for various plasmoid resistances. The final plasmoid position as a function of outer coil stray inductance is shown in (b). . . . . . . . . . . . . . . . . 327

9.2 Coil currents and midplane magnetic fields for Configuration \#1. The original circuit data is shown with the faint dotted line. . . . . . . . . . . 330

9.3 Magnetic field data for a $100 \mathrm{~J}, 4$ mTorr shot with the circuit in Configuration \#1. The data using the original circuit is indicated by the faint dotted lines. . . . . . . . . . . . . . . . . . 331

9.4 Magnetic pressure at the axial midplane for a $100 \mathrm{~J}, 4 \mathrm{mTorr}$ discharge using the $20 \mathrm{kHz}$ circuit in Configuration \#1. . . . . . . . . . . . . . . 332

9.5 Images of plasmoid formation for a $100 \mathrm{~J}, 4$ mTorr discharge using the 20 $\mathrm{kHz}$ circuit in Configuration \#1. Midplane magnetic fields are shown for reference. . . . . . . . . . . . . . . . . . 334

9.6 Coil currents and midplane magnetic fields for Configuration \#2. The original circuit data is shown with the faint dotted line. . . . . . . . . . 335

9.7 Magnetic field data for a $100 \mathrm{~J}, 4 \mathrm{mTorr}$ shot with the circuit in Configuration \#2. The data using the original circuit is indicated by the faint dotted lines. . . . . . . . . . . . . . . . . . . . . . 337

9.8 Magnetic pressure at the axial midplane for a $100 \mathrm{~J}, 4$ mTorr discharge using the $20 \mathrm{kHz}$ circuit in Configuration \#2. . . . . . . . . . . . . 338

9.9 Images of plasmoid formation for a $100 \mathrm{~J}, 4$ mTorr discharge using the 20

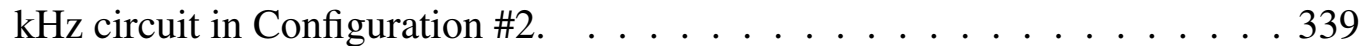

9.10 ADLP data at the axial midplane for Configuration \#2. The data collected by the probe is shown (A) along with the radial distribution along the channel (B), and midplane mangetic fields (C). . . . . . . . . . . . . . . 341

A.1 Vacuum data from a shot at $500 \mathrm{~J}$ and $10 \mathrm{kHz} \ldots \ldots . . . . . . . . .362$

A.2 Vacuum data from a shot at $100 \mathrm{~J}$ and $20 \mathrm{kHz}$. . . . . . . . . . . . . . 363

A.3 Current, magnetic field, and photometer data from a shot at $500 \mathrm{~J}$ and 10 $\mathrm{kHz}$, with a 4 mTorr fill of Argon. . . . . . . . . . . . . . . . 364

A.4 Current, magnetic field, and photometer data from a shot at $100 \mathrm{~J}$ and 20 $\mathrm{kHz}$, with a 4 mTorr fill of Argon. . . . . . . . . . . . . . . 365

A.5 Current and voltage traces from the ADLP at $\mathrm{z}=34.3 \mathrm{~cm}$. Curve fits to the data were constructed from ADLP theory. . . . . . . . . . . . . 366 


\section{List of Tables}

2.1 Pulsed inductive plasmoid thrusters. Asterisks denote projected numbers. . 14

3.1 History of AFRC devices . . . . . . . . . . . . . . . 46

4.1 Nominal inputs for design study . . . . . . . . . . . . . . . 74

4.2 XOCOT-T3 final design parameters . . . . . . . . . . . . 94

5.1 XOCOT-T3 circuit impedances . . . . . . . . . . . . . . . . . . . 127

5.2 External magnetic field probes' impedances, sensitivities, and proportionality constants. Impedances were measured at $10 \mathrm{kHz}$. . . . . . . 169

5.3 Internal magnetic field probes' impedances, sensitivities, and proportionality constants. Impedances were measured at $10 \mathrm{kHz}$. . . . . . . 172

6.1 XOCOT-T3 test conditions . . . . . . . . . . . . . . . . . 204

6.2 XOCOT-T3 vacuum coil currents as a function of energy for the $10 \mathrm{kHz}$ bank.207

6.3 XOCOT-T3 vacuum coil currents as a function of energy and charge voltage for the $20 \mathrm{kHz}$ circuit. . . . . . . . . . . . . . . . . 214

6.4 XOCOT-T3 calculated circuit resitances for the $10 \mathrm{kHz}$ circuit. . . . . . . . 227 



\section{Acknowledgments}

This research would not have been possible without the countless advice, technical input, and tireless support of many advisors, coworkers, friends, and family. Without your inspiration, guidance, and encouragement, this work would be a shadow of itself.

First and foremost, I would like to thank my advisor Brad King. You've taught me the most useful skills I have learned in this research: approach concepts with nothing but a pencil and paper. When things didn't make sense, you encouraged me to look from a new direction and follow the math. One of your greatest contributions to my career has been to make sure that all ideas are properly motivated and have a purpose and place. Thank you also for your patience and high standards, both which have shaped my future outlook on research.

I'd also like to thank the other members of my committee: David Kirtley, Brian Beal, and Jeffrey Allen. After compiling this document, I can only imagine the hours of review that must go into it. Thank you for your ideas and inputs in the early phases of this research when your creativity and realistic advice helped frame and ground the research. Thank you, David, for being a great mentor and sounding board for all things FRC-related. Without your advice on every facet of this project, it would have been an (even bigger) monster of metal, glass, wires.

Thank you to the program support at AFRL-Edwards. Thank you especially to Dan Brown, who worked tirelessly to make sure this project received the proper support to see it through. Your patience as I sat for months glued to a computer screen is greatly appreciated. I'd also like to thank my coworkers in the EP Lab. You are a special blend of people I'm not sure I could find anywhere else in this world. You were always willing to put down your tools to work on my problem.

Thank you to my many coworkers from the ISP Lab. My early days in the ISP Lab were a product of your input and advice on everything from coffee to leak-checking. Best of luck to the next generations of ISP'ers as you work toward that often elusive goal!

Finally, I'd like to thank my family and friends who cheered from the sidelines. Sasha, your refreshing fifth grade perspective reminded me that school can be fun. Emily, your friendship, advice, and humor were and still are a sanity-saver. And most of all Mike...thank you for seeing this through with me. I know you didn't bargain for my graduate student career to stretch out quite this far, but you never complained. I look forward to many dissertation-less years with you! 



\section{Nomenclature}

$\beta \quad$ Plasma beta; ratio of gas pressure to magnetic pressure

$\beta(\omega)$ B-dot probe's sensitivity or transfer function

$\Delta r_{m} \quad$ Separatrix width

$\varepsilon_{0} \quad$ Permittivity of free space

$\varepsilon_{B} \quad$ Electromotive force generated by a magnetic field

$\eta \quad$ System efficiency

$\eta_{\perp} \quad$ Resistivity perpendicular to $\mathbf{B}$

$\eta_{B} \quad$ Beam efficiency

$\eta_{E} \quad$ Energy efficiency

$\eta_{P} \quad$ Propellant efficiency

$\lambda_{D} \quad$ Debye length

$\lambda_{M} \quad$ Mean free path

$\langle\cos \theta\rangle_{m v}$ Momentum-weighted plume divergence

$\langle u\rangle_{m} \quad$ Mass-weighted velocity

T Thrust

$\mu_{0} \quad$ Permeability of free space

$\nabla p \quad$ Pressure gradient

$\Phi_{B} \quad$ Magnetic flux

$\sigma_{P} \quad$ Plasmoid conductivity 
$\tau_{B} \quad$ Magnetic field diffusion timescale

E Electric field

$\theta \quad$ Azimuthal coordinate

$A_{p} \quad$ Area of probe

$B_{z i} \quad$ Inner field

$B_{z o} \quad$ Outer field

$C_{M B} \quad$ Main bank capacitance

$E_{0} \quad$ Initial energy

$e_{c} \quad$ Elementary charge

$E_{\text {plasma }}$ Energy absorbed into plasma

$g \quad$ Acceleration due to gravity

$h \quad$ Time-step

$H(f)$ Transfer function

$I_{+}^{\text {sat }} \quad$ Ion saturation current

$I_{i} \quad$ Inner coil current

$I_{O} \quad$ Outer coil current

$I_{p} \quad$ Plasmoid current

$I_{b i t} \quad$ Impulse bit

$I_{e n} \quad$ Electron current

$I_{s p} \quad$ Specific impulse

$k \quad$ Coupling coefficient, $M_{12} \equiv k \sqrt{L_{1} L_{2}}$

$k_{B} \quad$ Stefan-Boltzmann constant

$k_{i p} \quad$ Plasmoid-inner coil coupling coefficient

$k_{o p} \quad$ Plasmoid-outer coil coupling coefficient

$l \quad$ Coil length

$L_{c} \quad$ Coil inductance 
$L_{e} \quad$ External circuit inductance

$L_{i} \quad$ Inner coil inductance

$L_{o} \quad$ Outer coil inductance

$L_{p} \quad$ Plasmoid inductance

$l_{s} \quad$ Separatrix length

$L_{e i} \quad$ Inner coil circuit external inductance

$L_{e o} \quad$ Outer coil circuit external inductance

$l_{s} \quad$ Separatrix length

$m \quad$ Mass

$m_{j} \quad$ Mass of the jth species

$M_{m} \quad$ Molar mass

$m_{\text {bit }} \quad$ Mass bit

$M_{i o} \quad$ Inner-outer coil mutual inductance

$M_{i p} \quad$ Inner coil-plasmoid mutual inductance

$M_{o p} \quad$ Outer coil-plasmoid mutual inductance

$n \quad$ Exponential power

n Number density

$n_{0} \quad$ Plasma density

$N_{A} \quad$ Avogadro's number

$n_{e} \quad$ Electron density

$N_{i} \quad$ Inner coil turns

$n_{i} \quad$ Plasma density

$N_{o} \quad$ Outer coil turns

$n_{i p} \quad$ Plasmoid-inner coil coupling exponent

$n_{o p} \quad$ Plasmoid-outer coil coupling exponent

$N A \quad$ B-dot probe's proportionality constant 


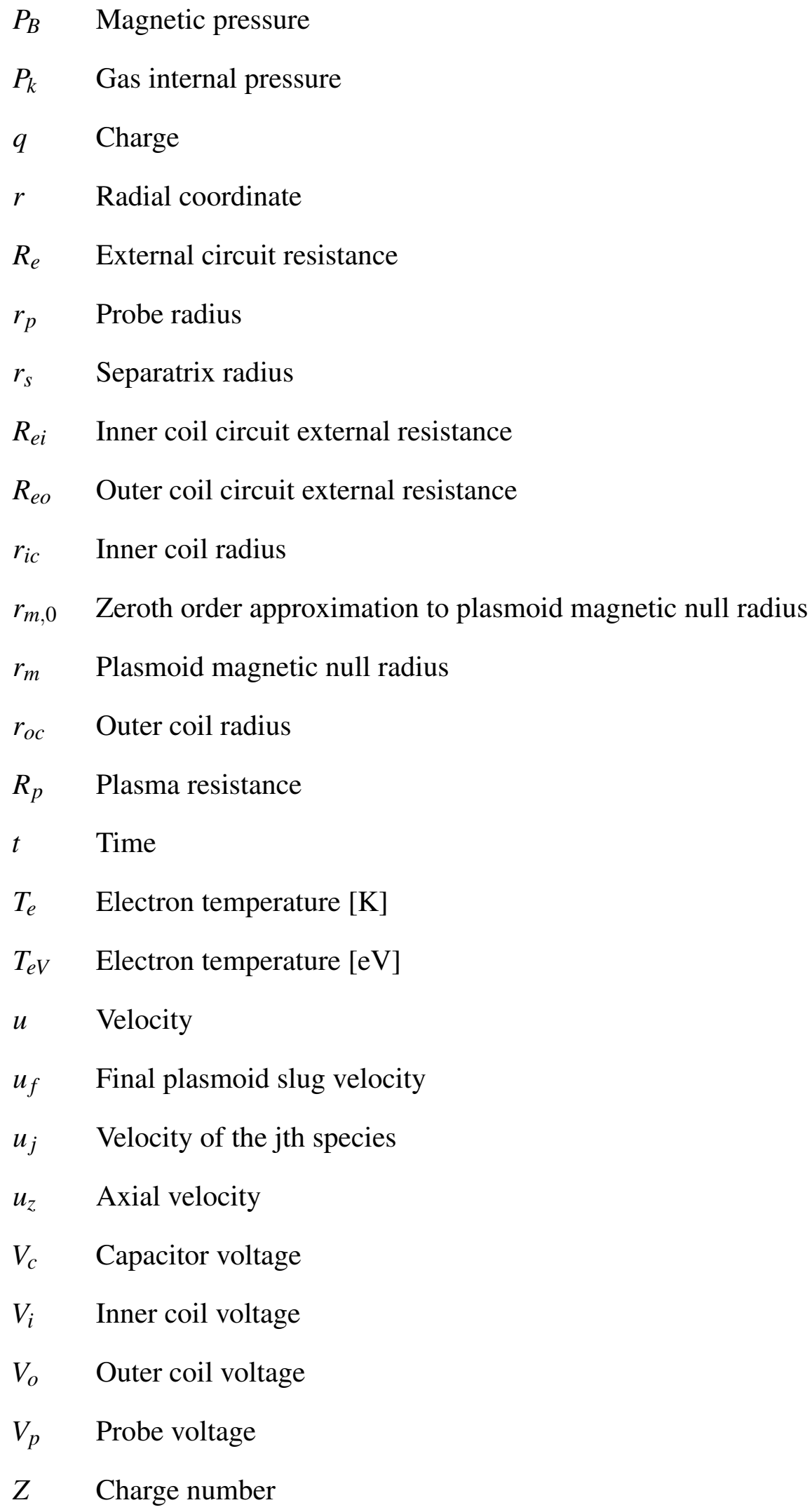


$z \quad$ Plasmoid slug position

$Z_{0} \quad$ Transmission line impedance

$Z_{L} \quad$ Termination (load) impedance

$Z_{p} \quad$ Probe impedance

$z_{i p} \quad$ Plasmoid-inner coil coupling scale length

$z_{o p} \quad$ Plasmoid-outer coil coupling scale length

$z_{\text {scale }}$ Coupling scale length or stroke length

$z_{\text {shift }}$ Positional offset of exponential profile

$z s_{i p} \quad$ Plasmoid-inner coil coupling positional offset

$z s_{o p} \quad$ Plasmoid-outer coil coupling positional offset

B Magnetic flux density or Magnetic field

J Current density

ADLP Asymmetric Double Langmuir Probe

AFRC Annular Field Reversed Configuration

AFRL Air Force Research Laboratory

B Magnetic flux density or Magnetic field

EP Electric Propulsion

FRC Field Reversed Configuration

$\ln \Lambda \quad$ Coloumb logarithm

PI Pre-ionization

PIPT Pulsed Inductive Plasmoid Thruster

R Resistance

RMF Rotating Magnetic Field

TOF Time-of-flight

X Reactance

XOCOT-T3 Experiment on Coaxial Toroids for Translation

Z Complex impedance 



\section{Abstract}

This research investigated annular field reversed configuration (AFRC) devices for high power electric propulsion by demonstrating the acceleration of these plasmoids using an experimental prototype and measuring the plasmoid's velocity, impulse, and energy efficiency.

The AFRC plasmoid translation experiment was design and constructed with the aid of a dynamic circuit model. Two versions of the experiment were built, using underdamped RLC circuits at $10 \mathrm{kHz}$ and $20 \mathrm{kHz}$. Input energies were varied from $100 \mathrm{~J} /$ pulse to 1000 $\mathrm{J} / \mathrm{pulse}$ for the $10 \mathrm{kHz}$ bank and $100 \mathrm{~J} / \mathrm{pulse}$ for the $20 \mathrm{kHz}$ bank. The plasmoids were formed in static gas fill of argon, from 1 mTorr to 50 mTorr. The translation of the plasmoid was accomplished by incorporating a small taper into the outer coil, with a half angle of $2^{\circ}$. Magnetic field diagnostics, plasma probes, and single-frame imaging were used to measure the plasmoid's velocity and to diagnose plasmoid behavior. Full details of the device design, construction, and diagnostics are provided in this dissertation.

The results from the experiment demonstrated that a repeatable AFRC plasmoid was produced between the coils, yet failed to translate for all tested conditions. The data revealed the plasmoid was limited in lifetime to only a few (4-10) $\mu \mathrm{s}$, too short for translation at low energy. A global stability study showed that the plasma suffered a radial collapse onto the inner wall early in its lifecycle. The radial collapse was traced to a magnetic pressure imbalance. A correction made to the circuit was successful in restoring an equilibrium pressure balance and prolonging radial stability by an additional $2.5 \mu \mathrm{s}$. The equilibrium state was sufficient to confirm that the plasmoid current in an AFRC reaches a steady-state prior to the peak of the coil currents. This implies that the plasmoid will always be driven to the inner wall, unless it translates from the coils prior to peak coil currents. However, ejection of the plasmoid before the peak coil currents results in severe efficiency losses. These results demonstrate the difficulty in designing an AFRC experiment for translation as balancing the different requirements for stability, balance, and efficient translation can have competing consequences. 



\section{Chapter 1}

\section{Introduction}

\subsection{Background}

Electric space propulsion provides a fuel-efficient way to move objects around in space, at the expense of inherently low thrust levels [4]. A branch of electric propulsion technology referred to as pulsed inductive plasmoid thrusters (PIPT) promises higher thrust levels than current technology with little change in efficiency. This class of thrusters form dense plasmoids using magnetic induction and accelerate them to a high velocity using electromagnetic forces. Plasmoids are magnetized structures of plasma, either in a toroid or sheet form. Pulsed inductive plasmoid thrusters can be throttled for flexibility, are compatible with a wide variety of propellants, and can be readily scaled to match power demands.

Several types of pulsed inductive plasmoid thrusters have been investigated over the years [5], [6], [7], [8], [9], [3]. These thrusters are at various stage of maturity, but have all 
shown that a plasmoid can be ejected with significant velocity. Most of the thrusters have a unique drawback, however. In some [8], the plasma initially touches the wall leading to large energy losses [10]. In others [5], [6], the plasmoid can eject before being fully formed [4] requiring large amounts of pulsed power for efficient operation [5]. While continued engineering may minimize the impacts of these losses, it is worthwhile to investigate a new thruster concept which promises to overcome some of the more traditional losses associated with other thrusters. Annular field reversed configurations (AFRCs) are one such concept.

An annular field reversed configuration is a plasma toroid formed in the annular region between two coaxial coils, as shown in Figure 1.1. The coils induce a toroidal (azimuthal) diamagnetic current in the plasma, setting up a closed poloidal B-field. The closed B-field confines the plasma and allows it to be accelerated as a whole from its formation chamber.

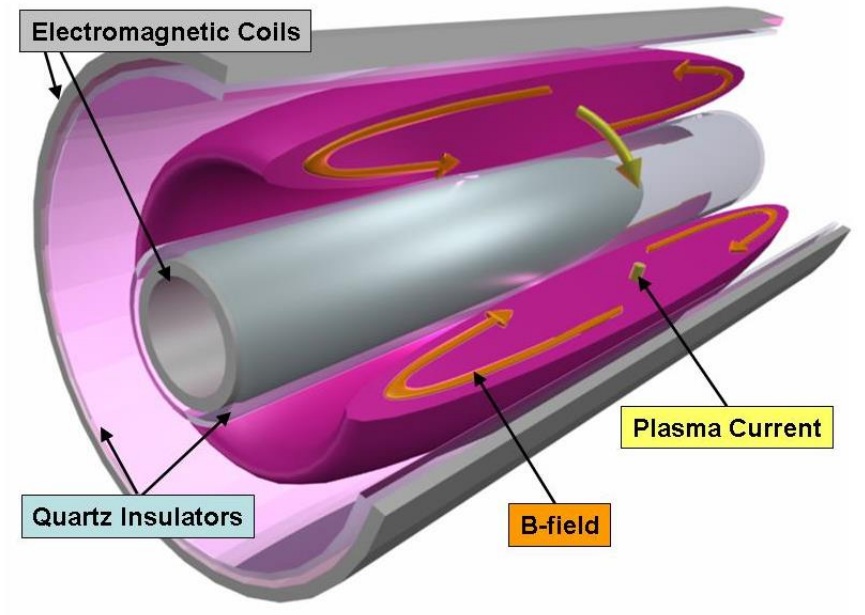

Figure 1.1: Annular field reversed configuration schematic. The poloidal B-field is supported by a toroidal (azimuthal) plasma current.

Annular field reversed configurations have been researched in the fusion community in several studies [11], [12], [13], [14], [10], [15], [16]. The formation of AFRCs has been well-documented using high densities in various configurations, on various timescales, and 
with high energy levels. The research has shown that magnetically detached, long-lived, and stable configurations can be formed using an annular source. The plasmoid forms off both walls, does not require the fast switching requirements of similar sources, and allows the plasmoid to form at low voltages (less than $1 \mathrm{kV}$ ). The presence of the inner coil allows magnetic flux to be continuously added to the plasmoid after formation to aid in current sustainment and to improve the lifetime of the AFRC plasmoid. The loss of magnetic flux in similar sources leads to a diminished lifetime [17]. Additionally, the plasmoid forms in a cylindrical geometry rather than with a planar coil suggesting that the plasmoid will only emerge from the coils after it is fully formed.

The AFRC formation studies suggest that an AFRC plasmoid source would be suitable for space propulsion technology, yet AFRC plasmoid translation (acceleration) has never been thoroughly investigated. An early fusion experiment by Alideres, et al [18] used an annular plasmoid source with permanent magnets to eject a deuterium plasmoid, showing that translation of AFRCs is possible. This design is not appealing for a space propulsion thruster due to its heavy mass. Furthermore, data published on this experiment suggests poor propellant utilization efficiency and no information is provided about the momentum and energy properties of the exhausting propellant. Both of these are important considerations for a spacecraft thruster. This single experiment is the only evidence of plasmoid motion in AFRC devices. Before AFRCs can be developed for electric propulsion, plasmoid translation must be studied in a suitable framework for space propulsion starting with a basic demonstration of plasmoid acceleration. 


\subsection{Aim and Scope}

The objective of this research was to demonstrate AFRC plasmoid translation using an experimental prototype, with design characteristics suitable for spacecraft propulsion. Rather than build a complete thruster hardware package, the experiment was built around the architecture of a spacecraft experiment using lightweight coils, long (20 $\mu \mathrm{s})$ timescales, heavy propellant, and plasmoid expansion into a field free region. If plasmoid translation was successful, the plasmoid's velocity and impulse were measured and the system's energy efficiency was estimated. If the plasmoid failed to translate, reasons for the failure were investigated.

While past studies on AFRCs and FRCs were instrumental to the design of this experiment, the design approach, constraints, and objectives for the prototype experiment differ from past AFRC work in three major ways. The first difference was that the outer coil was conical rather than using a cylindrical design with permanent magnets to accelerate the plasmoid. The second difference is the energy and density requirements and design of the experiment was based on the need to eject the plasmoid during its lifetime, compared with heating the gas to fusion conditions. The final difference is that this experiment intends to measure the directed momentum and energy efficiency of the accelerated plasmoid which has not been addressed in previous research.

The translation of an AFRC plasmoid in a fusion study [18] was achieved with a cylindrical geometry using permanent magnets to accelerate the plasmoid. Permanent magnets add significant mass to a propulsion system, limiting its attractiveness. Conical coils have been shown to accelerate a plasmoid in previous FRC literature [17] and these add no additional 
mass to the system. Other PIPTs have successfully used them to accelerate a plasmoid as well [8], [9], [3]. For AFRCs to be an attractive space propulsion technology, the translation of the plasmoid must be accomplished using a conical coil.

Past research on AFRCs has focused on using energy levels and gas densities suitable for fusion studies. While this information is not published in some sources, the energy levels that are published are nominally over $10 \mathrm{~kJ}[11]$, [13], [14], [10], [15]. Neutral fill densities are generally in excess of 50 mTorr, though formation studies are available for lower densities from 10-50 mTorr [10], [15]. These energy budgets and densities are sized to heat a gas for fusion reactions and are generally too high for a spacecraft thruster design. In a thruster, the energy and density are scaled to match the energy and plasmoid mass required to form and expel the plasmoid during a given time period. Therefore, it is important to design a propulsion experiment with these considerations in mind.

The majority of previous research on AFRCs has focused on stationary plasmoid experiments, where the plasmoid was not expelled from the coils. One AFRC study [18] accelerated the plasmoid from the coils deliberately. While exit velocities of $100-200$ $\mathrm{km} / \mathrm{s}$ were recorded by downstream diagnostics, no data were available for the directed momentum of the exhausting gas. This is one of the most important characteristics for a spacecraft thruster, along with the system efficiency for converting the gas into directed kinetic energy. As such, this research seeks to measure not only the directed velocity of the accelerated plasma, but the impulse delivered by the gas and energy conversion efficiency the AFRC prototype experiment attains.

This research is a critical step in directly evaluating AFRC thrusters for space propulsion. If translation of the plasmoid can be demonstrated, the findings of this research can help build a more realistic experiment for evaluating AFRC thruster technology. If translation of 
the plasmoid cannot be detected, the results will highlight some of the technical challenges in using AFRC devices for space propulsion.

\subsection{Structure of Dissertation}

This dissertation explores AFRCs as candidates for electric propulsion using an experimental demonstration of plasmoid translation. The background information in Chapter 2 provides an introduction to plasmoid propulsion as well as a review of other PIPT devices. Chapter 3 discusses AFRC formation physics and presents a review of AFRC formation studies. An engineering dynamic circuit model was developed to model basic plasmoid translation behavior and to aid in experimental design. This model and results from it are available in Chapter 4. The experimental setup constructed to test AFRC translation is presented in Chapter 5. Diagnostic tools used in measuring the plasmoid's behavior are also included. The data collected from the translation experiment are presented in Chapter 6, with full results following in Chapter 7. The translation study discovered the plasmoid did not escape from the coils and suffered a shorter than expected lifetime. An instability was the most likely reason for the abbreviated lifetime and a global instability study was conducted. The results from this study in Chapter 8 uncovered a radial motion of the plasmoid toward the inner wall. The nature of the radial imbalance is discussed in Chapter 9 , along with results from a study correcting for the radial imbalance in this experiment. The impact of these corrections on future experiments is also discussed. Conclusions are presented in Chapter 10, along with a discussion how the results from this experiment impact future studies on AFRCs for propulsion. 


\section{Chapter 2}

\section{Overview of Plasmoid Propulsion}

Annular field reversed configuration thrusters are a high power electric propulsion technology, belonging to the class of pulsed inductive plasmoid thrusters (PIPT). Plasmoid thrusters are inherently pulsed propulsion devices, where single plasmoids are formed using an electromagnetic coil and ejected with a Lorentz force. A stream of plasmoids can be ejected for continuous operation. Principles of pulsed propulsion are discussed in Section 2.1. An overview of plasmoid propulsion follows in Section 2.2. A comprehensive history of past and present plasmoid propulsion research is presented in Section 2.3 .

\subsection{Principles of Pulsed Propulsion}

Pulsed thrusters expel discrete slugs of propellant to create thrust, as opposed to steady state thrusters which stream mass continuously. The repetition rate can be varied to match the available power or desired thrust. This provides mission flexibility that steady-state devices 
cannot achieve. A continuously pulsed thruster is the best candidate for testing the full capability of many PIPT devices, however single-pulse behavior can be used as an initial baseline for performance assessments. Performance measurements in a single-pulse system include impulse-bit and energy efficiency of the plasmoid acceleration. Previously, these have been defined for a single-pulse thruster assuming little divergence of the exhausting plume and full utilization of the working gas [6]. An alternate derivation is presented here to account for these non-ideal effects in a manner similar to previous efficiency architecture for steady-state EP thrusters [19].

The impulse bit $I_{b i t}$ is the total momentum transfer per pulse the exhausting propellant delivers to a spacecraft parallel to its direction of travel. It includes the mass $m_{j}$ of all species, the average exhaust velocity of each species $u_{j}$, and the off-axis divergence of each species $\theta_{j}$. All of these quantities can vary with radial position $r$, leading to:

$$
I_{b i t}=\sum_{j=0}^{n} \iint m_{j}(r) \bar{u}_{j}(r) \cos \left(\theta_{j}(r)\right) r d \theta d r
$$

Mathematically, $I_{b i t}$ can be re-written as a product of the total mass of the propellant or mass-bit $m_{b i t}$ and weighted averages. The weighted averages are defined as a mass-weighted average velocity $\langle u\rangle_{m}$ and momentum-weighted divergence relation $\langle\cos \theta\rangle_{m v}$, where:

$$
\begin{gathered}
m_{b i t}=\sum_{j=0}^{n} \iint m_{j}(r) r d \theta d r \\
\langle u\rangle_{m}=\frac{\sum_{j=0}^{n} \iint m_{j}(r) u_{j}(r) r d \theta d r}{\sum_{j=0}^{n} \iint m_{j}(r) r d \theta d r}=\frac{\sum_{j=0}^{n} \iint m_{j}(r) u_{j}(r) r d \theta d r}{m_{b i t}}
\end{gathered}
$$




$$
\begin{aligned}
\langle\cos \theta\rangle_{m v} & =\frac{\sum_{j=0}^{n} \iint m_{j}(r) u_{j}(r) \cos \left(\theta_{j}(r)\right) r d \theta d r}{\sum_{j=0}^{n} \iint m_{j}(r) u_{j}(r) r d \theta d r} \\
& =\frac{\sum_{j=0}^{n} \iint m_{j}(r) u_{j}(r) \cos \left(\theta_{j}(r)\right) r d \theta d r}{\langle u\rangle_{m} m_{b i t}}
\end{aligned}
$$

so that

$$
I_{b i t}=m_{b i t}\langle u\rangle_{m}\langle\cos \theta\rangle_{m v}
$$

The thrust is the time rate of change of the total impulse in the direction of travel, $\hat{z}$ :

$$
\mathbf{T}=\frac{d I_{b i t}}{d t} \hat{z}
$$

Specific impulse $I_{s p}$ is defined as the thrust compared to the rate of propellant use at sea level [4]. In single-pulse thrusters, this equates to the impulse by:

$$
I_{s p}=\frac{I_{b i t}}{m_{b i t} g}
$$

The total system efficiency of a single-pulse thruster is a function of $I_{b i t}$ delivered by the propellant, the mass of the propellant $m_{b i t}$, and the initial energy $E_{0}$ required to operate the thruster, related by:

$$
\eta=\frac{I_{b i t}^{2}}{2 m_{b i t} E_{0}}
$$

The total efficiency $\eta$ can be distributed into various terms including the energy efficiency $\eta_{E}$, propellant efficiency $\eta_{P}$, and beam efficiency $\eta_{B}$. These terms can be defined by replacing $I_{b i t}$ in Equation 2.9 with Equation 2.5 and re-arranging terms:

$$
\eta=\frac{\frac{1}{2} m_{b i t}\langle u\rangle_{m}^{2}\langle\cos \theta\rangle_{m v}^{2}}{E_{0}}=\frac{\frac{1}{2}\left\langle m_{b i t} u^{2}\right\rangle_{m}}{E_{0}} \frac{\langle u\rangle_{m}^{2}}{\left\langle u^{2}\right\rangle_{m}}\langle\cos \theta\rangle_{m v}^{2}=\eta_{E} \eta_{P} \eta_{B}
$$


This research focuses on estimating the energy efficiency $\eta_{E}$ of the plasmoid's acceleration using plume measurements rather than measuring the complete system efficiency $\eta$. Total system efficiency measurements require direct impulse measurements using an isolated thruster fixed to a thrust balance inside a vacuum tank. The engineering challenges associated with developing a vacuum-compatible AFRC thruster and fast-response thrust balance are beyond the scope of this basic investigative research. Energy efficiency measurements in the plume provide an upper bound to system efficiency, when $\eta_{P}, \eta_{B}$ $=1$.

Propellant efficiency $\eta_{P}$, though not an investigative topic of this research, is an important consideration in pulsed gaseous thrusters because it includes the mass utilization of the propellant [19]. Mass utilization is the ratio of ionized propellant to injected propellant. In a pulsed gaseous thruster, propellant can stream out of the device while the coils are not energized between pulses. Unionized propellant can also escape on the leading and trailing edges of each pulse. If a large fraction of the propellant leaves the thruster without being ionized, it cannot be accelerated by the coils and presents a large loss to the system. For this reason, the system efficiency of any gaseous pulsed thruster must take into account the total propellant efficiency.

Impulse, thrust, and efficiency can be extrapolated to multi-pulse operation, if it is assumed that the pulses do not appreciably interfere and if the plasma slug maintains a coherent, mass conserving structure. Single pulse operation only provides a baseline assessment; characterization with multi-pulse operation is required to understand the full performance. 


\subsection{Plasmoid Propulsion}

Pulsed inductive plasmoid thrusters form magnetized plasma toroids by inductively driving large diamagnetic currents in a conductive plasma from an external coil. The magnetic field structure arising from the current induction keeps the plasma fully contained and detached from the coil's magnetic field. This means the entire plasmoid can be ejected (translated) from the formation coil as a complete entity using a Lorentz force $(\mathbf{J} \mathbf{X} \mathbf{B})$. The family of pulsed inductive plasmoid thrusters includes a diverse group of plasma sources; a few are shown in Figure 2.1. A brief description of this group is given in the text by Jahn [4].

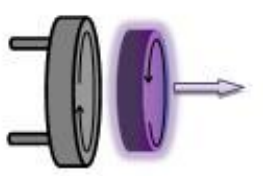

(a)

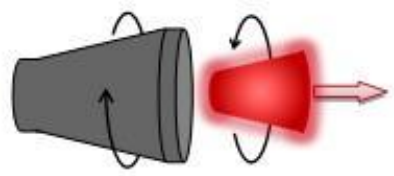

(b)

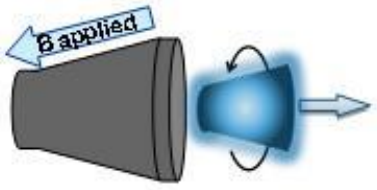

(c)

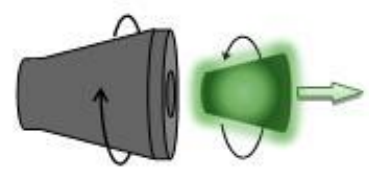

(d)

Figure 2.1: Family of pulsed indutive plasmoid thrusters, including (a) planar thruster, (b) conical $\theta$-pinch FRC thruster, (c) RMF-FRC thruster, (d) AFRC thruster.

Plasmoid thrusters can be constructed in either a planar or conical geometry. Translation in a planar device is a result of the azimuthal (toroidal) plasma current generated in the plasma interacting with the component of radial magnetic field from the flat coil. In a conical geometry, plasma acceleration is initiated by using a conical outer coil to create a component of radial magnetic field to interact with the azimuthal plasma current. Plasmoid acceleration can be accomplished using other methods such as impurity injection [20], 
magnetic kicker coils to introduce an axial instability, or permanent magnets to create a radial field component [18]. The conical outer coil is generally the easiest way to achieve translation and it provides considerable mass savings for propulsion systems.

Pulsed inductive plasmoid thrusters are attractive for high power spacecraft propulsion for several reasons. They create a high energy density plasmoid which can be accelerated to high velocities. Their pulsed nature gives them throttling capability, allowing them to cover a wide range of missions and power levels. By adjusting the pulse rate at a fixed energy per pulse, they can match the desired thrust with little change in efficiency and specific impulse. They operate on fast timescales, keeping non-conservative energy losses to a minimum. The inductive plasmoid formation increases the lifetime of the device, as it keeps hot plasma away from the walls, reducing erosion. Additionally, the inductive formation allows for the use of any variety of propellants since material compatibility issues are minimized.

The formation physics of the plasmoid is device dependant, though in each case the plasma currents are generated inductively as a result of Faraday's Law. Some devices choose to start with a seed plasma, which is created in the pre-ionization (PI) stage. This has been found in some devices to be critical to the formation [17], [21]. The level of initial ionization varies as does the method for pre-ionization; pre-ionization remains an empirical, device-dependant process. 


\subsection{Plasmoid Thruster Technology}

Pulsed inductive plasmoid thrusters can be crafted from closed-field devices or from planar current-sheet devices. Closed-field devices are generally referred to as compact toroids and include the workhorses of fusion research: the Field Reversed Configuration (FRC) [17], [21] and the Spheromak [22]. Several technologies have already been demonstrated as propulsion devices, with impulse measurements on single or multi-pulse prototypes. Others have only measured the velocity of the propellant downstream of the formation coil. Several more are currently in development. A summary of all known PIPT devices and studies is shown in Table 2.1.

The most notable pulsed inductive plasmoid device is the PIT, or pulsed inductive thruster. A comprehensive review of this program and its variants is available in a review paper by Polzin [6]. The PIT was developed in the 1960's by TRW Space Systems. Early work on basic physics in inductive plasma devices culminated in the early 1990's development of the PIT-MkVa propulsion system, currently the best performing thruster in PIPT [5]. The PIT-MkVa used a flat 1-m diameter coil, backed by $4.6 \mathrm{~kJ} / \mathrm{pulse}, 1 \mu$ s rise-time capacitor bank to ionize and accelerate a sheet of propellant. Single pulse testing with ammonia at maximum energy was reported for mass bits between $3.1 \mathrm{mg}$ to $0.69 \mathrm{mg}$. Impulse-bit measurements were $121 \mathrm{mN}-\mathrm{s}$ to $56 \mathrm{mN}-\mathrm{s}$, resulting in specific impulses between 4000-9000 s. System efficiencies were constant across this range at 50\%, deviating by $5 \%$. Testing at lower energies resulted in lower $\eta$ and lower $I_{s p}$. Results were also shown for simulated hydrazine, but efficiencies were reduced from the ammonia tests. The high pulse energies used in the PIT-MkVa were required for high efficiency. Lower energy 
Table 2.1

Pulsed inductive plasmoid thrusters. Asterisks denote projected numbers.

\begin{tabular}{|c|c|c|c|c|c|}
\hline Experiment & Type & $\mathbf{E}_{\text {pulse }}$ & Fill Gas & Characteristic & Refs \\
\hline $\begin{array}{l}\text { PTX } \\
(2003-2007)\end{array}$ & $\begin{array}{c}\text { Conical } \\
\text { Theta-Pinch }\end{array}$ & $379 \mathrm{~J}$ & $\begin{array}{c}\mathrm{H}_{2} \\
\mathrm{CH}_{4} \\
\mathrm{Ar}\end{array}$ & $\begin{array}{l}u_{e}: 23-27 \mathrm{~km} / \mathrm{s} \\
u_{e}: 16-18 \mathrm{~km} / \mathrm{s} \\
u_{e}: 13-19 \mathrm{~km} / \mathrm{s}\end{array}$ & [8] \\
\hline $\begin{array}{l}\text { PT-1 } \\
(2007-)\end{array}$ & $\begin{array}{c}\text { Conical } \\
\text { Theta-Pinch }\end{array}$ & $280 \mathrm{~J}^{*}$ & $\mathrm{n} / \mathrm{a}$ & $\begin{array}{c}\mathrm{T}: 200 \mathrm{mN}^{*} \\
\text { at } 30 \mathrm{~Hz}^{*}\end{array}$ & [23] \\
\hline $\begin{array}{l}\text { ELF-v1 } \\
(2008-2010)\end{array}$ & $\begin{array}{c}\text { Rotating } \\
\text { Magnetic Field }\end{array}$ & $50 \mathrm{~J}$ & $\begin{array}{l}\text { Air } \\
\mathrm{N}_{2} \\
\mathrm{O}_{2}\end{array}$ & $\begin{array}{c}I_{b i t}: 0.3 \mathrm{mN}-\mathrm{s} \\
I_{b i t}: 0.27 \mathrm{mN}-\mathrm{s} \\
I_{b i t}: 0.25 \mathrm{mN}-\mathrm{s}\end{array}$ & $\begin{array}{l}{[24} \\
25\end{array}$ \\
\hline $\begin{array}{l}\text { ELF-v2 } \\
(2012)\end{array}$ & $\begin{array}{c}\text { Rotating } \\
\text { Magnetic Field }\end{array}$ & $10-20 \mathrm{~J}$ & $\mathrm{CO}_{2}$ & $\begin{array}{l}u_{e}: 19 \mathrm{~km} / \mathrm{s} \\
u_{e}: 17 \mathrm{~km} / \mathrm{s}\end{array}$ & [9] \\
\hline $\begin{array}{l}\text { EMPT } \\
(2011-)\end{array}$ & $\begin{array}{c}\text { Rotating } \\
\text { Magnetic Field }\end{array}$ & $1.9 \mathrm{~J}$ & $\mathrm{Xe}$ & $\begin{array}{c}I_{b i t}: 0.02 \mathrm{mN}-\mathrm{s} \\
u_{e}: 20 \mathrm{~km} / \mathrm{s}\end{array}$ & [3] \\
\hline $\begin{array}{l}\text { RF-Fringe } \\
\text { Field Thruster } \\
\text { (1965-1968) }\end{array}$ & $\begin{array}{c}\text { Flat Plate } \\
\text { Current Sheet }\end{array}$ & $\begin{array}{l}41 \mathrm{~mJ} \\
23 \mathrm{~mJ}\end{array}$ & $\begin{array}{l}\mathrm{Hg} \\
\mathrm{Hg}\end{array}$ & $\begin{array}{c}I_{b i t}: 3.1 \mu \mathrm{N}-\mathrm{s} \\
\eta: 70 \% \\
I_{b i t}: 0.6 \mu \mathrm{N}-\mathrm{s} \\
\eta: 12 \%\end{array}$ & $\begin{array}{l}{[7]} \\
{[26} \\
{[27}\end{array}$ \\
\hline $\begin{array}{l}\text { PIT } \\
(1965-2005)\end{array}$ & $\begin{array}{c}\text { Flat Plate } \\
\text { Current Sheet }\end{array}$ & $4608 \mathrm{~J}$ & $\mathrm{NH}_{3}$ & $\begin{array}{c}m_{b i t}: 3.1 \mathrm{mg} \\
I_{b i t}: 121 \mathrm{mN}-\mathrm{s} \\
\eta: 50 \% \pm 5 \%\end{array}$ & [5] \\
\hline $\begin{array}{l}\text { FARAD } \\
(2004-)\end{array}$ & $\begin{array}{c}\text { Flat Plate } \\
\text { Current Sheet }\end{array}$ & $78.5 \mathrm{~J}$ & $\mathrm{Ar}$ & $u_{e}: 12 \mathrm{~km} / \mathrm{s}$ & [28] \\
\hline $\begin{array}{l}\mu \text {-FARAD } \\
(2008-2012)\end{array}$ & $\begin{array}{c}\text { Flat Plate } \\
\text { Current Sheet }\end{array}$ & $500 \mathrm{~J}$ & Ar & $I_{b i t}: 0.097 \mathrm{mN}-\mathrm{s}$ & 29] \\
\hline
\end{tabular}


testing at $2 \mathrm{~kJ} /$ pulse failed to exceed efficiencies above 30\%. Future generations of the PIT have not been able to match this performance; the PIT-MkVI and the $200 \mathrm{kWe}$ NuPIT suffered from switch failures and work has been discontinued.

The FARAD (Faraday accelerator with radio-frequency assisted discharge) is a device similar to the PIT, developed in 2004 at Princeton University [28]. The FARAD used an inductive RF pre-ionization (PI) source upstream of a $20 \mathrm{~cm}$ diameter flat coil. Using a PI-stage, the FARAD demonstrated that it was possible to create and accelerate a current sheet at $78.5 \mathrm{~J} /$ pulse. The resulting velocity using a 23 mTorr argon fill was $12 \mathrm{~km} / \mathrm{s}$.

After the original FARAD, four additional devices were created. The Conical Theta Pinch FARAD (CTP-FARAD) used a conical coil instead of the flat coil to accelerate the plasmoid [30]. Another used a pulsed PI and gas injection [31]. Princeton University is developing a single-stage FARAD (SS-FARAD) which combines an RF pre-ionization circuit with the main discharge circuit for a single-coil design [32]. Work on the SS-FARAD is currently in progress. A steady-state microwave PI source FARAD ( $\mu$-FARAD) [33], [29] was recently developed at NASA-Marshall. A conical discharge coil was substituted for the planar version to provide mass containment of the streaming propellant. The microwave-PI source was later discarded for a DC glow breakdown. Impulse measurements on the conical $\mu$-FARAD show impulse measurements of $0.097 \mathrm{mN}-\mathrm{s}$ at with input energy of $500 \mathrm{~J}$ and mass flow rate of $120 \mathrm{mg} / \mathrm{s}$. The poor performance on the $\mu$-FARAD was attributed to incomplete current sheet breakdown along the discharge coil.

Concurrent with the early years of PIT development, a similar device was also tested. The RF-Fringe Field Thruster used a set of small diameter $(15 \mathrm{~cm})$ coaxial flat coils to ionize and accelerate a mercury-vapor current sheet [7], [26], [27]. Permanent magnets behind the coils increased the radial magnetic field in front of the coils. The coils were powered by an 
external current supply at $240 \mathrm{kHz}$, with plasmoids ejected every half cycle. High power testing was conducted with water-cooled coils at $30 \mathrm{~kW}$ steady state and up to $134 \mathrm{~kW}$ in burst mode [7]. Thrust measurements of 1.5-2 $\mathrm{N}$ were recorded with a specific impulse of 2000-2500 seconds and an efficiency of 70\%. This corresponds to an impulse-bit of 3.1 $\mu \mathrm{N}-\mathrm{s}$ at $41 \mathrm{~mJ} /$ pulse. This performance could only be attained with water cooling through the coil. Low energy testing on a radiation cooled design at $10 \mathrm{~kW}$ produced thrust levels of $0.3 \mathrm{~N}$, with a specific impulse of 900 seconds and an efficiency of $12 \%$ [26], [27]. This is equivalent to a $0.6 \mu \mathrm{N}$-s impulse-bit at $23 \mathrm{~mJ} /$ pulse. It was suggested that these low ratings were due to insufficient ionization and poor mass utilization; the thruster accelerated only the ion mass fraction rather than the bulk propellant [7].

The PIT, FARAD, and RF-Fringe Field Thruster are current sheet accelerators. Breakdown and acceleration must happen very quickly in a planar geometry, as the plasma begins to push away from the coil as soon as it is formed. Without a pre-ionization, current sheet devices require high voltages to breakdown and accelerate the propellant quickly. A coil voltage on the PIT-MkVa of $32 \mathrm{kV}$ was required for maximum performance. Additionally, the current sheet must be impermeable to the neutral gas so that as it accelerates, it can sweep up the surrounding neutrals for higher mass utilization [6]. The high degree of ionization required implies that efficient, low-power and low-voltage operation is not feasible for planar devices. This has been demonstrated in both the PIT development and in the RF-Fringe Field thruster.

The closed-field PIPT devices allow the plasma to remain in contact with the coils for a longer duration, reducing some of the strain on high voltage and energy requirements. The closed-field structure is ideal for propulsion because the entire plasmoid mass can be ejected as a bulk and can interact magnetically with the coils. A comprehensive review of 
FRC technology for fusion research is available in the 2011 review paper by Steinhauer [21] and in the 1988 paper by Tuszewski [17]. Three formation techniques for FRCs are listed which would be compatible for propulsion: (1) $\theta$-pinch formation, (2) rotating magnetic field (RMF) formation, and (3) annular (coaxial) source formation. Propulsion concepts using each formation method have been tested or are currently under development.

The Plasmoid Thruster Experiment (PTX) was investigated at NASA-Marshall in the mid-2000s and used a $\theta$-pinch coil to form and accelerate a small $(1 \mathrm{~cm})$ plasmoid [34], [8]. The plasmoid's velocity was measured using an interferometeric time-of-flight array [8]. At coil energies of $9 \mathrm{~J}$ and coil voltages of $5.5 \mathrm{kV}$, plasmoids were ejected at 25 $\mathrm{km} / \mathrm{s}$ using hydrogen, $17 \mathrm{~km} / \mathrm{s}$ using methane, and $16 \mathrm{~km} / \mathrm{s}$ with argon. Significant circuit losses dissipated the remaining pulse energy of $370 \mathrm{~J}$. Performance measurements (impulse, efficiency) were speculated for this device at $1-100 \mathrm{mN}$ with 0.1 to $49 \%$ of thrust efficiency, but never directly measured. Thrust efficiency is defined as the ratio of final kinetic energy compared to coil energy. System efficiencies are much lower. The next-generation PTX (PT-1) was designed and constructed in 2007 to operate as a multi-pulse fully integrated thruster, but funding was not provided for full testing [23].

Rotating magnetic fields are an alternative formation scheme for propulsion FRCs. With $\mathrm{RMF}$, electron current is driven by radio-frequency waves, keeping the instantaneous current and voltages low enough for use with solid-state circuitry. The Electrodeless Lorentz Force (ELF) thruster was developed by MSNW in 2008 [24]. The ELF was a $50 \mathrm{~J} /$ pulse devices, $420 \mathrm{~mm}$ in length with an 8 degree cone and $26 \mathrm{~cm}$ exit diameter. Nominal circuit voltages were $300 \mathrm{~V}$, orders of magnitude below $\theta$-pinch FRCs. The first-generation ELF (ELF-v1) demonstrated single-pulse impulse measurements of $0.3 \mathrm{mN}-\mathrm{s}$ at an estimated $30 \%$ efficiency on air [25]. The corresponding plasmoid velocity 
was $15 \mathrm{~km} / \mathrm{s}$. Nitrogen and oxygen were also tested. A coherent plasmoid was measured up to $1 \mathrm{~m}$ downstream. Data in the formation region demonstrated that the formation and acceleration occur simultaneously.

Steady state operation of the next generation ELF (ELF-v2) was demonstrated in 2012 [9]. These experiments showcased steady-state operation of an FRC-thruster on Xenon and complex propellants, including water and Martian air $\left(95 \% \mathrm{CO}_{2}, 3 \% \mathrm{~N}_{2}, 2 \% \mathrm{Ar}\right)$. The second generation ELF was slightly smaller than the original ELF, with a reduced length of $200 \mathrm{~mm}$, cone angle of 12 degrees, and exit diameter of $20 \mathrm{~cm}$. Bus voltages of $300 \mathrm{~V}$ were used to drive the discharge capacitors at $2800 \mathrm{~V}$ using an MSNW-crafted power processing unit. Steady-state operation (9 or more pulses) with Martian air demonstrated average plasmoid velocities of $19 \mathrm{~km} / \mathrm{s}$. These numbers were based on a $4.8 \mathrm{~kW}$ power load, with a mass flow of $250 \mathrm{sccm}$, and a $1500 \mathrm{~Hz}$ repetition rate. Increasing the power to $5.8 \mathrm{~kW}$ and the flow rate to $500 \mathrm{sccm}$ dropped the plasmoid velocity to $17 \mathrm{~km} / \mathrm{s}$. Steady-state operation on water demonstrated that two populations emerged from the device. A fast population was seen, traveling in excess of $50 \mathrm{~km} / \mathrm{s}$ followed by a slower $17 \mathrm{~km} / \mathrm{s}$ population. The dual population was likely due to separation of the oxygen and hydrogen components, with the hydrogen components leaving at much higher velocities. The discrepancy in velocity made estimation of average plasmoid velocity using water propellant difficult. While the ELF-v2 was a $30 \mathrm{~kW}$-nominal thruster, testing at power levels exceeding $5.8 \mathrm{~kW}$ was not feasible at MSNW due to chamber and electronics limitations.

A small-scale RMF device has also been developed by MSNW for space propulsion [3]. The Electromagnetic Plasmoid Thruster (EMPT) is a nominal $1 \mathrm{~J} /$ pulse, $1 \mathrm{~kW}$ thruster, with a footprint of $44 \mathrm{~mm}$ in diameter by $100 \mathrm{~mm}$ in length. Preliminary testing with xenon demonstrated that 400 FRCs could be formed and ejected sequentially using a bus 
voltage of $250 \mathrm{~V}$ and repetition rate of $2800 \mathrm{~Hz}$. Average exit velocites under steady state operation of $20 \mathrm{~km} / \mathrm{s}$ were recorded at 1.9-2.1 J/pulse, corresponding to $5 \mathrm{~kW}$ of input power. Impulse bits of $0.02 \mathrm{mN}$-s were also measured for single-pulse operation with exit velocities of 10-40 km/s. Accurate impulse measurements under steady-state operation were not possible due to interaction of the ballistic pendulum with background gases, imperfect reflections, and other complications. Steady state operation of the EMPT highlighted several important considerations for FRC-based PIPTs. The EMPT tests found that with a high enough repetition rate $(2800 \mathrm{~Hz})$ only a single PI discharge was required at the beginning of the pulse train. The seed plasma remaining in the channel after the previous plasmoid departed was enough to initiate the next RMF-FRC. This required matching the repetition rate for the thruster with the neutral refill time. If additional gas was not added in time, there was not enough propellant left behind to form a second FRC. This indicates a high level of propellant utilization. Additionally, some conditions resulted in FRC formation on the first, third, and fourth pulses, but not on the second pulse. The steady state operation also found that the first 2 FRCs created were different in behavior from the remaining pulses. The first FRC appeared much faster and denser than the second FRC, as indicated by an order of magnitude decrease in signal on downstream probes. Steady state behavior of the thruster didn't appear until the third pulse. These findings underscore the importance of bringing thruster concepts past the single-pulse operation for full performance mapping.

The Star Thrust Experiment (STX) is often mistakenly considered to be another RMF thruster. The STX was a multi-megawatt concept was proposed in the late 1990's [35]. The idea behind the STX was to use an RMF-FRC stage to heat propellant so that it could be expelled from a gas nozzle at high velocity. The plasmoid itself was never intended to be accelerated from the chamber, making it an electrothermal thruster rather than an 
electromagnetic thruster. Thruster testing was never published, though formation results of the RMF stage are available [36].

The final type of closed field thruster is the annular FRC (AFRC). The annular geometry is intended to extend the formation timescales from less than $10 \mu \mathrm{s}$ to over $100 \mu \mathrm{s}$ and eliminates the need for high voltage technology. The annular geometry prevents the plasma from coming into contact with the walls at all times 1 , which can lead to severe energy losses early in the formation. This is the only FRC device which has not been demonstrated for propulsion. As discussed in Chapter 3, numerous formation studies have shown that stable AFRC can be produced on slow timescales (10's to 100's of $\mu \mathrm{s}$ ) suitable for ejecting a plasmoid at low energy as well as fast (less than $10 \mu \mathrm{s}$ ) timescales. A translation study in 1967 [18] demonstrated that a magnetized current sheet can be ejected at high velocity $(200 \mathrm{~km} / \mathrm{s}$ ) from an AFRC geometry using light gases, but that remains the only evidence of AFRC translation in literature.

Two design concepts have been proposed to mate acceleration attachments to closed field sources. Their purpose is to use a separate acceleration stage to boost the exit velocity of the plasmoid and to increase device efficiency. The Propagating Magnetic Wave Accelerator [37] proposed in 2000 projected a boost of efficiency from $50 \%$ to $90 \%$ at megawatt power levels. A prototype PMWAC was built, but the device was never tested with a plasma source. The Magnetically Accelerated Plasmoid (MAP) experiments were also intended for use with an additional acceleration stage [38], [39], [40]. Plasma tests using a $\theta$-pinch source demonstrated exit velocities of $180 \mathrm{~km} / \mathrm{s}$ at megawatt power levels.

\footnotetext{
${ }^{1}$ In the traditional theta-pinch FRC formation, the plasma comes in contact with the wall during the early stages before reversal.
} 


\subsection{Summary}

Pulsed inductive plasmoid thrusters form magnetized plasma toroids by inductively driving large diamagnetic currents in a conductive plasma from an external coil. The currents in the plasma react with the external coil magnetic field to drive the plasmoid out of the coils under a directed Lorentz force. PIPTs offer many benefits to electric propulsion including high thrust levels, mission flexibility, limited erosion, and a wide acceptance of various propellants.

The PIPT family represents a diverse group of thruster concepts, in various stages of development. Planar concepts include the PIT, the FARAD family, and the RF Fringe Field thruster. The PIT and FARAD demonstrated single pulse performance, while the RF Fringe Field thruster was operated at steady state. The PIT required massive amounts of energy $(4.6 \mathrm{~kJ} /$ pulse) for peak performance while the FARAD showed it was possible to drop the energy to under $100 \mathrm{~J} /$ pulse with the addition of a pre-ionization stage. Closed field concepts have also been tested including two version of FRC-based thrusters, conical $\theta$-pinch FRCs and RMF-FRCs. A conical $\theta$-pinch thruster was developed and tested at NASA-Marshall in two variations. The RMF-FRC thruster group, consisting of two scaled versions, is currently under development by a small company. The first thruster, the ELF, is a $30 \mathrm{~kW}$ thruster which has been operated on a wide variety of propellants. The second thruster, the EMPT, has shown that coherant FRCs can be formed for as little as $1 \mathrm{~J} /$ pulse. The RMF-FRC thrusters have both been operated in steady state and single pulse mode, making them the most developed of all PIPTs.

An additional formation scheme has been demonstrated for FRC-based thrusters, using 
coaxial coils to create a closed-field plasmoid. This formation method is referred to as the Annular Field Reversed Configuration and has received considerable attention in plasmoid formation studies. The formation studies have shown that it is possible to create a stable plasma toroid with a fairly long lifespan using electromagnetic coils without permanent magnets. While an AFRC-based thruster is possible, a thruster concept using AFRC formation techniques has never been developed. This research explores the viability of an AFRC thruster by demonstrating if an AFRC plasmoid can be translated from the coils. If the plasmoid does translate, the plasmoid's velocity, momentum, and acceleration efficiency will be measured for a basic characterization. 


\section{Chapter 3}

\section{Annular Field Reversed Configurations}

This chapter discusses annular field reversed configurations, including a basic description of the AFRC in Section 3.1, descriptions of the different formation methods for AFRCs in Section 3.2, and important AFRC physics in Section 3.3. A literature review on seven past AFRC experiments is presented in Section 3.4. Section 3.5 discusses translation considerations for AFRC devices.

\subsection{AFRC Basics}

AFRCs form an inductive, annular plasma toroid between an inner and outer coil. AFRCs resemble traditional FRCs which are formed without the inner coil [21]. AFRCs and FRCs get their name from the bi-directional magnetic field through their radial cross section; the magnetic field is reversed on the plasmoid's inner radius as compared to the outer radius. The bi-directional field forms a separatrix (magnetic boundary) with a magnetic null at the 
center. The FRC's separatrix radius is $r_{s}$ and separatrix length is $l_{s}$. The AFRC does not have a separatrix radius, rather it has a separatrix width $\Delta r_{m}$, a separatrix length $l_{s}$, and a magnetic null radius $r_{m}$. The anatomy of an AFRC and an FRC are shown in Figure 3.1.

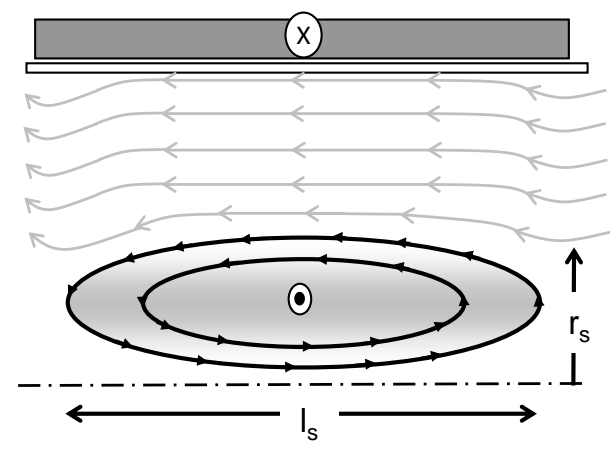

(a)

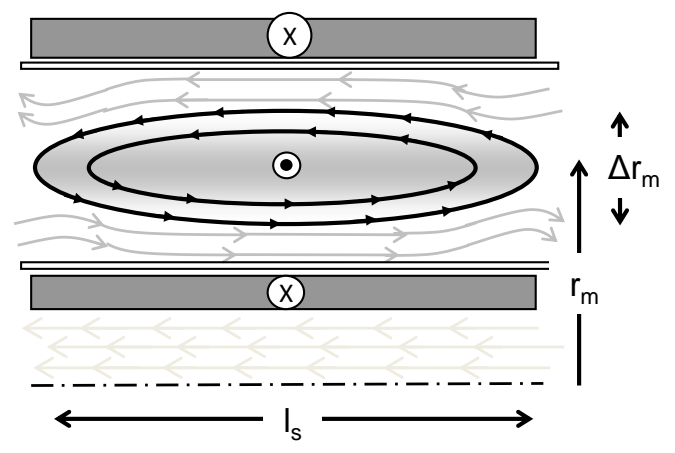

(b)

Figure 3.1: Principle dimensions of an (a) FRC and an (b) AFRC.

In theta-pinch FRCs, the reversed field configuration is formed by the tearing and reconnection of two opposing fields. The formation of an AFRC is different. The reversed field structure is formed by using the inner coil to induce a diamagnetic current in the plasmoid. The diamagnetic current forms a self-closing field structure to contain the plasma. The outer coil holds the plasma in balance with the inner coil to prevent the plasmoid's radial expansion. In this experiment, the outer coil also provides the accelerating Lorentz force by imposing a small radial magnetic field component. The radial magnetic field $\mathbf{B}_{\mathbf{r}}$ interacts with the azimuthal plasma current $\mathbf{J}_{\theta}$ to drive the plasmoid downstream. The axial force balance is shown in Figure 3.2.

Annular FRCs can be formed on fast (less than $10 \mu \mathrm{s}$ ) timescales and on slow (more than $50 \mu \mathrm{s}$ ) timescales. Slow formation is not possible with $\theta$-pinch FRC devices as the plasma comes in contact with the wall during formation and is subject to large energy and magnetic 


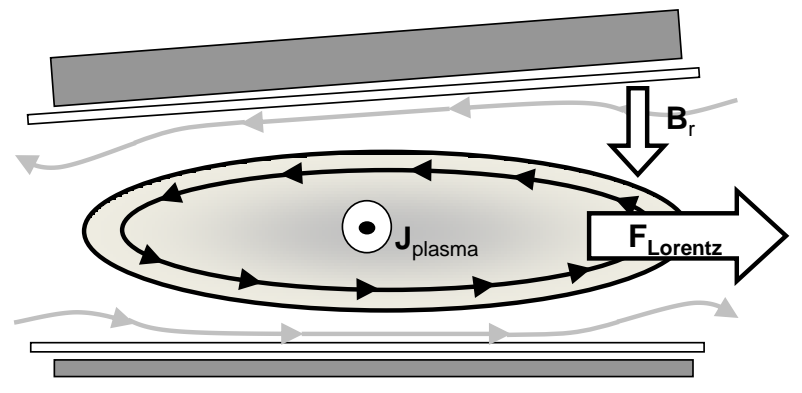

Figure 3.2: Force diagram for an AFRC translating from a conical coil.

flux losses. The reversal in a $\theta$-pinch FRC must be done very quickly (less than $5 \mu$ s) to prevent these energy and flux losses. As is shown in Section 3.2, the AFRC can form away the wall to allow the configuration to build on slower timescales.

Annular FRCs have three stages of life: formation, equilibrium, and termination. The formation stage begins with the start of the inner coil current. The equilibrium stage takes over when the configuration is fully formed, with a bi-directional field in the annulus. Equilibrium is eventually disturbed and the plasmoid configuration terminates and disappears. A brief description of each stage and the important physics is explained in Sections 3.2 and 3.3 .

\subsection{AFRC Formation Techniques}

The formation of an AFRC plasmoid is a complex process. The plasma is subject to drifts, collisions, shocks, instabilities, and rapidly changing magnetic topology. While these effects are essential to the formation, the bulk behavior of the plasma can be simplified by treating the plasmoid as a rigid conductor inductively driven between the coils. Formation 
can then be explained with a magnetic transformer analogy, using a primary circuit and secondary circuit. According to Faraday's Law, magnetic fields created by currents in the coils (primary circuits) induce currents in the plasmoid circuit (secondary circuit). The currents in the plasmoid circuit develop to oppose changing magnetic fields or magnetic flux created by the coil circuits. Magnetic flux $\Phi_{B}$ is defined as the net magnetic field passing through a surface or

$$
\Phi_{B}=\int_{A} \mathrm{~B} \cdot d \mathrm{~A}
$$

An important consequence of Faraday's Law in AFRCs is that the inner coil can actively drive current in the plasmoid, adding magnetic flux to the configuration after the magnetic fields close. This cannot be accomplished in traditional $\theta$-pinch FRC devices.

Annular FRC plasmoids are generally created between coils resembling very long solenoids, where the length of the coil is much greater than the radius of the coil. The magnetic field inside a solenoid is directed along the axis, with the magnetic field constant inside the solenoid and nearly zero outside. In the formation explanations provided here, it is assumed that the aspect ratio of both coils and the plasma currents can be approximated as semi-infinite solenoids. Magnetic fields are also super-imposable, meaning that the field configuration of two coaxial coils is simply the vector sum of their vacuum magnetic fields. The field generated by the plasma adds to this field, creating stronger fields outside the configuration than in the vacuum case.

Four different formation schemes are used to create an AFRC. A brief description of each formation method is provided in the following sections. Each method uses a pre-ionization stage, during which the propellant is partially ionized using any variety of techniques. This allows the propellant to respond to the currents in the coils. Though pre-ionization is a critical part of the discharge [17], it has been investigated for AFRCs elsewhere [41] and 
will not be the subject this work. Also, it should be clarified that formation of AFRCs remains an empirical process of which the physics have not been well-resolved. The plasma is a turbulent fluid, subject to complex magnetohydrodynamic effects requiring numerical plasma studies to resolve. End effects, which complicate this discussion but are very important to the confinement, only receive cursory treatment here.

The formation method for AFRCs varies depending on the coil configuration. The inner and outer coils can be operated in either parallel or independent mode. In parallel mode, the coils are attached to the same capacitor bank so that the voltages on each coil rise and fall at the same frequency. In independent mode, the coils are attached to separate capacitor banks and can be discharged independently from each other. Independent mode allows for the current ratio between the coils to be changed; in parallel, the current distribution is fixed by the circuit inductance and resistance. Additionally, both modes allow for the coils to be

operated in phase (synchronous) or out of phase (asynchronous). The formation scheme used in this research is presented in Section 3.2.1 the remaining formation schemes follow.

\subsubsection{Parallel Coil Mode: Synchronous}

Figure 3.3 illustrates the formation steps, circuit schematic, and current waveforms for the parallel formation with synchronous coils. This mode is referred to in other sources [15] as tearing formation. The formation begins by injecting propellant into the annulus between the coils. The propellant is then pre-ionized using any variety of techniques (Step 1). Following the pre-ionization (PI), the main bank switch is closed, generating large currents in the coils. The currents are in the same direction in both coils so that their magnetic fields are in the same direction. The solenoidal nature of the fields assures that the 
magnetic field in the annulus is a result of the outer coil alone (Step 2). Current sheets are generated in the plasma immediately following application of coil current. These current sheets form close to the inner and outer coils. Each current sheet attempts to keep the magnetic flux $\Phi_{B}$ through its cross section constant. If the current sheets form immediately, they will try to retain a $\Phi_{B}=0$ condition through their cross section. The plasma current close to the inner coil opposes the flux through the inner coil and the plasma current close to the outer coil opposes the flux from the outer coil. Since the flux through the inner plasma ring is generally much higher than the flux through the outer plasma ring, the current in the inner ring will be much higher than the current in the outer ring. This means that the plasma current is mostly due to the changing flux through the inner coil (Step 3). A radial Lorentz force $\left(F_{r}=J_{\theta} B_{z}\right)$ implodes both current sheets toward the annular centerline. The current sheets collide, resulting in single configuration with reversed fields on either side (Step 4). Magnetic tearing and reconnection is thought to occur at the ends of the configuration to improve confinement [15], yet evidence of this is unclear.

\subsubsection{Independent Coil Mode: Synchronous}

The inner and outer coils can be driven by different capacitor banks and different circuits. If each circuit is designed with the proper phase, the currents in the outer coil and inner coil can rise and fall in unison. The formation is identical that of the parallel synchronous mode in Section 3.2.1. The only difference is that the inner and outer coil currents can now be adjusted independently of each other by varying the circuit voltages. 


\subsubsection{Parallel Coil Mode: Asynchronous}

Figure 3.4 shows the circuit configuration, typical current waveforms, and the formation sequence for parallel coil operation with the coils operating out of phase. This mode is referred to in other sources [15] as programmed formation. It is different from the parallel formation circuit by the addition of a switch in the outer coil circuit. Before the start of the discharge, the area between the coils is filled with gas. At $t=0$, the inner coil circuit is discharged and a magnetic field through the inner coil is generated (Step 1). As this field reaches its maximum strength, the gas is pre-ionized (Step 2). The inner coil current and field begin to decrease. The conducting plasma tries to oppose this decrease in magnetic flux by developing a plasma current that is in the same direction as that of the inner coil. The outer coil circuit switch is then closed, creating a current in the opposite direction of the current in the inner coil and increasing the magnetic field inside the annulus. As a result, the plasma becomes strongly attracted to the inner coil (Step 3). The direction of the plasma current results in a magnetic field that reverses the field direction near the inner coil and sets up the closed B-field structure in the plasma. The field lines from the inner coil wrap around the outside of the plasma, such that the plasma separatrix is attached to the inner coil (Step 4). When the inner coil current passes through zero and the direction of the magnetic field from the inner coil changes, the plasma is forced off the wall by the current repulsion. The plasmoid detaches from the inner wall and the separatrix moves towards the center of the channel (Step 5). 

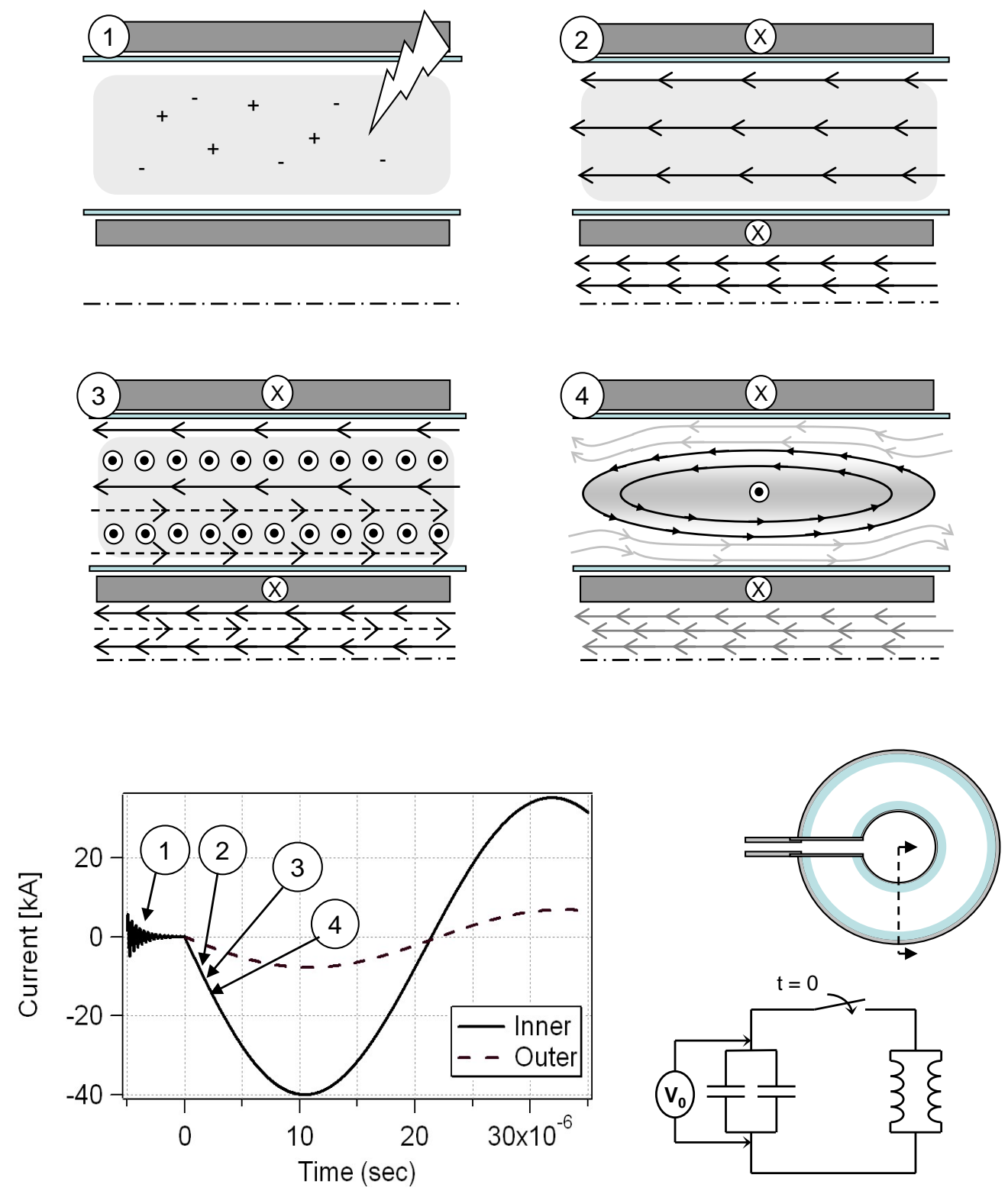

Figure 3.3: Formation schematic for an AFRC formed with the coils connected in parallel, operating in phase. 

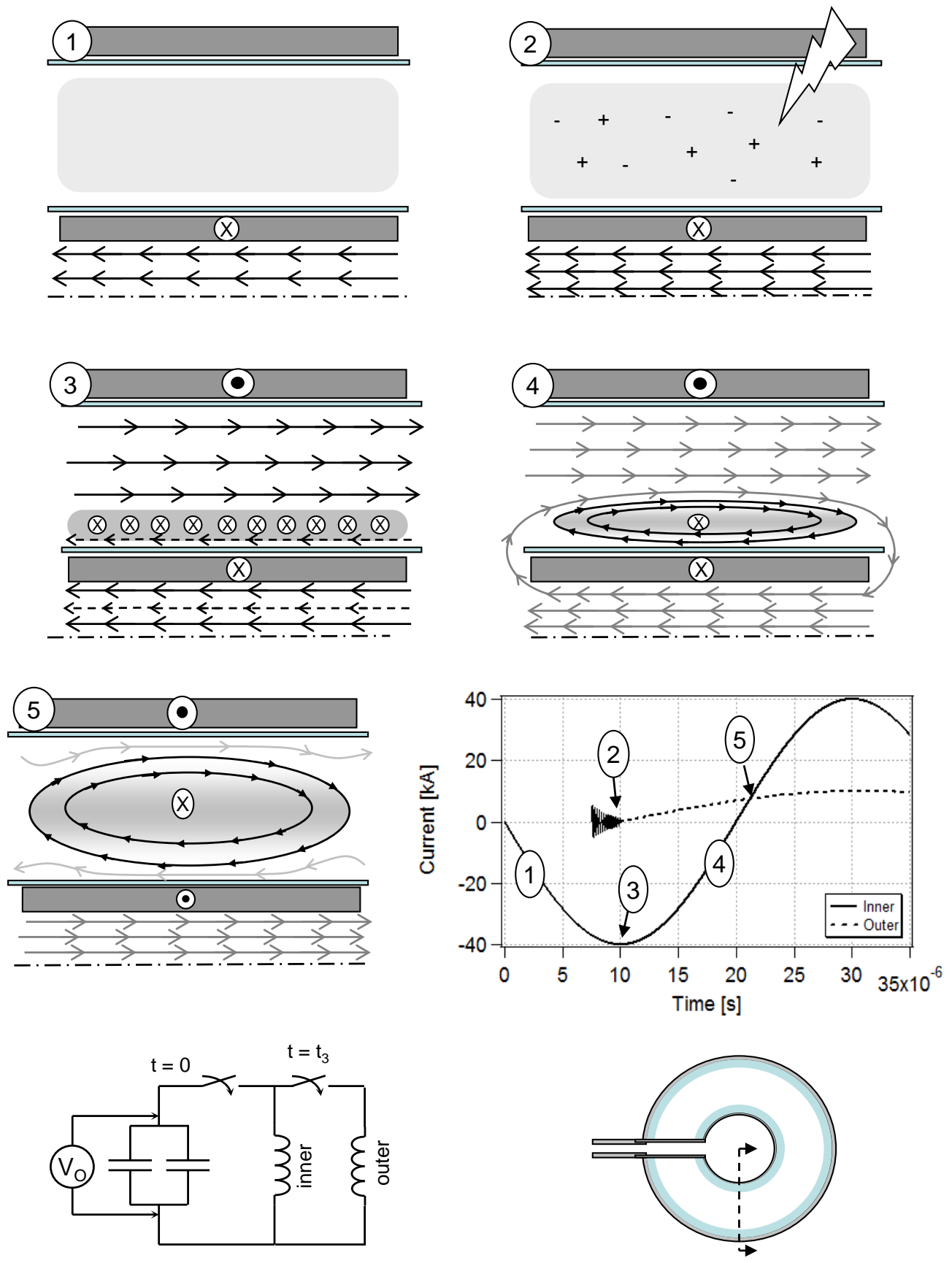

Figure 3.4: Formation schematic for an AFRC formed with the coils connected in parallel, operating asynchronously. 


\subsubsection{Independent Coil Mode: Asynchronous}

Figure 3.5 shows the circuit configuration, typical current waveforms, and the formation sequence for independent coil operation with the coils operating out of phase. The formation sequence is nearly identical to that of the parallel coil mode with asynchronous coils, with the difference arising from the two separate circuits for the outer and inner coils. Before the start of the discharge, the area between the coils is filled with gas. At $t=0$, the inner coil circuit is discharged and a magnetic field through the inner coil is generated (Step 1). As this field reaches its maximum strength, the gas is pre-ionized (Step 2). The inner coil current and field begin to decrease. The conducting plasma tries to oppose this decrease in magnetic flux by developing a plasma current that is in the same direction as that of the inner coil. The outer coil circuit is then discharged in the opposite direction as the current in the inner coil, increasing the magnetic field inside the annulus. As a result, the plasma becomes strongly attracted to the inner coil (Step 3). The direction of the plasma current results in a magnetic field that reverses the field direction near the inner coil and sets up the closed B-field structure in the plasma. The field lines from the inner coil wrap around the outside of the plasma, such that the plasma separatrix is attached to the inner coil (Step 4). When the inner coil current passes through zero and the direction of the magnetic field from the inner coil changes, the plasma is forced off the wall by the current repulsion. The plasmoid detaches from the inner wall and the separatrix moves towards the center of the channel (Step 5). 

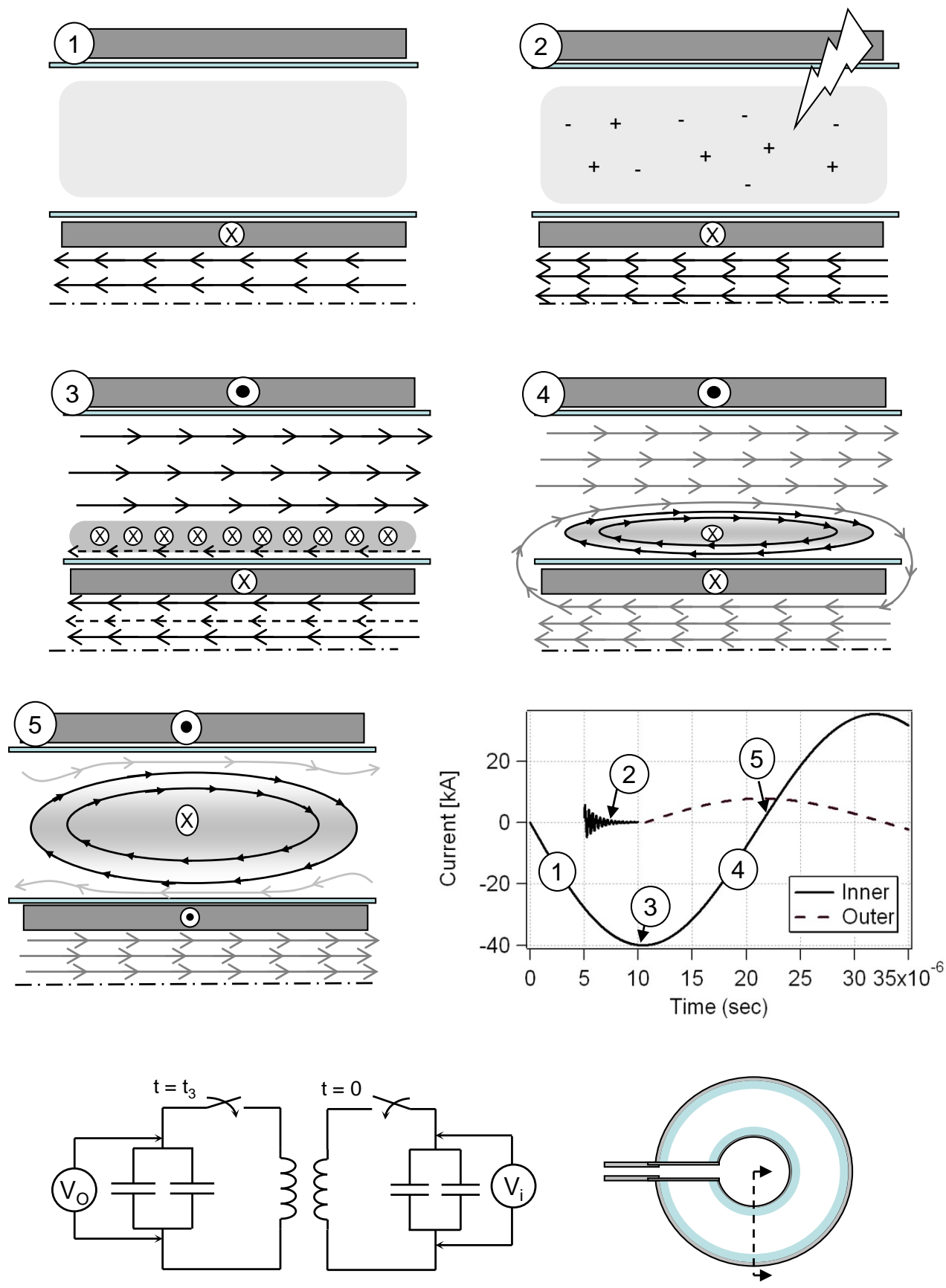

Figure 3.5: Formation schematic for an AFRC formed with the coils operating indepedently and asynchronously. 


\subsection{AFRC Physics}

While the formation physics of AFRCs and FRCs remain largely qualitative, the equilibrium physics for them are easier to define. Several models exist to explain the behavior of FRCs [21], but there is no single equilibrium solution in full agreement with experimental data. Rather than digressing into the extensive topic of AFRC/FRC equilibria, only a few salient topics will be covered in this section. These topics include deriving the radial balance of AFRCs, presenting measured profiles of the equilibrium plasmoid, discussing plasma heating through magnetic field diffusion, discussing plasma drifts and instabilities. Termination of AFRCs is also covered, though this topic remains largely device dependent.

Translation of the AFRC plasmoid in this research is accomplished by using a conical outer coil. The conical outer coil introduces a small radial magnetic field component to interact with the azimuthal plasmoid current and force the plasmoid out of the coils. This Lorentz force is the primary acceleration mechanism in an AFRC thruster. Unlike other propulsion technologies, a gas pressure gradient is not required for operation. The conical coil also lessens the magnetic mirror intensity on the large end of the coil as shown by the asymmetry in the axial magnetic field map in Figure 6.7 across the coil midplane $(\mathrm{z}=15 \mathrm{~cm})$. This allows the plasmoid to escape from the coils. As the plasmoid passes by the mirror at the large end of the coil, it experiences its peak acceleration as the strong radial magnetic fields act on the plasmoid. In formation studies, the magnetic mirrors are symmetric on both ends of the coil to confine the plasmoid to the annulus. A full discussion of translation physics and predictions of an AFRC plasmoid's trajectory is the topic of Chapter 4 . 


\subsubsection{Radial Balance}

The plasmoid must maintain a radial balance between the coils, dictated by the magnetic pressure on either side of the magnetic separatrix. Magnetic pressure $P_{B}$ is related to the square of the magnetic field $B$, according to:

$$
P_{B}=\frac{B^{2}}{2 \mu_{0}}
$$

The magnetic pressure on the inner radius of the plasmoid created by the reversed field pushes the plasmoid toward the outer wall and the magnetic pressure from the outer coil pushes the plasmoid toward the inner wall. The magnetic null radius $r_{m}$ corresponds to where these forces are equal.

The magnetic null radius $r_{m}$ can be predicted by portraying the plasmoid to be a rigid current sheet with forces acting on it. This treatment assumes the plasmoid is composed of infinitely hot electrons and cold ions to form an impermeable barrier between the plasma magnetic field and the coil magnetic field. The radial equation of motion for an annular plasma is then

$$
m \frac{d^{2} r_{m}}{d t^{2}}=2 \pi r_{m}\left(\frac{B_{z i}^{2}}{2 \mu_{0}}-\frac{B_{z o}^{2}}{2 \mu_{0}}\right)
$$

$B_{z i}$ is the axial field on the inner radius of the plasmoid, $B_{z o}$ is the axial field on the outer side, and $m$ is the plasmoid mass. The easiest approach to solve this differential equation is to make the assumptions that the fields are generated by ideal solenoids with well defined field distributions and inductances, as was done in References [42] and [43]. Additionally, the length of the plasmoid is equal to the length of the coils $l$. In this instance, the inner 
and outer fields are defined by:

$$
\begin{gathered}
B_{z i}=\frac{\mu_{0}\left(N_{o} I_{o}-I_{p}\right)}{l} \\
B_{z o}=\frac{\mu_{0}\left(N_{o} I_{o}\right)}{l}
\end{gathered}
$$

$N_{o}$ is the number of turns on the outer coil, $I_{o}$ is the current through the outer coil, and $I_{p}$ is the plasma current. The plasma current can be calculated by solving a circuit representation of the plasmoid, with self inductance $L_{p}$, mutual inductances $M_{o p}, M_{i p}$ and a resistance $R_{p}$ :

$$
\frac{d}{d t}\left(L_{p} I_{p}-M_{o p} I_{o}-M_{i p} I_{i}\right)+R_{p} I_{p}=0
$$

The inductances $L_{p}, M_{o p}$, and $M_{i p}$ are defined according to solenoid approximations:

$$
\begin{gathered}
L_{p}=\frac{\pi \mu_{0} r_{m}^{2}}{l} \\
M_{o p}=\frac{\pi \mu_{0} N_{o} r_{m}^{2}}{l} \\
M_{i p}=\frac{\pi \mu_{0} N_{i} r_{i c}^{2}}{l}
\end{gathered}
$$

where $r_{i c}$ is the inner coil radius. If the coil currents $I_{i}$ and $I_{o}$ are independent of each other, Equations 3.3 and 3.6 can be solved to find $r_{m}$. Kondoh [42] used a linearization of $I_{p}$ and $r_{m}$ to find zeroth-order approximations. This assumes that $d^{2} r_{m} / d t^{2}=0$. The linearization approximates the zeroth-order plasma current $I_{p, 0}$ and plasmoid radial position $r_{m, 0}$ to be

$$
\begin{gathered}
I_{p, 0}=2 N_{0} I_{0} \\
r_{m, 0}=\sqrt{\left(\frac{N_{i} I_{i} r_{i c}^{2}}{N_{o} I_{o}}-\frac{2 l}{\mu_{0} \pi I_{o}} \int_{t_{0}}^{t} R_{p} I_{o} d t\right)}
\end{gathered}
$$


Equation 3.11 shows that plasma resistance causes the radial plasmoid position to decay toward the inner wall. This was also seen in the experimental results [42]. Computer simulations by Kondoh [42] on the full ODE equation set confirm this finding, with larger resistances resulting in faster declines. A small radial oscillation is noted as well when the resistance is very low. When $R_{p}=0$, Equation 3.11 simplifies the radial position of the plasmoid to a function of the coil current ratio. By increasing the inner coil current, it is possible to drive the plasmoid toward the outer wall. Similarly, the plasmoid will move toward the inner wall if the outer coil current is too large. This was verified in experiments with independent coils [13].

The radial balance model for parallel coil operation was originally derived by Berger [43] and a variation on his approach is taken here. If the coils are connected in parallel so that $I_{o}$ and $I_{i}$ can no longer be varied independently, additional constraints on the radial balance model is required. The model must now include circuit equations for the outer and inner coil to relate $I_{i}$ and $I_{o}$. Neglecting the resistance of each circuit (which will generally be quite small) and any parasitic inductance, the circuit equations for the inner and outer coil circuits are

$$
\begin{aligned}
V_{o} & =\frac{d}{d t}\left(L_{o} I_{o}+M_{i o} I_{i}-M_{o p} I_{p}\right) \\
V_{i} & =\frac{d}{d t}\left(L_{i} I_{i}+M_{i o} I_{o}-M_{i p} I_{p}\right)
\end{aligned}
$$

The inductances can be represented by solenoid inductances, where $M_{i p}, M_{o p}$, have been described in Equations 3.9 and 3.8 and the remaining inductances are defined as

$$
\begin{aligned}
& L_{o}=\frac{\pi \mu_{0} N_{o}^{2} r_{o c}^{2}}{l} \\
& L_{i}=\frac{\pi \mu_{0} N_{i}^{2} r_{i c}^{2}}{l}
\end{aligned}
$$




$$
M_{i p}=\frac{\pi \mu_{0} N_{i} N_{o} r_{i c}^{2}}{l}
$$

Recognizing that parallel coil operation requires $V_{i}=V_{o}$, Equations 3.12 and 3.13 can be used along with their inductances to find the radial position of the plasmoid $r_{m}$. The plasmoid current is the same as it was in the independent model with $I_{p}=2 N_{o} I_{o}$ since the radial equation of motion (Equation 3.3) does not change. With these constraints, the coil current ratio becomes

$$
\frac{I_{i}}{I_{o}}=\frac{N_{o}^{2} r_{o c}^{2}+N_{i} N_{O} r_{i c}^{2}-2 N_{o}^{2} r_{m}^{2}}{N_{i}^{2} r_{i c}^{2}-N_{i} N_{o} r_{i c}^{2}}
$$

If the turns on the inner coil and outer coil are equal $\left(N_{o}=N_{i}\right)$, Equation 3.17 can be simplified to find the plasmoid position

$$
r_{m}=\sqrt{\frac{r_{i c}^{2}+r_{o c}^{2}}{2}}
$$

In this case, the plasmoid splits the annulus into equal areas. Experimental parallel coil operation [15] shows the plasmoid balanced between the coils at all times, though the exact plasmoid position in relation to the coils is not provided.

\subsubsection{Equilibrium Profiles}

After the volatile formation stage, the magnetic field internal to an AFRC develops a hyperbolic tangent profile to link the outer field with the inner field. The hyperbolic tangent behavior of this field has been measured in several studies [12], [16], [10], [42], and [14]. The representative structure of these fields has been reproduced in Figure 3.6, showing a change in field direction on the inner coil as compared to the outer coil. The width of the profile and location of $\mathrm{B}=0$ varies between experiments. 


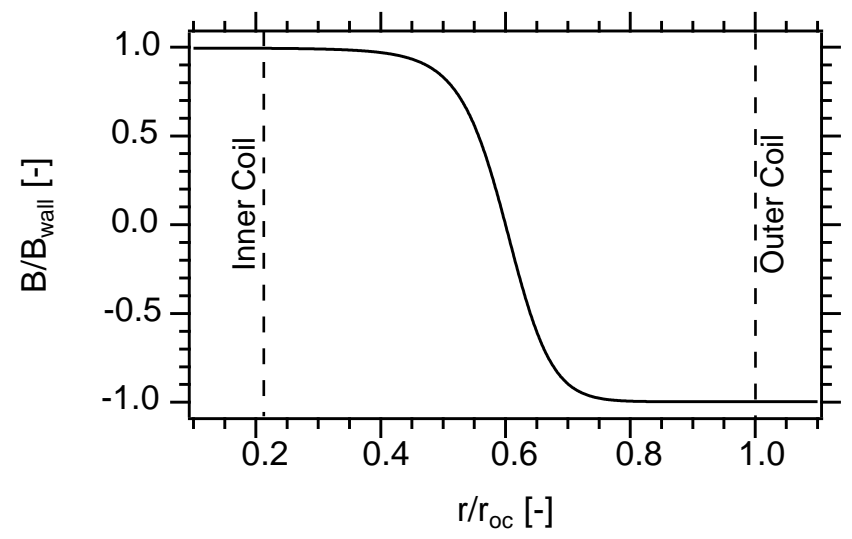

Figure 3.6: Representative internal magnetic field structure of AFRCs showing a reversed field on the inner coil as compared to the outer coil.

The electron density profile was measured in two experiments [10], [14], using holographic interferometry. The density profile follows a peaked structure, with the peak density along the $B=0$ centerline. Typical density profiles are displayed in Figure 3.7, scaled for peak quantities.

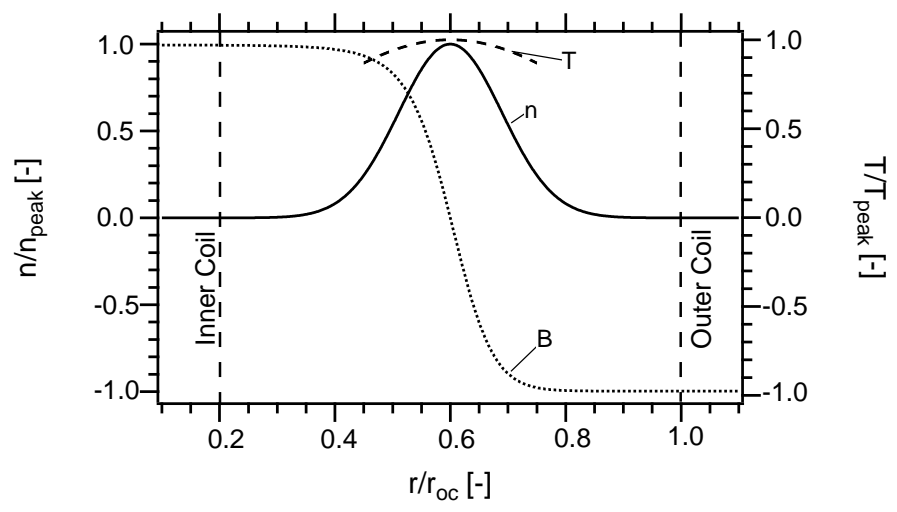

Figure 3.7: Representative internal electron density structure of AFRCs, showing a peak density at $\mathrm{B}=0$.

The temperature shown in Figure 3.7 was derived from a pressure balance in Reference [14]. The pressure balance in AFRCs and FRCs equates the internal pressure of an FRC to the external pressure from the coil fields $P_{B e}$. The internal pressure is a sum of the 
magnetic pressure $P_{B i}$ and the internal gas pressure from the hot plasma $P_{k}$. In equilibrium, the pressure balance between internal and external pressures becomes:

$$
\begin{gathered}
P_{k}+P_{B i}=P_{B e} \\
n k_{B} T_{t}+\frac{B_{z i}^{2}}{2 \mu_{0}}=\frac{B_{z e}^{2}}{2 \mu_{0}}
\end{gathered}
$$

In an AFRC, this assumes that the axial field on the outer side is equal to the pressure along the inner side. If the plasma is highly collisional, the electron temperature and the ion temperature will be approximately equal. Adequate temperature profiles for AFRCs are not available in literature, but FRC experiments have shown the temperature profile across the FRC is fairly flat [44].

The ratio of internal gas pressure to external field pressure is referred to as the plasma beta $\beta$, defined as:

$$
\beta=\frac{P_{k}}{B_{z e}^{2} / 2 \mu_{0}}
$$

FRCs have a very high $\beta$ from 0.8-0.95.

\subsubsection{Magnetic Field Diffusion}

AFRCs can be formed on slow timescales to deposit energy into the plasma after formation. Magnetic field diffusion through the plasma provides ohmic heating by eddy currents opposing the incoming field. This serves to increase the temperature and energy content of the configuration. The 1/e timescale for a magnetic diffusion through a plasma thickness $L$ 
is related to the plasma resistivity $\eta_{\perp}[45]$ :

$$
\tau_{B}=\frac{\mu_{0} L}{\eta_{\perp}}
$$

Another way to view $\tau_{B}$ is the total time it takes the magnetic field energy to be dissipated into Ohmic heat.

The resistivity $\eta_{\perp}$ perendicular to $\mathbf{B}$ in a fully ionized magnetized plasma is referred to as Spitzer resistivity [46]:

$$
\eta_{\perp}=1.30 \times 10^{-4} \frac{Z \ln \Lambda}{T_{e \nu}^{3 / 2}} \quad[\Omega-m]
$$

where $\ln \Lambda$ is the Coulomb logarithm, $Z$ is the ion charge, and $T_{e V}$ is the electron temperature in eV. In FRCs, the resisitivity can be 10-100 times the Spitzer (or classical) resistivity [47], [17]. However, for low temperature plasmas (less than $100 \mathrm{eV}$ ) as generated in slow timescale AFRC devices, the collisionality of the plasma suggests that anomalous effects are not important [48] and the resistivity is closer to Spitzer. For a $10 \mathrm{eV}$ plasma wtih a density of $1 \times 10^{20} \mathrm{~m}^{-3}$, the Spitzer resistivity is about $4 \times 10^{-5} \Omega-\mathrm{m}$. The magnetic field diffusion time for this plasma is $35 \mathrm{~ns}$ per $\mathrm{mm}$. The ability of the field to quickly diffuse through the plasma provides a rapid heating mechanism as the field energy is converted into Ohmic heat. From this simple calculation, it's apparent that creating a low temperature plasma on a slow timescale could provide the significant Ohmic heating via magnetic diffusion. 


\subsubsection{Plasma Drifts}

Plasma drifts exist in FRCs and AFRCs plasmas as evidenced by the appearance of strong rotational ion flows [21]. An AFRC experiment [16] recently measured an ion rotational velocity of $5-7 \mathrm{~km} / \mathrm{s}$ flowing with the current, though the origins of this drift were not discussed. While the lack of consensus on equilibrium models makes it difficult to predict the plasma drifts, the profiles of fields and gradients discussed in Section 3.3.2 can be used to provide a qualitative understanding of possible drifts.

Ion movement in an AFRC is dominated by the gyromotion of ions around magnetic field lines. The frequency at which ions orbit around a magnetic field line is known as the gyrofrequency $\Omega_{i}$ and is a function of the magnetic field strength $B$ and the ion mass $m_{i}$ :

$$
\Omega_{i}=\frac{q B}{m_{i}}
$$

For an argon ion in a $20 \mathrm{mT}$ field (as was measured in this research), the gyrofrequency ( 8 $\mathrm{kHz})$ is on the order of the discharge frequency $(10 \mathrm{kHz})$ indicating that motion of the ions is likely. The gyrofrequency alone cannot lead to significant ion currents as the ions are tied to a field line. However, the gyrofrequency gives rise to two plasma drifts including the $E \times B$ drift and the diamagnetic drift.

A common drift in plasma fluids is the $E \times B$ drift [45]. The $E \times B$ drift is defined by orthogonal electric and magnetic fields, according to Equation 3.25. Equilibrium models [49] predict a radial electric field $E_{r}$ in an FRC and it is likely that it also appears in AFRCs. The electric field points towards the coils, opposite the density gradient. With this field, the drift direction will be in the same direction as the plasma current. The drift of the ions will 
increase the current and the drift of the electrons will decrease the current.

$$
v_{E x B}=\frac{\mathbf{E} \times \mathbf{B}}{B^{2}}
$$

A diamagnetic drift is also likely in an AFRC [45]. The diamagnetic drift occurs when a pressure gradient is orthogonal to a magnetic field, defined by Equation 3.26. The equilibrium profiles in Section 3.3.2 show a density gradient, pointed toward the magnetic null. This represents a pressure gradient $\nabla \mathrm{p}$ in the radial direction. Combined with the axial field, this will result in a diamagnetic drift for ions in the current direction. The diamagnetic drift for electrons is in the counter current direction. The combination of the electron and ion diamagnetic drifts add to the current.

$$
v_{D}=\frac{\nabla p \times \mathbf{B}}{q n B^{2}}
$$

Another common drift in plasmas is the grad-B drift. It is not clear if these are important in AFRCs as they do not appear in Maxwellian fluids [45].

\subsubsection{Termination}

An AFRC is sustained as long as a reversed field appears on the inner radius as compared to the outer radius. The time duration of the field reversal is referred to as the configuration lifetime. An AFRC's configuration lifetime is device dependent, though it generally scales with the rise-time (quarter cycle) of the inner coil current. Several experiments have shown

that it is possible to sustain an AFRC slightly past the quarter cycle or rise-time of the 
coil currents [50], [42]. One AFRC experiment witnessed AFRC termination before the quarter-cycle [16].

Several instabilities in FRCs are known to limit configuration lifetime. These instabilities include rotational, tearing, and tilt instabilities. While these instabilities have been witnessed in AFRC experiments [15], they generally do not appear to limit the lifetime of an AFRC. In one instance [14], a tearing instability was blamed for the confinement degradation but evidence for this was limited.

The most common mode of termination for an AFRC has been a radial collapse onto the inner wall. This is not seen in traditional FRCs due to the lack of an inner boundary. A radial collapse of the plasmoid has been seen in at least five separate experiments [42], [50], [15], [16], [41]. In most cases [42], [50], [15], [16], the radial collapse was due to a limit in current induction. The AFRC was unable to generate enough current to hold itself off the inner coil indefinitely. In three of these experiments [42], [50], [15], the configuration lifetime surpassed the time of peak coil currents, though the reversed field began to decay shortly before the peak. In another experiment [16], the radial collapse occurred shortly before the coil currents peaked. The data from this experiment suggest that the plasmoid was driven into the inner wall by an unequal radial pressure balance. The pressure on the outer radius appeared to be much higher than the pressure on the inner coil. The mechanism for radial motion in the remaining experiment [41] is unclear as magnetic field data to support a pressure imbalance or limit in current induction is not available. The high occurrence of terminations due to radial plasmoid motion suggest that an AFRC plasmoid can only be sustained as long as it is being driven. This observation has been made in other sources as well [50], [16]. For a translation experiment, it is important to eject the AFRC before the radial collapse, within the first quarter cycle. 
An axial instability was also suspected in one instance [12]. The plasmoid was seen to disappear when the density reached a critical value. The time it took for this critical density to be reached was equal to the amount of time required for reducing the plasma density by an axial flow. No diagnostic evidence of plasma flow was provided, so it is unclear if the axial flow occurred.

\subsection{Literature Review of AFRCs}

Over seven different experiments and subsequent variants on AFRCs have been investigated for fusion research since 1963. Although some of these experiments refer to the annular plasmoid by a pseudonym (double theta pinch, flux-coil-generated FRC), they all use an inductive, coaxial geometry to generate the annular plasmoid. Annular FRC experiments, their years of operation, formation mode, and rise-time of the inner coil current are shown in Table 3.1

The earliest experiment on AFRCs was the "Slingshot" experiment by Phillips, Mather, and Wittman in 1963. Limited information is provided on the original Slingshot, but their 1981 proposal [11] provides details on a second version of the Slingshot tested in 1965. The Slingshot used an outer coil diameter of $27.5 \mathrm{~cm}$, inner coil diameter of $12.5 \mathrm{~cm}$, and coil length of $7.5 \mathrm{~cm}$. The peak energy was $9 \mathrm{~kJ}$, with a rise-time of $3 \mu \mathrm{s}$. Slingshot was tested at high fill pressures between 100-600 mTorr on argon and helium. At 600 mTorr and 300 mTorr, a radially centered plasma ring was noted to collapse to occupy $26 \%$ of the radial width during the first 1-1.5 $\mu$ s. The width remained constant during the remaining current rise. At 100 mTorr, the plasma ring collapsed quickly (within $0.8 \mu \mathrm{s}$ ) and then expanded steadily. At peak current, the ring occupied the entire width of the channel (4.5 
Table 3.1

History of AFRC devices

\begin{tabular}{|c|c|c|c|c|}
\hline Experiment & Year & Mode & RT & References \\
\hline Slingshot & $1963-65$ & $\begin{array}{c}\text { Parallel } \\
\text { Synchronous }\end{array}$ & $3 \mu \mathrm{s}$ & [11] \\
\hline French AFRC & $1967-68$ & $\begin{array}{c}\text { Parallel } \\
\text { Synchronous }\end{array}$ & $1-2 \mu \mathrm{s}$ & {$[18]$} \\
\hline Double Theta-Pinch & $1974-78$ & $\begin{array}{l}\text { Independent } \\
\text { Synchronous }\end{array}$ & $3.5-6.5 \mu \mathrm{s}$ & $\begin{array}{l}{[13]} \\
{[51]} \\
{[42]} \\
{[52]}\end{array}$ \\
\hline $\begin{array}{l}\text { Garching Poloidal } \\
\text { Field Belt-Pinch }\end{array}$ & 1975 & $\begin{array}{c}\text { Parallel } \\
\text { Synchronous }\end{array}$ & $\mathrm{n} / \mathrm{a}$ & [14] \\
\hline $\begin{array}{l}\text { Coaxial } \\
\text { Slow Source }\end{array}$ & $1985-87$ & $\begin{array}{l}\text { Independent } \\
\text { Asynchronous }\end{array}$ & $21 \mu \mathrm{s}$ & [10] \\
\hline CSS-Upgrade (CSSU) & $1987-89$ & $\begin{array}{l}\text { Independent } \\
\text { Asynchronous }\end{array}$ & $66 \mu \mathrm{s}$ & $\begin{array}{l}{[50]} \\
{[53]}\end{array}$ \\
\hline $\begin{array}{l}\text { CSS-Parallel (CSSP) } \\
\text { (tearing) } \\
\text { (programmed) }\end{array}$ & $\begin{array}{l}1989-93 \\
1989-93\end{array}$ & $\begin{array}{c}\text { Parallel } \\
\text { Synchronous } \\
\text { Asynchronous }\end{array}$ & $\begin{array}{c}39 \mu \mathrm{s} \\
46-140 \mu \mathrm{s}\end{array}$ & [15] \\
\hline ХOCOT & 2003-08 & $\begin{array}{c}\text { Parallel } \\
\text { Synchronous }\end{array}$ & $46-112 \mu \mathrm{s}$ & [41] \\
\hline Irvine FRC (IFRC) & 2004- & $\begin{array}{l}\text { Independent } \\
\text { Asynchronous }\end{array}$ & $\begin{array}{c}100 \mu \mathrm{s} \\
(\mathrm{est})\end{array}$ & $\frac{[16]}{[54]}$ \\
\hline $\begin{array}{l}\text { Flux-Coil-Generated FRC } \\
\text { (FCG-FRC) }\end{array}$ & $2010-$ & $\mathrm{n} / \mathrm{a}$ & $\mathrm{n} / \mathrm{a}$ & [55] \\
\hline
\end{tabular}

$\mathrm{cm})$. Magnetic field measurements suggested that the plasma current was about half of the total current. These measurements also noted a radial implosion of the plasma, followed by an axial expansion. While translation was proposed in the proposal, no mention of 
successful translation attempts were noted.

An experiment by a French group of researchers in 1967 demonstrated the first (and only) translation of an AFRC [18]. This experiment injected approximately $10 \mu \mathrm{g}$ of deuterium into an annular channel $18 \mathrm{~cm}$ long with an inner and outer diameter of $9 \mathrm{~cm}$ and $15 \mathrm{~cm}$. The coils were connected in parallel, backed by a $2 \mu$ s pulse from a $1 \mathrm{~J}$ capacitor bank. The total voltage drop across the coils was $10 \mathrm{kV}$. Two unconventional additions were added to the design. The first was a permanent magnetic field in the azimuthal (toroidal) direction of $0.65 \mathrm{~T}$. The second was a $0.03 \mathrm{~T}$ radial magnetic field created by a soft iron circuit. The radial field was added to provide the necessary $B_{r}$ for translation; the azimuthal field was added for stability. The experiment demonstrated translation of the configuration to a velocity of $200 \mathrm{~km} / \mathrm{s}$. A small translation delay of $0.8 \mu \mathrm{s}$ was noted, where resistive heating was noted to dissipate the magnetic energy into the sheet. A mass spectrometer located 110 $\mathrm{cm}$ downstream was used to measure the exhaust velocity of the particles. Two populations were noted; a fast population traveling around $200 \mathrm{~km} / \mathrm{s}$ and a slower population of $50 \mathrm{~km} / \mathrm{s}$. Note that the kinetic energy of $10 \mu \mathrm{g}$ of exhausted deuterium traveling at these velocities is several orders of magnitude higher than the $1 \mathrm{~J}$ input energy. This indicates that only a small fraction of the propellant was accelerated from the device to satisfy a basic energy balance. Low levels of impurities (less than 5\%) were observed. The French researchers noted that not all conditions resulted in a successful ejection of a magnetically confined plasma. If the gas fill was not uniform in the axial and azimuthal directions and was pre-ionized, only a turbulent current layer formed. The plasma still appeared to translate, but at a lower speed and with a high level of impurities, ingested from plasma-wall contact.

The French group did another AFRC experiment to test the prevailing theory of a tearing instability in FRCs. The second experiment [12] was different from their original 
translation experiment. The outer coil diameter was $16 \mathrm{~cm}$ with an inner coil diameter of $8 \mathrm{~cm}$ and coil length of $7 \mathrm{~cm}$. The coils were driven in parallel by a $2.0 \mathrm{kV}$ capacitor bank, with a quarter cycle of $1 \mu \mathrm{s}$. Deuterium and hydrogen were tested with fill pressures ranging from 40-120 mTorr. A bias field of 0.15 G was used as well. An annular plasma sheet was noted, with an estimated thickness of $3 \mathrm{~mm}$ and a reversed field on either side of the sheet. The sheet was observed to last for about $2 \mu \mathrm{s}$. This lifetime exceeded the tearing predictions. Instead of tearing, the sheet disappeared when a critical density $\left(6 \times 10^{21} \mathrm{~m}^{-3}\right)$ was reached. At low pressures (40-60 mTorr), the density would increase until it reached this critical value. At higher pressures, the density would reach a maximum before decaying to the critical density. The time required for the plasma sheet to reach the critical density was close to that required to reduce the plasma density by axial flow to the critical value, as predicted by a basic Lorentz force calculation. Axial flow measurements to confirm this finding were never recorded.

The Double Theta-Pinch experiments were conducted in Japan from 1974-1978 [13], [51], [42], [52]. The experiment was developed to create a high $\beta$ toroidal plasma stabilized by a toroidal magnetic field. The inner and outer coils were operated independently from each other, but the circuits were designed to keep the coil currents in phase. The inner coil had a diameter of $3 \mathrm{~cm}, 6$ turns with a length of $30 \mathrm{~cm}$. The outer coil was $14 \mathrm{~cm}$ in diameter, 40 turns, and $20 \mathrm{~cm}$ long. The experiments were conducted with various levels of energy from $5 \mathrm{~kJ}$ to $12 \mathrm{~kJ}$ per pulse, with full periods of $14 \mu \mathrm{s}$ to $26 \mu \mathrm{s}$ (quarter cycle of $3.5 \mu \mathrm{s}$ to $6.5 \mu \mathrm{s})$. All experiments were conducted using helium at 120 mTorr.

The first experiment on the Double Theta-Pinch [13] demonstrated that the radial location of the plasmoid could be controlled by tuning the coil currents. Current sheets were observed in streak photos to form on the inner and outer walls at early times and implode 
towards each other. Their final radial position was dictated by a ratio of the inner coil to outer coil currents. The next experiment on the Double Theta-Pinch was an experiment with a toroidal field or hard-core [51]. A hard-core is a DC, linear current on the axis of the device, which imposes a toroidal (azimuthal) field on the plasmoid. The toroidal field was found to improve the axial stability of the configuration. Without the toroidal field, the plasma was seen to tilt in the coils before the current peak. Once the issue of axial stability was remedied, the Double Theta-Pinch team investigated the radial motion of the AFRC between the coils [42]. Full results of this study are discussed in Section 3.3.1. The plasmoid's radial position was found to depend on the ratio of the coil currents, matching well with their radial-balance model. Plasma resistance was predicted to affect the radial motion as well. The plasmoid was noted to retain a reversed field for at least $5 \mu \mathrm{s}$, near the time of the peak coil currents. Throughout the lifecycle of the configuration, the plasmoid was observed to drift towards the inner wall. The plasmoid eventually terminated from contact with the inner wall. The lifetime was extended by a couple $\mu$ s by reducing the decay of the coil currents with power crowbarring. The final Double Theta-Pinch experiment [52] measured the internal magnetic field structure of the plasmoid. The magnetic field measurements were used to estimate the plasma equilibria properties, including internal plasma pressure, toroidal and poloidal currents, and the plasma $\beta$. The calculated $\beta$ was $0.23-0.30$, close to the predicted $\beta$ from ideal MHD calculations.

Concurrent with the Double Theta-Pinch experiments, an experiment on understanding the tearing instabilities of AFRC plasmas was being conducted [14]. The experiment, referred to as a Poloidal-Field Belt Pinch, was larger than the previous devices. The outer coil was $42 \mathrm{~cm}$ in diameter, with a length of $75 \mathrm{~cm}$. The inner coil was $17 \mathrm{~cm}$ in diameter, with a length of $75 \mathrm{~cm}$. The coils were driven in parallel by a $100 \mathrm{~kJ}$ capacitor bank, though no rise-time or capacitance information was provided. A toroidal field was imposed on the 
configuration for enhanced stability. Plasmoids were formed in a background fill between 10 mTorr and 70 mTorr; the type of gas is not mentioned. The configuration was noted to last for 10-30 $\mu$ s before an instability terminated the configuration. The instability was thought to arise from resistive tearing since the lifetime trends matched well with tearing predictions. This was never confirmed however, due to the uncertainties in the lifetime evaluation and the tearing theory.

The Coaxial Slow Source (CSS) was a large project started in 1985 to study if AFRCs could be formed on slow timescales for fusion applications. This was the first experiment with slowly-formed AFRCs. Previous experiments on AFRCs were conducted with fast rising currents ( $5 \mu \mathrm{s}$ and under). The CSS demonstrated that it was possible to create a plasma with rise-times in excess of $20 \mu \mathrm{s}$. The appeal of slowly building up the AFRC allowed for plasma resistive heating using magnetic diffusion (see Section 3.3.3). The CSS experiments intended to use this slow heating to achieve the plasma temperatures and densities required for fusion, at much lower coil voltages and longer timescales than traditional $\theta$-pinch formation techniques would allow. They also intended to demonstrate that flux could be continuously added to the formation, long after the formation phase ceased. The CSS program consisted of three major experimental variations: the original Coaxial Slow Source (CSS), the Coaxial Slow Source-Upgrade (CSSU), and the Coaxial Slow Source-Parallel (CSSP). A program summary is provided in the 1993 final report [56].

The original CSS [10] operated the inner coil and outer coil independently in an asynchronous fashion. The inner coil was $11.5 \mathrm{~cm}$ in diameter, $1 \mathrm{~m}$ long with 4 turns. (A 2-turn model was tested early on). The outer coil was $45 \mathrm{~cm}$ in diameter, $1 \mathrm{~m} \mathrm{long}$, and only a single turn. The outer coil capacitor was charged to $2 \mathrm{kV}$ and the inner coil was 
charged to $5 \mathrm{kV}$. Coil voltages were much lower than this, between 1-2 kV. The capacitors for each bank were $800 \mu \mathrm{F}$, meaning that $10 \mathrm{~kJ}$ was stored in the inner coil circuit and $1.6 \mathrm{~kJ}$ was stored in the outer circuit. The inner coil circuit was set to supply flux for 32 $\mu \mathrm{s}$, with a quarter cycle rise of $18 \mu \mathrm{s}$. The early experiments noted lifetimes of 10-40 $\mu \mathrm{s}$, with flux lifetimes of $15-20 \mu \mathrm{s}$. The four-turn coil was found to produce longer lifetimes than the 2-turn model. As was found in other studies with independent coils [13], the CSS experiment discovered that it was possible to push the plasmoid to either wall by changing the coil current ratios. A coil voltage ratio of 3 was required for keeping the plasma centered.

The original CSS was upgraded to the CSSU in 1987 [50], [53]. The upgrade consisted of increasing the flux delivery time (half-cycle) of the inner coil to $66 \mu \mathrm{s}$. The CSSU experiment found that a reversed field plasmoid could be sustained as long as the coil currents were changing. The configuration terminated when the circuit was crowbarred and the plasma was forced to the inner wall. Configuration lifetime was also seen to increase with pressure. Expected tilt instabilities were not observed. The overall energy content of the plasmoid was found to be quite low. Thomson scattering during the equilibrium phase of the discharge measured electron temperatures around $10 \mathrm{eV}$. An energy balance analysis [50] discovered that the CSSU plasma was radiation dominated. Line radiation losses from small impurities (carbon and oxygen) accounted for most of power originally deposited into Ohmic heating. This prevented the CSSU plasma from reaching higher temperatures. The troublesome impurities were introduced into the plasma through wall contact. This occurred early in the formation stage, when the plasma was attracted to the inner wall and during an axial contraction when the plasma expanded radially into the walls. Radial bouncing of the plasma due to mismatched coil waveforms created turbulence and enhanced transport of the impurities, worsening the global energy loss to the confinement. 
The severe radiation losses of the CSSU prompted a major design change in the CSS program. The design was reconfigured to connect the coils in parallel. The single turn outer coil was exchanged for a 4-turn outer coil, to match the 4-turn inner coil. This was done to ensure radial stability of the plasmoid (see Section 3.3.1). This new version of the CSS is referred to as the Coaxial Slow Source Parallel (CSSP)[15]. The CSSP was operated in both parallel modes, with the coils currents synchronized and asynchronized. These are referred to in CSS literature as the tearing mode and programmed mode, respectively. In tearing mode, a $3017 \mu \mathrm{F}$ bank was used, with a quarter cycle of $39 \mu \mathrm{s}$. Capacitor banks with 1520 to $7440 \mu \mathrm{F}$ were used for the programmed operation, resulting in a quarter cycle rise-times of 46-140 $\mu \mathrm{s}$. The circuits were not crowbarred and were allowed to ring down completely. Tearing formation was noted to form thin, sheet configurations with efficient Ohmic heating while programmed mode was found to form short, fat plasmas with low energy content. The parallel coil operation was observed to form radially stable plasmoids at much lower pressures and lower coil voltages than with the CSSU. The low pressure and low voltage formation (coil voltages below $100 \mathrm{~V}$ ) were attainable because the plasma remained off the walls at all times, eliminating energy losses due to quenching. Confinement times of $10-40 \mu$ s were observed in the tearing formation. Temperatures were measured between $10-20 \mathrm{eV}$, with an electron density of $2 \times 10^{21} \mathrm{~m}^{-3}$. Halfway through the lifetime, magnetic activity in the radial and azimuthal coordinates was seen. This corresponded to an instability, as seen through streak photography. The instability was not immediately disruptive, but the reversed field began to decay after its onset. Energy transport was enhanced through wall contact among other mechanisms leading to a loss of particles and loss of confinement. While a tearing instability was not seen in the programmed mode, a tilting instability appeared shortly after formation. The tilt did not appear to terminate the configuration but likely enhanced transport. Combined with the short configuration length, the programmed mode plasmoid was quite low in energy. While 
the parallel coil operation was successful in creating balanced plasmas with low radiation losses, the internal energies of the configurations were insufficient for fusion application and funding was discontinued.

The ability to form AFRCs at low coil voltages prompted a study into AFRCs for spacecraft propulsion. The first part of this study was a joint effort between the Air Force Research Laboratory and University of Michigan. It was a formation study into AFRCs with heavy gases (argon and xenon). This experiment, the XOCOT-S [41], is a predecessor to the experiment presented in this research, the XOCOT-T3. The XOCOT-S used parallel, single turn outer and inner coils to form a plasma. The outer coil had an inner diameter of $43.5 \mathrm{~cm}$ and the inner coil had an inner diameter of $15.8 \mathrm{~cm}$. Three timescales were experimented with in the XOCOT-S, using quarter cycles of $112 \mu \mathrm{s}, 62.5 \mu \mathrm{s}$, and 46.25 $\mu$ s. The circuit was not crowbarred, allowing the circuit to ring through several reversals. Coil voltages were kept low (less than $1 \mathrm{kV}$ ), with maximum discharge energies of $1.1 \mathrm{~kJ}$. In all discharges, plasma was observed through light emission and with a triple Langmuir probe but it is unclear if this corresponded to a fully reversed AFRC as inner coil magnetic field data were limited. The main conclusion from the XOCOT-S was that an insufficient pre-ionization stage resulted in poor formation during the first current rise. The second and third plasma formations (resulting from continued ringing of the capacitor) were much higher in density and registered more magnetic field activity on external magnetic field probes. Internal triple probe measurements for the shortest timescale experiments were collected at the axial midplane on the annulus centerline. Peak densities during the first formation cycle of $2 \times 10^{18} \mathrm{~m}^{-3}$ were measured for argon (14 mTorr) and lower for xenon ( 3 mTorr). During the second cycle, the plasma density increased to $3 \times 10^{19} \mathrm{~m}^{-3}$ for argon and $1 \times 10^{20} \mathrm{~m}^{-3}$ for xenon. Peak temperatures at midplane were $6-8 \mathrm{eV}$ for both argon and xenon. Termination of the plasmoid occured $20 \mu$ s after the onset of plasma activity in the 
annulus, appearing in images to collapse onto the inner wall.

The Irvine FRC (IFRC) experiment was conducted at University of California-Irvine beginning in 2004 [16], [54]. The IFRC was a fusion-focused experiment exploring the stability betatron orbits (large ion orbits) lend to an AFRC. The IFRC coils were operated independently. The outer coil was $80 \mathrm{~cm}$ in diameter, with a length of $60 \mathrm{~cm}$, and an initial voltage of $1.4 \mathrm{kV}$. The inner coil was fashioned as a solenoid with 22 turns $/ \mathrm{m}$, a diameter of $20 \mathrm{~cm}$, a length of $130 \mathrm{~cm}$, and a starting voltage of $5.4 \mathrm{kV}$. No information on the discharge circuit is provided, including energy or capacitance. The waveforms published from the experiment show a rise time of approximately $125 \mu \mathrm{s}$. The IFRC appears to be a moderate-energy experiment (less than $5 \mathrm{~kJ}$ ) based on the fairly low current through the coils. Instead of the traditional deuterium gas fill, the IFRC used ethylene $\left(\mathrm{C}_{2} \mathrm{H}_{4}\right)$ sputtered from the surface of a coaxial cable. Slight impurities were introduced as well for diagnostic purposes. No pressure information or neutral densities are provided. Field reversal on the inner coil is seen almost immediately after the inner coil turn-on. The reversal lasts for $70 \mu \mathrm{s}$ before disappearing. Internal magnetic probe measurements show the null radius of the plasma moves toward the inner coil steadily throughout its lifetime. The plasmoid terminates when it comes in contact with the inner wall. The authors [16] suggest a limit in inner coil rise-time as the source of the termination, but no additional information is provided. Electron temperatures, estimated by the line ratios of hydrogen, were between 2-4 eV for most of the discharge. Plasma current was measured with a small Rogowski coil to be $12-16 \mathrm{kA}$ during the stable part of the discharge. Drift calculations suggested that the total plasmoid current was canceled by competing ion and electron motion around the annulus, though the low fidelity of these estimates was unable to confirm this hypothesis.

The first task in the IFRC research was to determine how much of the plasma current 
was attributed to ion motion [16]. Visible emission spectroscopy was used to estimate the ion velocity by noting the Doppler shift of the impurities. Carbon and argon ions were measured to flow in the direction of the plasma current at a velocity of $5-7 \mathrm{~km} / \mathrm{s}$ for the majority of the discharge. Krypton accelerated to $5-7 \mathrm{~km} / \mathrm{s}$ but only after a delay of 50 $\mu \mathrm{s}$. This was likely a result of the longer Coulomb collisional (ion-ion) relaxation time for krypton than argon or carbon. The drift velocities of the ions was combined with a Rigid-Rotor equilibrium model to estimate the total contribution of current due to ions. This analysis estimated the ion current to be a full order of magnitude higher than the measured plasma current. The authors suggest that electron motion due to drifts is responsible for cancellation of some ion current. Their new estimate for plasma current, taking into account the rotation frequency of electrons from drifts, was only a factor of 3 different from the measured plasma current.

An experiment similar to the IFRC was conducted at the energy firm, Tri-Alpha. This experiment was referred to as the Flux-Coil-Generated FRC. Limited information on the experiment is available, however. Results in an interferometry paper [55] show slow rising $(100 \mu \mathrm{s})$ currents less than $100 \mathrm{kA}$. The plasma data show a reversed field on the inner wall lasting about $80 \mu$ s with average electron densities of $1 \times 10^{20} \mathrm{~m}^{-3}$.

\subsection{Propulsion Considerations}

AFRC experiments have demonstrated that a stable plasmoid can be sustained between two coils, provided an inductive current in the plasma can be maintained. This plasmoid has been noted to live until the rise-time of the coils for both slow $(>10 \mu \mathrm{s})$ and fast $(<10 \mu \mathrm{s})$ timescales. For a propulsion experiment, it is desirable to lower the instantaneous energy 
used to eject the plasmoid and to allow the plasmoid to fully form prior to expulsion from the coils. This implies that the plasmoid should be formed with fast-rising pulse followed by a slower pulse for translation. This arrangement, however, complicates the hardware. Also, it is unclear how the plasmoid's lifetime will be affected by this type of waveform. Since translation and formation likely occur simultaneously, based on results from a similar FRC experiment [24], slow timescales should allow the plasmoid to fully form prior to ejection. Since slow timescales also lower the energy requirement for the circuit, they will be used in this experiment.

Four operating modes are possible for an AFRC, but not all are suitable candidates for a propulsion experiment. While asynchronous coils may provide an extra level of control to accelerate the plamoid from the coils, it subjects the plasma to wall contact during a significant portion of the discharge. The plasmoid is formed on the wall and lifts off only after the configuration has been fully formed. Additionally, the plasmoid can bounce from the inner wall to the outer wall. Wall contact cools the plasma and causes the plasma to injest impurities which can radiate energy from the configuration. In a propulsion device with a hot plasma such as a plasmoid thruster, wall contact must be minimzed or eliminated; asynchronous formation modes are not a preferred method of formation for propulsion devices. The parallel synchronous mode is an ideal choice for an AFRC device. It eliminates wall contact and requires less hardware and tuning than the independent mode. For these reasons, this mode was chosen as the formation method for this research. 


\section{Chapter 4}

\section{Translation Model of an Annular Field}

\section{Reversed Configuration}

An annular electromagnetic launcher model was developed to aid in experimental design of the AFRC translation experiment. The principle goal of this modeling effort was to find an experimental design which would launch a plasmoid from the formation chamber during the plasmoid's expected lifetime. The secondary goal was to maximize the design's projected energy efficiency and the plasmoid's velocity. This annular electromagnetic launcher model simplifies the motion of the plasmoid using a circuit-based approach to calculate the trajectory of the annular plasmoid. The annular electromagnetic launcher model has been presented previously [57], with results on general scaling laws for AFRC devices. The results presented here focus on experimental design, providing specific dimensions for the required hardware.

The annular electromagnetic launcher model is presented in Section 4.1, including the 
model equations, methodology, and model validation studies. Design studies for the experiment using the model are presented in Section 4.2, along with expected trajectories from the final design and a sensitivity study on plasma conditions. Magnetic field predictions from the final design are presented in Section 4.3, showing the vacuum fields of the coil design and the expected magnetic field profiles of the translating plasmoid. A discussion of the results from the design study and conclusions about annular electromagnetic launchers are provided in Section 4.4

\subsection{Annular Electromagnetic Launcher Model}

Dynamic circuit models are a popular technique for analyzing pulsed inductive plasma thrusters [4], [58], [2], [59], and electromagnetic launchers [1]. These models couple the circuit equations with Newton's law, producing a set of simultaneous equations to describe the coupled electrical and dynamic behavior of the system. They simplify the device down to a collection of circuit elements which can be solved using first order ordinary differential equations rather than computationally intense particle codes. Dynamic circuit models for plasma thrusters remain unverified with experimental measurements and their treatment should be considered largely qualitative. Despite this limitation, their results can be used to gain general insight into the governing physics and scaling requirements of plasma thrusters.

Similar to other models, the annular electromagnetic launcher model developed for this research treats the plasmoid as a rigid conducting slug accelerated by a Lorentz force. This simplification masks the AFRC formation where the plasmoid is changing in shape as external magnetic field pressure balances with internal plasma pressure. Instead, it assumes 

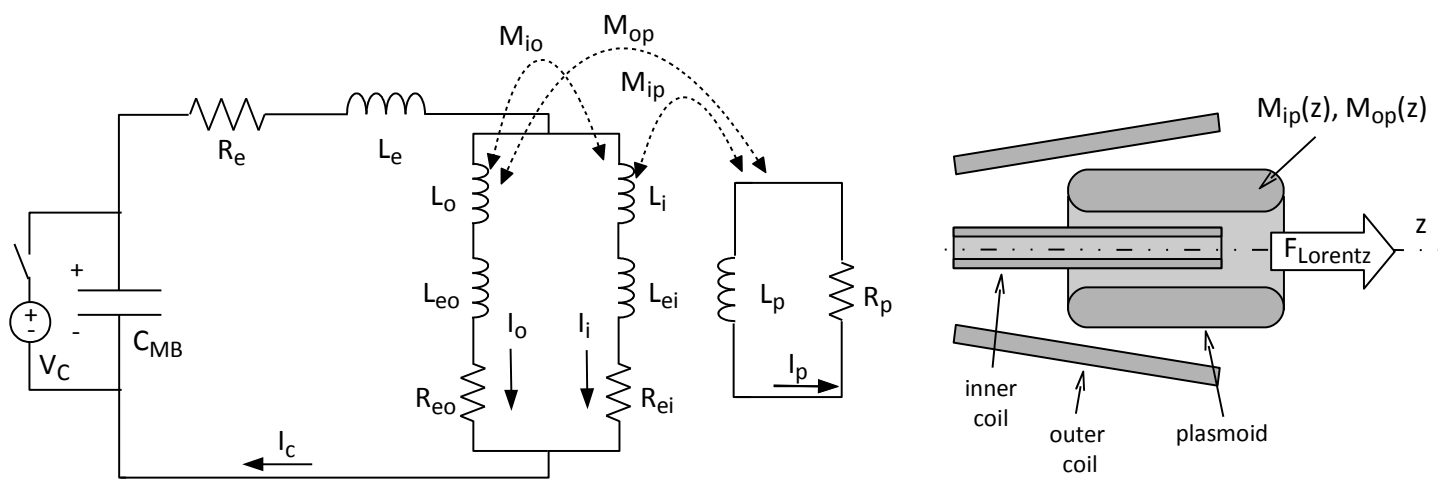

Discharge circuit

Plasmoid circuit

Figure 4.1: Schematic of the annular electromagnetic launcher model. The plasmoid is depicted with circuit elements $L_{p}$ and $R_{p}$, magnetically coupled to the coils through $M_{o p}$ and $M_{i p}$.

that equilibrium is reached and the plasmoid maintains a constant shape. The model also neglects the expansion that occurs when the plasmoid leaves the coils, since this expansion for an AFRC has not been investigated. Additionally, it assumes that all mass is entrained in the plasmoid confines and is accelerated as a bulk. All of these simplifications lead to optimistic results.

The AFRC plasmoid in the model forms inductively and is magnetically coupled to the coils through mutual inductance, which decays as it leaves the coils. The mutual inductance controls the Lorentz force by determining the interaction of the plasma current and the coil current. The currents can be calculated using circuit diagrams. Figure 4.1 provides a pictorial overview of the annular electromagnetic launcher model, operating in the parallel coil mode. A complete mathematical description of the model consists of 7 coupled non-linear ordinary differential equations: 5 circuit equations and 2 equations of motion. The system of equations is introduced in following sections. These equations can be solved using any variety of numerical integration methods. 
The equations of motion for the plasmoid can be derived by calculating the Lorentz force from $\mathbf{J x B}$, but this requires solving several intense integrals. A much easier and equivalent description can be found from conservation of energy, considering how the energy of the system changes as the plasmoid travels out of the coils. Magnetic field energy will change as the mutual inductance changes. This change in magnetic field energy can be related to Newton's 2nd Law through the work-kinetic energy theorem $\left(F_{z}=-\delta U / \delta z\right)$, giving:

$$
m \frac{d u_{z}}{d t}=-\frac{d M_{o p}}{d z} I_{o} I_{o}-\frac{d M_{i p}}{d z} I_{i} I_{p}
$$

where

$$
u_{z}=\frac{d z}{d t}
$$

In this model, position of the plasmoid $z$ is tracked with regard to the trailing edge and $z$ $=0$ occurs at the small cone end of the coils. Computing the total circuit current $I_{c}$, and the coil currents $I_{o}$, and $I_{i}$ requires a circuit analysis of the system, yielding the following equations:

$$
\begin{gathered}
V_{C}(t)=L_{e} \frac{d I_{c}}{d t}+R_{e} I_{c}+\left(L_{o}+L_{e o}\right) \frac{d I_{o}}{d t}+M_{i o} \frac{d I_{i}}{d t}+R_{e o} I_{o}-I_{p} \frac{d M_{o p}}{d z} u_{z}-M_{o p} \frac{d I_{p}}{d t} \\
I_{c}=I_{o}+I_{i} \\
0=\left(L_{o}+L_{e o}\right) \frac{d I_{o}}{d t}+M_{i o} \frac{d I_{i}}{d t}+R_{e o} I_{o}+\left(M_{i p}-M_{o p}\right) \frac{d I_{p}}{d t}+\left(\frac{d M_{23}}{d z}-\frac{d M_{13}}{d z}\right) u_{z} I_{3} \\
-R_{p} I_{p}+L_{p} \frac{d I_{p}}{d t}-M_{o p} \frac{d I_{o}}{d t}-I_{o} \frac{d M_{o p}}{d z} u_{z}-I_{i} \frac{d M_{i p}}{d z}-M_{i o} \frac{d I_{i}}{d t}-R_{e i} I_{i} \\
\frac{d V_{C}}{d t}=-\frac{I_{c}}{C_{M B}} \frac{d I_{i}}{d t}
\end{gathered}
$$


The time derivatives of $M_{o p}$ and $M_{i p}$ in Equations 4.3, 4.16, and 4.6 have been separated into a positional derivative and velocity using the chain rule. Their profiles $M_{o p}(z)$ and $M_{i p}(z)$ as well as the coil and plasmoid inductances $L_{o}, L_{i}$, and $L_{p}$ are determined from the coil and plasmoid geometry. Given the coil radii, lengths, cone angle, and plasmoid geometry, the mutual inductance profiles $M_{o p}(z)$ and $M_{i p}(z)$ and $L_{o}, L_{i}, L_{p}$ can be calculated using analytical equations, experimental methods, or electromagnetic field solvers. As multiple authors have discovered [58], [2],[60], the $M(z)$ profile satisfies an exponential distribution, similar to:

$$
M(z)=k_{0} \sqrt{L_{p} L_{c}} \exp \left(-\left(\frac{\left|z-z_{\text {shift }}\right|}{z_{\text {scale }}}\right)^{n}\right)
$$

or

$$
k(z)=k_{0} \exp \left(-\left(\frac{\left|z-z_{\text {shift }}\right|}{z_{\text {scale }}}\right)^{n}\right)
$$

using

$$
M(z)=k \sqrt{L_{p} L_{c}}
$$

where $L_{c}$ is the coil inductance and $L_{p}$ is the slug inductance. In transformer nomenclature, the coil is considered the primary inductor and the slug is the secondary inductor.

The difference in geometry between the outer coil-plasmoid combination and the inner coil-plasmoid combination will result in different coupling for both systems, meaning that for each geometry combination, the coefficients $k_{0}, z_{\text {scale }}, z_{\text {shift }}, n$ will have unique values $\left(k_{o p}, k_{i p}, z_{o p}, z_{i p}, z s_{o p}, z s_{i p}, n_{o p}, n_{i p}\right)$. It has been found that for large cone angles (greater than $\left.10^{\circ}\right), n$ approaches 1 and $z_{\text {shift }}$ is zero. For small cone angles, $n$ and $z_{\text {shift }}$ increase with decreasing cone angle.

The plasmoid's mass $m$ can be estimated by assuming a fully ionized plasma with uniform 
density $n_{i}$, constant volume $V$, and no mass crossing the plasmoid boundary. The plasmoid mass is then estimated as: $m=n_{i} V M_{m} / N_{A}$, where $M_{m}$ is the molar mass of the plasmoid gas, $N_{A}$ is Avogadro's number. The density is assumed to be equal to the initial background fill of gas, prior to the coil discharge. This profile underestimates the mass, since the density profile measured in experiments peaks on centerline (as shown in Figure 3.7), but it will sufficiently approximate the mass for this basic model.

The plasmoid's resistance $R_{p}$ can be estimated by assuming a uniform plasmoid conductivity, $\sigma_{p}$, a constant cross-sectional area $A$, and an arc length $2 \pi r_{m}$. The plasma resistance is then $R=2 \pi r_{m} / A \sigma_{p}$. The plasma conductivity in FRC's (and likely AFRCs) is difficult to predict, though classical Spitzer conductivity is often used as a best-case substitution. The temperature of an AFRC plasmoid formed with heavy gases is likely between $10 \mathrm{eV}$ and $50 \mathrm{eV}$. For the results presented here, an upper limit of $50 \mathrm{eV}$ was selected. A $50 \mathrm{eV}$ plasma with a density of $1 \times 10^{20} \mathrm{~m}^{-3}$ has a Spitzer conductivity of about $3 \times 10^{5} \mathrm{~S} / \mathrm{m}$. The actual value selected for the simulations was higher at $1 \times 10^{6} \mathrm{~S} / \mathrm{m}$. This overestimate was unlikely to have a drastic impact on the final results, as a senstivity study on plasma conductivity presented in Section 4.2 .8 showed only minor differences in the trajectory results between conductivities of $1 \times 10^{5} \mathrm{~S} / \mathrm{m}$ and $1 \times 10^{6} \mathrm{~S} / \mathrm{m}$.

Since this model assumes a non-expanding plasmoid with a static mass, the impulse bit of the transiting plasmoid is simply the product of the plasmoid mass and the terminal velocity $u_{f}$ :

$$
I_{b i t}=m u_{f}
$$

The energy efficiency of the plasmoid's acceleration can be defined as the final plasmoid kinetic energy compared to the energy initially stored in the capacitor bank. For the 
non-expanding plasmoid, this is equivalent to the system efficiency.

$$
\eta_{E}=\frac{\frac{1}{2} m u_{f}^{2}}{C_{1} V_{0}^{2}}
$$

The launcher model is an initial value problem, requiring the values for $I_{o}, I_{i}, I_{p}, V_{c}, u_{z}$, and $z$ to be specified at $t=0$. These 6 initial conditions, combined with the 7 independent circuit elements $\left(C_{M B}, L_{e}, R_{e}, L_{e o}, L_{e i}, R_{e o}, R_{e i}\right), 5$ coil geometry parameters (outer coil radius, outer coil cone angle, inner coil radius, coil length, coil turns), 2 plasma geometry parameters (height, width), and 2 plasma properties $\left(n_{i}, \sigma_{p}\right)$, require that 22 inputs be specified for the model. This large parameter set can take up to 30 minutes to solve on a single-core processor, with the bulk of the time consumed calculating $M_{o p}(z)$ and $M_{i p}(z)$.

\subsubsection{Numerical Approach}

The model equations (Equations 4.1 4.2 4.34 .4 4.16 4.6 4.7)were integrated using a Mathwork's Matlab ode45 solver. It is an explicit 4th order Runge-Kutta solver, ideal for medium-accuracy, non-stiff problems. The time-steps for the solver were determined internally, using an adaptive technique to minimize errors.

The coil inductances, slug inductance, and mutual inductances were calculated from the geometry of the coils and plasmoid using an electromagnetic field solver, as was done in Reference [2]. The electromagnetic field solver used in this work was COMSOL Multiphysics, with the AC/DC module. All simulations were completed with a 2D-axisymmetric, time-harmonic solver using an estimated frequency from the circuit

parameters. The domain surrounding the coils was expanded to mimic a free-field 
expansion. The coils and slug were modeled as single-turn entities, with multi-turn coils accounted for by multiplying each quantity by the appropriate number of turns $\left(L \propto N^{2}\right)$. The slug was modeled with circular ends to approximate the expected profile for an AFRC and an FRC. A sample geometry is displayed in Figure 4.2 a, with a typical mesh shown in Figure 4.2 b. As is shown in Figure 4.2 b, the mesh for the solver was refined so that at least 3 elements covered a single skin-depth. The number of elements was increased to 10 for important features such as corners. While this increased the computational time for each simulation, resolving the skin-depth was important for accurate results.

COMSOL calculates the inductance directly for single, isolated coils. The inductance for the inner and outer coil was calculated by simulating each coil in an isolated setting, with no surrounding conductors. The inductance of the slug could not be calculated directly by COMSOL, however. Modeling an isolated slug in COMSOL produced a current ring on the inner surface alone, whereas the true inductance of the slug is dictated by current rings on the inner and outer surfaces. To properly resolve these rings, both coils were modeled as the primary inductors with the slug as a secondary inductor. The time-harmonic circuit equations were used with the total simulated current in each coil to calculate $L_{p}$. The time-harmonic equations for the system (neglecting resistance) are

$$
\begin{gathered}
V_{o}=j \omega L_{o} I_{o}+j \omega M_{i o} I_{i}-j \omega k_{o p} I_{p} \\
V_{i}=j \omega L_{i} I_{i}+j \omega M_{i o} I_{o}-j \omega k_{i p} I_{p} \\
0=j \omega L_{p} I_{p}-j \omega M_{o p} I_{o}-j \omega k_{i p} I_{i}
\end{gathered}
$$

Equations 4.134 .15 were re-arranged to find an expression for $L_{p}$, using math software (Sage [61]) to expedite the process. The slug inductance $L_{p}$ calculated from the 


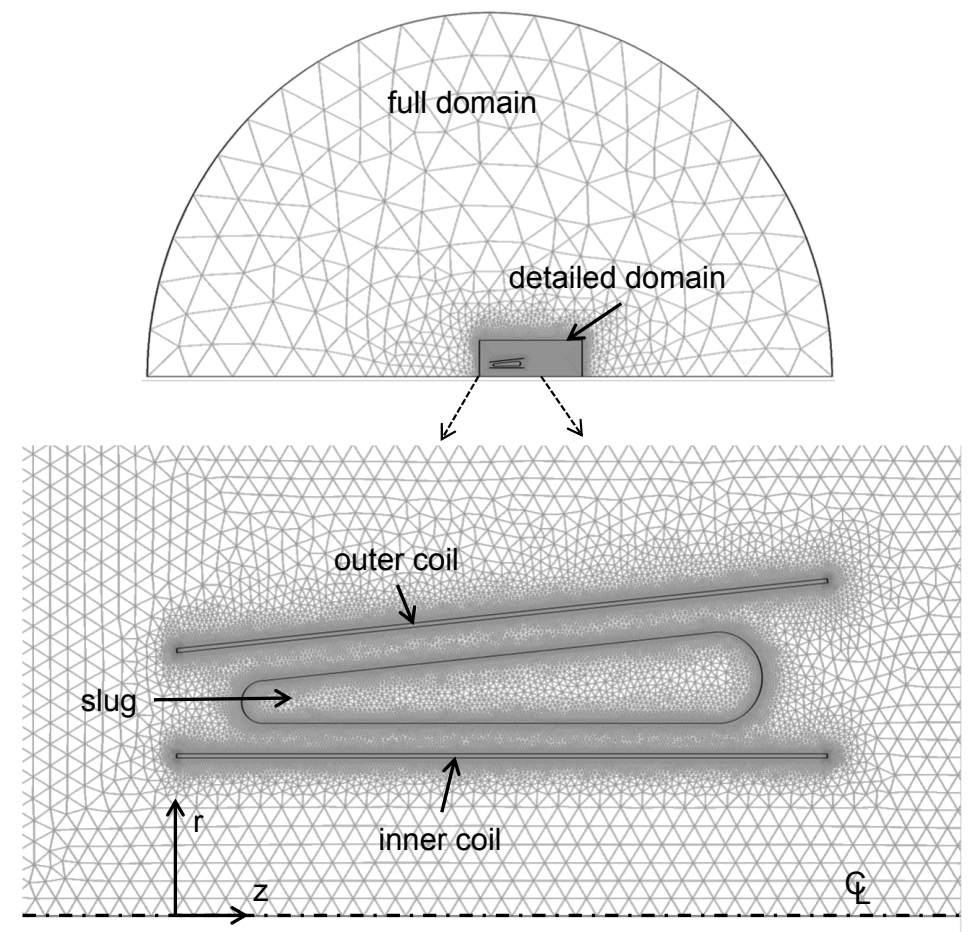

(a)

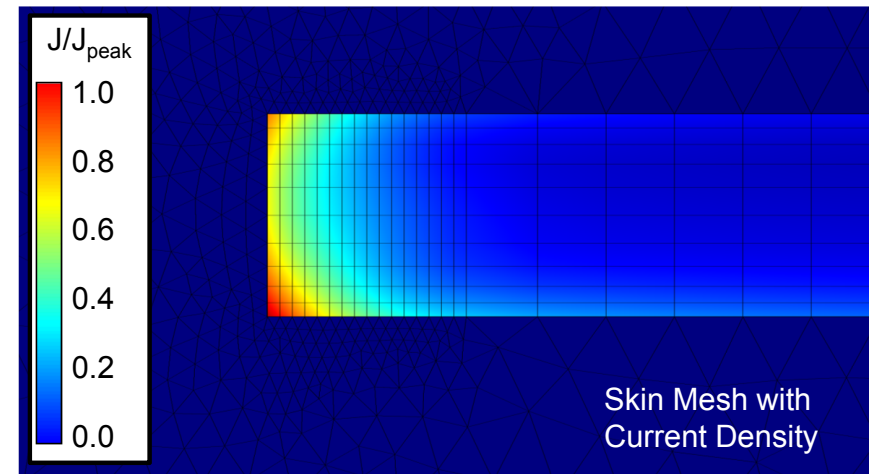

(b)

(c)

Figure 4.2: Geometry and mesh used for the inductance calculations in COMSOL. The coil meshes were refined to resolve the skin depth of the current in the coils. 
time-harmonic circuit equations is

$$
L_{p}=\frac{1}{j \omega I_{p}^{2}}\left(j \omega 2 M_{i o} I_{o} I_{i}+j \omega L_{o} I_{o}^{2}+j \omega L_{i} I_{i}^{2}-V_{o} I_{o}-V_{i} I_{i}\right)
$$

This approach is only valid when the slug is inside the coils. Outside the coils, large numerical errors develop and the slug inductance approaches infinity.

COMSOL also calculates the effective inductance of coupled conductors. The effective inductance $L_{e f f}$ of two conductors can be shown to be [5]

$$
L_{e f f}=L_{1}-\frac{M^{2}}{L_{2}}
$$

where $L_{1}$ is the inductance of the primary coil, $L_{2}$ is the inductance of the secondary coil, and $M$ is their mutual inductance. In transformer nomenclature, the primary coil is the driving coil and the secondary coil is the non-driven coil. The effective inductance can be re-written to express the mutual inductance in terms of the coupling coefficient $k$ [2] so that

$$
L_{e f f}=L_{1}\left(1-k^{2}\right)
$$

The effective inductance of the inner and outer coil was calculated in COMSOL and Equation 4.17 was used to calculate $M_{i o}$. The effective inductance between each coil and slug was also calculated in COMSOL, with each coil as the primary inductor. The effective inductance as a function of position was calculated by moving the slug to several axial locations. The coupling coefficients $k_{o p}(z)$ and $k_{i p}(z)$ were calculated from these data using Equation 4.18 .

The technique for calculating the coupling coefficients $k_{o p}(z)$ and $k_{i p}(z)$ was not ideal, 
since it did not properly resolve the inner and outer current rings in the slug. A more accurate technique might have been to calculate the coupling coefficients $k_{i p}$ and $k_{o p}$ from the time-harmonic equations (Equations 4.13-4.15) for the full system. However, large numerical errors produced when the slug is immediately outside the coils lead to gross errors in the calculated trajectories using this method. This method calculated the mutual inductances $M_{o p}$ and $M_{i p}$ increasing towards infinity, leading to rapidly increasing Lorentz forces. In reality, the Lorentz force decays as the slug moves away from the coil since the magnetic fields between the coils and slug no longer interact.

The coupling coefficient data $k_{i p}(z)$ and $k_{o p}(z)$ were used with a curve-fitting tool in $\operatorname{MatLab}(\mathrm{R})$ to estimate coefficients for the empirical fits, described by Equation 4.9. These coefficients were used with an analytical expression to calculate the positional derivatives $d M_{o p} / d z$ and $d M_{i p} / d z$ as well.

\subsubsection{Model Validation}

Validation of the AELM model was limited, since experimental results for comparison are not available for the annular launcher configuration. Despite this, an effort was made to ensure the numerical techniques and model equations were comparable to results from other single coil launcher studies. Two different studies were conducted to this end: a study to check that the equations of motion agreed with results from a single-coil launcher study and a study using COMSOL's electromagnetic modeling to ensure that it could calculate a reasonable coupling profile.

The first validation study compared the annular electromagnetic launcher model equations 
against results from a numerical electromagnetic launcher study with a single flat coil with a rigid flat armature by Novac, et al [1]. Novac's flat coil launcher was modeled using a similar circuit equation approach to the one presented here and compared it against experimental results for terminal velocity. The annular electromagnetic launcher model was tested with the experimental inputs provided in Table 1 by Novac, et al [1]. Current through the inner coil circuit for the annular launcher was minimized by setting the resistance $R_{e i}$ to $100 \Omega$. The mutual inductance between the inner coil and slug was turned off by setting the inner coil inductance $L_{2}$ to zero. This reduced the dual coil model to a single coil launcher. The mutual inductance profile was not provided in Reference [1], but other authors [2] used $k_{o p}=0.98, z_{o p}=0.005, n_{o p}=1.0$ in a separate validation study with success. These values for the coupling profile were used in this study as well. The results with the modified annular electromagnetic launcher using the circuit parameters from [1] are shown in Figure 4.3. The current, velocity, and position history shown in Figure 4.3 are in good agreement with the original results. Novac, et al report a terminal velocity of $4.0 \mathrm{~km} / \mathrm{s}$ for their experimental study. The terminal velocity predicted with the modified annular model was $3.78 \mathrm{~km} / \mathrm{s}$, within $6 \%$ of the reported results. This validation study ensures that the equations of motion and modified circuit equations from the annular electromagnetic launcher are in agreement with published experimental data from single-coil launcher studies.

The second validation study was completed to ensure that COMSOL software could be used to predict a reasonable coupling profile for a single coil and projectile. The target coupling profile was from an experimental demonstration of coil-slug coupling by Martin and Eskridge [2]. In this experiment, a slug of aluminum was moved away from a conical discharge coil and the resulting effective inductance of the coil was measured. Martin and Eskridge's geometry was duplicated in COMSOL and the effective inductance was 


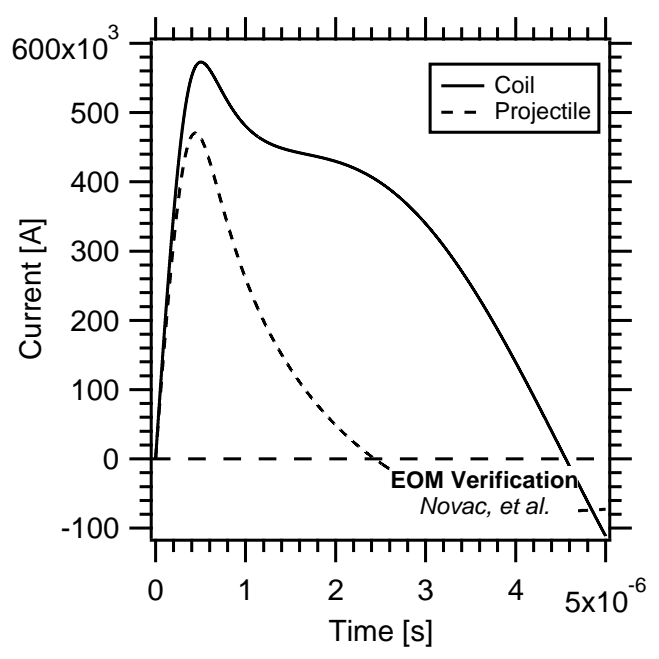

(a)

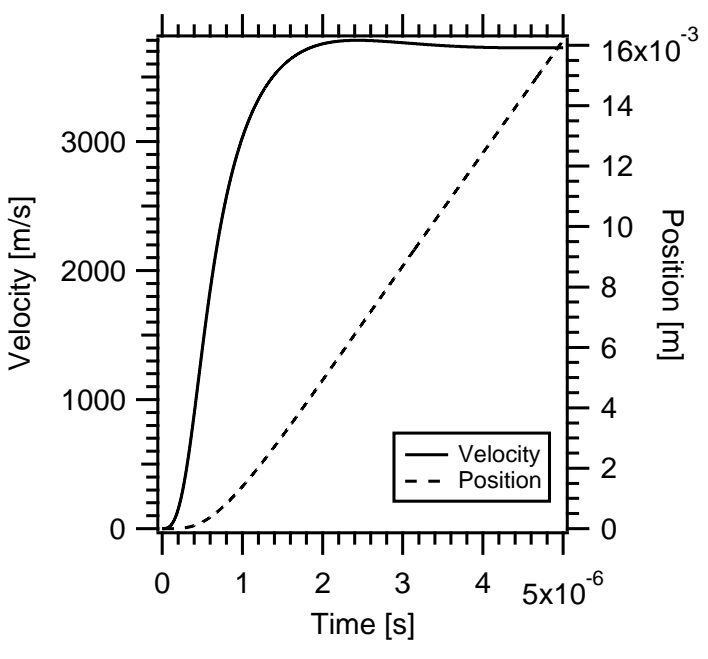

(b)

Figure 4.3: Results from the modified annular electromagnetic launcher model, using a test case presented by Novac, et al [1].

computed. The slug dimensions were not provided, so a $2 \mathrm{~mm}$ offset was used to model the slug. A comparison of the COMSOL results with Martin and Eskridge's experimental results are compared in Figure 4.4. A curve fit to the COMSOL data shows good agreement with Martin and Eskridge's experimental data, with a $12 \%$ difference between the experimental data and the profile predicted by COMSOL. The curve-fit produced the coefficients for the fit to the exponential $L_{e f f}$ profile: $L_{c}=27 \mathrm{nH} \pm 68 \mathrm{pH}, k_{o}=89 \pm 0.004$, $z_{0}=52 \mathrm{~mm} \pm 1 \mathrm{~mm}$. A sizeable difference between the COMSOL data and experimental data published by Martin and Eskridge is likely due to a slight difference in the modeled slug geometry, since this was not provided by the authors. However, the agreement between the two results supports the use of COMSOL for computing coupling profiles for single-coil geometries. 


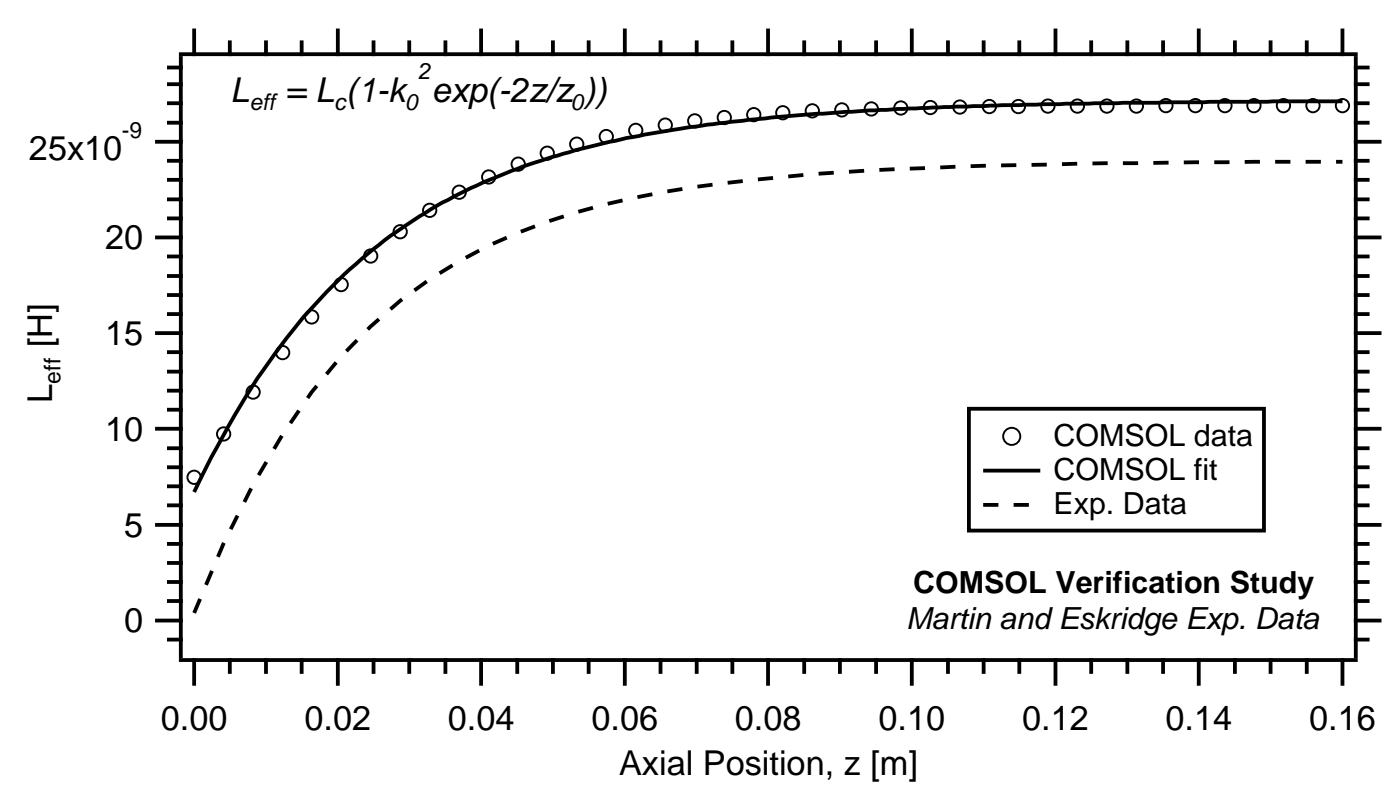

Figure 4.4: Effective inductance of a slug-coil geometry calculated using COMSOL. Experimental data from Reference [2] are shown with the dotted line.

\subsection{Design Studies for the XOCOT-T3 Experiment}

The annular electromagnetic launcher model was used to make design decisions for the XOCOT-T3 AFRC translation experiment. Various input parameters were used with the model to estimate the trajectory of a plasmoid and acceleration efficiency. Input parameters favorable to plasmoid ejection from the coils were considered in the final design. The design process was simplified by constraining several inputs to the model based on existing hardware or hardware limitations. These inputs included outer coil dimensions, capacitance values, and current limits. The remaining inputs for the external circuit, including the half-angle of the outer coil, inner coil dimensions, charge voltages, coil inductances, stray inductances, and resistances were varied across a limited range of feasible values to study their effects. A final design was selected from the range of inputs tested. The primary goal of this study was to find an experimental design which would expel the plasmoid from 
the formation chamber during the plasmoid's expected lifetime or before the magnetic confinement of the plasmoid is expected to disappear. If the plasmoid configuration terminates prior to expulsion from the coils, it will produce no directed impulse. Therefore, it is critical to eject the plasmoid before the end of its lifecycle. The secondary goal was to maximize the design's projected energy efficiency and the plasmoid's velocity. Sensitivity studies on the plasmoid geometry and properties were conducted to estimate their impacts to the plasmoid's trajectory with the final design.

One of the most important considerations in the design process was that the final design must result in plasmoid ejection from the coils prior to the expected lifetime of the plasmoid. As stated previously, it is critical to eject the plasmoid prior to its termination so it can impart impulse on the coils. If the plasmoid extinguishes inside the coils, it will produce little thrust. Previous results on AFRC formation studies found that the lifetime of an AFRC plasmoid was limited to the rise-time or quarter-cycle of the coil currents. This is discussed in Section 3.3.5. The model therefore required that a plasmoid must leave the coils within the first quarter cycle of the circuit discharge for a successful outcome. The quarter cycle for each case was determined by simulating a stationary plasmoid coupled the coils. This ensures the plasma-coil coupling determines the frequency rather than relying on a vacuum (no plasma) waveform. The two cases can be very different. The lifetime definition is displayed graphically in Figure 4.5. A vacuum waveform is shown along with waveforms when the plasma is present in the coils (stationary and translating case). The plasmoid inductively shorts the coils, leading to a higher frequency and larger current than in the vacuum case. This lifetime limit assumes that the plasmoid maintains coherency and stability after it ejects from the coils. Both assumptions are unlikely to hold in the translation experiment where a free-field expansion is expected to alter the downstream plasma dynamics considerably, though their effects must be determined experimentally. 


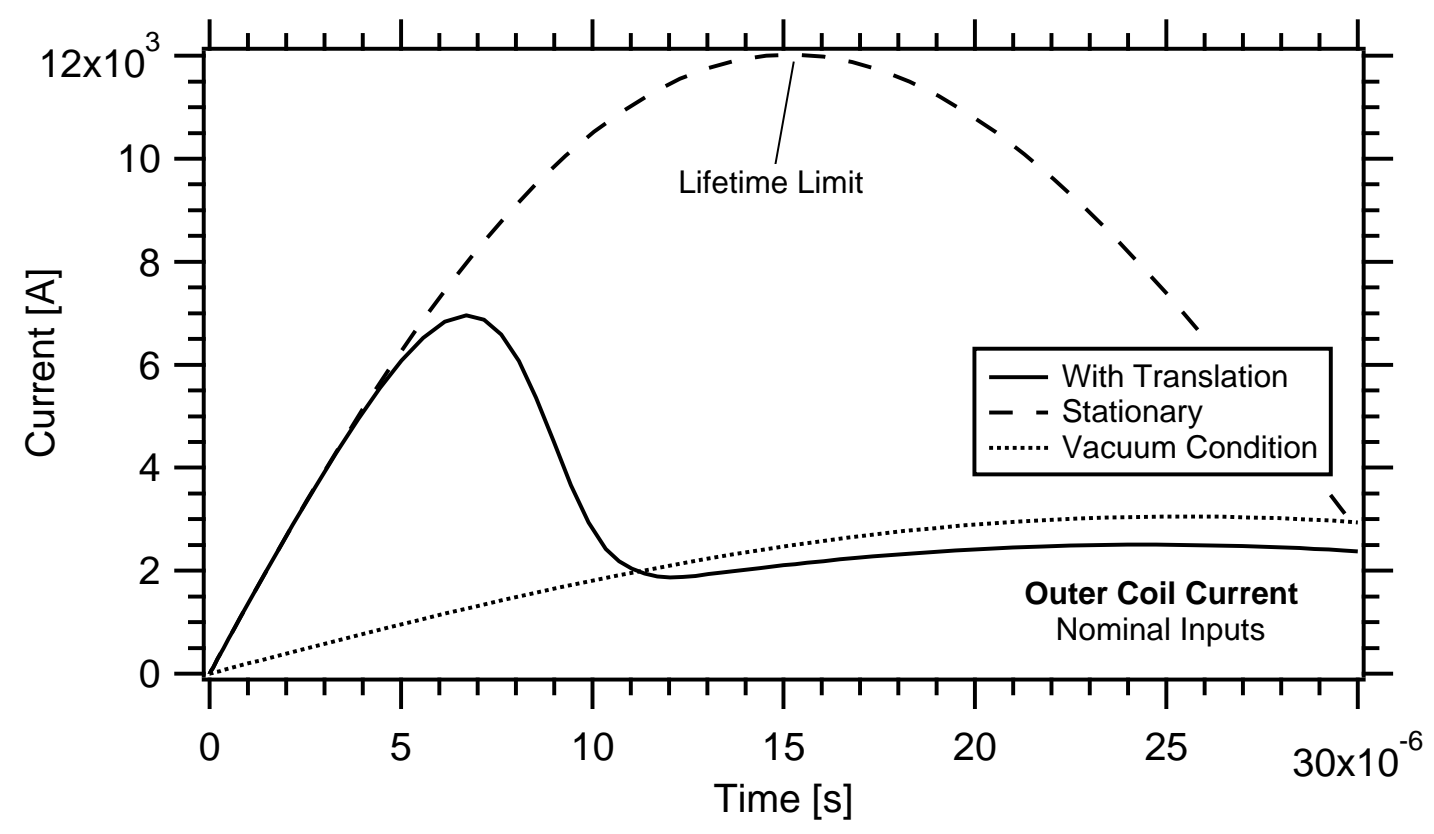

Figure 4.5: Definition of the plasmoid lifetime limit, as determined by the peak in the coil-plasma coupling case where the plasmoid is not allowed to translate.

The expansive design space of the experiment was significantly narrowed by applying design constraints to the problem. Most of these design constraints originated from limits or dimensions of existing or readily available hardware. The design constraints were as follows:

1. Capacitances should be either $225 \mu \mathrm{F}$ or $43.5 \mu \mathrm{F}$.

2. The outer coil should be $30 \mathrm{~cm}$ in length and $24 \mathrm{~cm}$ in diameter to match insulator and vacuum tank dimensions. The cone angle should not exceed $12^{\circ}$.

3. The inner and outer coils must have the same number of turns for a radially balanced plasmoid and should be equal in length.

4. Charge voltages cannot exceed $15 \mathrm{kV}$.

5. Total currents cannot exceed $30 \mathrm{kA}$ (for the $225 \mu \mathrm{F}$ capacitor) and $50 \mathrm{kA}$ (for the 


\section{$43.5 \mu \mathrm{F}$ capacitor).}

The design space was further reduced by applying limits to several parameters, based on their expected ranges and what could be attained with realistic hardware. These limits were as follows:

1. $L_{e}, L_{e o}, L_{e i}: 100 \mathrm{nH}$ to $1 \mu \mathrm{H}$

2. $R_{e o}, R_{e i}: 1 \mathrm{~m} \Omega$ to $30 \mathrm{~m} \Omega$

3. $R_{e}: 10 \mathrm{~m} \Omega$ to $50 \mathrm{~m} \Omega$

4. $r_{i}: 2.5 \mathrm{~cm}$ to $7.2 \mathrm{~cm}$

The plasmoid dimensions for each case were scaled according to the coil dimensions. The plasmoid length and thickness were specified as fractions of the coil length and annular channel width, respectively. The plasmoid was assumed to start its trajectory at $10 \%$ of the coil length (or $3 \mathrm{~cm}$ in from the small end of the coil). The plasmoid's radial position was centered between the coils. This is in contrast to the equilibrium position derived in Section 3.3.1, which found that the radial position of the plasmoid splits the annulus into equal areas. The assumptions required for this derivation, namely that the dimensions of all inductors resemble ideal solenoids and the plasmoid is infinitesimally thin, are not valid for the plasmoid description in the launcher model. The actual plasmoid position in the experiment will be governed by the reversed field the plasmoid generates as well as the thickness of the plasmoid. These are difficult to predict beforehand, so it was appropriate to fix the plasmoid conditions arbitrarily. A sensitivity study was conducted to determine how the plasmoid trajectory was affected by the plasmoid conditions (thickness, length, conductivity). 
Nominal input parameters were chosen to simplify the design studies. This allowed the effects of one or two parameters to be studied individually. Nominal input parameters to the model are displayed in Table 4.1

Table 4.1

Nominal inputs for design study

\begin{tabular}{lcc}
\hline Component & Symbol & Value \\
\hline Capacitor & $C_{M B}$ & $225 \mu \mathrm{F}$ \\
& & $43.5 \mu \mathrm{F}$ \\
External inductance & $L_{e}$ & $200 \mathrm{nF}$ \\
Outer stray inductance & $L_{e o}$ & $100 \mathrm{nF}$ \\
Inner stray inductance & $L_{e i}$ & $100 \mathrm{nF}$ \\
External resistance & $R_{e}$ & $10 \mathrm{~m} \Omega$ \\
Outer stray resistance & $R_{e o}$ & $1 \mathrm{~m} \Omega$ \\
Inner stray resistance & $R_{e i}$ & $1 \mathrm{~m} \Omega$ \\
\hline Outer coil radius & $r_{o}$ & $12 \mathrm{~cm}$ \\
Outer coil length & $l_{o}$ & $30.5 \mathrm{~cm}$ \\
Outer coil half-angle & $\alpha$ & $6^{\circ}$ \\
Coil turns & $N_{i}, N_{o}$ & 4 \\
Inner coil radius & $r_{i}$ & $7.2 \mathrm{~cm}$ \\
Inner coil length & $l_{i}$ & $30.5 \mathrm{~cm}$ \\
Outer stray resistance & $R_{e o}$ & $1 \mathrm{~m} \Omega$ \\
Inner stray resistance & $R_{e i}$ & $1 \mathrm{~m} \Omega$ \\
\hline Plasma density & $n_{i}$ & $1.29 \times 10^{20} \mathrm{~m}$ \\
Plasma conductivity & $\sigma_{p}$ & $1 \times 10^{6} \mathrm{~S} / \mathrm{m}$ \\
Plasma length & $l_{p}$ & $80 \% l_{c}$ \\
Plasma width & $\Delta r_{m}$ & $40 \%\left(r_{o}-r_{i}\right)$ \\
Plasma radius & $r_{m}$ & $\left(r_{o}+r_{i}\right) / 2$ \\
\hline Charge voltage & $V_{c}$ & $1.9 \mathrm{kV}$ \\
(225 $\mu F$ ) & & $400 \mathrm{~J}$ \\
Charge voltage & $V_{c}$ & $5.2 \mathrm{kV}$ \\
(43.5 $\mu F$ ) & & $600 \mathrm{~J}$ \\
& &
\end{tabular}




\subsubsection{Results for Nominal Case}

The plasmoid trajectories and coil currents from the annular electromagnetic launcher model were computed using the nominal inputs displayed in Table 4.1. This was done to gain a basic understanding of the behavior of a translating plasmoid slug. The time history of coil currents, plasma current, plasmoid velocity, capacitor voltage, and plasmoid axial position is shown in Figure 4.6. Vacuum coil currents (no plasma in coils) are shown for comparison. The plasmoid adds an inductive load to the circuit, indicated by the increased frequency and increased coil currents compared to the vacuum case. The plasma current is much higher than the coil currents due to its lower inductance. The plasmoid gains velocity quickly, within $2 \mu$ s of the simulation start. The coil and plasma currents start to decay at $7 \mu \mathrm{s}$ as the leading edge of the plasmoid passes the end of the coil. The inductive load begins to decrease at this time, though the velocity continues to climb. The plasmoid slug completely exits the coil $(\mathrm{z}=0.3 \mathrm{~m})$ at $10 \mu \mathrm{s}$ and terminal velocity is reached $2 \mu \mathrm{s}$ later. The plasma current returns to zero and the coil currents return to their vacuum frequency. The plasmoid slug is no longer able to inductively couple with the coils this far downstream, leading to the decay in plasma current and loss of increased inductive loading on the coils. The plasmoid slug reaches its lifetime limit at $15 \mu \mathrm{s}$, terminating at $0.8 \mathrm{~m}$ downstream.

The positional dependence of the the mutual inductance gradient $(\mathrm{dM} / \mathrm{dz})$ and the Lorentz force for the nominal case is displayed in Figure 4.7. From this graph, it is apparent that the inner coil is not responsible for the initial acceleration of the plasmoid; the initial plasmoid acceleration is due to the outer coil alone. The mutual inductance gradient for the inner coil is less than the mutual inductance gradient of the outer coil. The outer coil mutual inductance gradient peaks before the inner coil and is greater in magnitude. This indicates 

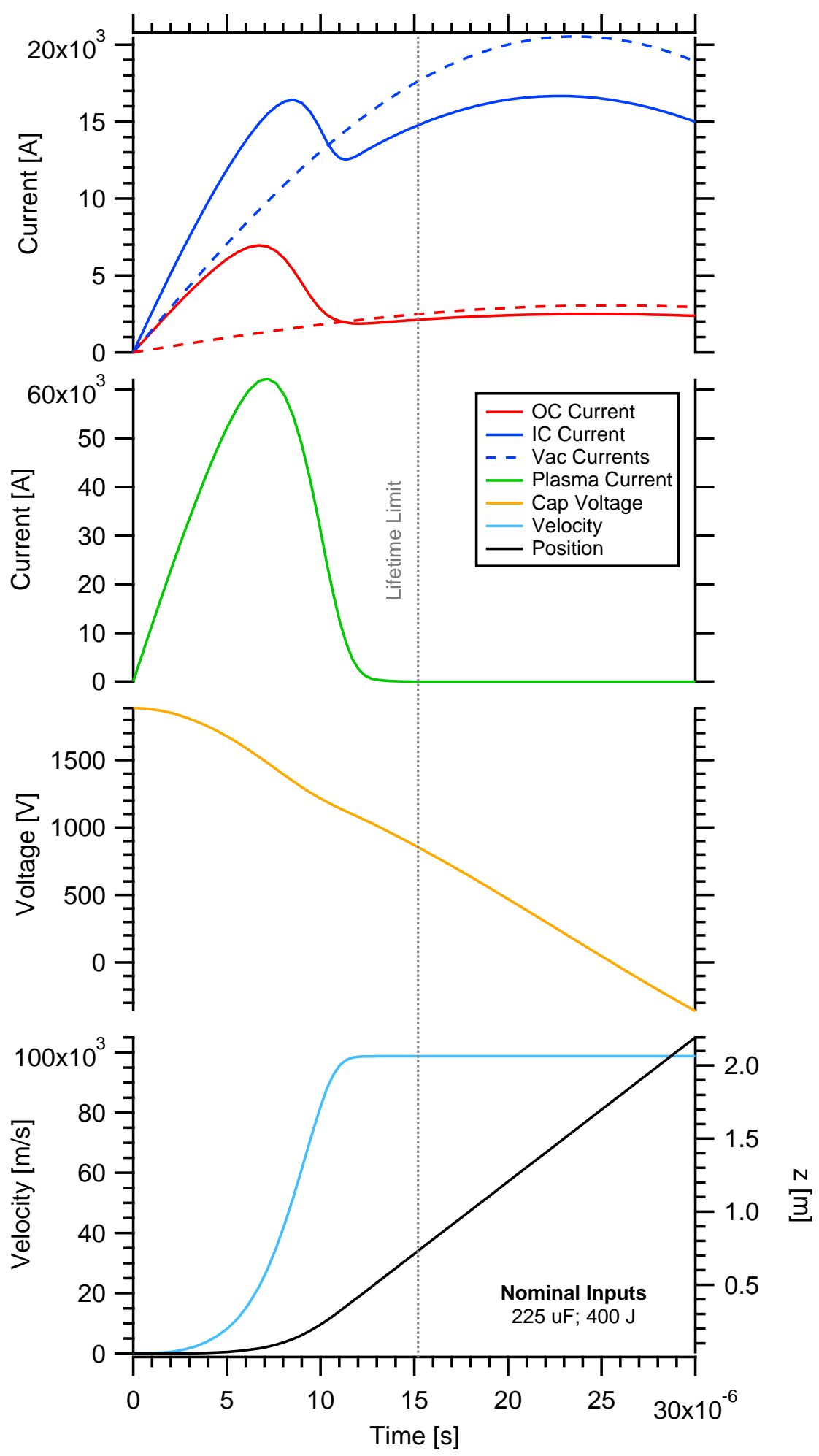

Figure 4.6: Results from the launcher model, including coil currents, plasma current, capacitor voltage, plasmoid velocity, and axial plasmoid position as a function of time for nominal inputs. 

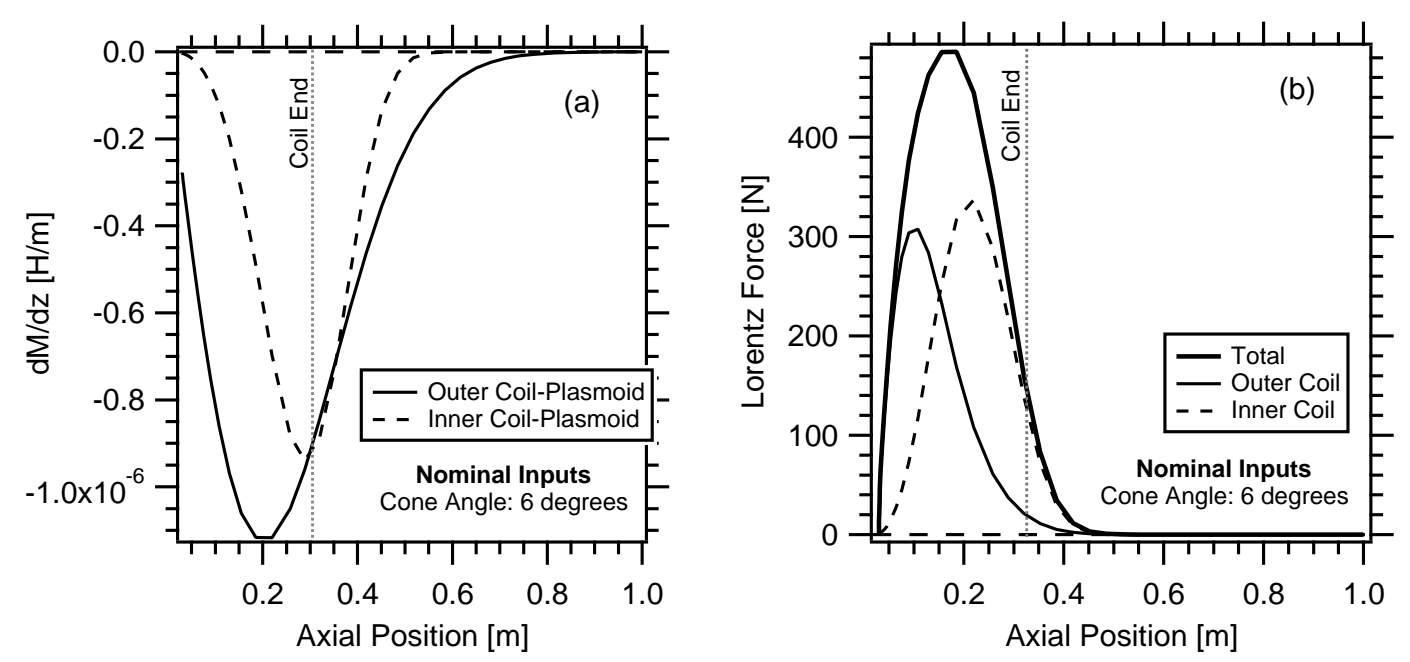

Figure 4.7: Positional dependence of the mutual inductance gradient for the inner and outer coil and the combined Lorentz force.

that both coils will push on the plasmoid slug at different times. Examining the plot of the Lorentz force, this is readily apparent. The outer coil is responsible for the acceleration during the first half of the coil and the inner coil largely accelerates the plasmoid through the second half of the coil. The peak Lorentz force occurs somewhere between these zones. This disparity in acceleration zones is likely a result of the cone angle from the outer coil. The effects of cone angle on effective acceleration distance is discussed in Section 4.2.2.

The results in Figure 4.7 make it clear that the inner coil delivers a Lorentz force even though it is not conical. While this is explained by the mutual inductance gradient displayed in Figure 4.7, an alternate explanation is offered from a vector perspective. The inner coil produces a radial magnetic field at the ends of the coil similar to magnetic mirror. As the plasmoid travels past the end of the coil, the radial magnetic field and the azimuthal plasma current exert an axial Lorentz force on the slug. The delayed acceleration is apparent in Figure 4.7. When the plasmoid nears the end of the coil, the inner coil has a greater effect. At the beginning of the coil, the inner coil exerts very little axial force on the plasmoid. 
The momentum delivered by the exhausting plasmoid is $2.29 \mathrm{mN}$-s. The efficiency of the system (calculated using Equation 4.12) for the nominal inputs was $27 \%$. The low efficiency can be explained by observing the coil current waveforms. After the plasmoid slug leaves the coils, the coil currents continue to climb. They do not decay to zero like the plasmoid current. The additional coil currents indicate that not all of the energy from the capacitor was able to couple with the plasmoid before it exited the coils. Examining the voltage history, it is apparent that the plasmoid slug left the coils while there was still a significant voltage across the capacitor. The efficiency can be increased if the plasmoid remains inside the coils for a longer duration, exiting when the coil currents are at their peak to maximize the Lorentz force. This requires either increasing the plasmoid's mass, decreasing the charge voltage to decrease the coil currents, or reducing the cone angle to lower the radial magnetic field. These effects slow the plasmoid's trajectory for optimal efficiency. However, if the plasmoid is allowed to remain in the coils for too long it can terminate before ejecting. This discussion makes it clear that even in an ideal accelerator like the one described by this model, fine tuning is required to maximize efficiency.

\subsubsection{Cone Angle Study}

The effect of cone angle was studied for the limited range of $2^{\circ}$ to $12^{\circ}$. The maximum cone angle of 12 degrees was determined based on the dimensions of the mating vacuum port for the experiment. The model was used with varying cone angles and nominal inputs to compute the final position, velocity, and energy efficiency of the plasmoid as a function of cone angle. This was done for 4 different inner coil radii. The results for both capacitor banks are displayed in Figure 4.8. The smallest cone angle results in the longest trajectory, highest velocity, and greatest efficiency. This is the case for several different inner coil 

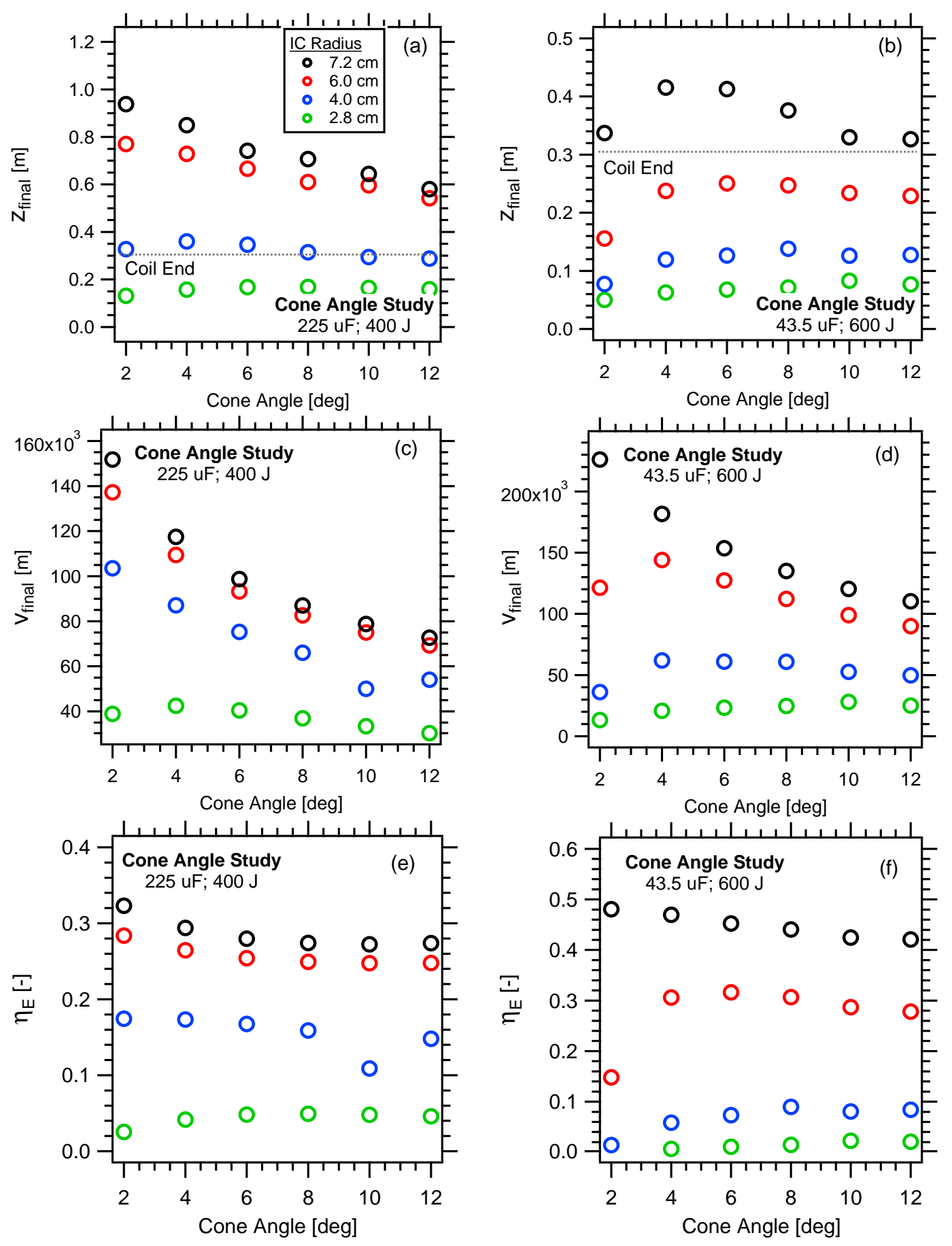

Figure 4.8: Results from the cone angle study, including the final plasmoid position, final velocity, and energy efficiency. Results are shown for 2 different capacitor banks. 

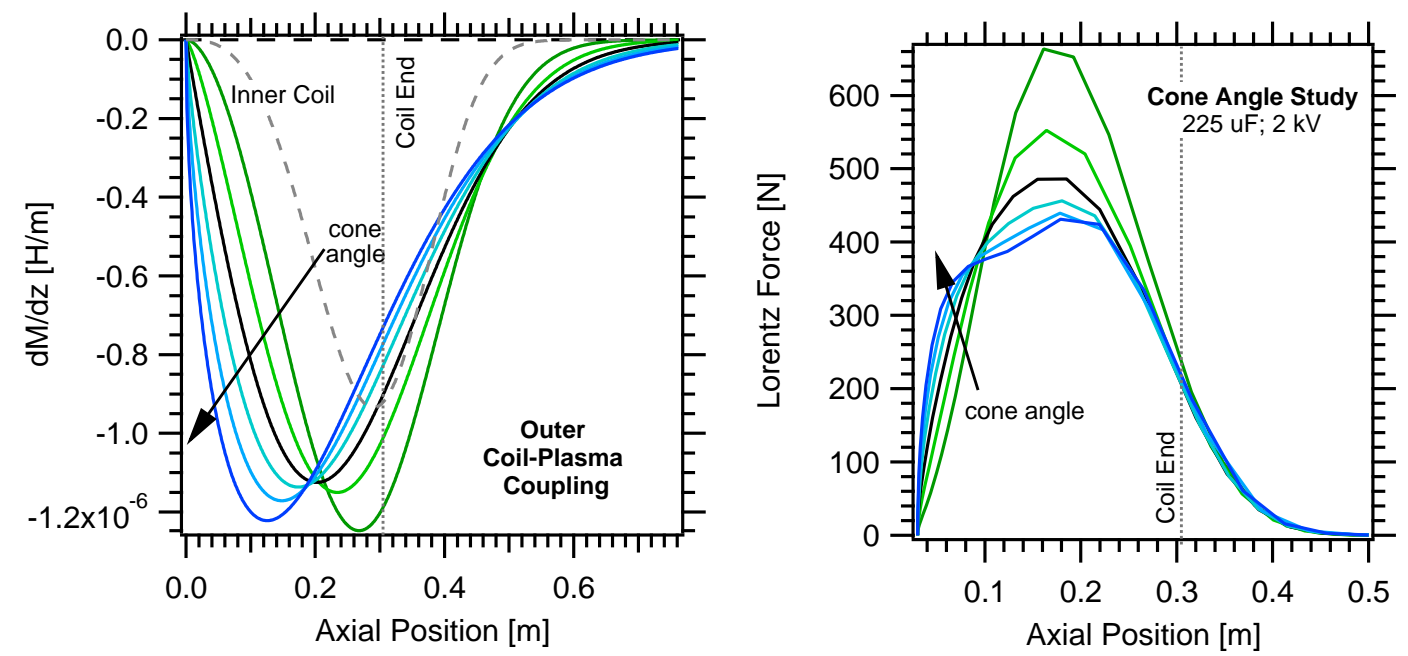

Figure 4.9: Mutual inductance gradient and Lorentz force as a function of slug position. Several different cone angles are shown, increasing in the arrow direction.

radii. This behavior holds only if the plasmoid has sufficient energy to escape the coils completely. The results from the $43.5 \mu \mathrm{F}$ case illustrate this for the largest inner coil radius $(7.2 \mathrm{~cm})$. The 2 degree cone was not sufficient to accelerate the plasmoid far enough from the coils in the allotted time and its final position fell short of the larger cone angle cases. The 4 degree cone launched the plasmoid farther than the larger cone angles.

The increase in plasma position with decreased cone angle is not expected. Larger cone angles exert greater Lorentz forces on the plasmoid slug due to the increased radial magnetic field and this means they should expel it further. To understand why the results for the coaxial geometry were different than expected, the $\mathrm{dM} / \mathrm{dz}$ profiles and Lorentz force profiles were displayed as a function of axial distance from the small end of the coil $(\mathrm{z}=0)$. The results are displayed in Figure 4.9. As is apparent in Figure $4.9 \mathrm{a}$, a larger cone angle exerts a larger Lorentz force on the slug for the far upstream regions ( $\mathrm{z}$ $<0.1 \mathrm{~m}$ ). This is due to a greater $\mathrm{dM} / \mathrm{dz}$ in the upstream regions of the coil. The greater $\mathrm{dM} / \mathrm{dz}$ means the larger cone angles will accelerate the plasmoid sooner than smaller cone 
angles. However, maximizing the Lorentz force is about timing the peak coil currents with the peak $\mathrm{dM} / \mathrm{dz}$ for each coil. The peak $\mathrm{dM} / \mathrm{dz}$ for smallest cone angle aligns with the peak $\mathrm{dM} / \mathrm{dz}$ for the inner coil. This means the combined Lorentz force (inner and outer) will be greatest for the smallest cone angle, provided the plasmoid can accelerate to this region within its lifetime. The greater Lorentz force results in greater terminal velocities, greater acceleration efficiencies, and longer trajectories. For an annular FRC thruster, these results suggest that small cone angles are better for maximizing performance than large cone angles. Small cone angles allow the regions of peak $\mathrm{dM} / \mathrm{dz}$ for the inner and outer coil to overlap so that the maximum Lorentz force can be achieved.

\subsubsection{Inner Coil Radius Study}

The effect of the inner coil radius on the plasmoid trajectory was investigated for four different coil radii: $2.8 \mathrm{~cm}, 4.0 \mathrm{~cm}, 6.0 \mathrm{~cm}$, and $7.2 \mathrm{~cm}$. The smallest radius was chosen to match the aspect ratio of the CSS design[15]. The largest coil radius was selected as the maximum radius that would allow clearance for quartz insulators and a $2.5 \mathrm{~cm}$ wide channel. The model was used with these different inner coil radii to compute the final position, velocity, and energy efficiency of the plasmoid as a function of cone angle. The results for both capacitor banks are displayed in Figure 4.10, As was discovered in Section 4.2 .2 , the largest inner coil radius creates the longest trajectory, highest velocity, and greatest energy efficiency. The smallest radius fails to eject the plasmoid from the coils for both capacitor banks indicating that more energy will be required for successful translation with this radius. The inner coil radius improves the performance of the annular launcher for two reasons: improved coupling and increased Lorentz forces. These are illustrated in Figure 4.11. The larger radius increases the coupling $k$ between the inner coil 

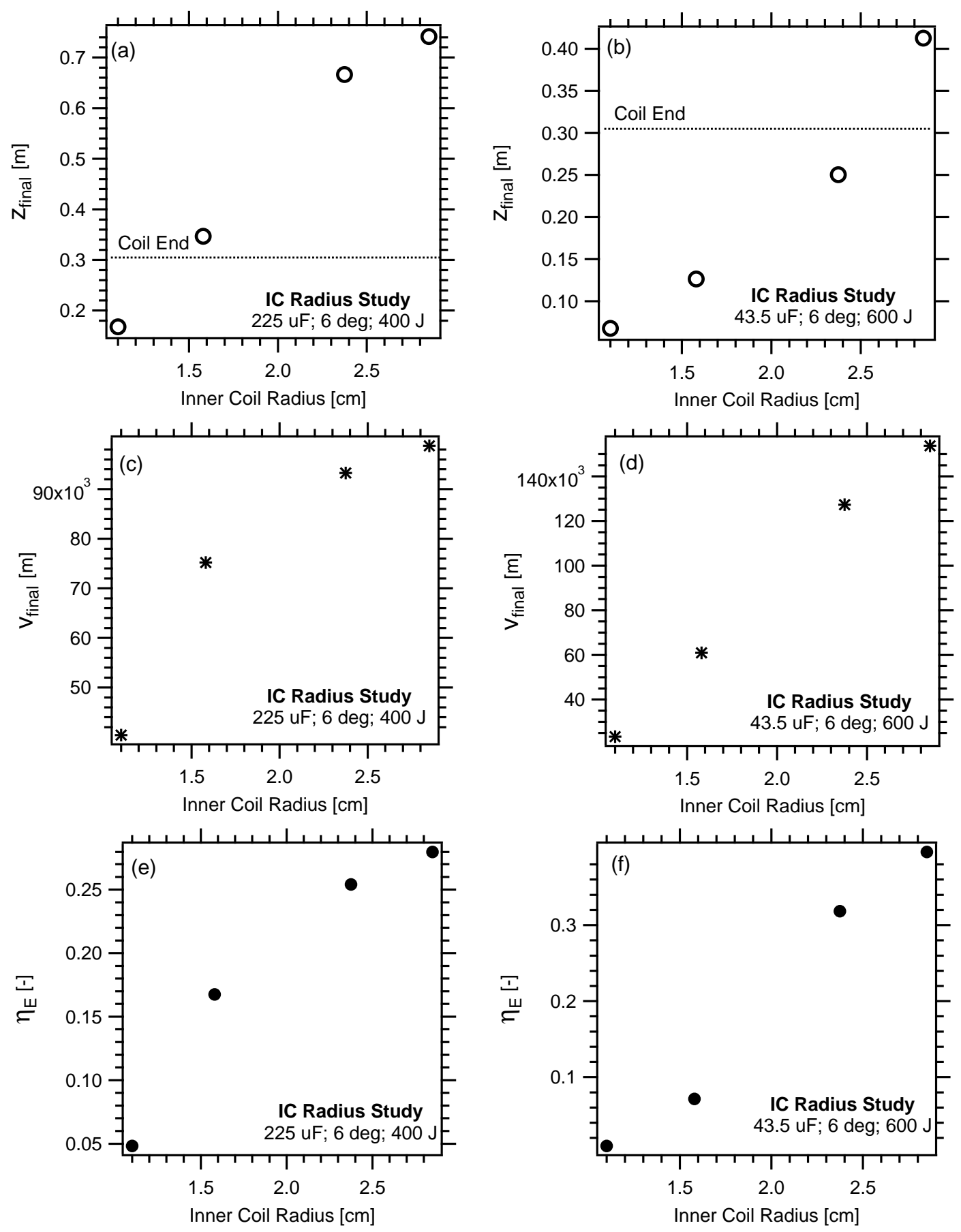

Figure 4.10: Results from the inner coil radius study, including the final plasmoid position, final velocity, and energy efficiency. Results are shown for 2 different capacitor banks. 

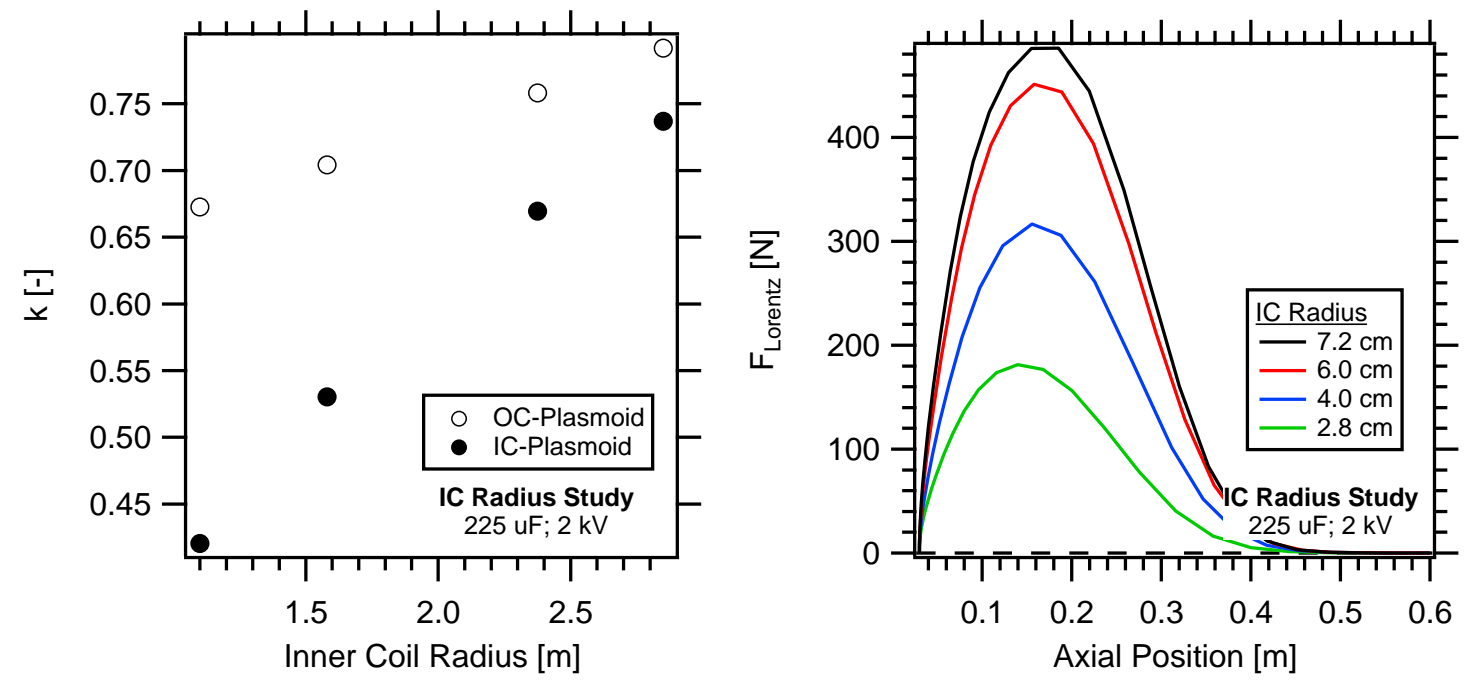

Figure 4.11: Coupling coefficients and Lorentz force computations for several inner coil radii.

and plasmoid and the outer coil and plasmoid, as the conductors are brought into closer proximity. The Lorentz force also increases with increased inner coil radius. This is the result of more effective coupling between the coils and plasmoid and the larger inner coil inductance $L_{i}$. An increased $L_{i}$ increases $d M_{i p} / d z$ which is directly proportional to the Lorentz force and also drives more current to the outer coil improving the outer coil's effectiveness. These findings conclude that an annular FRC thruster should maximize the inner coil radius for improved performance.

\subsubsection{Initial Energy Study}

The initial energy for the system was studied to determine its effect on the plasmoid trajectory, final velocity, and system efficiency. The range of energies studied was restricted to a narrow range, based on maximum current ratings of each circuit. The maximum energy for the $225 \mu \mathrm{F}$ capacitor circuit was $500 \mathrm{~J}$ and the maximum energy for the $43.5 \mu \mathrm{F}$ circuit 
was $750 \mathrm{~J}$. These ratings were based on current levels attained when a plasmoid was present in the coils; the vacuum current levels were much lower. The results for final plasmoid position, final velocity, and energy efficiency are shown in Figure 4.12.

The results from the initial energy study show the plasmoid travels a longer distance and with a greater velocity when the initial energy stored in the capacitor is increased. If the plasmoid travels past the end of the coils, the efficiency will be greatest for the lowest energy settings. This is most apparent in the $225 \mu \mathrm{F}$ data. The $100 \mathrm{~J}$ setting contains too little energy to expel the plasmoid from the coils in the allotted time. When the initial energy is increased to $200 \mathrm{~J}$, the plasmoid is able to escape the coils and does so with a greater efficiency than the $300 \mathrm{~J}$ and higher settings. The drop in efficiency with increased energy can be understood by examining the Lorentz force equation, Equation 4.1. Maximizing the force and energy delivered to the plasmoid requires timing the peak $\mathrm{dM} / \mathrm{dz}$ with the peak currents. The peak $\mathrm{dM} / \mathrm{dz}$ occurs at the far end of the coils, as shown in Figure 4.7. Higher discharge energy settings cause the plasmoid to travel past the end of the coils before the coil currents peak. This finding underscores the importance of optimizing the timing for electromagnetic launchers to achieve peak efficiency. To maximize effiency it is critical to time the plasmoid exit with the peak coil currents.

The results from the initial energy study also show that the minimum energy required to eject the plasmoid from the coils is different for different size capacitor banks. The $225-\mu \mathrm{F}$ bank requires a minimum of $200 \mathrm{~J}$ (under otherwise nominal conditions) to eject the plasmoid and the $43.5-\mu \mathrm{F}$ capacitor bank requires at least $600 \mathrm{~J}$ to eject the plasmoid. The disparity in minimum energy for successful translation can be explained by noting that the discharge frequency for the smaller bank is twice that of the larger bank. The quarter cycle for the $43.5 \mu \mathrm{F}$ bank is half of the quarter cycle for the $225 \mu \mathrm{F}$ bank. This means the 

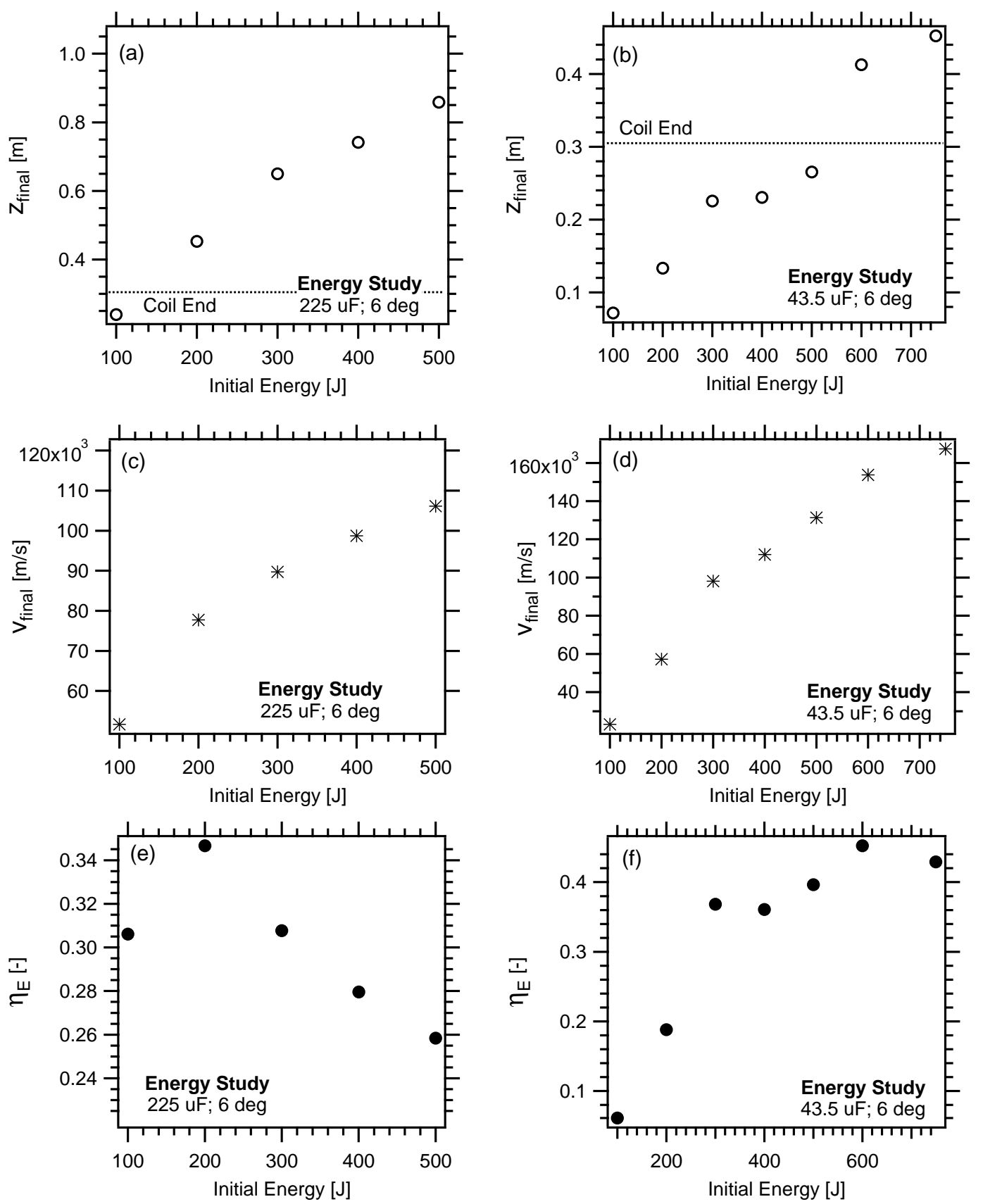

Figure 4.12: Results from the initial energy study, including the final plasmoid position, final velocity, and energy efficiency. Results are shown for 2 different capacitor banks.

$43.5 \mu \mathrm{F}$ bank must use more instantaneous energy to expel the plasmoid in half the time of the $225 \mu \mathrm{F}$ bank. Additionally, the $225 \mu \mathrm{F}$ bank is able to successfully launch a plasmoid 
over a larger range of input energies than the $43.5 \mu \mathrm{F}$ bank. The $225 \mu \mathrm{F}$ bank can operate between $200 \mathrm{~J}$ and $500 \mathrm{~J}$ while the smaller $43.5 \mu \mathrm{F}$ bank may only work at 600 to 750 J. This finding indicates that plasmoid launchers with a low frequency should be used if energy consumption is to be minimized.

\subsubsection{Coil Inductance Study}

The coil inductance should be maximized for peak efficiency. This ensures that most of the initial energy from the capacitor bank is transferred to the coils from where it can be transferred to the plasma. This lowers the energy budget for the system since the initial energy stored in the capacitor can be used more efficiently. A basic study on the effect of coil inductance was conducted using the nominal inputs in this design study. The coil inductance was varied by changing the number of turns for the inner and outer coil. Coil inductance is related to the number of turns $N$ by $N^{2}$. Since the results from the initial energy study in Section 4.2.4 demonstrated that the efficiency was sensitive to initial energy, the initial energy for each coil inductance case was optimized individually for peak efficiency rather than using the nominal setting. The optimization was done using the fmincon function, available in MatLab. The results from the coil inductance study are shown in Figure 4.13 The results in Figure 4.13 show the expected trends. Energy efficiency increases with coil inductance and the minimum initial energy decreases with coil inductance. These results conclude that a large number of turns is optimal for reducing the required input energy and increasing system efficiency.

The launcher model does not address how plasmoid formation is impacted by a multi-turn coil. Multi-turn coils can create significant artifacts in the magnetic field, which may lead 

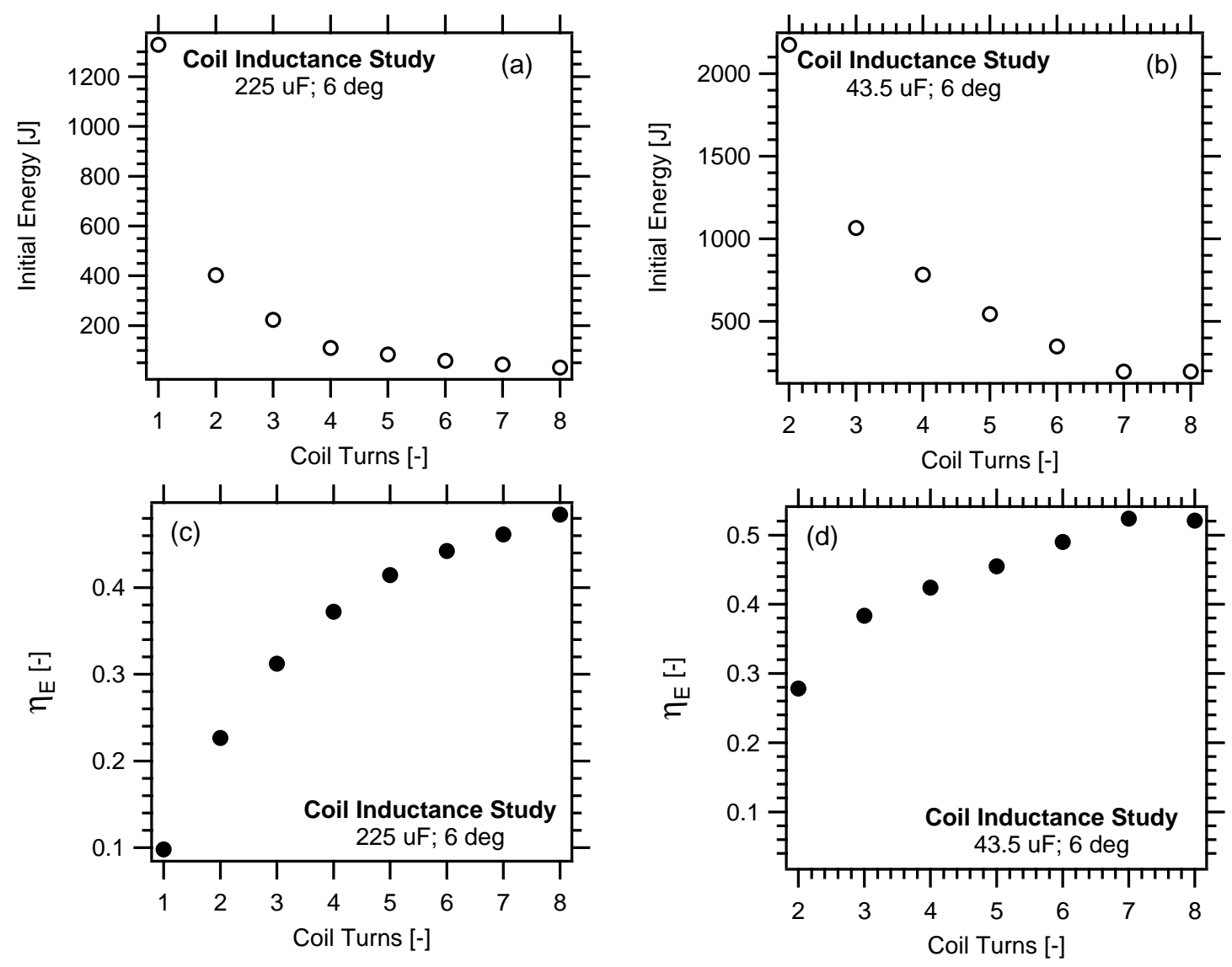

Figure 4.13: Results from the coil inductance study, showing energy efficiency and minimum required input energy as a function of coil turns.

to plasma instabilities. For this reason, a modest number of turns should be used for both coils. In this design study, four turns is a modest compromise. The data in Figure $4.13 \mathrm{a}$ and c illustrate that four turns does not significantly alter the required input energy compared to five or more turns and decreases the efficiency of an 8-turn coil by only 10\%. A 4-turn coil also allows the $30 \mathrm{~cm}$ long coil to be segmented into $7.5 \mathrm{~cm}$ wide strips, providing sufficient spacing between junctions in the coil which may introduce deviations in the magnetic field. 


\subsubsection{Parasitic Inductance and Resistance Study}

Reducing parasitic impedances in the discharge circuit is essential to maximizing energy transfer from the capacitor into the coils and plasma load. However, parasitic inductance and resistance are inherent to most experiments in non-negligible quantities so it is important to understand how much of an effect they will have on the overall performance of an electromagnetic launcher. The effect of parasitic elements was studies for the annular electromagnetic launcher model in four separate studies. In the first study, the outer coil stray inductance was varied from $100 \mathrm{nH}$ to $1 \mu \mathrm{H}$ to examine its effect. In the second study, the inner coil stray inductance was varied across the same range. Both coil inductances were varied across this range in the third study, with matching inductances. In the fourth study, the stray resistance was varied for both coil circuits from $1 \mathrm{~m} \Omega$ to $10 \mathrm{~m} \Omega$. All other inputs were left at their nominal setting. The results from each study are displayed in Figures 4.14 4.15, showing final plasmoid position, final velocity, and energy efficiency. The results for the stray inductance show a surprising trend. Increasing the inner coil stray inductance $L_{e i}$ increases the final plasmoid position and decreases the energy efficiency. The same effect happens when both $L_{e o}$ and $L_{e i}$ are increased. The increase in plasmoid position can be explained by observing that increased inductances decrease the frequency and extend the lifetime. This is shown in Figure 4.16, increasing the stray inductance increases the plasmoid lifetime (as defined in this model). The longer-lived plasmoid is able to travel a greater distance. The efficiency and velocity decrease, however, since less energy is available to the discharge coils and for the acceleration process. The results in plasmoid position and energy efficiency are different when only the outer coil stray inductance $L_{e o}$ is increased. The plasmoid position decreases and the energy efficiency either stays constant or slightly increases. Increasing $L_{e o}$ diverts more current to the inner 

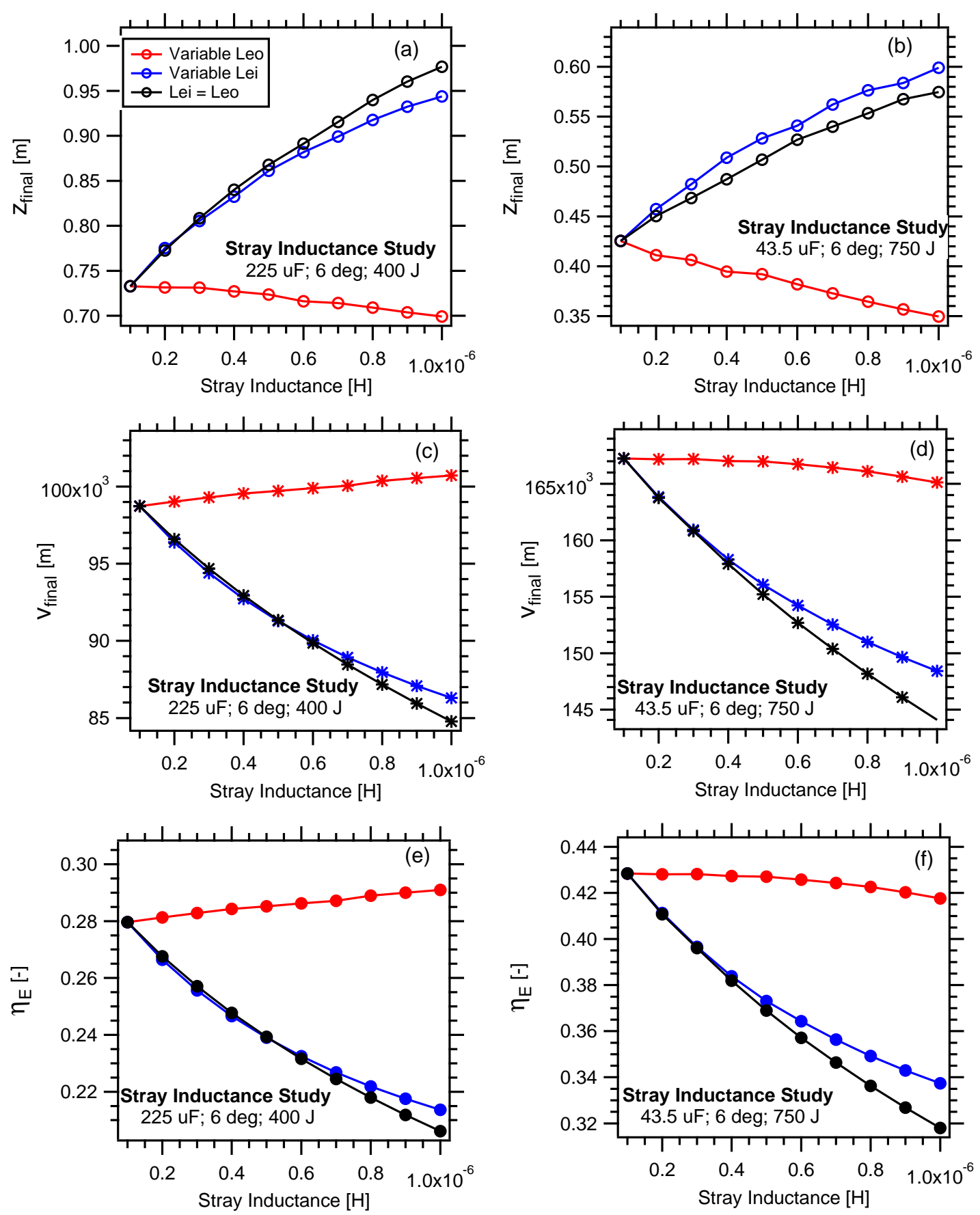

Figure 4.14: Results from the stray inductance study, including the final plasmoid position, final velocity, and energy efficiency as a function of stray inductance. 

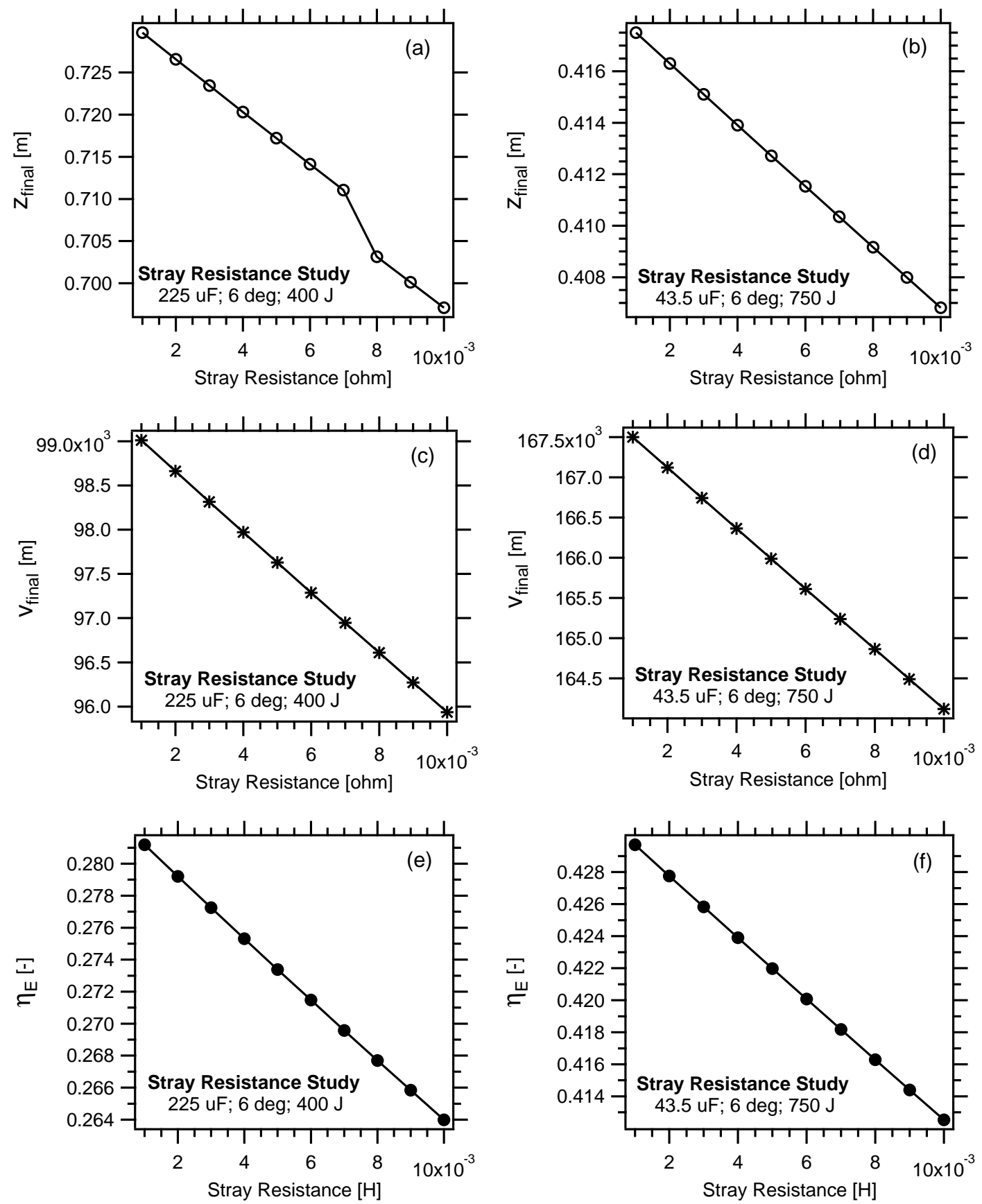

Figure 4.15: Results from the stray study, including the final plasmoid position, final velocity, and energy efficiency as a function of stray resistance. 

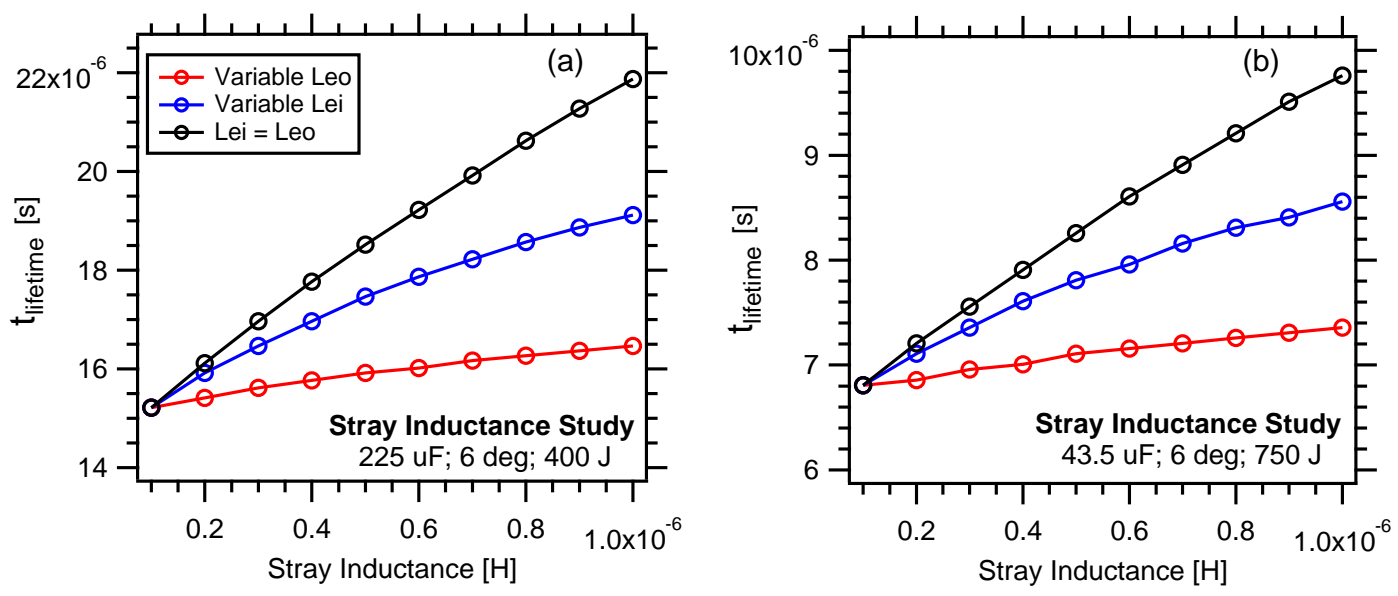

Figure 4.16: Plasmoid lifetime (related to rise time of circuit) as a function of stray inductance.

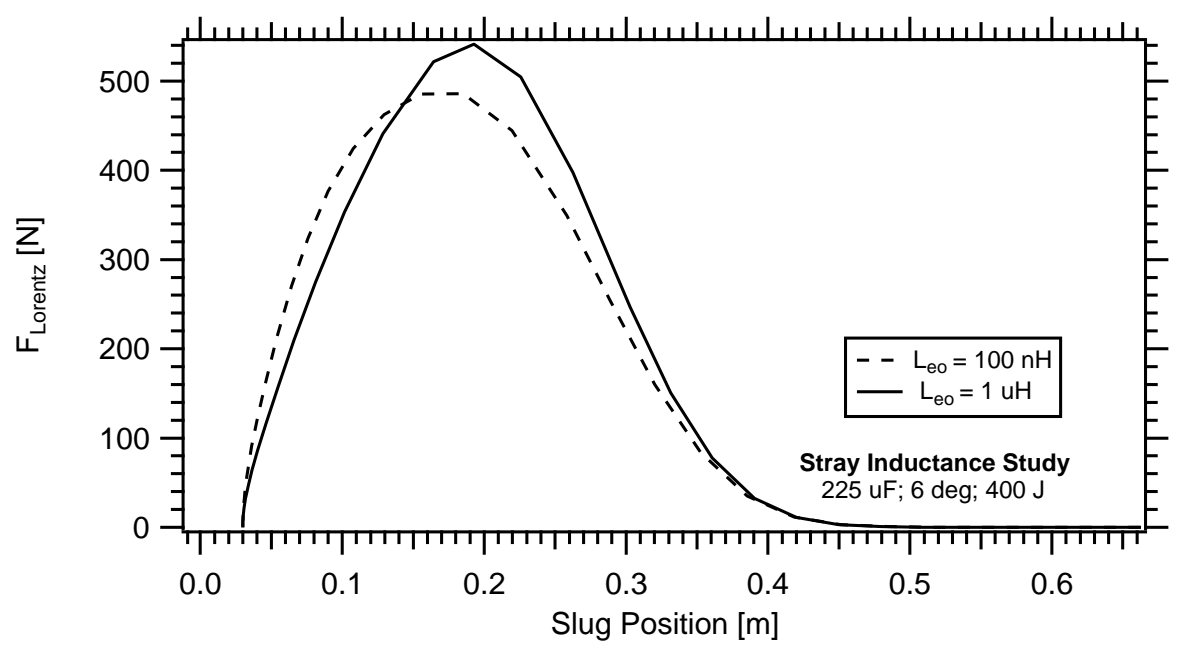

Figure 4.17: Lorentz force as a function of slug position for two stray outer coil inductances. The larger stray inductance results in a larger Lorentz force.

coil so that the outer coil is unable to provide as much initial acceleration to the plasmoid. This shifts the maximum location of the Lorentz force closer to the edge of the coil, as shown in Figure 4.17. This effect decreases the final plasmoid position since the plasmoid's initial acceleration is delayed. However, the delayed acceleration results in a more efficient acceleration since the peak Lorentz force is now higher. The longer residence time inside the coils allows the plasmoid to eject closer to the time of peak coil currents. The results 
from the stray resistance study are displayed in Figure 4.15. The results from this study are expected; larger resistances decrease the plasmoid position and decrease the system efficiency since more energy is absorbed by Ohmic heating and unavailable for translation. The effect for the range of parameters tested in this study does not show a large change in results.

\subsubsection{Final XOCOT-T3 Experiment Design}

The results from the initial phase of the design study offered several insights into annular electromagnetic launchers, including guidance on coil angles, inner coil radii, and coil inductance. These findings were used to finalize a design for the XOCOT-T3 experiment. The final design is presented in this section along with predicted trajectories of the resulting plasmoid at various initial energy levels.

The cone angle study found that small cone angles are preferred for annular launchers; a cone angle of 2 degrees was selected for the XOCOT-T3 experiment. The design study on sizing the inner coil found that the inner coil should be as large as possible. The outer coil radius of the XOCOT-T3 was fixed by available hardware to $12 \mathrm{~cm}$ so the maximum inner coil radius must not exceed $7.2 \mathrm{~cm}$. This provides clearance for the insulators and allows $3 \mathrm{~cm}$ of channel width for plasmoid formation. The coil inductance study concluded that multi-turn coils lower the minimum energy required to eject the plasmoid, though it remains unclear what impact multi-turn coils will have on plasmoid formation. For this reason, four turns for each coil was selected as a compromise between a high-inductance coil design and lowered risk for detrimental plasma effects. The study on initial system energy discovered that plasmoid launchers with a low frequency should be used if energy 
consumption is to be minimized. Additionally, it found that the $225 \mu \mathrm{F}$ capacitor bank has a larger operating range than the $43.5 \mu \mathrm{F}$ bank. The $225 \mu \mathrm{F}$ bank can operate between $200 \mathrm{~J}$ and $500 \mathrm{~J}$ while the smaller $43.5 \mu \mathrm{F}$ bank may only work at 600 to $750 \mathrm{~J}$. For these reasons, the $225 \mu \mathrm{F}$ bank was used for most of the translation studies. The $43.5 \mu \mathrm{F}$ bank was used as an alternate to the $225 \mu \mathrm{F}$ bank, in the event of capacitor failure.

The remaining design study investigated the effect of parasitic impedance. The results from these design studies show that additional parasitic impedance on the outer coil circuit only slightly decreases the trajectory of the plasmoid and impacts the terminal velocity and energy efficiency only marginally. Additional impedance on both the inner and outer coil circuit will increase the trajectory, but decrease the velocity and energy efficiency. This means that parasitic impedance should be minimized, if possible. The design target for the conceptual design studies was $100 \mathrm{nH}$, which is the minimum inductance for a basic prototype experiment similar to the one developed in this research. The actual stray inductance in the XOCOT-T3 experiment is likely to be higher than this figure, as longer line lengths, bends, and gaps in the lines are required for diagnostic access. For this reason, the target inductance in the XOCOT-T3 design projections was elevated to $500 \mathrm{nH}$ per coil circuit leg. The final design parameters for the XOCOT-T3 experiment are displayed in Table 4.2 ,

The annular electromagnetic launcher model was used to predict the trajectories of the plasmoid slug with the XOCOT-T3 design parameters. This was done to ensure the final design would successfully eject the plasmoid from the coils and to predict the exit velocities of the translating plasmoid. The predicted coil currents, plasma current, capacitor voltage, velocity, and plasmoid position are displayed in Figure 4.18 for a $200 \mathrm{~J}$ discharge with the $225 \mu \mathrm{F}$ capacitor. As indicated by the results, the $200 \mathrm{~J}$ discharge successfully ejects the 
Table 4.2

XOCOT-T3 final design parameters

\begin{tabular}{lcc}
\hline Component & Symbol & Value \\
\hline Capacitor & $C_{M B}$ & $225 \mu \mathrm{F}$ \\
Alternate & & $43.5 \mu \mathrm{F}$ \\
External inductance & $L_{e}$ & $200 \mathrm{nF}$ \\
Outer stray inductance & $L_{e o}$ & $500 \mathrm{nF}$ \\
Inner stray inductance & $L_{e i}$ & $500 \mathrm{nF}$ \\
External resistance & $R_{e}$ & $10 \mathrm{~m} \Omega$ \\
Outer stray resistance & $R_{e o}$ & $1 \mathrm{~m} \Omega$ \\
Inner stray resistance & $R_{e i}$ & $1 \mathrm{~m} \Omega$ \\
\hline Outer coil radius & $r_{o}$ & $12 \mathrm{~cm}$ \\
Outer coil length & $l_{o}$ & $30.5 \mathrm{~cm}$ \\
Outer coil half-angle & $\alpha$ & $2{ }^{\circ}$ \\
Coil turns & $N_{i}, N_{o}$ & 4 \\
Inner coil radius & $r_{i}$ & $7.2 \mathrm{~cm}$ \\
Inner coil length & $l_{i}$ & $30.5 \mathrm{~cm}$ \\
Outer stray resistance & $R_{e o}$ & $1 \mathrm{~m} \Omega$ \\
Inner stray resistance & $R_{e i}$ & $1 \mathrm{~m} \Omega$ \\
\hline Plasma density & $n_{i}$ & $1.29 \times 10^{20} \mathrm{~m}$ \\
Plasma conductivity & $\sigma_{p}$ & $1 \times 10^{6} \mathrm{~S} / \mathrm{m}$ \\
Plasma length & $l_{p}$ & $80 \% l_{c}$ \\
Plasma width & $\Delta r_{m}$ & $40 \%\left(r_{o}-r_{i}\right)$ \\
Plasma radius & $r_{m}$ & $\left(r_{o}+r_{i}\right) / 2$ \\
\hline Charge voltage & $V_{c}$ & $1.3 \mathrm{kV}$ \\
(225 $\mu F$ ) & & $200 \mathrm{~J}$ \\
Charge voltage & $V_{c}$ & $5.3 \mathrm{kV}$ \\
(43.5 $\mu F$ ) & & $600 \mathrm{~J}$ \\
& &
\end{tabular}

plasmoid before its allotted lifetime $(18.6 \mu \mathrm{s})$. It reaches a terminal velocity of $110 \mathrm{~km} / \mathrm{s}$ during this time. The plasmoid's mass of $1.1 \times 10^{-8} \mathrm{~kg}$, results in a directed impulse bit of $1.2 \mathrm{mN}$-s. The energy efficiency in this case is $33 \%$. The effect of input energy on the final velocity, momentum, and energy efficiency was examined for the XOCOT-T3 design. The results from this study are shown in Figure 4.19. As expected, the plasmoid trajectory is sensitive to the initial energy. Higher energies result in longer trajectories and faster velocities, at the expense of energy efficiency. This effect was also noted in the results in 


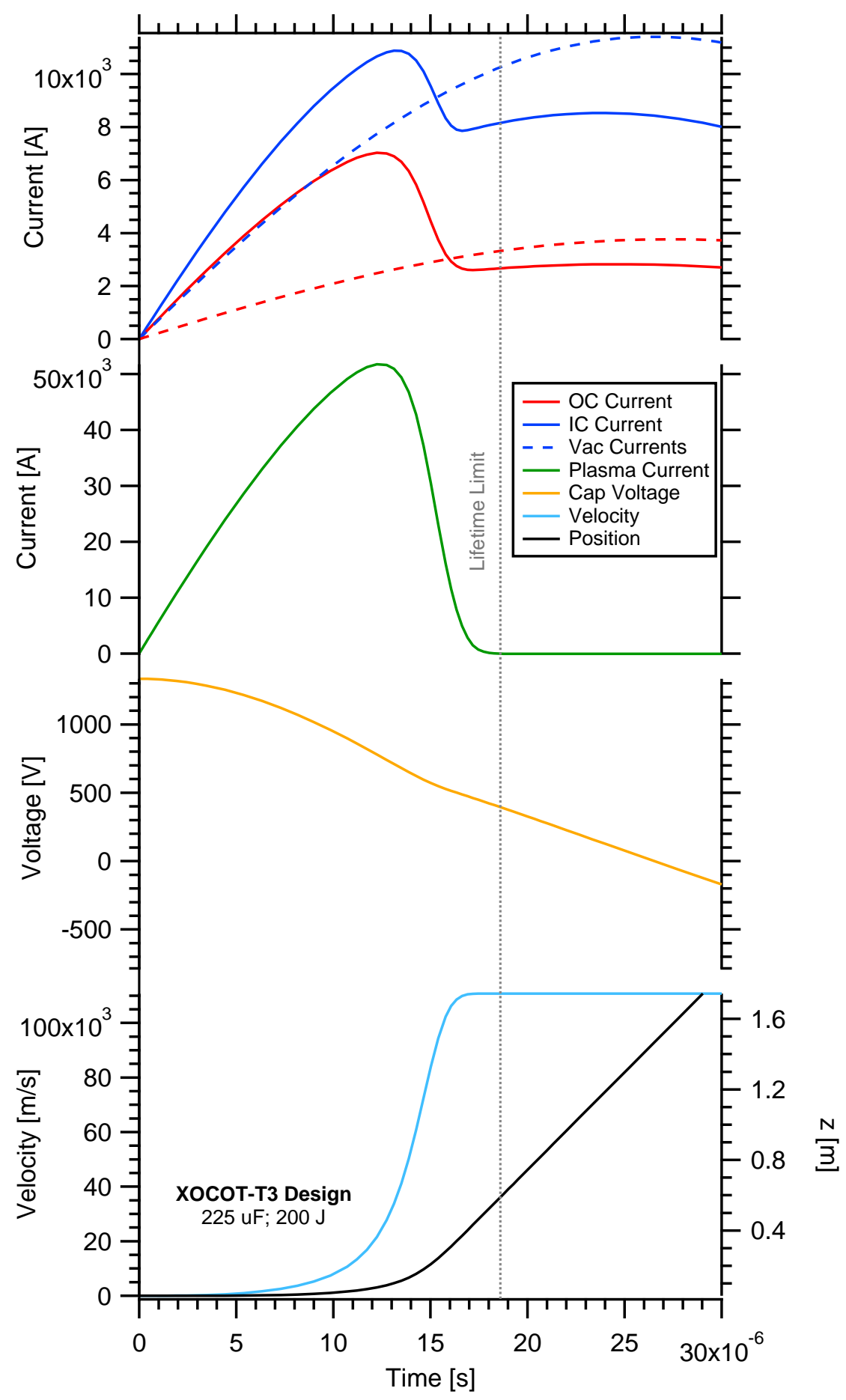

Figure 4.18: Results from the launcher model for the XOCOT-T3 design inputs, including coil currents, plasma current, capacitor voltage, plasmoid velocity, and axial plasmoid position as a function of time for nominal inputs. 
Section 4.2.4. The results in Figure 4.19 indicate that the plasmoid for the $225 \mu \mathrm{F}$ bank can be expected to translate from the coils at $200 \mathrm{~J}$ to $500 \mathrm{~J}$ of initial energy for the $225 \mu \mathrm{F}$ capacitor bank. The plasmoid should translate from the coils using the $43.5 \mu \mathrm{F}$ bank from $600 \mathrm{~J}$ to $1 \mathrm{~kJ}$ of input energy. The upper energy limit is set by current ratings for circuit components.

\subsubsection{Plasmoid Sensitivity Studies}

The design studies presented for the XOCOT-T3 design use fixed geometry and conditions for the plasmoid. The sensitivity of these inputs was examined to see how alternate plasmoid geometries would impact the results. Four sensitivity studies were conducted, focusing on plasmoid dimensions (minor radius and length), plasmoid mean radius, and plasmoid conductivity. All other inputs were set to the XOCOT-T3 design parameters, outlined in Table 4.2.

The effect of the plasmoid width (radial direction) was studied by varying the width from $0.5 \mathrm{~cm}$ to $2.7 \mathrm{~cm}$, at two intermediate values. The results from this study are displayed in Figure 4.20. They show that the plasmoid is able to translate from the coils for all possible widths with the $225 \mu \mathrm{F}$ bank and for most possible widths with the $43.5 \mu \mathrm{F}$ bank. The thickest plasmoid in the higher frequency bank $(43.5 \mu \mathrm{F})$ is too massive to translate from the coils in the allotted time. The results also show the plasmoid position and velocity decreases for thicker plasmoids. This effect is due to the larger plasmoid mass contained in the thicker plasmoids. The efficiency generally improves with plasmoid thickness, provided the plasmoid is able to exit the coils. Since efficiency is primarily a function of timing, the slower velocity of the thicker plasmoids keeps the plasmoid contained in the coils for longer 

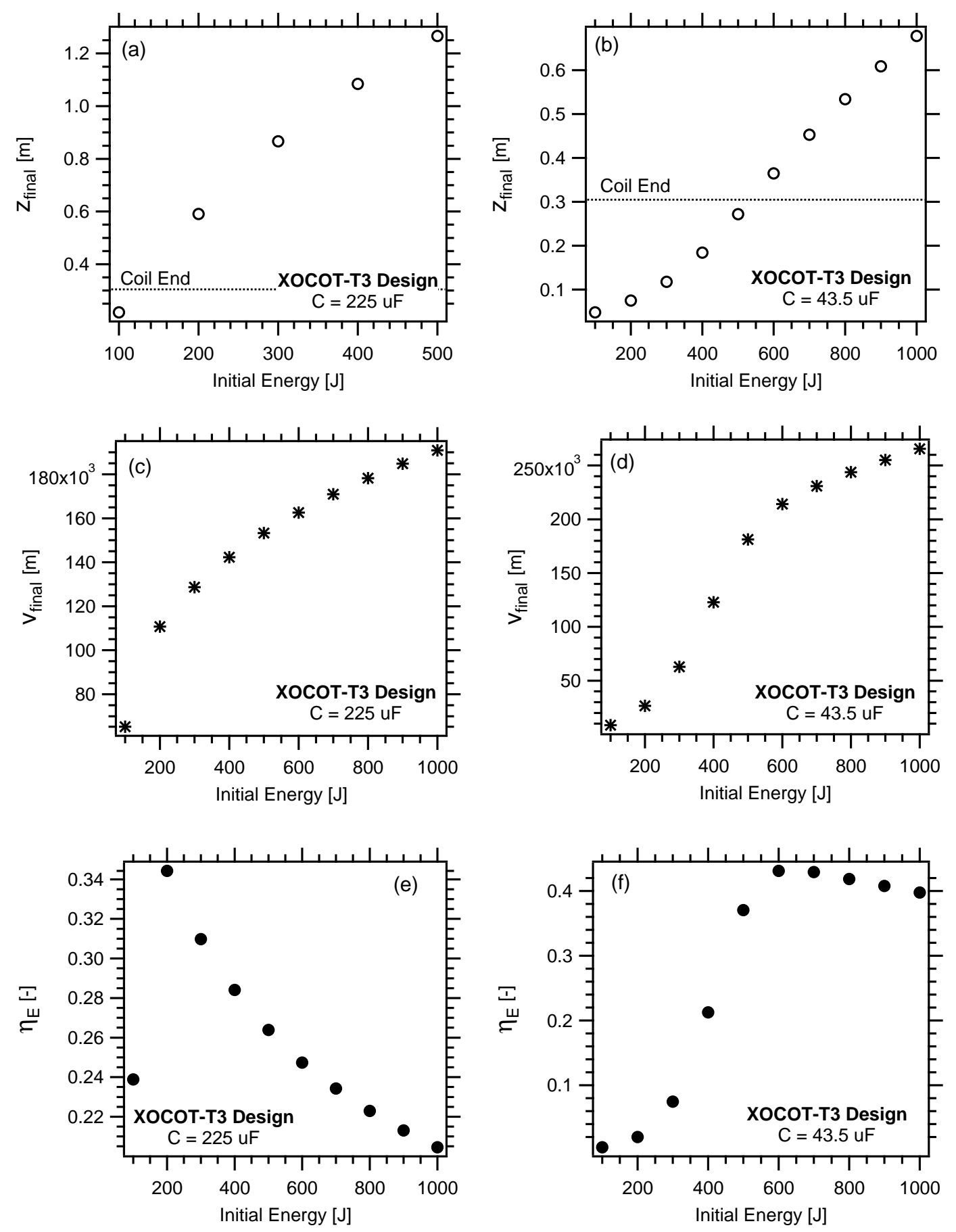

Figure 4.19: Results from the XOCOT-T3 design study on initial energy, including the final plasmoid position, final velocity, and energy efficiency. Results are shown for the primary bank $(225 \mu \mathrm{F})$ and the alternate bank (43.5 $\mu \mathrm{F})$. 
duration so that its exit can be timed with higher coil currents.

The effect of plasmoid length was studied by varying the length of the plasmoid from $3 \mathrm{~cm}$ to $25 \mathrm{~cm}$ (representing $10 \%$ to $80 \%$ of the coil length). The results are shown in Figure 4.21. The sensitivity of the plasmoid's trajectory to plasmoid length is readily apparent in these results. Shorter plasmoids are less likely to translate from the coils in their lifetime compared to longer plasmoids. They do not couple as well to the coils as their longer counterparts and they must travel a greater distance before encountering the region of peak $\mathrm{dM} / \mathrm{dz}$ at the end of the coils where they receive most of their kinetic energy. This means that more energy will be required to translate the plasmoid if its length does not extend more than half of the coil length. Provided a plasmoid is able to escape the coils during its lifetime, shorter plasmoids can accumulate more kinetic energy than longer plasmoids. Their lower mass allows them to be accelerated to higher velocities for the same amount of transferred energy. The efficiency does not change appreciably with plasmoid length, indicating that each plasmoid has the same final kinetic energy.

The plasma conductivity's effect on the plasmoid's trajectory was investigated for a range of values from $10^{3} \mathrm{~S} / \mathrm{m}$ to $10^{6} \mathrm{~S} / \mathrm{m}$. This spans the range of Spitzer conductivities for a $10 \mathrm{eV}$ and $100 \mathrm{eV}$ plasmoid. While temperatures of $100 \mathrm{eV}$ are unlikely in an AFRC, a Spitzer conductivity of $10^{6} \mathrm{~S} / \mathrm{m}$ represents an absolute upper bound. The results from the conductivity study are displayed in Figure 4.22. These results show that plasma conductivity (except for very low values) does not impact the translation of the plasmoid out of the coils; the plasmoid is able to escape the coils with sufficient conductivity. Conductivity does, however, impact the terminal velocity and system efficiency. In this limited study, increased conductivity improves the energy efficiency and increases the final velocity. Increased conductivity improves the coupling between the coils and plasmoid by 

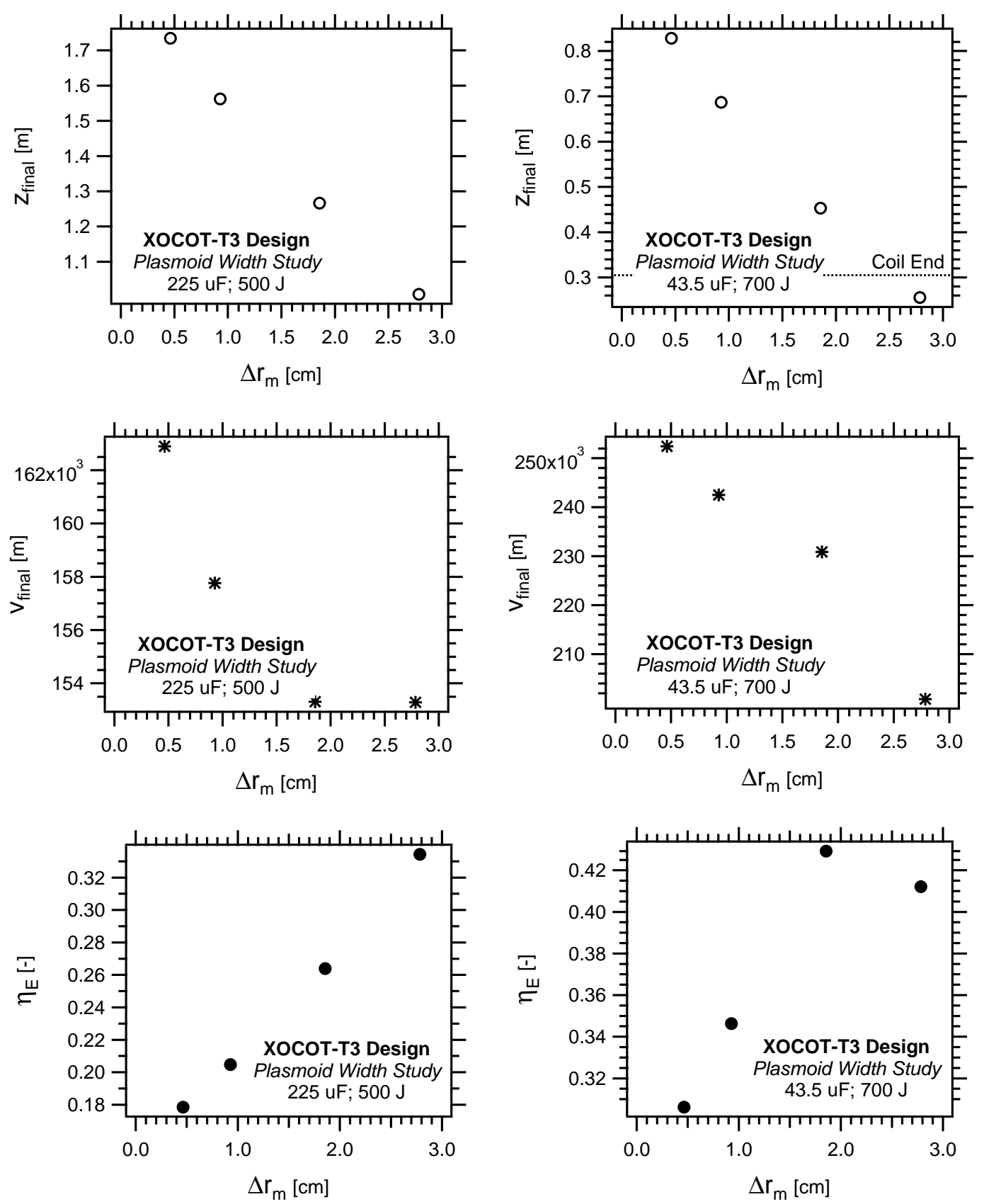

Figure 4.20: Sensitivity results from the XOCOT-T3 design study on plasmoid width, including the final plasmoid position, final velocity, and energy efficiency. Results are shown for the primary bank $(225 \mu \mathrm{F})$ and the alternate bank $(43.5 \mu \mathrm{F})$. 

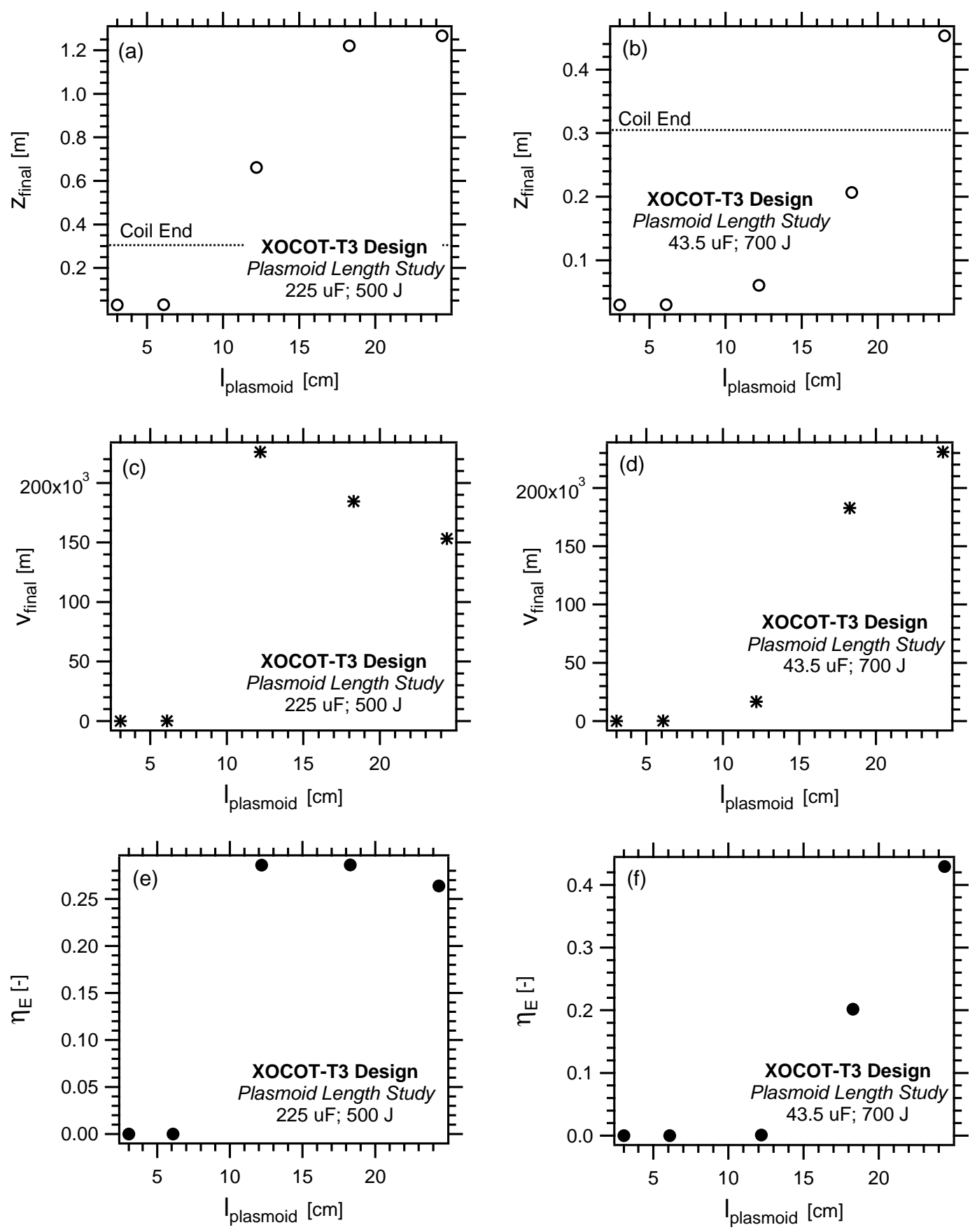

Figure 4.21: Sensitivity results from the XOCOT-T3 design study on plasmoid length, including the final plasmoid position, final velocity, and energy efficiency. Results are shown for the primary bank $(225 \mu \mathrm{F})$ and the alternate bank $(43.5 \mu \mathrm{F})$. 
generating thinner skin currents which couple more effectively to the coils. This results in more efficient energy transfer between the plasmoid and coils, boosting the terminal kinetic energy and system efficiency. These results are promising for the XOCOT-T3 design, as low conductivities are unlikely due to the significant temperature $(10 \mathrm{eV})$ and density $\left(10^{20}\right.$ $\mathrm{m}^{-3}$ ). The effect of the plasmoid's radial position was studied in the final sensitivity study. The radius of the plasmoid $r_{m}$ was moved to five radial locations between the inner and outer coil. The results from this study are displayed in Figure 4.23. The effect of the radial position does not appear to change the plasmoid's trajectory and energy efficiency appreciably, unless the plasmoid is located very close to the outer wall. In the $43.5 \mu \mathrm{F}$ case, the plasmoid does not translate from the coils if very close to the outer wall. Despite the small change in results, it appears that plasmoids located closer to the inner wall attain a greater velocity than those closer to the outer wall. The Lorentz force is higher for the inner coil, due to its larger current. Improved coupling between the plasmoid and inner coil will further increase the Lorentz force resulting in a larger velocity. Energy efficiency appears to decrease as the plasmoid moves toward centerline. However, the change in efficiency among all the results is small (less than 10\%) so the effect of the plasmoid's radial location on energy efficiency is likely to be minimal.

The sensitivity studies conclude that the plasmoid's trajectory and the energy efficiency are highly sensitive to the plasmoid's dimensions (length and width). If the plasmoid is too wide or too short, it may not be able to translate from the coils during its lifetime. The initial energy of the system will need to be increased to ensure the plasmoid can translate. The plasmoid's conductivity and initial radial position have less of an impact on the plasmoid's trajectory and energy efficiency. The extreme cases (very low conductivity and plasma very close to outer wall) were the only cases which indicated issues with plasmoid ejection and these extremes are unlikely in the XOCOT-T3. 

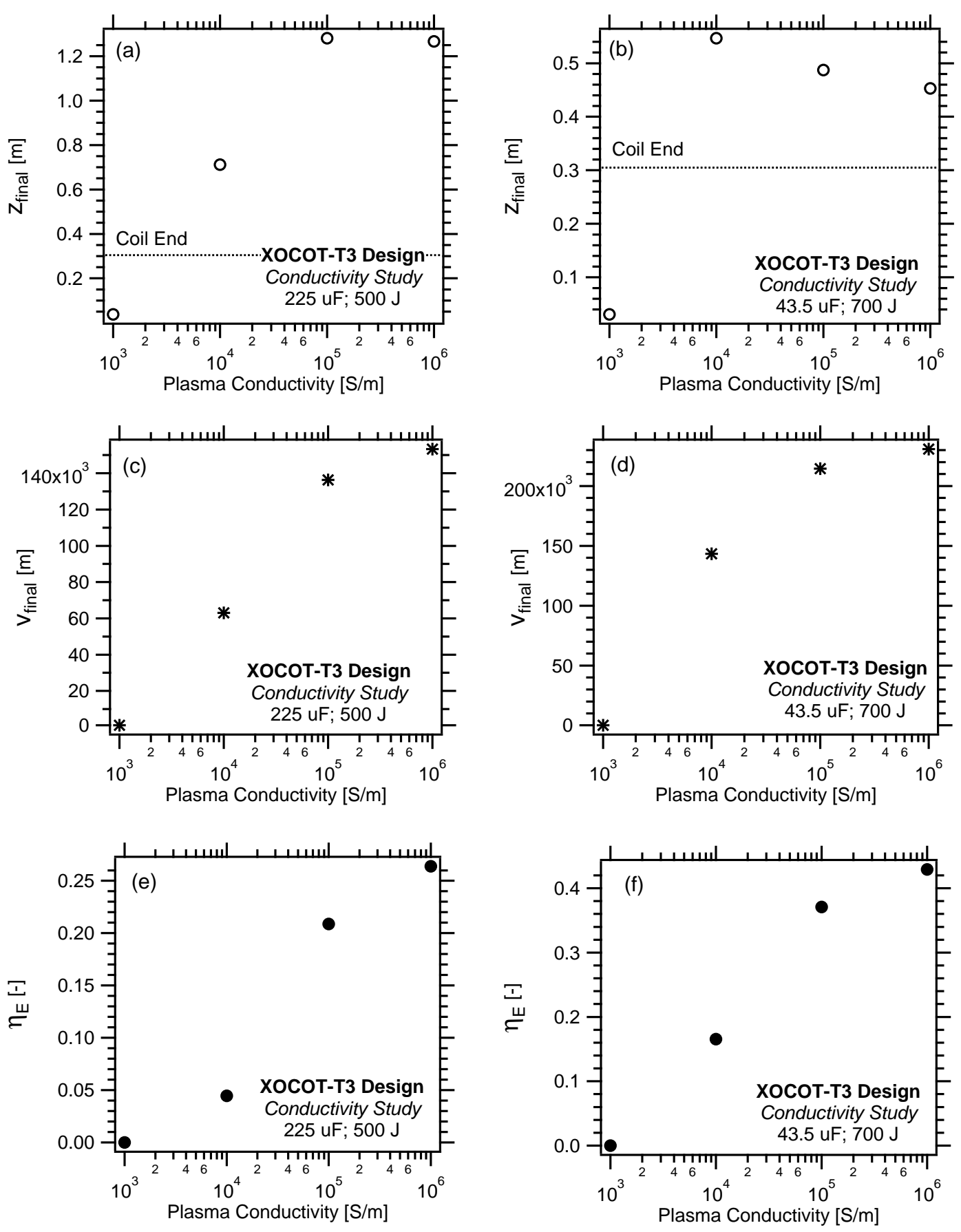

Figure 4.22: Sensitivity results from the XOCOT-T3 design study on plasma conductivity, including the final plasmoid position, final velocity, and energy efficiency. Results are shown for the primary bank $(225 \mu \mathrm{F})$ and the alternate bank $(43.5 \mu \mathrm{F})$. 

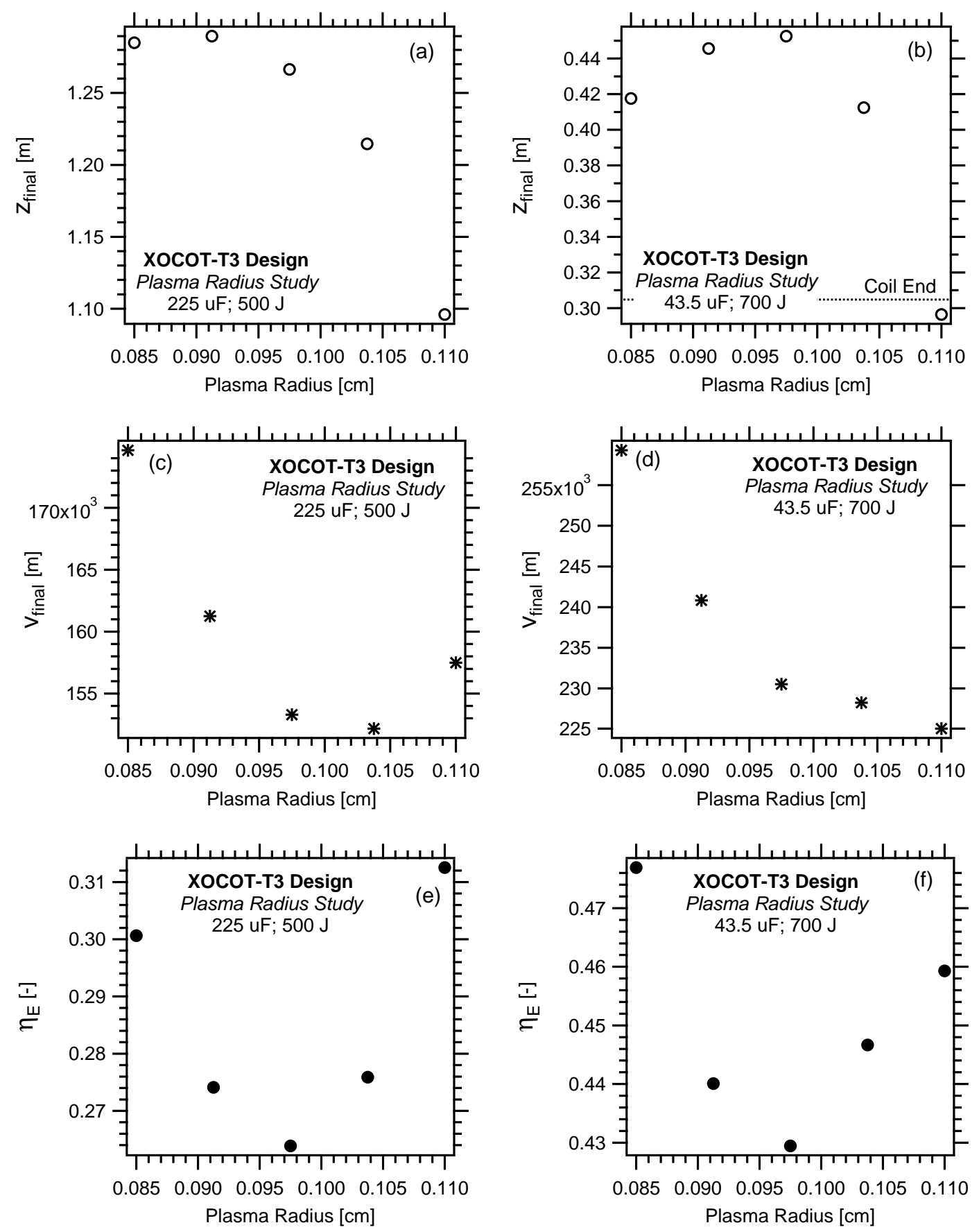

Figure 4.23: Sensitivity results from the XOCOT-T3 design study on plasmoid radius, including the final plasmoid position, final velocity, and energy efficiency. Results are shown for the primary bank $(225 \mu \mathrm{F})$ and the alternate bank $(43.5 \mu \mathrm{F})$. 
The only sensitivity study not completed for this design was the effect of the starting axial position of the plasmoid. The effect of the initial plasmoid axial position can be qualitatively understood by examining the mutual inductance profiles shown in Figure 4.7 . These profiles show that if the plasmoid starts near $\mathrm{z}=0$, the transit time through the coils will increase since the Lorentz force near the narrow end of the coil is small. If the plasmoid is formed further downstream toward the wide end of the coil, the plasmoid can eject well before the peak coil currents. This means the plasmoid will reach a lower terminal velocity and the system's efficiency will be lower. These arguments conclude that the plasmoid trajectory is highly sensitive to the plasmoid's initial axial position inside the coils.

\subsection{Magnetic Field Modeling of XOCOT-T3}

The magnetic field of the XOCOT-T3 design was calculated using COMSOL's electromagnetic field solver with the AC/DC module (version 4.2a). The magnetic field was calculated for the vacuum case and for the plasmoid-coupling case with a stationary plasma. The axial magnetic field profiles at several axial locations for a translating plasmoid were also calculated. This section contains the magnetic field calculations and field profiles, along with a brief discussion of the methods used in each case.

The vacuum magnetic fields were calculated using a time-dependent solver in COMSOL, with a 2D axisymmetric geometry and circuit interface for current calculations. The final geometry (see Table 4.2) was modeled with flat, four-turn coils for the inner and outer coils. The coils were connected to an external circuit, with stray circuit elements defined by the final design values. Modeling the problem in this fashion allowed the current through each coil to be influenced by the external circuit and coil-coil coupling, as it would be in an 


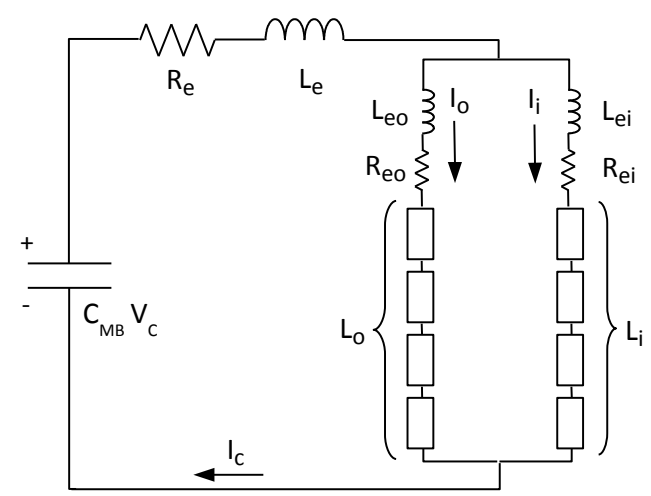

(a)

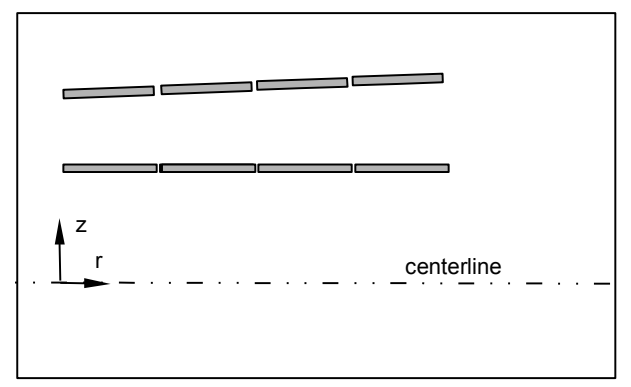

(b)

Figure 4.24: Coil connections, circuit connections, and coil geometry used to calculate the vacuum fields. The rectangles in (a) represent FEM objects in COMSOL. The geometry used in COMSOL to represent the multi-turn coils is drawn in (b).

experiment. Figure 4.24 a shows the coil and circuit connections used in the COMSOL model. Figure $4.24 \mathrm{~b}$ depicts the geometry used to model the coils. The multi-turn coil is modeled in a segmented design, with minimal spacing between the coil turns. The current in each segment is entirely in the azimuthal direction. The rectangles in Figure 4.24a represent the coils in COMSOL, connected the external circuit as shown. The vacuum fields were calculated using the circuit parameters outlined in the XOCOT-T3 final design (see Table 4.2). The calculated vacuum fields and coil currents are shown in Figure 4.25, As expected, the vacuum fields are in phase with the coil currents. The magnetic field between the coils is in the same direction as the magnetic field in the inner coil, though lower in magnitude. The magnetic field along the length of the coils is not as uniform as expected. A solenoidal coil (radius < length) has a uniform axial field through the center of the coil, with curvature near the ends. The axial field distribution shown in Figure 4.25 , is fairly irregular with distortions seen every $7 \mathrm{~cm}$ or so. These distortions line up with the gaps between the coils, suggesting the multi-turn coils are the source of the non-uniformities. A topographical map of the magnetic field confirms this theory. This map is displayed in 
Figure 4.26. Detail views of the area between the coils shows strong magnetic fields created between subsequent turns of the multi-turn coil. These fields are created by interaction between current repulsion in the nearby turns. These distortions extend away from the coil, resulting in the non-uniform field distribution shown in Figure 4.25r. Following computation of the vacuum fields, the magnetic fields created by a conductor (plasma) between the coils were calculated by adding a conductive region between the coils. The simulation was completed using the same methods and inputs as the vacuum magnetic field studies. A plasmoid was draw between the coils, $1 \mathrm{~cm}$ wide and centered between the coils. The conductivity of this region was fixed to $10^{6} \mathrm{~S} / \mathrm{m}$. The resulting current and magnetic fields are displayed in Figure 4.27. The plasmoid adds an inductive load to the circuit, as noted by the change in frequency of the plasmoid-coupling case and greater currents as compared to the vacuum case. The magnetic field between the coils also changes, increasing in magnitude. The inner field is now in the opposite direction as the vacuum field, creating a reversed field on either side of the plasmoid. The field changes directions inside the plasmoid, as shown in Figure 4.27k. The appearance of a reversed field is consistent with magnetic field measurements from previous AFRC experiments, as detailed in Section 3.3.2. Magnetic field modeling was also completed in COMSOL to estimate the magnetic field profiles of a translating plasmoid. The simulations were completed using the same methods as the vacuum field calculations, 2D axisymmetric geometry with coil currents specified using a circuit framework. The moving plasmoid was modeled as a sliding rectangular region between the coils with a time-dependant conductivity $\sigma_{p}(t)$. The rectangular region of plasma conductivity, bound by $z_{1}$ and $z_{2}$, moved along $z$ with a constant velocity $u_{z}$. Additionally, the plasmoid conductivity definition used a finite turn-on delay $\tau_{p}$ to mimic the finite ionization time of a plasmoid. The plasmoid conductivity relation is described by Equation 4.19. The geometry of the coils and plasmoid zone is 

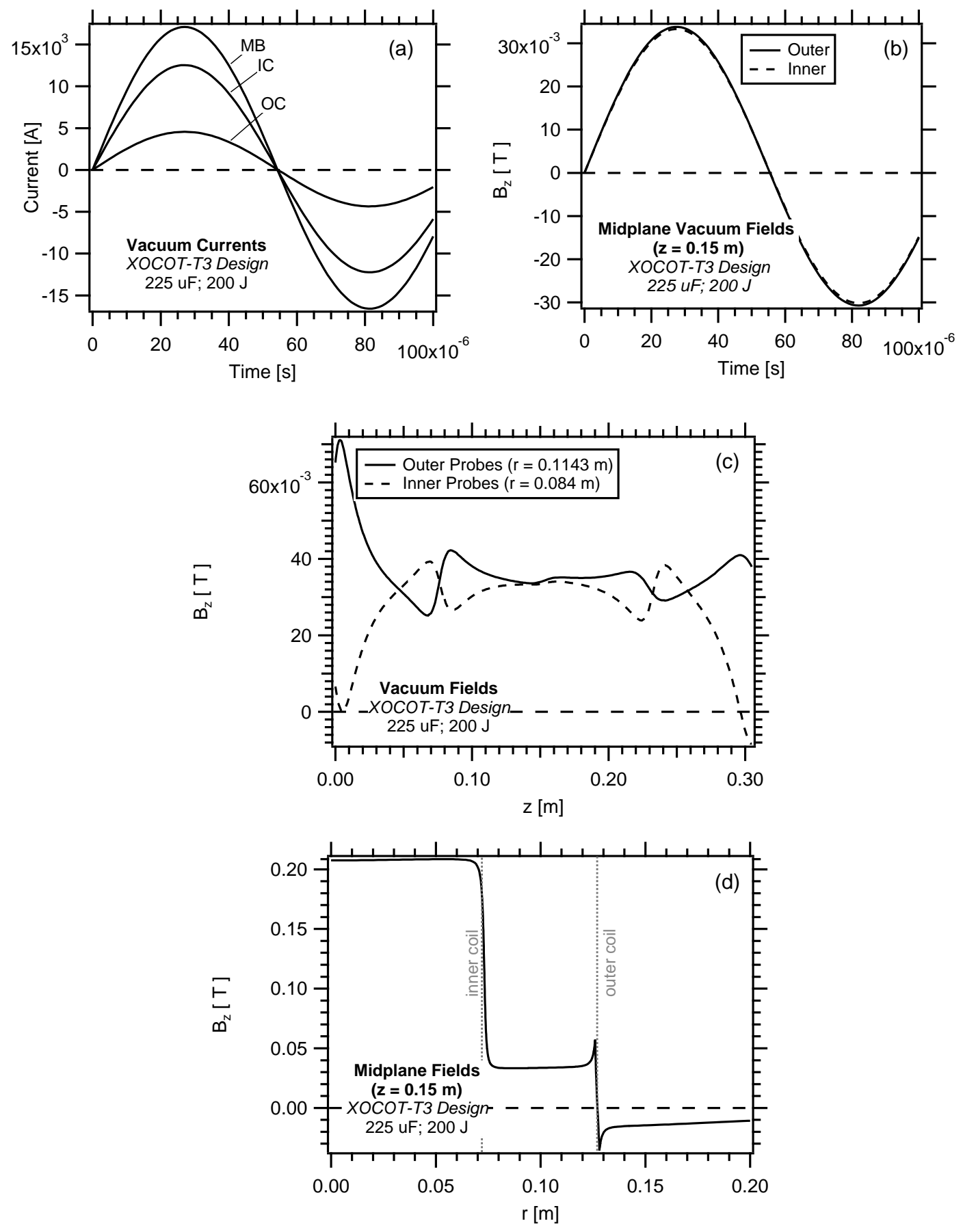

Figure 4.25: COMSOL results for the vacuum case using the XOCOT-T3 design, including (a) coil currents, (b) time history of midplane axial fields, (c) axial magnetic fields as the length of the coil, and (d) midplane axial field across the radial cross section. 

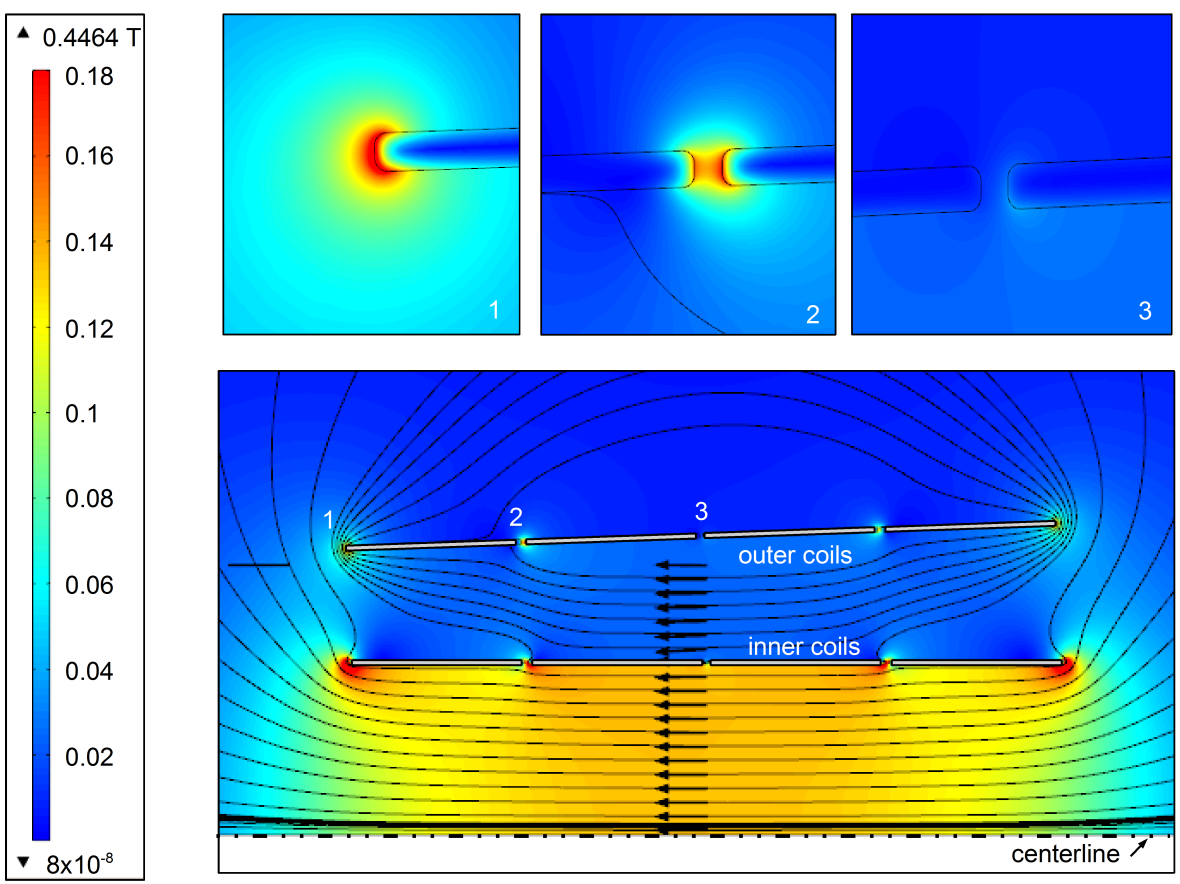

Figure 4.26: Magnetic field map from the XOCOT-T3 design, computed in COMSOL. Arrows denote field direction. Detail views of the magnetic field between coil turns is displayed in the smaller figures.

shown in Figure 4.28, along with the circuit connections.

$$
\sigma_{p}(t)=\left\{\begin{aligned}
\sigma_{p}\left(1-e^{-\left(t / \tau_{p}\right)}\right) & \text { if } z_{1}<z<z_{2} \\
0 & \text { otherwise }
\end{aligned}\right.
$$

The moving plasmoid could have been modeled in COMSOL with a moving mesh instead of the sliding conductivity region, but the moving mesh simulations were numerically unstable and computationally expensive. The sliding conductivity simulations were found to be slightly more stable, with total computation time around 12 hours. The simulation was conducted with the following variables $t a u_{p}=500 \mathrm{~ns}, u_{z}=50 \mathrm{~km} / \mathrm{s}, \sigma_{p}=10^{6} \mathrm{~S} / \mathrm{m}$. Magnetic field probes were position at several axial locations $5 \mathrm{~cm}$ apart on either side of 


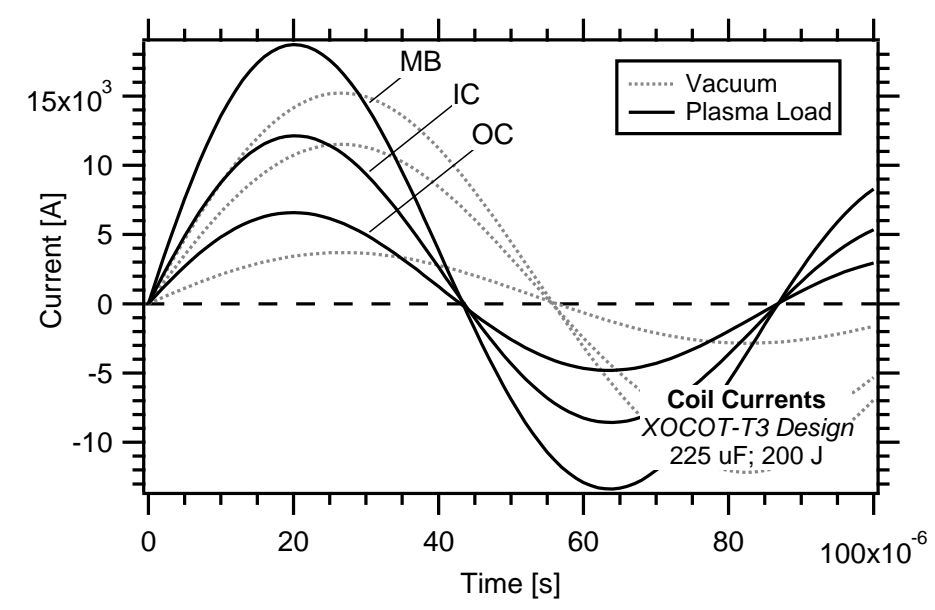

(a)

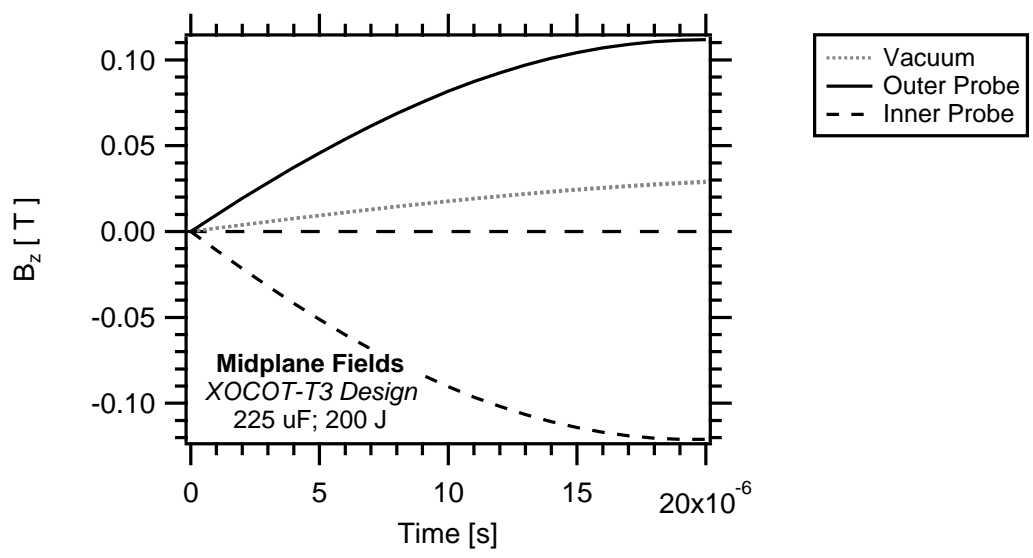

(b)

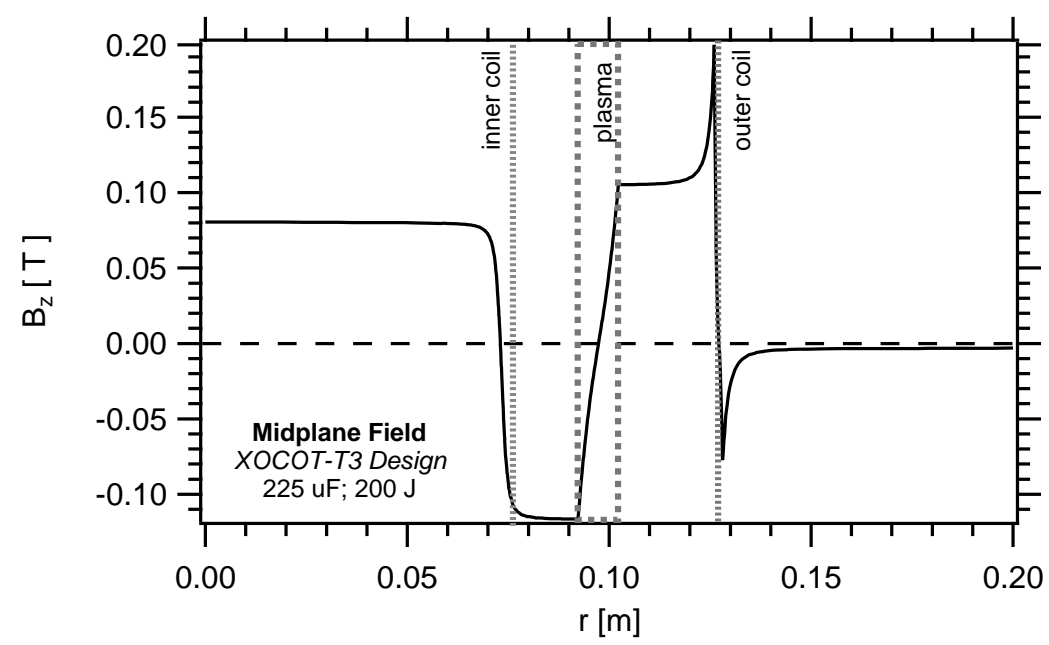

(c)

Figure 4.27: COMSOL results for the plasmoid-coupling case using the XOCOT-T3 design, including (a) coil currents, (b) time history of midplane axial fields, and (c) midplane axial field across the radial cross section. 


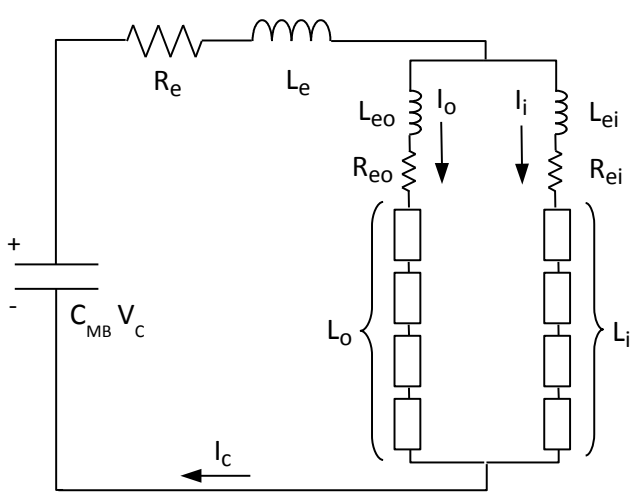

(a)

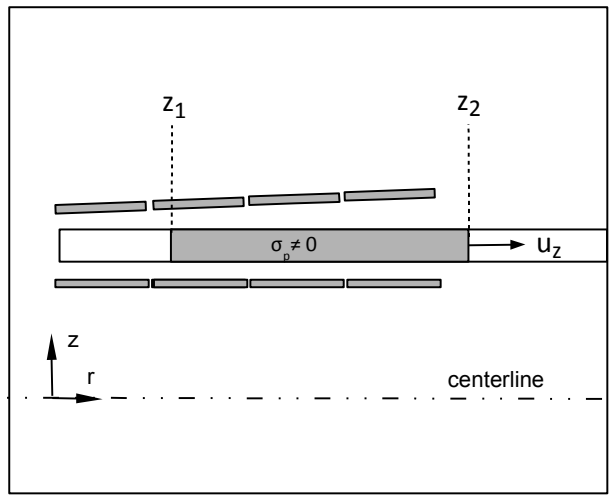

(b)

Figure 4.28: Coil connections, circuit connections, and coil geometry used to calculate the translating plasmoid fields. The rectangles in (a) represent FEM objects in COMSOL. The moving plasmoid in COMSOL is specified by the sliding region of conductivity in (b).

the plasmoid and downstream of the coils in the path of the plasmoid. The resulting coil currents and magnetic field signatures are displayed in Figure 4.29 These results show the clear translation of the plasmoid past each axial location on the outer and inner surfaces. As the trailing edge of the plasmoid passes each probe, the magnetic field peaks and then returns to the vacuum case. The magnetic field on the inner probes change sign and the outer probes resume a vacuum frequency. Once the plasmoid transits out of the coils, the coil currents return to their vacuum signature, with a reduced frequency and amplitude. The downstream probes, directly in the path of the transiting plasmoid show a flat signature as the probe passes over the top of them. The transiting signatures on each probe is staggered in accordance with a plasmoid translating at $50 \mathrm{~km} / \mathrm{s}$, with $1 \mu$ second between neighboring probes $(5 \mathrm{~cm}$ apart) on the outer and inner probe arrays. In the experiment, the plasmoid's velocity is expected to change with time, based on the velocity profile shown in Figure 4.18. This means the signatures seen in Figure 4.29 will likely be spaced further apart with irregular spacing, but the general behavior of a plasmoid translating past a magnetic probe should still be apparent. 


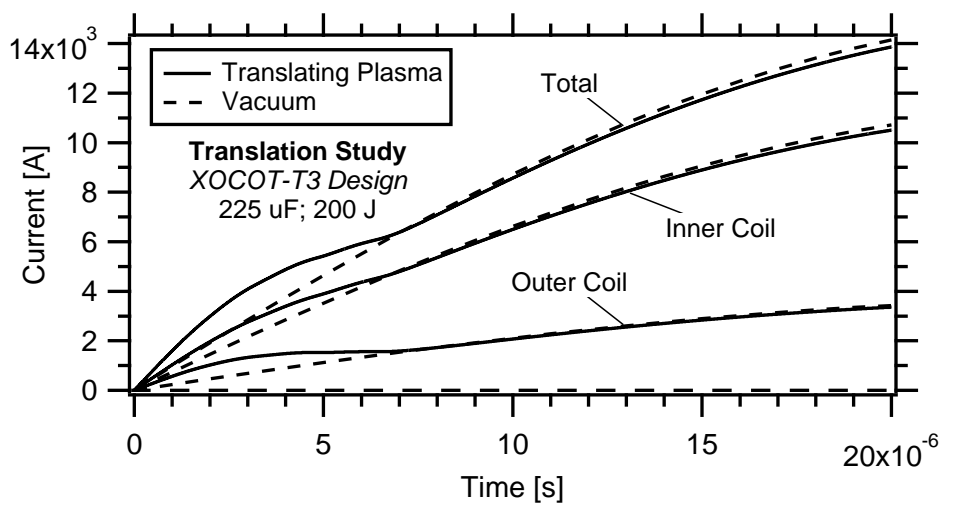

(a)

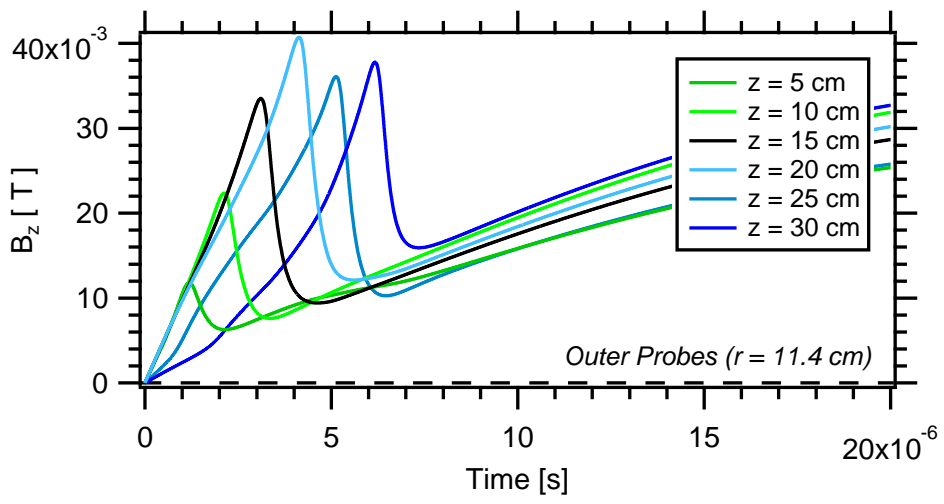

(b)

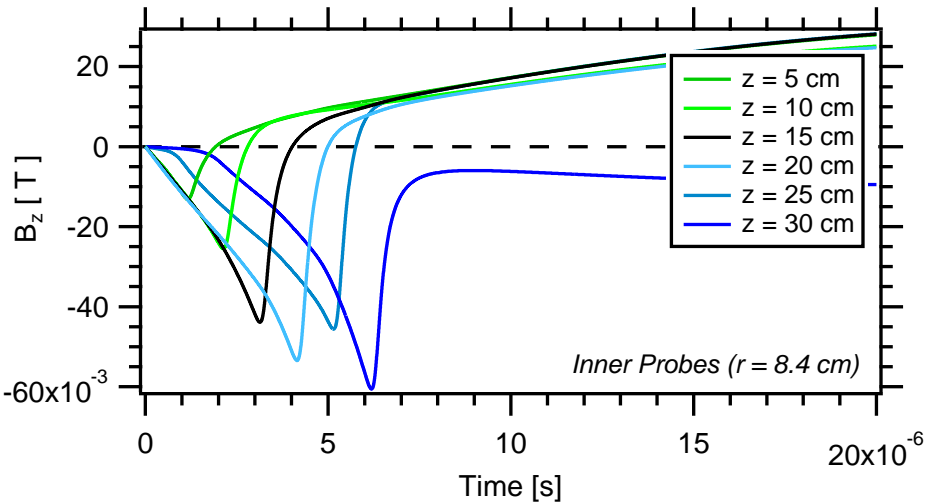

(c)

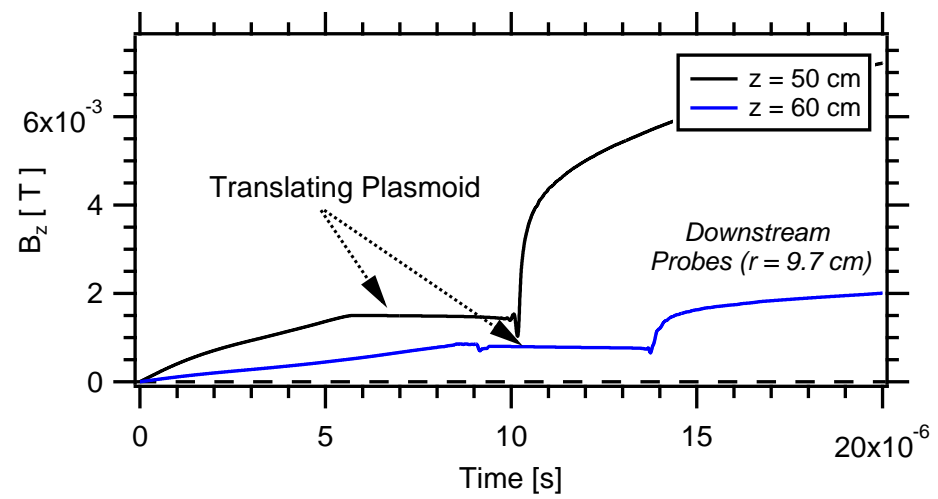

(d)

Figure 4.29: Coil currents and magnetic field predicted by COMSOL for a plasmoid translating at $50 \mathrm{~km} / \mathrm{s}$. 


\subsection{Summary and Discussion of Results}

An annular electromagnetic launcher model was developed to simplify the physics of a translating plasmoid using a circuit-based approach. The plasmoid was approximated as a rigid conducting slug, expelled from the coils by a Lorentz force. A series of design studies was conducted to understand how inputs for the model such as cone angle and inner coil radius affected the resulting plasmoid's trajectory and the energy efficiency of the translation process. Using the results from these studies, a design was selected for the XOCOT-T3 and tested with the launcher model to ensure the plasmoid was able to translate from the discharge coils during its lifetime. The final design selected the $225 \mu \mathrm{F}$ capacitor bank for a discharge frequency of $10 \mathrm{kHz}$, with the $43.5 \mu \mathrm{F}, 20 \mathrm{kHz}$ bank as the alternate. The cone angle was minimized to 2 degrees and the inner coil radius was maximized at $7.2 \mathrm{~cm}$. The minimum energy required for translation with the $225 \mu \mathrm{F}$ bank was $200 \mathrm{~J}$; the minimum energy using the $43.5 \mu \mathrm{F}$ bank was $600 \mathrm{~J}$. The expected plasmoid velocity and energy efficiency of the final design was estimated at $100-150 \mathrm{~km} / \mathrm{s}$ with an energy efficiency of $26-35 \%$. The simulated plasmoid mass of $1.1 \times 10^{-8} \mathrm{~kg}$, resulted in a directed impulse bit (momentum) of $1.1 \mathrm{mN}-\mathrm{s}$ to $1.7 \mathrm{mN}$-s.

The final XOCOT-T3 design selected four turns for the outer and inner coil. Magnetic field simulations in COMSOL revealed that the coil introduced distortions in the field from current interactions between nearby turns. Magnetic field simulations were conducted with a conducting plasma between the coils, producing a reversed field magnetic field on the inner wall compared to the vacuum field. The appearance of this reversed field in the experiment will provide evidence that a conducting reversed-field plasmoid is present in annulus. Further simulations with a moving conductive region between the coils, 
mimicing a translating plasmoid, demonstrated typical magnetic field signatures expected by a translating plasmoid. These signatures will aid in diagnosing plasmoid motion in the experiment.

The design studies uncovered new insights into the design of annular launchers, including the need for small cone angles on the outer coil. Shallow cone angles are required for efficient annular launchers as they align the region of peak acceleration $(\mathrm{dM} / \mathrm{dz})$ of the outer coil with the inner coil. Larger cone angles extended the region of peak acceleration for the outer coil back towards the throat of the coil; while the region of peak acceleration for the inner coil remains near the downstream end of the coil. Aligning the regions of peak acceleration for the inner and outer coil maximizes the total $\mathrm{dM} / \mathrm{dz}$ of the system, which is directly proportional to the Lorentz force. This means that the maximum possible Lorentz force can be achieved by timing the passage of the plasmoid through this region when the coil currents and plasmoid current are at their peak. Maximizing the Lorentz force is a direct payoff to energy efficiency, since it allows the plasmoid to exit with the maximum velocity.

Maximum efficiency also arises when the inner coil is the largest possible diameter. This brings the coil in closer contact with the plasmoid for improved coupling, increases the inductance of the inner coil, and drives more current to the outer coil to improve its effectiveness. All three effects work to maximize the Lorentz force and thus, energy efficiency. The drawback to larger inner coil diameters is that it increases the chance of wall contact with the plasma since finite thickness insulators must be placed over the coils for electrical protection. Wall contact is to be avoided in pulsed inductive plasma systems as it leads to massive cooling of the plasma and disruption of the current configuration.

The results from the design study also demonstrated the sensitivity of electromagnetic 
launcher geometries to perfect timing. For maximum efficiency, the plasma must arrive at the region of peak acceleration (peak $\mathrm{dM} / \mathrm{dz}$ ) when the coil currents and plasma current peak. This was discovered in the energy study for the annular model, but can be expanded to other geometries as well. The timing can be adjusted by changing the initial energy to control the initial plasmoid acceleration. Unpredictable factors such as a variation in mass of the plasmoid and a variation in plasmoid size can alter the optimal input energy from model predictions. It is highly likely that empirical studies will be required to find the optimal energy for each device to attain maximum efficiency.

The sensitivity of energy efficiency to timing of the plasmoid's trajectory through the region of peak acceleration is unfortunate for AFRCs. Experimental studies have demonstrated that the lifetime of an AFRC is limited to the quarter-cycle of the discharge. This lifetime definition is based on when magnetic field reversal is lost on the inner wall. In reality, the reversed field begins to decay before this time implying that the plasma current begins to degrade as well and the true lifetime is shorter than the quarter-cycle of the discharge. This means that an AFRC must be ejected from the coils when the plasma currents peak, sometime before the coil currents peak. This severely limits the energy efficiency of the system, since all three currents must be at their peak together for maximum efficiency. These observations are not limited to the annular geometry. Conical $\theta$-pinch launchers have similar acceleration physics and may be sensitive to a similar timing constraint. While launcher-types studies have been previously conducted for conical $\theta$-pinch designs [2], lifetime considerations were not included in this analysis.

The exit velocity of the AFRC plasmoid predicted by these studies is much higher than other PIPT devices. The range of exit velocities demonstrated in other experiments discussed in Section 2.3 is between $10-20 \mathrm{~km} / \mathrm{s}$. The exit velocities predicted by the 
launcher model were in excess of $100 \mathrm{~km} / \mathrm{s}$. These extreme exit velocities are the consequence of a fairly high discharge frequency $(10 \mathrm{kHz})$. The plasmoid must travel the full length of the coil in less than $25 \mu \mathrm{s}$, accelerating from a standstill only when the currents are high enough to result in a sufficient Lorentz force. Lengthening the quarter cycle by reducing the frequency will likely lower the exit velocity, though the competing effect of slower-building plasma currents may result in a longer initial translation delay.

Despite the numerous insights the annular electromagnetic launcher model results have uncovered, many of these are based around the assumption of a rigid plasmoid-slug which maintains its shape even as it travels downstream. This assumption is unlikely to hold in a plasma experiment, as changing fluid properties will dominate the overall structure of the plasmoid. Experimental hardware is required to fully test the capability of an AFRC-based thruster. Data from the experiment can be compared to the model to understand how well this model predicts plasmoid behavior. 


\section{Chapter 5}

\section{Experiment, Facilities, and Diagnostics}

This chapter discusses the experimental setup, facilties, and diagnostics used in this research. Section 5.1 describes the XOCOT-T3 experiment, including specific details about the discharge circuit, the pre-ionization circuit, switching hardware, gas feed system, vacuum systems, safety considerations, and experimental operating procedures. Detailed information about the coil construction is provided, along with impedance measurements of discharge circuit components. The diagnostics used in this work are discussed in Section 5.2 The diagnostic tools include current and voltage probes, magnetic field diagnostics, plasma probes, and single frame photography. An indepth look at magnetic probe theory is provided in Section 5.2 .2 along with a discussion of probe calibration techniques and probe construction. Plasma probes, or Langmuir probes, are discussed in Section 5.2.3. The implementation of these diagnostics in measuring the plasmoid's velocity, impulse-bit, and energy efficiency is discussed in Section 5.3 . 


\subsection{Experimental Apparatus}

The XOCOT-T3 was a single pulse demonstration of AFRC formation and translation, connected to Chamber 5B at the U.S. Air Force Research Laboratory-Edwards. A photograph of the experiment and chamber is shown in Figure 5.1

The experiment used two separate circuits for the pre-ionization and main bank discharge. The inductive formation allowed all circuit components to remain at atmosphere. The plasmoid was formed inside a pair of vacuum-sealed quartz liners. The current pulse required to form the plasmoid was created by releasing the energy stored in a discharge capacitor into main electromagnetic coils through a triggered ignitron switch. The plasmoids were formed in a static backfill of gas, rather than using a puffed gas fill. An antenna upstream of the main coils was used for pre-ionization. A cross-sectional

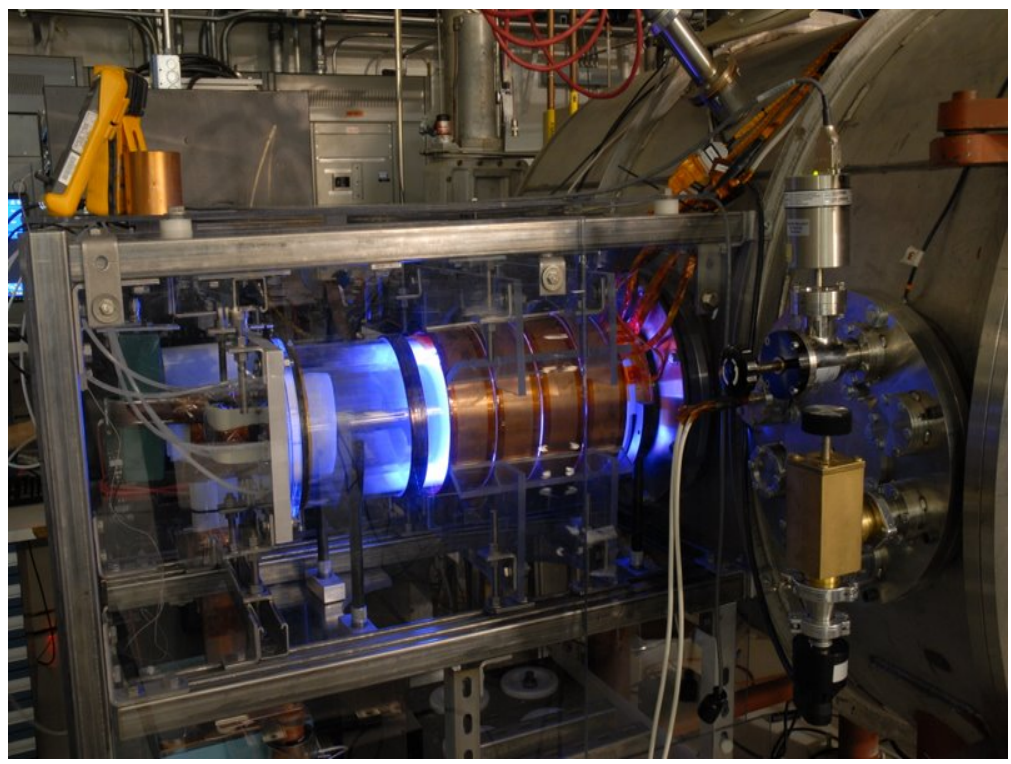

Figure 5.1: The XOCOT-T3 experiment, connected to Chamber 5B. Image is a long exposure photograph of a single pulse discharge in argon. The pre-ionization antenna is shown as the thinner coil upstream (left) of the main 4-turn coils. 


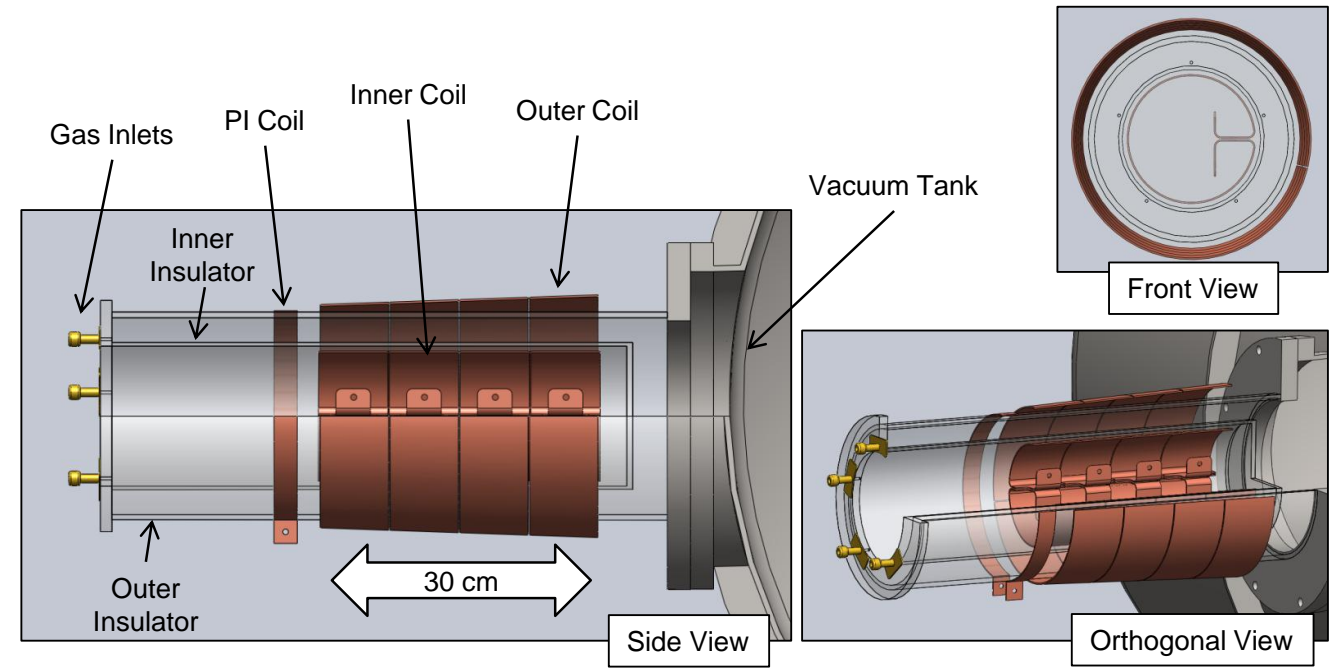

Figure 5.2: A dimensional drawing of the XOCOT-T3 experiment from several perspectives, connected to Chamber 5B. The coils were concentric, separating an annular space with quartz insulators.

dimensional drawing of the experiment from several perspectives is shown in Figure 5.2 A detailed circuit schematic in Figure 5.3 shows the layout of the entire experiment.

\subsubsection{Electromagnetic Coils}

The XOCOT-T3 main coils were concentric 4-turn coils, constructed from copper sheet. The coils were designed based on the translation predictions from Section 4.2. The outer coil had a nominal diameter of $24 \mathrm{~cm}$, the inner coil diameter was $14 \mathrm{~cm}$, and both coils were $30.5 \mathrm{~cm}$ long. The outer coil was tapered to $2^{\circ}$ to provide the Lorentz acceleration force, while the inner coil remained cylindrical. Each turn on the outer coil was tapered to provide a smooth magnetic field gradient. The thickness of the inner coil was $1.5 \mathrm{~mm}$ and the outer coil thickness was $2 \mathrm{~mm}$. Both coil thicknesses were sized to allow for 2-3 skin depths of current penetration at $10 \mathrm{kHz}$. 


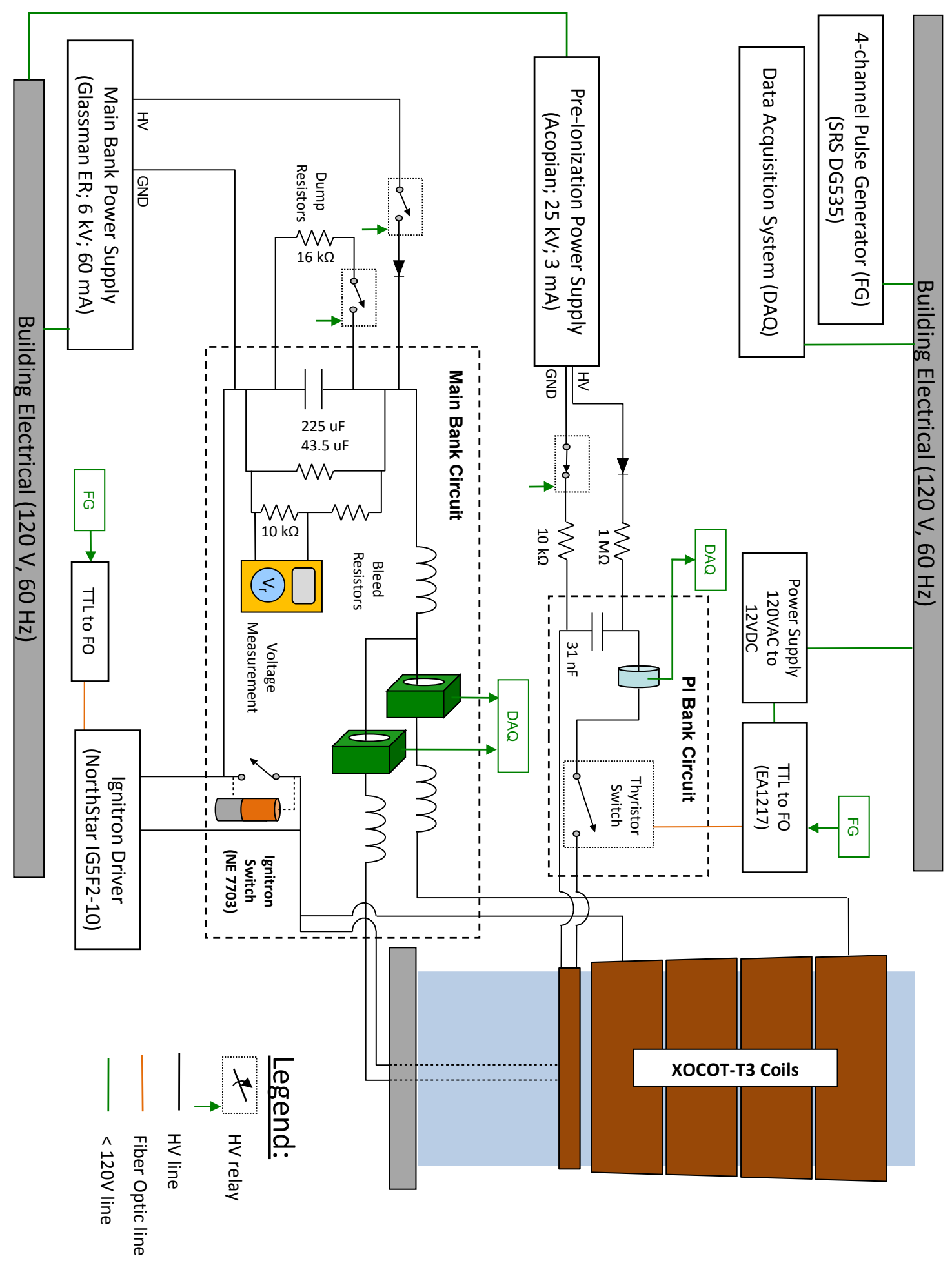

Figure 5.3: A system view of the XOCOT-T3 experiment. 


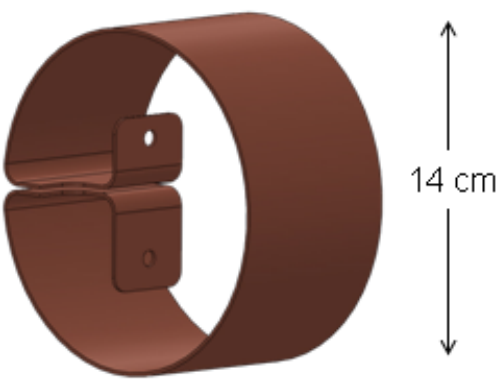

(a)

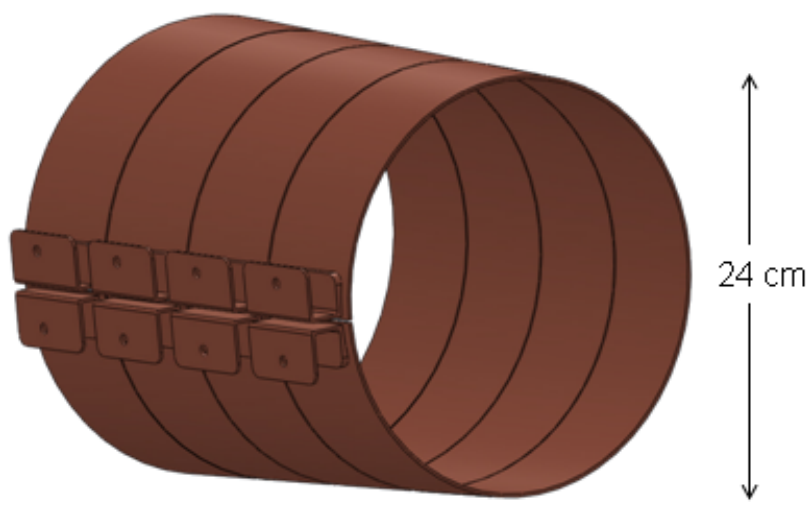

(b)

Figure 5.4: XOCOT-T3 discharge coils, showing (a) a single turn of the inner coil and (b) the outer coil assembly.

The coils were hand wrapped from a flat copper sheet, annealed for workability. A mandrel was machined for each coil and the coil turn cutouts were wrapped around it. The edges of the coils were sanded to minimize high-voltage arcing. Connections to the coils were made away from the walls to reduce local disturbances to the magnetic field. The connection points were placed in the center of each coil, rather than at the corners to eliminate axial coil currents. For the outer coil, connection plates were silver-soldered to the coil surface and copper jumpers were bolted between coils. The inner coil turns were made from a continuous sheet of copper, with the connection leads bent inwards. Custom copper jumpers connected the turns in series. The inner coil was cantilevered from the back plate with a plastic support structure to facilitate plasmoid translation. Drawings of the coils are shown in Figure 5.4 .

The coils were insulated to minimize arcing between the coils. Two layers of polymide tape and PTFE spacers provided insulations between the outer coil turns. The inner coil turns were insulated with polymide tape and 2 layers of clear, PVC heat shrink. The inner coil was originally intended to be exposed to vacuum, but failures in the insulation at high voltages required the custom inner insulator shown in Figure 5.2 to keep the inner coil at 
atmosphere.

\subsubsection{Quartz Insulators}

Quartz insulators were used to protect the coils from the plasma for inductive formation. The quartz insulators were custom fabricated to minimize the distance between the plasma and the coils. The drawing in Figure 5.2 shows the arrangement of the insulators with respect to the coils. The outer insulator had an inner diameter of $21.6 \mathrm{~cm}$, wall thickness of $6.35 \mathrm{~mm}$, and a length of $61 \mathrm{~cm}$. Rather than match the small angle taper on the outer coil, it remained cylindrical. Since the cone angle on the outer coil was small $\left(2^{\circ}\right)$, the plasmoid was not expected to contact the cylindrical outer wall during its exit. Image results in Chapter 8 confirm the plasma remained off the outer wall. A $6 \mathrm{~mm}$ gap between its outer insulator surface and the small diameter of the outer coil was retained to provided diagnostic access for the outer coil probes. The inner insulator sat directly on the inner coil assembly. It was $16.2 \mathrm{~cm}$ on the outer diameter with a wall thickness of $4 \mathrm{~mm}$. The inner insulator extended $2.5 \mathrm{~cm}$ past the end of the coil.

The outer insulator was sealed against atmosphere using L-gaskets on the upstream and downstream end. Small holes in the end plate of the inner insulator provided access for the gas feed. Custom brass gas ports were epoxied to the inner insulator for gas hose attachment. The gasket and gas feed seals were leak tested with a helium leak detector and no detectable leaks were found. 


\subsubsection{Main Bank Discharge Circuit}

The current pulse required to form the plasmoid was created by releasing the energy stored in a discharge capacitor into the main coils, through an ignitron switch. The resulting underdamped RLC circuit rang through 2-4 cycles, depending on the charge voltage. The simplifed circuit schematic for the XOCOT-T3 main circuit is shown in Figure 5.5. The coils are represented by $L_{o}$ and $L_{i}$, with parasitic elements indicated with the subscript $e$. Photographs of the main bank circuit components are shown in Figure 5.6.

Two versions of the circuit were designed: a $10 \mathrm{kHz}$ circuit and a $20 \mathrm{kHz}$ circuit. The $10 \mathrm{kHz}$ circuit was created first, using a single $225-\mu \mathrm{F}(+15 \%-0 \%)$ discharge capacitor (Maxwell p/n 32634). The actual frequency of the $10 \mathrm{kHz}$ circuit was measured to be 9.4 $\mathrm{kHz}$. The second version of the circuit was created by exchanging the $225-\mu \mathrm{F}$ for a $43.5-\mu \mathrm{F}$ bank. This bank was constructed from three 14.5- $\mu \mathrm{F}$ (p/n CJE101) connected in parallel. The second discharge circuit, designed for $20 \mathrm{kHz}$ operation, rang at $19.4 \mathrm{kHz}$.

Low inductance transmission lines, connecting the capacitor bank to the coils, were

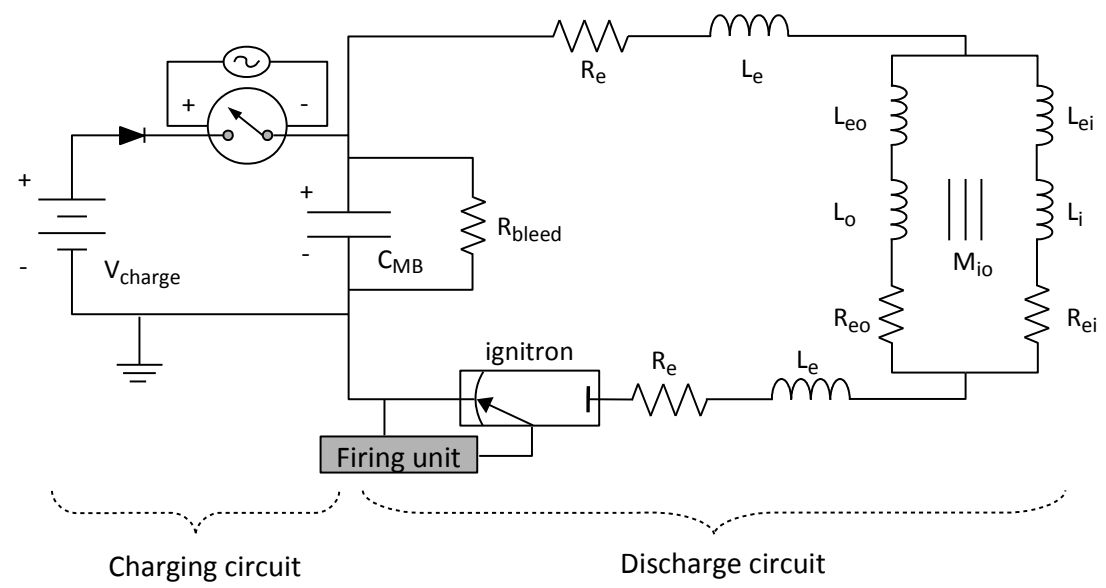

Figure 5.5: Main Bank Discharge Circuit Schematic. 
constructed from $2 \mathrm{~mm}$ thick copper strips, $7 \mathrm{~cm}$ wide. The high voltage line and ground line were sandwiched together to minimize their parasitic inductance and insulated with polymide tape and PVC heat shrink. These are marked as $R_{e}$ and $L_{e}$ in Figure 5.5. The inner coil and outer coil transmission lines connected to the main lines and were made from $1.5 \mathrm{~mm}$ thick, $2.5 \mathrm{~cm}$ wide copper strap. These lines were sandwiched together as much as possible to reduce stray inductance and still accomodate the Rogowski coils, used for measuring the current. The inner and outer coil lines were insulated with 2 layers of heat shrink insulation and 2 layers of polymide tape. The inner lines are noted in Figure 5.5 as $L_{e i}$ and $R_{e i}$; the outer lines are marked as $L_{e o}$ and $R_{e o}$.

The ignitron switch (National Electronics p/n 7703) closed when a column of mercury plasma was created between the anode and cathode. The firing unit (Northstar $\mathrm{p} / \mathrm{n}$ IG5F2-10) provides the seed electrons required for the breakdown. Ignitron switches can be extremely tempermental, but are robust solutions for switching high currents. They are not intended to be used in ringing applications as they favor a positive voltage at the anode and their operation around the zero-voltage point (current peak) creates significant EMI. They are temperature sensitive and will not operate reliably at slightly elevated temperatures (above $25 \mathrm{C}$ ) unless the cathode is actively cooled. The switch used in this experiment produced a healthy dose of EMI, but did not seem to be severely affected by bi-polar operation.

The main bank capacitor(s) were welded metal can capacitor(s) with a fairly high inductance (100 $\mathrm{nH}$ or more). These capacitors use a dedicated terminal for the high voltage connection and another (the casing for the $225 \mu \mathrm{F}$ capacitor) as the ground connection. The ground terminal of the capacitor was fixed to the ignitron cathode, connected to ground through its firing unit. During a discharge, this was the only relative ground connection on 

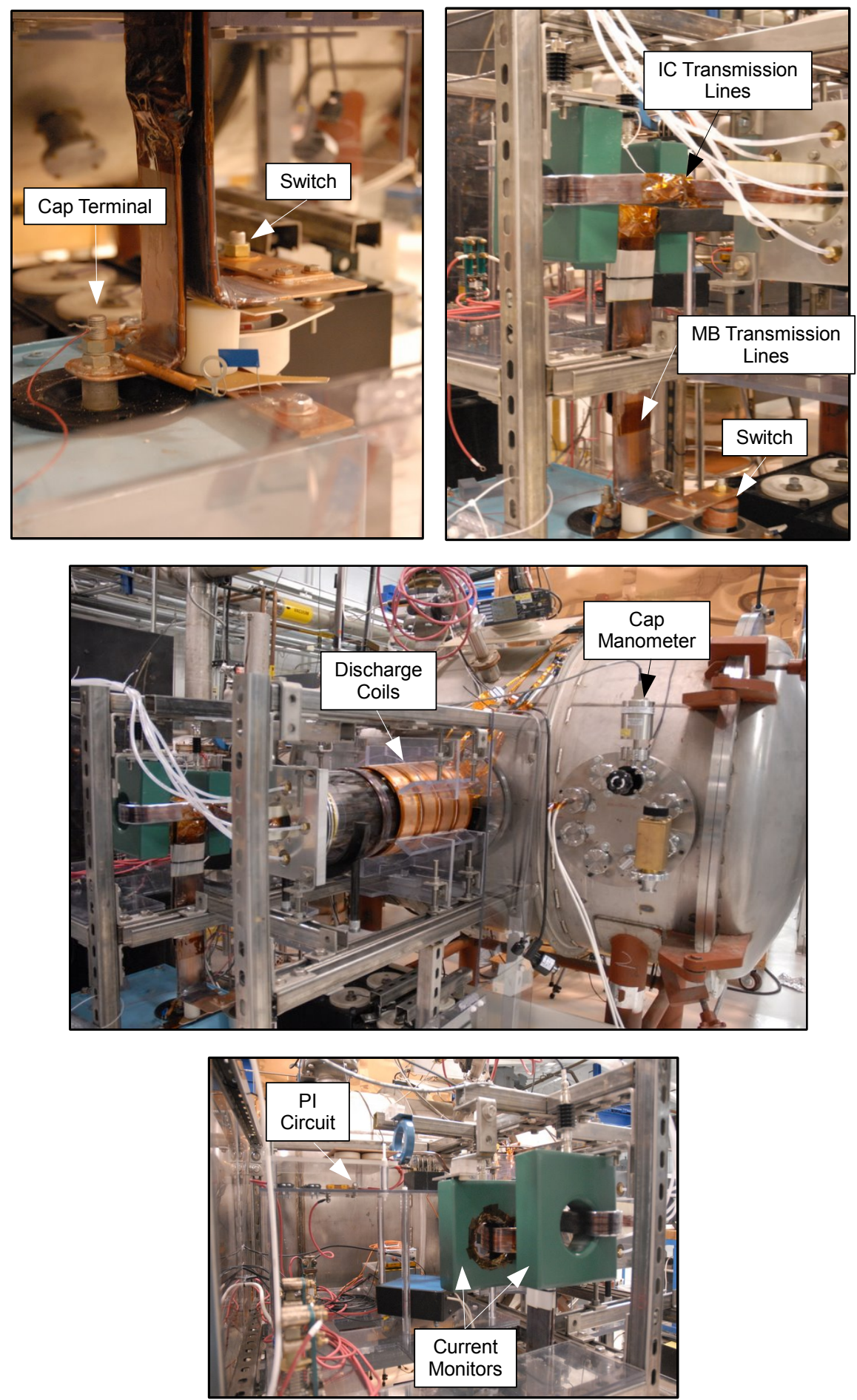

Figure 5.6: Photographs of the XOCOT-T3 main bank circuit. 
the entire circuit. The capacitor was charged using a high voltage power supply (Glassman $\mathrm{p} / \mathrm{n} \mathrm{ER}, 6 \mathrm{kV}, 60 \mathrm{~mA})$. The power supply was connected to the capacitor terminals through a normally-open high voltage relay to keep high voltage transients during circuit firing from traveling back to the power supply. The power supply was also turned off during circuit firing for additional protection.

The impedances of the transmission lines and coils were determined using a combination of electromagnetic modeling, vacuum discharge tests, SPICE modeling, and an Agilent 4294A impedance analyzer. The coils were impossible to isolate from any parasitic inductance, so their inductances $L_{o}$ and $L_{i}$ were computed using COMSOL (see Section 4.2.7). The outer coil line inductance $L_{e o}$ was computed by measuring the total inductance of the outer coil with the impedance analyzer and lines and subtracting $L_{o}$. The geometric constraints of the experiment prevented any other impedance measurements from being collected. Vacuum discharges were used to calculate the remaining inductances by measuring the frequency $f$ and using Equation 5.1 to solve for the system inductance. The capacitance was assumed to be the nominal rating with no additional parasitics. Rather, the parasitic elements associated with the capacitor bank were lumped together with the impedances of the tranmission lines since they were difficult to isolate. The outer coil circuit was tested alone to determine the external inductance $L_{e}$. The inner coil circuit was then tested alone to estimate $L_{e i}$. The mutual inductance between the inner and outer coil was determined by connecting both coils to the circuit and measuring the current ratios between them. Equation 5.2 derived in Section 4.2 was used along with the current ratio to calculate $M_{i o}$. Finally, SPICE modeling was used to check the results and to determine the total resistance using an interative approach. The current waveforms and SPICE waveforms agreed within $1 \%$. The impedence results are shown in Table 5.1. 


$$
\begin{gathered}
f=\frac{1}{2 \pi \sqrt{L C}} \\
\frac{\dot{I}_{o}}{\dot{I}_{i}}=\frac{L_{e i}+L_{i}-M_{i o}}{L_{e o}+L_{o}-M_{i o}}
\end{gathered}
$$

Table 5.1

XOCOT-T3 circuit impedances

\begin{tabular}{lccccc}
\hline Component & Symbol & R & L & C & Remarks \\
\hline Capacitor & $C_{1}$ & - & - & $225 \mu \mathrm{F}$ & \\
& & - & - & $43.5 \mu \mathrm{F}$ & \\
Main & $R_{e}$ & $13 \mathrm{~m} \Omega$ & - & - & $(20 \mathrm{kHz})$ \\
Lines & $L_{e}$ & - & $112 \mathrm{nH}$ & - & $(20 \mathrm{kHz})$ \\
& & & & & \\
Inner Coil & $R_{e i}$ & $1 \mathrm{~m} \Omega$ & - & - & \\
Lines & $L_{e i}$ & - & $744 \mathrm{nH}$ & - & \\
Outer Coil & $L_{e o}$ & - & $600 \mathrm{nH}$ & - & \\
Lines & $R_{e o}$ & $1 \mathrm{~m} \Omega$ & - & - & \\
Outer Coil & $L_{o}$ & - & $2.282 \mu \mathrm{H}$ & - & \\
Inner Coil & $L_{i}$ & - & $857 \mathrm{nH}$ & - & \\
& & & & &
\end{tabular}

\subsubsection{Pre-Ionization Source}

The pre-ionization (PI) plasma was created using a 2 Joule, $1 \mathrm{MHz}$ ringing theta discharge through a $2.5 \mathrm{~cm}$ wide antenna. The placement of the antenna is shown in Figure 5.2 , located $2.5 \mathrm{~cm}$ upstream of the main coils. The PI capacitor bank was six 5-nF ceramic 


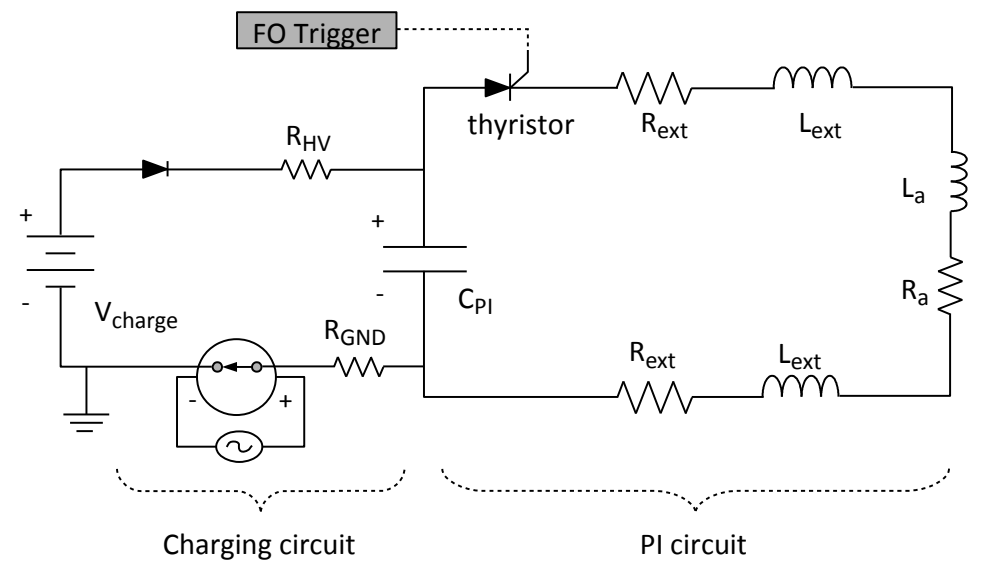

Figure 5.7: A schematic of the pre-ionization circuit.

capacitors connected in parallel to retain a high-Q pulse. The bank was switched through a 16 kV, 9 kA compact thyristor stack (Applied Pulsed Power p/n S33A-4), triggered by an optical pulse. The feedlines were made from thin copper strips, tightly sandwiched together to mimimize parastic inductance. The power supply for the PI circuit (Acopian; $25 \mathrm{kV}$; 3 $\mathrm{mA}$ ) was connected through $201-\mathrm{kV}$ diodes and high impedance resistors, including a 5 $\mathrm{k} \Omega$ resistor between ground and the low voltage side of the capacitor to protect the power supply. The PI power supply remained connected to the circuit and turned on during the tests since the PI capacitor bank was unable to hold a constant voltage for more than a couple seconds. The pre-ionization circuit diagram is shown in Figure 5.7. Photographs of the PI circuit are shown in Figure 5.8

An additional PI source was used to increase the effectiveness of the ringing PI. Seed electrons were created using a simple 2-kV DC glow discharge into the main chamber. The DC glow was required as the PI plasma and AFRC plasmoid would not form without it. This secondary source is also referred to as the pre-pre-ionization or PPI. These methods were selected based on earlier work with the XOCOT-S [41]. 

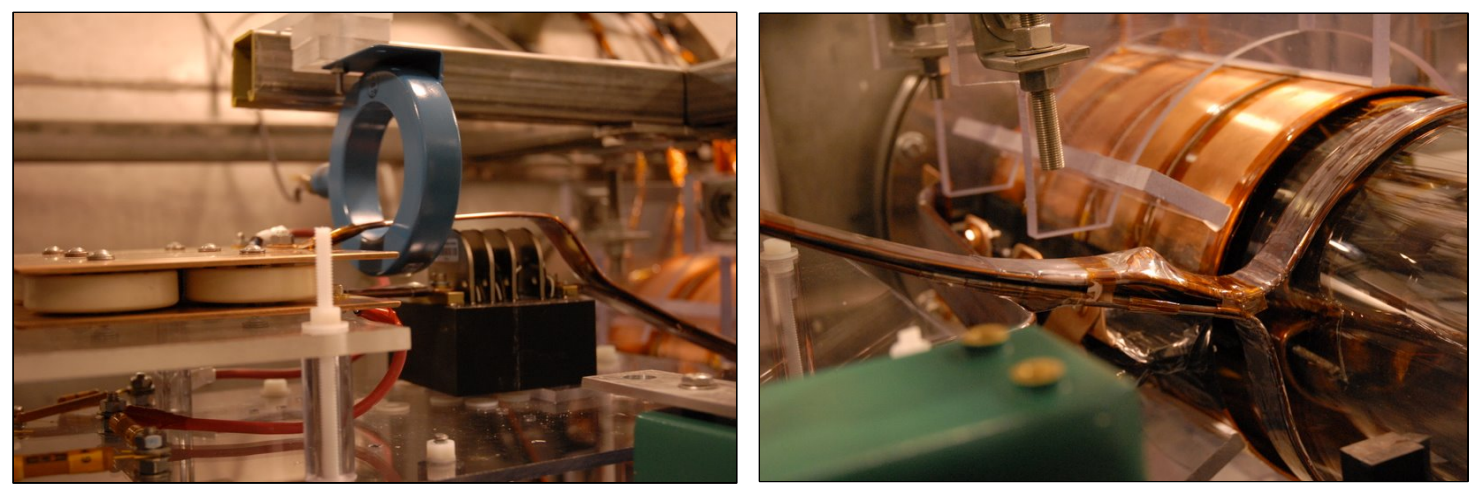

Figure 5.8: Photographs of the pre-ionization circuit. The capacitor bank (far left) is connected to the antenna (far right) through a thyristor stack (middle). The current in the circuit is measured with the Rogowski coil directly in front of the capacitor bank.

\subsubsection{Safety Considerations}

The high voltages used in this experiment posed significant safety concerns for the operator. Several engineering controls were implemented to minimize the risk of personnel or equipment injury, including fiber optically isolated switches, two energy drain systems, and restricted physical access to the experiment. These engineering controls are considered the minimum required for safe operation; future experiments should consider stricter controls such as door interlocks and fiber optically isolated power supplies.

The PI switch and main bank switch were triggered with a fiber optic signal. The signal line responsible for triggering each switch was connected to a optical signal convertor and then to a long fiber optical cable. The fiber optic cable then connected to the switch. These fiber optic controls prevented any high voltage transients in the switches from traveling back along the lines to the user. 
High energy storage capacitors can build up charge over time, even when not connected to a power source. In this experiment, excess charge was drained by attaching high impedance resistors across the terminals. Two parallel high resistance resistors were connected across the terminals for redunancy. The high impedance assured that they would not affect experiment performance.

The energy stored in the capacitor also needs to be drained very quickly in the event of an emergency. A load of three parallel $50 \mathrm{k} \Omega$ non-inductive resistors were connected across the capacitor terminals through a normally-closed gravity activated switch. The switch was opened during experiment firing and then closed again after the circuit discharge. The resulting RC time constant of this circuit was 3.6 seconds (for the $10 \mathrm{kHz}$ circuit).

The experiment was enclosed in a polycarbonate shroud on all sides to prevent physical access to the experiment during operation. The shroud required several tools and about 5 minutes to open, giving plenty of time for the energy dump bank to dissipate all the energy from the capacitor. Continuous high voltage cabling fed through the shroud before connecting to the capacitors. The polycarbonate also functioned as a UV-blocker for the plasma optical radiation.

\subsubsection{Vacuum Facilities and Gas Injection}

The XOCOT-T3 experiment is connected to Chamber 5B. Chamber 5B is a stainless steel vacuum chamber 1 meter in diameter and 2 meters in length. It is nominally evacuated with twin diffusion pumps, backed by a $10 \mathrm{hp}, 300 \mathrm{cfm}$ roughing pump and Roots blower. For these tests, the vacuum facility was equipped with a 6-inch turbomolecular pump, backed 


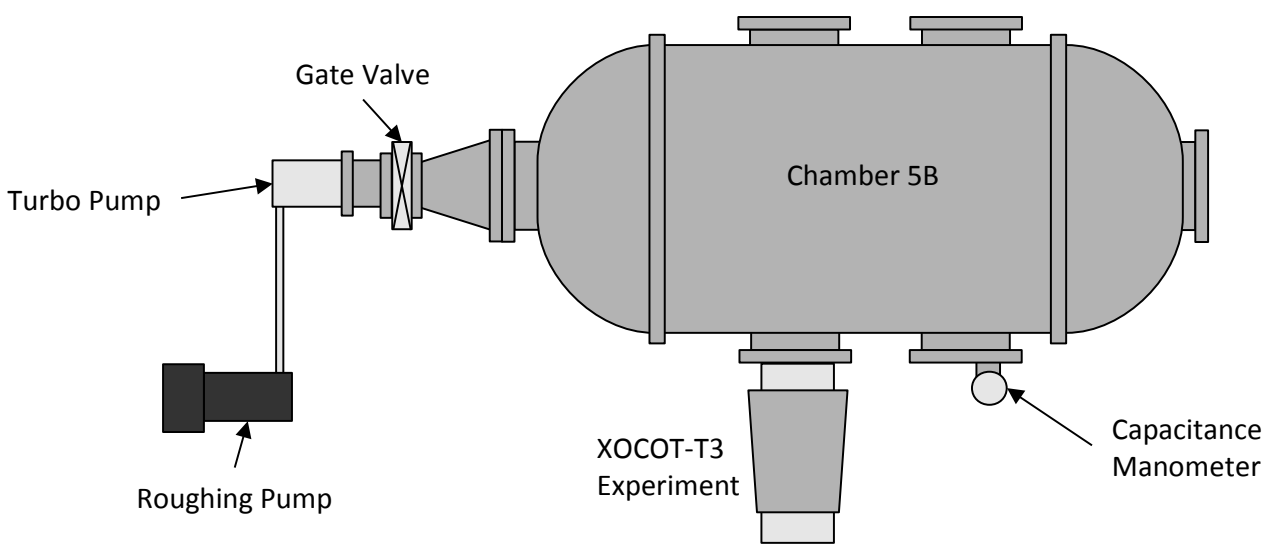

Figure 5.9: A schematic of the vacuum facilities used in the XOCOT-T3 testing. This diagram is not drawn to scale.

by a Varian Tri-Scroll 300 roughing pump. The turbomolecular pump was protected during the test by a mostly-closed gate valve to create a high-conductance path to the pump. The pressure differential across the valve allowed the tank to be at millitorrs of pressure, while the turbo-pump inlet remained in a safe operating range. The complete vacuum system is shown in Figure 5.9.

The XOCOT-T3 formed the plasmoid into a static background gas fill of argon, seeded with $2 \%$ hydrogen. The gas was dispensed through five ports in the backplate, with the flow rate adjusted by a mass flow controller. The pressure of the gas was monitored on a calibrated MKS Baratron Capacitance Manometer ( $\mathrm{p} / \mathrm{n}$ 627B) connected to the main chamber. During testing, the gas feed rate was adjusted to within $2 \%$ percent of the pressure desired for the test point.

A 2-axis translation stage was configured in Chamber 5B for recording data downstream of the XOCOT-T3 coils. The stage was setup to sweep in the $\hat{r}$ and $\hat{z}$ directions. A manual base provided adjustments of the third axis, when the system was at atmosphere. The stage had a total range in the $\hat{z}$ direction of $75 \mathrm{~cm}$ and $50 \mathrm{~cm}$ in the $\hat{r}$ direction. 


\subsubsection{Experiment Operation}

Several hours before testing, the chamber was pumped to $2 \times 10^{-5}$ Torr to remove impurities. The low pressure measurement was recorded with a hot ion gauge and cold cathode gauge. The capacitance manometer was turned on at least 4 hours before testing to ensure accurate readings. The remaining instruments were powered on for at least 1 hour prior to testing.

A 4-channel pulse generator (Stanford Research System p/n DG535) was used to control the experiment timing. The data acquisition system and pre-ionization circuit were triggered concurrently. The main bank switch was triggered after the pre-ionization. Timing between all channels on the function generator could be varied from shot-to-shot.

The testing sequence for a single test point consisted of twelve steps: (1) Turn on the propellant, using the mass flow controller equipment. (2) Monitor the pressure on the capacitance manometer read-out until the pressure stabilizes around the desired test point. (3) Set the desired PI voltage and main bank voltage on the power supplies. (4) Turn on the PI supply to charge PI bank. (5) Turn on the PPI supply. (6) Open the relay to disconnect the dump resistors from the main bank charge supply. (7) Turn on the main bank supply to charge the main bank. (8) When the capacitor is fully charged, open the charge relay, and turn off the main bank power supply. (9) Wait for capacitor to drain to the desired test voltage (typically 4-6 seconds). (10) Manually trigger the function generator to discharge the experiment. (11) Turn off the PI supply and turn off the relay to reconnect the dump resistors. (12) Collect the data on a central computer and reset the operating conditions.

The gas was left on for multiple tests, with the number of shots determined by maintaining a safe temperature on the turbo-pump. The discharge circuit was not designed to dissipate 
heat loads since it was meant to be a single-shot experiment. An overly-conservative five minutes between shots was set as the minimum wait time.

\subsection{Diagnostics}

An objective of this research was to measure the velocity, momentum, and energy efficiency of an AFRC plasmoid in the XOCOT-T3 experiment. The plasmoid velocity was measured with a time-of-flight (TOF) array, consisting of two probes spaced apart a fixed distance. Both magnetic field (b-dot) probes and Langmuir plasma probes were used in the TOF array. Momentum was inferred by combining the velocity measurements with a mass estimate from a density map of the plume. The density of the plume was measured with Langmuir probes in the translation region of the experiment. Energy efficiency was then estimated by comparing the kinetic energy of the plasmoid to the initial energy stored in the capacitor.

Additional diagnostics were also incorporated, though they were not essential to the performance characterization of the experiment. These diagnostics were still important for monitoring the health of the experiment, the shot-to-shot repeatibility, and for diagnosing plasmoid behavior. Coil current measurements were made on the inner and outer coil circuits. External magnetic field probes adjacent to the outer coil provided non-intrusive information about the magnetic field topology of the plasmoid. The external probes were also a qualitative TOF array upstream of the main TOF array in the translation region. Fast single-frame photography was used for mapping a qualitative radial plasma distribution inside the formation chamber. An asymmmetric double Langmuir probe was used for mapping the radial distribution of plasma inside the confinement region. The arrangement 


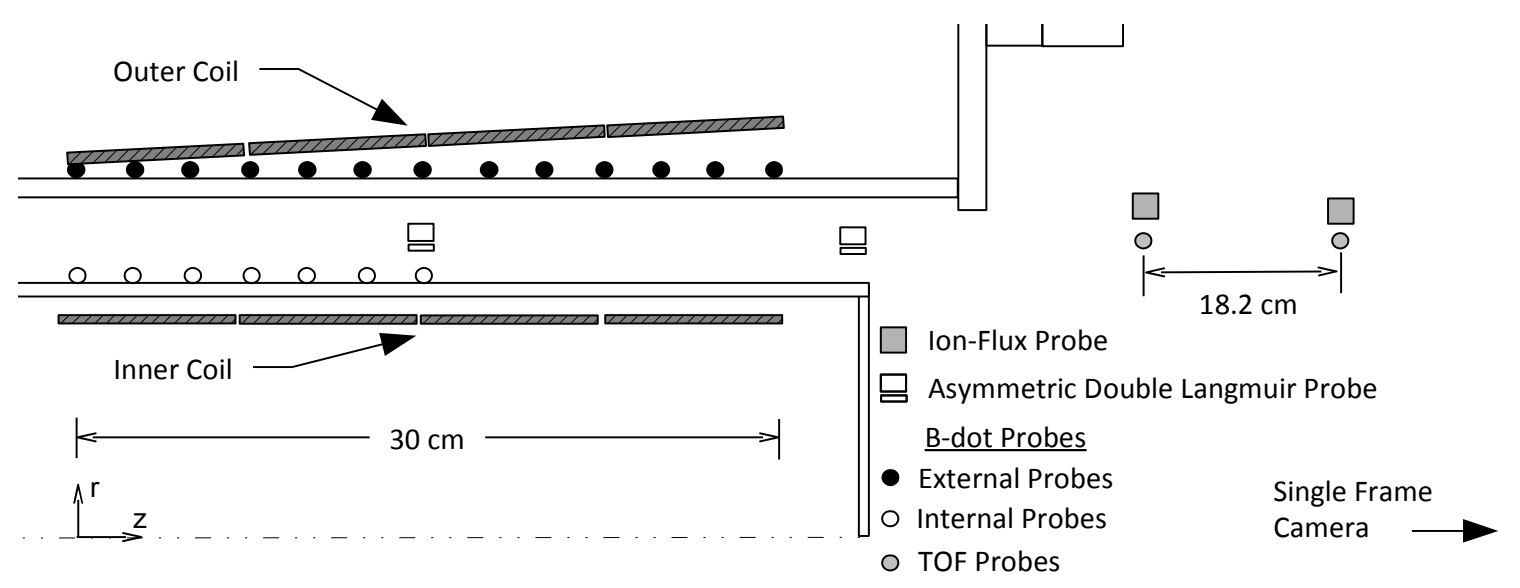

Figure 5.10: Layout of the diagnostics used in the XOCOT-T3. The single frame camera is located outside the chamber, capturing images from an end-on view. Current and voltage monitors are not shown in this figure and are located on the external circuit.

of the diagnostics are shown in Figure 5.10.

For most of the tests, the XOCOT-T3 data acquisition (DAQ) system was a 16-channel, high-speed oscillscope network, with two Tektronix 8-bit, 4-channel, $125 \mathrm{MS} / \mathrm{s}$ oscilloscopes and one Nicolet Sigma 8-channel, 12-bit, $100 \mathrm{MS} / \mathrm{s}$ oscillscope. The Tektronix oscilloscopes (p/n TDS 7104 and TDS 5104) were set to record 50,000 samples at 125 MSamples per second (8 ns per point). The Nicolet Sigma (p/n LDS 100) recorded 50,000 points at $100 \mathrm{MS} / \mathrm{s}$ (10 ns per point). The sample rates were more than adequate to capture phenomena well below the Nyquist frequency $(50 \mathrm{MHz})$. The Nicolet and Tektronix oscilloscopes recorded $10 \%$ of the data before the oscilloscope triggered. This provided a clean DC response, used to correct for oscilloscope offsets. The oscilloscopes were located inside a grounded screen room behind Chamber 5B. The screen room was clad in solid sheet copper $0.6 \mathrm{~mm}$ thick. This corresponds to a single skin depth at $10 \mathrm{kHz}$, effectively blocking all higher frequency radiated EMI. A secondary DAQ component was added to the system during the $20 \mathrm{kHz}$ tests. This was a 24 channel PXI system with 12-bit, 
$60 \mathrm{MS} / \mathrm{s}$ digitizers (NI-5105). The trigger on this system was also set to record a pre-trigger record length of $10 \%$. The sampling frequency was set to $60 \mathrm{MS} / \mathrm{s}$, with a record length of 20,000 points.

\subsubsection{Current and Voltage Monitors}

The outer and inner coil currents were measured using wide band Rogowski coils (Pearson Electronics $\mathrm{p} / \mathrm{n} 301 \mathrm{x}$ ) on the outer and inner transmission lines. Placement is shown in Figure 5.3. The Pearson current monitors had a sensistivity of $0.01 \mathrm{~V} / \mathrm{A}+1 \%$, a $3 \mathrm{~dB}$ bandwidth from $5 \mathrm{~Hz}$ to $2 \mathrm{MHz}$, a useable risetime of $200 \mathrm{~ns}$, a droop rate of $3.0 \% /$ millisecond, and peak current ratings of $50 \mathrm{kA}$. A bi-directional $20 \mathrm{~dB}$ attenuator (Weinschel p/n 34-20-34) was added to the output of the current monitor to decrease the sensitivity of the current monitor to 0.001 V/A. The attenuator had a deviation of \pm 0.6 $\mathrm{dB}$ and bandwidth of DC - $4 \mathrm{GHz}$. The current monitors and attenuators were used well within recommended operating ranges; no significant errors were expected from these measurements.

The PI current was measured with a small-profile Rogowski coil (Stangenes Industries p/n 2-0.1WA). The PI current monitor had an output sensitivity of $0.1 \mathrm{~V} / \mathrm{A} \pm 0.5 \%$, a droop rate of $0.0008 \% / \mu \mathrm{s}$, an rise time of $20 \mathrm{~ns}$, a $3 \mathrm{~dB}$ bandwidth from $1 \mathrm{~Hz}$ to $20 \mathrm{MHz}$, and a peak current of $5 \mathrm{kA}$. The rise time and bandwidth of the PI current monitor was insufficient to capture the initial rise of plasma current at $1 \mathrm{MHz}$, though this information was not required in this experiment. The PI circuit characteristics and waveforms were not a focus of this research, so the significant errors introduced by using a non-ideal current monitor had little impact on the results. 
Initial capacitor voltage was recorded using a battery isolated multimeter, connected to a voltage divider circuit between the terminals of the capacitor as shown in Figure 5.11, This circuit used two high impedance resistors (bleed resistors) in parallel and a small 10 $\mathrm{k} \Omega$ resistor in series with one high impedance resistor. The terminals of the small resistor were connected to an isolated multimeter (Fluke $\mathrm{p} / \mathrm{n}$ 189) for the voltage readout. The voltage divider circuit was calibrated at DC from 30 volts to 250 volts using a high-voltage Tektronix 6015A probe and a Fluke multimeter (p/n 189) at 47 voltage points. A linear curve fit to the data resulted in the following relation: $\mathrm{V}_{\text {cap }}=\mathrm{CF}^{*} \mathrm{~V}_{\text {resistor }}$. Each circuit used different bleed resistors. The $10 \mathrm{kHz}$ circuit used two parallel $2.66 \mathrm{M} \Omega$ (Caddock p/n MS 313) bleed resistors and the $20 \mathrm{kHz}$ circuit used two parallel $23 \mathrm{M} \Omega$ resistors. The voltage divider calibration factor for the $10 \mathrm{kHz}$ circuit was calculated to be $266.47 \pm$ 0.90. The calibration factor for the $20 \mathrm{kHz}$ circuit was $2466.93 \pm 3.42$. This includes the measurement error and the standard deviation from the linear fit.

The circuit was triggered manually by waiting for the resistor voltage on the voltage divider circuit to reach a certain level. Error analysis revealed that a tolerance of $10 \mathrm{mV}$ on this reading was allowed to maintain a $1 \%$ or less deviation in capacitor energy for the 10 $\mathrm{kHz}$ circuit. A tolerance of $1 \mathrm{mV}$ was required for the $20 \mathrm{kHz}$ circuit. Normal operation came well within these bounds so the error in capacitor energy using a manual trigger was expected to be small.

Time-dependent voltage measurements are difficult to record on pulsed high-frequency experiments since an accurate and well-known ground reference must be known. High ground potentials can develop during the discharge, resulting in an inconsistent voltage offset that cannot be accounted for. Voltage measurements also require direct contact to the circuit which presents a high voltage danger to the operator. For these 


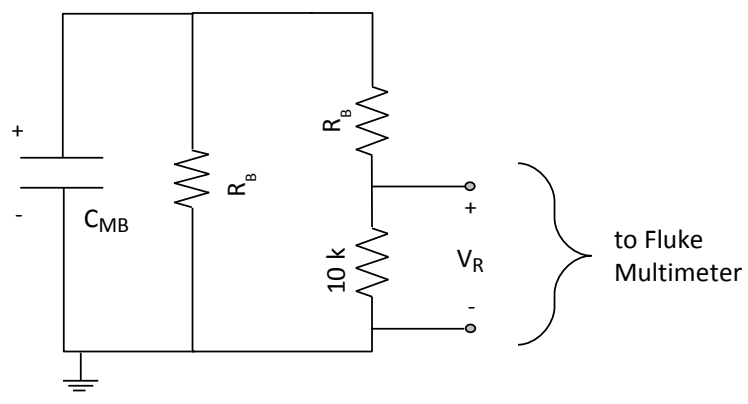

Figure 5.11: Capacitor Voltage Divider Circuit. The capacitor voltage is measured across the $10 \mathrm{k} \Omega$ resistor with an isolated Fluke multimeter.

reasons, time-dependant voltages were not directly measured on the XOCOT-T3. The time-depedendent voltage could be estimated by fitting the current measurements to an RLC circuit description of the device.

\subsubsection{Magnetic Field Probes}

The principle diagnostics for this work were magnetic field probes (b-dot probes). The XOCOT-T3 used 13 z-axis b-dot probes on the outer coil, 6 z-axis internal b-dot probes, and 2 z-axis downstream TOF probes, as shown in Figure 5.12. The external b-dot probes were placed between the outer coil and quartz insulator with a probe spacing of $2.5 \mathrm{~cm}$. The internal b-dot probes were also spaced $2.5 \mathrm{~cm}$ apart and were set on the inner insulator for most of the tests. The TOF probes were located inside the translation region, spaced $18.2 \mathrm{~cm}$ apart. The near probe was at $\mathrm{z}=49.5 \mathrm{~cm}$ and the far probe was at $\mathrm{z}=67.7 \mathrm{~cm}$. 


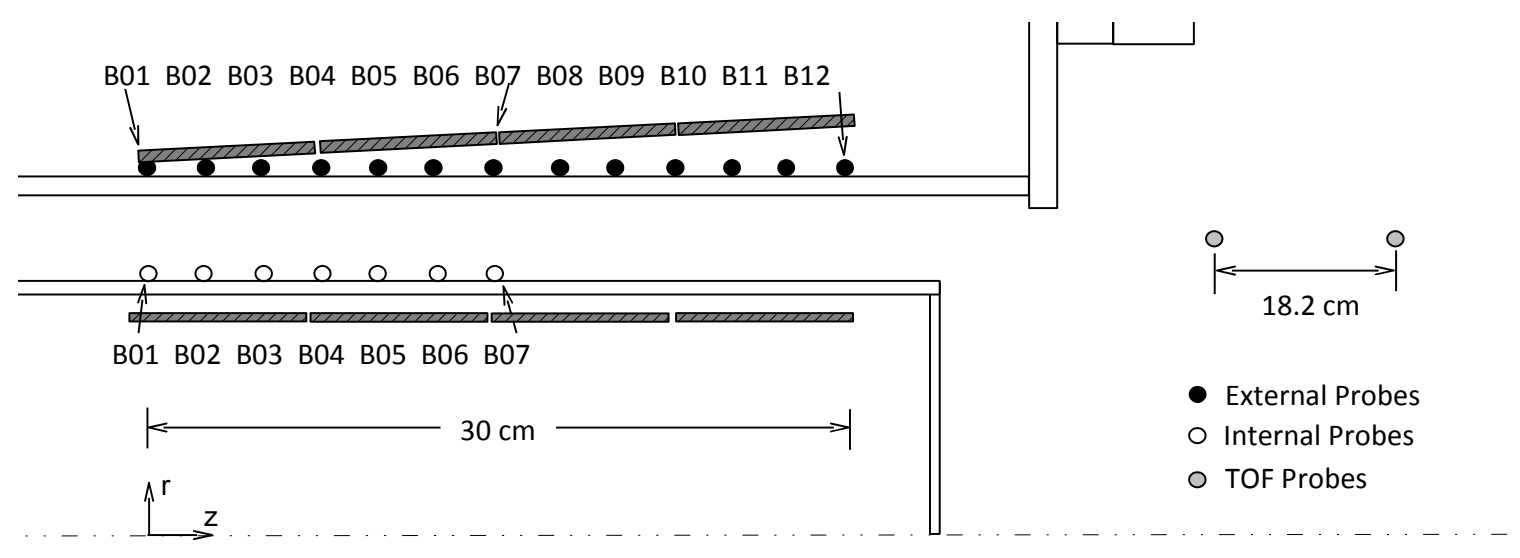

Figure 5.12: Magnetic field probe locations in the XOCOT-T3 experiment. External b-dot probes run the length of the discharge coils. The placement of the internal b-dot probes shown here is typical for most of the testing. Probe-to-probe spacing is $2.5 \mathrm{~cm}$ apart on the external and internal arrays.

\subsubsection{B-dot Probe Theory}

B-dot probes are loops of wire wrapped in a single plane. The loops enclose a cross-sectional area $A$ with a total number of turns $N$. When exposed to a time-changing magnetic field, they detect an electromotive force $\varepsilon_{B}(\mathrm{EMF})$ proportional to the rate of change of magnetic flux $\Phi_{B}$ normal to $A$ as a result of Faraday's Law:

$$
\varepsilon_{B}=-N \frac{d \Phi_{B}}{d t}=-N \frac{d B \cdot A}{d t}=-N A \frac{d B}{d t}
$$

The proportionality constant $N A$ relates the voltage produced by the EMF to the changing magnetic field. The EMF is quite small, so large proportionality constants are used to amplify the signal. The b-dot probe is generally connected to transmission lines and the output voltage produced by the probe assembly $V_{p}$ is measured at the end of the lines, shown in Figure 5.13. Most elementary treatments equate the EMF generated by the fluctuating magnetic field $\varepsilon_{B}$ to the probe voltage $V_{p}$ (i.e. $\varepsilon_{B}=V_{p}$ ). This relationship is only true for the 


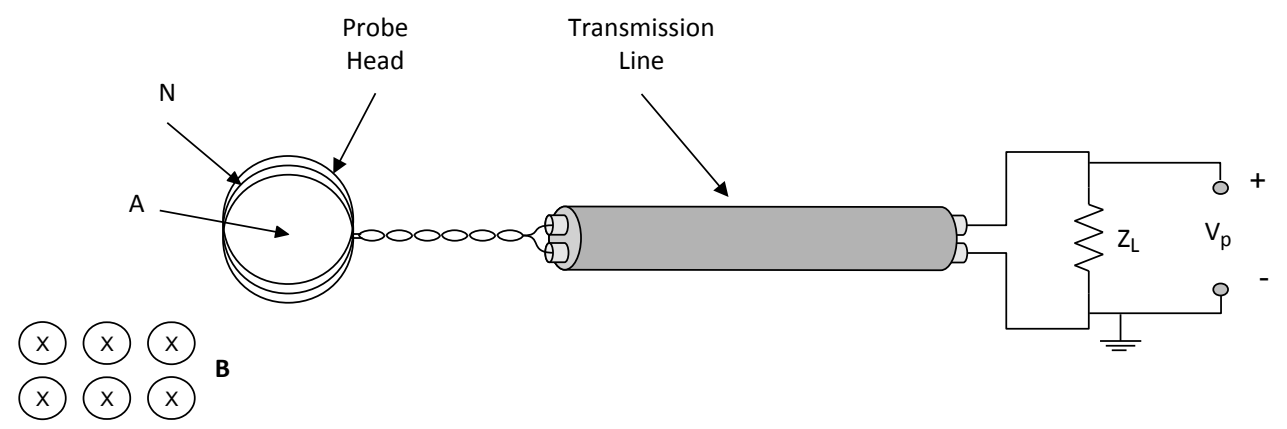

Figure 5.13: Illustration of a magnetic field probe. The probe head is connected to a transmission line and $V_{p}$ is measured at the end of the transmission line.

ideal case, which rarely exists. The transmission lines and the probe have non-negligible impedance which become significant at high frequency. Additionally, the probe assembly can interact with nearby ground planes and conductors, shunting capacitive current away from or to the probe. These parasitic impedances distort $\varepsilon_{B}$ by the time it arrives at the measurement device, reducing the amplitude and shifting the phase.

The distortion of the signal can be regarded as a transfer function which transforms the field fluctuations $d B / d t$ into $V_{p}$ [62]. The transfer function $\beta(\omega)$ includes the proportionality constant $N A$ as well as the impedance effects and external influences, resulting in the following relation:

$$
V_{p}=-\beta(\omega) \frac{d B}{d t}=-\beta(\omega) \frac{\varepsilon_{B}}{N A}
$$

Messer, et al introduced this notation in their broadband b-dot probe calibration paper [63], referring to $\beta(\omega)$ as the sensitivity parameter. An expanded derivation of $\beta(\omega)$ is derived here to include additional capacitive effects.

The probe assembly's transfer function $\beta(\omega)$ can be modeled by considering the circuit equivalent of a probe assembly, shown in Figure 5.14a. The probe head (circuit elements 
with subscript $\mathrm{p}$ ) is connected to a balanced transmission line such as a twisted, shielded pair. The EMF from field fluctuations creates a source voltage $\varepsilon_{B}$. The probe and transmission lines contain some resistance, inductance, capacitance, and conductance which alter the source voltage by the time it is measured across a termination load $Z_{L}$. The finite resistivity of the lines and probe dissipate some of the source voltage as ohmic loss. The inductance converts the voltage into magnetic field temporarily and releases it a short time later. Capacitance behaves similar to inductance, but it converts the voltage to an electric field instead of a magnetic field. Conductance shunts some of the current into alternative paths. At frequencies below $1 \mathrm{MHz}$, capacitance effects can have very little effect but if the probe is located close to a high-voltage source, the large potential difference can cause the relatively small capacitance to have a significant coupling effect. This effect is largely neglected in all treatments of $b$-dot probe behavior.

The probe assembly shown in Figure 5.14a represents a distributed impedance diagram where the transmission line is treated as a collection of individual small impedances. If the wavelength is much longer than the transmission line, the net effect of the individual impedances can be lumped together into one common element $\mathbf{Z}$. This approximation is sufficient for the low frequencies considered in this work (10-500 kHz). The upper limit of $500 \mathrm{kHz}$ was based on the expected transit time between the probes for a $100 \mathrm{~km} / \mathrm{s}$ plasmoid velocity. Furthermore, at low frequency the effective impedace of the capacitive elements between the lines is sufficiently high $(Z=-j \omega C)$ so $C$ and $G$ in Figure 5.14 can be neglected. Then, the net impedance $\mathbf{Z}$ is a complex quantity with resistance $R$ and reactance $X$, equivalent to $\mathbf{Z}=R+j X$. The equivalent circuit using lumped elements is shown in Figure 5.14p.

Applying Kirchoff's current and voltage laws to the equivalent probe circuit shown in 


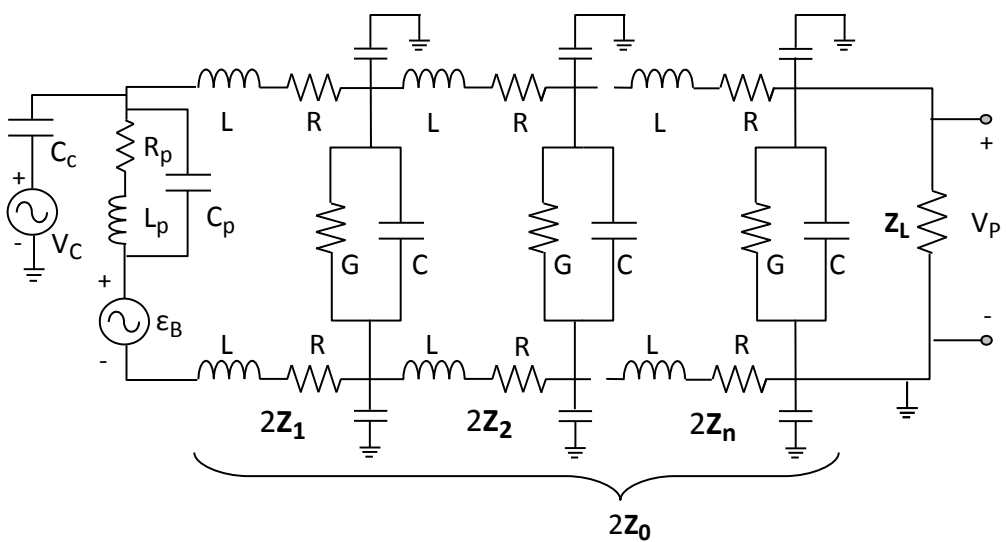

(a)

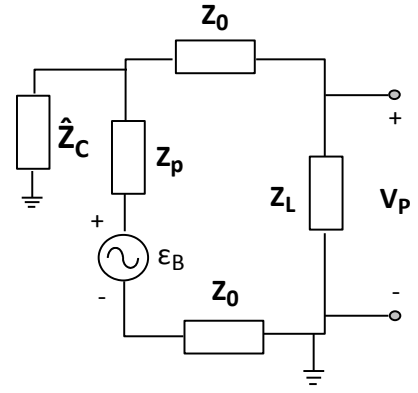

(b)

Figure 5.14: The circuit schematic for a b-dot probe connected to transmission lines. The complete circuit (a) can be simplifed to equivalent lumped impedances for low frequency (b) as probe impedances $Z_{P}$, line impedances $Z_{0}$, termination impedance $Z_{L}$, and high voltage capacitive coupling impedance $\hat{Z}_{c}$.

Figure $5.14 \mathrm{p}, V_{p}$ and $\varepsilon_{B}$ are related by:

$$
\frac{V_{p}}{\varepsilon_{B}}=\frac{Z_{L}}{Z_{L}+2 Z_{0}+Z_{P}+\hat{Z}_{c}^{-1}\left(Z_{L} Z_{p}+Z_{0} Z_{L}+Z_{0} Z_{p}+Z_{0}^{2}\right)}
$$

Combining Equations 5.4 and 5.5 , the probe's transfer function $\beta(\omega)$ is:

$$
\frac{V_{p}}{\dot{B}}=\beta(\omega)=N A \frac{Z_{L}}{Z_{L}+2 Z_{0}+Z_{P}+\hat{Z}_{c}^{-1}\left(Z_{L} Z_{p}+Z_{0} Z_{L}+Z_{0} Z_{p}+Z_{0}^{2}\right)}
$$

The high voltage capacitive coupling impedance $\hat{Z}_{c}$ is defined as the voltage at the probe side of the capacitor $C_{c}$ with respect to ground divided by the current through the capacitor:

$$
\hat{Z}_{c}=\frac{Z_{c} V_{c}}{\left(V_{c}-V_{H V}\right)}
$$

The voltage at the capacitor is equivalent to the combined voltage drop across $Z_{0}$ and $Z_{L}$. 
This creates the following expression for $\hat{Z}_{c}$ :

$$
\hat{Z}_{c}=\frac{\frac{V_{p}\left(Z_{0}+Z_{L}\right)}{Z_{L}}}{Z_{c}^{-1}\left(\frac{V_{p}\left(Z_{0}+Z_{L}\right)}{Z_{L}}-V_{H V}\right)}=\frac{Z_{c}\left(Z_{0}+Z_{L}\right)}{\left(Z_{0}+Z_{L}\right)-Z_{L} \frac{V_{H V}}{V_{p}}}
$$

It should be stressed that Equation is only valid for low frequency, where inductive effects in the transmission lines dominate the capacitive effects and the cable length is much, much longer than the frequency wavelength. If either condition does not hold, a different formation of Equation 5.2.2.1 must be used.

In some cases, the high voltage capacitive coupling impedance $\hat{Z}_{c}$ can be neglected. This is true if the capacitance between the coil and probe is very, very small and the voltage differential between the probe and nearby conductors are small. Removing this element from the circuit leaves the probe head and transmission lines connected in series so their equivalent impedances $Z_{P}$ and $Z_{0}$ add. Their combined series impedances form a single complex expression: $R+j X$. The probe's transfer function for this case resembles that found by other authors [63]:

$$
\frac{V_{p}}{\frac{d B}{d t}}=\beta(\omega)=N A \frac{Z_{L}}{Z_{L}+2 Z_{0}(\omega)+Z_{P}(\omega)}=N A \frac{Z_{L}}{Z_{L}+R(\omega)+j X(\omega)}
$$

The probe's transfer function is frequency dependant, as noted by the functional form of $\beta(\omega)$. The proportionality constant $N A$ does not change with frequency, but the complex impedance components $R$ and $X$ do. Resistance will increase with frequency as the skin depth decreases. The reactance will also increase with frequency as $X \equiv \omega L$ for inductive 


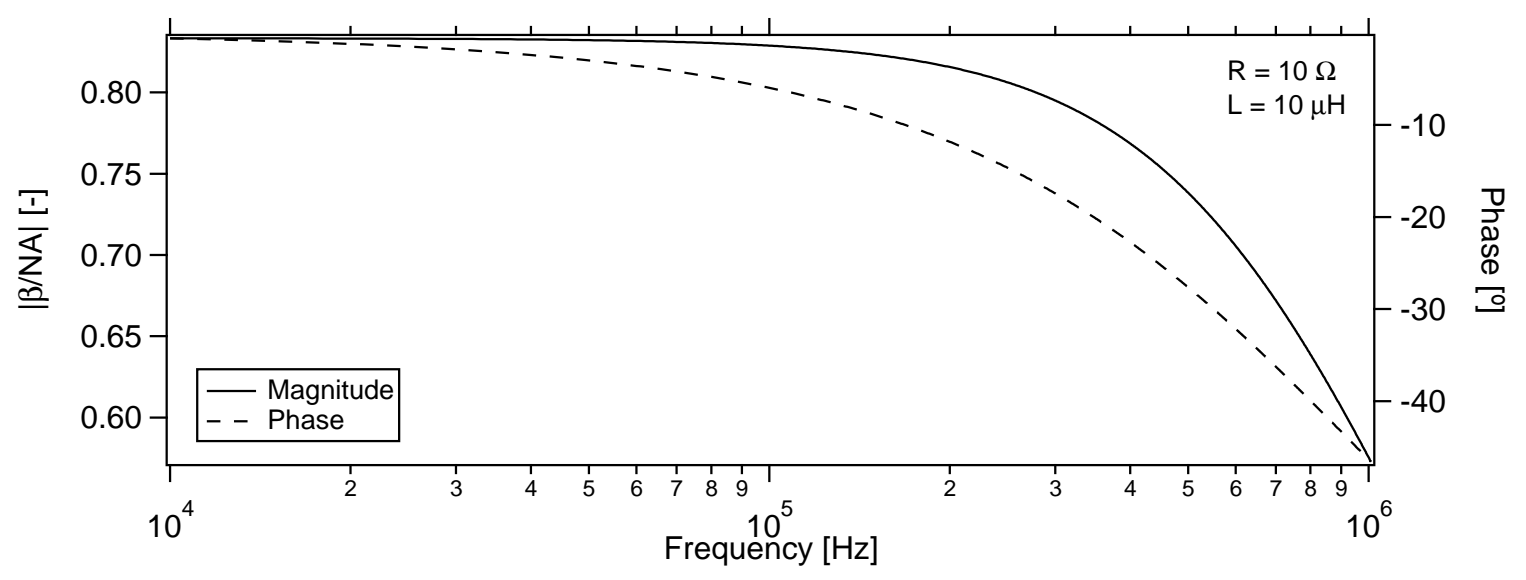

Figure 5.15: The frequency response (gain and phase) of a simulated probe assembly with negligible capacitance, $10 \mu \mathrm{H}$ of inductance, and $10 \Omega$ of resistance from $10 \mathrm{kHz}$ to $1 \mathrm{MHz}$.

circuits and $X \equiv \frac{-1}{\omega C}$ for capacitive circuits. Combined, $R$ and $X$ dictate the phase shift and attenuation of $\beta(\omega)$ over a range of frequencies. For illustration of this, a sample frequency response of $\beta$ is shown in Figure 5.15. The magnitude and phase of $\beta(\omega)$ shown in Figure 5.15 were computed using Equation 5.9 from $10 \mathrm{kHz}$ to $1 \mathrm{MHz}$ for a typical probe assembly with neglibible capacitance, an inductance of $10 \mu \mathrm{H}$, and a resistance of $10 \Omega$, measured across a $50 \Omega$ load.

The results in Figure 5.15 show that the signal recorded at the end of the probe assembly is only $85 \%$ of the signal produced by the field fluctuations $\varepsilon_{B}$, at $10 \mathrm{kHz}$. The values chosen for this example are realistic representations of a b-dot probe used in plasma research, with a high inductance $(8 \mu \mathrm{H})$ probe head and 4 meters of twisted coax cable. The magnitude and phase of $\beta(\omega)$ as shown in Figure 5.15 stays constant for $10 \mathrm{kHz}$ to $100 \mathrm{kHz}$. After $100 \mathrm{kHz}$, the magnitude and phase shift drop substatially. This means that for the typical case presented here, different frequency signals will arrive at the probe with different phase delays and with different attenuation levels. This illustrates that for probes exposed to a wide bandwidth, it is important to resolve $\beta(\omega)$ across the entire bandwidth and to account 
for the significant attenuation caused even at a single frequency.

The effect of the high voltage capacitive coupling impedance can be illustrated by plotting the transfer functions with and without $\hat{Z}_{c}$ (Equations 5.6 and 5.9 , respectively) and comparing the output with a circuit model. A SPICE-based circuit simulation software (LTspice IV v4.12a) was used to model a representative probe connected to 3.05 meters of twisted-shielded pair transmission lines. The SPICE circuit is shown in Figure 5.16. The probe's EMF was modeled as $10 \mathrm{kHz}$ sinusoidal voltage source $V_{s}$, using a $8.2 \mu \mathrm{H}$ inductor with $6.483 \Omega$ of series resistance and $2.9 \mathrm{pF}$ of parallel capacitance. The transmission lines were modeled as circuit elements with a pair-to-pair inductance of $587 \mathrm{nH} / \mathrm{m}$, capacitance of $87.6 \mathrm{pF} / \mathrm{m}$, and resistance of $0.427 \Omega / \mathrm{m}$. The capacitance from one conductor to the shield was set as $149 \mathrm{pF} / \mathrm{m}$. A capacitor $C_{c}$ was connected between the probe elements and a high voltage voltage sinusoidal source $V_{c}$. Three different simulations were conducted. The first simulation set the high voltage source to an amplitude of $1 \mathrm{kV}$ at a frequency of $10 \mathrm{kHz}$ and swept the capacitor $C_{c}$ from $1 \mathrm{fF}$ to $100 \mathrm{pF}$. The second simulation fixed the capacitor $C_{c}$ at $10 \mathrm{pF}$ and varied the high voltage source from $100 \mathrm{~V}$ to $10 \mathrm{kV}$ at $10 \mathrm{kHz}$. The final simulation set the capacitor $C_{c}$ to $10 \mathrm{pF}$ and swept the high voltage source from $-100 \mathrm{~V}$ to $-10 \mathrm{kV}$. The transfer function for each case was calculated using Equations 5.6 and 5.9. The gain and phase for each SPICE simulation is displayed with the calculated phase and gain in Figure 5.17. The solid lines are from including $\hat{Z}_{c}$ in the analysis and the dashed lines are from neglecting $\hat{Z}_{c}$.

The effect of the high voltage capacitive coupling impedance is apparent from Figure 5.17 . For the limited range of parameters shown, $\hat{Z}_{c}$ does not dramatically alter the gain of the probe's transfer function $\beta(\omega)$ but it can introduce significant phase changes if the capacitance is sizeable or if the potential difference between the probe and high voltage 


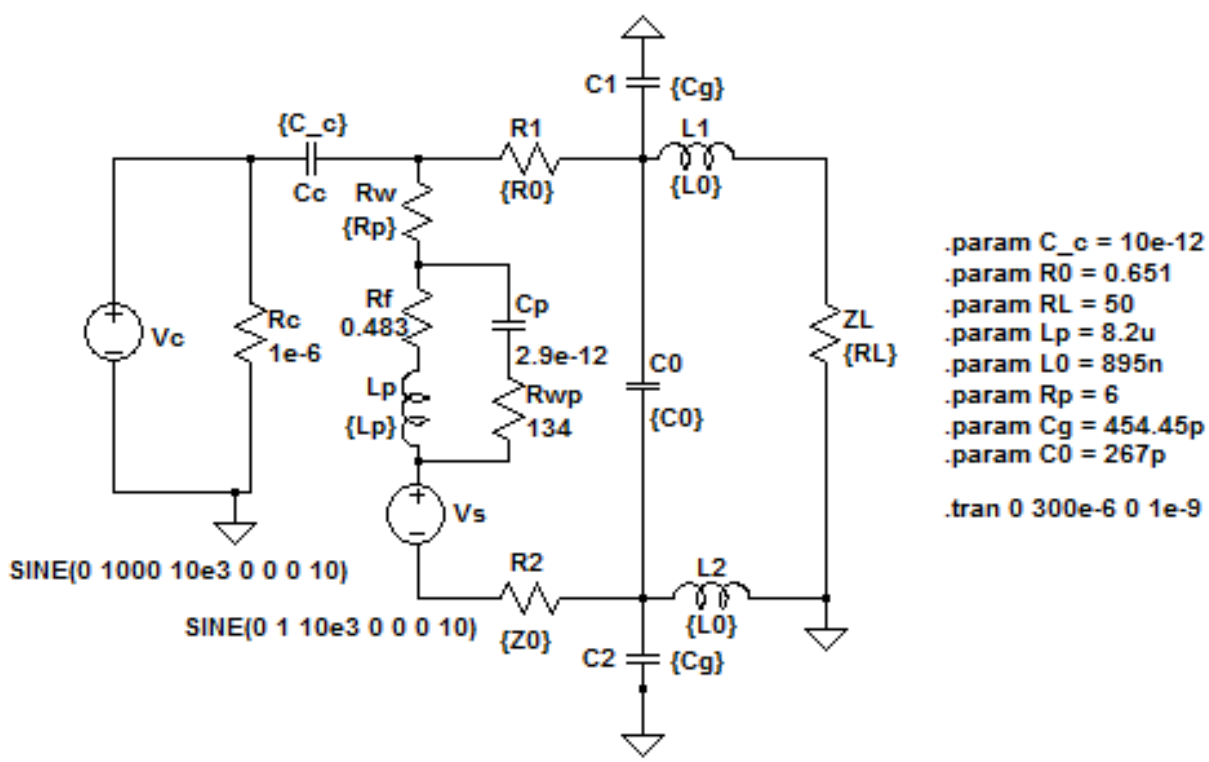

Figure 5.16: A SPICE circuit representation of a magnetic field probe used for examining the effect of the high voltage capacitive coupling on the probe's transfer function $\beta(\omega)$.

source is large. The sign of the phase shift indicates that the high voltage coupling can either cause the probe signal to lead the field fluctuations (when the potential difference is positive) or to lag them (when the potential difference is negative). The relatively constant gain across the span of $V_{c}$ and $C_{c}$ shows that the magnitude of the signal should not change due to the effect of $\hat{Z}_{c}$. This behavior is expected as pure capacitance does not remove energy from a source, it merely displaces it in time. Note that the results shown in Figure 5.17 are for a single frequency.

The frequency dependance of the probe's transfer function disappears if the load impedance $Z_{L}$ is set to a large value (i.e. $1 \mathrm{M} \Omega$ ). As tempting as it is to terminate the signal into 1 $\mathrm{M} \Omega$ to greatly simplify the analysis, this should be avoided. Impedance matching requires that the source impedance and the load impedance $Z_{L}$ are equivalent. This ensures that all the power from the source is deposited into the load and is not reflected back to the 


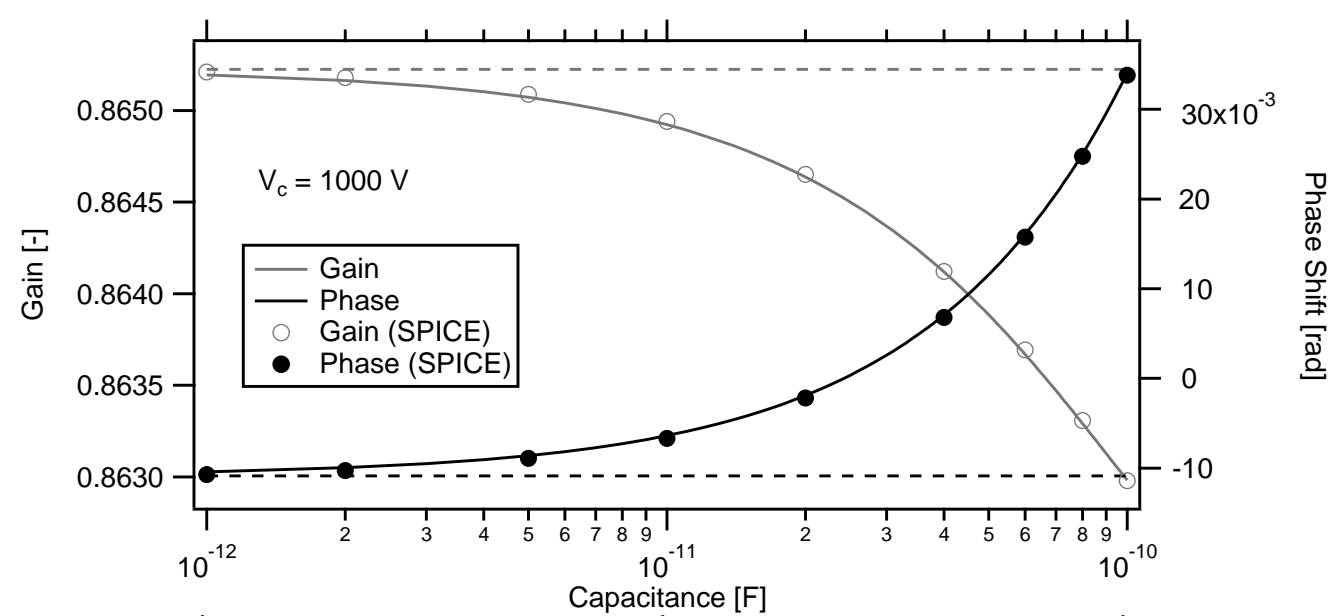

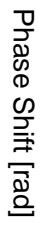
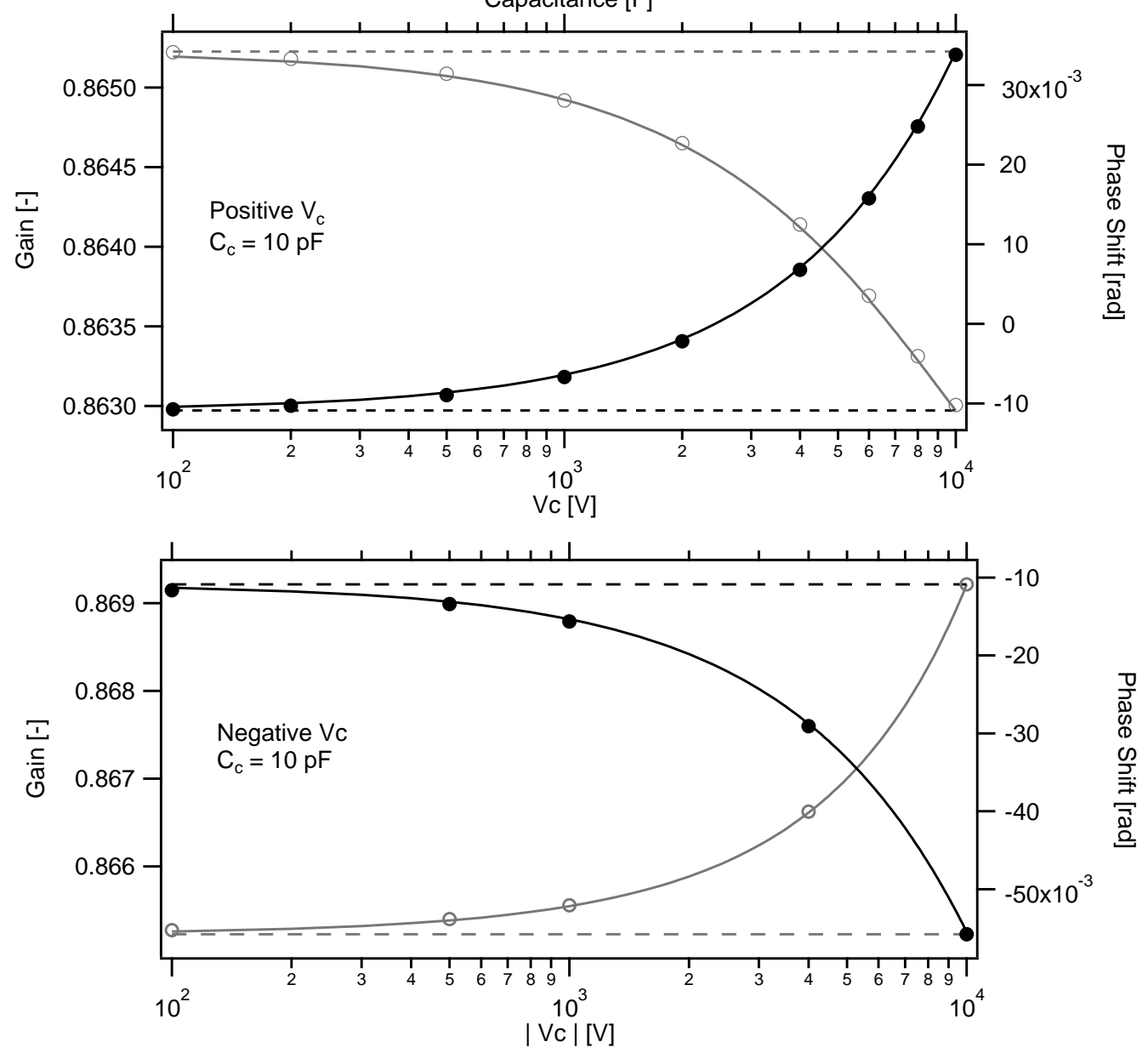

Figure 5.17: Gain and phase changes of a b-dot probes transfer function $\beta(\omega)$ due to high voltage capacitive coupling. The solid lines are from including $\hat{Z}_{c}$ in the transfer function and the dashed lines are from neglecting $\hat{Z}_{c}$. Results from a SPICE simulation are shown as hollow (gain) dots and solid (phase) dots. 
source. Failure to match impedances can result in inaccurate measurements especially at of higher frequency. Multiple reflections of the same signal are recorded, leading to errors during integration. Since the probe and transmission lines are low impedance, magnetic field probes should always be terminated with a low impedance load, conventionally 50 ohms.

The theory presented in this section is considerably more involved that classical treatment of b-dot probes, however it is essential in obtaining high accuracy measurements from broadband probe signals. Failure to account for the frequency dependent behavior of the probe assembly can result in significant errors. This is especially evident by examining the sample probe response shown in Figure 5.15. In this example, failure to account for impedance effects at low frequency can result in an field magnitude error of $15 \%$. Assuming a constant response for the probe from $10 \mathrm{kHz}$ to $500 \mathrm{kHz}$ results in an error of over $40 \%$ for the high frequency portion of the signal. While the actual error will be dependent on the probe design, this example illustrates the importance of resolving the probe's response across the entire frequency range of measured signal. A full error analysis of b-dot probe methods is provided in Section 5.2.2.5.

\subsubsection{Differential B-dot Probe Theory}

A differential b-dot probe arrangement is commonly used to cancel capacitive effects between the probe head and nearby conductors, including plasmas. Two probe heads are

wound over the same form, in opposite directions. A schematic of a differential probe assembly is shown in Figure 5.18 .

Each probe head records the same magnetic field, though the resulting EMF for each probe 


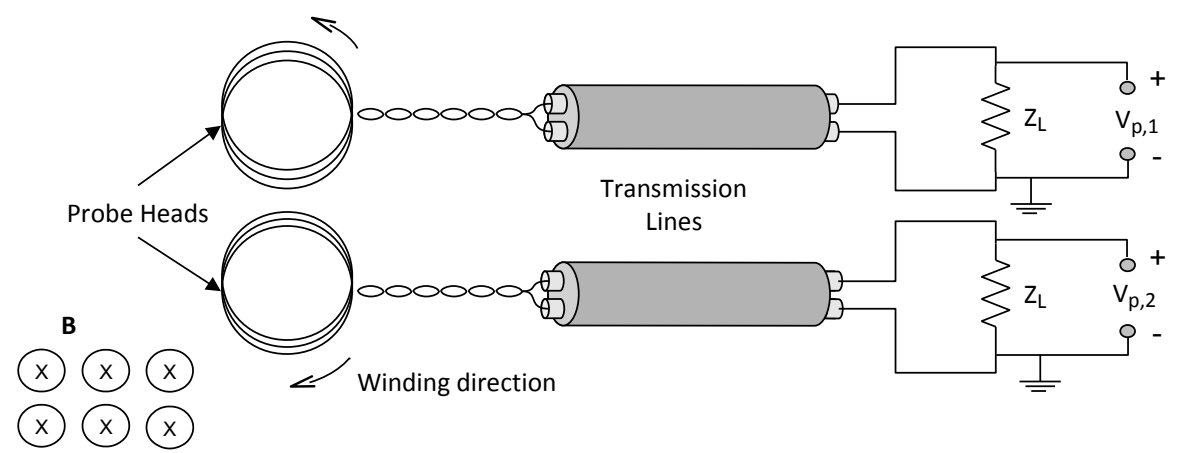

Figure 5.18: Illustration of a differential magnetic field probe. Each probe head is wound in a separate direction.

has the opposite sign. The capacitive effect measured by each probe is in the same direction and has the same magnitude. The probe voltages $V_{p 1}$ and $V_{p 2}$ are subtracted from each other so that the total voltage is twice the field, with the capacitive effects canceling, as shown in Equation 5.10 .

$$
V_{p}=V_{p 1}-V_{p 2}=\left(V_{p 1}^{B}+V_{p 1}^{C}\right)-\left(V_{p 2}^{B}+V_{p 2}^{C}\right)=\left(V_{p 1}^{B}+V_{p 1}^{C}\right)-\left(-V_{p 1}^{B}+V_{p 1}^{C}\right)=2 V_{p 1}^{B}
$$

Differential b-dot probes behave much the same as their traditional single-winding counterparts, with one exception. The windings on each differential probe couple to each other with a mutual inductance. Each probe winding works to oppose the change of flux through its probe-pair. An equivalent circuit diagram of the probe head, showing the mutual inductance between the windings (using the dot-convention), is displayed in Figure 5.19.

The effect of the mutual inductance between the windings will add to the inductive impedance of the probe assembly, increasing the phase delay and the attenuation. Including the mutual inductance into the transfer function results in a modified expression for $\beta(\omega)$ :

$$
\beta(\omega)=N A \frac{Z_{L}}{Z_{L}+R(\omega)+j(X(\omega)+\omega M)}
$$




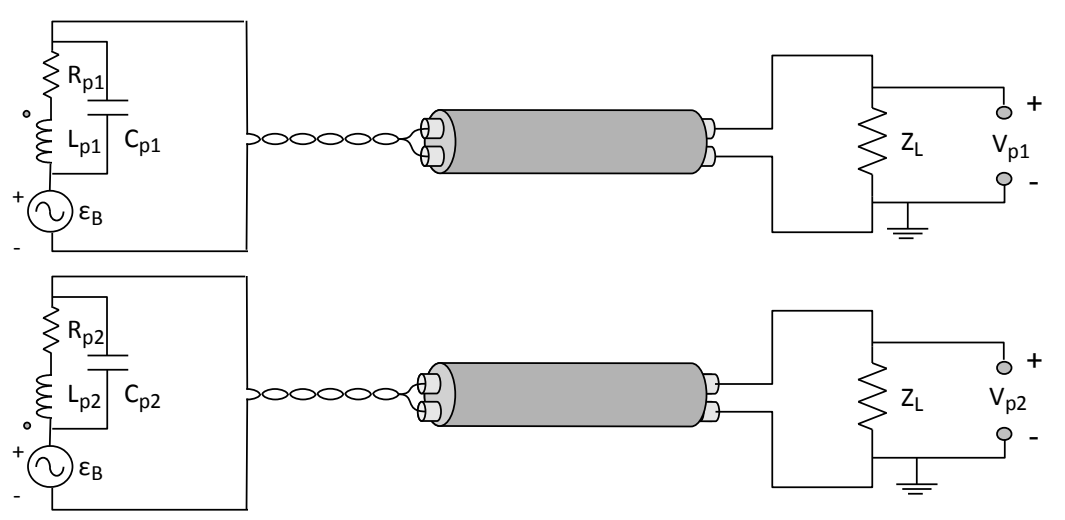

Figure 5.19: Circuit diagram of a differential b-dot probe. Mutual inductance between probe heads is indicated using the dot convention.

where $R$ and $X$ are the resistive and reactive impedance of a single probe in the differential pair and $M$ is their mutual coupling. This equation assumes both probes in the differential pair are identical (as they should be).

\subsubsection{Calibration of B-dot Probes}

B-dot probes must be calibrated prior to use to determine their sensitivity. If the probe is to be used across a range of frequencies, its sensitivity as a function of frequency must also be calibrated. There are three ways to calibrate a b-dot probe:

1. Measure $N$ and $A$ directly, then measure the complex impedance as a function of frequency.

2. Measure the output voltage of the probe when it is placed into a known AC field. Change the frequency of the AC field to determine the frequency response of $\beta(\omega)$.

3. Measure the output voltage of the probe when it is placed into a known AC field. Measure the complex impedance as a function of frequency independently. 
The first method listed is suitable if $N$ and $A$ can be measured with a great deal of accuracy. A ceramic core surface-mount (SM) inductor is the ideal candidate for this method [64]. The inductors are machine fabricated with very thin wire so that $N$ and $A$ can be known to a high degree of certainty. Since inductance is related to $N A$, deviations from the target $N A$ can be easily detected by measuring the inductance. Most SM inductors have an inductance tolerance of $5 \%$ or less; the deviation from the reported $N A$ can be much lower. Once $N A$ is known, the probe assembly's impedance can be measured across the probe's required bandwidth using an impedance analyzer, network analyzer, or similar tool.

The second method can be implemented by tuning the source frequency and measuring the probe's output [65]. Alternatively, one can use a network analyzer to measure the scattering parameters of the source and the probe to calculate the transfer function [66] [63]. Tuning the source frequency works well for limited bandwidth situations, but can get cumbersome when the required bandwidth spans several orders of magnitude. Magnetic field sources can have limited frequency responses and often several different sources are required to cover the entire frequency range.

The third method requires calibration at a single frequency to determine $N A$. The remaining variables in the transfer function $\beta(\omega)$ can be measured using an impedance analyzer. These measurements are straight-forward and equipment to take these measurements is readily available. This is the calibration method selected for this research.

The second and third method are similar in that they require that the magnetic field source is well behaved and well known. Most calibration experiments measure the magnetic field at DC with a Hall-probe for a given current level and then assume that it behaves identically at higher frequencies. This assumption must be rigorously checked if small errors in the measurement are required, as skin effect and the proximity effect can lead to magnetic field 
deviations at elevated frequencies. Other experiments simply calculate the magnetic field from the geometry and current, using formularies or electromagnetic field solvers. This is sufficient if high accuracy (less than $10 \%$ ) measurements are not required.

The most popular source for the magnetic field in methods \#2 and \#3 is a Helmholtz coil [67]. A Helmholtz coil consists of two identical loops of coils, connected in series with their spacing equal to the coil radius. The magnetic field inside a Helmholtz coil is in a single direction, extremely uniform in the center of the coil, and defined by a simple formula:

$$
B_{z}=\left(\frac{4}{5}\right)^{3 / 2} \frac{\mu_{0} N I}{a}
$$

In equation 5.12, $N$ is the number of turns per coil, $I$ is the current through the coil, and $a$ is the coil radius. The magnetic field of a Helmholtz coil can be measured at DC using a Hall-probe. It is often assumed to have the same current-to-field ratio at low frequency. The AC current through the Helmholtz coil can be measured using a low impedance resistor or a Rogowski coil. If the frequency characteristics of the Helmholtz coil are well-behaved in the neighborhood of the measurements, it can provide an ideal magnetic field source for probe calibration.

There are some pitfalls to using a Helmholtz coil as a magnetic field source at moderate (10 $\mathrm{kHz}-100 \mathrm{kHz}$ ) frequencies. Since the probe voltage is proportional to $d B / d t$, the magnetic field created by the Helmholtz coil must be high enough to produce a readable probe voltage. Common laboratory function generators generally have maximum current rating of $200 \mathrm{~mA}$ (10 V into $50 \mathrm{ohm})$, which is too low to create a significant magnetic field. This current can be boosted by an external amplifier, but these can be expensive for frequencies above $10 \mathrm{kHz}$. Higher current levels can also cause the coil to overheat. The number of loops on the coil can be increased, but doing so also increases the inductance. The 
inductance and resistance form a low-pass filter, with a cutoff frequency of $f_{c}=R /(2 \pi L)$. A Helmholtz coil generally has an inductance of several hundred $\mu \mathrm{H}$ and paired with a 50 ohm resistor for load balancing, this results in a cutoff frequency of $10-30 \mathrm{kHz}$. The area of the coil can be made smaller to increase the magnetic field, but this limits the physical probe size that can be placed in the coil. The uniform axial magnetic field shown in Equation 5.12 only applies to the very center of the coil and begins to drop off substantially at $20 \%$ of the coil's diameter. Balancing all of these restrictions requires special care when designing a Helmholtz coil for moderate frequency use.

At higher frequencies (above $100 \mathrm{kHz}$ ), the current distribution through the Helmholtz coil turns becomes affected by the skin depth and proximity effect as mentioned previously. Detailed studies of these effects are necessary to determine if the magnetic field inside the Helmholtz coil remains consistent with DC measurements. Above $1 \mathrm{MHz}$, a simple Helmholtz coil calibration becomes difficult. Small details such as the connection of wires to the magnetic field source can have a big impact on its frequency response [64]. Capacitive effects between the turns can also start to take effect, providing an alternative path for current. The length of coil windings also becomes important as the wavelength decreases. Some authors choose not to calibrate the probe at all at high frequencies, relying instead on having probe with a well-known proportionality constant [64]. Other authors attempt to correct for the high frequency effects by calculating the proportionality constant from the scattering matrix of the probe-coil pair, obtained using a network analyzer [63].

The probes used in the XOCOT-T3 experiment were calibrated using a combination of methods outlined above and were probe-dependant. High frequency calibration of magnetic field probes was found to be unnecessary for this research, as the upper frequency limit of the probes was determined to be $500 \mathrm{kHz}$ (see Section 5.2.2.4). A full explanation 
of the calibration methods used is provided in the probe-specific sections.

\subsubsection{Analysis of B-dot Data}

The signals from b-dot probes can be integrated digitally to calculate the time-dependant magnetic field, $\mathbf{B}(\mathrm{t})$. If the phase delay created by the probe and line impedances is not significant, a simple scaled, numerical integration method can be applied. The probe voltage can be divided by the magnitude of $\left|\beta\left(\omega_{0}\right)\right|$ at the principle frequency $\omega_{0}$ and integrated:

$$
B(t)=\frac{1}{\left|\beta\left(\omega_{0}\right)\right|} \int V_{p}(t) d t
$$

This is referred to as the standard integration method.

Equation 5.13 is only valid for signals with constant attenuation and phase shift across their applied bandwidth. If $R$ and $X$ vary over the frequency spectrum of the signal and contribute to a significant frequency dependant phase shift and attenuation in the probe signal, their variation with frequency should be considered. This can be done by converting the time domain signal $V_{p}(t)$ into the frequency-domain using a Fourier transform algorithm, such as

a fast Fourier transform (FFT). The frequency-domain signal $V_{p}(\omega)$ can then be multiplied by $\beta(\omega)$ [62]. An inverse Fourier transform, such as an inverse Fast Fourier Transform (IFFT) can be used to convert the scaled signal back to the time domain for the integration. This sequence of operations is illustrated in Equation 5.14 through 5.17 .

$$
\begin{gathered}
V_{p}(t) \Rightarrow F F T \Rightarrow V_{p}(\omega) \\
\dot{B}(\omega)=V_{p}(\omega) \cdot \beta(\omega)
\end{gathered}
$$




$$
\begin{gathered}
\dot{B}(\omega) \Rightarrow \operatorname{IFFT} \Rightarrow \dot{B}(t) \\
B(t)=\int \dot{B} d t
\end{gathered}
$$

This method is referred to as the phase correction method in this work. It has been referred to as the hybrid method in other sources [65].

Alternatively, the signal can be integrated in frequency space [62]. Singularities introduced by the discrete Fourier transform at $\omega=0$ due to offsets, non-zero field components, and other factors makes this approach less attractive [65].

If a b-dot probe is used with a filtering circuit or an amplifier, the transfer function $H(f)$ of the amplifier or circuit should be measured across the range of frequencies prior to use. The effect of the amplifier can then be accounted for in the phase-correction method by multiplying $H(f)$ by the probe's transfer function $\beta(\omega)$ in Equation 5.15 in the method of cascaded transfer functions.

In some cases, high frequency signals present in the data are unwanted or cannot be properly characterized. Some filtering techniques suggest adding an analog filter in-line with the probe to remove the high frequency components. Simple analog filters can decrease the amplitude of a signal by 10x at the filter frequency, but they also introduce a phase shift at frequencies an order of magnitude above and below the filter frequency. This wide rolloff band is unattractive if the frequency to be filtered is close (within an order of magnitude) to frequencies of interest.

Digital filters can also be used to attenuate high frequency signals. Digital filters are ideal as they can be applied after the data has been collected so their effects on the raw data can be reversed. They can also be designed to have much sharper rolloffs than analog 
filters and introduce no phase shifts. In this research, the frequency of the EMI created by the ignitron switch was within an order of magnitude of frequency components created by plasma activity. For these reasons, digital filters were chosen to be used in this work instead of their analog counterparts.

Oscilloscopes and other DAQ systems can introduce a small DC-offset to the signal. This offset must be corrected for prior to the integration. The oscilloscopes in this research recorded $50 \mu$ s of data prior to firing the PI circuit. The average voltage of the data in this quiescent delay window was subtracted from all the data points to correct for the offset.

The digital integration used for calculating magnetic field requires that a high level of digitization precision is used to collect the probe signals used for b-dot probes. Traditional oscilloscopes normally use an 8-bit analog-to-digital convertor. This low resolution will introduce significant uncertainty in the data. The small DC-offset error in the digitzer causes some of the data to be discretized incorrectly. This error in discretization cannot be recovered even by removing the offset. The error propagates in time through the integration, leading to a positive or negative droop which worsens in time. If the data is discretized with low resolution, it is readily apparent that this can lead to gross deviations. To illustrate this effect, two ideal digitizers were simulated in Igor Pro to sample a clean analog signal. One digitizer used a 8 bits of resolution, the other used 12 bits. A small offset ( $10 \%$ of a bin level) was added to the data prior to sampling. The offset was removed after sampling and prior to integration. The analog and digital signals were integrated and plotted along with the integrated analog signal. The results are shown in Figure 5.20 . From these results, it is apparent that small offsets on a low resolution digitizer will lead to trending errors in integrated data.

Random events, such as digitizer noise and periodic EMI, can also cause errors in the 


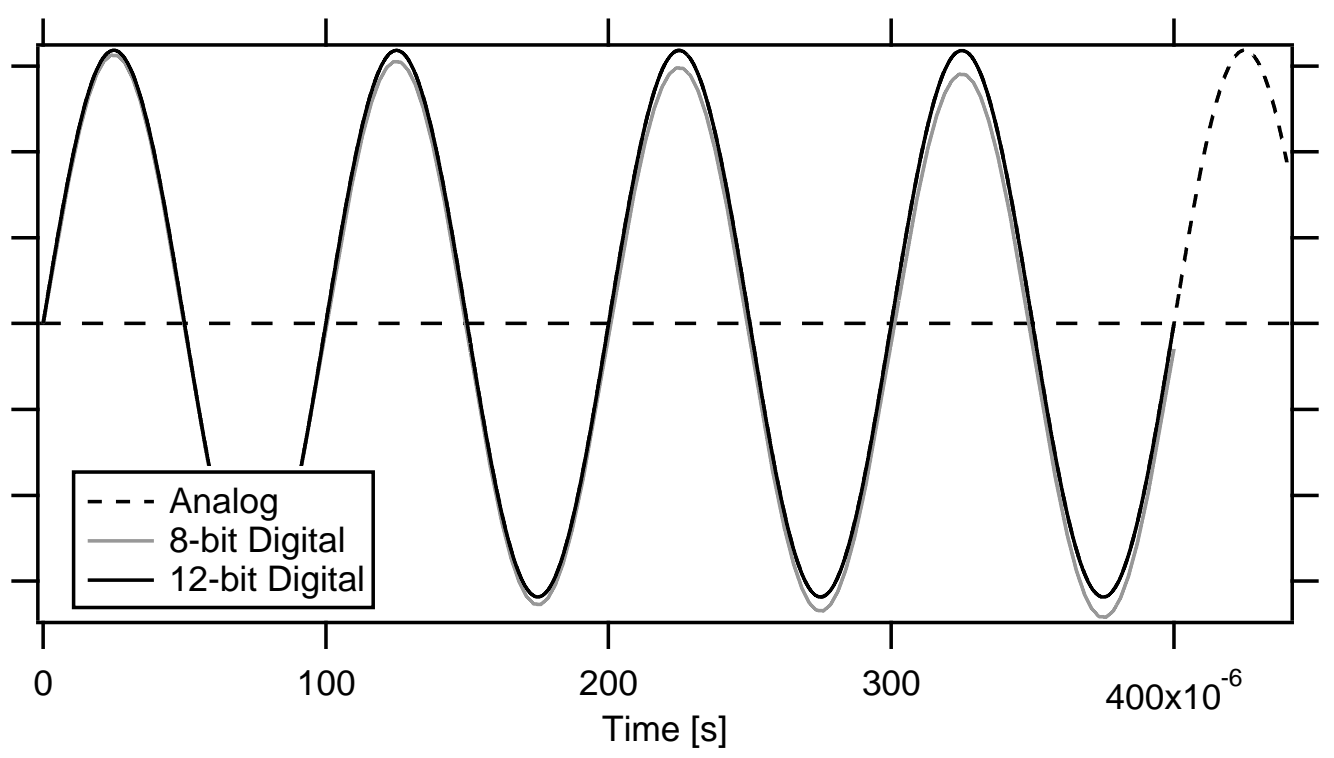

Figure 5.20: Integrated digital signals sampled from an analog waveform using an 8-bit digitizer and a 12-bit digitizer.

probe signal. However, if the sampling rate is very high and the digitization of the signal is also high, the average of the noise over a short time interval will be very small. When the signal is integrated, the errors introduced by the noise will smooth out and have only a small impact on the integrated signal. For these reasons, the data from the external and internal b-dot probes was recorded at $100 \mathrm{MS} / \mathrm{s}$ using the 12-bit Nicolet Sigma 100 oscilloscope. The TOF probes were recorded on a 8-bit system to accomodate a commercial pre-amplifier.

\subsubsection{Error Analysis for B-dot Probes}

Errors in b-dot probes come from several sources:

$\dagger$ Transfer function errors $\left(\sigma_{\beta}\right)$

$\dagger$ Integration errors $\left(\sigma_{I N T}\right)$ 
$\dagger$ Instrument errors $\left(\sigma_{\text {inst }}\right)$

$\dagger$ Electrostatic pickup errors $\left(\sigma_{c a p}\right)$

$\dagger$ EMI error $\left(\sigma_{E M I}\right)$

$\dagger$ Statistical averaging errors $\left(\sigma_{\text {stat }}\right)$

$\dagger$ Probe alignment errors $\left(\sigma_{\text {align }}\right)$

Errors accumulated during the calibration process to calculate the transfer function $\sigma_{\beta}$ are process dependent, but generally due to several common sources. These include uncertainty in the calibration magnetic field, instrument imperfections, and background interference. Computation of calibration errors in the XOCOT-T3 research will be discussed in the probe-dependent sections below.

Integration errors occur because the signal is approximated using finite timesteps during digitization. Integrating across these timesteps $h$ result in errors as the small local errors amass into a global error. The local error (per timestep) is generally quite small, on the order of $h^{3}$ for trapezoid integration [68]. This error is shown in Equation 5.18. When this error is summed across all points, it results in total integration error $\sigma_{I N T}$ shown in Equation 5.20 .

$$
\begin{gathered}
\Delta E_{i}=-\frac{h^{3}}{12} \frac{d^{2} V_{p}}{d t^{2}} \\
\sigma_{I N T}=\frac{t_{f}-t_{i}}{12} h^{2} \frac{d^{2} V_{p}}{d t^{2}}
\end{gathered}
$$

The total error is generally $\mathrm{O}\left(\Delta t^{2}\right)$ or slightly higher for noisy derivative signals. If the timestep is kept small, the integration error can be neglected. 
Errors can also arise from small uncertainties in the instrument used to digitize and record the probe signal. Instrument errors in digitizers are random and Gaussian in nature and small in amplitude. If the data is sampled at a much faster rate than changes in the signal and a high resolution digitizer is used, their net effect in the total integrated signal will cancel out so that $\sigma_{\text {inst }}$ will be small.

Electrostatic pickup errors $\sigma_{c a p}$ arise from fluctuating plasma potentials near the probe or from nearby high voltage sources. The capacitance this interaction is usually quite low (10 $\mathrm{pF}$ ), but the rapid fluctuations of a plasma or high voltages in a nearby coil can result in several $\mathrm{mV}$ of signal on the probe. If the probe is built with a high proportionality constant, the magnetic field amplitude will be much higher than the electrostatic coupling. In this case, the electrostatic pickup errors $\sigma_{c a p}$ can be neglected. If magnetic field contribution is very low, then a differential probe arrangement [64] should be used to minimize the $\sigma_{\text {cap }}$.

Background interference with the probe, such as that from noisy circuit components can create real errors in the integrated probe signal. This type of noise is typical in ringing RLC circuits. Background noise or EMI cannot be predicted, measured, or corrected for since there is not a completely clean signal to compare against. Using a probe with a high proportionality constant can reduce the effect of integrated EMI into the signal, but $\sigma_{E M I}$ should be considered in the computation.

Repeatibility errors are common for pulsed experiments. These errors can be calculated by measuring the statistical spread in repeated data sets. The standard devation of these data sets provides a figure for $\sigma_{\text {stat }}$. 
Probe alignment errors $\left(\sigma_{\text {align }}\right)$ occur when the probe is off axis from the measured field. Trigonometric relations show that the alignment error is

$$
\sigma_{\text {align }}=B_{\text {measured }}\left(\frac{1}{\cos \theta}-1\right)
$$

where $\theta$ is the angle of incidence between the probe's normal vector and the magnetic field. Small angle approximations assure that this error will be less than $2 \%$ for angles smaller than 10 degrees and less than $0.5 \%$ for angles less than 5 degrees. With properly placed probes, orientation and alignment errors can be neglected.

Instrument errors, electrostatic pickup errors, and background EMI errors affect how the probe signal is recorded by the digitizer. These errors can be combined with the integration error per timestep to form a single error in the integrated voltage signal $\sigma_{\text {Vint }}$. These errors are small in comparison to the probe signal, gaussian, and random in nature, meaning their effect on the integrated signal will be small as well. An error of 1-2\% can be used for a conservative estimate.

The error in voltage integration $\sigma_{V i n t}$ and calibration error $\sigma_{\beta}$ compute magnetic field together, combining into a single uncertainty in calculated field $\sigma_{B c a l c}$. The error can be computed considering how all the component errors propogate through the calculation for B. This is done using the method of partial derivatives [69]. In the method of partial derivatives for error computation, the partial derivative of the function $f\left(x_{1}, x_{2}, \ldots x_{n}\right)$ with respect to each variable $x_{n}$ is used along with the uncertainty of each variable to compute the total error. The partial derivatives are calculated as:

$$
\theta_{n}=\frac{\partial f}{\partial x_{n}}\left(x_{1}, x_{2}, x_{3}\right)
$$


Their contribution to the total error in $f$ or $\sigma_{f}$ is

$$
\sigma_{f}=\sqrt{\theta_{1}^{2} \sigma_{1}^{2}+\theta_{2}^{2} \sigma_{2}^{2}+\ldots+\theta_{n}^{2} \sigma_{n}^{2}}
$$

where $\sigma_{n}$ is the error in $x_{n}$. Using this method, it is apparent that the calculated error in $B$ due to $\beta$ and $V_{\text {int }}$ is

$$
\sigma_{B c a l c}=\sqrt{\theta_{\beta}^{2} \sigma_{\beta}^{2}+\theta_{V i n t}^{2} \sigma_{V i n t}^{2}}
$$

The partial derivatives can be calculated using the slope method with a point $3 \sigma$ away in all directions. This will provide a conservative estimate. The calculated error $\sigma_{B c a l c}$ combines with the other sources of error using the method of uncorrelated errors so that the total error in $B$ is $\sigma_{B}$ is:

$$
\sigma_{B}=\sqrt{\sigma_{B c a l c}^{2}+\sigma_{\text {stat }}^{2}+\sigma_{\text {align }}^{2}}
$$

\subsubsection{XOCOT-T3 External B-dot Probes}

The magnetic field in the XOCOT-T3 experiment is primarily in the axial direction. Axial magnetic field probes were installed between the outer coil and outer insulator to measure the time-history of this field with and without a plasmoid. The high levels of EMI from this experiment combined with the low discharge frequency required maximizing the proportionality constant $N A$ of the probes for maximum resolution. The external probes were selected to have an $N A=1 \times 10^{-3}$ turns- $\mathrm{m}^{2}$. To match this number and maintain physical clearance between the outer coil and the outer insulator, the external axial probes were wound on flexible thin, $2.5 \mathrm{~cm}$ wide plastic forms with small gauge magnet wire. A photograph of the probes is shown in Figure 5.21. 


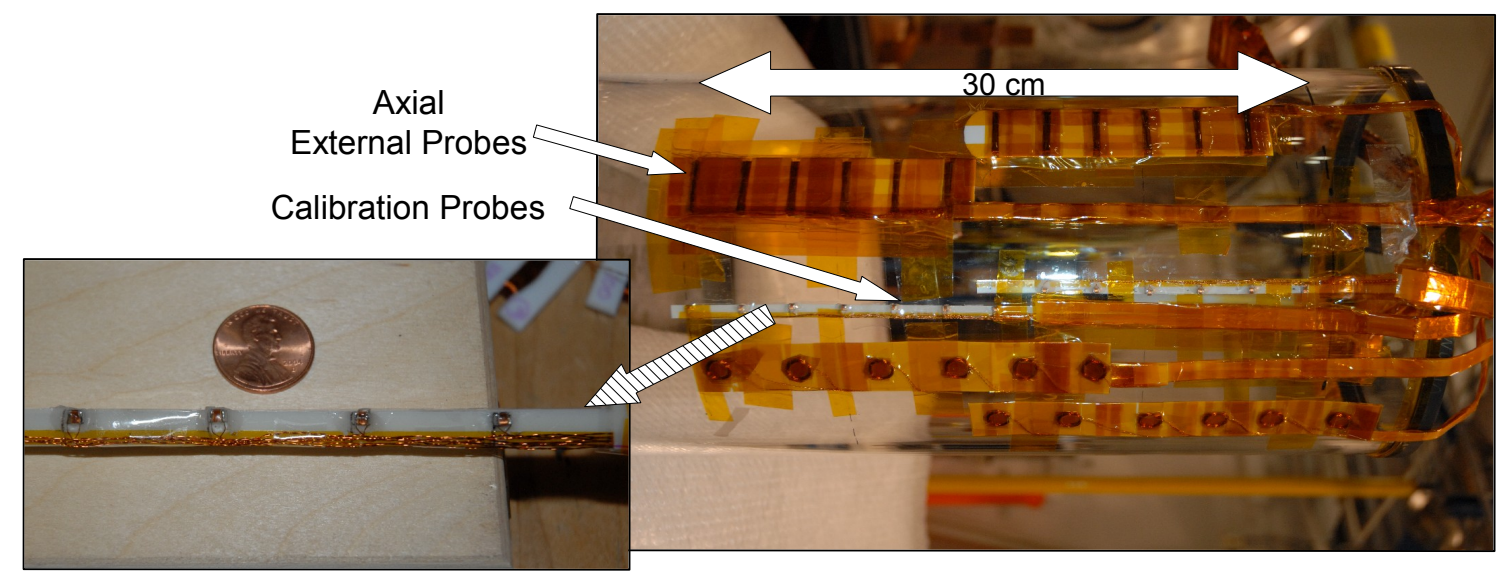

Figure 5.21: External magnetic field probes and their calibration probes, secured to the quartz insulator. Two arrays of external probes and calibration probes span the length of the electromagnetic coils.

Probe spacing between each probe was $2.5 \mathrm{~cm}$ and measured with a digital caliper. Six probes were wound on a continuous form and the form was secured to the outer quartz insulator with polymide tape. All probe ends were twisted tightly and connected to M27500 twisted-shielded pair ( $83 \Omega$ ) outside of the coils, extending to the data acquisition system inside the screen room. A BNC-breakout box inside the screen room connected the twisted pair to a BNC connector. The shield from the twisted pair was grounded to the BNC breakout box, which was connected to the screen room ground. A coaxial RG58 cable connected the breakout box to the desired oscilloscope channel. A 50-ohm terminator (Pasternack p/n PE6008-50) was added to the end of the cable before connecting to the oscilloscope. A schematic of the external b-dot probe assembly with cable lengths is shown in Figure 5.22 .

The external probes were calibrated in-situ since their long length and low calibration frequency required an actively cooled Helmholtz coil. Commercial surface mount (SM) inductors with a known proportionality constant $N A$ were placed alongside the probes, epoxied to a Mylar strip. The calibration probes were $8.2 \mu \mathrm{H}$ wire-wound, ceramic core 


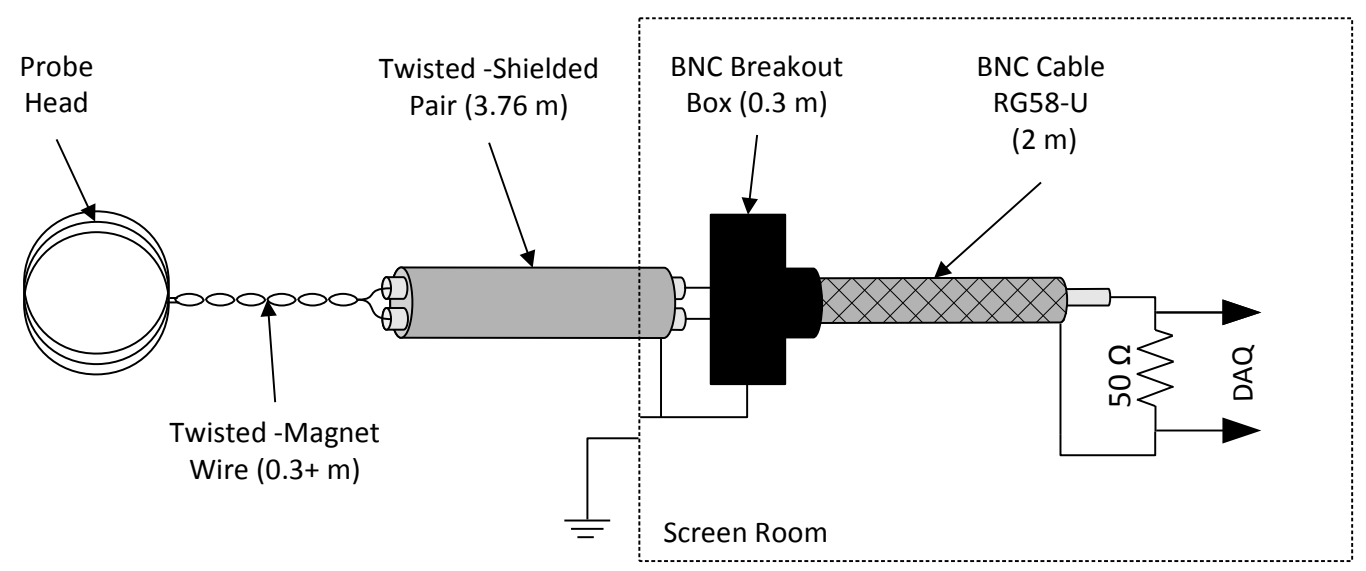

Figure 5.22: External magnetic field probe wiring diagram.

SM inductor (Coilcraft p/n 1008CS-822XJLC), chosen for their small physical dimension ( $2 \mathrm{~mm}$ by $2 \mathrm{~mm}$ ) and high inductance. Their $N A$ was provided by the manufacturer as $2.13 \times 10^{-4}$ turns $-\mathrm{m}^{2}$ and was the largest $N A$ for the probe size. The tolerance on the inductance is $5 \%$ and since inductance is directly proportional to $N^{2} A$, this tolerance was a very conservative estimate for $N A$ as well. Twisted magnet wire leads were soldered to the SM inductor solder pads and these were connected to 4.60 meters of M27500 twisted shielded pair cable and a BNC breakout box in the same manner as the external probes (see Figure 5.22,

The impedances of the probe assemblies were measured with an impedance analyzer to provide the resistance $R$ and reactance $X$ for the probe's transfer function $\beta(\omega)$. The impedance analyzer was an Agilent 4294A Precision Impedance Analyzer with a total bandwidth of $40 \mathrm{~Hz}$ to $100 \mathrm{MHz}$, capable of sweeping through 800 points across any subrange of frequencies, with a base accuracy of $0.08 \%$. For the probe characterization, the 4294A was set to record $R$ and $X$ at 800 points for four frequency intervals of interest: $(1 \mathrm{kHz}-10 \mathrm{kHz}),(10 \mathrm{kHz}-100 \mathrm{kHz}),(100 \mathrm{kHz}-1 \mathrm{MHz})$, and $(1 \mathrm{MHz}-5 \mathrm{MHz})$. The coaxial cable of each probe assembly was connected to an Agilent test fixture (p/n 16047E) on the 


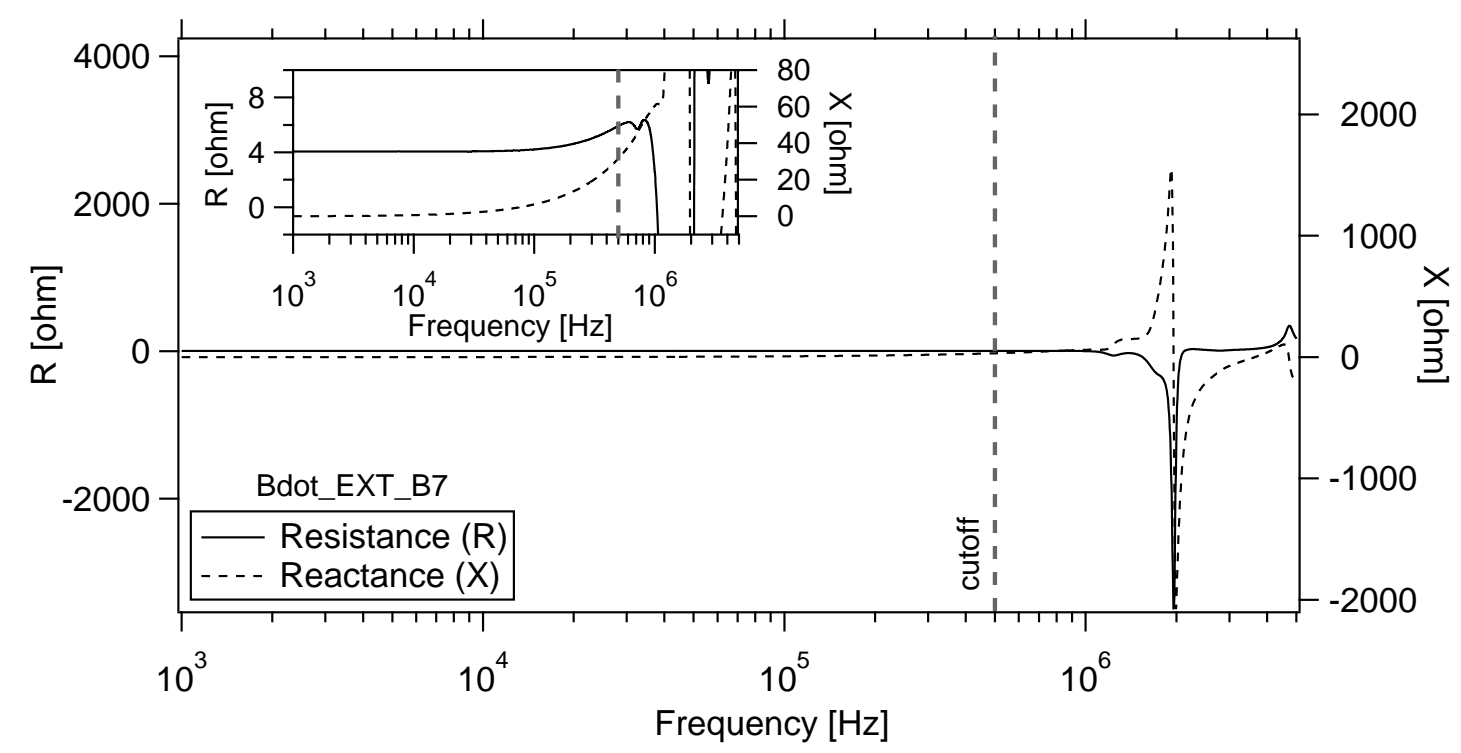

Figure 5.23: The complex impedance of an external probe assembly (B007) from $1 \mathrm{kHz}$ to $5 \mathrm{MHz}$, measured with an impedance analyzer. An inset graph shows the detail from 1 to $500 \mathrm{kHz}$.

impedance analyzer using a BNC-to-banana adapter. The $R$ and $X$ measurements for one external probe (B07) are shown in Figure 5.23 at all measured frequencies; an inset graph shows a detail view of the measurements at low frequency.

The frequency response shown in Figure 5.23 is well-behaved at frequencies below 500 $\mathrm{kHz}$. The resistance varies only slightly with frequency and the reactance follows an $X=$ $2 \pi f L$ behavior. The probe self-resonates around $2 \mathrm{MHz}$, noted by the sharp peaks in the $R$ and $X$ traces. The waveforms of $R$ and $X$ in the vicinity of $2 \mathrm{MHz}$ was found to change drastically just by moving the cable slightly. This could be due to non-ideal ground planes changing the probe lines capacitance to ground, slight insulation defects, or some other high-frequency artifact. This behavior was seen in all the probes for configurations with the shield connected and disconnected from ground. To remove this source of uncertainty, the probe's upper bandwidth limit was set at $500 \mathrm{kHz}$, where the frequency response did not change from cable movements. Signals above this frequency were removed with a digital 
filter prior to integration.

The impedance measurements recorded with the impedance analyzer were not capable of isolating the high-voltage capacitance coupling $\hat{Z}_{c}$ to the XOCOT-T3 outer coil. The capacitance between each probe head and the coil was estimated to be very small $(\approx 10 \mathrm{pF})$ using theoretical calculations for a wire close to a wall. While the impedance analyzer was able to take accurate measurements in this range, it required a very clean setup with short, fixed leads and firm connections. Since the physical distances separating the probe head, coil connection point, and analyzer were over $0.5 \mathrm{~m}$ apart it was impossible to record clean measurements. Based on the findings in Section 5.2.2.1 and Figure 5.17, the high-voltage capacitance is responsible only for an added phase shift. If the signal spans a narrow bandwidth, a simple time-shift in the data can account for $\hat{Z}_{c}$ and the simplifed transfer function in Equation 5.9 can be applied to the signals.

The proportionality constant $N A$ was determined for the external probes by discharging the XOCOT-T3 main bank circuit through coils and recording the voltages generated by the external probes and the calibration probes. These tests were performed at vacuum, with no plasma present. A 12-bit, $100 \mathrm{MS} / \mathrm{s}$ oscilloscope was used to record the probe voltages. The signals were terminated into $50 \mathrm{ohms}$ prior to digitization. Twenty shots were conducted at $500 \mathrm{~J}$ with the external probe and corresponding calibration probe recording simultaneously. Ten shots were taken with midplane probe (B07) and its calibration probe to establish repeatibility. For all shots, the current through the inner and outer coils was also recorded. The probe voltages and their frequency spectra are shown in Figure $5.24 \mathrm{a}$ and Figure 5.24 b.

The probe signals were corrected for the frequency dependant impedance effects to find the unscaled EMF produced by the magnetic field for each probe, using Equations 5.14-5.16 

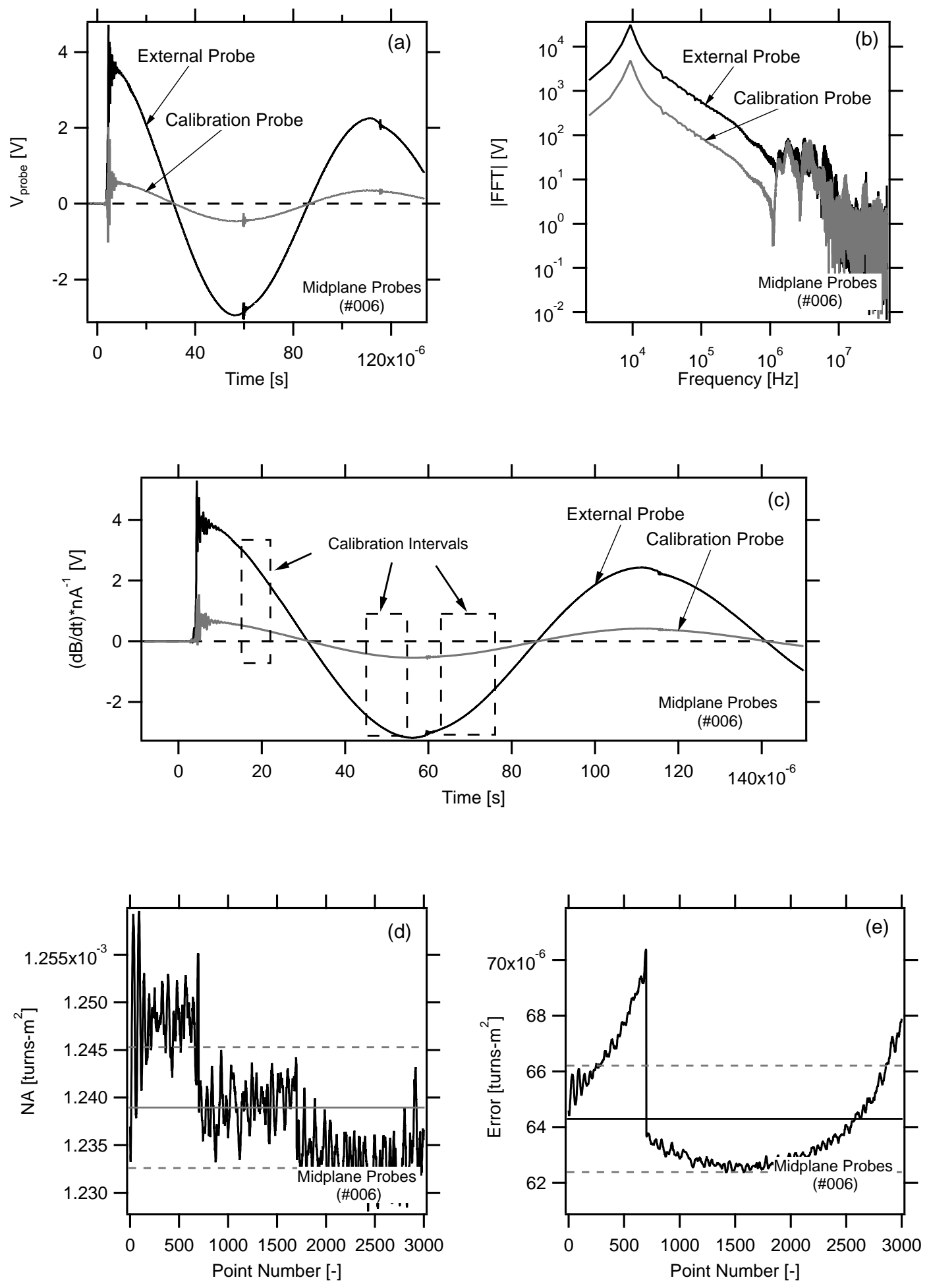

Figure 5.24: Sample calibration waveforms for external probe array, including (a) raw b-dot probe signals, (b) corresponding FFTs, (c) intervals of data used for calibration, (d) NA waveform for three intervals, and (e) error in NA. 


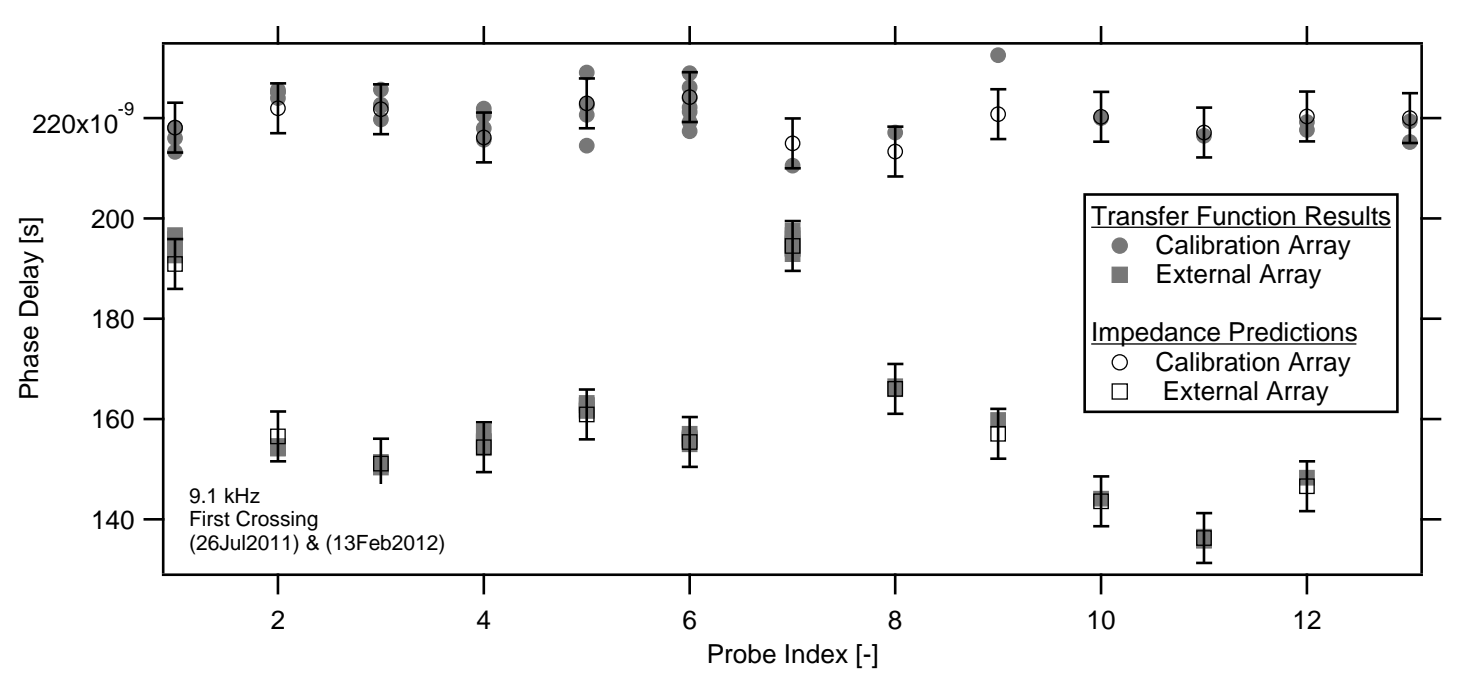

Figure 5.25: Measured phase delay at $9.1 \mathrm{kHz}$ of external probes, compared to their predicted delay.

and setting the proportionality constant to unity. No filters were used in order to keep the error estimates conservative. The resulting unscaled EMF signals from the midplane probes are shown in Figure 5.24c. The phase correction method was checked by comparing the original signals to the phase-corrected signals to determine if the expected phase shift from the impedance measurements was accounted for in the data analysis. The comparison of the corrected phase shift to the predicted phase shift at $9.1 \mathrm{kHz}$ is shown in Figure 5.25. For the external array data, the corrected phase shift lies within 1 sample period of the predicted phase shift (marked with error bars). The calibration array shows the phase delay fits within 2 sample periods. This is due to the lower voltage signals and greater noise susceptibility. These results indicate that the phase-correction data analysis is able to correct the phase of the primary frequency component.

Despite the phase correction, the external probe's signal did not have the same phase as its corresponding calibration probe; the phase of the calibration probe lagged the phase of the external probe. The phase difference was likely caused by a mismatched high voltage coupling capacitance $\hat{Z}_{c}$ due to the different capacitances and different voltage levels of 
the signals. If left uncorrected, dividing the probe signals by the calibration signals would result in large errors. Since $\hat{Z}_{c}$ does not cause an attenuation of the signal and the frequency spectrum of each signal illustrates that the $10 \mathrm{kHz}$ frequency component is several orders of magnitude higher than other frequencies, the calibration signals were shifted in time to align with the probe signals.

After the phase corrections were applied, the external probes' proportionality constants were calculated by dividing the unscaled EMF signals from the external probes by the corresponding calibration probe and multiplying by the calibration probe's proportionality constant $N A$, as illustrated in Equation 5.25. This was done for three time intervals in the data, to prevent the portions of the signal with poor signal-to-noise ratios from artifically inflating the error estimate. These time intervals are bracketed in Figure 5.24k. Figure 5.24d shows the resulting trace from the calibration of the midplane probe. The proportionality constants were calculated from the average of this trace (marked by a solid line) and the standard deviation of the average $N A$ (dotted lines) was recorded for the error estimate.

$$
\left.N A\right|_{\text {ext }}=\left.N A\right|_{c a l} \frac{\left.\frac{d B}{d t}\right|_{e x t}}{\left.\frac{d B}{d t}\right|_{c a l}}
$$

The experimental uncertainty in the calibration process were due to several sources, including the uncertainty in the calibration probes $N A$, the uncertainty in the transfer function, and he uncertainty in the measurement of the probe signals. The transfer functions combined uncertainty was estimated using the uncertainty in the calibration probe's $N A$, the uncertainty in the impedance measurements, and the uncertainty in the termination resistance. The calibration probes have a $5 \%$ uncertainty in their inductance. Inductance is proportional to $N^{2} A$ so the specified uncertainty can be considered a very conservative estimate for $N A$. The $4924 \mathrm{~A}$ impedance analyzer specifies a tolerance of $0.08 \%$ on the 
impedance measurements for the range of values measured. The $50 \mathrm{ohm}$ terminators used for $Z_{L}$ were assumed to have a $1 \%$ tolerance, as this information was not provided by the manufacturer. The uncertainty in the probe signals was set by the accuracy of the digitizer, specified as $0.25 \% \pm 3$ least significant bits.

Using the method of partial derivatives and transmission of error [69], the combined experimental uncertainty across the calibration interval was computed for each probe. The results for the midplane probe are shown in Figure 5.24. The average experimental calibration error $\sigma_{\exp }$ was combined with the statistical error from the data spread calibration calculation $\sigma_{\text {stat }}$, shown in Figure 5.24d, to determine the total error in the calibration process. This was done using the equation for uncorrelated errors:

$$
\sigma_{c a l}=\sqrt{\sigma_{e x p}^{2}+\sigma_{s t a t}^{2}}
$$

The full results from the calibration are shown in Table 5.2, including the complex impedance for each probe at $10 \mathrm{kHz}$, the magnitude of each probe assembly's transfer function at $10 \mathrm{kHz}$, and the proportionality constant for each probe assembly. From these results, it is shown that the uncertainty in the calibration probes' $N A$ dominates the error estimate, suggesting that for more accurate measurements using this calibration method an inductor with tighter tolerances should be used.

The resulting proportionality constants were multiplied by the integrated EMFs to calculate the magnetic field. The magnetic field at the midplane is shown in Figure 6.7.

The total error in the magnetic field measurement was calculated by combining the uncertainty due to integration and the uncertainty in the transfer functions of the external probes. While it was difficult to quantify the integration errors since there is no clean, 
Table 5.2

External magnetic field probes' impedances, sensitivities, and proportionality constants. Impedances were measured at $10 \mathrm{kHz}$.

\begin{tabular}{|c|c|c|c|c|}
\hline Probe & $\begin{array}{c}\mathbf{R}+\mathbf{j} \mathbf{X} \\
{[\Omega]}\end{array}$ & $|\beta / \mathrm{NA}|$ & $\begin{array}{c}|\beta| \\
\left.\text { [turns-m }^{2}\right]\end{array}$ & $\begin{array}{c}\text { NA } \\
{\left[\text { turns-m }^{2}\right]}\end{array}$ \\
\hline \multicolumn{5}{|c|}{ External Probes } \\
\hline B01 & $3.950+\mathrm{j} 0.647$ & 0.927 & $1.271 \times 10^{-3}$ & $1.371 \times 10^{-3} \pm 5.0 \%$ \\
\hline B02 & $4.425+\mathrm{j} 0.535$ & 0.919 & $1.010 \times 10^{-3}$ & $1.099 \times 10^{-3} \pm 5.1 \%$ \\
\hline B03 & $4.193+\mathrm{j} 0.514$ & 0.923 & $1.045 \times 10^{-3}$ & $1.133 \times 10^{-3} \pm 5.4 \%$ \\
\hline B04 & $3.709+\mathrm{j} 0.521$ & 0.931 & $1.194 \times 10^{-3}$ & $1.283 \times 10^{-3} \pm 5.2 \%$ \\
\hline B05 & $3.637+\mathrm{j} 0.542$ & 0.932 & $1.236 \times 10^{-3}$ & $1.326 \times 10^{-3} \pm 5.2 \%$ \\
\hline B06 & $3.632+\mathrm{j} 0.524$ & 0.932 & $1.165 \times 10^{-3}$ & $1.250 \times 10^{-3} \pm 5.2 \%$ \\
\hline B07 & $4.055+\mathrm{j} 0.661$ & 0.925 & $1.143 \times 10^{-3}$ & $1.235 \times 10^{-3} \pm 5.2 \%$ \\
\hline B08 & $3.825+\mathrm{j} 0.562$ & 0.929 & $1.029 \times 10^{-3}$ & $1.107 \times 10^{-3} \pm 5.5 \%$ \\
\hline B09 & $4.082+\mathrm{j} 0.534$ & 0.924 & $1.156 \times 10^{-3}$ & $1.250 \times 10^{-3} \pm 5.3 \%$ \\
\hline B10 & $3.650+\mathrm{j} 0.484$ & 0.932 & $1.122 \times 10^{-3}$ & $1.204 \times 10^{-3} \pm 5.4 \%$ \\
\hline B11 & $3.532+\mathrm{j} 0.458$ & 0.934 & $9.302 \times 10^{-4}$ & $9.959 \times 10^{-4} \pm 5.5 \%$ \\
\hline B12 & $3.535+\mathrm{j} 0.493$ & 0.934 & $1.024 \times 10^{-3}$ & $1.096 \times 10^{-3} \pm 5.3 \%$ \\
\hline \multicolumn{5}{|c|}{ Calibration Probes } \\
\hline B01 & $9.417+\mathrm{j} 0.814$ & 0.841 & $1.79 \times 10^{-4}$ & $2.13 \times 10^{-4} \pm 5.0 \%$ \\
\hline B02 & $9.527+\mathrm{j} 0.830$ & 0.840 & $1.79 \times 10^{-4}$ & $2.13 \times 10^{-4} \pm 5.0 \%$ \\
\hline B03 & $9.328+\mathrm{j} 0.827$ & 0.843 & $1.79 \times 10^{-4}$ & $2.13 \times 10^{-4} \pm 5.0 \%$ \\
\hline B04 & $9.190+\mathrm{j} 0.804$ & 0.845 & $1.80 \times 10^{-4}$ & $2.13 \times 10^{-4} \pm 5.0 \%$ \\
\hline B05 & $9.186+j 0.829$ & 0.845 & $1.80 \times 10^{-4}$ & $2.13 \times 10^{-4} \pm 5.0 \%$ \\
\hline B06 & $9.155+\mathrm{j} 0.833$ & 0.845 & $1.80 \times 10^{-4}$ & $2.13 \times 10^{-4} \pm 5.0 \%$ \\
\hline B07 & $9.129+\mathrm{j} 0.799$ & 0.846 & $1.80 \times 10^{-4}$ & $2.13 \times 10^{-4} \pm 5.0 \%$ \\
\hline B08 & $8.935+\mathrm{j} 0.790$ & 0.848 & $1.81 \times 10^{-4}$ & $2.13 \times 10^{-4} \pm 5.0 \%$ \\
\hline B09 & $9.151+\mathrm{j} 0.820$ & 0.845 & $1.80 \times 10^{-4}$ & $2.13 \times 10^{-4} \pm 5.0 \%$ \\
\hline B10 & $8.876+\mathrm{j} 0.815$ & 0.849 & $1.81 \times 10^{-4}$ & $2.13 \times 10^{-4} \pm 5.0 \%$ \\
\hline B11 & $8.774+\mathrm{j} 0.802$ & 0.851 & $1.81 \times 10^{-4}$ & $2.13 \times 10^{-4} \pm 5.0 \%$ \\
\hline B12 & $8.782+\mathrm{j} 0.814$ & 0.851 & $1.81 \times 10^{-4}$ & $2.13 \times 10^{-4} \pm 5.0 \%$ \\
\hline B13 & $8.757+\mathrm{j} 0.812$ & 0.851 & $1.81 \times 10^{-4}$ & $2.13 \times 10^{-4} \pm 5.0 \%$ \\
\hline
\end{tabular}

stable signal to reference, a few observations were used to estimate the error in integration. As discussed in Section 5.2.2.5, the small errors in digitizer accuracy can be assumed to have little impact on the magnetic field results as these errors appear to be equally random throughout the signal. The sampling rate was much higher than the EMI seen in the 
frequency spectrum (consult Figure 5.24 p) and a 12-bit digitizer was used to further reduce the uncertainty from these effects. The midplane external probe signal is relatively free of EMI; the noise level of the 1-10 MHz signal components shown in the FFT trace of Figure $5.24 \mathrm{p}$ is two orders of magnitude below the principle frequency. With the phase correction method, the magnetic field matches the period and phase of the outer coil current to within a few timesteps. When compared with significantly noisier calibration probe signal, the difference in magnitude of the first and second magnetic field peaks is only $0.6 \%$. All of these clues suggest that the external magnetic field probes have a low level of integrated error (less than 1\%) for the first period of a vacuum shot, so a conservative error of $1 \%$ was used. The error is expected to grow in time, but this is of little relevance as only the first cycle of data from the experiment will be used in the analysis.

\subsubsection{XOCOT-T3 Internal Magnetic Field Probes}

Internal b-dot probes must be small to minimize perturbations to the plasmoid and retain a large NA. Commercial surface mount (SM) inductors were chosen for the internal b-dot probes since they use many turns around a very small area. The SM inductor with the highest available $N A$ was found to be the same inductor used for the external calibration probes (in Section 5.2.2.6). This is a $8.2 \mu \mathrm{H}$ wire-wound, ceramic core SM inductor (Coilcraft $\mathrm{p} / \mathrm{n} 1008 \mathrm{CS}-822 \mathrm{XJLC}$ ) with an $N A$ provided by the manufacturer as $2.13 \times 10^{-4}$ turns- $\mathrm{m}^{2} \pm 5 \%$. The proportionality constant is well below the target $N A$ of $1 \times 10^{-3}$ turns- $\mathrm{m}^{2}$, but a larger area probe would create larger plasma perturbations. It was decided to accept the larger uncertainty at the expense of minimizing plasma perturbations.

An array of seven SM inductors were spaced $2.5 \mathrm{~cm}$ apart and sheathed inside a $7 \mathrm{~mm}$ 
Figure 5.26: Photograph of internal magnetic field probes.

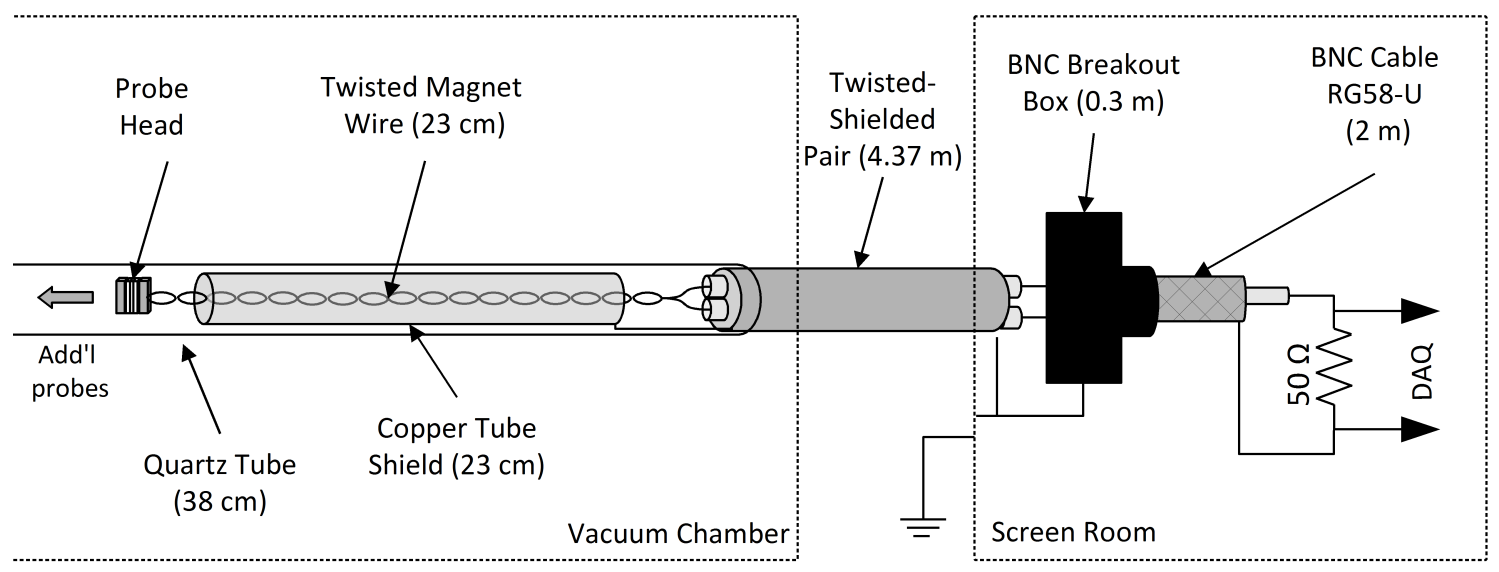

Figure 5.27: Internal magnetic field probes wiring diagram.

diameter, $38 \mathrm{~cm}$ long quartz tube. Small diameter magnet wire was soldered to the connection pads and these leads were twisted tightly until the end of the quartz tube. A small diameter copper pipe was inserted into the quartz tube for EMI shielding. The twisted leads and shield were connected to twisted-shielded cable (M27500) and fed through the vacuum chamber using an isolated DB15 pin connector. The twisted-shielded pair continued until it reached a BNC-breakout box inside the screen room. The shield was grounded at the screen room. A photograph of the probes is shown in Figure 5.26. A diagram of the probe construction is shown in Figure 5.27. For all of the data presented in this research, the internal probes were placed directly outside the inner insulator at the upstream end of the coils to check for magnetic field reversal. Figure 5.12 shows this placement, with the first probe in the array located at $\mathrm{z}=0$. 
The probes were not calibrated for $N A$ since it was provided by the manufacturer. The impedance of each probe assembly was measured using the Agilent 4294A Impedance Analyzer, as was done for the external probes in Section 5.2.2.6. This was used to calculate each probe's transfer function $\beta(\omega)$. The results are shown in Table 5.3. Similar to what was found with the external probes, the internal probes resonated at $1 \mathrm{MHz}$. The impedance above $500 \mathrm{kHz}$ was found to change drastically with probe position, movements of the cable, and shielding attachment. To remove this source of uncertainty, the probe's upper bandwidth limit was set at $500 \mathrm{kHz}$, where the frequency response did not change from cable movements. Signals above this frequency were removed with a digital filter prior to integration.

\section{Table 5.3}

Internal magnetic field probes' impedances, sensitivities, and proportionality constants. Impedances were measured at $10 \mathrm{kHz}$.

\begin{tabular}{ccccc}
\hline Probe & $\begin{array}{c}\mathbf{R}+\mathbf{j X} \\
{[\Omega]}\end{array}$ & $|\beta / \mathrm{NA}|$ & $\left.\begin{array}{c}|\beta| \\
{[\text { turns-m }}\end{array}{ }^{2}\right]$ & $\begin{array}{c}\text { NA } \\
{\left[\text { turns-m }^{2}\right]}\end{array}$ \\
& \multicolumn{5}{c}{ Internal Probes } \\
B01 & $9.356+\mathrm{j} 0.886$ & 0.842 & $1.79 \times 10^{-4}$ & $2.13 \times 10^{-4} \pm 5.0 \%$ \\
B02 & $9.370+\mathrm{j} 0.883$ & 0.842 & $1.79 \times 10^{-4}$ & $2.13 \times 10^{-4} \pm 5.0 \%$ \\
B03 & $9.283+\mathrm{j} 0.893$ & 0.843 & $1.80 \times 10^{-4}$ & $2.13 \times 10^{-4} \pm 5.0 \%$ \\
B04 & $9.276+\mathrm{j} 0.865$ & 0.843 & $1.80 \times 10^{-4}$ & $2.13 \times 10^{-4} \pm 5.0 \%$ \\
B05 & $9.255+\mathrm{j} 0.919$ & 0.844 & $1.80 \times 10^{-4}$ & $2.13 \times 10^{-4} \pm 5.0 \%$ \\
B06 & $9.134+\mathrm{j} 0.876$ & 0.845 & $1.80 \times 10^{-4}$ & $2.13 \times 10^{-4} \pm 5.0 \%$ \\
B07 & $9.154+\mathrm{j} 0.891$ & 0.845 & $1.80 \times 10^{-4}$ & $2.13 \times 10^{-4} \pm 5.0 \%$
\end{tabular}

The uncertainty in the probes' transfer function $\sigma_{\beta}$ was calculated using the method of partial derivatives [69]. The internal probes had a 5\% uncertainty in their inductance, according to the data sheet provided by the manufacturer. Since inductance is proportional to $N^{2} A$ the uncertainty in inductance is a very conservative estimate for $N A$. The $4924 \mathrm{~A}$ impedance analyzer specified a tolerance of $0.08 \%$ on the impedance measurements for the 
range of values measured. The $50 \mathrm{ohm}$ terminators were assumed to have a $1 \%$ tolerance, as this information was not provided by the manufacturer. The uncertainty in the probe signals was set by the accuracy of the digitizer, specified as $0.25 \% \pm 3$ least significant bits.

The error in the calculated field of the internal probes $\sigma_{B c a l c}$ was calculated using the method of partial derivatives, combining the error in the transfer function $\sigma_{\beta}$ with the error in the integrated probe signal $\sigma_{V i n t}$ (see Equation 5.23). The probe's smaller $N A$ made them more susceptible to noise pickup, so a $2 \%$ error was used for $\sigma_{V i n t}$. Orientation errors $\sigma_{\text {align }}$ were considered trivial, since the probes' normal axes were misaligned by no more than 5 degrees. Statistical errors $\sigma_{\text {stat }}$ were taken into account by averaging several shot together as warranted.

\subsubsection{XOCOT-T3 TOF Magnetic Field Probes}

The TOF b-dot probes required a very large $N A$ since they were located far downstream of the main discharge coils. The upstream probe in this array was selected to have an $N A$ of $1 \times 10^{-3}$ turns- $\mathrm{m}^{2}$ and the downstream probe had an $N A=1 \times 10^{-2}$ turns $-\mathrm{m}^{2}$. The probes were wound on a notched, cylindrical Macor mandrel with small gauge magnet wire. The upstream probe had mandrel dimensions of $2.48 \mathrm{~mm}$ by $17.73 \mathrm{~mm}$ and 50 winding turns. The downstream probe had mandrel dimensions of $3.33 \mathrm{~mm}$ by $26.65 \mathrm{~mm}$ and 120 winding turns. Since the probes would be exposed directly to the plasma environment with relatively small magnetic fields, the probes were wound in a differential configuration. During construction, two lengths of wire were wrapped around the mandrel simultaneously to provide nearly identical configurations for the two windings. The leads of each winding 


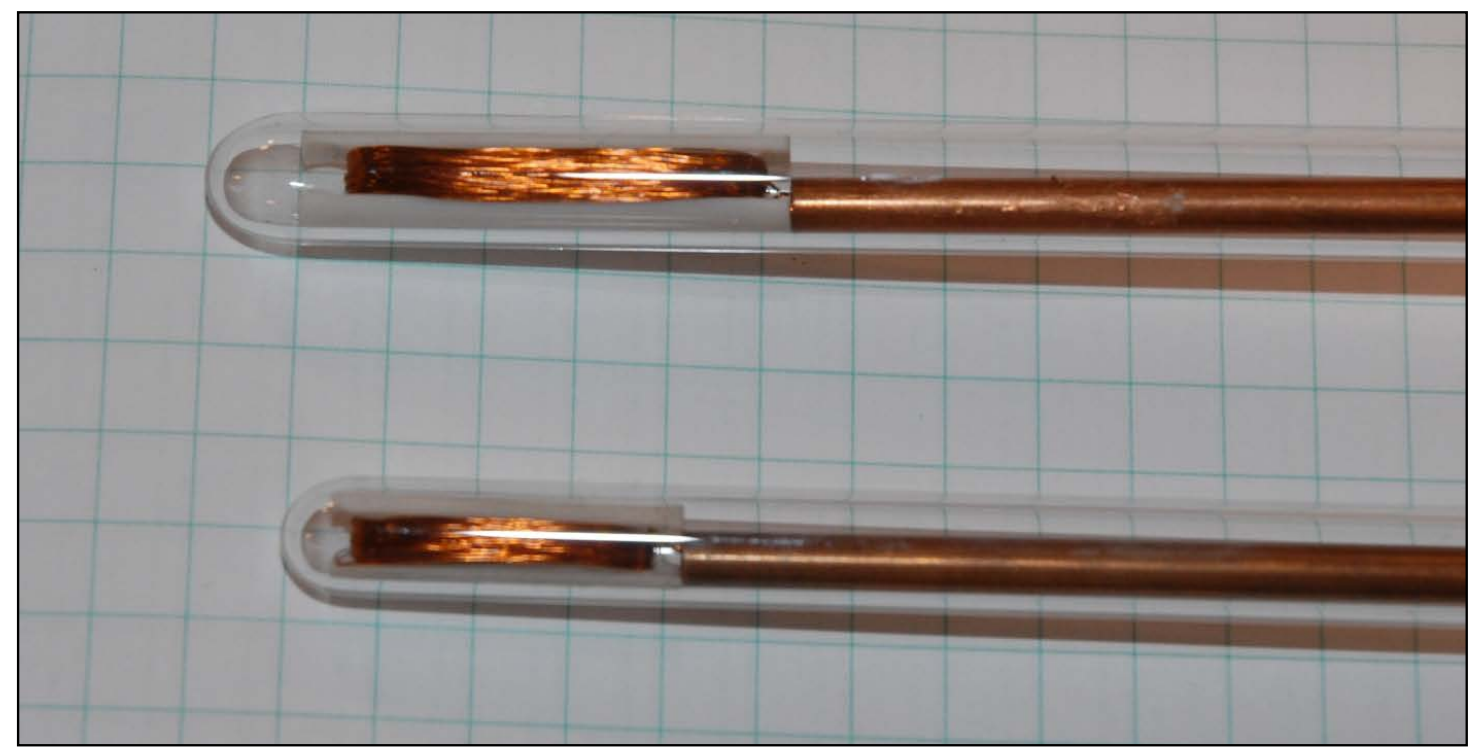

Figure 5.28: Photograph of TOF magnetic field probes. The downstream probe is the larger probe (top) and the upstream probe is the smaller probe (bottom).

were twisted together and then the twisted leads were twisted with each other. The probe head and twisted magnet wire leads were inserted into a $30 \mathrm{~cm}$ long quartz tube and a copper tube was inserted between the leads and tube to provide a ground shield. The twisted leads were connected to 2.36 meters of twisted-shielded cable (M27500). The shields of the cables were soldered to the copper shields over the twisted magnet wire. The twisted-shielded cable was connected to a vacuum feedthrough and joined to an additional $1.17 \mathrm{~m}$ of twisted-shielded cable (M27500) outside the vacuum tank. The twisted cable connected to the screen room electrical panel with a BNC connector. An additional $2 \mathrm{~m}$ of RG-58U cabling inside the screen room was required to attach the probe signals to a DAQ unit. The probe cabling was terminated into $50 \mathrm{ohm}$ in-line terminators prior to digitization (or amplification). Photographs of both probes are shown in Figure 5.28, A wiring diagram of the probe is shown in Figure 5.29.

The impedance of each probe assembly was measured using an Agilent 4294A impedance 


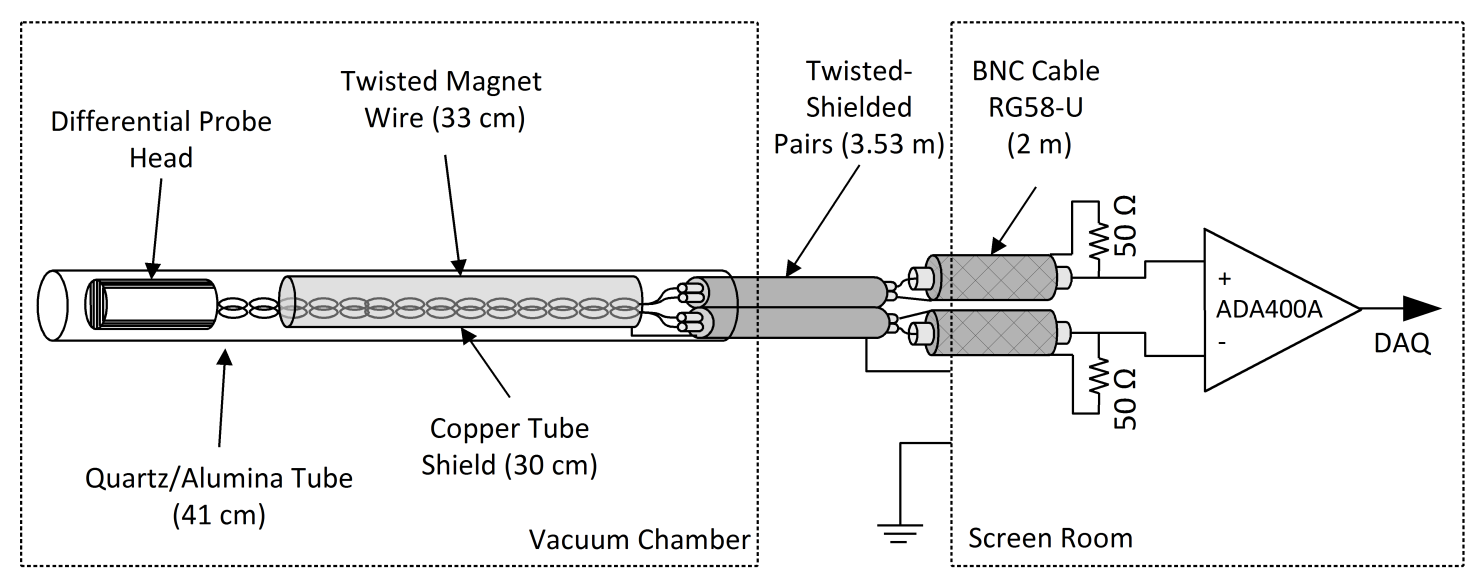

Figure 5.29: TOF magnetic field probes wiring diagram.

analyzer. Each winding of each assembly was measured separately to check for deviations between each differential probe winding. The impedances of each probe in the differential assembly was found to agree by less than $2 \%$, meaning that each probe for a differential pair was nearly identical. The impedance across the range of frequencies is shown in Figure 5.30 for both windings. One winding is indicated with a (+) and the other with a (-). The TOF b-dot probes reached a resonance at a much lower frequency, due to the large inductance of the probe heads. The upstream probe had a resonance around $900 \mathrm{kHz}$ and the downstream probe had a resonance at $600 \mathrm{kHz}$. The cutoff frequency for each probe was calculated where the resistance of the assembly deviated by $3 \mathrm{~dB}$ from the resistance at $10 \mathrm{kHz}$. For the upstream probe this was at $360 \mathrm{kHz}$ and for the downstream probe this was at $250 \mathrm{kHz}$. All frequencies above this point were removed with a digital filter prior to integration.

The impedance of the differential assembly was considered from a single probe winding only. This did not include the mutual inductance between the differential pairs. Since there was no easy way to measure the mutual inductance, an estimate was made for how the mutual inductance would affect the time-response of the probe. The transfer function for 


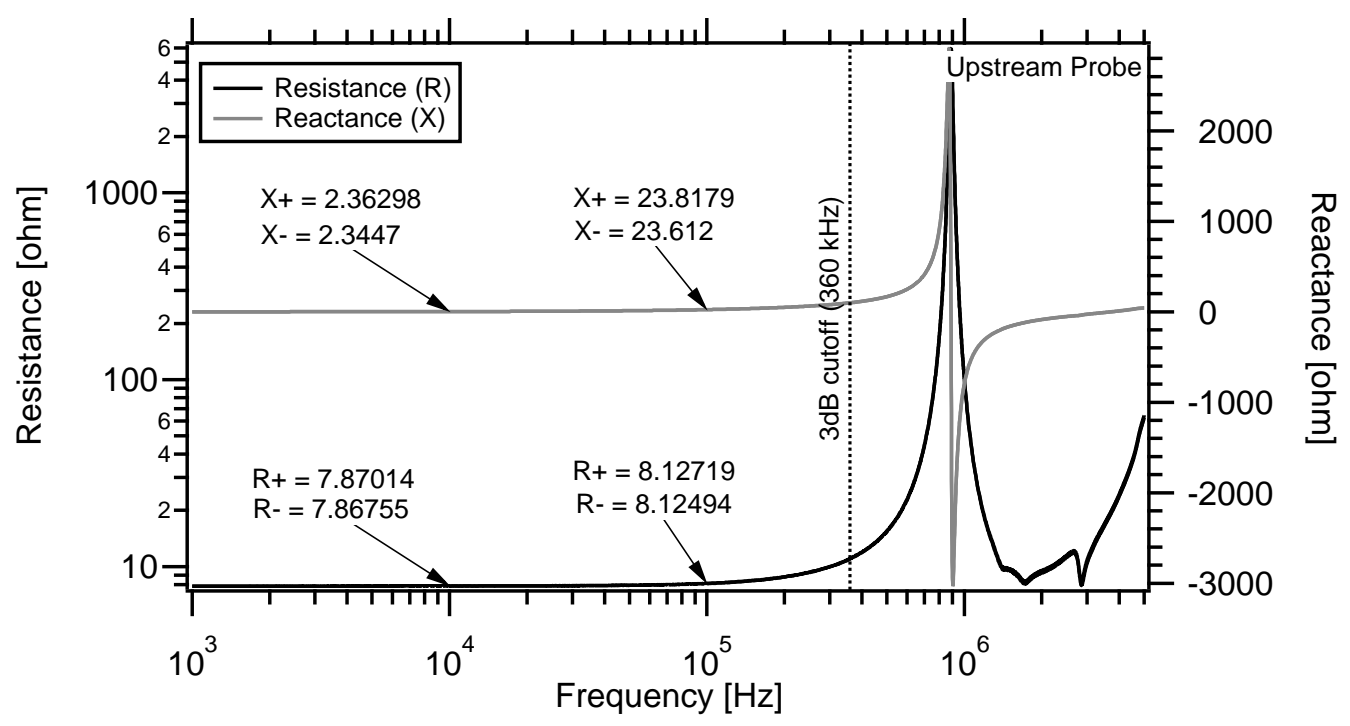

(a)

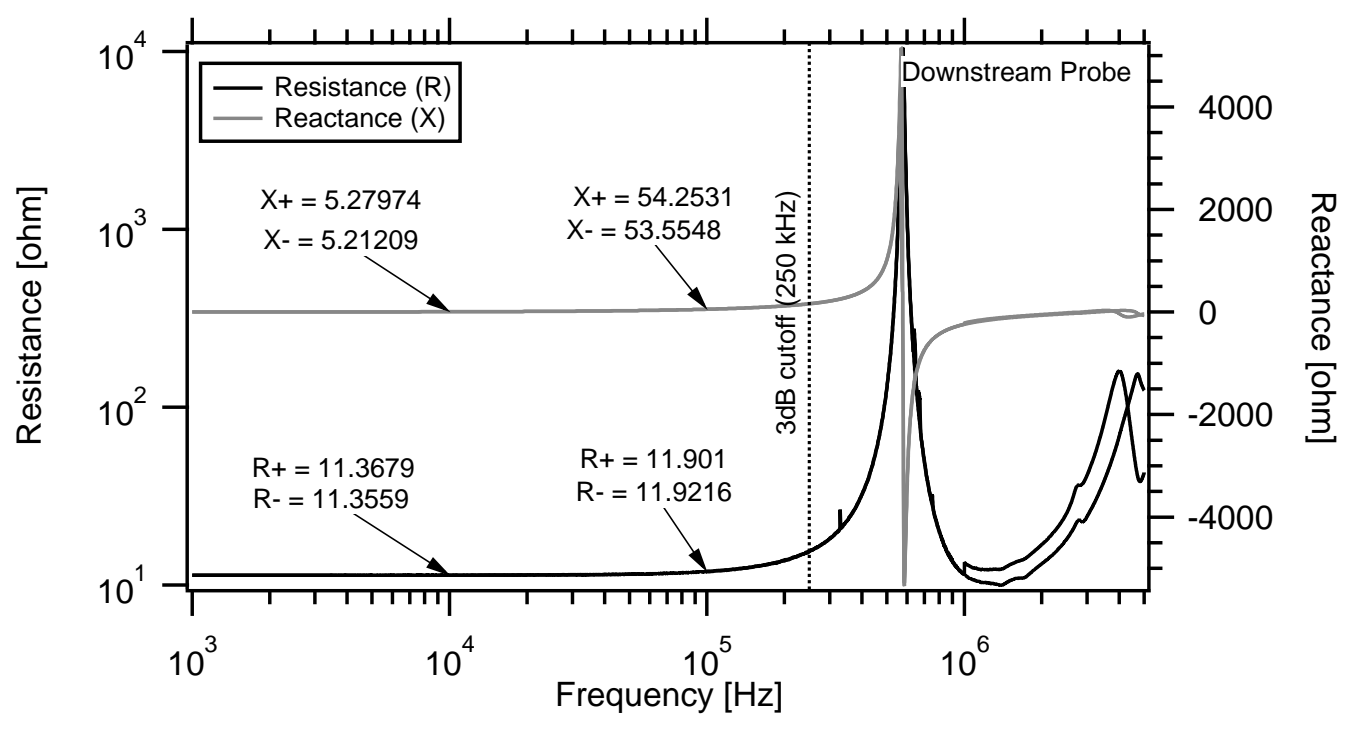

(b)

Figure 5.30: TOF magnetic field probes' impedance as a function of frequency for the (a) upstream probe and (b) downstream probe.

the probe assembly was calculated using Equation 5.11, with $R$ and $X$ from a single probe in the differential pair. The mutual inductance between the probe heads required for this equation was estimated by calculating using the self-inductance of the probe head. This is valid since both probes are co-wound for maximum coupling coefficient $k=1.0$ and by noting that $M=k \sqrt{L_{p 1} L_{p 2}}=k L_{p}$. The upper limit on self-inductance of the probe head was calculated by subtracting the cable impedance from the total probe assembly reactive 
impedance (shown in Figure 5.30). The inductance of the upstream probe was calculated to be $35 \mu \mathrm{H}$ and the downstream probe was calculated to be $81.4 \mu \mathrm{H}$. The added mutual inductance for the upstream probe would increase the phase-delay of the probe by $600 \mathrm{~ns}$ (at $10 \mathrm{kHz}$ ). The downstream probe's estimated phase-delay due to mutual coupling between probe heads would increase by $1.303 \mu \mathrm{s}$ (at $10 \mathrm{kHz}$ ). The resulting phase delay between the probes that would result by not factoring in the phase shift due to mutual coupling in the differential assembly was $703 \mathrm{~ns}$. For a plasmoid traveling at $100 \mathrm{~km} / \mathrm{s}$ between the probes, the measured velocity would be slower by $28 \%(28 \mathrm{~km} / \mathrm{s})$. The substantial error accumulated by neglecting the mutual inductance of the probe heads required that the velocity measurement must be cross-checked with a measurement from plasma probes, as described in Section 5.3.1.

The TOF b-dot probes were not calibrated to find their proportionality constants for two reasons. The first reason was that the TOF b-dot probes were used to record the time-history of the magnetic field for measuring plasmoid velocity; the absolute magnetic field was of little importance to this measurement. The second reason was that a calibration setup with a traditional Helmholtz coil required a specialty high-current amplifier for accurate results. A quick scaling study on sizing a Helmholtz coil for the upstream probe (17.73 $\mathrm{mm}$ long) required that the diameter of the Helmholtz coil be $89 \mathrm{~mm}$, so that the probe occupy no more than $20 \%$ of the interior of the coil. (The field inside a Helmholtz coil is only uniform across $20 \%$ of the coil diameter.) The minimum voltage that should be recorded by the probe ( $N A$ of $1 \times 10^{-3}$ turns- $\left.\mathrm{m}^{2}\right)$ is $100 \mathrm{mV}$, assuming a $1 \mathrm{mV}$ noise floor. This dictates that the minimum field at $10 \mathrm{kHz}$ is $1.6 \mathrm{mT}$. This requires that product of the turns on the Helmholtz coil and the current satisfy 79 turns-amps. The peak current available from an available low-noise RF amplifier at $10 \mathrm{kHz}$ was $800 \mathrm{~mA}$ (Kronheit 7500). The number of turns required to match $800 \mathrm{~mA}$ was 100 turns. The total inductance of a Helmholtz 
coil matching this description would be $3 \mathrm{mH}$. The cutoff frequency of the Helmholtz coil with a $50 \mathrm{ohm}$ series resistor is $2.7 \mathrm{kHz}$. At $10 \mathrm{kHz}$, the current would drop by $10 \mathrm{~dB}$ (or more) meaning the actual current driven through the Helmholtz coil would be $70 \%$ of the rated $800 \mathrm{~mA}$. Increasing the turns on the Helmholtz coil would increase the field, but it would also increase the inductance by $N^{2}$. Increasing the current to reach $800 \mathrm{~mA}$ (or above) would require a custom amplifier. For these reasons, it was decided not to calibrate the TOF probes to find their proportionality constant and scale the resulting waveforms to display $B / N A$.

A differential pre-amplifier (Tektronix p/n ADA400A) was sometimes used to measure the differential signal between the probes prior to digitization. The amplifier was used when the signal-to-noise ratio was too poor for adequate digitization. A 1-MHz commericial preamplifier was chosen, with a variable gain of 100x or 10x. The transfer function for the differential amplifiers was measured by applying two sinusoidal signals from a function generator (Tektronix p/n AF3102) with opposite polarity to the inputs of the amplifier. The frequency of the signals was changed to map the response of the amplifier to various frequencies from $10 \mathrm{kHz}$ to $1 \mathrm{MHz}$. The transfer function for the amplifier was $H(f)$ was calculated by dividing the amplifier output by the input according to

$$
H(f)=\frac{\mathbf{V}_{\text {out }}}{\mathbf{V}_{\text {in }}}=\frac{\left|\mathbf{V}_{\text {out }}\right|}{\left|\mathbf{V}_{\text {in }}\right|} \angle\left(\theta_{\text {out }}-\theta_{\text {in }}\right)
$$

Note that in Equation 5.27, $\mathbf{V}_{\text {out }}$ and $\mathbf{V}_{\text {in }}$ are phasors with an amplitude and phase. To simplify the math, an FFT was performed on the input and output signals to calculate their respective amplitudes and phases. A smoothing spline was used to interpolate the transfer function at intermediate frequencies. The transfer function of each amplifier unit is displayed in Figures 5.31 and 5.32. It should be noted that this method is not the 


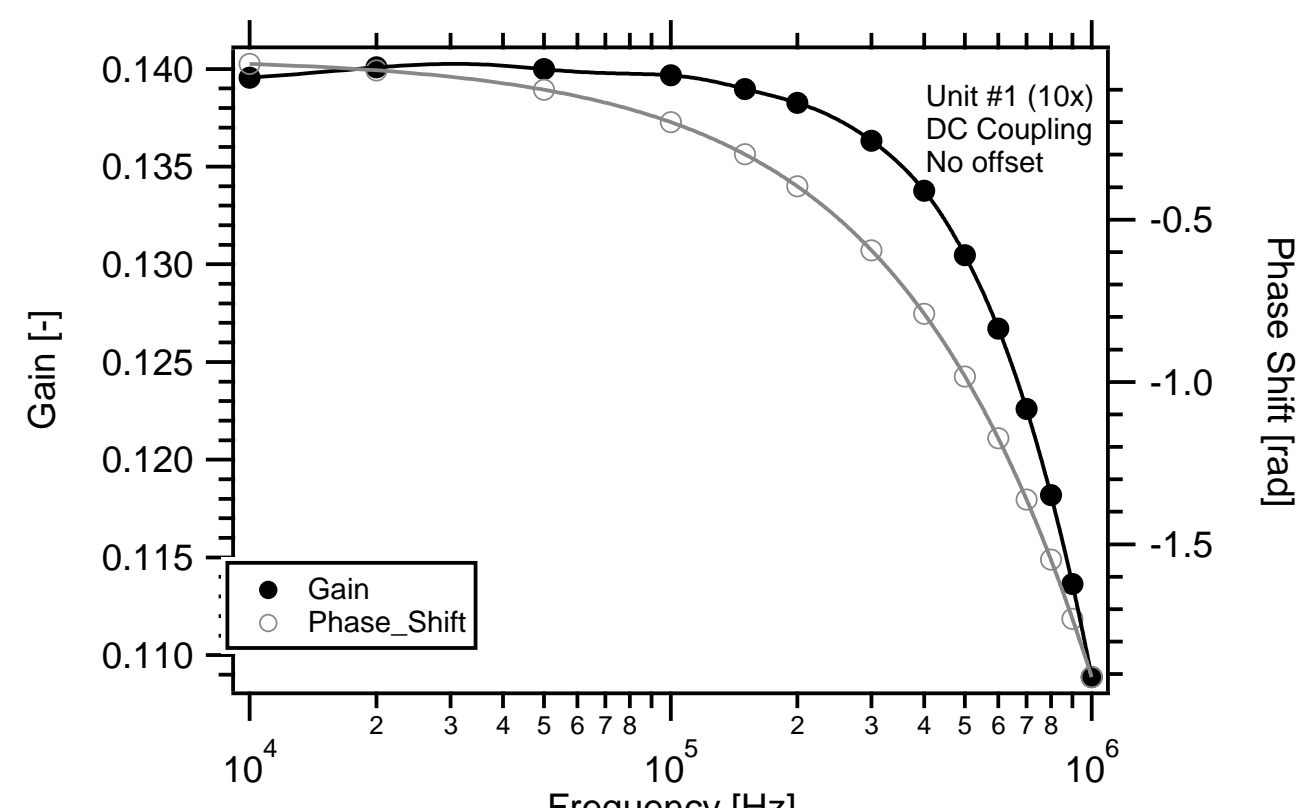

(a)

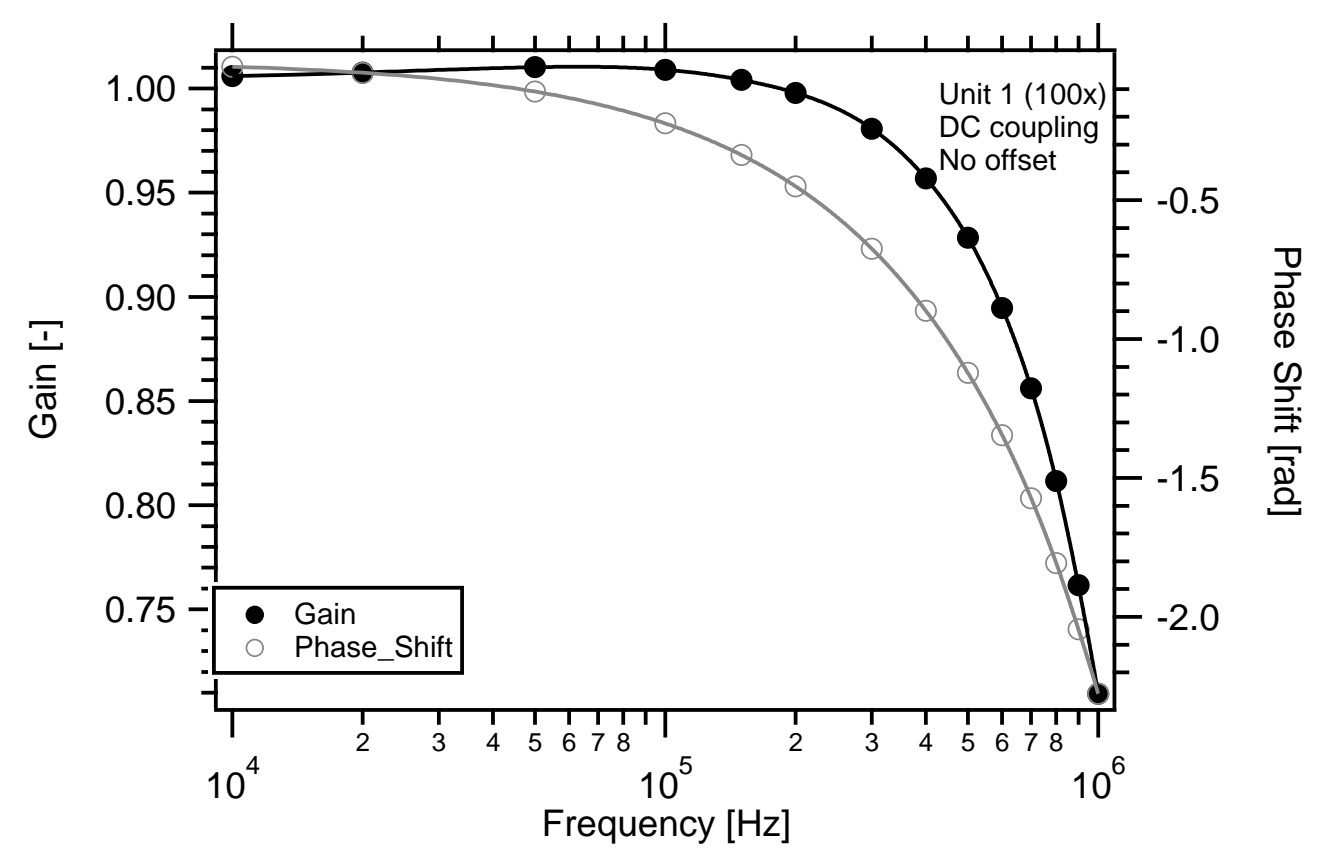

(b)

Figure 5.31: Measured frequency response of preamplifier \#1 from $10 \mathrm{kHz}$ to $1 \mathrm{MHz}$ for (a) 10x gain and (b) 100x gain.

preferred method for measuring the transfer function of the differential amplifiers. Ideally, chirp signals would be used as inputs for the amplifiers so that all frequencies of the desired range could be tested at once. However, the signal generator used in this instance was not 


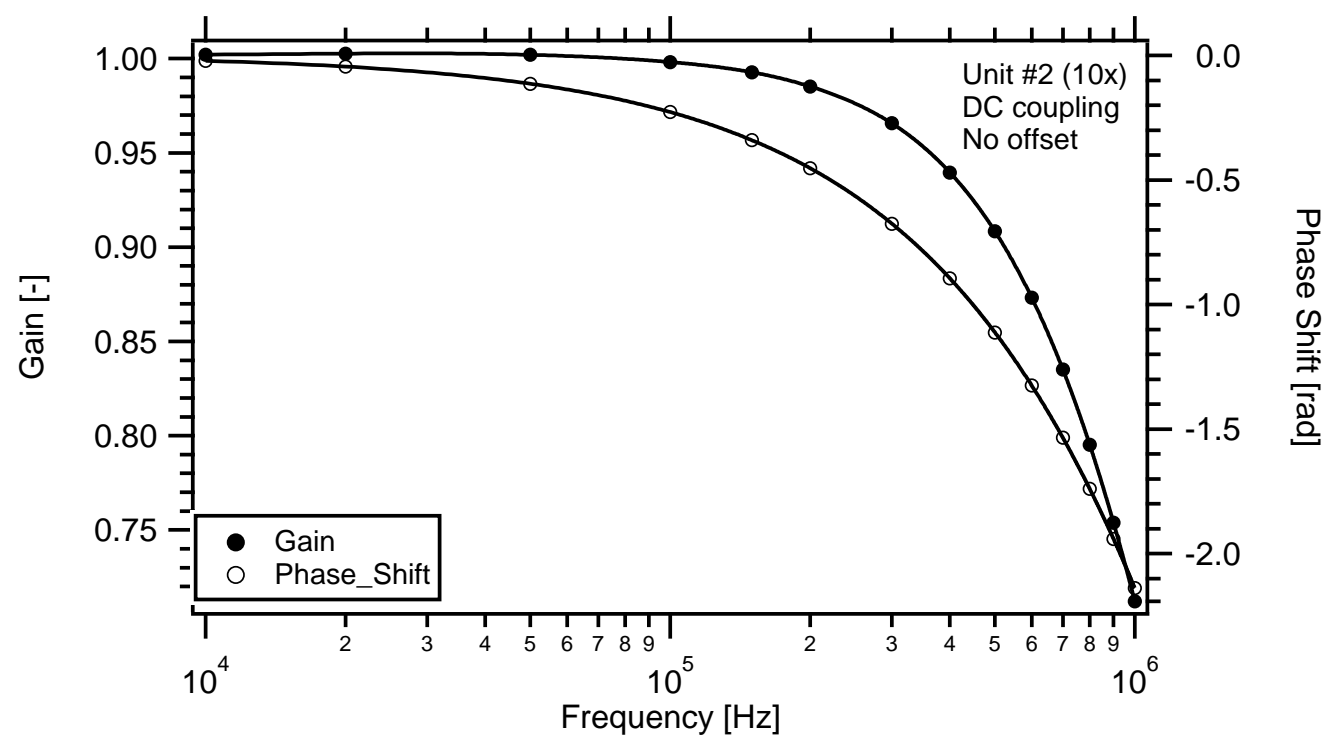

(a)

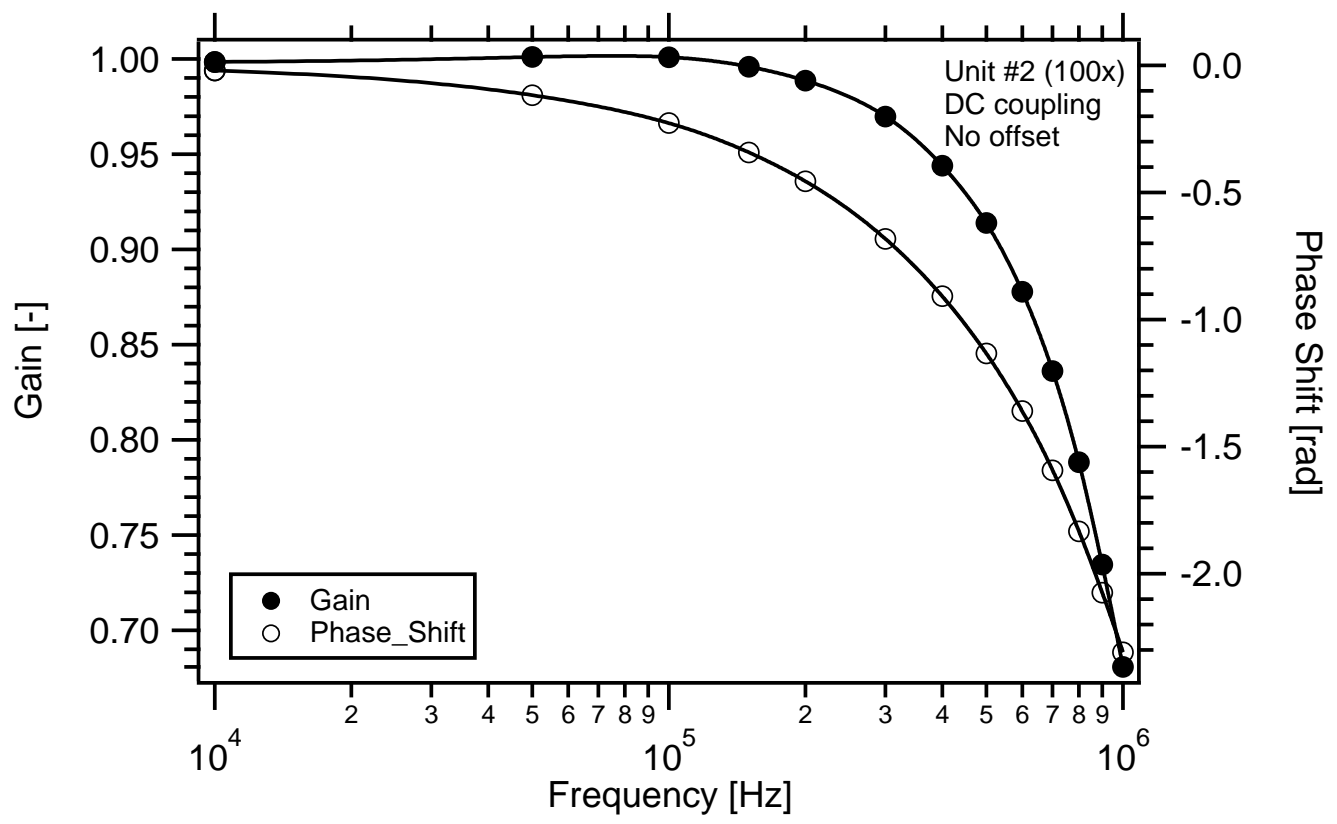

(b)

Figure 5.32: Measured frequency response of preamplifier \#2 from $10 \mathrm{kHz}$ to $1 \mathrm{MHz}$ for (a) 10x gain and (b) 100x gain.

capable of sourcing 2 simulatanenous chirps with opposite polarity.

The transfer function for the differential amplifier was multiplied by the transfer function for probe assembly to find the overall transfer function of the TOF b-dot probe signals. 


\subsubsection{Plasma Probes}

Two different types of plasma probes were used in this research. The first type of plasma probe was a single Langmuir probe fixed to a negative bias voltage. This is referred to in this research as an ion flux probe. An asymmetric double Langmiur probe (ADLP) was also used. This section explains how both probes were used in this research including basic probe theory, probe construction and geometry, associated circuitry, and data analysis.

A single Langmuir probe is a conductive electrode emersed in a plasma, biased to a potential [70], [71], [72]. The voltage applied to the probe is swept through a range of voltages and the current collected to the probe is monitored as function of voltage. This information can be used to infer plasma density, electron temperature, plasma potential, and floating potential. The behavior of the probe current as a function of applied voltage is referred to as an I-V characteristic. A illustration of a typical I-V characteristic is shown in Figure 5.33, At low bias voltages (negative), the probe only collects ions. All electrons are repelled. The current collected to the probe is constant in this region (Region I in Figure 5.33 as the ions surrounding the probe in the Debye layer shield out the probe's potential from the nearby plasma. This region is referred to as the ion saturation region. At high bias voltages (positive), the probe only collects electrons. Ideally, the current here would be constant as well, though in reality expanding electron sheaths cause an increase in current with voltage. This region (Region III in Figure 5.33) is referred to as the electron saturation region. The intermediate region (Region II in Figure 5.33) between the ion and electron saturation regions is a transition region where the probe collects both ions and electrons. Assumptions about the plasma can be applied to the data so that it can be used to infer certain plasma properties. All three regions are required to build a complete picture, which 


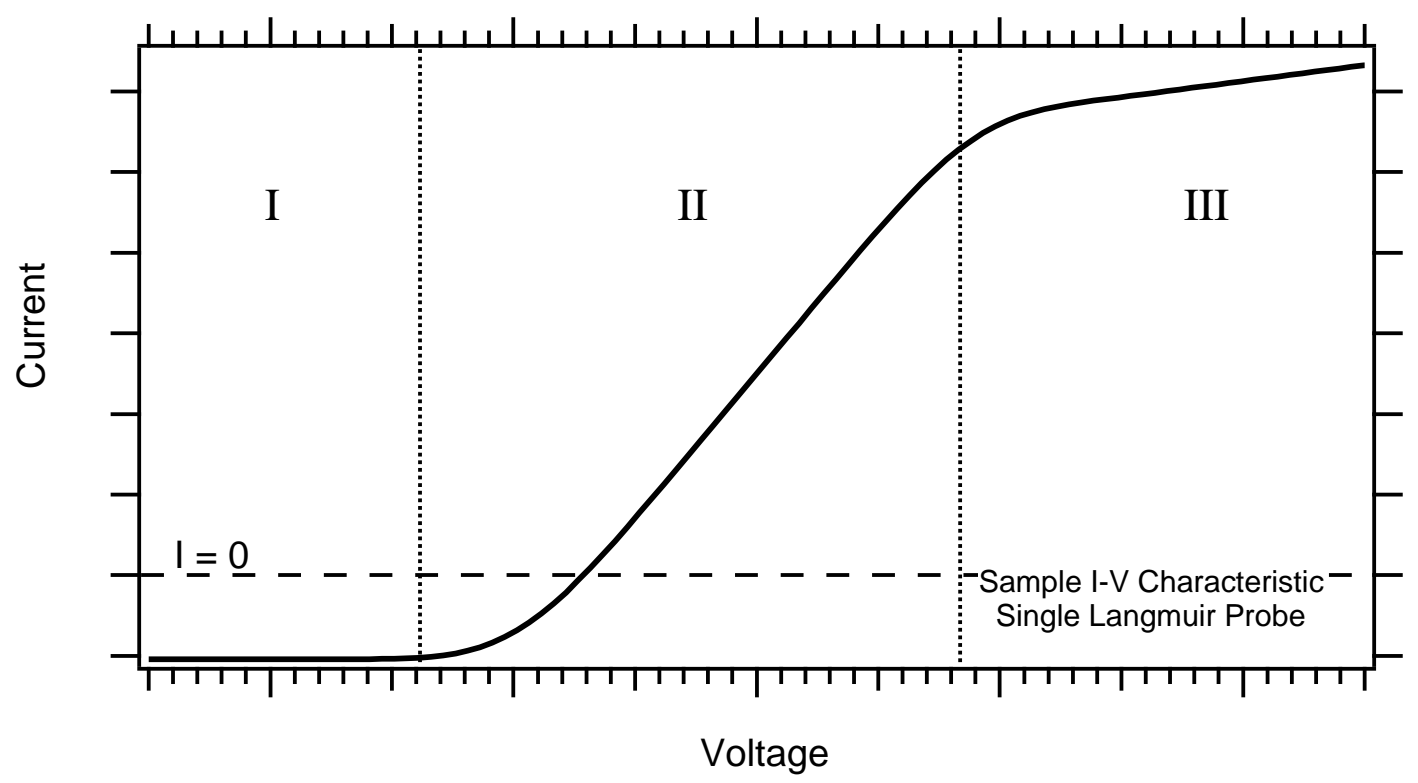

Figure 5.33: Sample I-V characteristic for single Langmuir probe.

is why sweeping the probe through a range of voltages is necessary for measuring plasma properties.

The fast timescales of the XOCOT-T3 experiment made sweeping the probe difficult. The sweeping method would require novel, custom probe circuitry to satisfactorily sweep the probe through a range of voltages on a timescale comparable to plasma fluctuations (greater than $5 \mathrm{MHz}$ ). Instead of sweeping, the probe was fixed to a single bias in the ion saturation region where repeatable data and repeatable probe behavior was most likely. Limited information is available about a probe in ion saturation and generally only probe currents can be reported with any accuracy. However, if certain assumptions can be made about the plasma, density or temperature measurements can be inferred from the data.

If the kinetic energy of the plasma relative to the probe's collection surface is much greater than its thermal energy $\left(1 / 2 m_{i} u_{i}^{2} \gg k_{B} T\right)$, the ion saturation current is related to the local plasma density $n_{0}$, the velocity of the ions $u_{i}$, the charge number of the ions $Z$, and the 
probe area $A_{p}$ by a simple flux relation [73]:

$$
I_{+}^{s a t}=A_{p} e_{c} n_{0} Z u_{i}
$$

This relation is valid for planar probes normal to the velocity vector and for cylindrical probes transverse to the flow vector. For a planar probe situated in a $10 \mathrm{eV}, 100 \mathrm{~km} / \mathrm{s}$ argon plasma, the kinetic energy $\left(3 \times 10^{-16} \mathrm{~J}\right.$ or $\left.1900 \mathrm{eV}\right)$ is two orders of magnitude higher than the thermal energy $\left(2 \times 10^{-18} \mathrm{~J}\right.$ or $\left.10 \mathrm{eV}\right)$. Since the AFRC plasma in the XOCOT-T3 was expected to stream past the probes at $100 \mathrm{~km} / \mathrm{s}$ with thermal temperatures less than $10 \mathrm{eV}$, this means that Equation 5.28 can be used to estimate the plasmoid's density if the velocity is known.

The ion flux probes used in this research determined while plasma was measured at the probe's collection surface, it did not correspond to a flowing plasma (see Section 7.2.3). Rather, it was due to background ionization of the neutral fill gas. In this case, the density of the plasma was estimated by assuming an ion temperature $T_{i}$ equivalent to the electron temperature $T_{e}$. Furthermore, the plasma was assumed to have no net drift velocity impinging on the probe's collecting surface, a Maxwellian energy distribution, and equal number of ions and electrons (quasi-neutral). In this case, the ion saturation current related to plasma density through [74]:

$$
I_{+}^{s a t}=e_{c} n_{0} A_{p} Z \sqrt{\frac{k_{B} T_{e}}{2 \pi m_{i}}} i_{i}(X)
$$

where $m_{i}$ is the mass of the ions, $i_{i}(X)$ is a correction factor to account for collisional effects, expanding collection areas, and probe potentials. Equation 5.2.3 is also valid when a cylindrical probe is placed with its axis parallel to the flow vector [74]. In a stationary, fully ionized (collisionless), and dense plasma, $i_{i}(X)$ is unity since these effects are negligible. 
A thin ion sheath surrounds the probe, screening its potential from the rest of the plasma. The thickness of this sheath, is given by the Debye length [45]

$$
\lambda_{D}=\sqrt{\frac{\varepsilon_{0} k_{B} T_{e}}{n_{0} e_{c}^{2}}}
$$

When the sheath is much, much smaller than the probe radius, the area of the sheath is approximately equal to the area of the probe. All ions with enough thermal energy to enter the sheath are collected by the probe. This is commonly referred to as the thin-sheath approximation. For the high density plasma created in the XOCOT-T3 $\left(10^{18}-10^{20} \mathrm{~m}^{-3}\right.$ and $1-10 \mathrm{eV}), \lambda_{D}$ is only a few microns. Using a probe radius $r_{p}$ of $1 \mathrm{~mm}$ or so will satisfy the thin-sheath approximation. If the sheath grows to the limit of $r_{p}<45 \lambda_{D}$, the sheath expansion will need to be considered and a more elaborate form for $i_{i}(X)$ will be required [75], [76].

Some sources [71] replace the ion thermal velocity $(1 / 2 \pi)^{(1 / 2)}$ with a Bohm factor of $\exp (-1 / 2)$ to tweak for presheath interactions. This changes the $(1 / 2 \pi)^{(1 / 2)}$ coefficient in Equation from 0.40 to 0.61 . However, it has been shown elsewhere [77] that a coefficient of 0.40 provides better agreement to classic analytical work on Langmuir probes [78].

The single Langmuir probes (or ion flux probes) in the XOCOT-T3 experiment were used in the downstream region. Two probe assemblies were fixed $18.2 \mathrm{~cm}$ apart in the axial direction at a 10 degree azimuthal spacing. The first probe assembly was located at $\mathrm{z}=$ $49.5 \mathrm{~cm}$ and the second assembly was located at $\mathrm{z}=67.7 \mathrm{~cm}$. Each assembly consisted of two identical probes, with one insulated from the plasma for a EMI monitor. The non-insulated (active) probe's signal was compared to its insulated mate (the null probe) to ensure the signal reading corresponded to a plasma flux rather than a noise event. The probes were planar probes, with only a front face exposed. Each probe was machined 


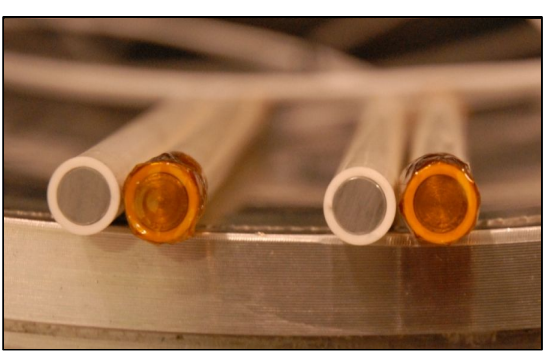

(a)

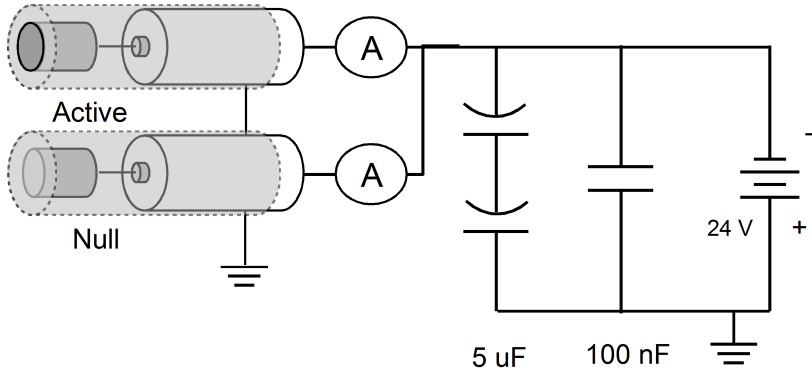

(b)

Figure 5.34: Ion flux probes, including (a) a photograph of the probes and (b) circuit diagram

from molybedenum and covered in by an alumina tube. The exposed area was $9.52 \mathrm{~mm}$ in diameter, with a total area of $71.18 \mathrm{~mm}^{2}$. Short BNC cables (RG-188, $2 \mathrm{~m}$ long) connected the probes to the tank feedthrough with an additional 0.5 meters of cabling (RG-58) outside the tank for connection to the power supply. The probes were biased to a negative voltage relative to ground to ensure electrons were repelled and ions were collection. Photographs and drawings of the probes are shown in Figure 5.34a.

Two negative bias voltages were tested on the ion flux probes to determine the correct voltage for ion saturation. Potentials of $-6 \mathrm{~V}$ and $-24 \mathrm{~V}$ relative to the chamber ground were applied to the probe. The current density measured by the probes for each bias setting is shown in Figure 5.35. It is noted that despite a large change in probe voltage, little difference was seen in the current collected to the probe. This indicates that a bias voltages of $-6 \mathrm{~V}$ and $-24 \mathrm{~V}$ both correspond to the ion saturation region. Since $-24 \mathrm{~V}$ is farther into the ion saturation region, this voltage was used for the ion flux probe bias.

Each probe assembly was connected to a $24 \mathrm{~V}$ (nominal) battery array, with the planar electrode negatively biased to collect ions. The positive terminal of the battery was 


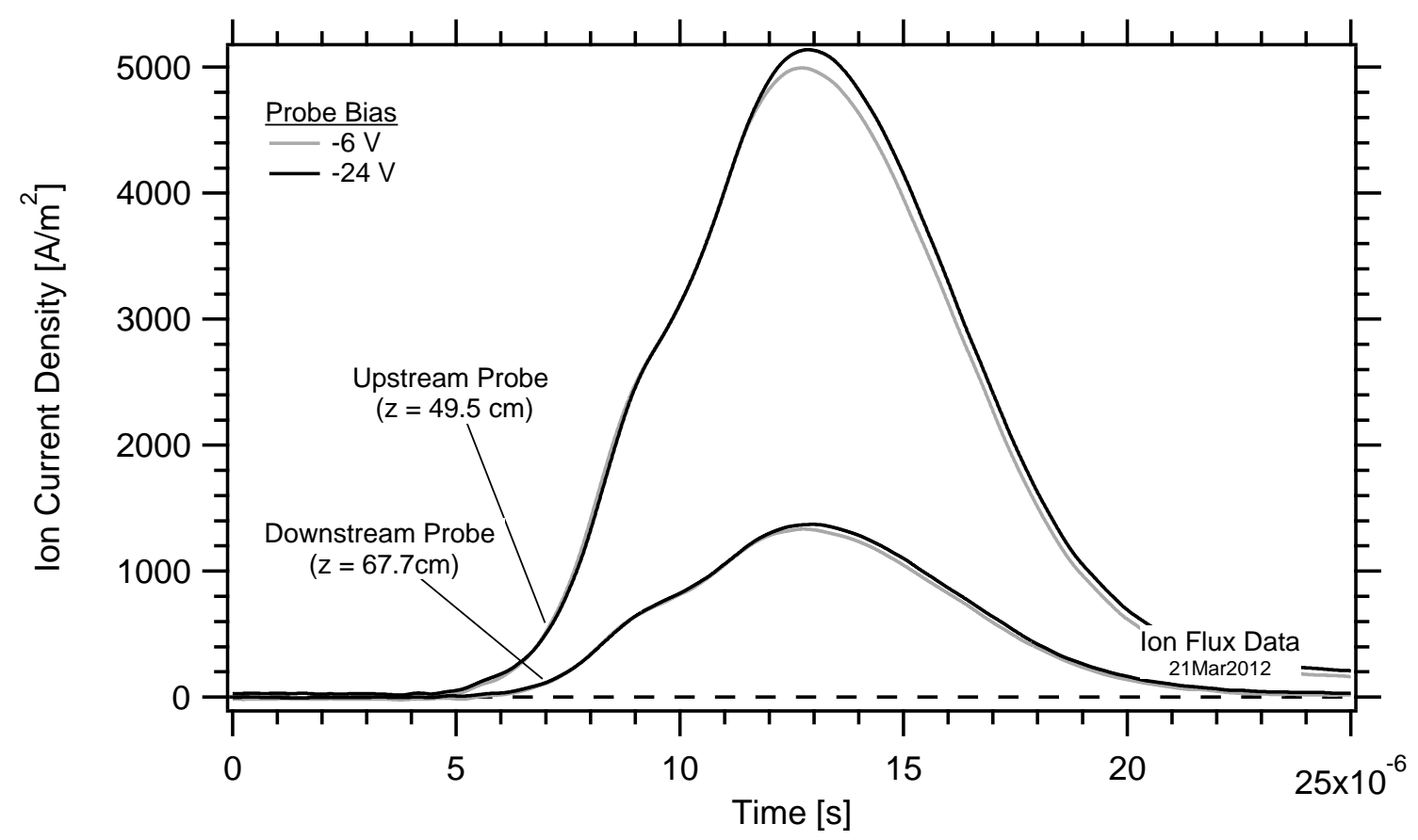

Figure 5.35: Current density measured by the ion flux probes for a $-6 \mathrm{~V}$ bias and $-24 \mathrm{~V}$ bias.

connected to the vacuum tank. Current monitors (Pearson $\mathrm{p} / \mathrm{n} 4100$ and Stangenes $\mathrm{p} / \mathrm{n}$ 0.5-1.0) were used to measure the current through each probe. The signal lines were wrapped through the current monitors 10 times for a 10x amplification. The current monitors were terminated into $50 \Omega$ prior to digitization, dropping the amplification to $5 x$. The current monitors and wire wrappings were calibrated with a function generator to measure the actual amplification, so it could be corrected for in data processing. The orientation of each monitor was checked to ensure the current was an ion current and not an electron current. Since batteries can have a slow rise-time, a small capacitor array was connected across the terminals to improve the probe's response. Two $10 \mu \mathrm{F}$ tantalum capacitors were connected in series, along with a $100 \mathrm{nF}$ ceramic (X7R) capacitor. The layout of the probe circuit is displayed in Figure 5.34b.

There was no accurate way to test the frequency response of the ion flux probes, due to 
the uncertainty of the plasma's ground path. This current path is necessary to resolve the frequency response since it determines the resistance and inductance of the probe during a measurement. The frequency response of the probe was made as high as possible by keeping the lines to the ion flux probes short, with the circuitry connected directly outside the tank. To minimize the uncertainty of the timing delays between probes, each probe was made with identical line lengths and similar circuitry to ensure similar frequency responses.

The signals from each probe were scaled to calculate the ion current measured by each probe. The null probe current was subtracted from the active probe current to remove electrostatic pickup. A digital filter $(500 \mathrm{kHz}$ low pass) was then applied to remove digitizer noise. The scaled signals from each probe assembly were compared to measure the plasmoid's velocity. For most tests in this research, this was the limit of the data analysis for the ion flux probes.

The asymmetric double Langmuir probe was used primarily for comparative internal plasma measurements of probe current across the plasma's radius, though it was intended to be used in the plume for density mapping. A double Langmuir probe [79] is a variation on the single Langmuir probe, but uses two electrodes instead of one. A bias voltage is applied between the electrodes so that neither electrode is tied to ground. This allows the probe to float with the plasma floating potential (which can change rapidly in rf plasmas), avoids the need for stable reference ground, and limits the current collected to the probe. While most double Langmuir probes are used in a symmetric configuation with both electrodes identical in size, the asymmetric probe uses two electrodes of different sizes. The smaller electrode is biased positively to collect mostly electrons and the larger electrode is negatively biased to collect mostly ions. The smaller electrode's potential is somewhere above the floating potential and the larger electrode's potential is below the 


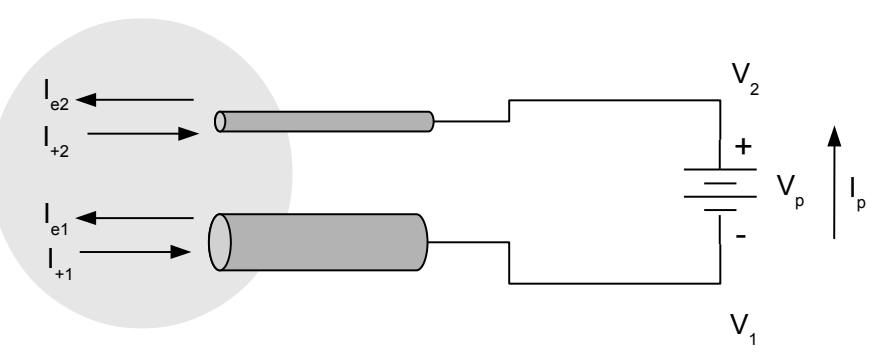

Figure 5.36: Illustration of an asymmetric double Langmuir probe.

floating potential. An illustration of the ADLP is displayed in Figure 5.36, The appeal of using an asymmetric probe over a symmetric probe is that it samples more of the electron energy distribution function providing a more accurate temperature [80] and is known empirically to lower noise signals issues with the probe.

An asymmetric double probe can be used in a similar fashion to a symmetric double probe, though the interpretation of the probe data between the two probes is different. An asymmetric probe collects both ion and electron currents to its electrodes, as illustrated in Figure 5.36. The current collected to the probe $I_{p}$ is a sum of the electron current $I_{n e}$ and ion currents $I_{+n}$

$$
I_{p}=I_{+1}-I_{e 1}=I_{e 2}-I_{+2}
$$

meaning

$$
I_{+1}-I_{e 1}-I_{e 2}+I_{+2}=0
$$

The probe voltage $V_{p}$ is the potential difference between $V_{2}$ and $V_{1}$

$$
V_{p}=V_{2}-V_{1}
$$


As in a single Langmuir probe, when the probe is placed in a Maxwellian, quasineutral plasma with minimal flow relative to the collection area, the ion and electron currents for singly charged species can be written as [74]

$$
I=e_{c} n_{0} A_{n} Z \sqrt{\frac{k_{B} T_{e}}{2 \pi m}} i(X)
$$

where $A_{n}$ is the probe area of electrode $n, m$ is the species mass, and $i(X)$ a correction factor, with $X \equiv e_{c} V_{n} / k_{B} T_{e}$. For electron currents, $i(X)$ is the exponential function of $X$ from Boltzman's law so that

$$
I_{e n}=e_{c} n_{0} A_{n} \sqrt{\frac{k_{B} T_{e}}{2 \pi m}} \exp (X)=e_{c} n_{0} A_{n} \sqrt{\frac{k_{B} T_{e}}{2 \pi m}} \exp \left(\frac{e_{c} V_{n}}{k_{B} T_{e}}\right)
$$

For ion currents, $i(X)$ is more complicated as it depends on collisional effects, expanding collection areas, and probe potentials. Generally, the ion current is given as

$$
I_{+n}=e_{c} n_{0} A_{n} \sqrt{\frac{k_{B} T_{e}}{2 \pi m}} i_{i}(X)
$$

Combining Equations 5.32 and 5.35 and dividing both sides by $I_{e 1}$, the resulting equation becomes

$$
\frac{I_{+1}+I_{+2}}{I_{e 1}}-1-\frac{A_{2}}{A_{1}} \exp \left(\frac{e_{c} V_{p}}{k_{B} T_{e}}\right)=0
$$

Substituting in $I_{e 1}=I_{p}+I_{+1}$, Equation 5.37 transforms to

$$
1+\frac{A_{2}}{A_{1}} \sqrt{\frac{k_{B} T_{e}}{2 \pi m}} \exp \left(\frac{e_{c} V_{p}}{k_{B} T_{e}}\right)=\frac{I_{+1}+I_{+2}}{I_{+1}-I_{p}}
$$

Solving for $I_{p}$

$$
I_{p}=I_{+1}-\frac{I_{+1}+I_{+2}}{1+\frac{A_{2}}{A_{1}} \exp \left(\frac{e_{c} V_{p}}{k_{B} T_{e}}\right)}
$$


High density plasmas generally ensure that the $I_{+n}$ can be approximated to a thin-sheath formulation so that $i_{i}(X)$ is unity. This is valid if the Debye length $\lambda_{D}$ is much smaller than the probe radius $r_{p}$. If $3<r_{p} / \lambda_{D}<50$, thin-sheath behavior no longer applies and $i_{i}(X)$ becomes a transition between thin-sheath and orbital-limited-motion (OML) theory. Empirical fits for this region is defined elsewhere [81], [82]. For thin-sheath behavior $\left(r_{p} / \lambda_{D}>50\right)$, the ion current to each electrode is defined as

$$
\begin{aligned}
& I_{+1}=e_{c} n_{0} A_{1} \sqrt{\frac{k_{B} T_{e}}{2 \pi m_{i}}} \\
& I_{+2}=e_{c} n_{0} A_{2} \sqrt{\frac{k_{B} T_{e}}{2 \pi m_{i}}}
\end{aligned}
$$

The I-V characteristic for an asymmetric double probe represents a shifted hyperbolic tangent. A sample I-V characteristic for several temperatures is displayed in Figure 5.37. At low temperatures, the characteristic saturates at low voltages. The saturation current and voltage increase with temperature and density. The voltage required for saturation increases with temperature. While most double probes only draw a few $\mathrm{mA}$ at most from the plasmas, in the high-density plasmas of the XOCOT-T3, it is not unusual to see currents on the order of a few amps. The electron temperatures in Figure 5.37 are expressed in electron-volts (eV) which are non-SI units, as is common in plasma physics. An electron-volt is a unit of energy. To convert electron temperatures in Kelvin $T_{e}$ to electron volts $T_{e V}$, the following relation is employed

$$
T_{e V}=\frac{k_{B} T_{e}}{e_{c}}
$$

The relations presented in Equations 5.34 through 5.41 are only valid for negligible flow velocity relative to the probe's collection surface. They apply to cylindrical probes with a length much greater than the radius and when the axis of the probe is oriented normal to 


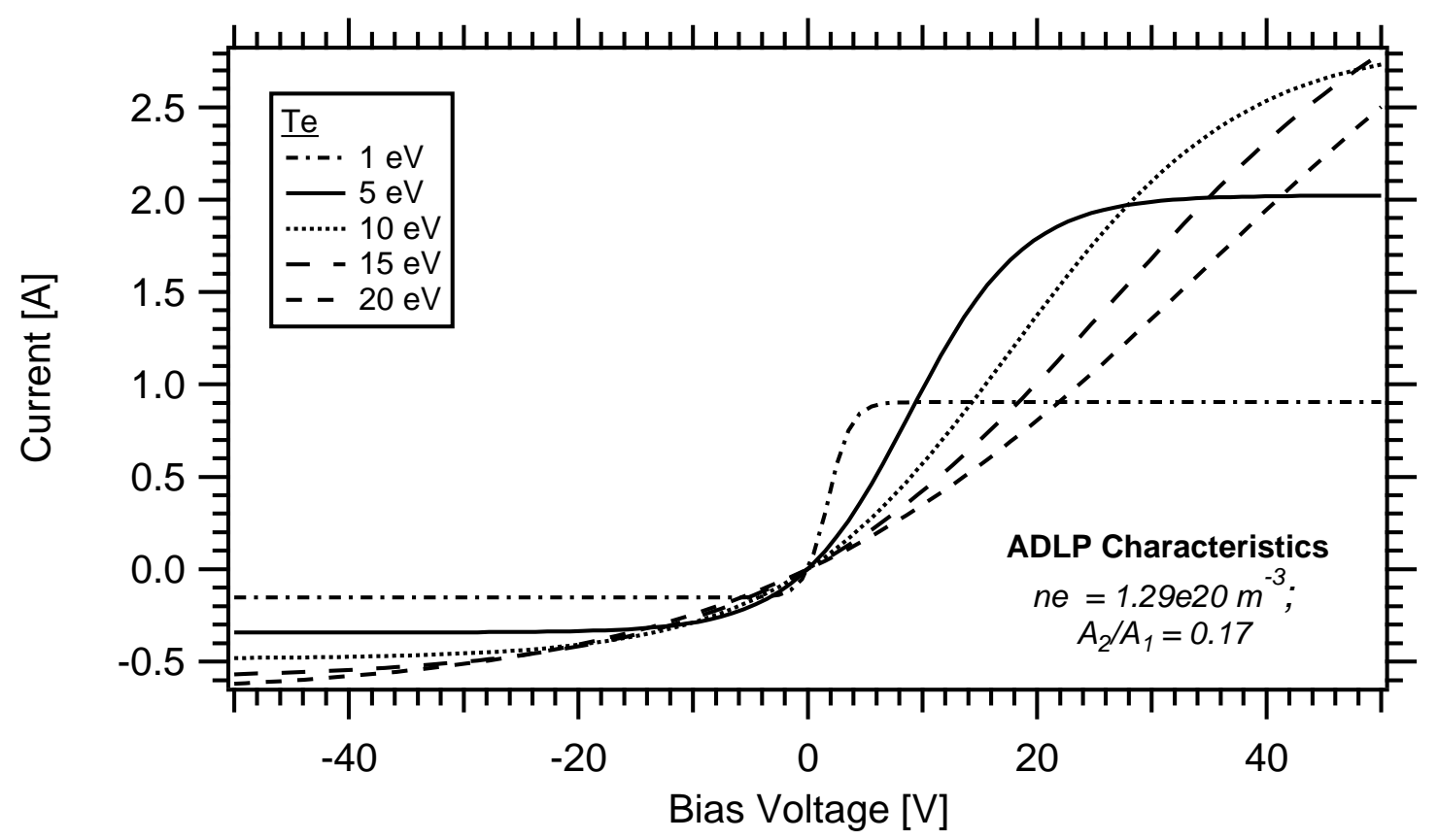

Figure 5.37: Asymmetric double Langmuir probe I-V characteristics for several different electron temperatures.

the flow direction [74]. The ADLP data presented in this research did not always satisfy this condition; internal measurements across the probe radius were collected with the flow vector transverse to the probe's collection surface. In this instance, the magnitude flow vector of the plasmoid's ion and electron currents were unknown. While the angular velocity of the electrons was likely well in excess of $500 \mathrm{~km} / \mathrm{s}$ (greater than $10 \mathrm{kA}$ of current), the ion velocity was only a few $\mathrm{km} / \mathrm{s}$ if present at all. This means that the ion kinetic energy is similar to the ion's expected thermal energy $(10 \mathrm{eV})$ and Equations 5.34 through 5.41 cannot be used to estimate the plasma's density. A form of Equation 5.28 is not valid, either. The probe current instead is a combination of the ion velocity and the plasma density.

Langmuir probe theory and behavior are fraught with experimental uncertainty, leading to over predictions of plasma density and electron temperature by 30\% [82]. These uncertainties include errors in fitting the data to Equation 5.39, errors in estimating the 
collection area, thermal expansion of the probe, uncertainty in ion temperatures, end effects, and the kinetic energy of the incoming ions in flowing plasmas. These uncertainties are accounted for in the data analysis.

The ADLP in the XOCOT-T3 experiment was used in two locations. The probe was first placed $3.8 \mathrm{~cm}(\mathrm{z}=34.28 \mathrm{~cm})$ outside of the discharge coils. The probe was then moved internally to the plasma to take measurements at the axial midplane $(\mathrm{z}=15 \mathrm{~cm})$. A photograph of the ADLP is displayed in Figure 5.38a. The ion collection probe was made from a tungsten rod with a diameter of $1.5 \mathrm{~mm}$ in diameter by $15 \mathrm{~mm}$ long, yielding an area of $22 \mathrm{~mm}^{2}$. The electron collector was a thin tungsten wire $0.254 \mathrm{~mm}$ in diameter by 15 mm long. BNC cables (RG-188, 2 m long) connected the probes to the tank feedthrough with an additional 2 meters of cabling (RG-58) outside the tank connecting the probes to the power supply. The bias voltage between the probes was fixed by an isolated power supply (Stanford Research System p/n SIM 928) or battery array. An array of capacitors was used to hold the bias voltage steady throughout the discharge. Using three different types of capacitors ensured that the slow, moderate, and fast risetimes could be resolved. The voltage across the battery/capacitor array was monitored with a high frequency differential voltage probe to ensure the voltage did not drop less than $10 \%$.

The voltage applied between both electrodes of the double Langmuir probe is traditionally swept by changing the bias voltage across the probe and measuring the current collected to the probe. The fast timescales of the XOCOT-T3 experiment made it difficult to operate the probe in this fashion, so the probe bias was fixed to a single level for each shot. Only one measurement was taken at each voltage setting, necessitated by the largely qualitative nature of the data collected from the diagnostics paired with the large number of tests required to map a complete $\mathrm{I}-\mathrm{V}$ characteristic. Repeating the trace 50 times would require 


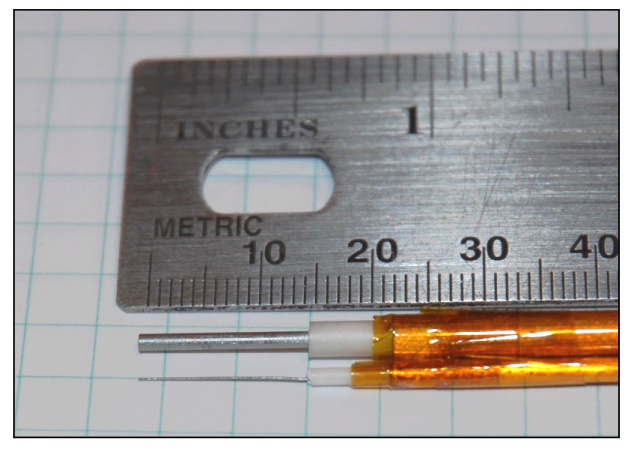

(a)

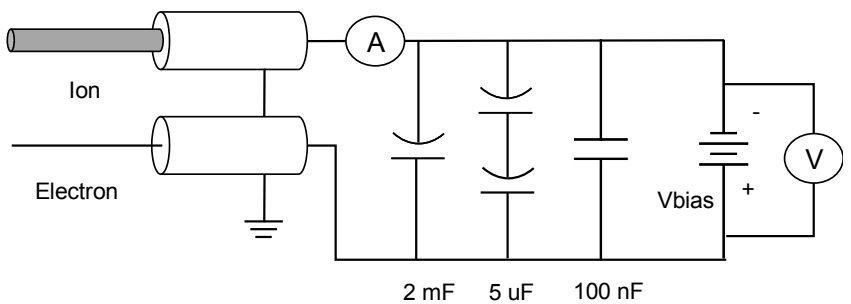

(b)

Figure 5.38: Asymmetric double Langmuir probe, including (a) a photograph of the probe and (b) circuit diagram.

several days of testing and produce excess wear on the experiment's discharge circuit. Repeated measurements were collected, though at a single probe voltage $\left(V_{p}=6 \mathrm{~V}\right)$ to ensure the current collected to the probe did not fluctuate appreciably. Additionally, the probe was only biased in one polarity, with the larger electrode collecting ions. This was done to reduce the number of shots required to build the I-V characteristic and provide better refinement of the characteristic.

Probe cleanliness was an issue in this experiment for the ADLP. It was noted that the current collected to the probe would decay by $10 \%$ with every 10 shots. Dielectric contaminents (likely quartz) would build up on the probe's surface, leading to a drop in effective area. An insitu cleaning technique was used to remove the contaminents. The probe was operated in a glow-discharge mode by applying a high voltage $(2 \mathrm{kV}, 1 \mathrm{~mA})$ across the probe for 1 minute using 50 mTorr of background gas. The bias was changed across the probe to clean the other side. The bombardment of argon ions was generally sufficient to remove the contaminents, though a $10 \%$ error in probe signal levels at a repeated condition was still noted. Cleaning the probe more often might have lowered this uncertainty, but would have resulted in longer test times and more stress on the vacuum equipment. Cleaning the 
probe for longer would have damaged the probe. This $10 \%$ uncertainty in probe area was accounted for in the error analysis of the probe data.

\subsubsection{Single Frame Digital Camera}

An intensified CCD single-frame digital camera was used to image the plasma formation. The digital camera is a DiCAM-2, with a minimum exposure time of $5 \mathrm{~ns}$. A $480 \mathrm{~nm}, 10$ $\mathrm{nm}$ bandwidth filter was added to camera to isolate a set of Ar II emission lines. The camera was pointed along a line parallel to the $\mathrm{z}$-axis of the experiment so that an end-on view of the plasmoid could be imaged. The camera had to be positioned to view only a portion of the plasmoid; the perspective imposed by inner coil prevented the entire plasmoid from being imaged.

\subsection{Translation Measurements}

One objective of this research was to measure the velocity, momentum, and energy efficiency of the plasmoid as it traveled downstream from the coils. The measurements were collected or designed to be collected using the diagnostics outlined in Section 5.2. The velocity diagnostics are presented in Section 5.3.1. Using these techniques, the velocity results in Chapter 7 show that the plasmoid failed to translate from the coils. Given this observation, the design of the momentum and efficiency diagnostics are presented in Sections 5.3.2 and 5.3.3 as they may have been implemented if plasmoid translated. Section 5.3.4 describes the techniques used to estimate the global energy deposition into the plasma. 


\subsubsection{Velocity Measurements: Time-of-Flight Array}

The plasmoid velocity was measured using a time-of-flight (TOF) array. A TOF array uses two or more probes spaced a fixed distance apart in the direct path of the plasmoid. The time history of their signals is recorded as the plasmoid travels past. By comparing the signals from the two probes, a mean velocity between the two probes can be measured.

The TOF array in the XOCOT-T3 consisted of both b-dot probes and plasma probes. The plasmoid was a magnetically confined, dense plasma so that a translating plasmoid should appear simultaneously on a plasma probe and a b-dot probe. If only one type of probe is used, false readings can result. The magnetic field distortions from the formation of the plasmoid can produce misleading signals on the b-dot probes. For example, an axial growth in the AFRC can appear as a velocity on the downstream probes. Similarly, hot plasma escaping the confinement can appear on the downstream plasma probes as a translating plasma. Additionally, the phase error between the TOF b-dot probes as discussed in Section 5.2 .2 .8 results in a $30 \%$ measurement uncertainty on the b-dot probes. This means the measurement on the b-dot probes must be cross-checked against the signals on the plasma probes for accuracy. For these reasons, it was important to include both types of probes in a TOF array.

In this research, the plasmoid's velocity was measured with a TOF array for each operating condition (fill pressure and energy). This information provided a characterization of the experiment across its entire range. In the event of a translating plasmoid, the characteristic magnetic signature should resemble a variant of Figure 4.29 , with the magnetic field data on the axially separated probes showing a distinct trend, staggered in time. The plasma probes 

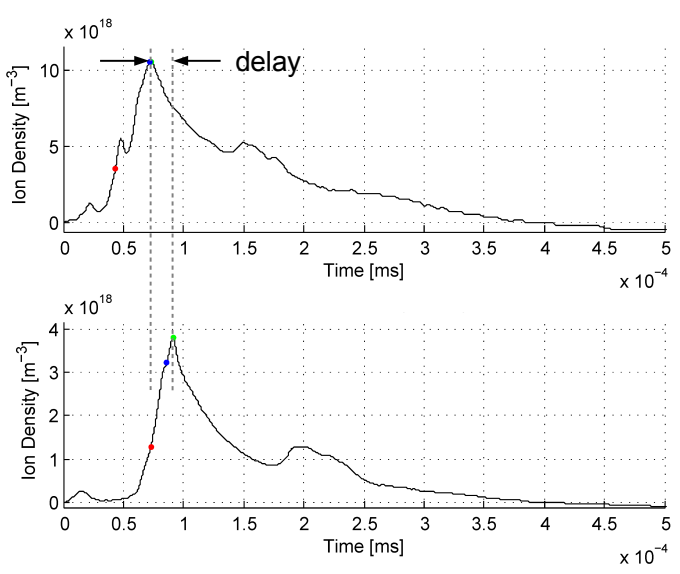

Figure 5.39: Plasma probe data from a time-of-flight array in a PIPT experiment [3].

should appear as sharp peaks, separated in time. Data from a similar plasmoid translation experiment [3] shown in Figure 5.39 illustrates an expected delay.

The plasmoid was assumed to have reached a terminal velocity so no acceleration or deceleration occured between the probes. Terminal velocity was demonstrated with the annular electromagnetic launcher model to occur in the XOCOT-T3 design at $\mathrm{z}=40$ $\mathrm{cm}$, about $10 \mathrm{~cm}$ downstream of the coils (see Section 4.2.7). This trajectory is likely to be complicated with encounters with the cold background gas, recombination, and thermal-to-kinetic energy conversions. All of these non-ideal effects lead to acceleration or decceleration of the plasmoid. Additionally, the plasmoid was assumed to be non-deforming along its direction of travel so that the velocity of the leading edge of the plasma matched the tail end. Expansion of the plasmoid into a field free region would alter this assumption. If the discharge is fairly repeatable, the validity of these assumptions can be checked by repeating the test with the probes at new locations in the plume. 


\subsubsection{Momentum Measurements}

Momentum measurements are ideally recorded with a measurement device such as a ballistic pendulum or thrust stand. A ballistic pendulum captures the momentum of the plasmoid downstream as it impacts the target, displacing the pendulum in accordance with conservation of momentum. Thrust stands are directly attached to the thruster, measuring the impulse the thruster produces during firing. Thrust stands require the entire system to be mounted to the displacement sensor. The XOCOT-T3 is a benchtop demonstration experiment and use of a thrust stand to take momentum measurements is not practical in this instance. Ballistic pendulums are a better choice for impulse measurements in this experiment, but development of such a diagnostic is prohibitively time-consuming for the basic characterization this research is seeking.

The momentum of the plasmoid's ejection was instead designed to be inferred from spatial plume measurements, specifically density and velocity. Plasma density was to be measured with an ADLP in ion saturation, as described in Section 5.2.3. Axial velocity measurements were to be collected from a time-of-flight array of two axially separated ADLPs, outlined in Section 5.3.1. Single point measurements were likely insufficient for resolving the density of the plasmoid. The probe array was to be swept in the radial direction, across the entire width of the plasmoid for a full density profile. Axisymmetry was to be assumed so the momentum $\left(I_{b i t}\right)$ of the plasmoid could be calculated from a 2D integration of the velocity and density measurements as shown in Equation 5.43 . The number of measurement points along the radial axis should be fine enough to resolve radial density gradients and were to 
be determined after a baseline set of measurements is collected.

$$
I_{b i t}=\int\left(\frac{M_{m} 2 \pi}{N_{A}} \int n_{i}(r, t) u_{z}(r) r d r\right) d t
$$

Measuring the density with an ADLP in ion saturation would require an estimate for the temperature of the plasma. While the temperature could be measured by changing the probe voltage at repeated conditions, it is noted from Figure 5.37 that the ion saturation current is less sensitive to temperature than density. Increasing the temperature by $5 x$ only doubles the calculated density. This means that small errors in temperature are unlikely to add signficant error to the momentum measurement. A modest estimate for temperature was to be made in this experiment by collecting two full I-V traces at different radii in the plume, one on channel centerline and the other on the edge of the plume. The temperature at intermediate points was to be estimated by fitting a line to the temperatures at the two points.

Several assumptions factor into this momentum analysis. The momentum calculation assumes that the velocity of the plasma is constant at the point of the measurement. It neglects any momentum the neutrals deliver to the thuster. It also assumes that no additional plasma is created downstream of the thruster. These assumptions, along with the large uncertainties in densities calculated from Langmuir probe theory, are likely to result in significant errors in the calculated momentum. Errors would exceed 50\% with at least $30 \%$ of the error due to uncertainty in the density measurement. The errors in the data were to be estimated using the method of partial derivatives [69], at each data point. As momentum measurements were never collected from the experiment (Chapter 7 shows the plasmoid did not translate from the coils), actual error estimates were not completed. 


\subsubsection{Efficiency Estimates}

The energy efficiency of the XOCOT-T3 was designed to be calculated by comparing the final kinetic energy in the axial direction to the stored energy in the capacitor. This estimate would include divergences losses or beam efficiency $\eta_{B}$ by measuring only the axial directed kinetic energy, but would not include propellant efficiency $\eta_{P}$

$$
\eta_{E}=\frac{m_{b i t} u_{z}^{2}}{C_{M B} V_{c}^{2}}
$$

The mass of exhausted propellant $m_{b i t}$ was to be calculated from the density map compiled from momentum measurements, described in Section 5.3.2, where

$$
m_{b i t}=\int\left(\frac{M_{m} 2 \pi}{N_{A}} \int n_{i}(r, t) r d r\right) d t
$$

The velocity $u_{z}$ was also designed to be calculated from the momentum measurements, using TOF array.

The error in estimated efficiency was predicted to be significant (at least $50 \%$ ), primarily due to the uncertainty in plasma density inherent with Langmuir probe measurements (30\%). The total error was to be calculated using the method of partial derivatives [69] to propagate the error due to each measured quantity. 


\subsubsection{Energy Analysis}

Coil currents were used to estimate the energy deposition into the plasma by comparing the difference between plasma and vacuum currents. Energy dissipated in the plasma was calculated by a simple energy balance: $E_{0}=E_{\text {circuit }}+E_{\text {plasma }}$. The input energy $E_{0}$ is the energy intially stored in the capacitor and the circuit energy $E_{\text {circuit }}$ is the energy dissipated by the circuit's ohmic heating $\int I^{2} R d t$. The circuit resistance $R$ was calculated from the vacuum current (the current in the circuit without a plasma load). The energy into the plasma was then calculated by:

$$
E_{\text {plasma }}=\frac{1}{2} C_{M B} V\left(t_{0}\right)^{2}-R \int_{t 0}^{t f}\left(I_{i}+I_{o}\right)^{2} d t-\frac{1}{2} C_{M B} V\left(t_{f}\right)^{2}
$$

In Equation 5.46, $I_{\text {inner }}$ and $I_{\text {outer }}$ are the currents through the coils with a plama load, $V\left(t_{0}\right)$ is the initial charge voltage on the capacitor, $C_{M B}$ is the main bank circuit capacitance, and $V\left(t_{f}\right)$ is the voltage remaining on the capacitor after the desired time interval. The energy dissipated by the plasma calculated by this method includes all losses (ionization, radiation, inelastic collisions, etc) and estimates the global energy deposition and losses. A more refined energy analysis requires more sophisticated measurement techniques and models.

Equation 5.46 can be rearranged to estimate the circuit resitance for the vacuum case, where $E_{\text {plasma }}=0$. This is a quick way to estimate the resistance $R$, required to calculate the energy into the plasma. 


$$
R=\frac{\frac{1}{2} C V\left(t_{0}\right)^{2}-\frac{1}{2} C V\left(t_{f}\right)^{2}}{\int_{t 0}^{t f}\left(I_{\text {inner }}+I_{\text {outer }}\right)^{2} d t}
$$

The error in $R$ and $E_{\text {plasma }}$ are largely dependent on the uncertainty in the charge voltage and integrated coil currents. The total error for each term was calculated using the method of partial derivatives [69] and is presented alongside the data in Chapter 6 . 


\section{Chapter 6}

\section{Experimental Data: Translation Study}

This chapter discusses the experimental data collected in this research, used for the translation analysis in Chapter 7. The full set of tested operating conditions is presented in Section 6.1. Operating conditions refer to static gas pressure, discharge energy, pre-ionization voltage, and timing between the pre-ionization and main bank trigger. Vacuum results are presented in Section 6.2. These results include coil current and magnetic field measurements without a plasma or gas fill. Results from plasma studies begin in Section 6.3, starting with a detailed discussion of how plasmoid formation can be inferred from current and magnetic field measurements. Section 6.3 also discusses the results from basic device characterization studies, including a limited pre-ionization study, optimal timing study, and the shot-to-shot repeatibility studies. Full data sets from all test

conditions are provided in Section 6.4. These data sets include measurements from the external and internal magnetic field probes and downstream magnetic field probes. Results from the plasma probes are also given, when available. 


\subsection{Test Conditions}

The XOCOT-T3 experiment was tested with both discharge circuits (10 kHz and $20 \mathrm{kHz}$ ) at 6 different fill pressures with various discharge energies. The fill pressure was adjusted to change the plasmoid mass. Discharge energy was changed to control the Lorentz force acting on the plasmoid. Both adjustments should affect the velocity and momentum results directly, in accordance with Newton's second law.

The XOCOT-T3 was designed for operation at two separate discharge frequencies: $10 \mathrm{kHz}$ and $20 \mathrm{kHz}$. The $10 \mathrm{kHz}$ version was tested from $100 \mathrm{~J}$ to $1 \mathrm{~kJ}$ of input energy, while the $20 \mathrm{kHz}$ version was tested at $100 \mathrm{~J}$. For each setting, the plasmoid was formed in a static background gas of argon with the fill pressure ranging from $1 \mathrm{mTorr}\left(3.2 \times 10^{19} \mathrm{H}_{-} \mathrm{m}^{-3}\right)$ to 50 mTorr $\left(1.61 \times 10^{21} \#-\mathrm{m}^{-3}\right)$. Table 6.1 summarizes the XOCOT-T3 test matrix.

Table 6.1

XOCOT-T3 test conditions

\begin{tabular}{|c|c|c|c|}
\hline & $100 \mathrm{~J}$ & $500 \mathrm{~J}$ & $1 \mathrm{~kJ}$ \\
\hline \multirow{6}{*}{$10 \mathrm{kHz}$} & 1 mTorr & $1 \mathrm{mTorr}$ & $1 \mathrm{mTorr}$ \\
\hline & 4 mTorr & 4 mTorr & 4 mTorr \\
\hline & 10 mTorr & 10 mTorr & 10 mTorr \\
\hline & 14 mTorr & 14 mTorr & 14 mTorr \\
\hline & 20 mTorr & 20 mTorr & 20 mTorr \\
\hline & 50 mTorr & 50 mTorr & 50 mTorr \\
\hline \multirow{6}{*}{$20 \mathrm{kHz}$} & $1 \mathrm{mTorr}$ & - & - \\
\hline & 4 mTorr & - & - \\
\hline & 10 mTorr & - & - \\
\hline & 14 mTorr & - & - \\
\hline & 20 mTorr & - & - \\
\hline & 50 mTorr & - & - \\
\hline
\end{tabular}


While the range of input energies for each circuit was largely chosen using the translation predictions in Section 4.2.7, it was modified after experimental testing. The translation predictions expected the plasmoid to translate out of the coils using $200 \mathrm{~J}$ to $500 \mathrm{~J}$ of input energy with the $225 \mu \mathrm{F}$ bank. However, during testing it was found that the maximum current of $30 \mathrm{kA}$ occurred at $1 \mathrm{~kJ}$ instead of $500 \mathrm{~J}$. The coil-plasmoid coupling predicted by the model was optimistic when compared with experimental results, leading to lower peak currents. For this reason, the maximum energy for the $225 \mu \mathrm{F}$ bank was elevated to $1 \mathrm{~kJ}$. The $43.5 \mu \mathrm{F}$ bank was expected to translate a plasma starting at $600 \mathrm{~J}$ of initial energy from model predictions. However, the circuit was only operated at $100 \mathrm{~J}$ of discharge energy to increase the lifetime of the capacitor. The decision to decrease the minimum energy for the $43.5 \mu \mathrm{F}$ circuit was based off of failures with the $225 \mu \mathrm{F}$ capacitor. During high energy testing, the $225 \mu \mathrm{F}$ capacitor was damaged internally from the large voltage reversals at peak current. Testing of the $43.5 \mu \mathrm{F}$ capacitor at $600 \mathrm{~J}$ likely would have resulted in the same internal damage as the current load was expected to be $30 \mathrm{kA}$ with higher discharge voltages of $5.25 \mathrm{kV}$. For this reason, the total energy for the circuit was derated. Operating at reduced energy was unlikey to produce a translating plasmoid, however it was still sufficient for studies of plasmoid behavior.

The range of fill pressures was selected to minimize the amount of mass the discharge circuit would have to expel from the coils. Since plasmoid formation is likely dependent on the initial neutral gas density, a range of parameters was selected across an order of magnitude. The higher density fills were not expected to result in translation, but were included in the test matrix in the event they resulted in a more stable plasmoid.

Additional settings for the XOCOT-T3 included the pre-ionization energy and the delay between the pre-ionization bank trigger and the main bank trigger. The pre-ionization 
energy was set to $2.4 \mathrm{~J}$ (12.5 $\mathrm{kV}$ charge voltage) following the study results detailed in Section 6.3.2. The main bank delay was fixed to $10 \mu \mathrm{s}$, based on energy deposition into the plasma and level of field reversal as seen by the inner coil probes. Further details on the selection of the main bank timing delay are available in Section 6.3.3.

\subsection{Vacuum Characterization}

The XOCOT-T3 experiment was tested without plasma to determine the total coil currents and vacuum magnetic fields as a function of discharge energy. At least 10 shots were conducted at each nominal energy setting (consult Table 6.1) and the processed data for each probe was averaged to compute an average waveform. The statistical spread between shots was calculated and combined with the experimental error to determine the total error in each result. Results for the $10 \mathrm{kHz}$ circuit are provided in Section 6.2.1, and the $20 \mathrm{kHz}$ circuit results are in Section 6.2.2.

\subsubsection{Vacuum Data: $10 \mathrm{kHz}$}

A current waveform of the coil currents at $500 \mathrm{~J}$ for the $10 \mathrm{kHz}$ circuit is shown in Figure 6.1. The waveform shows that the circuit rings at $9.5 \mathrm{kHz}$ with $26 \%$ of the current passing through the outer coil. The waveforms for the $100 \mathrm{~J}$ and $1 \mathrm{~kJ}$ cases are similar to the data shown in Figure 6.1, with different amplitudes. Raw b-dot traces and processed magnetic field data for the $500 \mathrm{~J}, 10 \mathrm{kHz}$ vacuum circuit are available in Figure A.1 (in Appendix A).

The average coil currents as a function of discharge energy were compiled by averaging the 


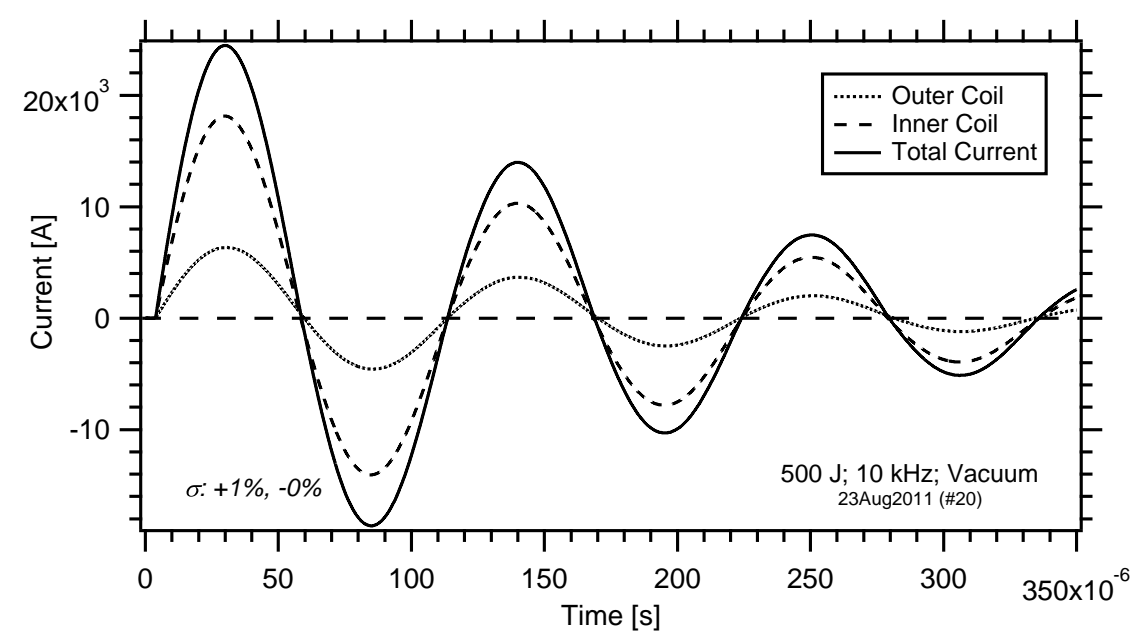

Figure 6.1: Coil currents as a function of time for the vacuum shot using the $10 \mathrm{kHz}$ bank.

Table 6.2

XOCOT-T3 vacuum coil currents as a function of energy for the $10 \mathrm{kHz}$ bank.

\begin{tabular}{lcrrr}
\hline Energy & $V_{\text {cap }}$ & $I_{\text {total }}$ & $I_{\text {outer }}$ & $I_{\text {inner }}$ \\
{$[\mathrm{J}]$} & {$[\mathrm{V}]$} & {$[A]$} & {$[A]$} & {$[A]$} \\
\hline $100 \mathrm{~J}$ & $942 \pm 4.2 \mathrm{~V}$ & $10,575+128$ & $2,740+33$ & $7,835+95$ \\
& & -72 & -18 & -53 \\
$500 \mathrm{~J}$ & $2108 \pm 7.6 \mathrm{~V}$ & $24,507+275$ & $6,340+73$ & $18,167+202$ \\
& & -124 & -35 & -89 \\
$1000 \mathrm{~J}$ & $2981 \pm 10.4 \mathrm{~V}$ & $33,736+505$ & $8,772+132$ & $24,964+374$ \\
& & -376 & -98 & -279 \\
\hline
\end{tabular}

current data at least 10 shots at each major energy setting (100 J, $500 \mathrm{~J}, 1 \mathrm{~kJ})$. Five shots at intermediate energy settings were also processed. The average waveform at $500 \mathrm{~J}$ is shown in Figure 6.2 $\mathrm{a}$. The peak average coil currents as a function of discharge energy and coil voltage follow in Figure $6.2 \mathrm{p}$ and Figure 6.2k. The average peak coil current at each energy is tabulated in Table 6.2. Total errors for the average current are quite small (1\%) and have been omitted from Figure 6.2 for clarity.

Figure 6.2 shows that the current throughput is proportional to the square root of the 
(a)

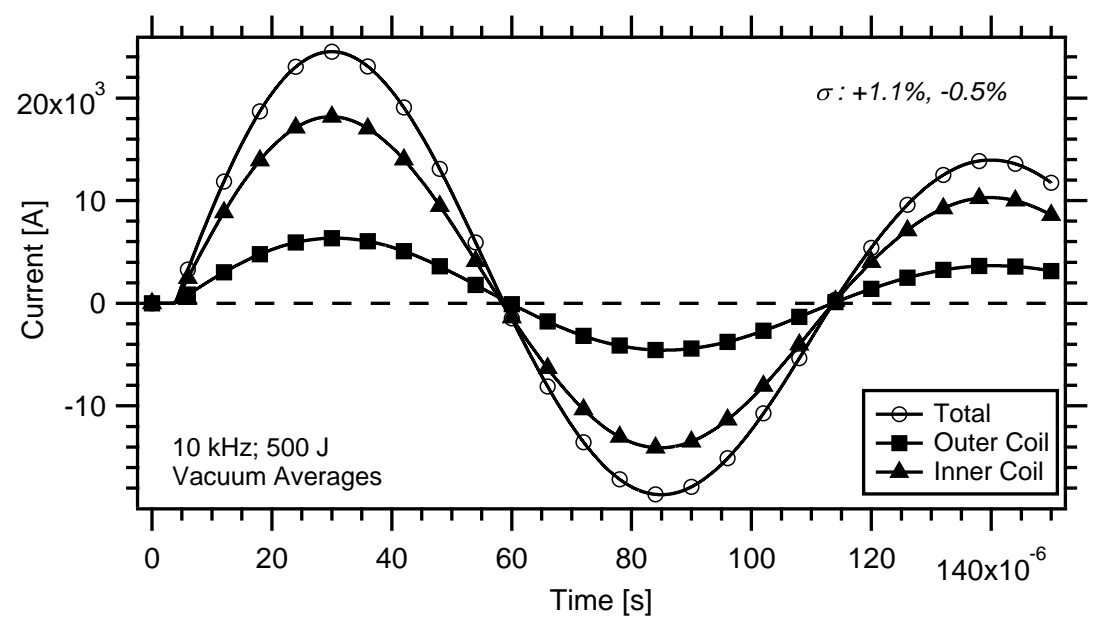

(b)
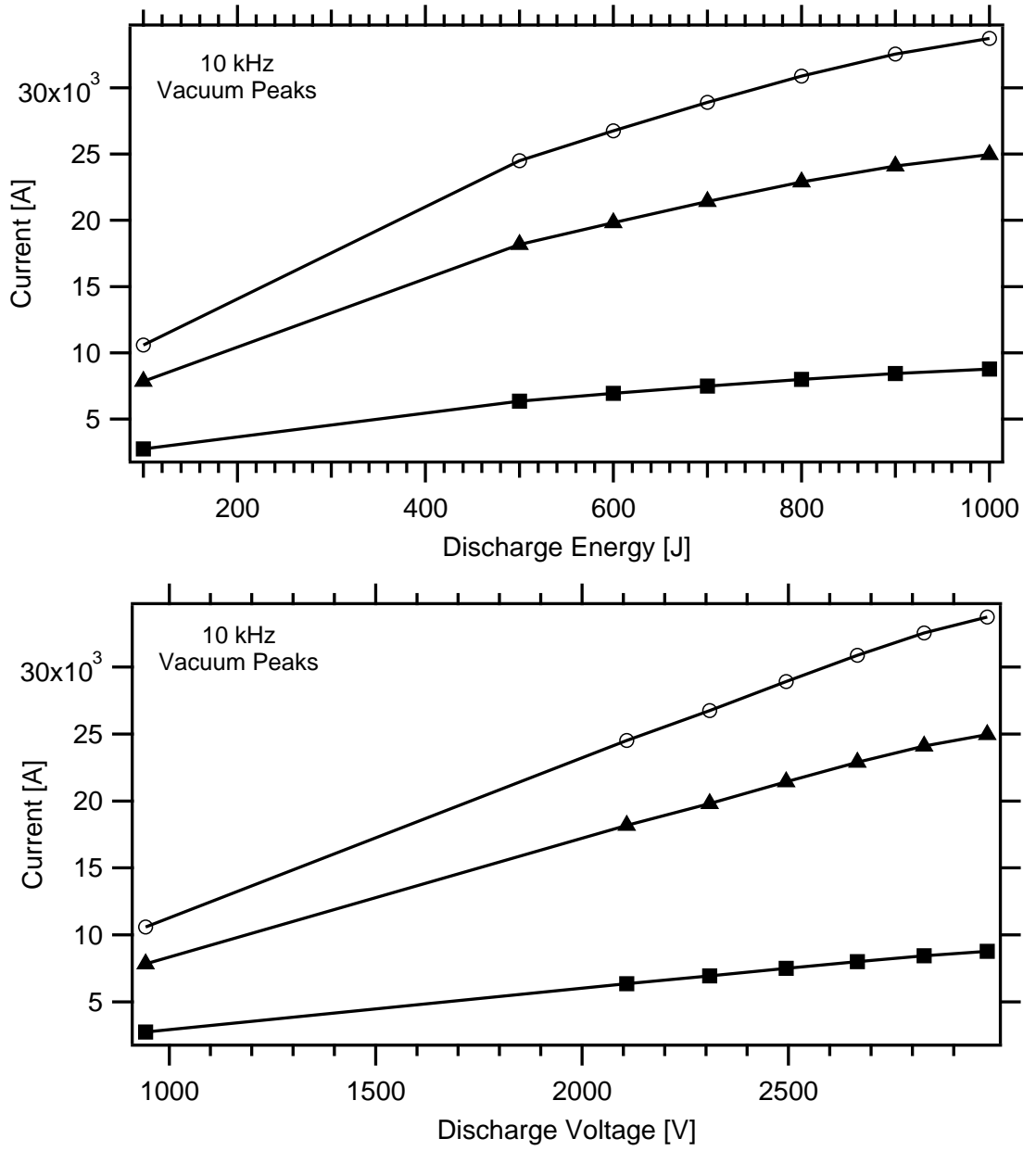

Figure 6.2: Vacuum coil current data for the $10 \mathrm{kHz}$ bank, including (a) average waveforms for the $500 \mathrm{~J}$ setting, (b) peak currents versus energy, and (c) peak currents versus discharge voltage. 
discharge energy, as expected. The spread in the data is small for the $100 \mathrm{~J}$ and $500 \mathrm{~J}$ case $(0.6 \%)$, indicating very repeatible coil currents despite manual triggering. The deviation in the vacuum data for $1 \mathrm{~kJ}$ is moderately larger $(1.5 \%)$, resulting from degradation of the capacitor. The capacitor for the $10 \mathrm{kHz}$ circuit was fault-limited to a voltage-reversal of $80 \%$ and $30 \mathrm{kA}$. Operating this bank at $1 \mathrm{~kJ}$ of input energy was at the maximum limit for both ratings and likely damaged the capacitor. The data from these conditions is still useable, at the expense of larger shot-to-shot deviations.

The average vacuum magnetic fields at each probe location were also calculated. The resulting waveform averages for the magnetic field at the coil midplane at each energy setting are shown in Figure $6.3 \mathrm{a}$ and $\mathrm{b}$. The probe locations correspond to the outer and inner surfaces of the annulus. The magnetic field along the discharge coil at $30.5 \mu \mathrm{s}$ is displayed in Figure 6.3 for the $500 \mathrm{~J}$ case. Magnetic fields calculated using COMSOL, scaled from the results presented in Section 4.3, are displayed as well. The magnetic field measured by the probes is within $10 \%$ of the fields predicted by COMSOL.

From these plots, it is readily apparent that the vacuum field inside the annulus is in a single direction and magnitude of the magnetic field near the inner coil is only slightly lower than near the outer coil. As expected, the vacuum magnetic field increases monotonically with current and follows the time-behavior of the current. These plots also confirm that the axial vacuum magnetic field is not uniform along the coil, as predicted by the electromagnetic field simulations in Section 4.3 . The non-uniformity was discovered in COMSOL to be an artifact of the multi-turn coils and this finding is substantiated by the experimental data. The shot-to-shot deviation in the magnetic field results is similar to the current data (1-2\%). The majority of the experimental error is due to the uncertainty in the probes' proportionality constants. The average vacuum traces are shown with the plasma shot data in the remaining 
(A)

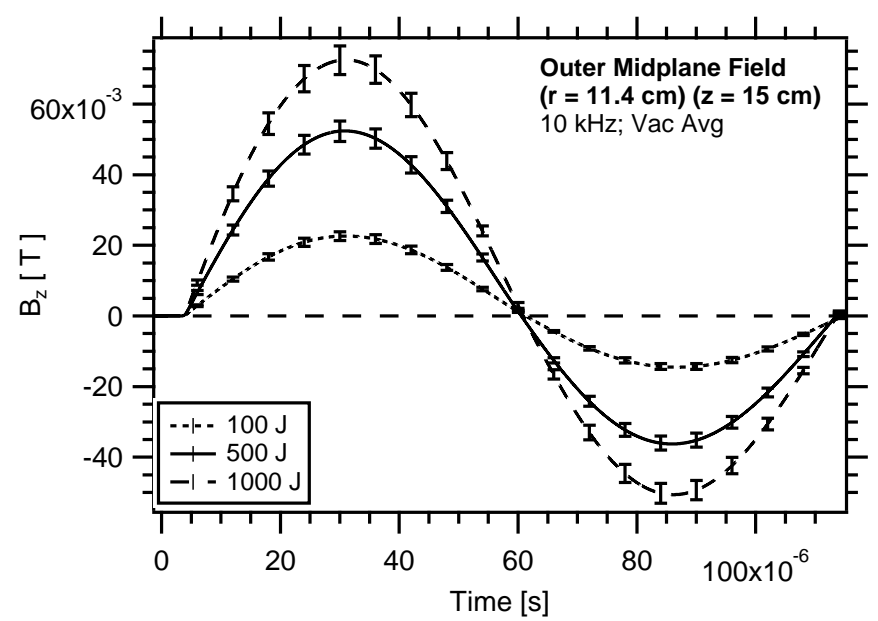

(B)

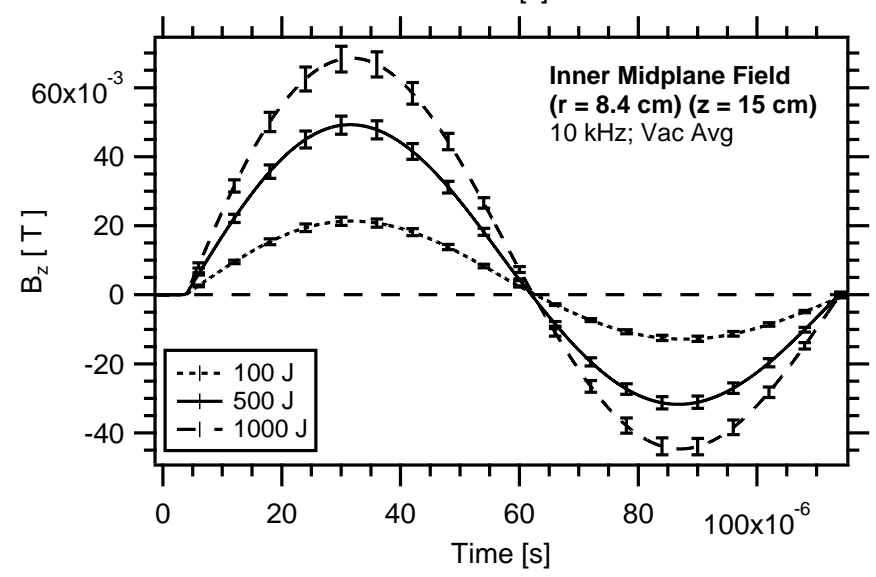

(C)

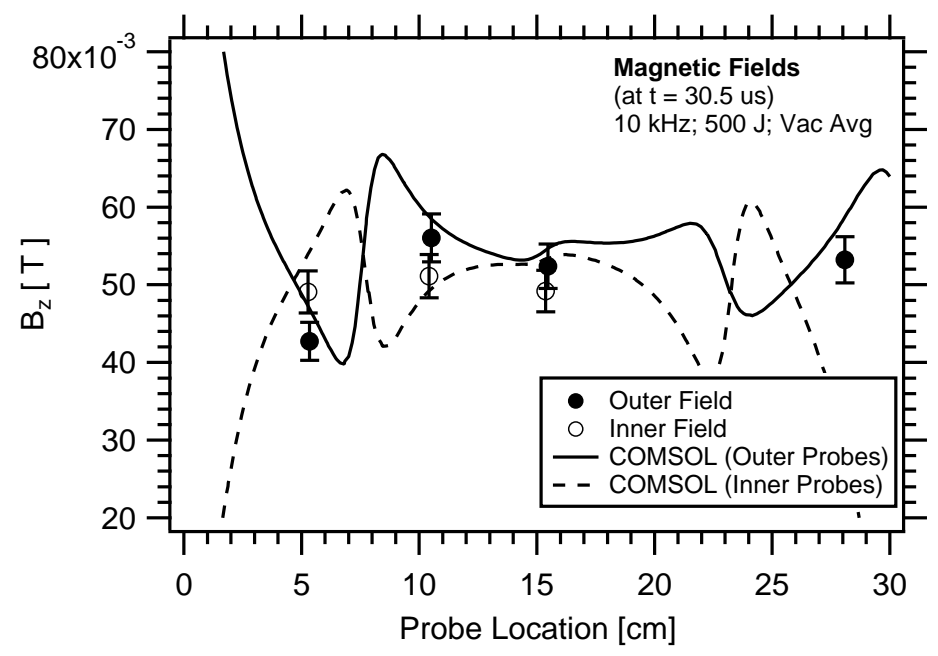

Figure 6.3: XOCOT-T3 vacuum magnetic fields as a function of discharge energy for the $10 \mathrm{kHz}$ circuit. 
sections of this Chapter for comparison.

The downstream magnetic fields were also measured during the vacuum shots. The data was collected using a commerical 1-MHz differential amplifier ( $\mathrm{p} / \mathrm{n}$ Tektronix ADA400A), as discussed in Section 5.2.2.8. The amplifier was required due to the poor signal-noise resolution of the downstream probes. Several shots were also conducted without the amplifier, connecting both probes to the oscilloscope directly with $50 \Omega$ terminations. A comparison of the data obtained from these techniques is shown in Figure 6.4, including the raw signals and processed data. Figure 6.4 shows that the amplifier has difficulty measuring the differential voltage correctly during the noise burst at $\mathrm{t}=4 \mu \mathrm{s}$, leading to an artificial sharp peak in the differential amplifier signal. This imperfection results in errors in the calculated magnitude of the magnetic field, as it contributes a non-physical offset. However, it does not affect the shape of the waveform several timesteps past the glitch. Since the purpose of the downstream probes was to measure the time-history of the translating plasma and not necessarily the magnetic field magnitude, the integrated signals can be peak-normalized. This removes the magnitude error far from the glitch event and retains the necessary time-history information required to resolve the plasmoid's velocity. While the normalization process may seem unnecessary for the relatively small errors in the calculated vacuum field, the introduction of plasma noise into the signal worsens this effect by over $20 \%$.

The peak-normalized downstream magnetic field data was averaged across all available shots to generate the typical waveform at each downstream probe location. The resulting traces are displayed in Figure 6.5, with a sketch of the total current. It is apparent that the downstream probes are not in phase with the current or with the upstream probes. The first upstream probe peaks at $41.04 \mu$ s and the second probe peaks at $50.46 \mu$ s, while the current 

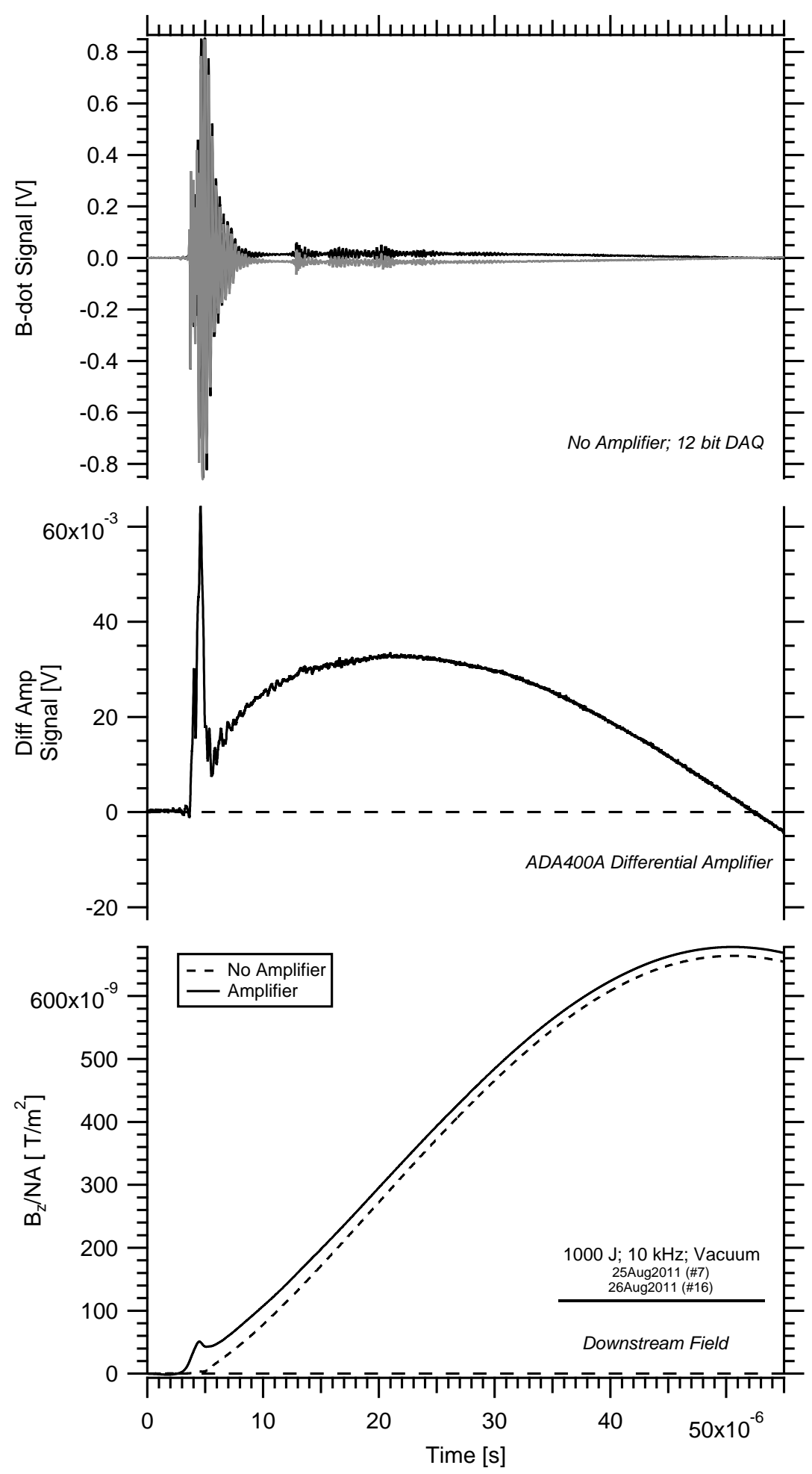

Figure 6.4: Downstream magnetic field signals measured with and without a differential amplfier. 
peaks much sooner at $30.27 \mu \mathrm{s}$. This delay is likely due to finite magnetic-soakthrough time of the vacuum facility; the magnetic diffusion time for a 1/4 inch plate of stainless steel is around $70 \mu \mathrm{s}$. Since the downstream field is a superposition of the field from the coils and the diffused field crossing the tank boundary, it can appear to lag from the coil field. The difference in delay times can be explained by noting that the magnetic diffusion time-constant is proportional to the square of the material thickness. An extra 1/8 inch of metal would double the delay. The probes are located in different areas of the tank and the variation in geometry may be enough to change the relative delay between the probes. Regardless, this delay will not impact the measurements of the translating plasmoid, as translation measurements occur local to the probe.

\subsubsection{Vacuum Data: $20 \mathrm{kHz}$}

The current data from the $20 \mathrm{kHz}$ circuit was averaged over 10 or more vacumm shots to calculate the mean current for each coil for the $100 \mathrm{~J}$ case. The resulting waveform at 100 $\mathrm{J}$ for the $20 \mathrm{kHz}$ circuit is shown in Figure 6.6. Peak coil currents as a function of input energy is shown in Figure 6.6p, scaled in Figure 6.6 against discharge energy. Table 6.3 displays the peak average current for the $50 \mathrm{~J}$ and $100 \mathrm{~J}$ cases, along with the total error or combined shot-to-shot deviation and exerimental error. Single-shot data for a $100 \mathrm{~J}$ test is available in Appendix A, Figure A.2.

The current has start delay of $3 \mu \mathrm{s}$, resulting from a finite switch closing time. The circuit rings through 2 complete cycles with a measured frequency of $19.5 \mathrm{kHz}$. The coil current ratios remain identical to the $10 \mathrm{kHz}$ bank, with $26 \%$ of the current passing though the outer coil. The $100 \mathrm{~J}, 20 \mathrm{kHz}$ bank produces similar current levels to the $100 \mathrm{~J}, 10 \mathrm{kHz}$ bank, at 

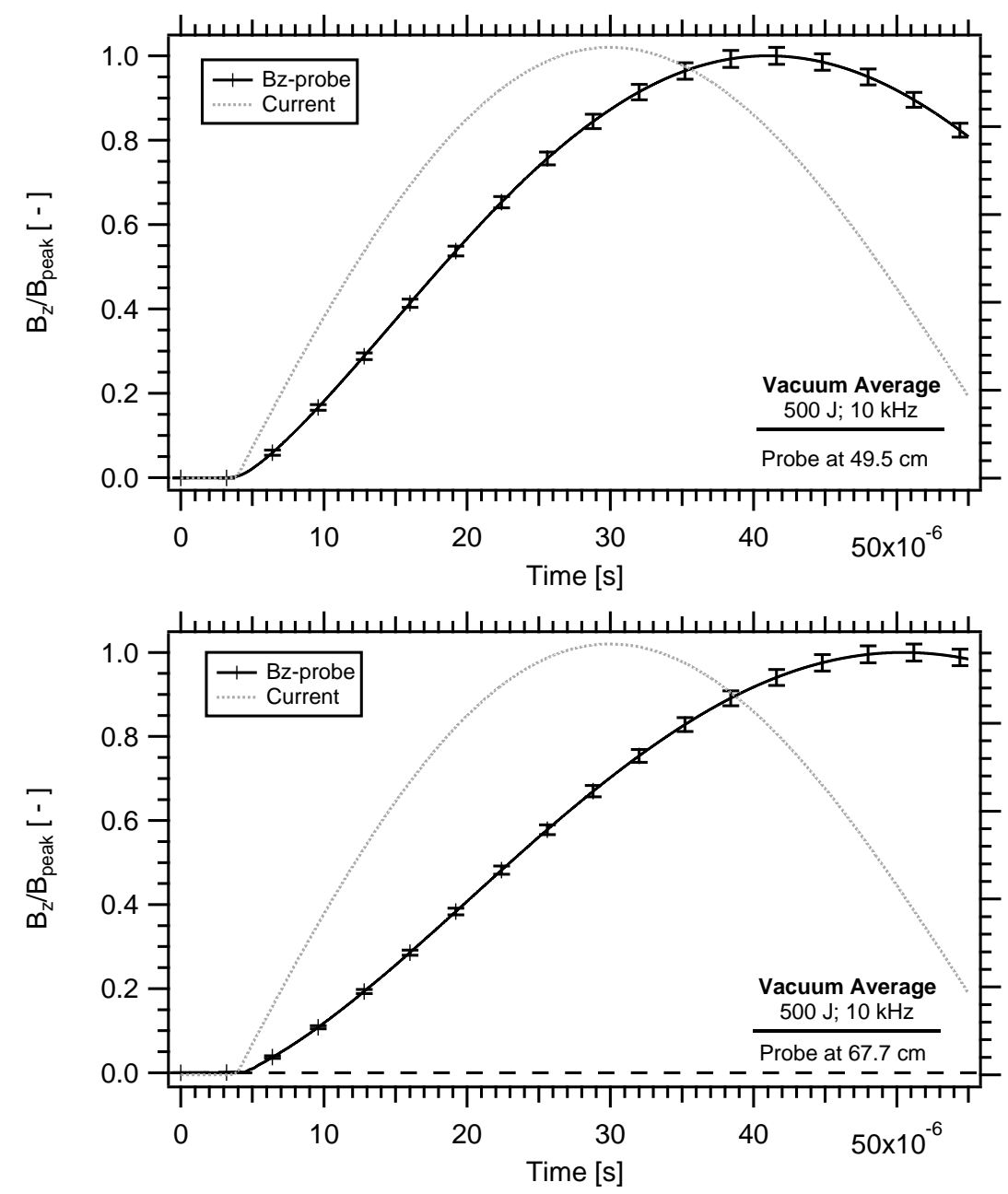

Figure 6.5: TOF peak-normalized vacuum magnetic field traces for the $10 \mathrm{kHz}$ circuit, including average magnetic field waveforms for the (A) upstream probe and the (B) downstream probe. The coil current waveform is shown for comparison.

Table 6.3

XOCOT-T3 vacuum coil currents as a function of energy and charge voltage for the $20 \mathrm{kHz}$ circuit.

\begin{tabular}{lcrrr}
\hline Energy & $V_{\text {cap }}$ & $I_{\text {total }}$ & $I_{\text {Outer }}$ & $I_{\text {inner }}$ \\
{$[\mathrm{J}]$} & {$[V]$} & {$[A]$} & {$[A]$} & {$[A]$} \\
\hline $100 \mathrm{~J}$ & 2144 & $10751+115$ & $2816+30$ & $7936+85$ \\
& & -41 & -10 & -31 \\
\hline
\end{tabular}


the expense of higher coil and circuit voltages. The total experimental error for the peak currents is very small for both energy settings and is comprised mostly of the experimental uncertainty in the current measurement $(+1 \%)$. Shot-to-shot deviations are less than $0.5 \%$.

The magnetic fields across the entire coil length and the vacuum magnetic flux at coil midplane were also measured at vacuum conditions, at every probe location. The average magnetic field and flux at $100 \mathrm{~J}$ is shown in Figure 6.7. The midplane waveforms for the first complete cycle near the outer coil are shown in Figure 6.7a. Figure 6.7b displays the midplane magnetic field data near the inner coil. The magnetic fields along the coil at peak current are shown in Figure 6.7c, with the predicted magnetic fields from COMSOL. Uncertainty in each measurement is a combination of the statistical error between shots and the experimental error in each measurement.

The vacuum magnetic field for the $20 \mathrm{kHz}$ case is similar to the $10 \mathrm{kHz}$ case, with the magnetic field in the annulus in a single direction and following the time history of the coil currents. Peak vacuum magnetic fields are approximately $20 \mathrm{mT}$ for each midplane probe at $100 \mathrm{~J}$. From Figure 6.7c, it is apparent that the magnetic field along the coil length is not uniform. This is an artifact of the segmented multi-turn coil design. As discussed in Section 4.3, this design increases the current density at the edges of each turn resulting in magnetic field disturbances along the coil length. The shot-to-shot deviation in the magnetic fields was calculated to be small, less than $0.5 \%$. This means that the total uncertainty in each measurement is primarily a result of the uncertainty in the measurement (5-6\%). The COMSOL predictions agree with the experimental data within $10 \%$, showing with more clarity the axial distortions in the field caused by the multi-turn geometry.

The TOF vacuum magnetic field for the $20 \mathrm{kHz}$ case is shown in Figure 6.8, for the probes at $\mathrm{z}=49.5 \mathrm{~cm}$ and $\mathrm{z}=67.7 \mathrm{~cm}$. The data is shown for the vacuum averages at $100 \mathrm{~J}$, 
(a)

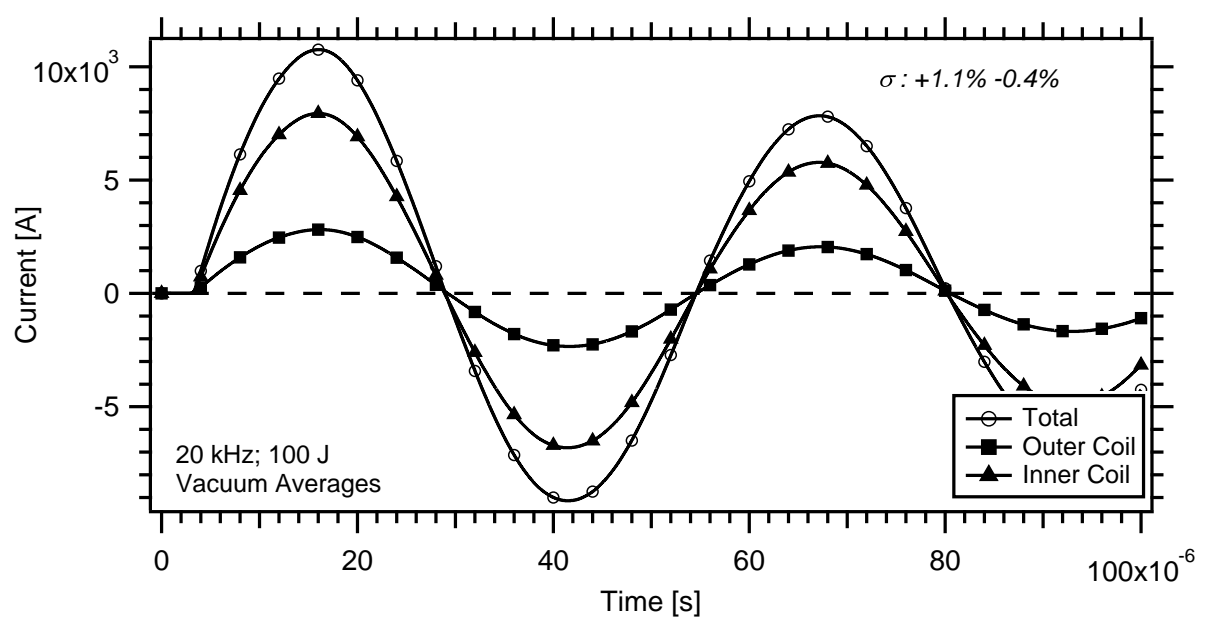

(b)

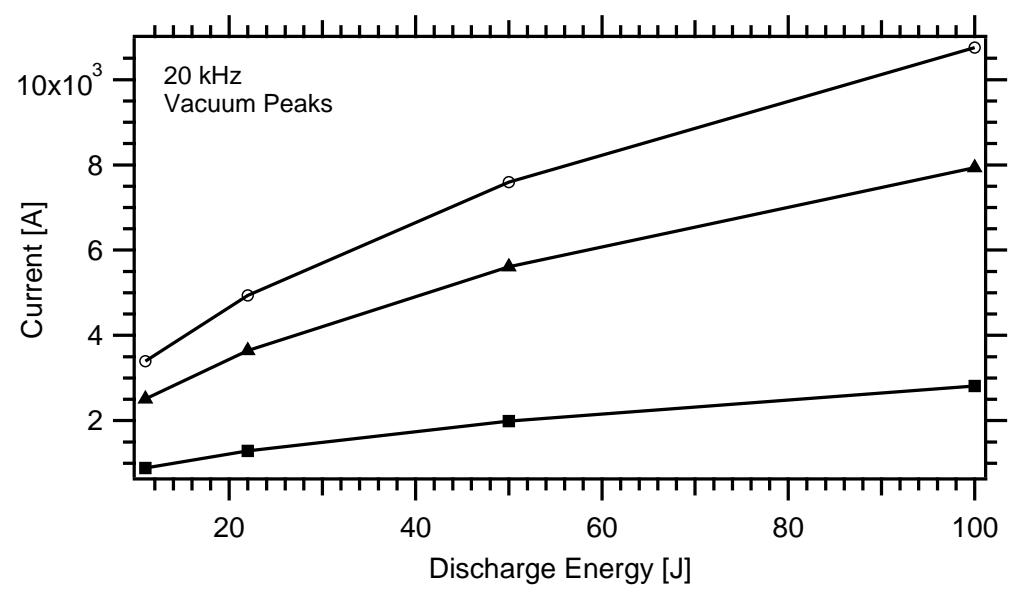

(c)

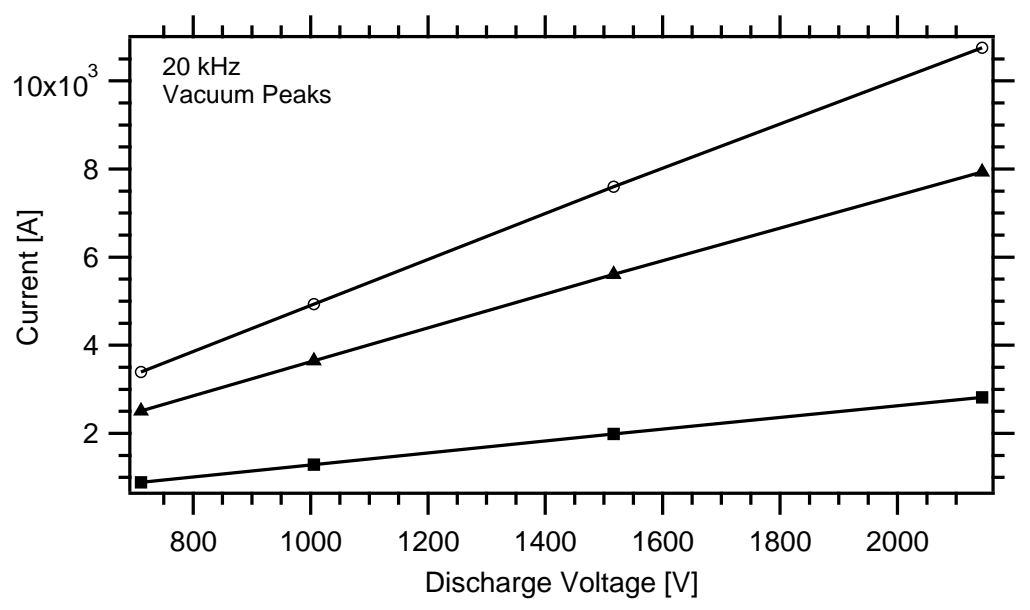

Figure 6.6: Vacuum coil currents for the $20 \mathrm{kHz}$ circuit, including (A) the time history of the coil currents, (B) peak coil currents compared to input energy, and (C) peak currents versus discharge voltage. Uncertainty for all measurements is $1 \%$. 
(A)

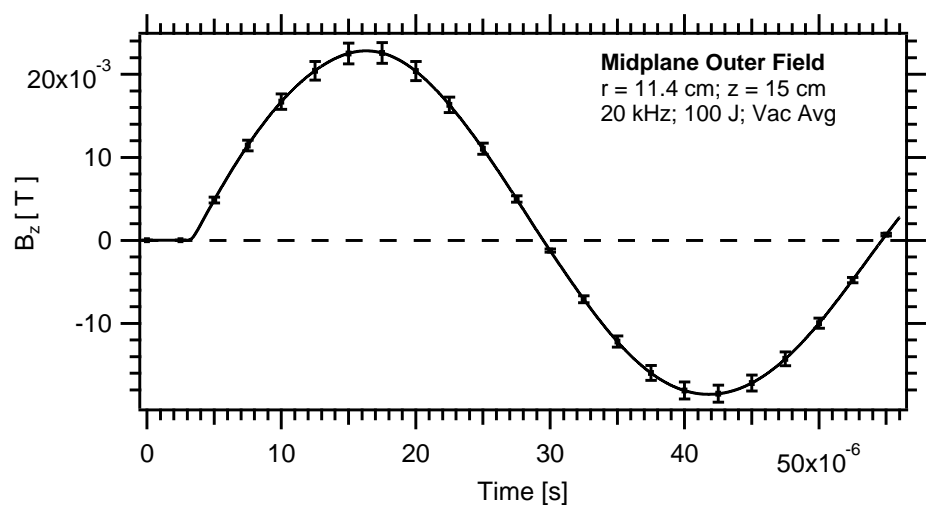

(B)

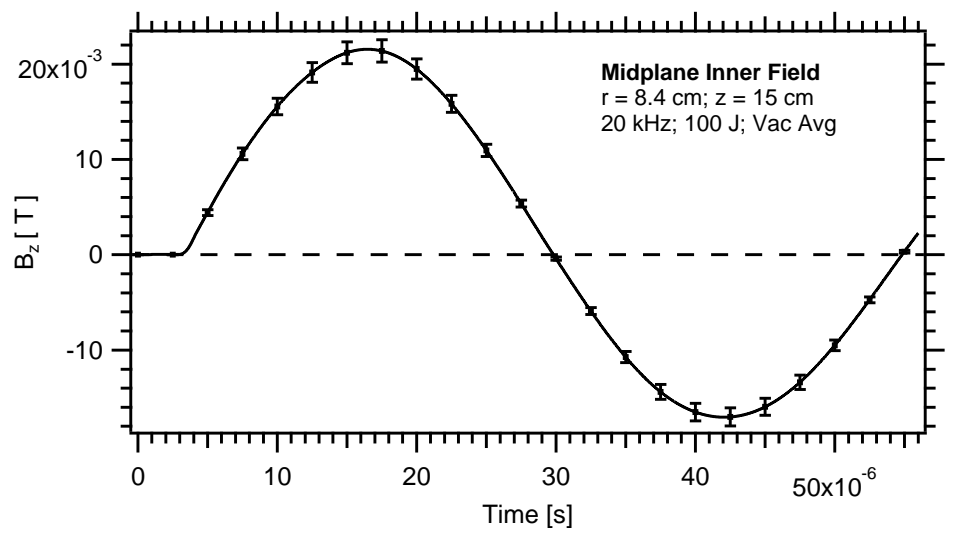

(C)

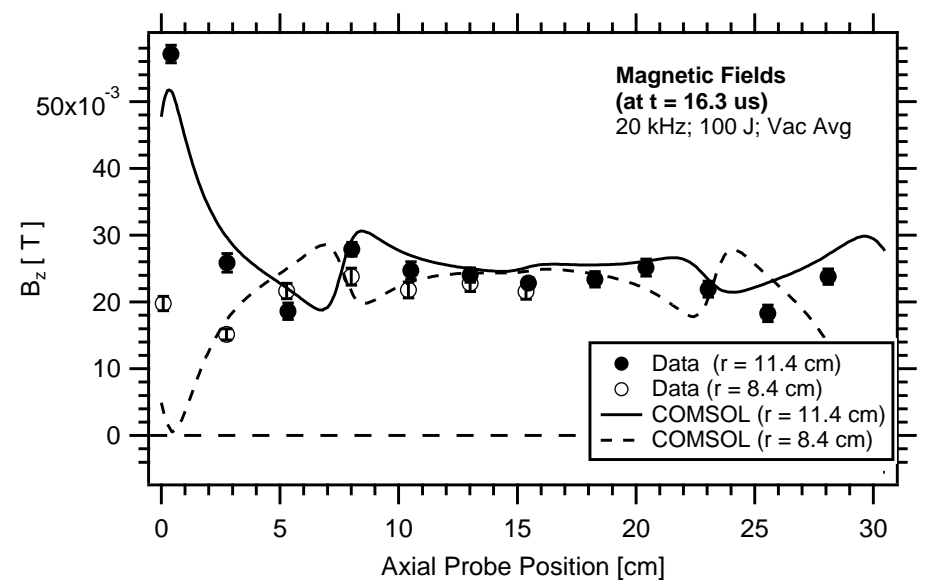

Figure 6.7: Vacuum magnetic fields for the $20 \mathrm{kHz}$ circuit, including average magnetic field waveforms at the coil midplane on the outer wall (A), the inner wall (B), and the average magnetic field at peak coil current along the coil length $(\mathrm{C})$. 
(A)

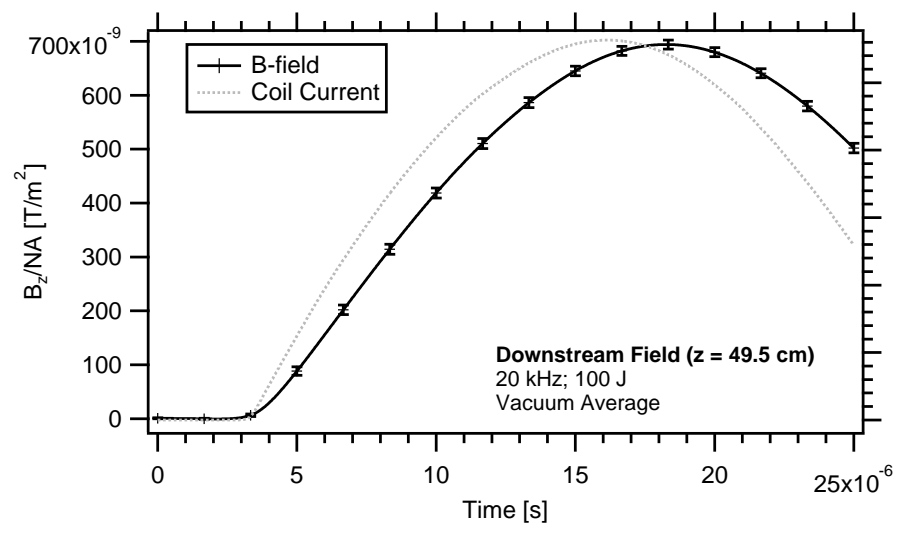

(B)

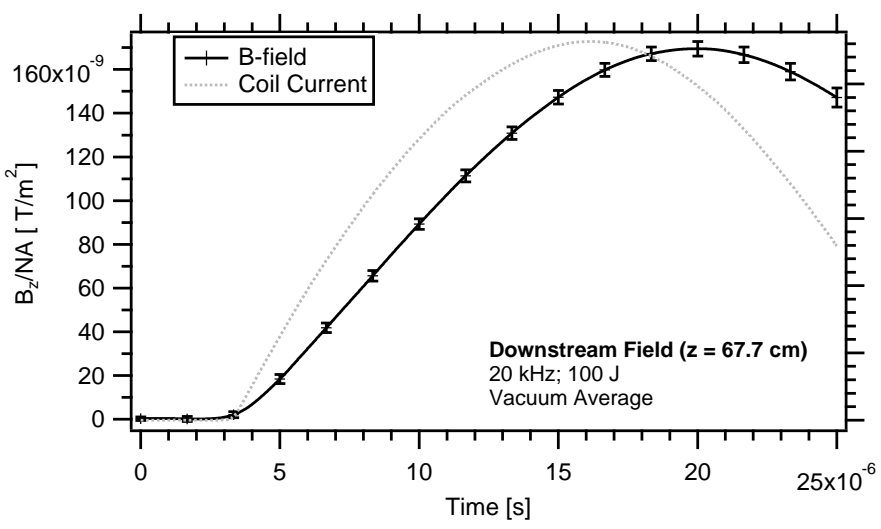

Figure 6.8: TOF vacuum magnetic fields for the $20 \mathrm{kHz}$ circuit, including average magnetic field waveforms for the (A) upstream probe and the (B) downstream probe. The coil current waveform is also shown.

scaled against each probe's proportionality constant $N A$. The statistical spread in the TOF magnetic field data is comparable to the data from inside the coils, with a $0.4 \%$ deviation in the upstream TOF probe and a 1.7\% spread for the downstream TOF probe. The magnetic field measured by both TOF probes is significantly delayed from the coil currents. The upstream TOF probe lags the coil current by $1.8 \mu$ s and the downstream TOF probe lags by 3.6 $\mu$ s. As mentioned in Section 6.2.1, this is likely an artifact of the finite magnetic field diffusion through the stainless steel tank boundary. 


\subsection{Plasmoid Formation Characterization}

The first step towards plasmoid translation is to create a fully reversed plasmoid inside the annulus. Plasmoid formation in the XOCOT-T3 experiment was tested by discharging the coils into a gas fill to create the magnetized AFRC toroid. Results demonstrating plasmoid formation are presented in Section 6.3.1.

Plasmoid formation can be enhanced by optimizing the pre-ionization settings, including the pre-ionization input energy and the time interval between the PI and the main bank trigger. Optimization of the PI pulse is discussed in Section 6.3.2. Timing results are discussed in Section 6.3.3.

The XOCOT-T3 is a pulsed experiment and the data can vary from shot-to-shot. Repeatibility results are presented in Section 6.3.4 to establish that while small deviations between shots occur, the overall behavior of the XOCOT-T3 and AFRC experiment are highly repeatable.

\subsubsection{Formation Results}

The XOCOT-T3 experiment was tested at $10 \mathrm{kHz}$ and $20 \mathrm{kHz}$, across the range of energies and fill pressures shown in Table 6.1. At each setting, AFRC plasmoids were formed between the coils. A single data set from the $10 \mathrm{kHz}$ circuit and the $20 \mathrm{kHz}$ circuit are

presented in this subsection to discuss the key pieces of evidence indicating plasmoid formation. Full results for each discharge energy at all background pressure are presented 
in Section 6.4 .

Coil current, midplane magnetic field, and photometer data is shown for a single shot with the $10 \mathrm{kHz}$ circuit in Figure 6.9 and with the $20 \mathrm{kHz}$ circuit in Figure 6.10. A full time-history is shown, through several discharge cycles. Vacuum traces are provided for comparison. Raw data is included in Appendix A, Figures A.3 and A.4.

Evidence of plasmoid formation requires a magnetic field reversal on the inner wall and inductive coupling in the coil currents. This indicates that current in the plasma is large enough to reverse the magnetic field from the coils and to magnetically couple with the coils. While some discharge circuits are constructed to only produce a single plasmoid, the ringing of the XOCOT-T3 circuit produces several plasmoids through the duration of the discharge. This occurs at each $\mathrm{dI} / \mathrm{dt}$ event, where a reversed magnetic field is seen on the inner wall along with a change in coil currents. This also corresponds to a peak in light output measured from the downstream photometer, signifiying an intense burst of ionization. In the data shown in Figure 6.9 and 6.10, it can be observed that 5 AFRCs are formed with diminishing intensities. The actual number of AFRCs formed during any given discharge is dependant on the input energy, pressure, and circuit frequency.

The data also shows that plasmoid formation sinks energy from the main bank circuit. This is most readily seen in the current data for the $20 \mathrm{kHz}$ circuit (top graph of Figure 6.10). The vacuum circuit rings through 4 complete cycles, dropping to 2.5 during a plasma shot. This behavior is expected and is a qualitative indication of the energy absorbed during the formation process. Ideally the bulk of the energy should be deposited in the first quarter cycle and should be carried downstream by the ejected plasmoid. Inefficiencies in the circuit and a lossy or ineffective translation will leave some remaining energy in the formation chamber for the subsequent pulses. 
(a)

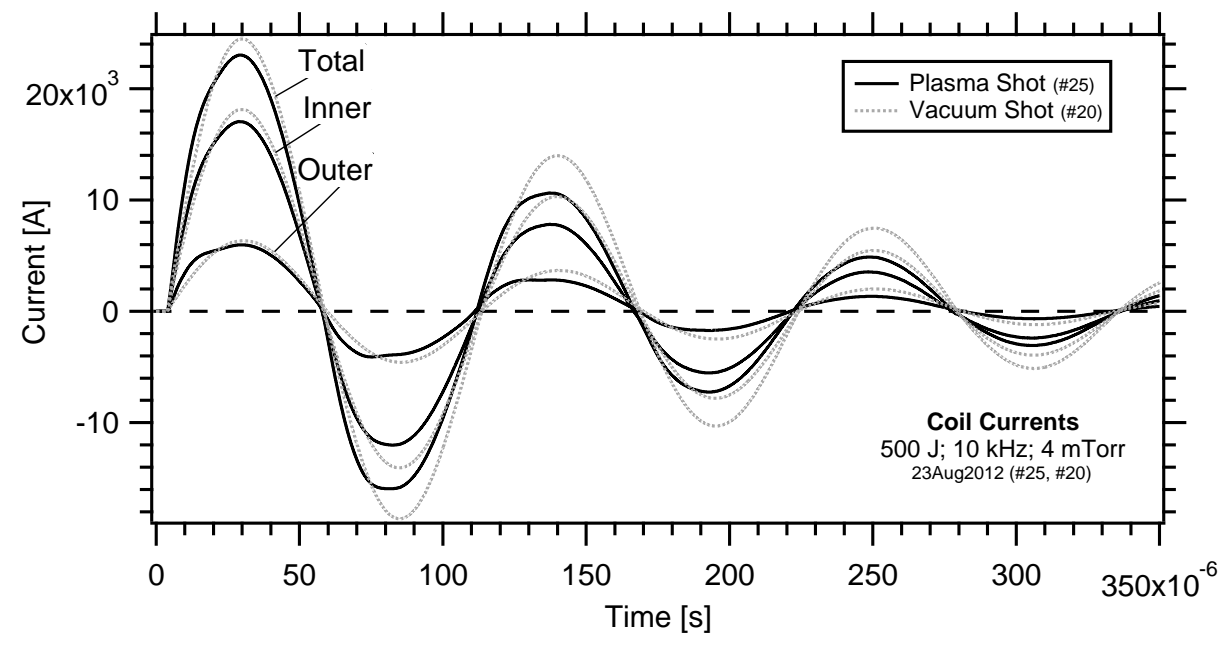

(b)
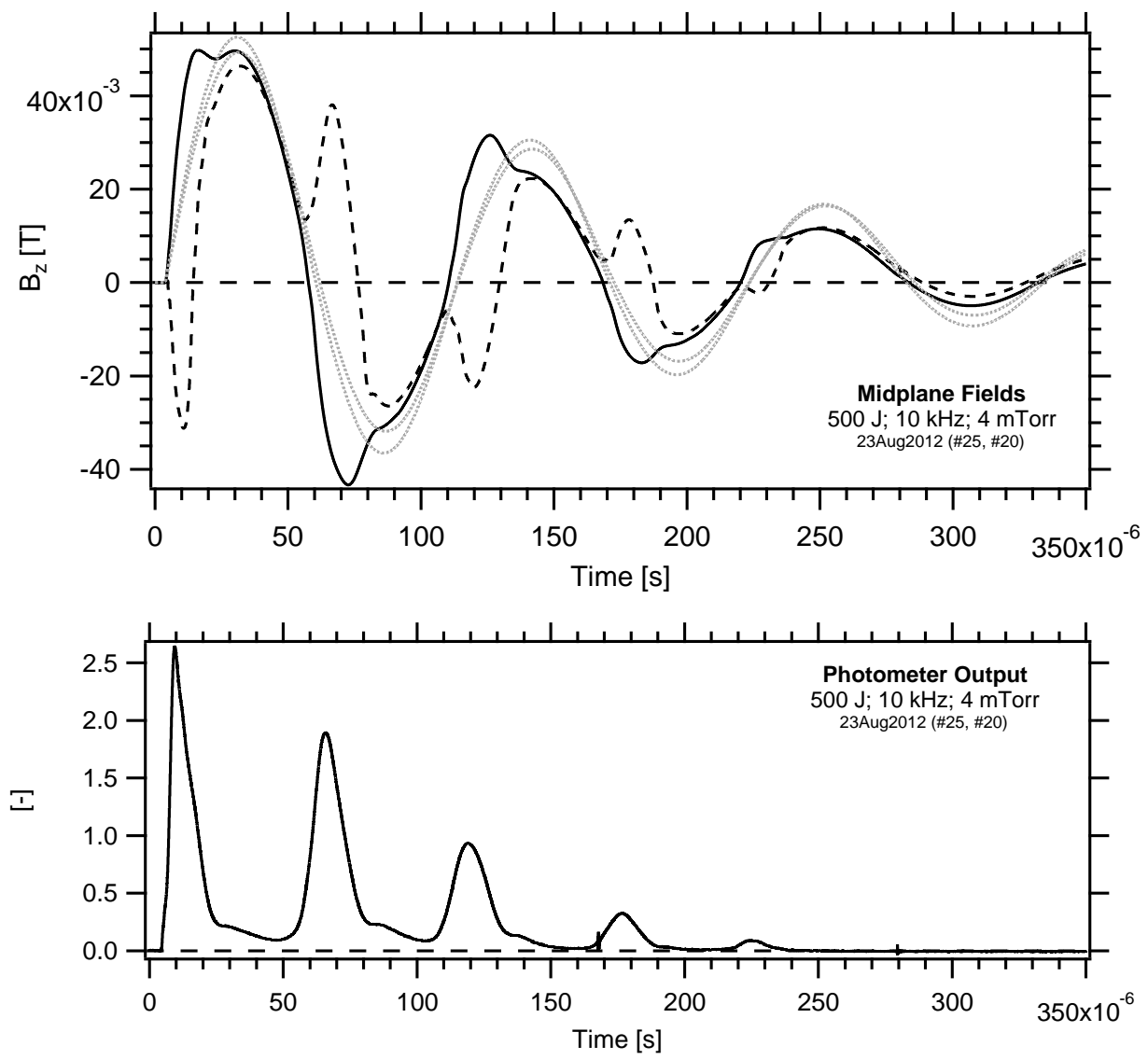

Figure 6.9: Typical plasmoid formation data for the $10 \mathrm{kHz}$ circuit at 500 $\mathrm{J}$ with a $4 \mathrm{mTorr}$ fill. A full time-history is shown with vacuum and plasma data for the main bank current, midplane magnetic fields, and light output measured by a photometer. 
(a)

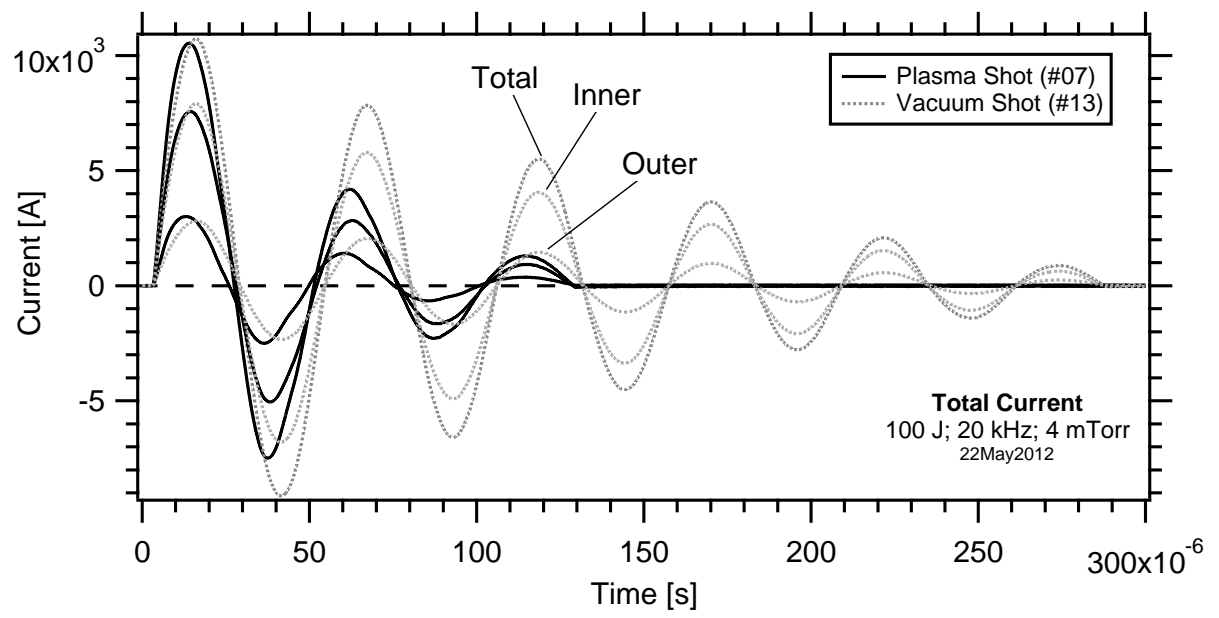

(b)

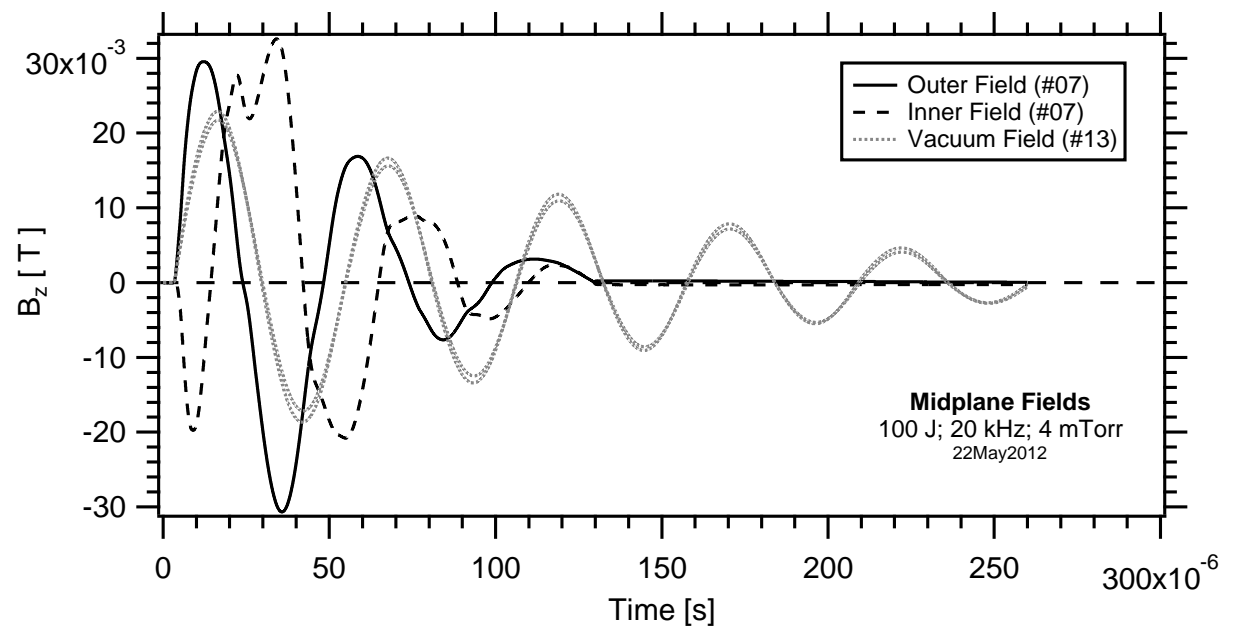

(c)

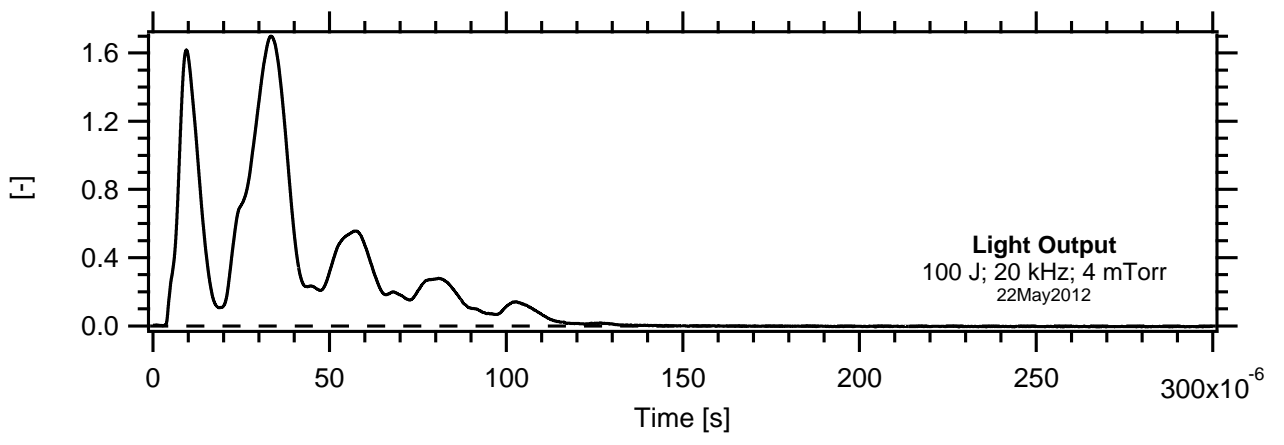

Figure 6.10: Typical plasmoid formation data for the $20 \mathrm{kHz}$ circuit at 100 $\mathrm{J}$ with a $4 \mathrm{mTorr}$ fill. A full time-history is shown with vacuum and plasma data for the (A) main bank current, (B), midplane magnetic fields, and (C) light output measured by a photometer. 
It is interesting to note that while the higher frequency bank is operated at $1 / 5$ of the energy of the $10 \mathrm{kHz}$ bank, plasmoid formation occurs on the same timescale. The field reversal in the $20 \mathrm{kHz}, 100 \mathrm{~J}$ pulse occurs within $1.1 \mu \mathrm{s}$ after the switch closes; the $500 \mathrm{~J}, 10 \mathrm{kHz}$ pulse reverses somewhere between $800 \mathrm{~ns}$ and $1.4 \mu \mathrm{s}$. Both circuits have nearly identical voltages so these findings are somewhat expected. It is well known that coil voltage (proportional to $\mathrm{dI} / \mathrm{dt}$ ) is responsible for ionization in pulsed devices. Therefore, one would expect matching coil voltages would produce similar results. Additionally, the plasmoid formation sinks much more energy in the $20 \mathrm{kHz}$ bank than the $10 \mathrm{kHz}$ bank. This finding is also not surprising; ionization improves with increased frequency. The $20 \mathrm{kHz}$ bank should be more effective at ionization than the $10 \mathrm{kHz}$ bank for matched coil voltages. While a full study on the effects of frequency and coil voltage on AFRC formation are beyond the scope of this research, these findings provide an interesting design note for future experiments.

The remaining data in this research will study only the first AFRC plasmoid. The focus of this experiment is plasmoid translation, which is expected to happen with the first AFRC when the fields and currents are at their peaks. Additionally, the secondary AFRCs are often nonrepeatable as they depend on the leftovers from the first AFRC which may change drastically from shot-to-shot.

\subsubsection{Pre-Ionization}

The pre-ionization circuit was first tested without a plasma from $5 \mathrm{kV}$ to $15 \mathrm{kV}$ to determine the current waveform of the circuit. A typical trace using a $12 \mathrm{kV}$ charge voltage is shown by the dashed line in the top graph of Figure 6.11. The current monitor output as a function of charge voltage is shown in the second graph of Figure 6.11. The PI circuit rang at 
1.1 $\mathrm{MHz}$ through 18 cycles before the energy was dissipated into ohmic heat. The upper limit of the current monitor used to measure the signal was $4 \mathrm{MHz}$. This presented two problems: the rated sensitivity for the measured waveform was invalid and the full risetime of the signal could not be accurately captured. For these reason, the current monitor data was left unscaled.

Following the vacuum tests, the pre-ionization circuit was tested using a 4 mTorr gas fill with a $5 \mathrm{kV}$ to $15 \mathrm{kV}$ charge voltage. A typical trace using a $12 \mathrm{kV}$ charge voltage is shown by the solid line in the top graph of Figure 6.11. The current monitor waveform for the plasma trace shows that the plasma absorbs a significant portion of energy from the discharge circuit, indicated by a severe damping. The PI plasma also seems to inductively couple to the PI circuit, noting that the frequency increases from the vacuum case. The vacuum and plasma shot current monitor data were integrated across their full ranges to estimate the energy absorbed by the plasma. The results are shown as a function of charge voltage in the bottom graph of Figure 6.11. The results were also scaled against the charge voltage to represent the fraction of energy absorbed by the plasma. Uncertainty estimates are from repeated tests at $12 \mathrm{kV}$, yielding a $10 \%$ spread in the data.

The results in Figure 6.11 show that the PI is ineffective at charge voltages below $8 \mathrm{kV}$, when less than $10 \%$ of the energy is absorbed by the plasma. The pre-ionzation is most effective at $15 \mathrm{kV}$ when the plasma absorbs $1.1 \mathrm{~J}$ of energy from the circuit. Assuming an ionization cost of $100 \mathrm{eV} /$ ion, $1.1 \mathrm{~J}$ of energy is enough to ionize $6.86 \times 10^{16} \#-\mathrm{m}^{-3}$ of the fill gas. This is still several orders of magnitude below the neutral fill density $\left(1.2 \times 10^{20}\right.$ $\#-\mathrm{m}^{-3}$ ), indicating the PI only weakly ionizes the initial gas fill.

The PI circuit was designed with an absolute upper limit of $15 \mathrm{kV}$. While this seems to produce the highest fraction of ions, operating consistently at $15 \mathrm{kV}$ severely derates the 

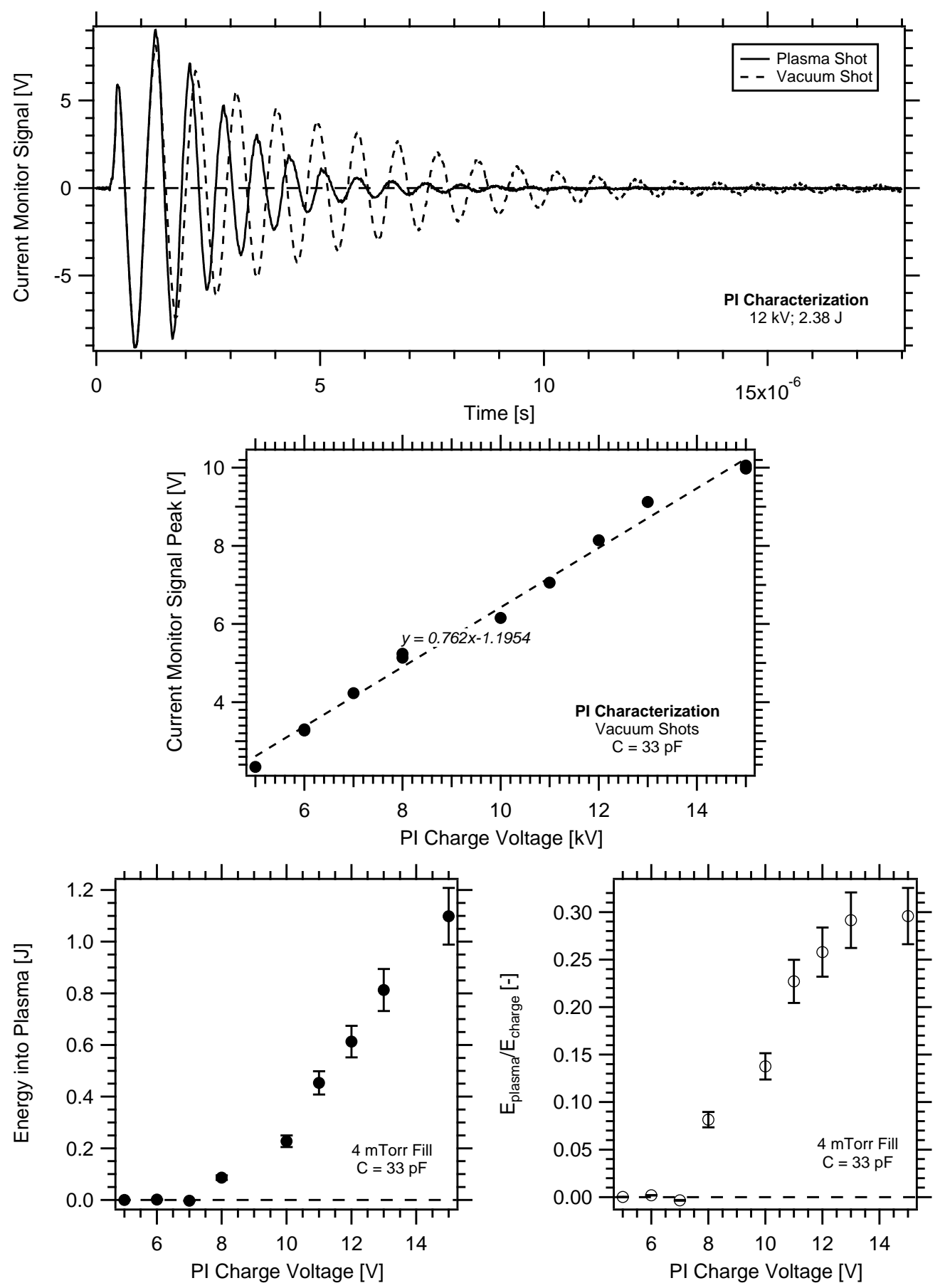

Figure 6.11: Pre-ionization study data, including current waveforms for the vacuum and plasma case, coil current compared to charge voltage, and energy absorbed by the plasma versus charge energy. 
lifetime of the capacitors and switch. A compromise was made to operate the circuit at a $12 \mathrm{kV}$, where the energy absorbed by the plasma was about $0.6 \mathrm{~J}$ and the resulting plasma

density was estimated at $3.82 \times 10^{16} \#-\mathrm{m}^{-3}$. This is only half of the density produced at 15 $\mathrm{kV}$, but it is still roughly the same ionization fraction (ions compared to initial neutral fill).

\subsubsection{Main Bank Timing}

The optimal timing between the pre-ionization and the main bank circuit trigger of the experiment was tested by adjusting the time delay between the pre-ionization trigger and the main bank trigger. The delay times tested include $10 \mu \mathrm{s}, 20 \mu \mathrm{s}, 30 \mu \mathrm{s}, 50 \mu \mathrm{s}, 100 \mu \mathrm{s}$, $150 \mu \mathrm{s}$, and $200 \mu \mathrm{s}$. This was done for all nominal discharge energies using a 4 mTorr fill pressure. The energy absorbed by the plasma was calculated for each shot, using the methods described in Section 5.3.4. The magnetic fields at the midplane were also analyzed and compared for each delay interval. The results from the $10 \mathrm{kHz}$ circuit and $20 \mathrm{kHz}$ circuit tests are discussed in Sections 6.3.3.1 and 6.3.3.2, respectively. From these results, it was determined that a delay of $10 \mu$ s was sufficient for all test conditions. The results also show that timing was not critical for the $20 \mathrm{kHz}$ discharges or the $10 \mathrm{kHz}$ discharges at $500 \mathrm{~J}$ and above.

\subsubsection{Timing: $10 \mathrm{kHz}$}

The timing study for the $10 \mathrm{kHz}$ circuit was conducted at $100 \mathrm{~J}, 500 \mathrm{~J}$, and $1000 \mathrm{~J}$ using a 4 mTorr gas fill. Midplane magentic fields were examined from each shot to check for field reversal and the coil currents were recorded to estimate the energy absorbed by the plasma. 
Since the time-period of interest involves only the first $1 / 4$ cycle, the energy absorbed during the first $1 / 2$ cycle was calculated. The first $1 / 2$ cycle was chosen for comparison as it is easier to calculate than the first 1/4 cycle when energy is stored in unknown inductances. The energy absorbed by the plasma during the first $1 / 2$ cycle was calculated for each data set, using Equation 5.46. Circuit resistance is a required input for this equation and was calculated by solving Equation 5.47, using the circuit current from the vacuum traces. The calculated resistances and associated errors are shown in Table 6.4. The timing results for each energy setting are shown in Figures 6.12, 6.13, and 6.14. Uncertainty estimates for the energy calculations include calculated error as well as statistical error from repeated shots with a $10 \mu$ s delay.

\section{Table 6.4}

XOCOT-T3 calculated circuit resitances for the $10 \mathrm{kHz}$ circuit.

\begin{tabular}{lcccc}
\hline $\begin{array}{l}\text { Energy } \\
{[\mathrm{J}]}\end{array}$ & $\begin{array}{c}R_{\text {circuit }} \\
{[\mathrm{m} \Omega]}\end{array}$ & $\begin{array}{c}\sigma_{\text {total }} \\
{[\mathrm{m} \Omega]}\end{array}$ & $\begin{array}{c}\sigma_{\text {sdev }} \\
{[\mathrm{m} \Omega]}\end{array}$ & $\begin{array}{c}\sigma_{\text {meas }} \\
{[\mathrm{m} \Omega]}\end{array}$ \\
\hline $100 \mathrm{~J}$ & 14.0 & 1.3 & 0.6 & 1.2 \\
$500 \mathrm{~J}$ & 11.0 & 1.3 & 0.4 & 1.2 \\
$1000 \mathrm{~J}$ & 12.6 & 1.2 & 0.7 & 1.7 \\
\hline
\end{tabular}

The timing results for the $10 \mathrm{kHz}$ circuit indicate that a $10 \mu$ s delay is acceptable for all cases. At $100 \mathrm{~J}$, the energy absorbed into the plasma is slightly higher than delays of 100 $\mu$ s or more. The field reversal also is highest with a $10 \mu$ delay. Larger discharge energy settings $(500 \mathrm{~J}$ and $1 \mathrm{~kJ}$ ) results show that the timing delay is not essential to the onset of reversal or to energy absorbtion. This suggests that the PI is more critical for low energy than high energy settings. Testing without the PI at high energy demonstrated that it was still possible to form an AFRC, though it did not appear until after the first 1/4 cycle and it created weaker magnetic fields. AFRCs at $100 \mathrm{~J}$ and $10 \mathrm{kHz}$ failed to form without the PI.

The uncertainty in the energy absorbed into the plasma is substantial. All significant errors 

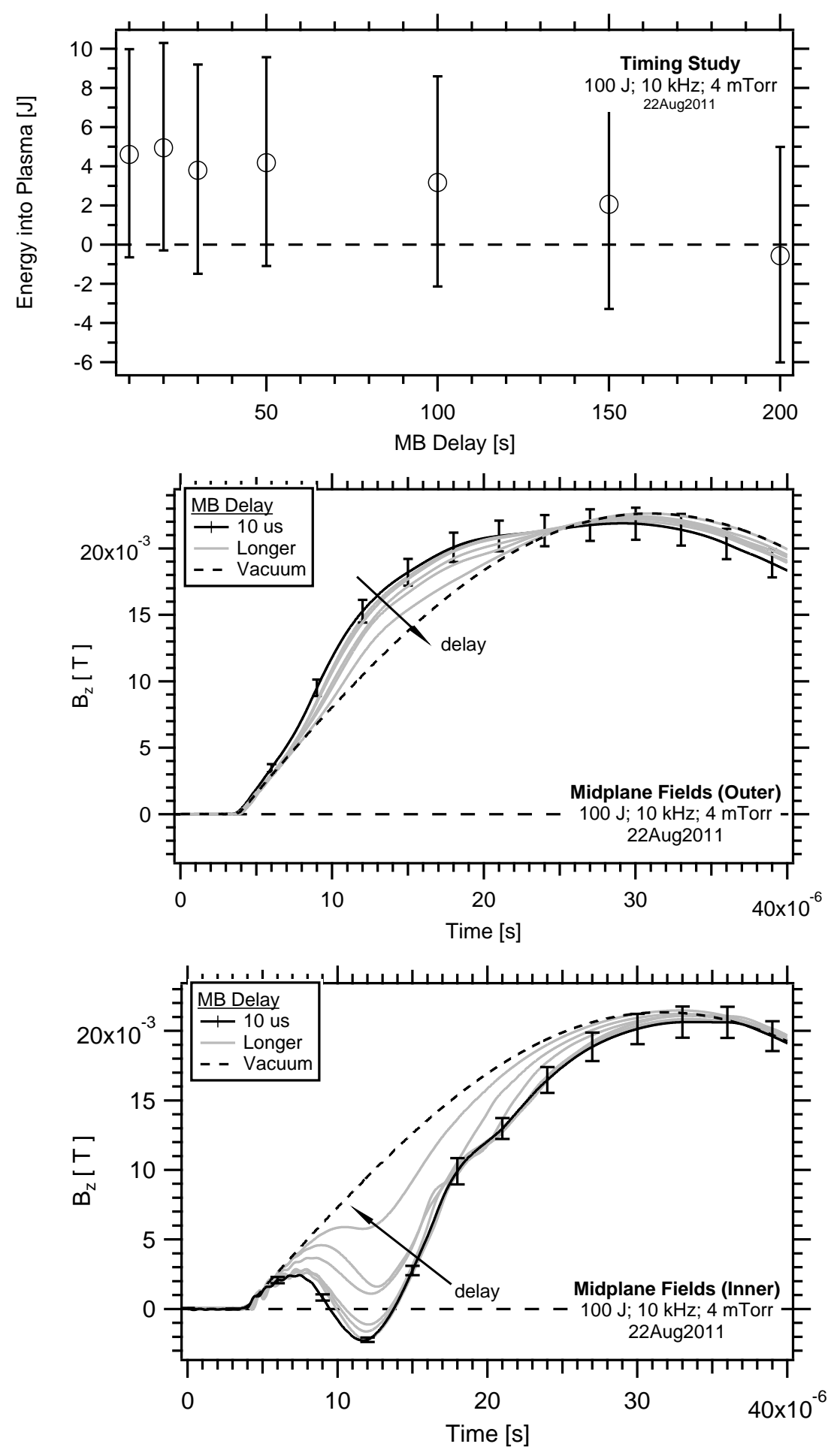

Figure 6.12: Timing results for the $20 \mathrm{kHz}, 100 \mathrm{~J}$ tests, showing energy absorbtion into the plasma (top) and midplane magnetic fields for various delay intervals. 

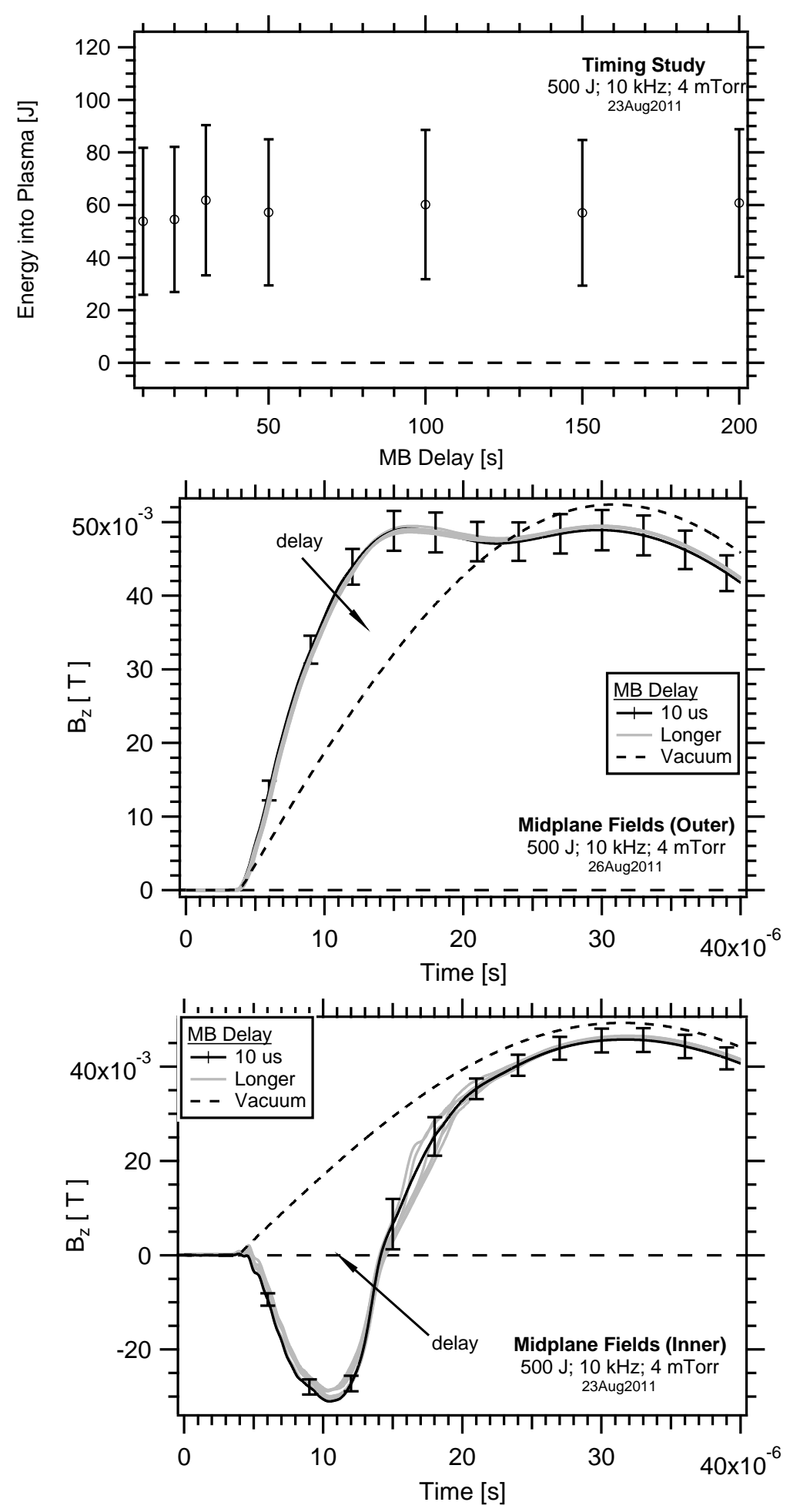

Figure 6.13: Timing results for the $20 \mathrm{kHz}, 1000 \mathrm{~J}$ tests, showing energy absorbtion into the plasma (top) and midplane magnetic fields for various delay intervals. 

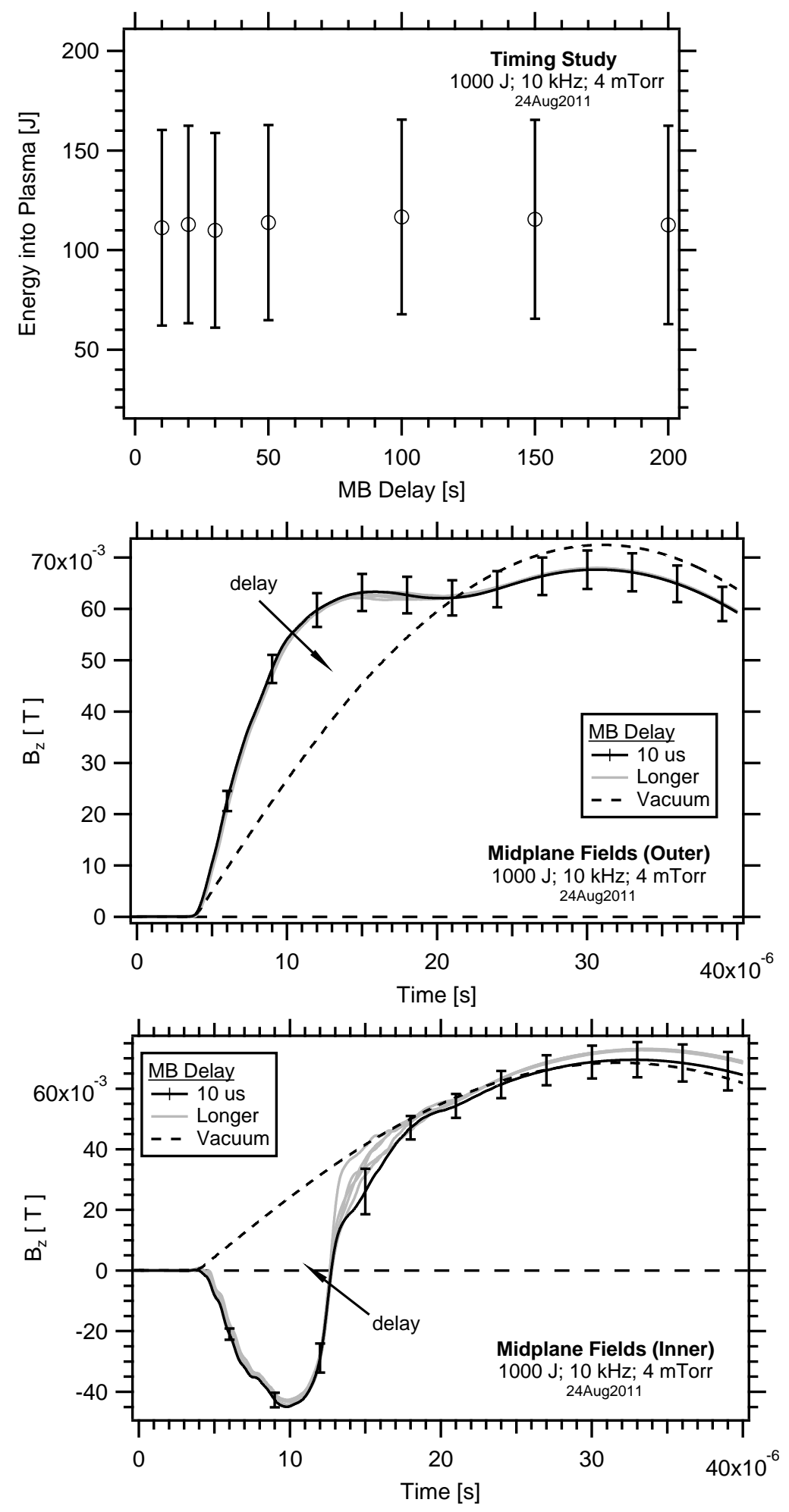

Figure 6.14: Timing results for the $20 \mathrm{kHz}, 1000 \mathrm{~J}$ tests, showing energy absorbtion into the plasma (top) and midplane magnetic fields for various delay intervals. 
in this calculation point back to the $1 \%$ error in the current measurement. While this is a fairly low uncertainty, the current measurement is used extenstively through the calculation for $E_{\text {plasma }}$ and is often squared and integrated which increases the error substantially. It also appears in the calculation for the circuit resitance, contributing heavily to its $10 \%$ error. The standard devation in absorbed energy among repeated shots only accounts for a small portion of the error. In the $10 \mathrm{kHz}, 100 \mathrm{~J}$ circuit, the standard devation between shots is $1.13 \mathrm{~J}$.

\subsubsection{Timing: $20 \mathrm{kHz}$}

The timing study results for the $20 \mathrm{kHz}$ circuit at $100 \mathrm{~J}$ are shown in Figure 6.15. The midplane magnetic fields are displayed along with the energy absorbed by the plasma during the first $1 / 2$ cycle of the discharge. Uncertainty intervals show the combined error due to the measurement and repeated data sets with a $10 \mu$ s delay.

The energy absorbed by the plasma does not change with respect to the main bank timing delay. The energy absorbed for a $10 \mu$ s delay is $23.6 \mathrm{~J} \pm 5.4$ joules. Timing seems to have a minor effect on the magnetic fields as well. Slightly stronger magnetic fields and a somewhat faster reversal are seen for the $10 \mu$ s case compared to the longer delays. These results indicate that timing of the main bank trigger is not vital to plasmoid formation, up to $200 \mu \mathrm{s}$, and operating with a shorter delay yields only minor improvements. Using these results as a guide, the timing delay between the pre-ionization trigger and main bank trigger was set to $10 \mu$ s for the $20 \mathrm{kHz}, 100 \mathrm{~J}$ tests. 

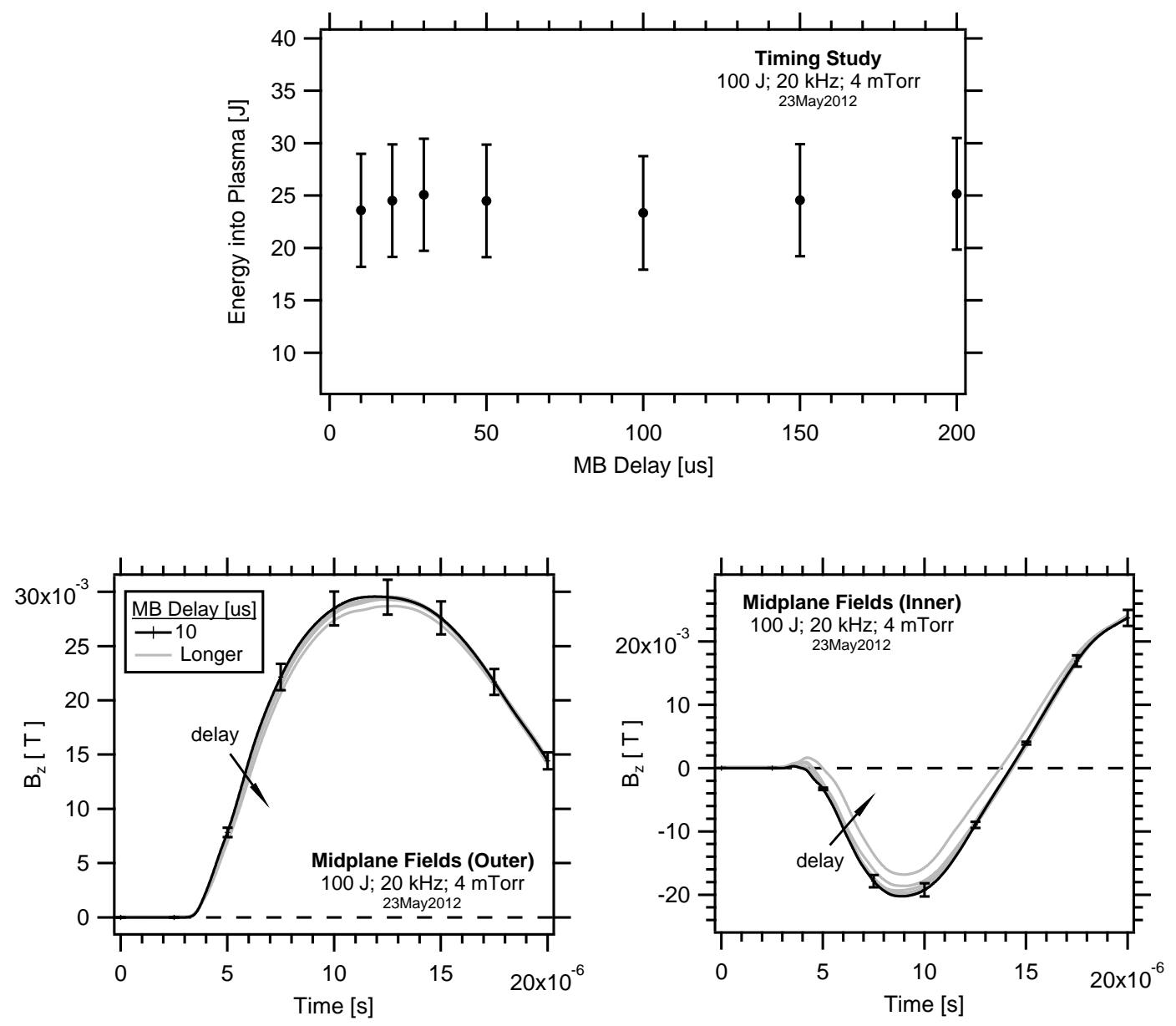

Figure 6.15: Timing results for the $20 \mathrm{kHz}, 100 \mathrm{~J}$ tests, showing energy absorbtion into the plasma (top) and midplane magnetic fields for various delay intervals.

\subsubsection{Repeatibility}

The XOCOT-T3 experiment was tested repeatedly at single test condition for the $10 \mathrm{kHz}$ circuit and the $20 \mathrm{kHz}$ circuit to establish shot-to-shot repeatibility. This was done for the plasma discharges at one fill pressure (4 mTorr) with discharge energies of $100 \mathrm{~J}, 500 \mathrm{~J}$, and $1000 \mathrm{~J}$ for the $10 \mathrm{kHz}$ circuit. The $20 \mathrm{kHz}$ repeatibility study was performed at $100 \mathrm{~J}$ and 4 mTorr. Repeatibility was established by averaging the data from each probe for 10 
or more shots at each discharge energy.

\subsubsection{Repeatibility: $10 \mathrm{kHz}$}

The repeatibility study for the $10 \mathrm{kHz}$ circuit was completed for each energy setting, including $100 \mathrm{~J}, 500 \mathrm{~J}$, and $1 \mathrm{~kJ}$ discharges. All tests were conducted using a 4 mTorr background fill. At least 10 shots at each energy setting were recorded. The average waveform for each diagnostic was computed along with the standard deviations from the average. Figures 6.16, 6.18, and 6.20 show the results for the coil currents and upstream magnetic fields. Figures 6.17, 6.19, 6.21 display the data for the downstream magnetic fields and plasma probes. Average waveforms are shown, along with the corresponding history of standard deviation. Error bars are a combination of the statistical deviation and the measurement uncertainty.

Overall the XOCOT-T3 at $10 \mathrm{kHz}$ creates fairly repeatible plasma discharges from the reference of the magnetic probes. The first couple microseconds of the discharge show the most deviation, as the plasma currents forms and ionizes the neutral fill. After this period, the deviation on most probes drops to less than $10 \%$. The coil currents and outer coil fields show the highest repeatibility, with no more than $4 \%$ devation between shots.

The inner field probes are the best indicator of repeatable plasmoid behavior, as these are the most direct measurement of plasmoid current. Using the inner probes as a guide, it can be inferred that the high energy cases $(500 \mathrm{~J}$ and $1 \mathrm{~kJ})$ produce more repeatable plasmoids than the low energy case. The deviation on the inner probe data for the high energy cases does not exceed $10 \%$. The plasmoid behavior is somewhat less predictable at $100 \mathrm{~J}$, due to low energy content. This is apparent in the longer reversal time and low reversed field. 

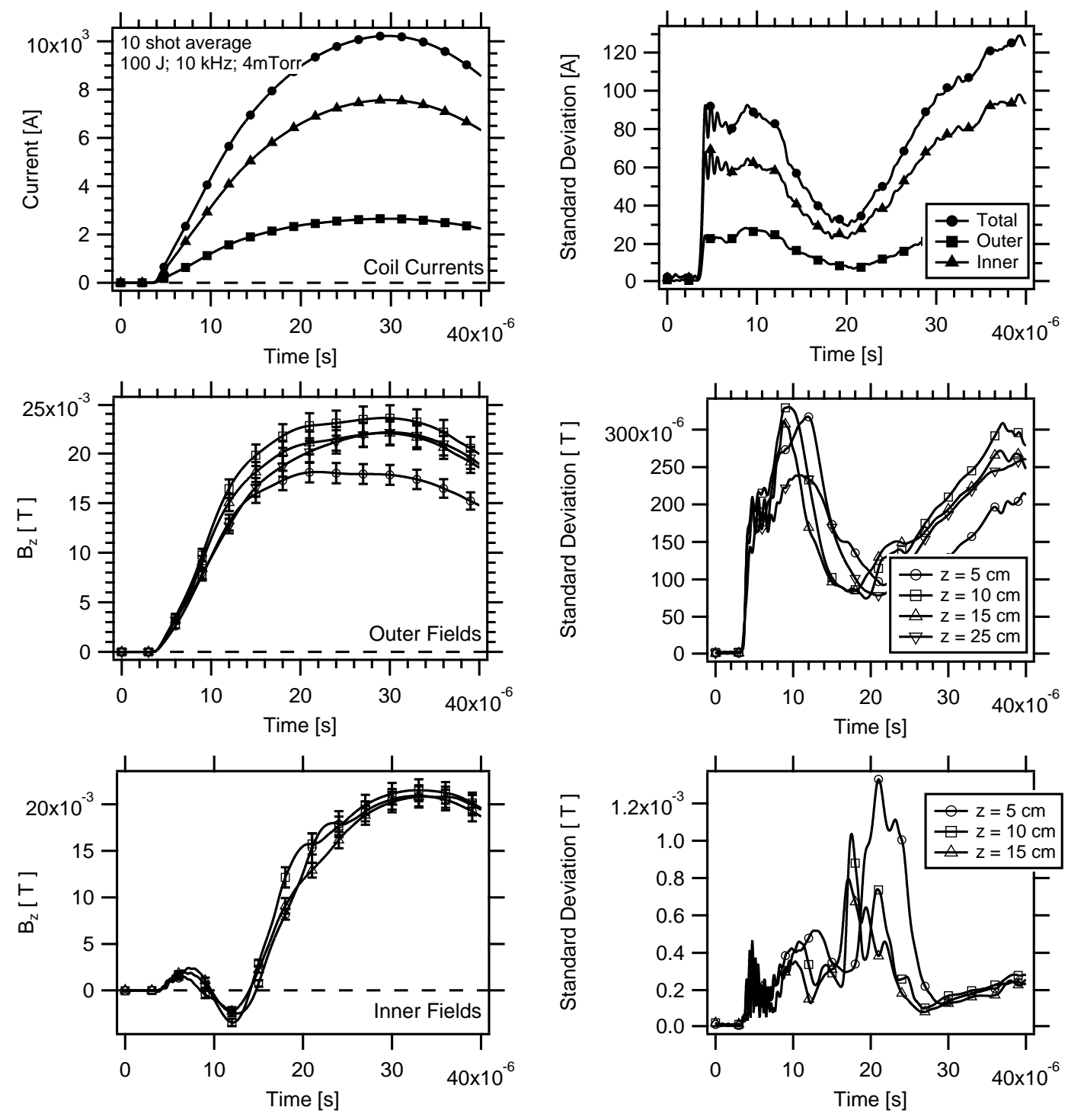

Figure 6.16: Repeatibility study data for the $10 \mathrm{kHz}$ circuit at $100 \mathrm{~J}$, including coil currents and upstream magnetic field measurements. The standard deviation of repeated data sets for each diagnostics is shown on the right.

However, the repeated data sets even in the $100 \mathrm{~J}$ case do not differ in by more than $15-20 \%$.

The downstream TOF probes show more shot-to-shot variation than the upstream probes. The probe at $\mathrm{z}=49.5 \mathrm{~cm}$ produces repeatable waveforms, with deviations between shots of less than $6 \%$. The probe at $\mathrm{z}=67.7 \mathrm{~cm}$ is more susceptible to variation, showing deviations 

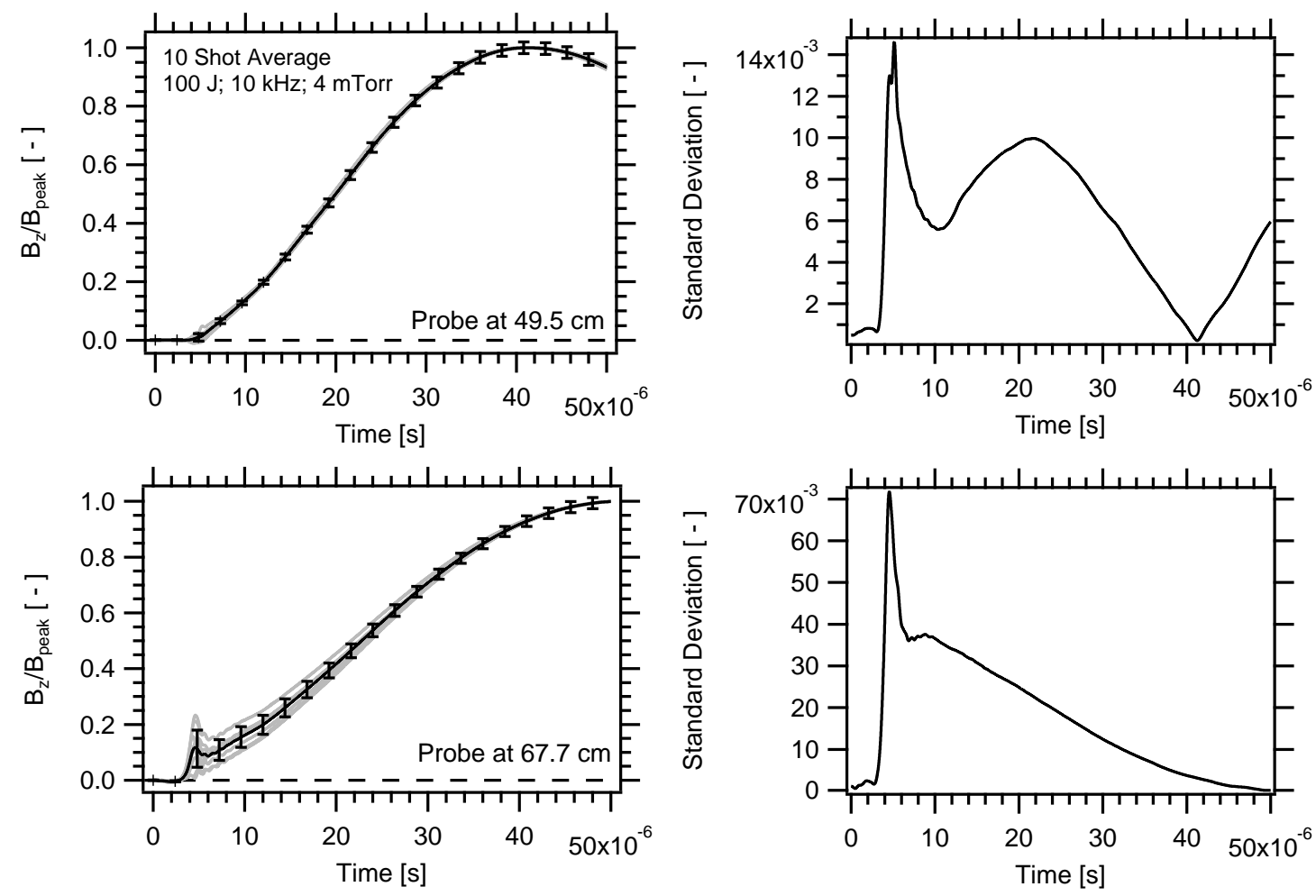

Figure 6.17: Downstream results from the repeatibility study for the $10 \mathrm{kHz}$ at $100 \mathrm{~J}$, including the measurements recorded from the TOF probes and the standard deviations among repeated shots.

of $15 \%$ at $100 \mathrm{~J}, 8 \%$ at $500 \mathrm{~J}$, and $20 \%$ at $1 \mathrm{~kJ}$. The magnetic fields at this probe location are quite small so the variation is somewhat expected.

\subsubsection{Repeatibility: $20 \mathrm{kHz}$}

The repeatibility data for the $20 \mathrm{kHz}$ circuit was conducted in 2 parts. First, 32 current and magnetic field data sets were collected at $100 \mathrm{~J}$ of discharge energy and 4 mTorr of background pressure. Downstream plasma probe data was collected during a second set of testing using 10 shots of the same repeated conditions. The average waveform for each diagnostic was computed along with the standard deviations of the each waveform from 

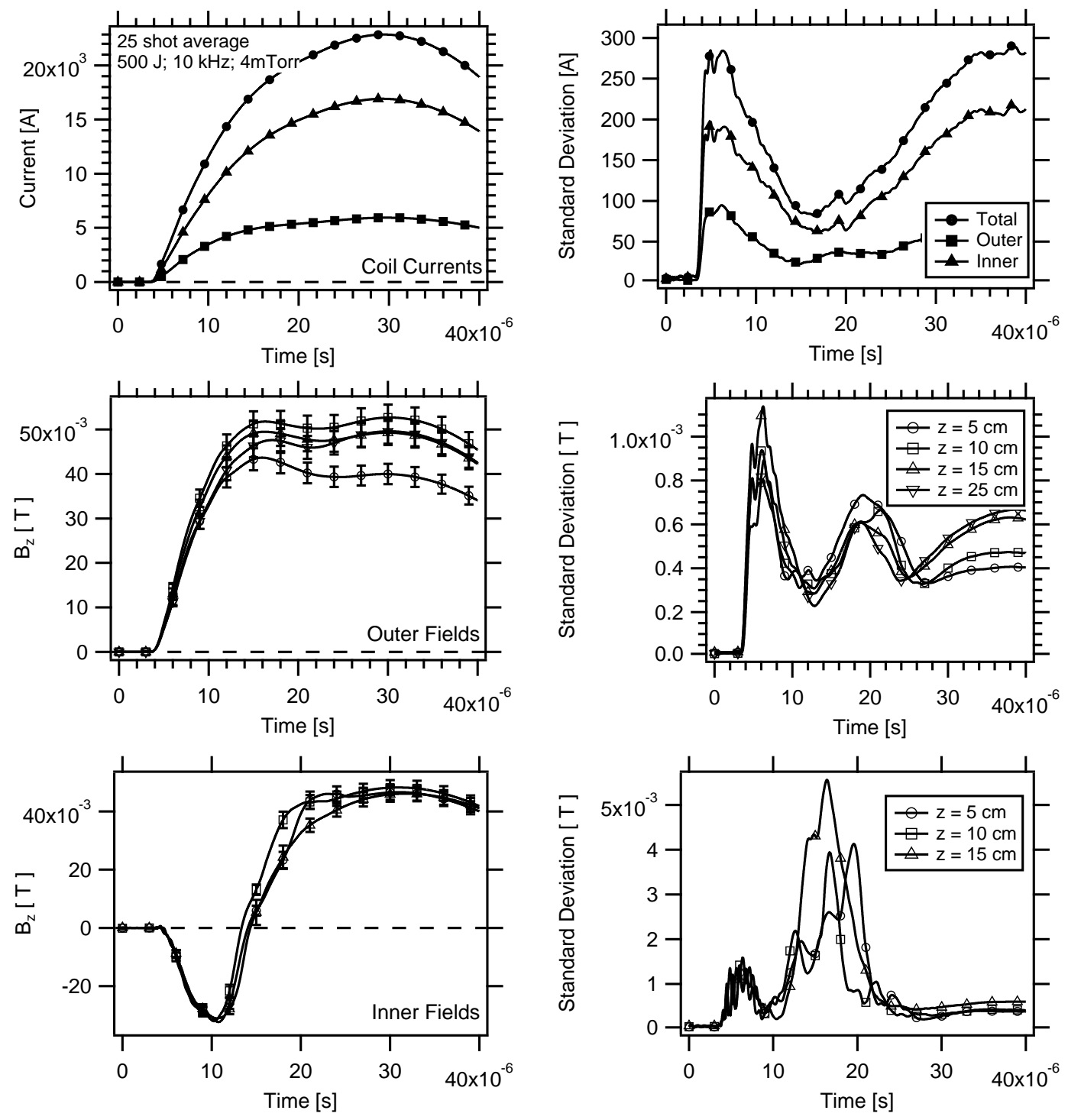

Figure 6.18: Repeatibility study data for the $10 \mathrm{kHz}$ circuit at $500 \mathrm{~J}$, including coil currents and upstream magnetic field measurements. The standard deviation of repeated data sets for each diagnostics is shown on the right.

the average. Figure 6.22 shows the results for the coil currents and upstream magnetic fields. Figure 6.23 displays the data for the downstream magnetic fields and plasma probes. Average waveforms are shown, along with the corresponding history of standard deviation. Error bars are a combination of the statistical deviation and the measurement uncertainty. 

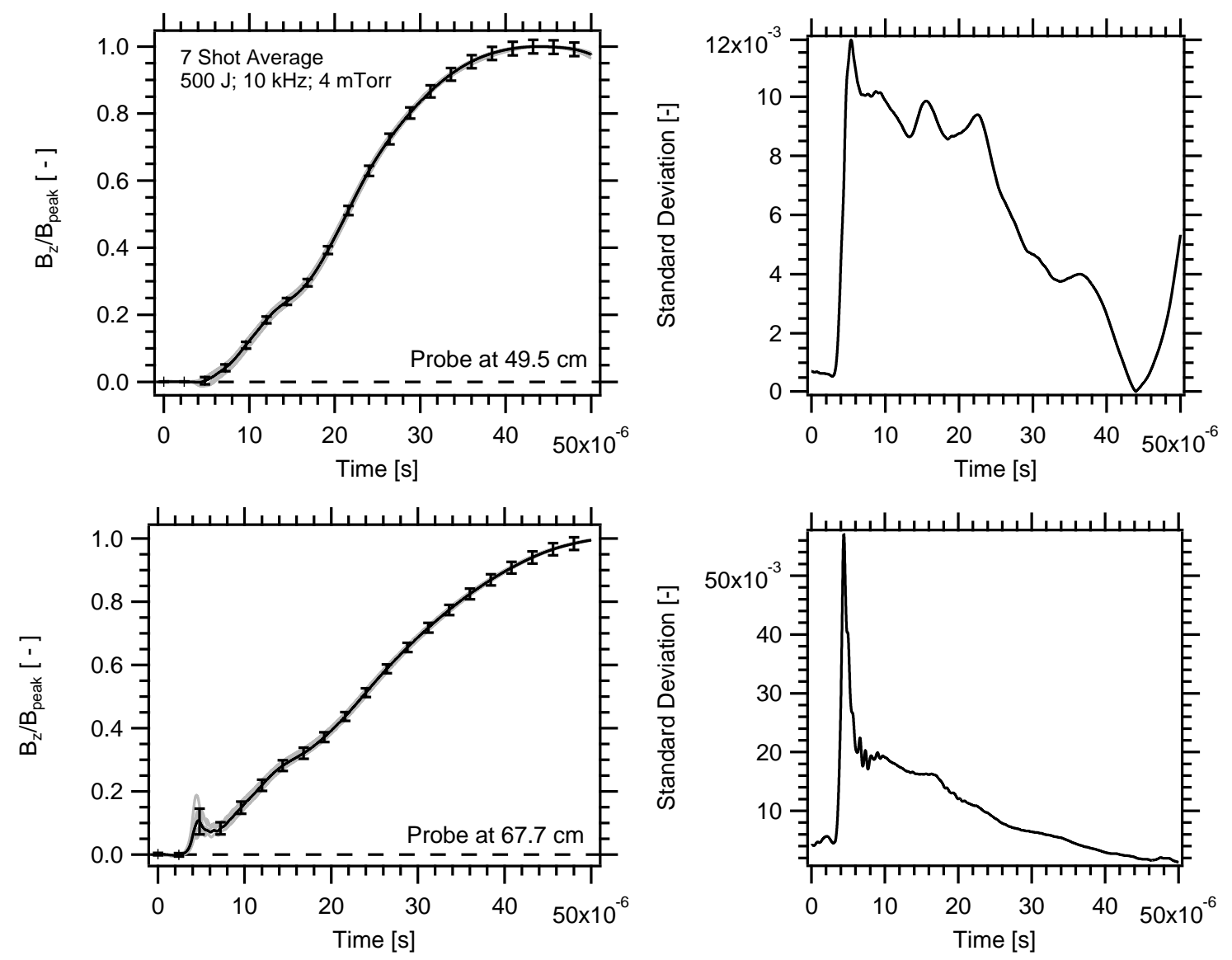

Figure 6.19: Downstream results from the repeatibility study for the $10 \mathrm{kHz}$ at $500 \mathrm{~J}$, including the measurements recorded from the TOF probes and the standard deviations among repeated shots.

The repeatibility study data demonstrates that the XOCOT-T3 experiment operating at 100 $\mathrm{J}$ and $20 \mathrm{kHz}$ produces repeatible data at each probe location. The current measurements deviate between shots by less than $2 \%$ and the outer magnetic field measurements differ by around $2.5 \%$. The inner magnetic fields are somewhat more sporadic during the initial ionization phase, differing by $12 \%$ during the first several $\mu$ s, but leveling off to around $2 \%$ around the local minimum. The downstream magnetic field data is similarily repeatable, with only a few percent difference between shots. The plasma flux data is less repeatible than the magnetic field measurements, differing between shots by $10-15 \%$. 

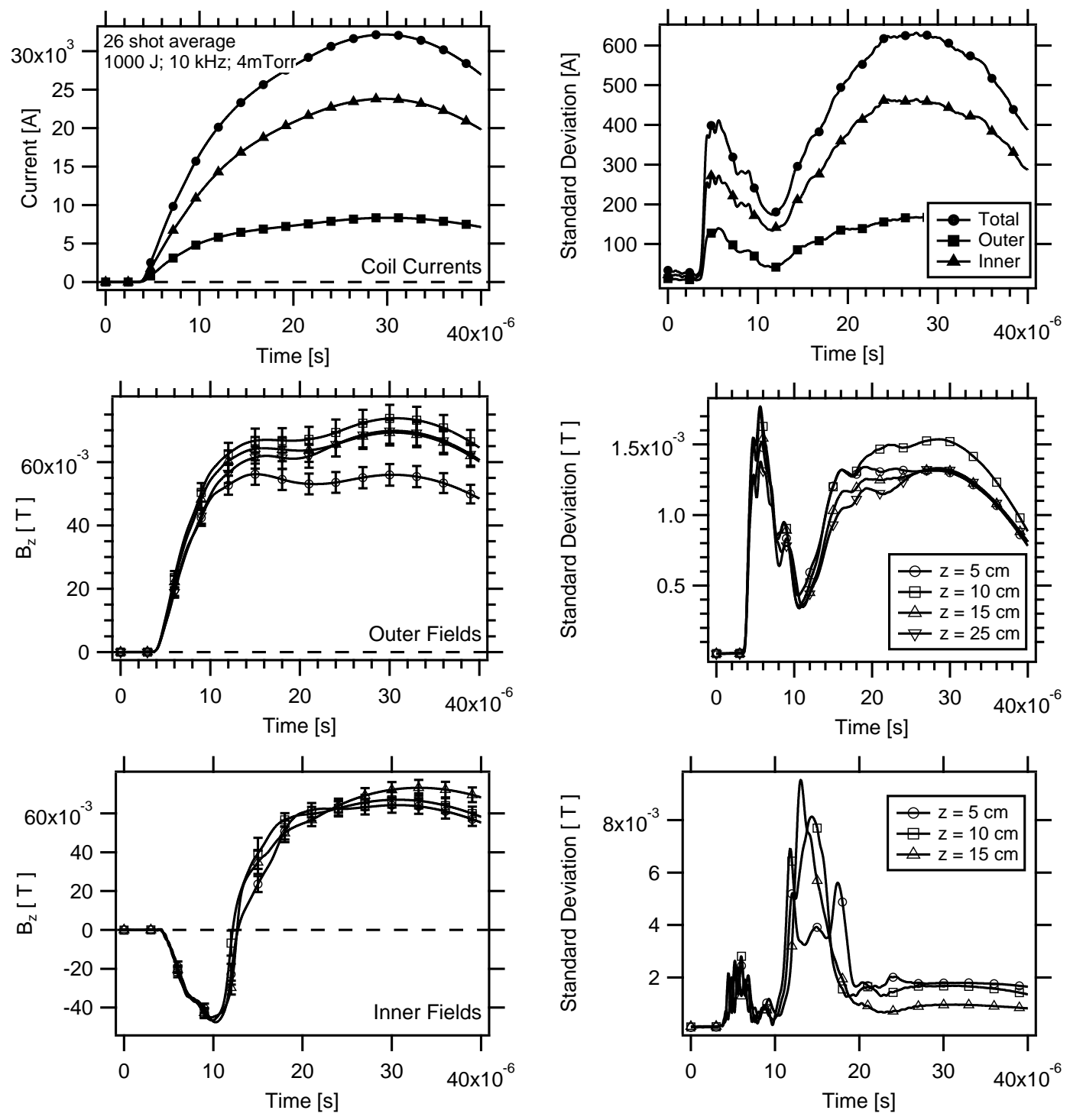

Figure 6.20: Repeatibility study data for the $10 \mathrm{kHz}$ circuit at $1000 \mathrm{~J}$, including coil currents and upstream magnetic field measurements. The standard deviation of repeated data sets for each diagnostics is shown on the right.

The highly repeatible nature of the XOCOT-T3 plasmoid formation made it possible to reduce the number of shots required to establish behavior. Analysis was conducted to determine how the average waveform varied with sample size. Subsets of the original 32 data sets were averaged in a random fashion and the average and standard devation of the subset was calculated at one point $(\mathrm{t}=7 \mu \mathrm{s})$ during the initial formation, away from the 

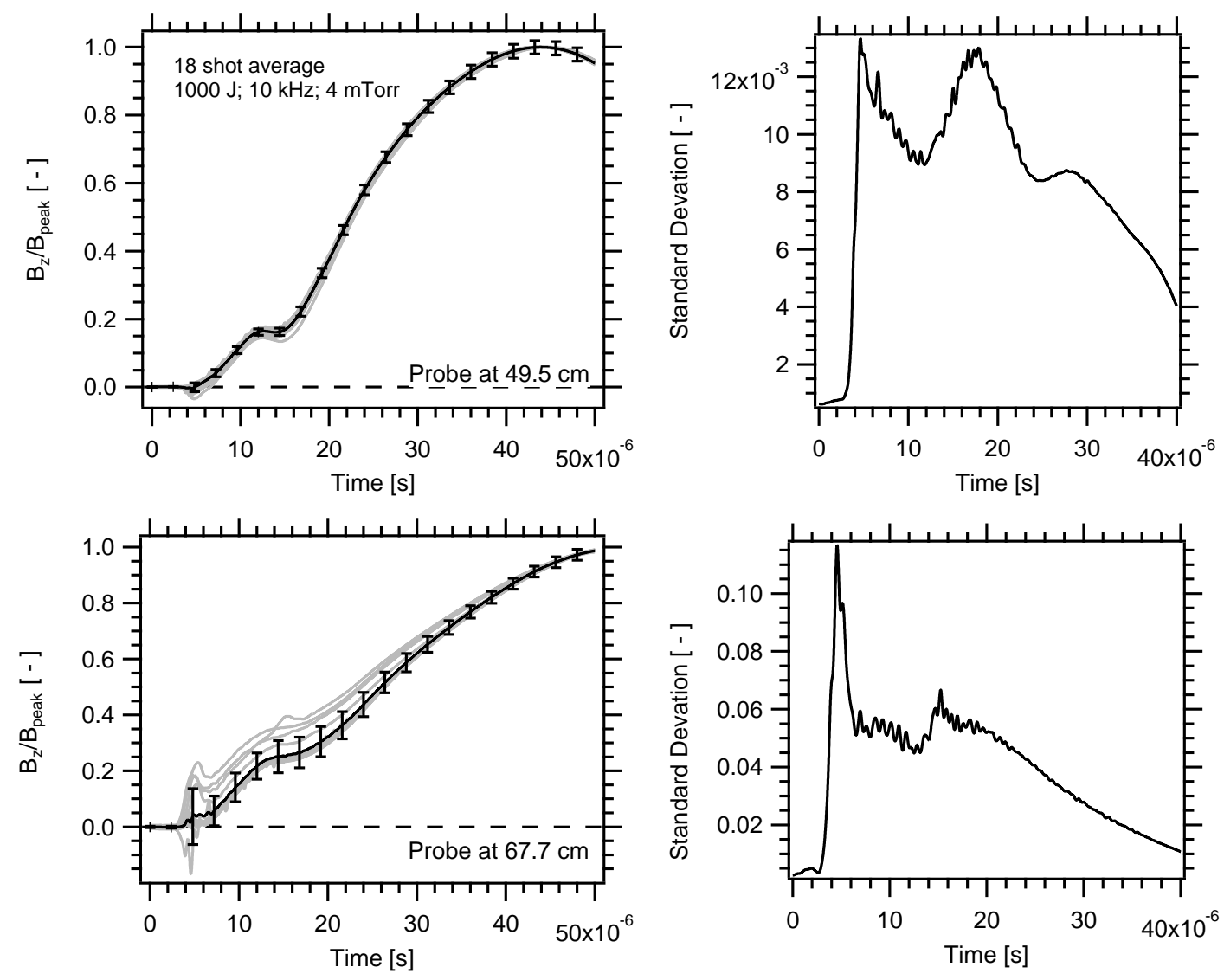

Figure 6.21: Downstream results from the repeatibility study for the $10 \mathrm{kHz}$ at $1000 \mathrm{~J}$, including the measurements recorded from the TOF probes and the standard deviations among repeated shots.

startup transients. The results are displayed in Figure 6.24.

The results from this study shows that the averaged subset of current and midplane fields fall within $2 \%$ of the set average, regardless of sample size. This suggests that only a few shots are necessary to provide a characteristic of plasmoid behavior, assuming this trend does not change appreciably with pressure and energy. A few shots at each setting may not produce a statistically sound data set, they will be sufficient to determine under what conditions the plasmoid translates. A larger sample set can then be collected at the conditions where translation is evident. A reduced sample size for initial testing is also warranted for ensuring the entire operating matrix can be explored before the discharge 

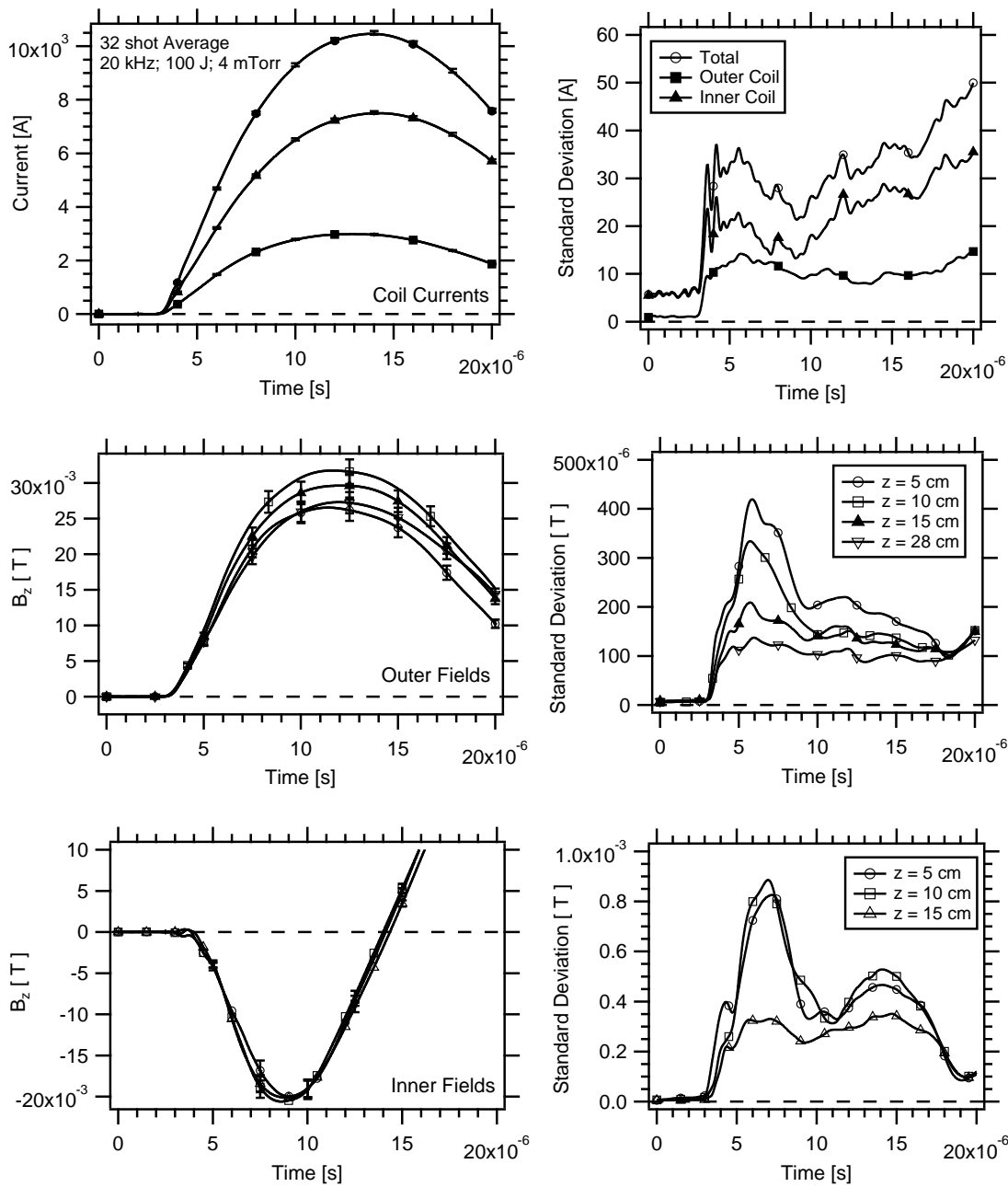

Figure 6.22: Repeatibility study data for the $20 \mathrm{kHz}$ circuit at $100 \mathrm{~J}$, including coil currents and upstream magnetic field measurements. The standard deviation of repeated data sets for each diagnostics is shown on the right.

circuit wears out. This was especially important in the XOCOT-T3 experiment, in which the discharge capacitors were subject to severe wear during testing. 

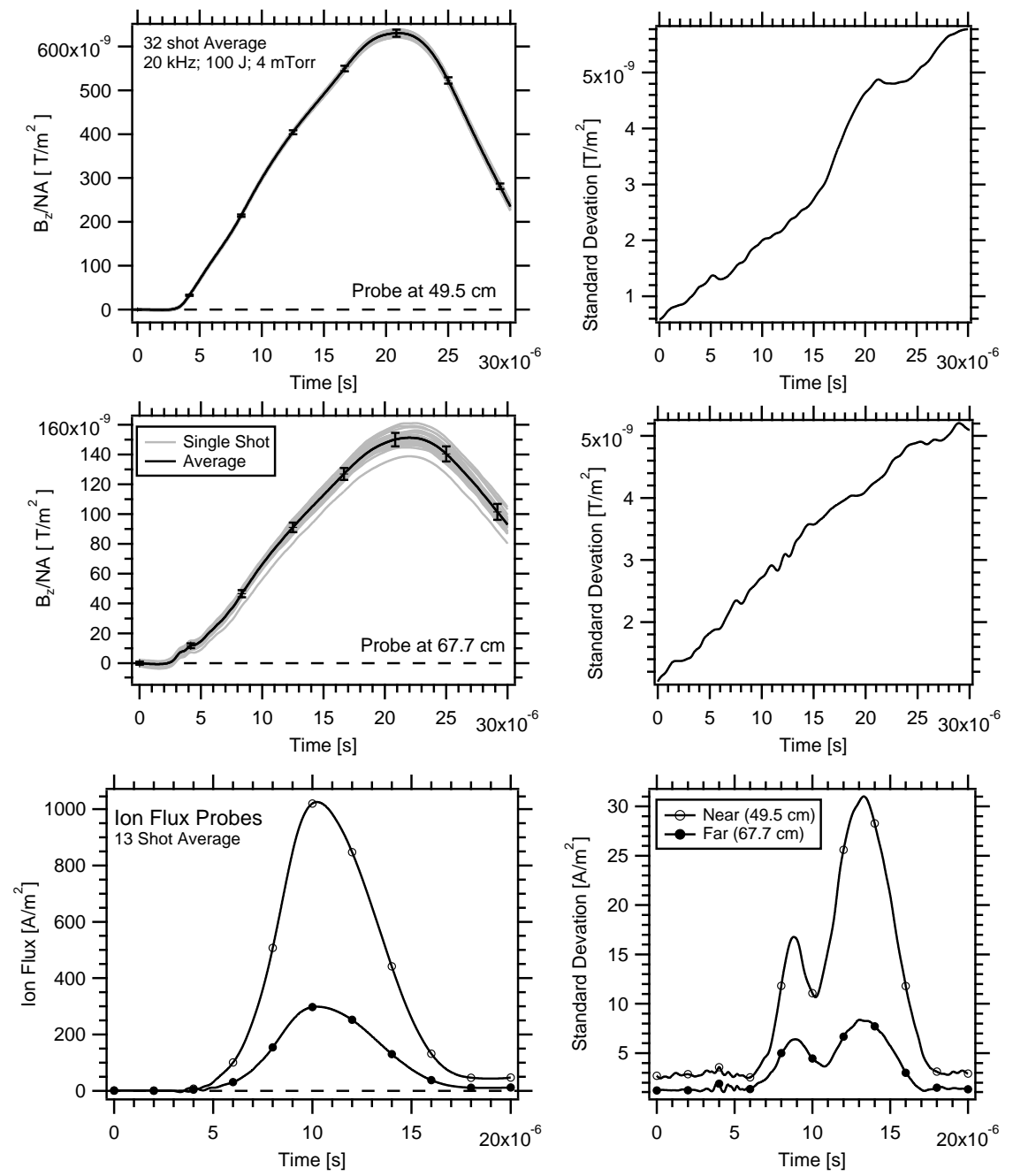

Figure 6.23: Downstream results from the repeatibility study for the $20 \mathrm{kHz}$ at $100 \mathrm{~J}$, including the measurements recorded from the TOF probes and the standard deviations among repeated shots.

\subsection{Translation Study Data}

The XOCOT-T3 experiment was tested in both circuit configurations at 6 different fill pressures and various discharge energies. The fill pressure was adjusted to change the plasmoid mass. Discharge energy was changed to control the Lorentz force acting on the plasmoid. Both adjustments should affect the velocity and momentum results directly, in 

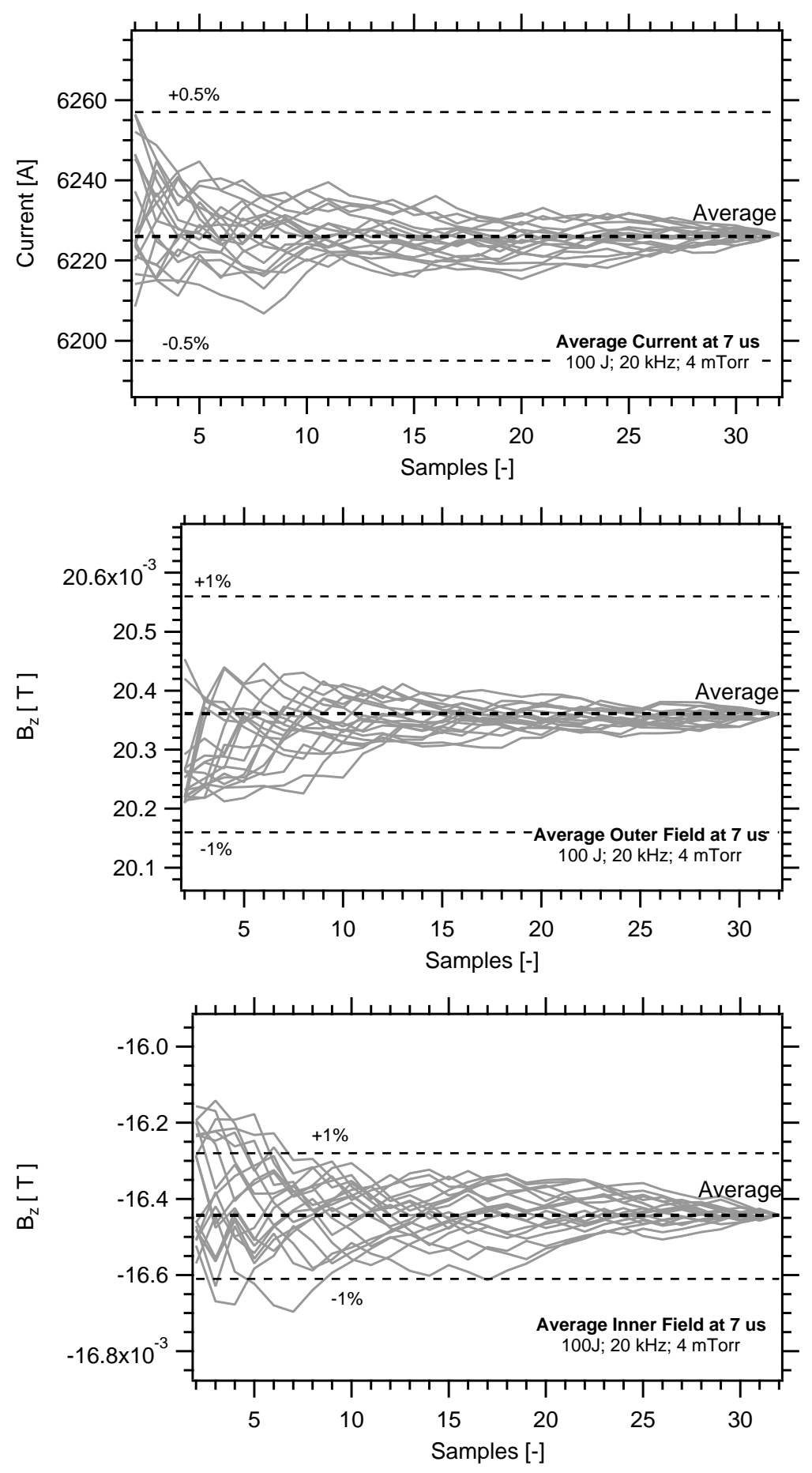

Figure 6.24: Average currents and magnetic fields at $7 \mu$ s compared to number of samples included in average. 
accordance with Newton's second law and the translation predictions in Chapter 4 .

The pressure and energy setttings chosen for this research are presented in Table 6.1. The fill pressure range varied from $1 \mathrm{mTorr}$ to 50 mTorr, including $1 \mathrm{mTorr}, 4 \mathrm{mTorr}, 10 \mathrm{mTorr}$, 14 mTorr, 20 mTorr, and 50 mTorr. The energy for the $10 \mathrm{kHz}$ circuit also covered a full magnitude from $100 \mathrm{~J}$ to $1 \mathrm{~kJ}$, with a stop at $500 \mathrm{~J}$. The $20 \mathrm{kHz}$ circuit was tested at each fill pressure for the $100 \mathrm{~J}$ energy setting. Low energy testing of the $20 \mathrm{kHz}$ circuit was conducted at $50 \mathrm{~J}$ with $4 \mathrm{mTorr}$ of background pressure. All tests were conducted using an Argon fill, a $10 \mu$ s main bank trigger delay and the PI configuration outlined in Section 6.3 .2 .

The data collected from these experiments include magnetic field signatures, coil currents, and downstream plasma flux measurements. Magnetic field data is collected from three primary locations, including upstream measurements on the outer wall, upstream measurements on the inner wall, and downstream measurements in the translation region. Plasma flux probes were recorded at the same axial locations as the downstream magnetic probe, spaced 180 degrees away.

The data from the $10 \mathrm{kHz}$ circuit configuration is presented in Section 6.4 .1 and the $20 \mathrm{kHz}$ data is presented in Section 6.4.2. Plasmoid translation measurements using these data sets are discussed in Chapter7

\subsubsection{Translation Data: $10 \mathrm{kHz}$}

The XOCOT-T3 was tested with the $10 \mathrm{kHz}$ circuit at $100 \mathrm{~J}, 500 \mathrm{~J}$, and $1 \mathrm{~kJ}$ using a static backfill of Argon. The coil currents and upstream magnetic field at all tested pressures at 
$100 \mathrm{~J}$ is displayed in Figures 6.25-6.27. This data was averaged from 3 to 6 shots at each background pressure. Figure 6.25 displays the current data and the magnetic fields (inner and outer) at the coil midplane $(\mathrm{z}=15 \mathrm{~cm})$. Figure 6.25 shows the magnetic field data upstream of the midplane (at $\mathrm{z}=5 \mathrm{~cm}$ and $\mathrm{z}=10 \mathrm{~cm}$ ) and at the far end of the coil $(\mathrm{z}=$ $25 \mathrm{~cm}$ ). Downstream magnetic field signatures on the TOF probes are displayed in Figure 6.27. Confidence intervals for all traces is a combination of the experimental uncertainty in each diagnostic and the statistical spread in the data among shots.

The $100 \mathrm{~J}, 10 \mathrm{kHz}$ circuit produces a plasma current at all pressures, except for $50 \mathrm{mTorr}$. This is noted by a reversed field on the inner wall, an excluded field on the outer probes, and inductive coupling in the circuit current. The strength of the plasma current, evident primarily by the inner field data, is strongly dependant on fill pressure. Low fill pressures create a stronger current than the high pressure cases. The midplane inner field data (see Figure 6.25) shows that the axial field crosses through zero only for the low pressure cases of $1 \mathrm{mTorr}$ and $4 \mathrm{mTorr}$. The field at higher pressures (other than $50 \mathrm{mTorr}$ ) attempts to reverse, but the current is not strong enough to fully oppose the outer coil field. This trend is also seen in the outer probes, where the excluded field increases with decreasing pressure. The dependancy of plasma current on fill pressure can be understood by noting that plasma resistance is proportional to density.

While the low pressure cases create a reversed field, the reversal is late and short lived. At midplane, the field reversal takes $6 \mu$ s to develop and stops growing $2 \mu$ s later. The field also fails to fully reverse, which would create the same field strengths on the inner coil as the outer coil. This suggests that the AFRC in the $100 \mathrm{~J}, 10 \mathrm{kHz}$ circuit is weak and unlikely to translate.

The late reversal of the $100 \mathrm{~J}$ data makes it apparent that the field reversal begins at the 

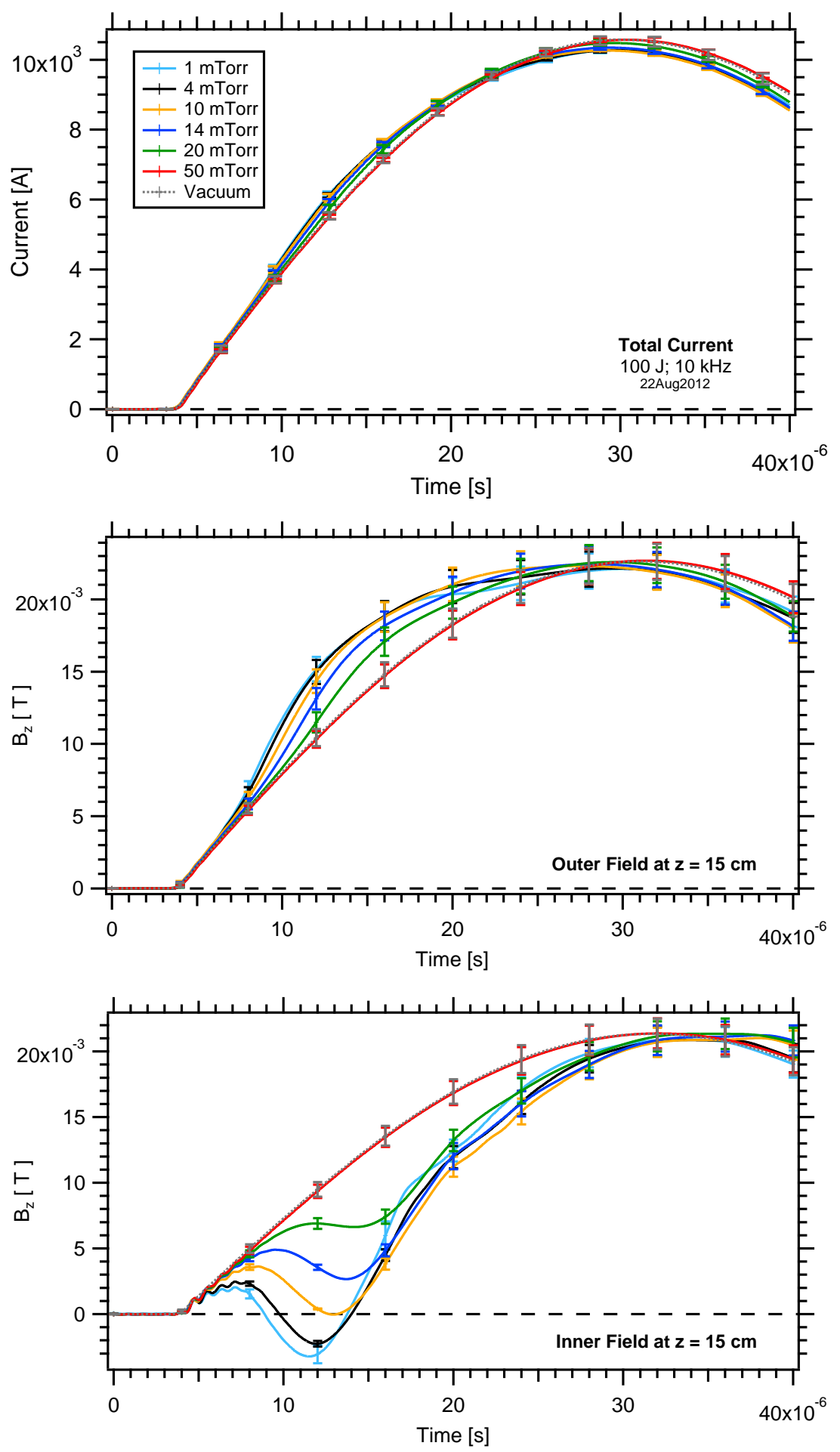

Figure 6.25: Total current and midplane magnetic field data at various fill pressures for the $100 \mathrm{~J}, 10 \mathrm{kHz}$ circuit. Vacuum traces are shown at each diagnostic by dotted lines. 

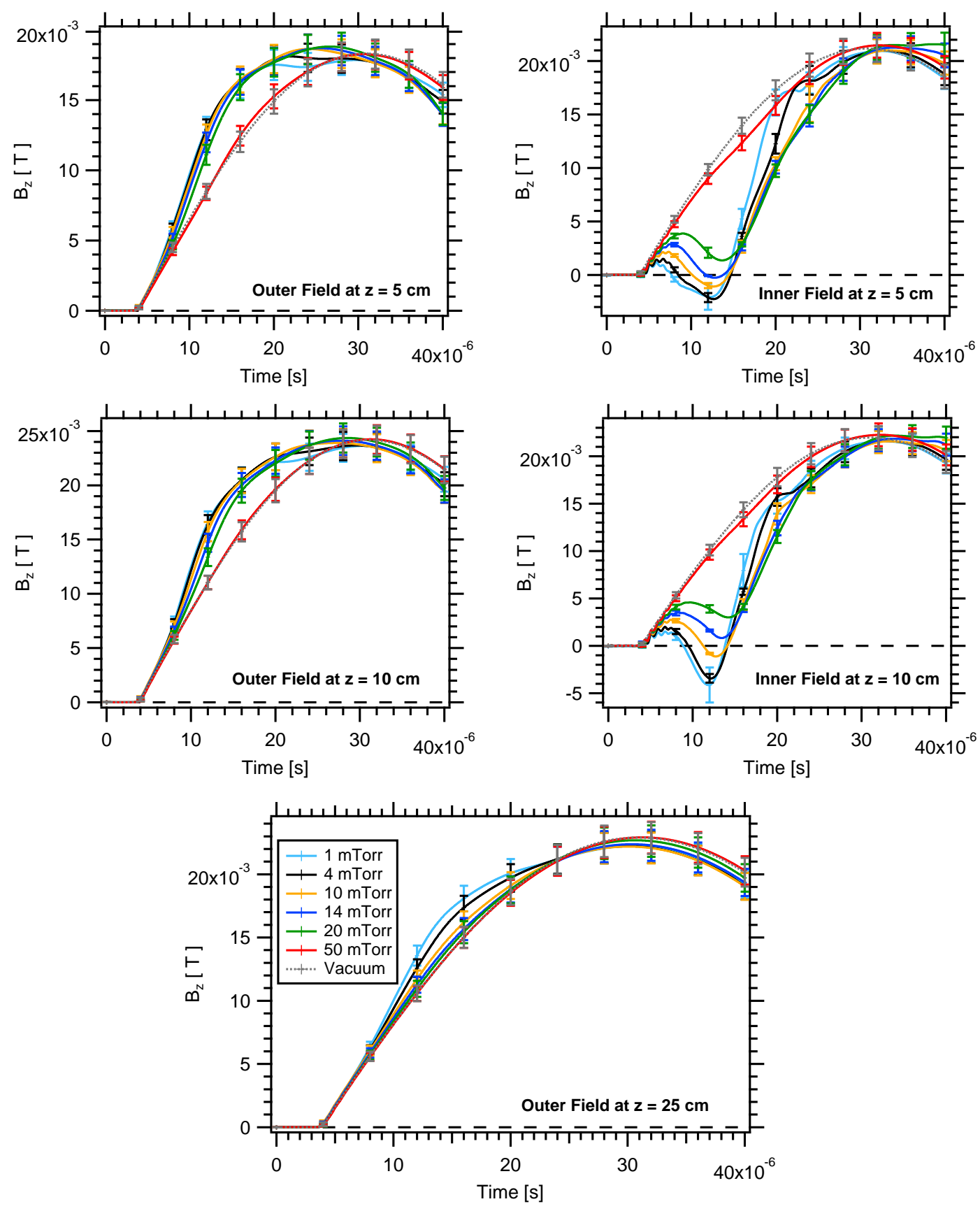

Figure 6.26: Magnetic field data at various fill pressures and locations for the $100 \mathrm{~J}, 10 \mathrm{kHz}$ circuit. Vacuum traces are shown at each diagnostic by dotted lines. 

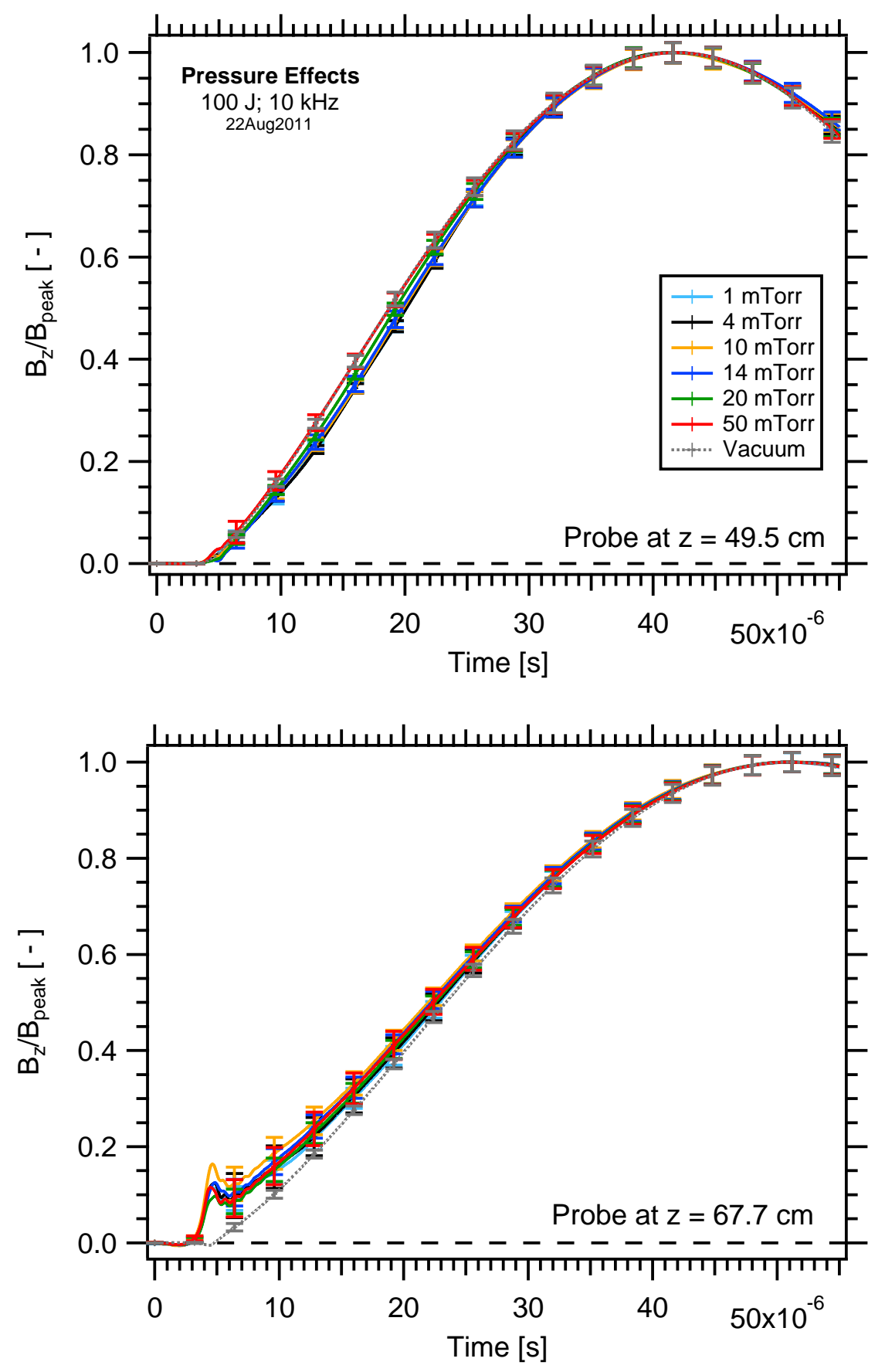

Figure 6.27: Peak normalized downstream magnetic field data at various fill pressures for the $100 \mathrm{~J}, 10 \mathrm{kHz}$ circuit. Vacuum traces are shown at each diagnostic by dotted lines. 
narrow end of the coil initially. Reversal on the probes at $\mathrm{z}=5 \mathrm{~cm}$ and $\mathrm{z}=10 \mathrm{~cm}$ appears before reversal on the midplane probe. This trend is seen in other conical FRC geometries as well.

The downstream magnetic field signatures for the $100 \mathrm{~J}, 10 \mathrm{kHz}$ data show no signficant change from their vacuum pattern. The downstream signatures also show little noticeable changes with pressure. The probe signatures measured closer to the coils (at $\mathrm{z}=49.5$ $\mathrm{cm})$ appear to deflect very slightly with decreasing pressure, but this is likely due to the changing field inside the coils.

The current and magnetic field signatures for the $500 \mathrm{~J}, 10 \mathrm{kHz}$ circuit are shown in Figures 6.28 - 6.30. Downstream plasma flux data is presented in Figure 6.30 for 1 mTorr, 4 mTorr, and 50 mTorr only. The $500 \mathrm{~J}$ data was collected from at least 3 shots at each background pressure. Confidence intervals for all traces is a combination of the experimental uncertainty in each diagnostic and the statistical spread in the data among shots.

The large $\mathrm{dI} / \mathrm{dt}$ in the $500 \mathrm{~J}$ circuit compared to the $100 \mathrm{~J}$ circuit produces a much stronger plasma current at all pressures, even at 50 mTorr. The plasma current is only weakly dependant on plasma pressure up to 20 mTorr, suggesting a fully ionized current with low resistance from neutral collisions. The reversal also happens much faster in the $500 \mathrm{~J}$ case than the $100 \mathrm{~J}$ case, within $600 \mathrm{~ns}$ of the switch closing. The 50 mTorr case shows a delayed reversal of $3.5 \mu \mathrm{s}$.

Despite the fast development of the plasma current, the reversal only lasts $9.3 \mu \mathrm{s}$. This is true for all pressures. Following the loss of reversal, the excluded field signature disappears on the outer probes. The field returns to the vacuum cases. The disappearance of the 

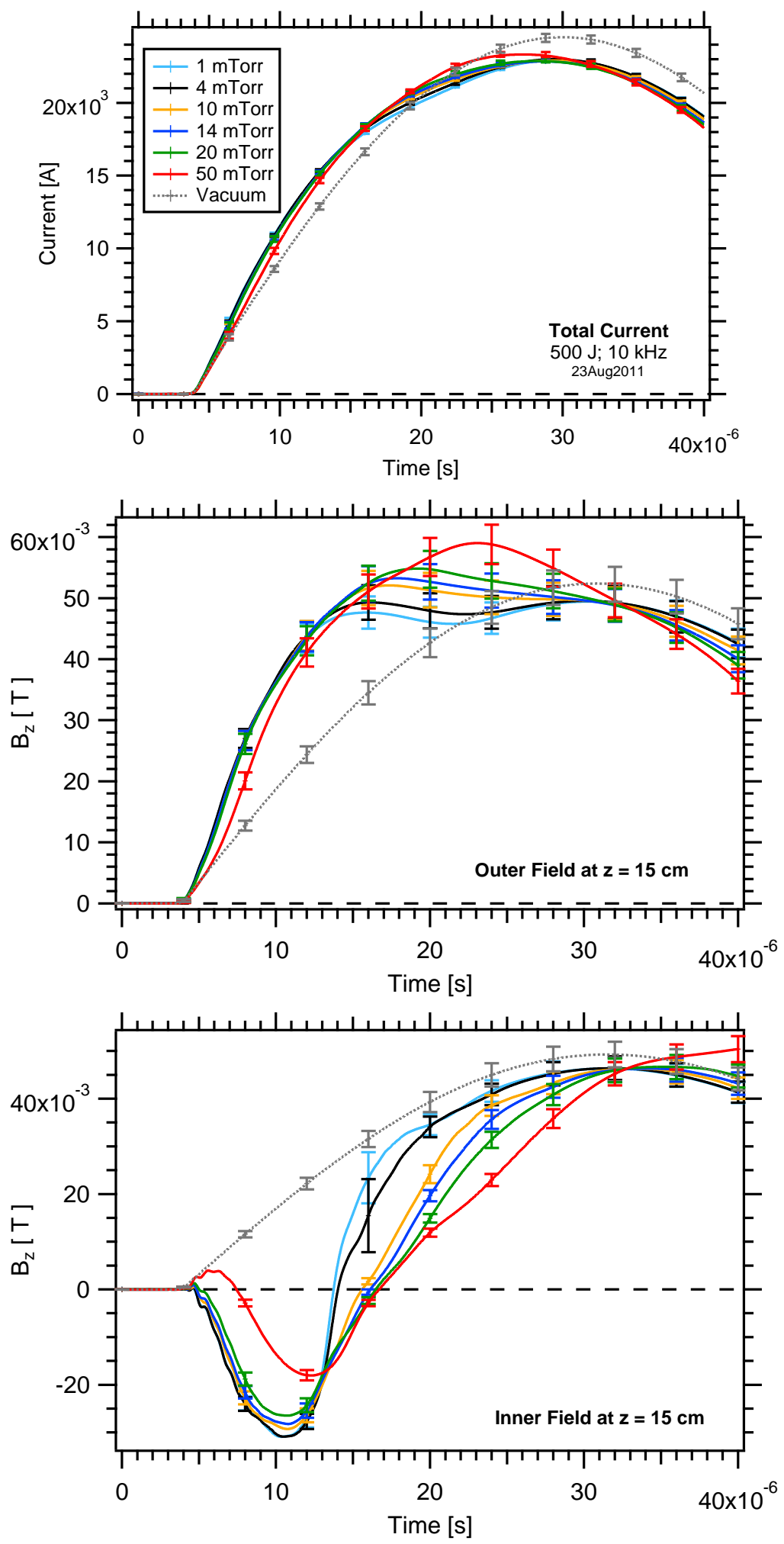

Figure 6.28: Total current and midplane magnetic field data at various fill pressures for the $500 \mathrm{~J}, 10 \mathrm{kHz}$ circuit. Vacuum traces are shown at each diagnostic by dotted lines. 
inductive load either indicates plasmoid termination or a translating plasmoid. This topic will be discussed in more detail in Chapter 7 .

The downstream magnetic field signatures for the $500 \mathrm{~J}, 10 \mathrm{kHz}$ data show a slight change when compared to their vacuum counterpart. The first probe shows a convex feature and the second probe shows a concave nature. Neither feature changes drastically with pressure. The downstream plasma flux data for $500 \mathrm{~J}$ is shown in the bottom graphs of Figure 6.30. The plasma flux peaks on both probes at $12 \mu \mathrm{s}$ for the low pressure case and at $17 \mu \mathrm{s}$ for the 50 mTorr case. The 1 mTorr fill creates a higher ion flux than the lower pressures, with over $6 \mathrm{kA} / \mathrm{m}^{2}$ collected by the probe. This trend will be discussed in more detail in Chapter 7

The current and magnetic field signatures for the $1 \mathrm{~kJ}, 10 \mathrm{kHz}$ circuit are shown in Figures 6.31- 6.33. The data was collected from at least 3 shots for the $1 \mathrm{mTorr}$ through $14 \mathrm{mTorr}$ case. A circuit fault prevented more than 2 shots at 20 mTorr and 50 mTorr. Confidence intervals for all traces is a combination of the experimental uncertainty in each diagnostic and the statistical spread in the data among shots.

The results from the $1 \mathrm{~kJ}$ tests are similar in behavior to the $500 \mathrm{~J}$ circuit. The field reversal happens quickly, within $200 \mathrm{~ns}$ of switch closing. Little change in field signatures is seen with increasing pressure, except for the 50 mTorr fill. The reversal lasts $8.7 \mu \mathrm{s}, 600 \mathrm{~ns}$ shorter than the $500 \mathrm{~J}$ case. After reversal is lost, the field returns to the vacuum state. As mentioned in the $500 \mathrm{~J}$ discussion, the disappearance of the inductive load either indicates plasmoid termination or a translating plasmoid and will be discussed in more detail in Chapter 7

The downstream magnetic field signatures for the $1 \mathrm{~kJ}, 10 \mathrm{kHz}$ data show a substantial 
change from the vacuum traces. The first probe shows a flat profile in the magnetic field rise at $12 \mu \mathrm{s}$, lasting $4 \mu \mathrm{s}$. This feature is most apparent in the low pressure data. The severe noise in the probe at $\mathrm{z}=67.7 \mathrm{~cm}$ makes it difficult to determine the corresponding feature, but it appears to follow the same behavior. The comparison of these features with the upstream probes will be analyzed in Chapter 7 . 

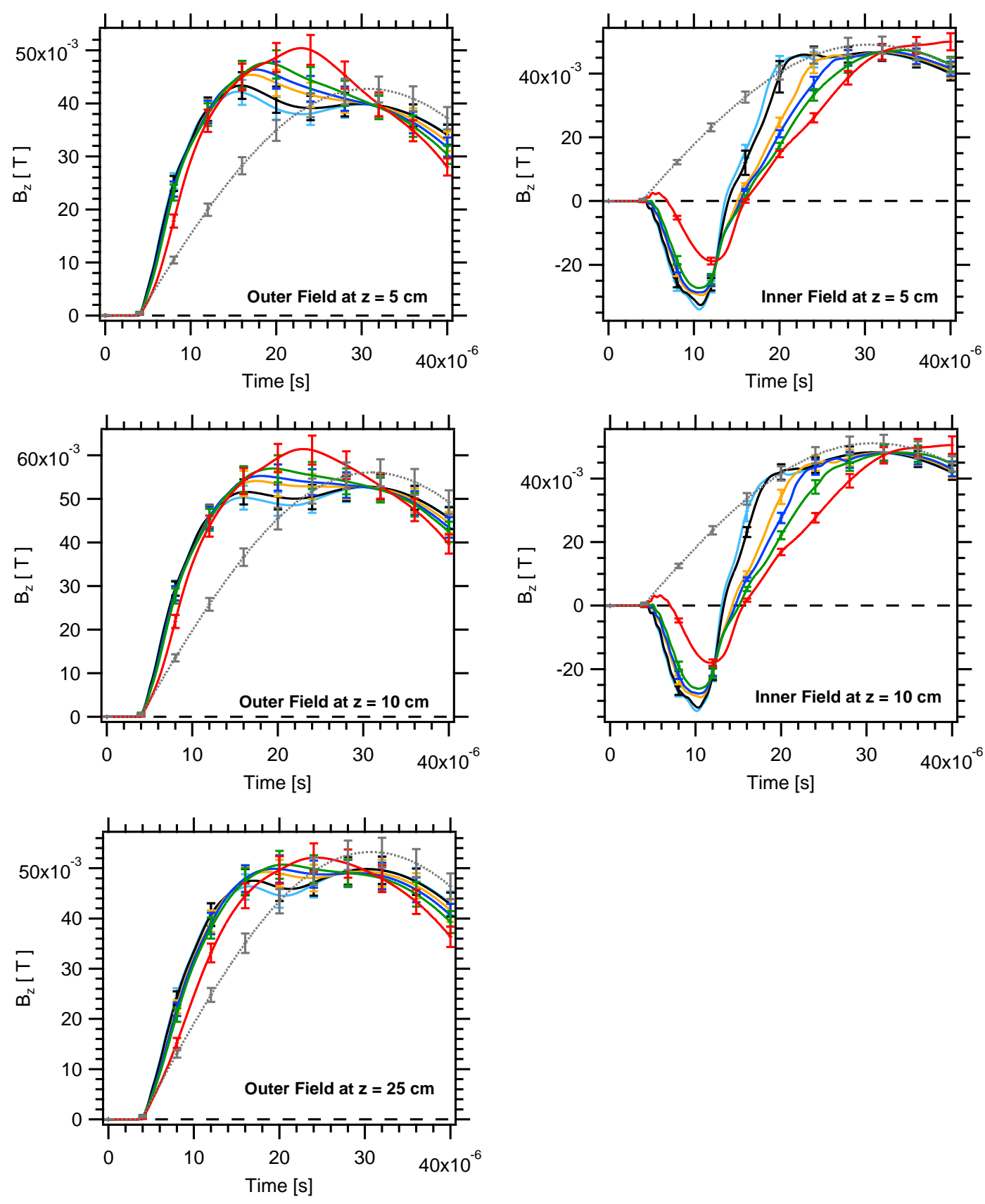

Figure 6.29: Magnetic field data at various fill pressures and locations for the $500 \mathrm{~J}, 10 \mathrm{kHz}$ circuit. Vacuum traces are shown at each diagnostic by dotted lines. 

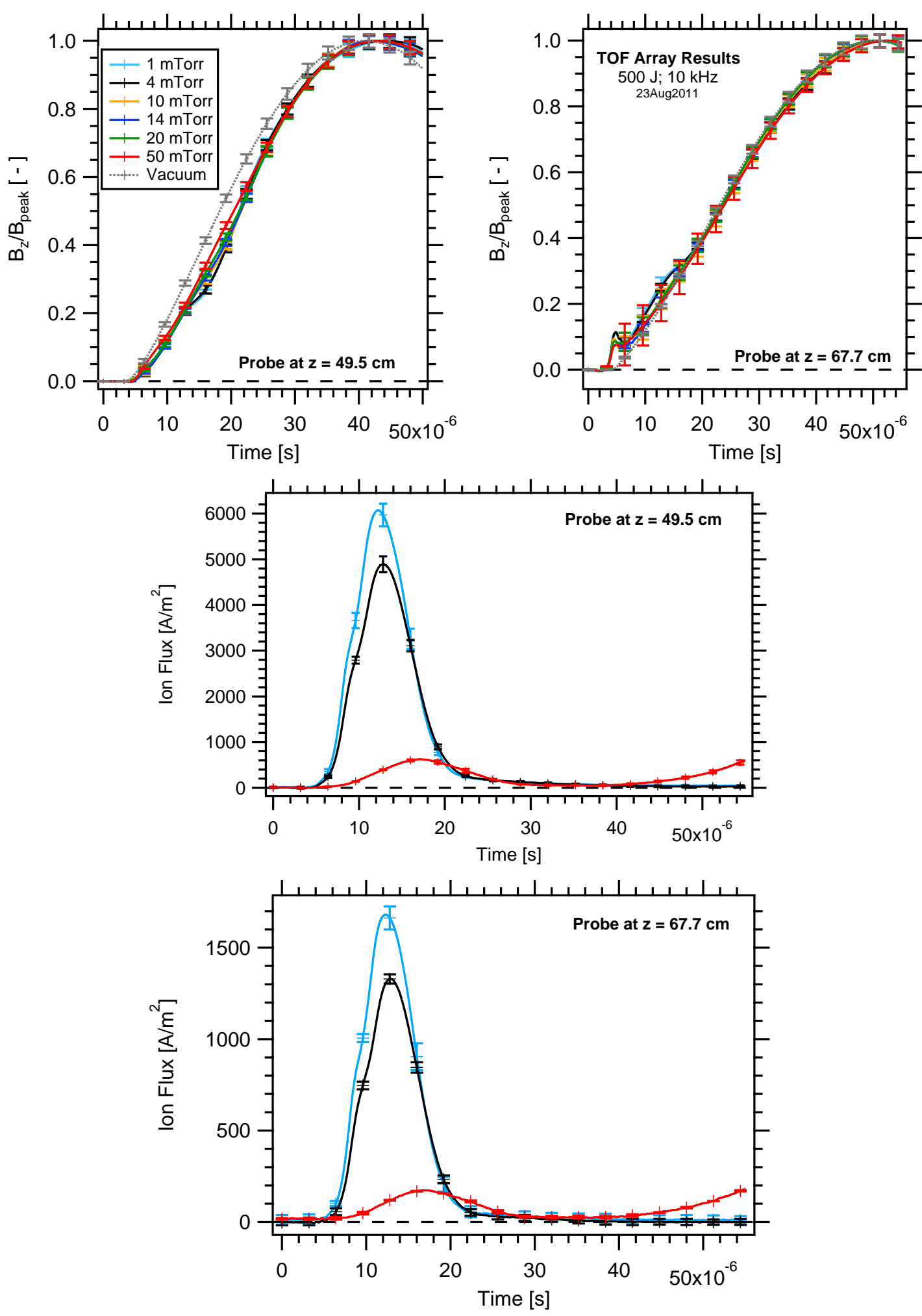

Figure 6.30: TOF probe data at various fill pressures for the $500 \mathrm{~J}, 10$ $\mathrm{kHz}$ circuit, including peak normalized downstream magnetic field data and plasma flux measurements. Vacuum traces are shown at each diagnostic by dotted lines. 

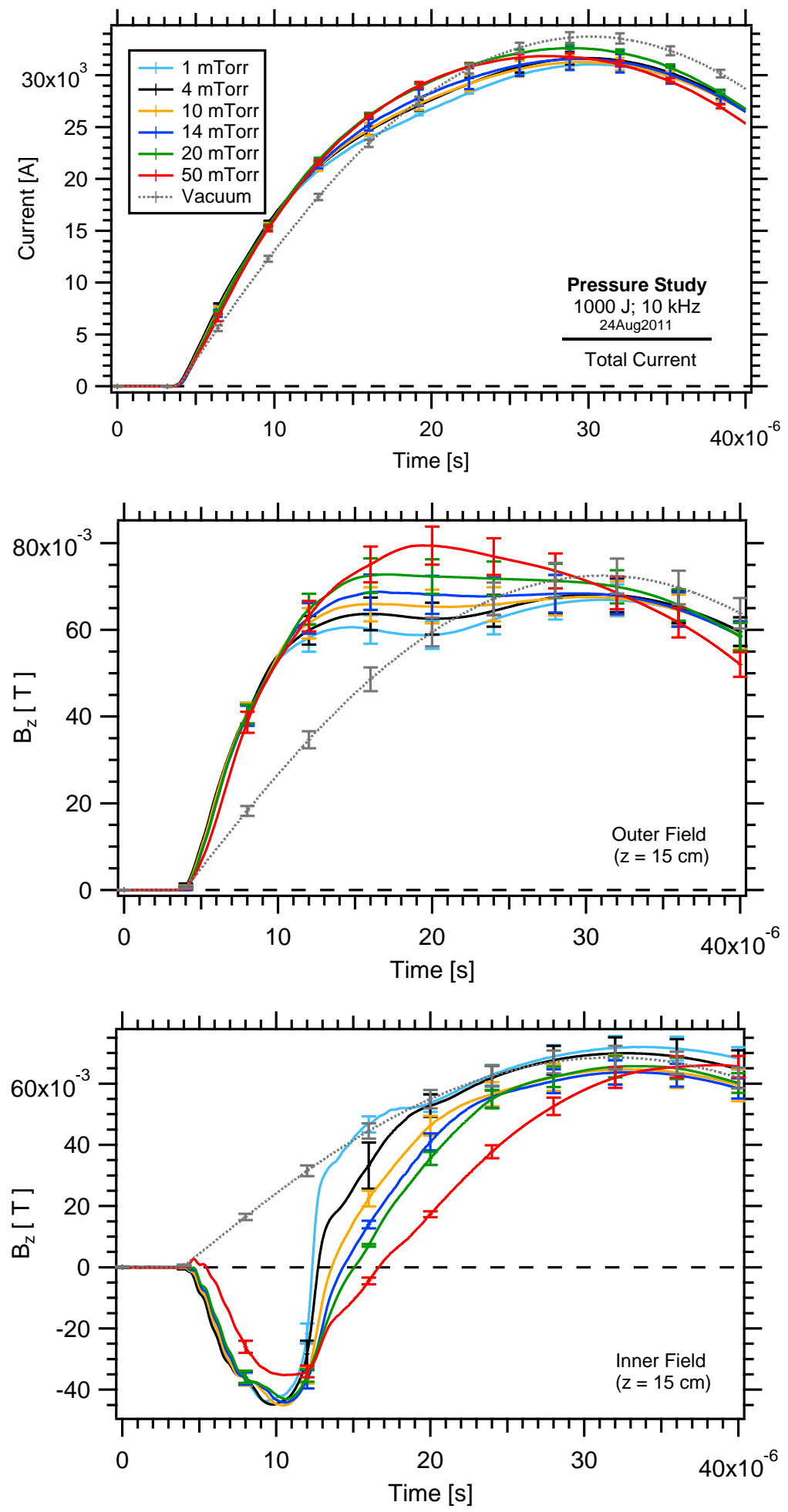

Figure 6.31: Total current and midplane magnetic field data at various fill pressures for the $1000 \mathrm{~J}, 10 \mathrm{kHz}$ circuit. Vacuum traces are shown at each diagnostic by dotted lines. 

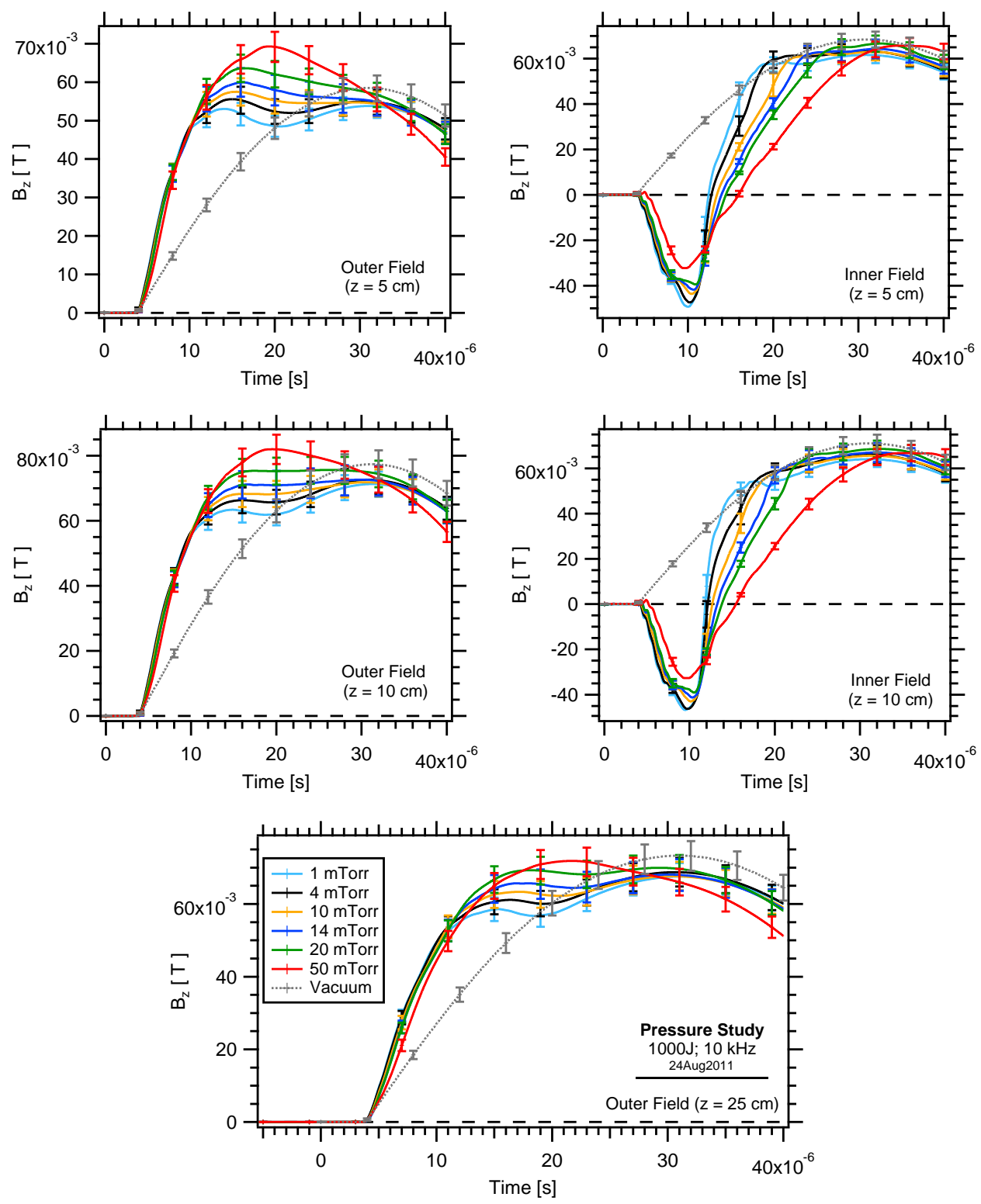

Figure 6.32: Magnetic field data at various fill pressures and locations for the $1000 \mathrm{~J}, 10 \mathrm{kHz}$ circuit. Vacuum traces are shown at each diagnostic by dotted lines. 

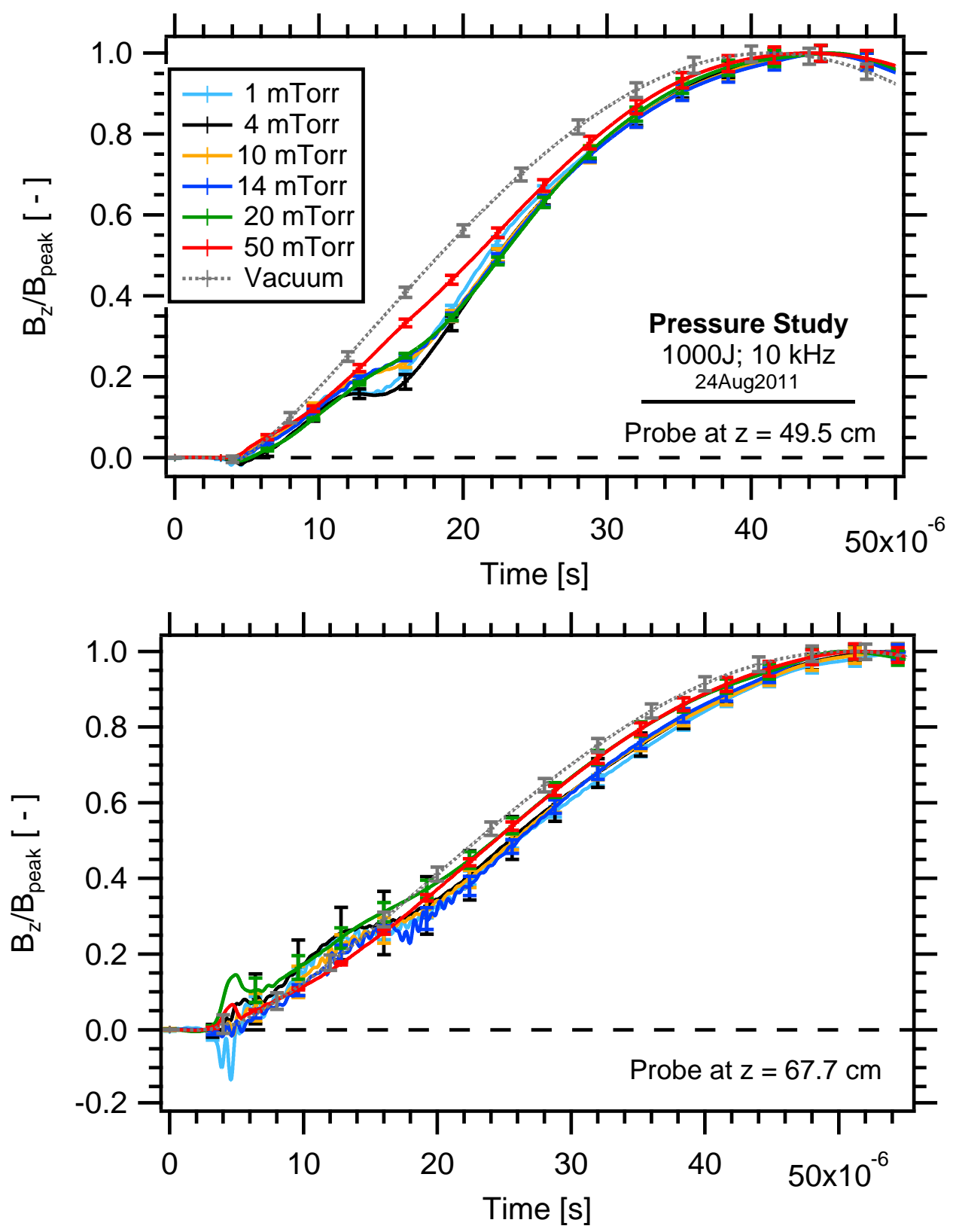

Figure 6.33: Peak normalized downstream magnetic field data at various fill pressures for the $1000 \mathrm{~J}, 10 \mathrm{kHz}$ circuit. Vacuum traces are shown at each diagnostic by dotted lines. 


\subsubsection{Translation Data: $20 \mathrm{kHz}$}

The current and upstream magnetic field at all tested pressures for the $20 \mathrm{kHz}$ circuit at $100 \mathrm{~J}$ is shown in Figures 6.34 and 6.35. This data was averaged from 5 shots at each background pressure. Figure 6.34 displays the current data and the magnetic fields (inner and outer) at the coil midplane $(\mathrm{z}=15 \mathrm{~cm})$. Figure 6.35 shows the magnetic field data upstream of the midplane (at $\mathrm{z}=5 \mathrm{~cm}$ and $\mathrm{z}=10 \mathrm{~cm})$ and at the far end of the coil $(\mathrm{z}=25$ $\mathrm{cm})$. Confidence intervals for all traces is a combination of the experimental uncertainty in each diagnostic and the statistical spread in the data among shots. The statistical spread in the data is quite small (2-3\%) with the uncertainty in magnetic field proportionality constants comprising the largest source of error.

The effect of background pressure on plasmoid formation is especially evident in the inner magnetic field data. With the exception of a fill pressure at 50 mTorr, the change in current and outer magnetic field between pressures is only a few percent. The inner magnetic field data shows more distinct plasmoid behavior at different pressures. At low pressures, higher magnetic fields are generated on the inner wall signaling larger plasma currents. This trend is somewhat expected; low fill pressures create less resistance for plasmoid current since the current carriers have fewer interactions with the neutral gas. These results suggest that plasmoid translation is more likely at low fill pressures (1-4 mTorr) due to the stronger Lorentz force resulting from higher plasmoid currents and lower plasmoid mass.

The inner field for the $100 \mathrm{~J}, 10 \mathrm{kHz}$ circuit reverses $1 \mu \mathrm{s}$ after switch closing for the 4 mTorr case. The reversal lasts $10.3 \mu \mathrm{s}$, which is $1 \mu \mathrm{s}$ longer than the $500 \mathrm{~J}$ and $1 \mathrm{~kJ}$ cases for the $10 \mathrm{kHz}$ circuit. 

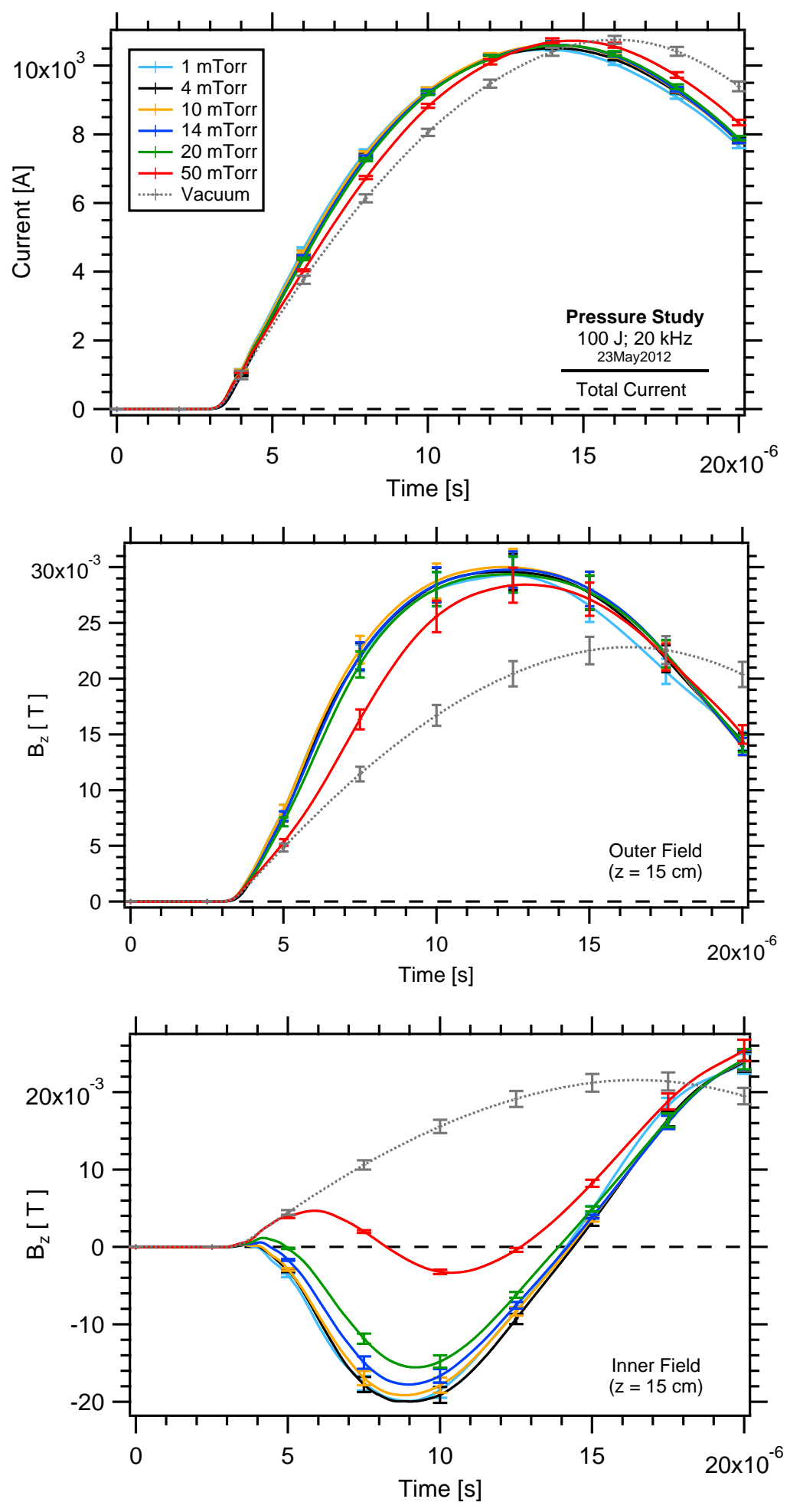

Figure 6.34: Total current and magnetic field data at various fill pressures for the $100 \mathrm{~J}, 20 \mathrm{kHz}$ circuit. Vacuum traces are shown at each diagnostic by dotted lines. 

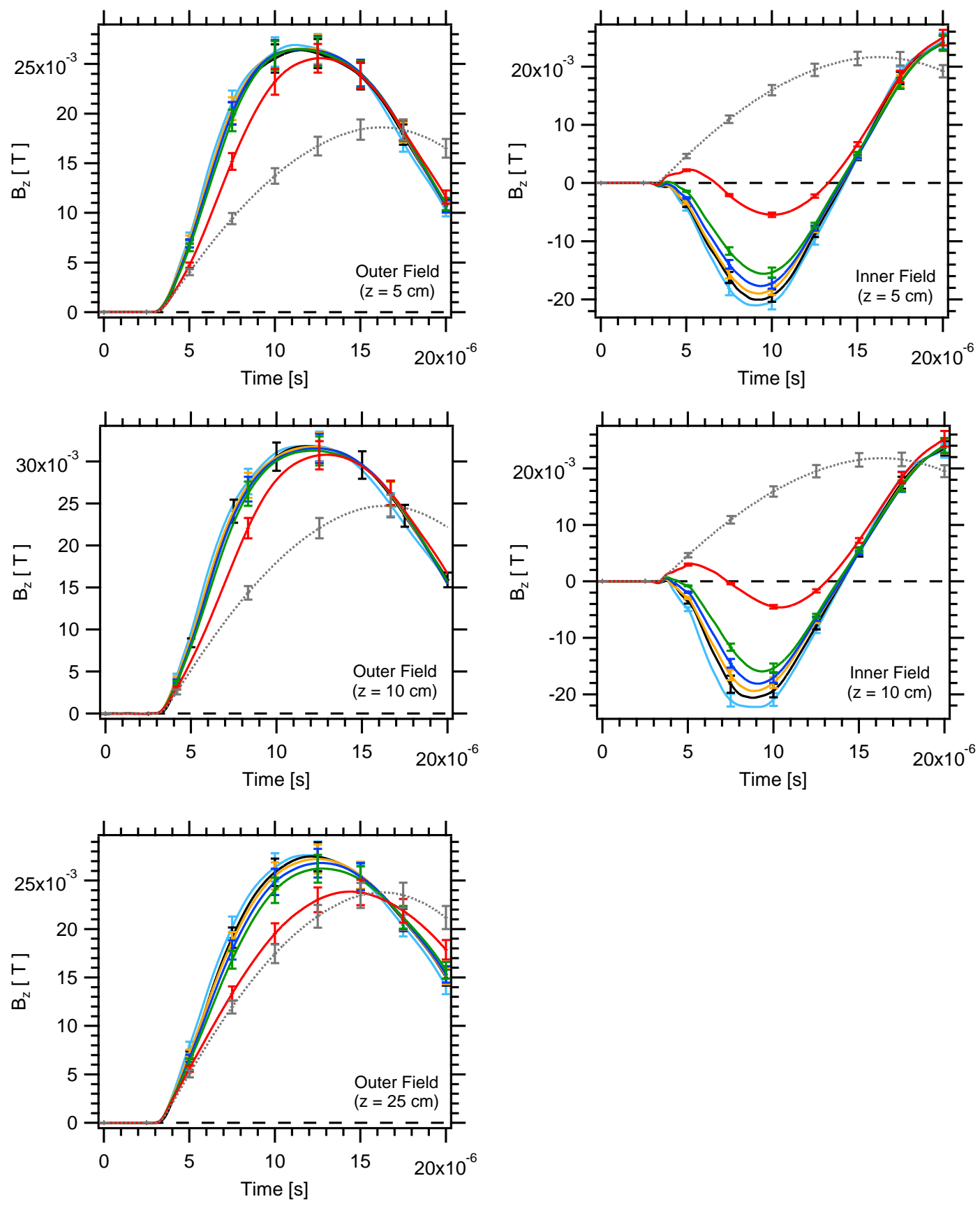

Figure 6.35: Magnetic field data at various fill pressures for the $100 \mathrm{~J}, 20$ $\mathrm{kHz}$ circuit. Vacuum traces are shown for each diagnostic by a dotted line.

A full set of downstream data was collected for the $100 \mathrm{~J}, 20 \mathrm{kHz}$ study, shown in Figure 6.36. The downstream magnetic field data did not have to be normalized for amplifier glitches, though a correction for the probes' proportionality constants were not made. The 

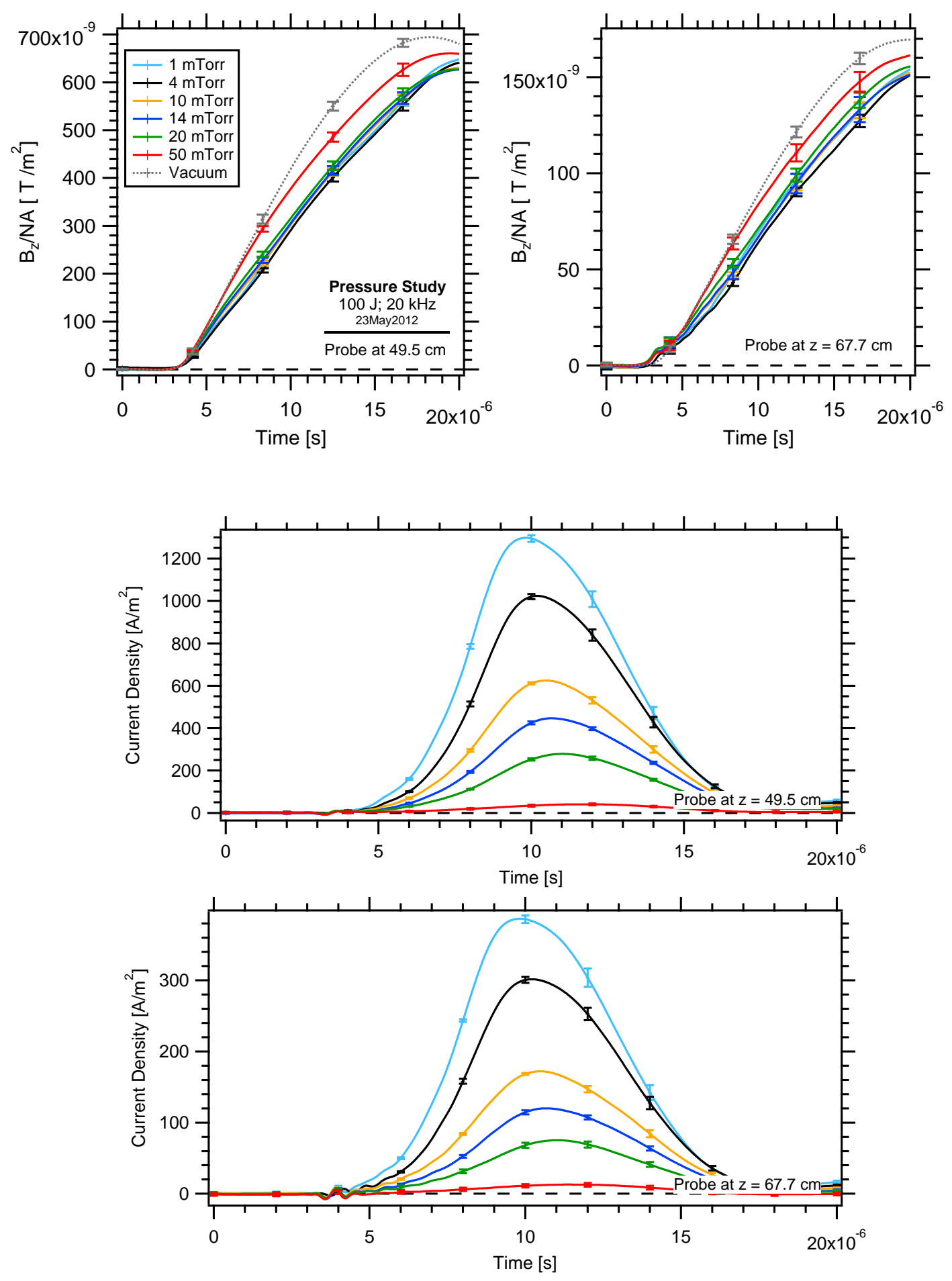

Figure 6.36: TOF probe data at various fill pressures for the $100 \mathrm{~J}, 20$ $\mathrm{kHz}$ circuit, including peak normalized downstream magnetic field data and plasma flux measurements. Vacuum traces are shown at each diagnostic by dotted lines. 
plasma flux at each location was also collected for each pressure. The magnetic field data does not change appreciably with pressure, with the exception of the 50 mTorr case. The plasma shot data for both probes show a lower peak field than the vacuum data. However, no additional features are apparent deviating significantly from the vacuum traces.

The downstream plasma flux data shows that the current density measured by the probe decreases with pressure, topping $1 \mathrm{kA} / \mathrm{m}^{2}$ with a $4 \mathrm{mTorr}$ fill. The peaks also seem to lag with increasing pressure. The plasma flux profiles follow a bell-shaped behavior without any strong peaks visible. While this is more indicative of a thermal plasma spilling out of the device than a translating plasmoid; full evidence of translation will be discussed in Chapter7. 


\section{Chapter 7}

\section{Translation Results}

This chapter discusses the translation results from the experimental data presented in Chapter 6. Section 7.1 describes the data reduction applied to the data to estimate plasmoid translation velocities. Section 7.2 presents the plasmoid velocity results, demonstrating that the plasmoid failed to translate from the formation region for all test conditions. Section 7.3 discusses possible causes for the failed translation, including a limit in inductive current, insufficient conductivity, and gross instabilities.

\subsection{Translation Data Reduction}

The experimental data presented in Chapter 6 is sufficient to resolve the plasmoid's velocity at each operating point. The external probes are used as the first time-of-flight (TOF) array, with the downstream probes forming the secondary array. Axial staggered signatures on both arrays are a clear indicator of a translating magnetized plasmoid. Data from plasma 
probes can be used to confirm that the magnetized structure contains a dense plasma. Small switching delays between data sets can cause errors in velocity measurements. Velocity data was collected from single shots when possible.

A single shot of data from the $20 \mathrm{kHz}, 100 \mathrm{~J}$ circuit is shown in Figure 7.1. Uncertainty estimates are omitted from this graph for clarity. This figure shows that each probe recorded a different magnitude of field. The velocity measurements from this data required comparing propagating features in axially-separated probes and the different field magnitudes made it difficult to pick out features among different probes. The data was peak-normalized to remove the differences in field magnitudes, making it easier to compare features and trends in all probes.

The first step in processing the data for this research was to establish that the plasmoid traveled out of the coils with a detectable velocity. Once the velocity was established, plasma probe sweeps were to be used to estimate momentum and translation efficiency. The results presented in Section 7.2 show that the plasmoid failed to translate under all tested conditions. This means it was not necessary to estimate the plasmoid momentum and translation efficiency.

\subsection{Velocity Results}

Plasmoid velocity can be measured by comparing when certain features appear at different axial locations along the velocity vector. Several examples of this have been provided previously in this dissertation. Characteristic magnetic probe signatures using electromagnetic modeling for a constant-velocity plasmoid were computed in Section 4.3 , 

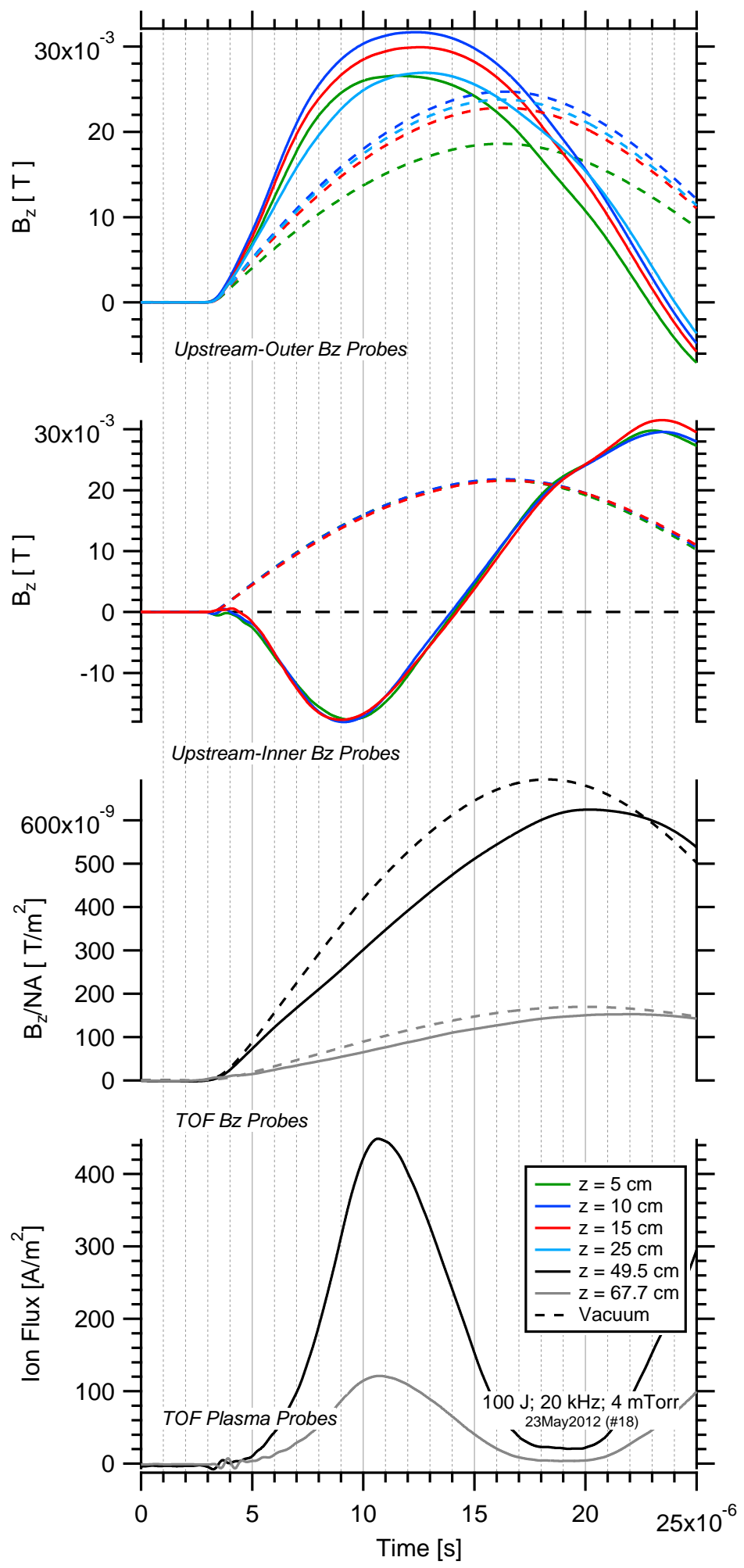

Figure 7.1: Full data set from a single shot at $100 \mathrm{~J}$ with the $20 \mathrm{kHz}$ bank and a 4 mTorr gas fill. 
Data from a TOF plasma probe array from a similar RMF-FRC translation experiment were presented in Figure 5.39. The trends from these illustrations are discussed in detail here so that this information can be used to decipher the translation data from the XOCOT-T3 experiments.

Magnetic field modeling in Section 4.3 was used to calculate the magnetic field from a translating plasmoid. The results in Figure 4.28 show characteristic magnetic field signatures for a plasmoid traveling at $50 \mathrm{~km} / \mathrm{s}$ through the coils. The signatures were measured using probes spaced $5 \mathrm{~cm}$ apart inside the coils and by probes placed in the path of the plasmoid downstream of the coils. A peak field was recorded on the coil probes as the trailing edge of the plasmoid passed by. The time delay between these peak fields was $1 \mu \mathrm{s}$, corresponding to a velocity of $50 \mathrm{~km} / \mathrm{s}$. The peaks on the outer probes and inner probes for the same axial location occurred at the same time, as well. The downstream probes recorded a flat signature as the plasmoid traveled over it, lasting $5 \mu$ s for a plasmoid $25 \mathrm{~cm}$ in length.

The constant velocity in the magnetic field model was used for numerical stability, though in reality the plasmoid should accelerate through the coil. This is apparent in the calculated trajectory from the annular electromagnetic model shown in Figure 4.18. The plasmoid's acceleration through the coils will produce different delays than the uniform delay shown in Figure 4.28, with shorter delays occurring with increasing distance from the beginning of the coil. The peak velocity is not expected until the plasmoid is $10 \mathrm{~cm}$ outside the coil, according to the trajectory shown in Figure 4.18. This means that TOF probes should record a velocity comparable to the terminal velocity, but the coil probes should record a lower velocity. The peak velocities for $500 \mathrm{~J}, 10 \mathrm{kHz}$ circuit are expected to be $160 \mathrm{~km} / \mathrm{s}$, according the predictions in Figure 4.19. This means that the time delay between features 
on probes spaced $5 \mathrm{~cm}$ apart inside the coil should be greater than $300 \mathrm{~ns}$. The time delay between the probe located at $\mathrm{z}=25 \mathrm{~cm}$ and the first probe in the TOF array is expected to be around $1.25 \mu$ s and the delay between probes in the downstream TOF array should be 1 $\mu$ s apart.

The plasma flux measurements appearing on the downstream plasma probes in a TOF array appear as sharp peaks, spaced by a delay inversely proportional to the plasmoid velocity. This was seen in an RMF-FRC translation experiment [3], with the data reproduced in Figure 5.39. The first sharp peak in each signal corresponds to the arrival of the leading edge of the plasmoid. The dense plasma front traveling at high velocity has only a brief encounter with the probe, which is why it appears as a sharp peak. Additional signal follows the initial peak as the remainder of the plasmoid passes by the probe.

Magnetic field data from single shot at each pressure and energy is presented in this Section for the $10 \mathrm{kHz}$ and $20 \mathrm{kHz}$ discharge circuits. Plasma flux measurements are included, when available. This data was used to estimate the plasmoid velocity. Each trace was peak-normalized for the first quarter cycle. Error bars are omitted for clarity.

\subsubsection{Velocity Results: $10 \mathrm{kHz}$}

The results for the $10 \mathrm{kHz}$ circuit at $100 \mathrm{~J}$ are presented in Figures 7.2 through Figure 7.7. A single shot from each pressure and energy setting is shown, with all traces peak normalized to show trends in the data.

The translation results at $100 \mathrm{~J}$ for each pressure do not show a translating plasmoid. The switch closes at $4 \mu$ s and the inner field begins to reverse several $\mu$ s later. If the reversal 

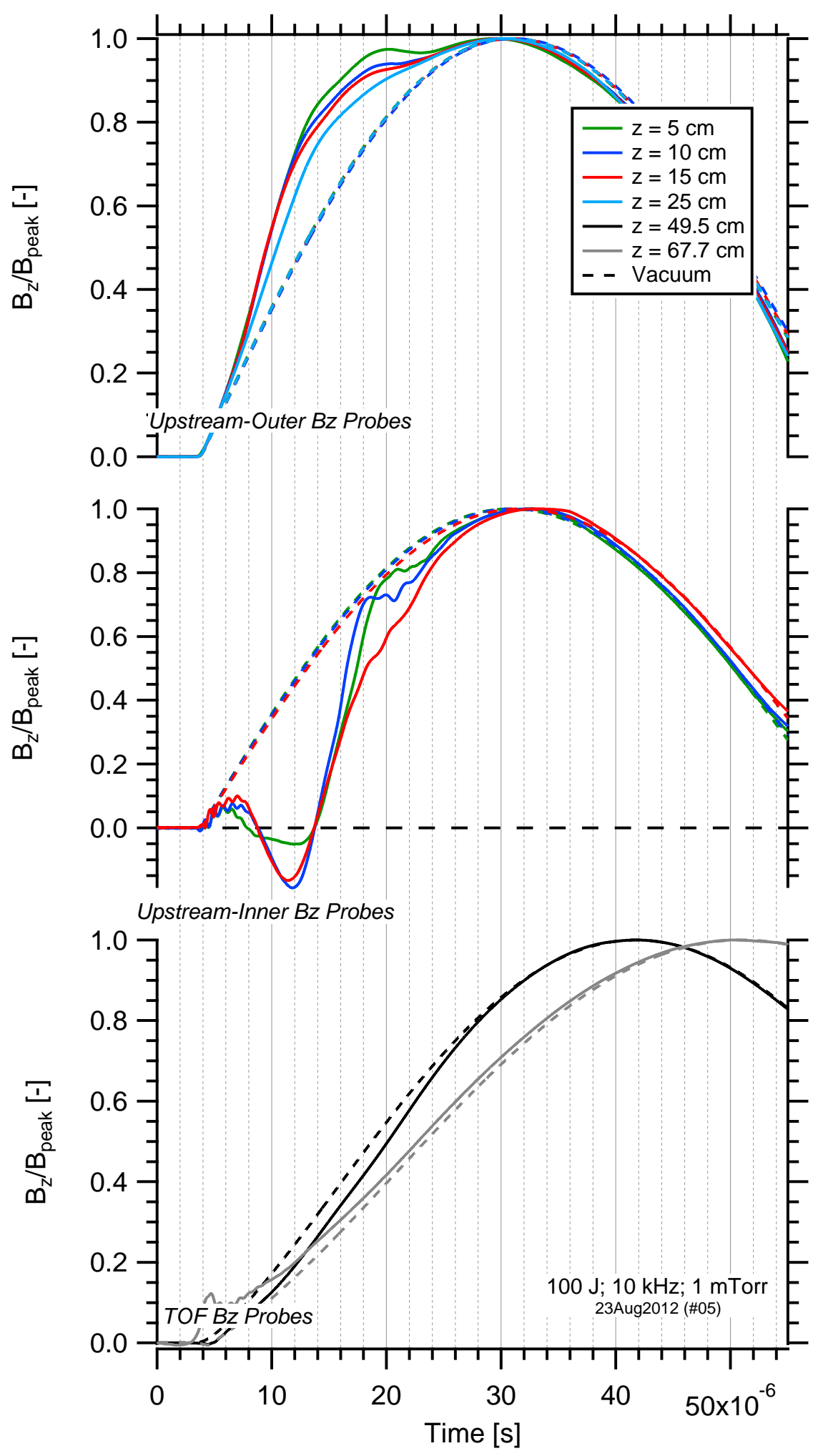

Figure 7.2: Translation results for the $10 \mathrm{kHz}$ circuit at $100 \mathrm{~J}$, with a 1 mTorr fill pressure. 

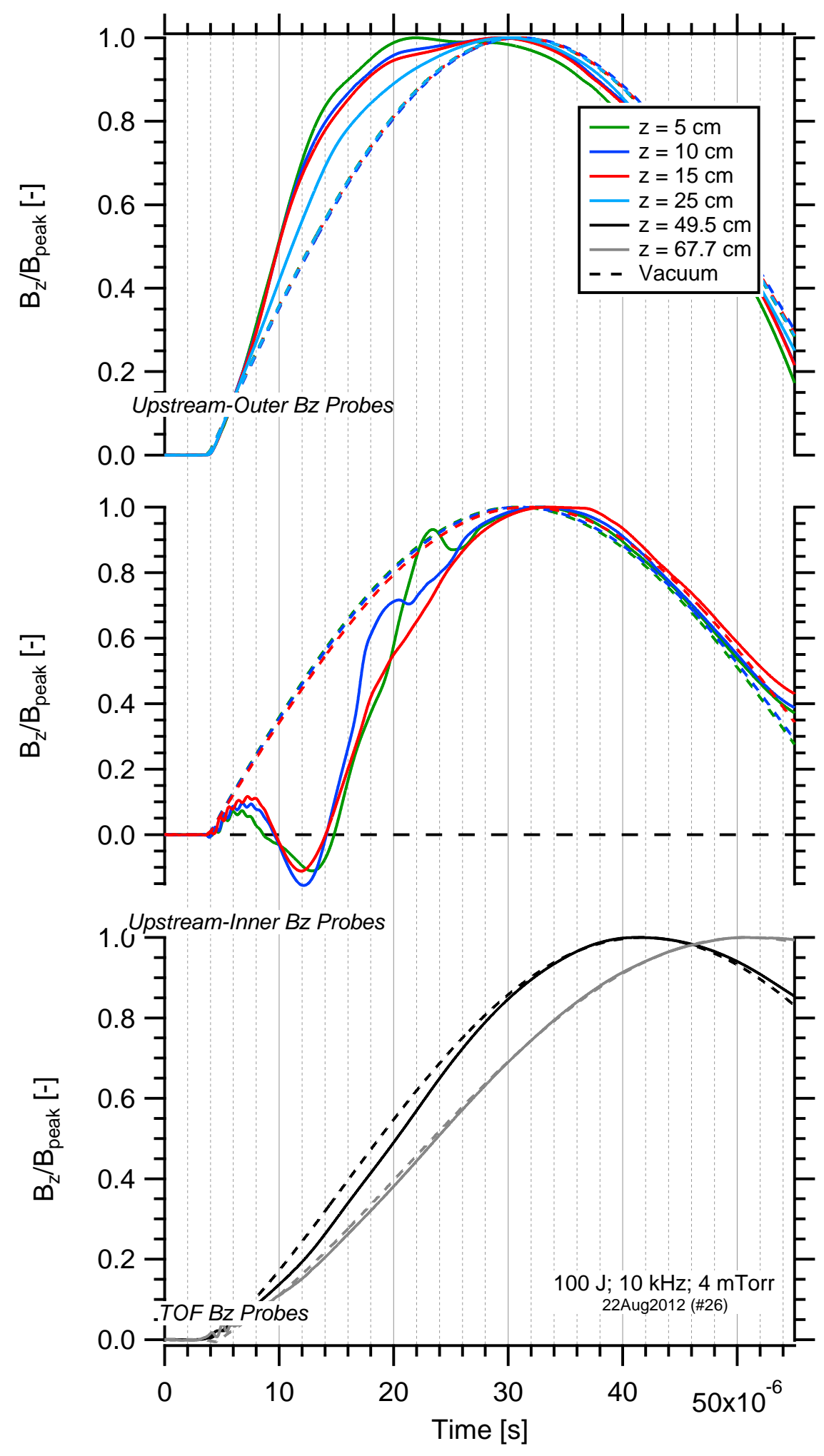

Figure 7.3: Translation results for the $10 \mathrm{kHz}$ circuit at $100 \mathrm{~J}$, with a 4 mTorr fill pressure. 

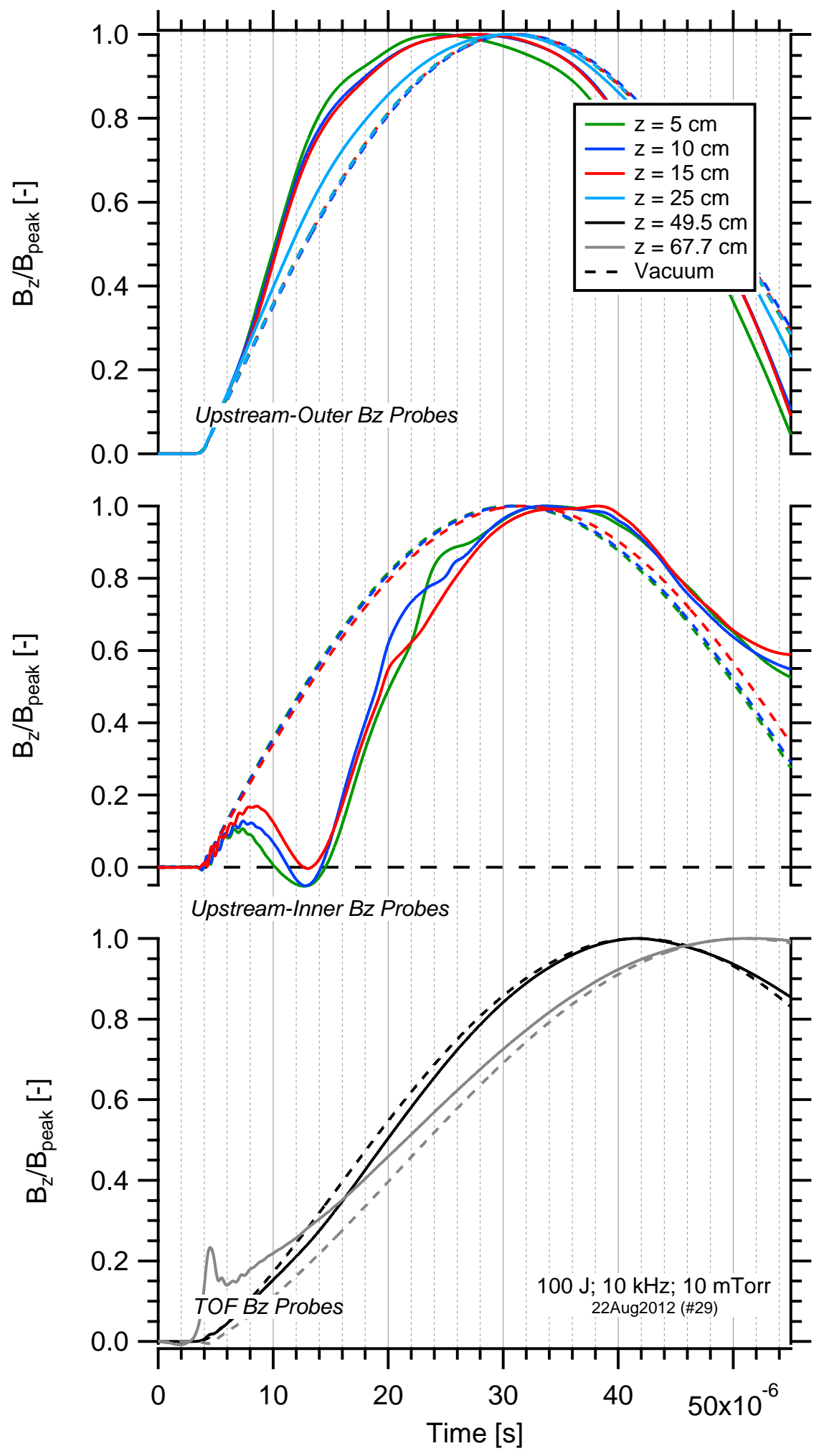

Figure 7.4: Translation results for the $10 \mathrm{kHz}$ circuit at $100 \mathrm{~J}$, with a 10 mTorr fill pressure. 

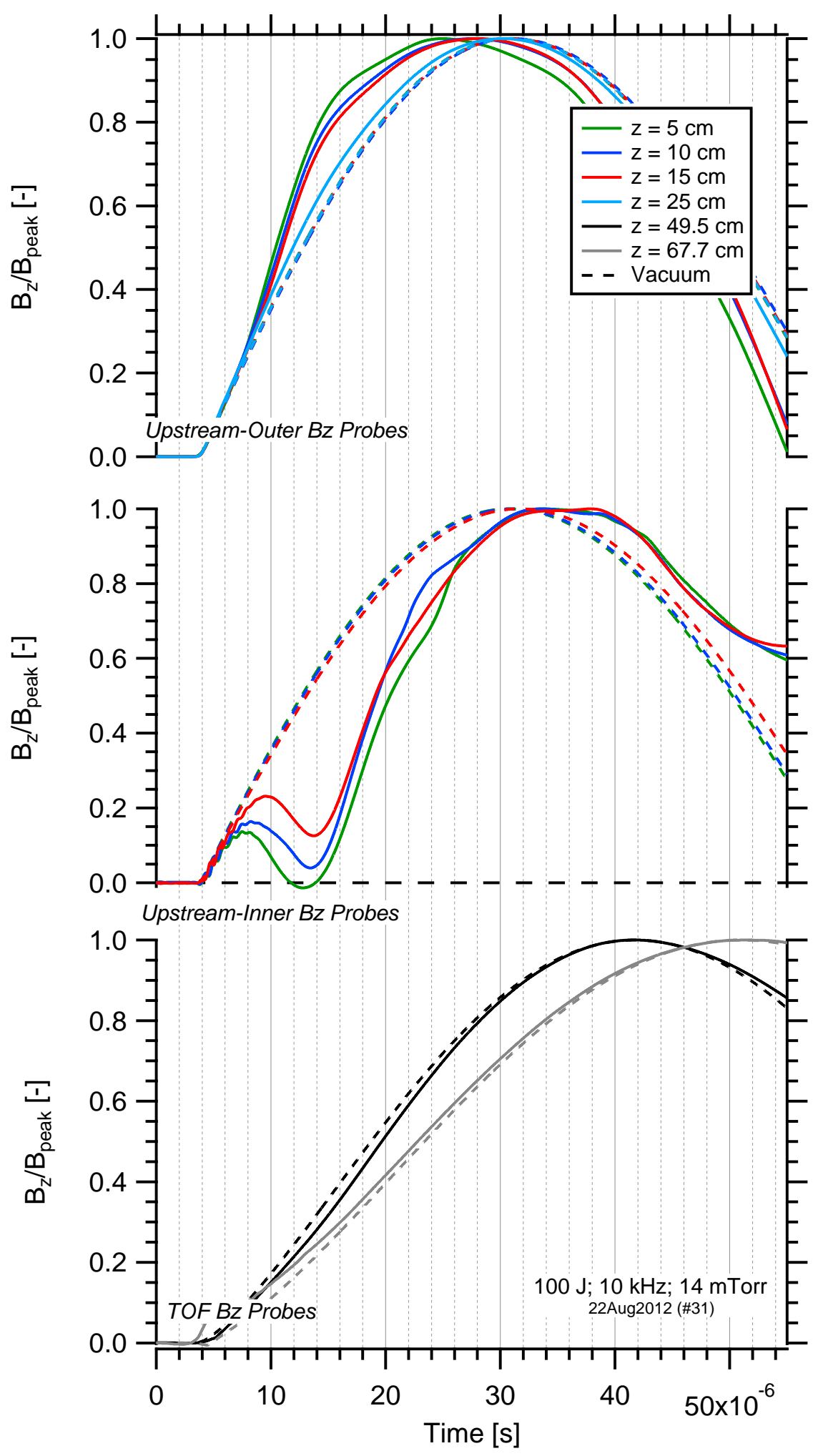

Figure 7.5: Translation results for the $10 \mathrm{kHz}$ circuit at $100 \mathrm{~J}$, with a 14 mTorr fill pressure. 

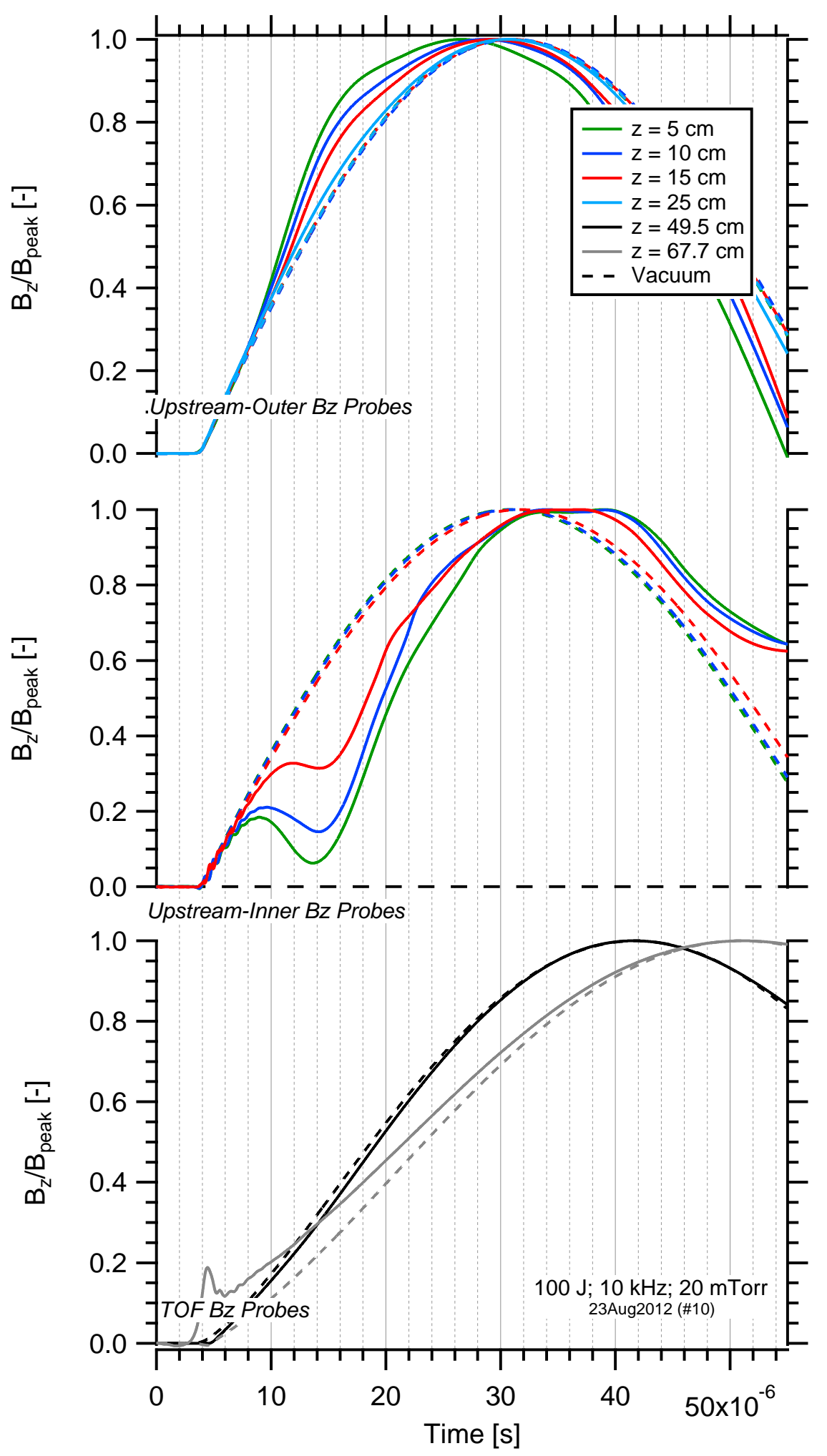

Figure 7.6: Translation results for the $10 \mathrm{kHz}$ circuit at $100 \mathrm{~J}$, with a 20 mTorr fill pressure. 

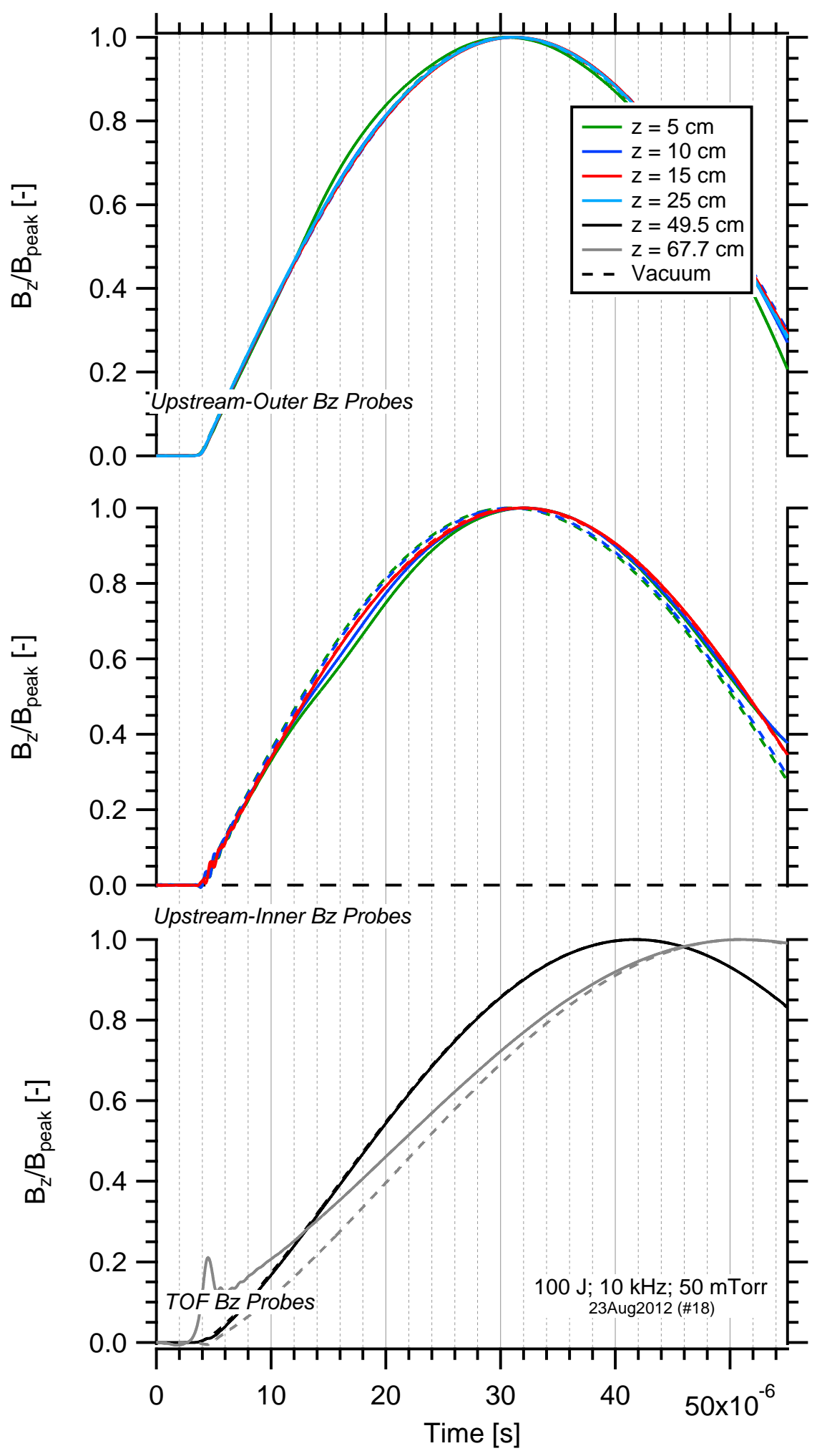

Figure 7.7: Translation results for the $10 \mathrm{kHz}$ circuit at $100 \mathrm{~J}$, with a 50 mTorr fill pressure. 
happens at all it is short lived, lasting at most $2 \mu$ s before changing back toward the vacuum field. The peak reversed field on the inner probes does not correspond to any distinct peak on the outer probe. The outer probes do not show any staggered features along their array; they rise and fall in unison. Additionally, the downstream probes show no magnetic field structure passing by. It's clear from all these observations that even when a reversed field plasmoid is formed, it never leaves the coils. The short-lived and relatively small reversal suggests that the energy content of the plasmoid is too low form a sufficient current for the Lorentz force.

The results for the $10 \mathrm{kHz}$ circuit at $500 \mathrm{~J}$ are presented in Figures 7.8 through Figure 7.13. A single shot from each pressure and energy setting is shown, with all traces peak normalized to show trends in the data. Data from the downstream plasma flux probes is presented for the $1 \mathrm{mTorr}, 4 \mathrm{mTorr}$, and $50 \mathrm{mTorr}$ data sets.

The translation results at $500 \mathrm{~J}$ show that while the field reversal happens very quickly, the plasmoid does not appear to translate from the coils. The field reversal peaks 6-7 $\mu \mathrm{s}$ after the switch closes, but the upstream inner probe $(\mathrm{z}=5 \mathrm{~cm})$ peaks after the probes at $\mathrm{z}=10 \mathrm{~cm}$ and $\mathrm{z}=15 \mathrm{~cm}$. It also crosses through $\mathrm{B}=0$ a full $\mu$ s after the downstream probes. Furthermore, as the reversal peaks the field on the outer coil probes continue to climb and don't reach a peak until $5 \mu$ s later. The inner and outer probes at the same axial location should show the same behavior in time with a translating plasmoid. The probe at $\mathrm{z}=25 \mathrm{~cm}$ reaches its peak $1 \mu \mathrm{s}$ after the midplane probe and while this may also be a translation indicator corresponding to the expected velocity $(150 \mathrm{~km} / \mathrm{s})$, the downstream magnetic field probes peak don't agree. They appear to have an inflection several $\mu \mathrm{s}$ before the outer probes peak and concurrent with the zero-crossing of the inner coil field.

The plasma flux measurements support this conclusion. The plasma flux (shown in Figures 

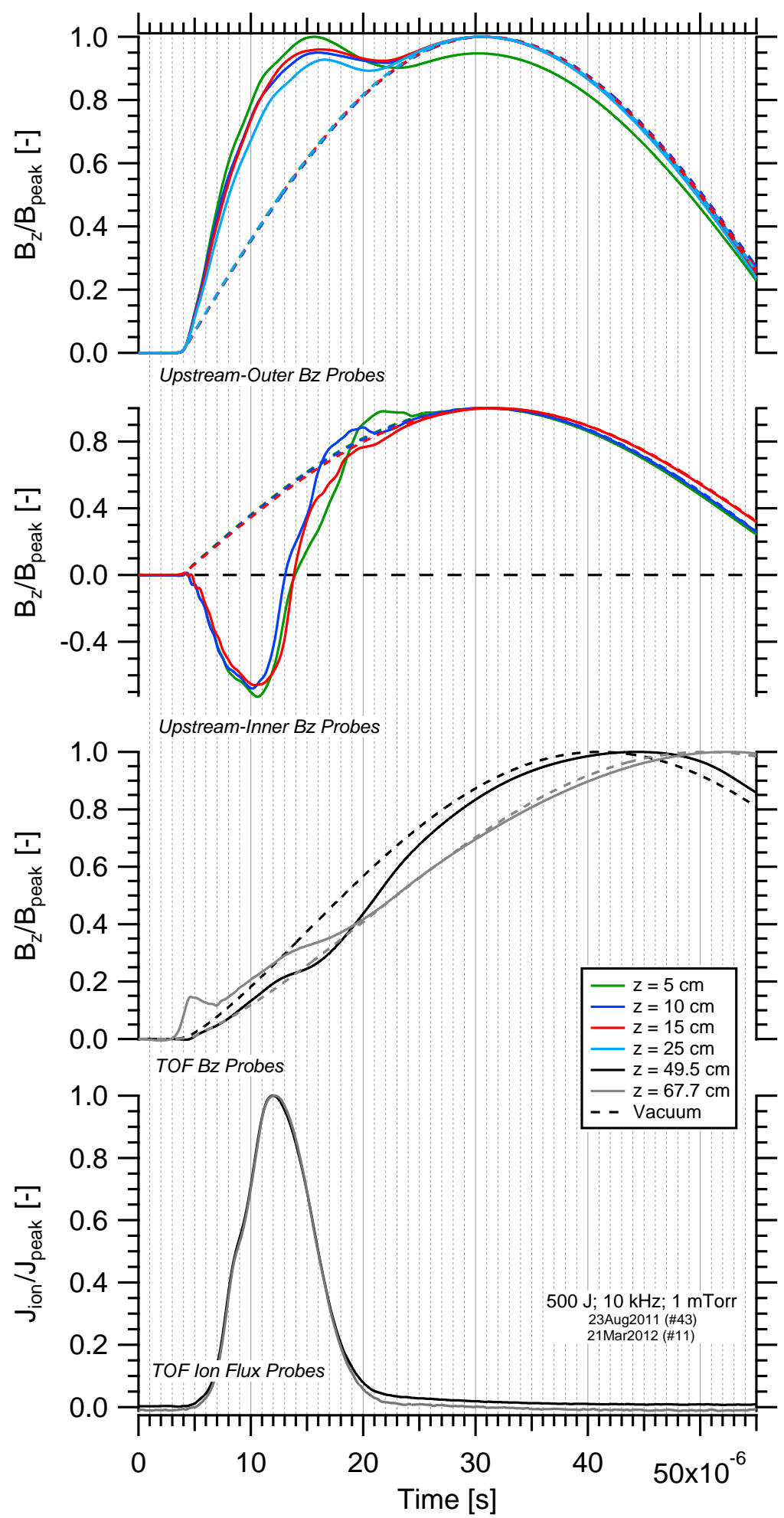

Figure 7.8: Translation results for the $10 \mathrm{kHz}$ circuit at $500 \mathrm{~J}$, with a 1 mTorr fill pressure. 

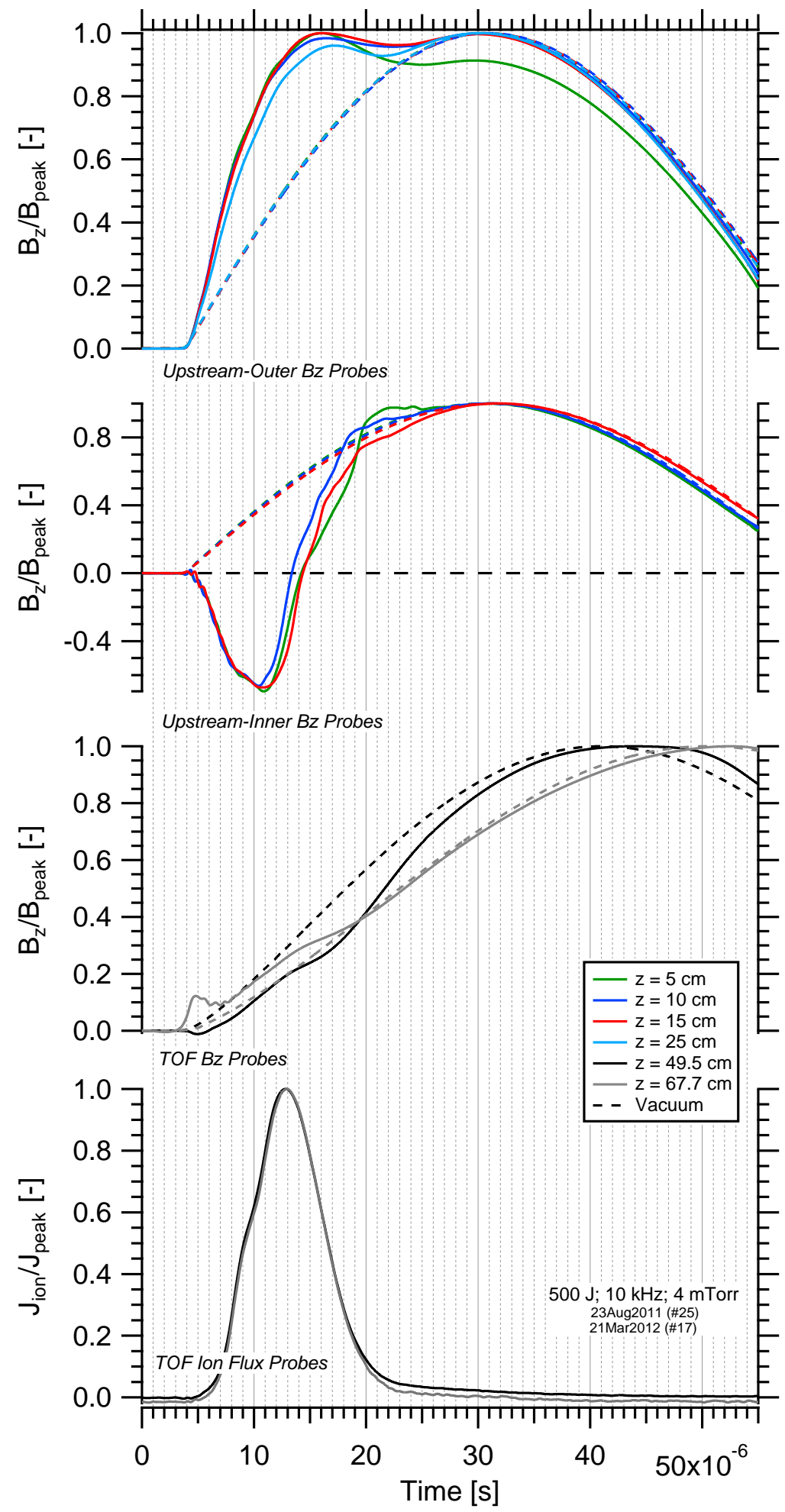

Figure 7.9: Translation results for the $10 \mathrm{kHz}$ circuit at $500 \mathrm{~J}$, with a 4 mTorr fill pressure. 

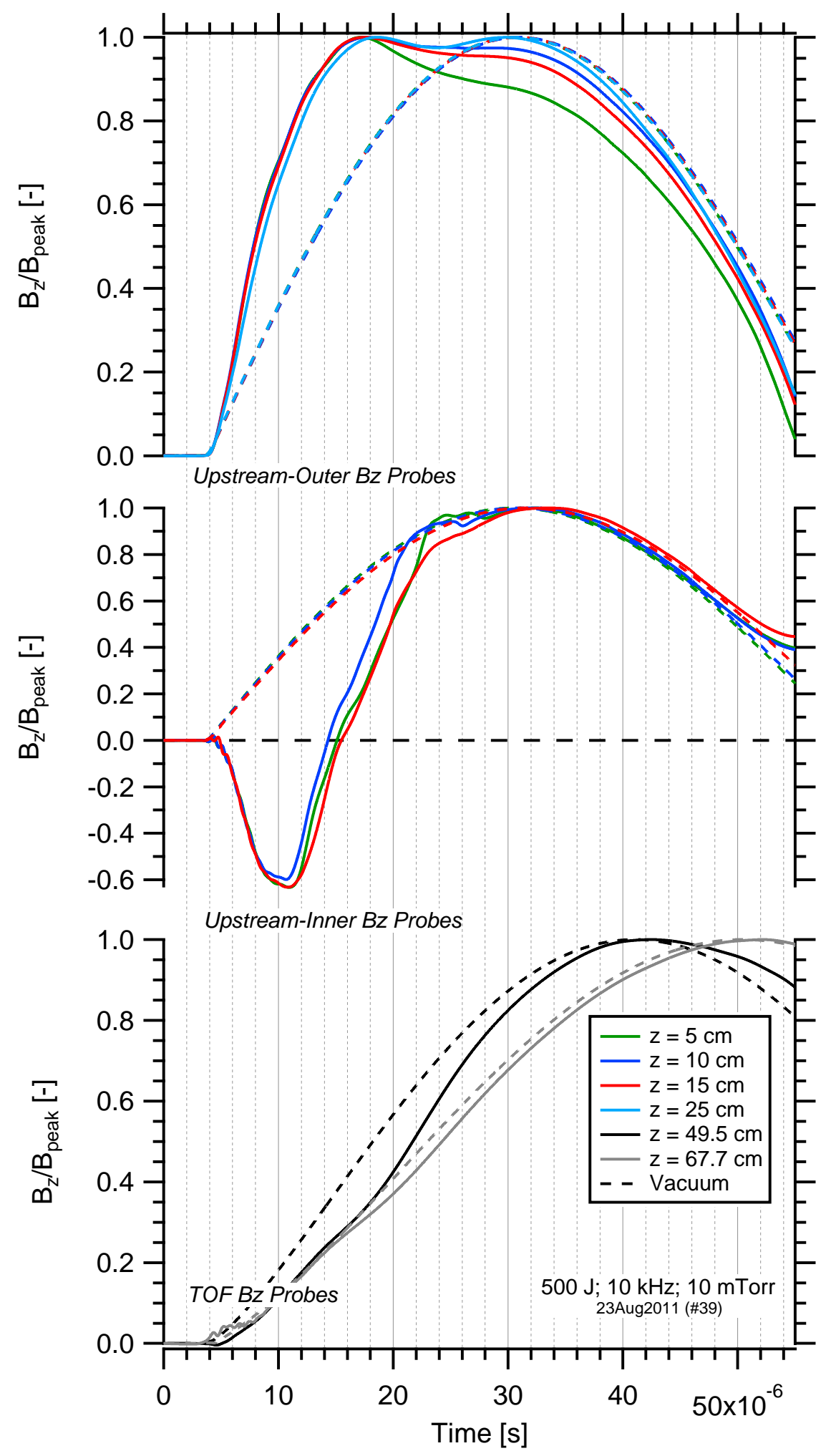

Figure 7.10: Translation results for the $10 \mathrm{kHz}$ circuit at $500 \mathrm{~J}$, with a 10 mTorr fill pressure. 

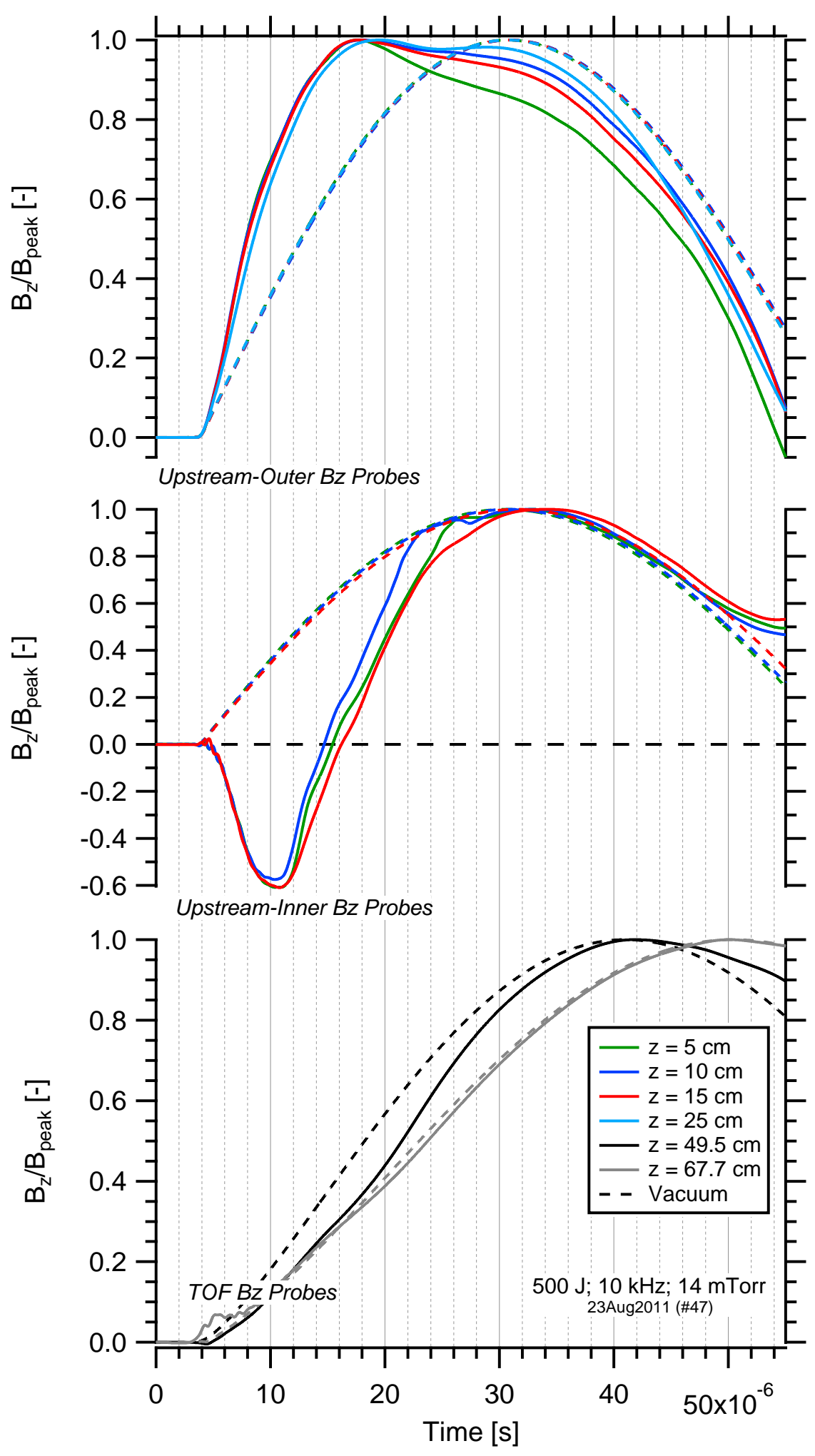

Figure 7.11: Translation results for the $10 \mathrm{kHz}$ circuit at $500 \mathrm{~J}$, with a 14 mTorr fill pressure. 

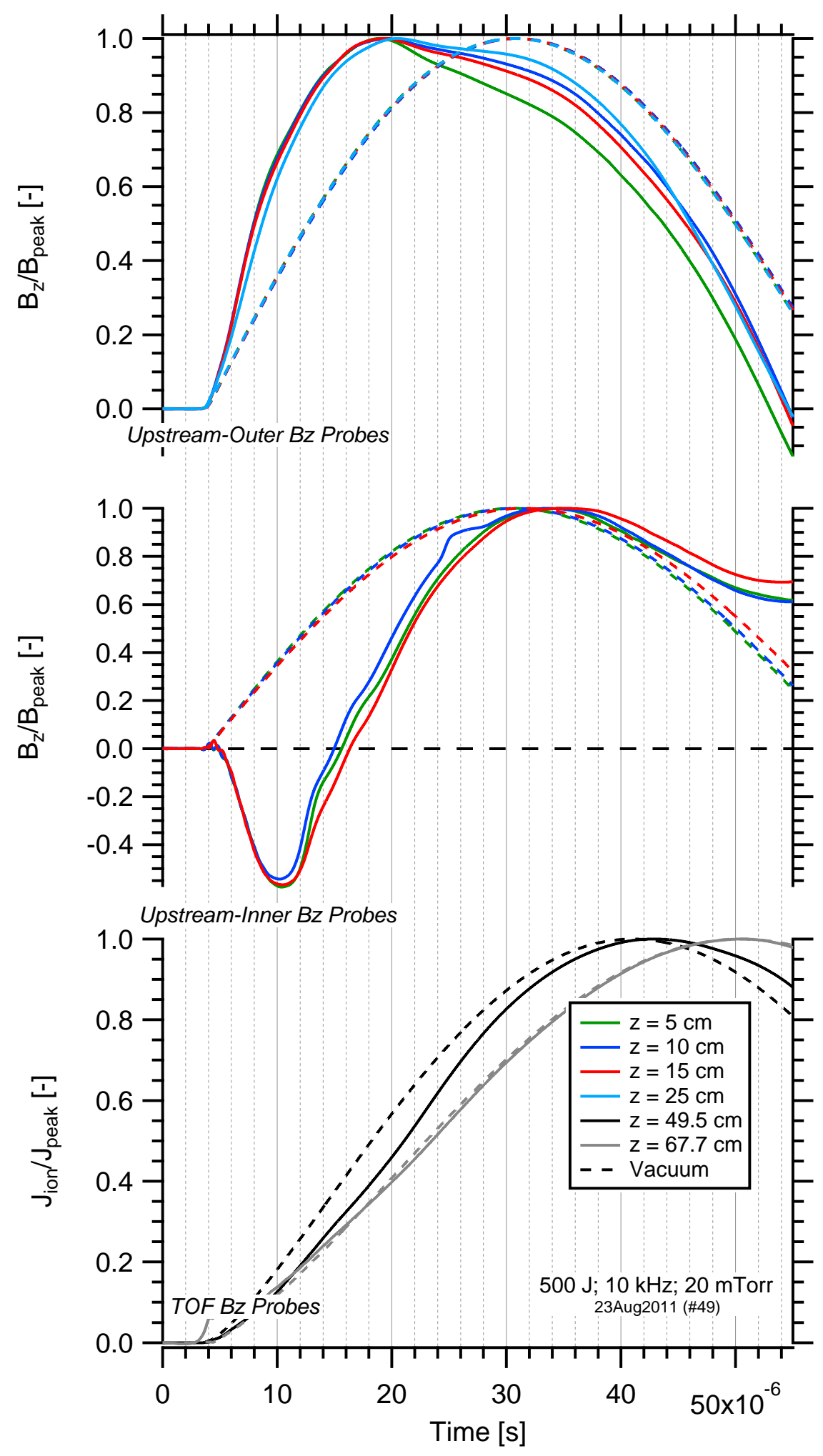

Figure 7.12: Translation results for the $10 \mathrm{kHz}$ circuit at $500 \mathrm{~J}$, with a 20 mTorr fill pressure. 

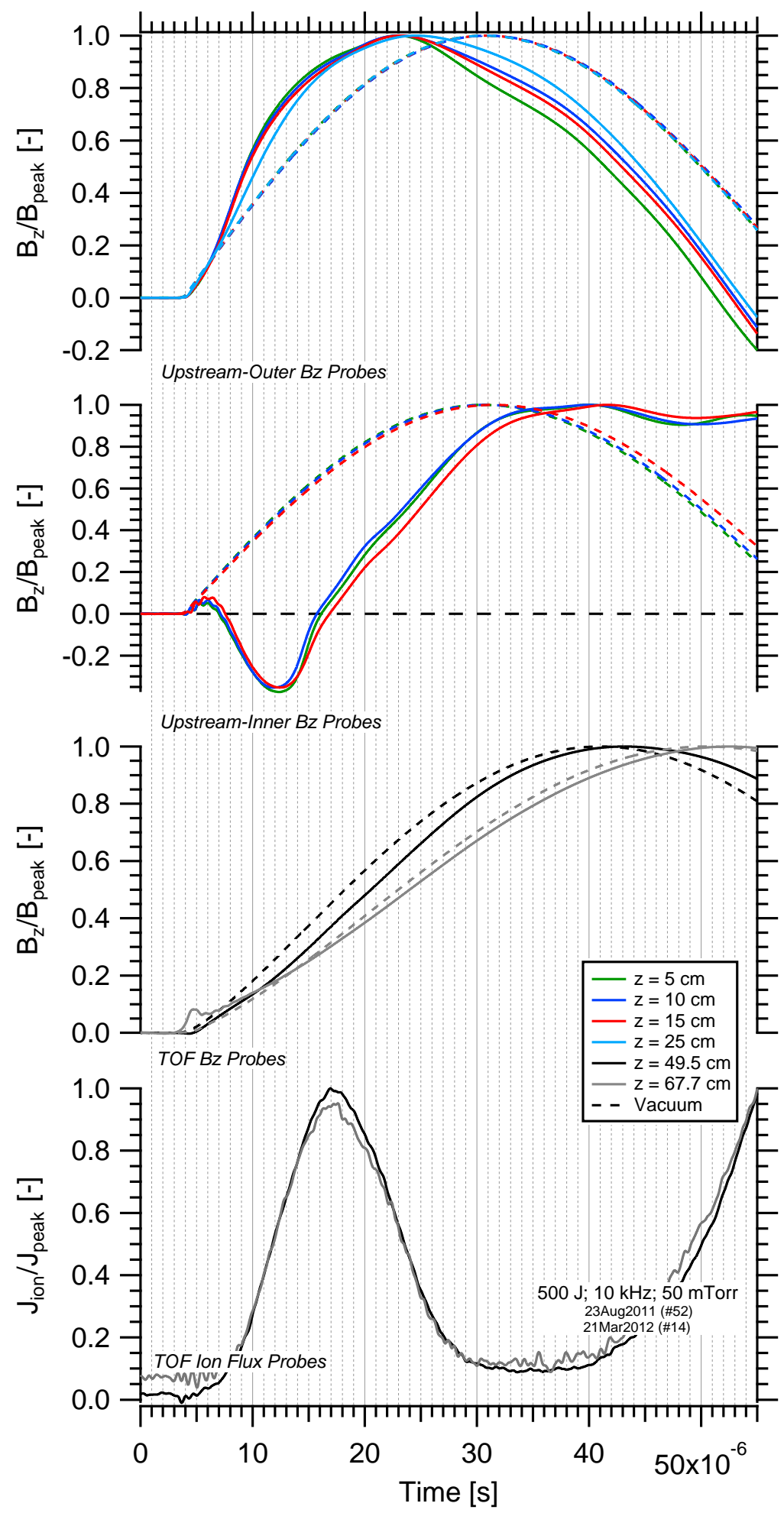

Figure 7.13: Translation results for the $10 \mathrm{kHz}$ circuit at $500 \mathrm{~J}$, with a 50 mTorr fill pressure. 
7.8, 7.9, and 7.13 appears on both probes simultaenously. The peak flux to the probe happens $2 \mu$ s after the inner fields peak and 1-2 $\mu$ s before the outer coil probes peak. Even if the plasmoid was traveling at a velocity around $160 \mathrm{~km} / \mathrm{s}$, a very sharp set of peaks spaced $1 \mu \mathrm{s}$ apart should appear on the plasma probes. Assuming the plasma is $25 \mathrm{~cm}$ in length, the signal should last on each probe for only $1.6 \mu \mathrm{s}$. The signature appears instead to be a very elongated structure with FWHM of $8 \mu \mathrm{s}$. The source of the plasma on the downstream probes is discussed in Section 7.2.3. The overwhelming verdict for the $500 \mathrm{~J}$ data is that the plasmoid does not translate from the coils.

The results from the high energy tests at $1 \mathrm{~kJ}$ with the $10 \mathrm{kHz}$ circuit are shown in Figures 7.14 through Figure 7.19. A single shot from each pressure and energy setting is presented, with the data peak normalized for velocity measurements.

The results for the $1 \mathrm{~kJ}$ circuit are similar in nature to the $500 \mathrm{~J}$ circuit, with no clear sign of translation seen in the magnetic field data. The field reverses quickly and peaks 6-7 $\mu$ s after the switch closes. The point of maximum field reversal corresponds to an insignificant inflection in the outer probe data; the full inflection occurs $5 \mu$ s later. The probe at the far end of the coil reaches a peak after the probes further upstream and by a single $\mu$ s. However, the downstream magnetic probes show a strong inflection about 2 $\mu$ s after the inner probes peak and $1 \mu$ s before the outer coil probes peak. The inflection appears to be slightly staggered between the TOF probes, by a delay of less than $1 \mu \mathrm{s}$. This would agree with the expected velocity of $200 \mathrm{~km} / \mathrm{s}$, but because it appears before the outer coil probes peak it is likely just an artifact. As was also shown by the $500 \mathrm{~J}$ data, the $1 \mathrm{~kJ}$ circuit fails to translate the plasmoid from the coils for each fill pressure tested. 

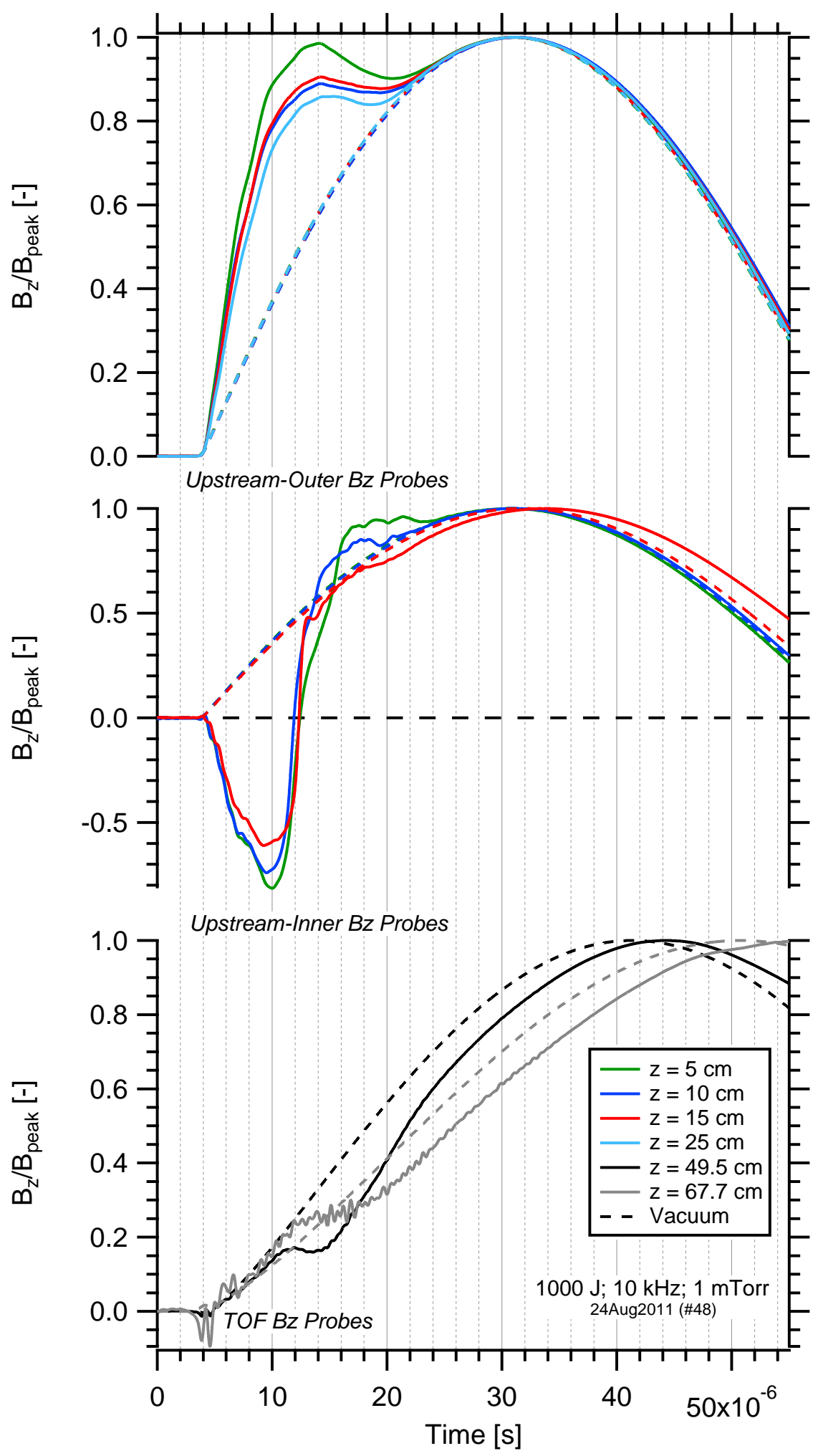

Figure 7.14: Translation results for the $10 \mathrm{kHz}$ circuit at $1000 \mathrm{~J}$, with a 1 mTorr fill pressure. 

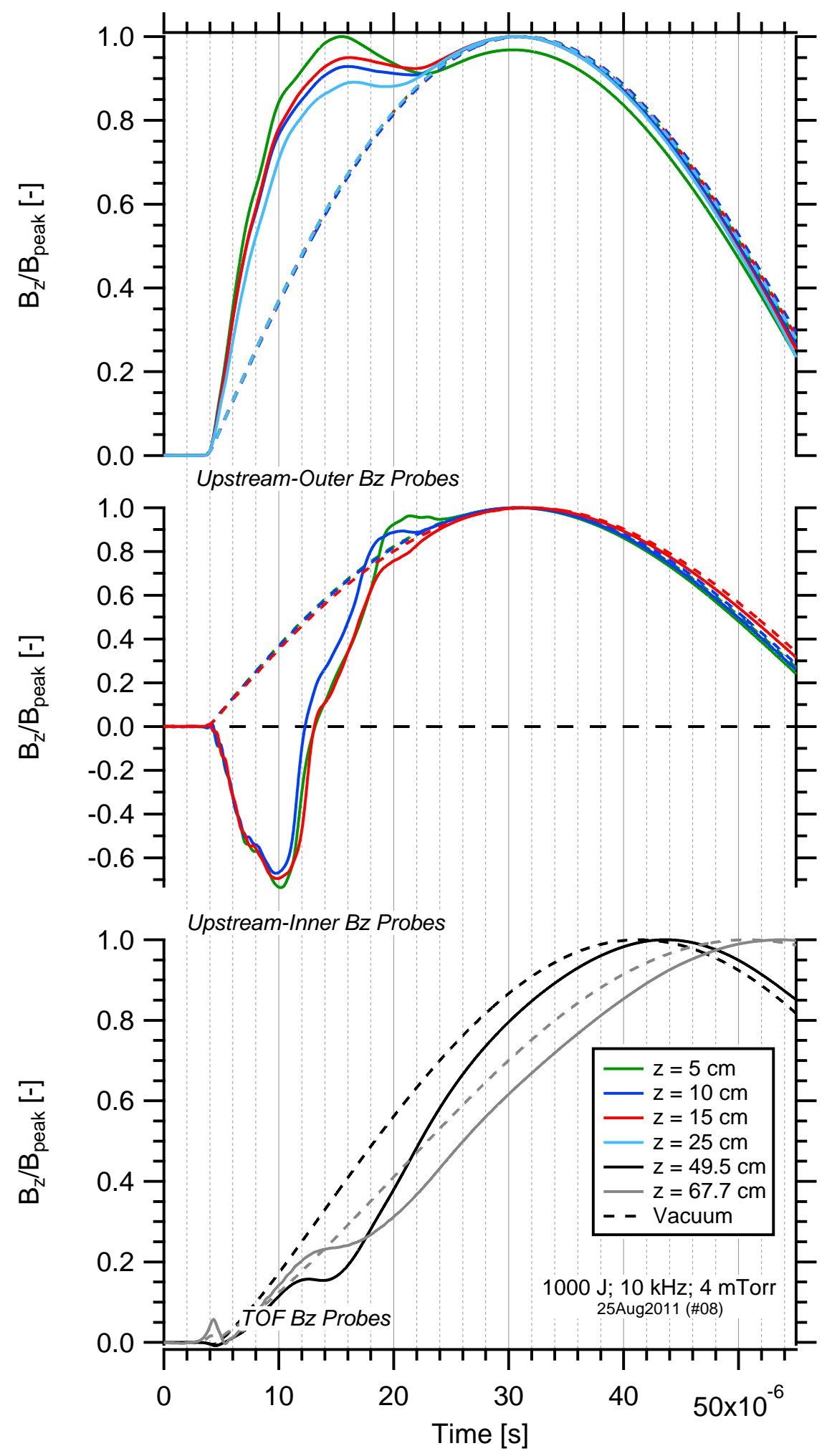

Figure 7.15: Translation results for the $10 \mathrm{kHz}$ circuit at $1000 \mathrm{~J}$, with a 4 mTorr fill pressure. 

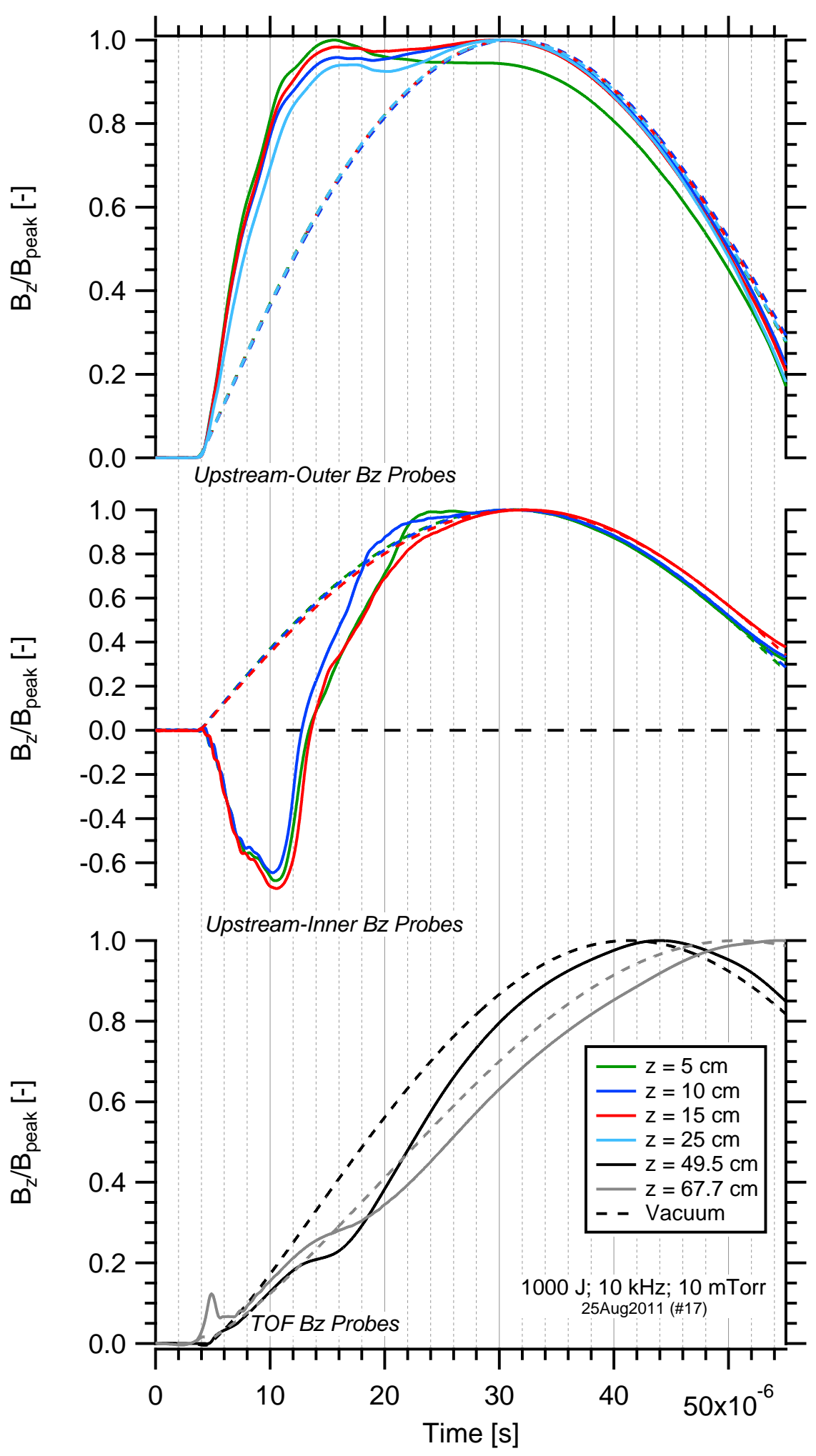

Figure 7.16: Translation results for the $10 \mathrm{kHz}$ circuit at $1000 \mathrm{~J}$, with a 10 mTorr fill pressure. 

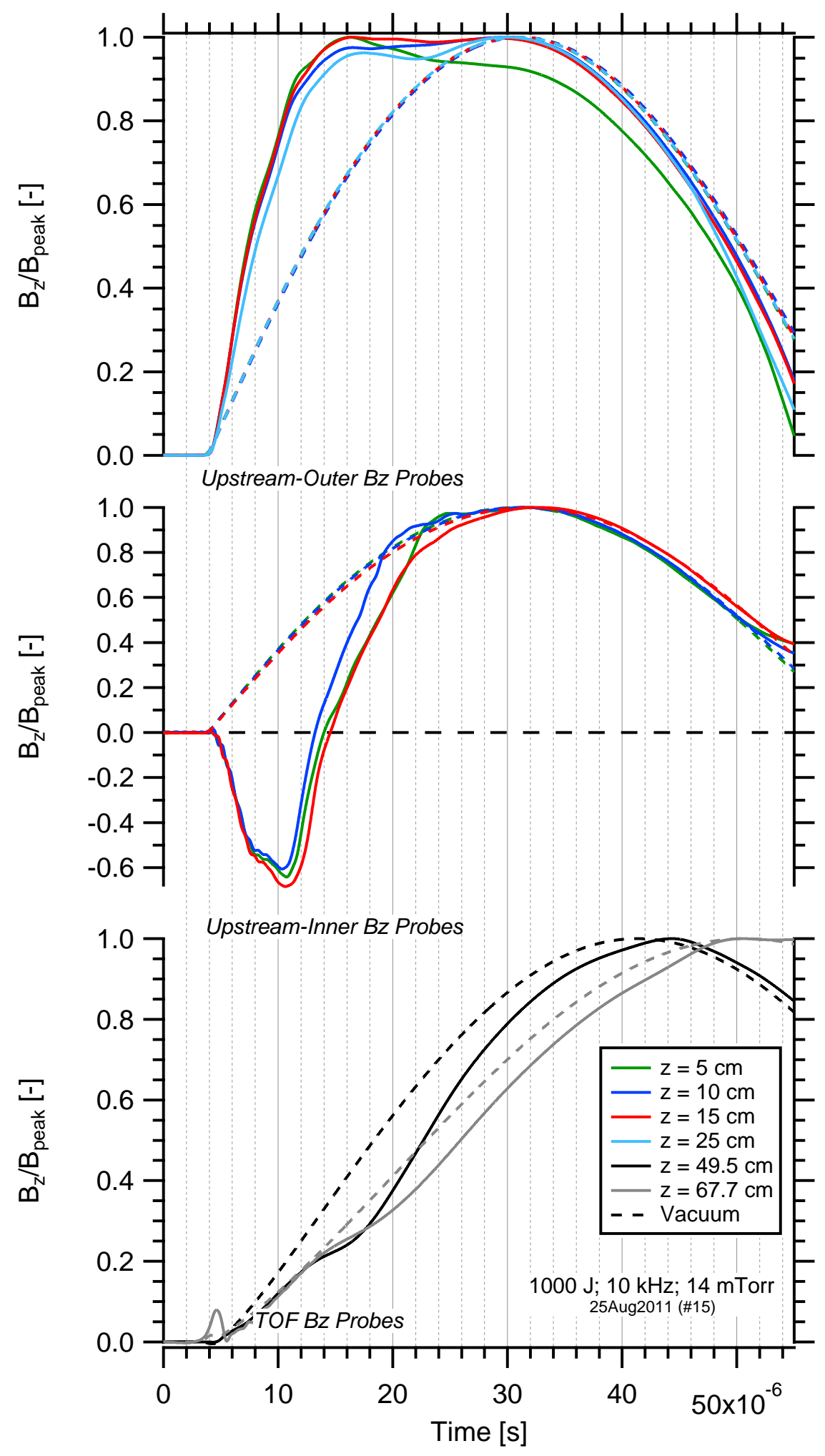

Figure 7.17: Translation results for the $10 \mathrm{kHz}$ circuit at $1000 \mathrm{~J}$, with a 14 mTorr fill pressure. 

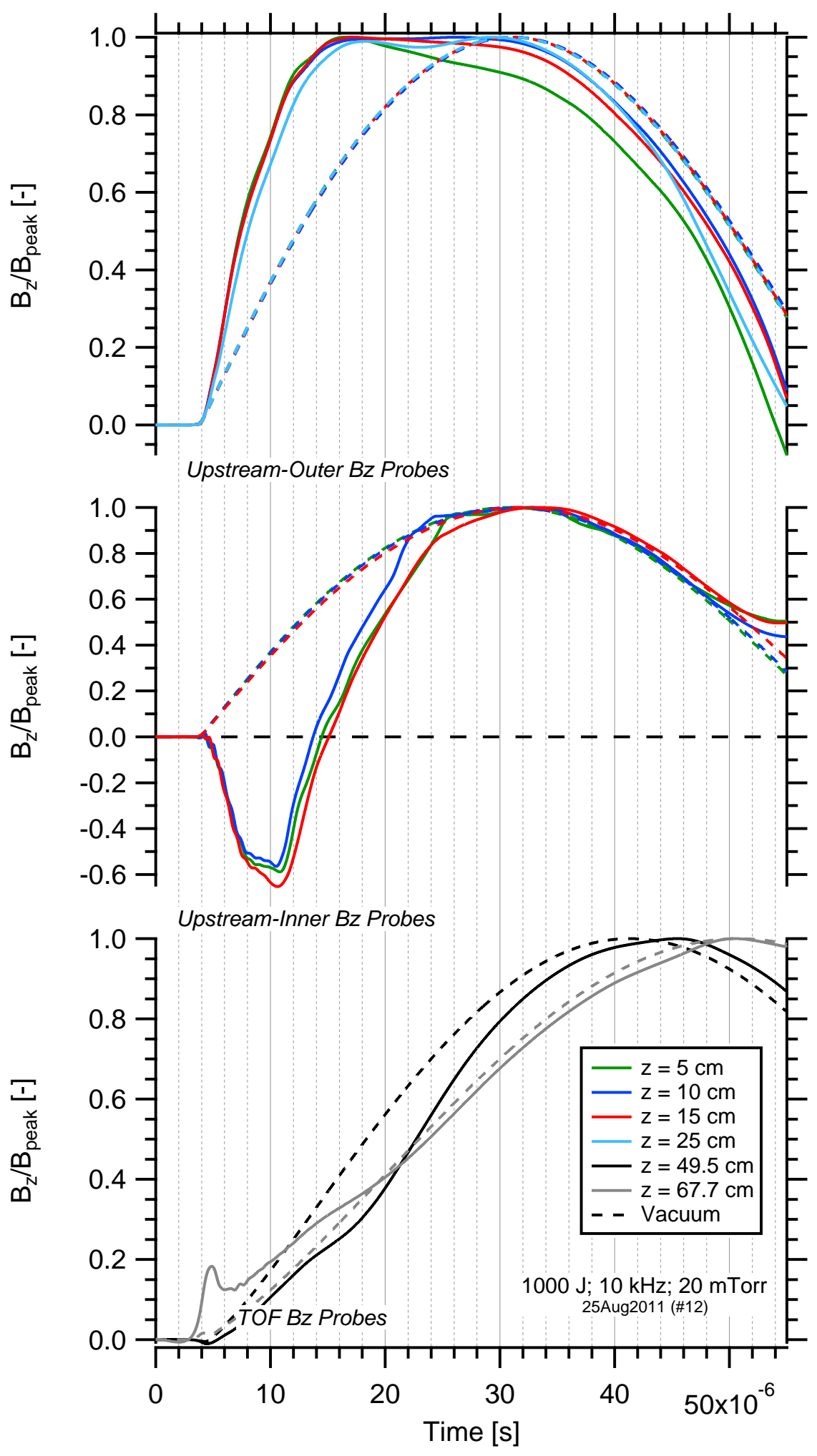

Figure 7.18: Translation results for the $10 \mathrm{kHz}$ circuit at $1000 \mathrm{~J}$, with a 20 mTorr fill pressure. 

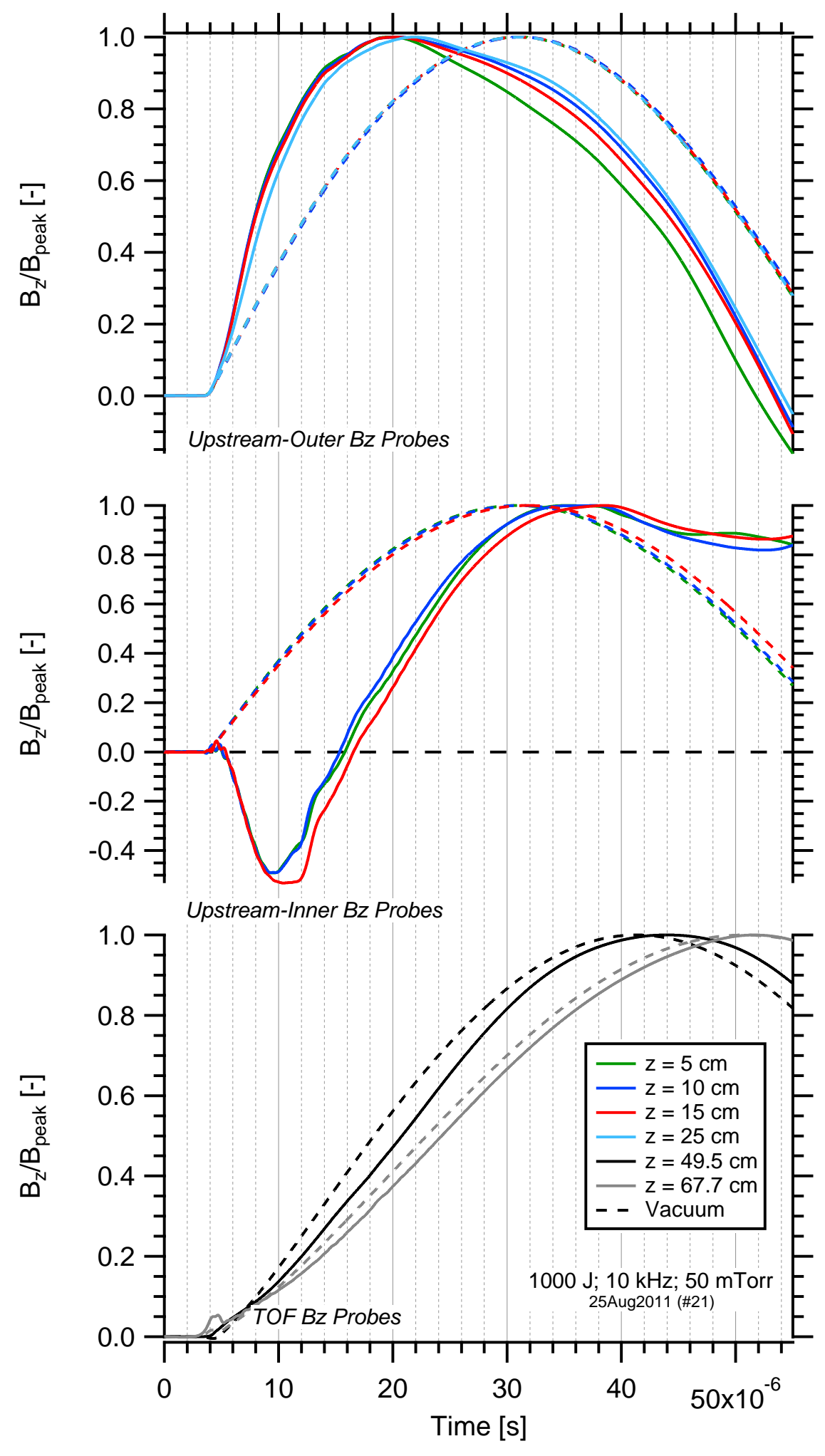

Figure 7.19: Translation results for the $10 \mathrm{kHz}$ circuit at $1000 \mathrm{~J}$, with a 50 mTorr fill pressure. 


\subsubsection{Velocity Results: $20 \mathrm{kHz}$}

The $20 \mathrm{kHz}$ circuit was operated at $100 \mathrm{~J}$. The $100 \mathrm{~J}$ energy setting was unlikely to result in translation due to an extremely low energy content, however the results are presented here for completeness. These results are presented in a similar fashion to the results in Section 7.2.1, with a single shot from each pressure and energy setting. All traces have been peak normalized to show transient trends in the data. Plasma flux data at each downstream location is presented for each pressure setting.

The translation results at $100 \mathrm{~J}$ are similar in nature to the $500 \mathrm{~J}, 10 \mathrm{kHz}$ results. The switch closed at $3 \mu \mathrm{s}$ and the inner field began to reverse $1 \mu \mathrm{s}$ later. The reversal peaked at $9 \mu \mathrm{s}$, but no corresponding inflection was seen on the outer probes. These probes peaked $3 \mu$ s later. Again, the probe at $\mathrm{z}=25 \mathrm{~cm}$ reached its peak after the midplane probe but the delay did not necessarily indicate translation in light of the outer probe data. The downstream magnetic probes witnessed no major inflection, though a deviation from their vacuum pattern was noted. The peak plasma flux appeared on both downstream probes simultaneously, less than $500 \mathrm{~ns}$ behind the peak field reversal, and about $1 \mu$ s before the outer coil probes peaked. A significant signal on the plasma probes was recorded by both probes as the plasmoid fields were still forming. As was discussed in the $10 \mathrm{kHz}$ results, the data recorded by all the probes does not represent a translating plasmoid as there were no temporally related features among all the probes. The plasmoid fails to translate in the $100 \mathrm{~J}, 20 \mathrm{kHz}$ circuit. The nature of the downstream plasma is discussed in Section 7.2.3. 

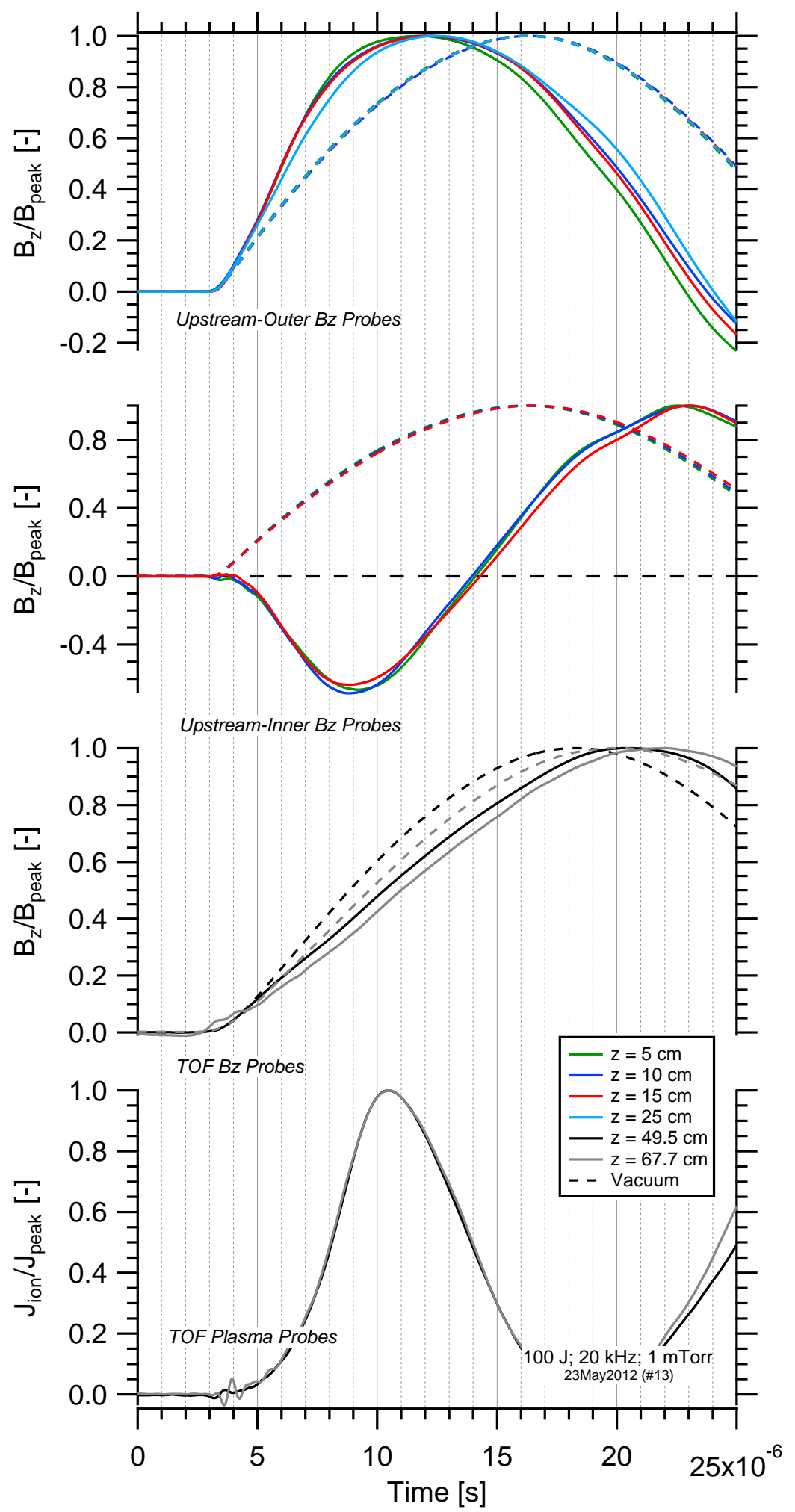

Figure 7.20: Translation results for the $20 \mathrm{kHz}$ circuit at $100 \mathrm{~J}$, with a 1 mTorr fill pressure. 

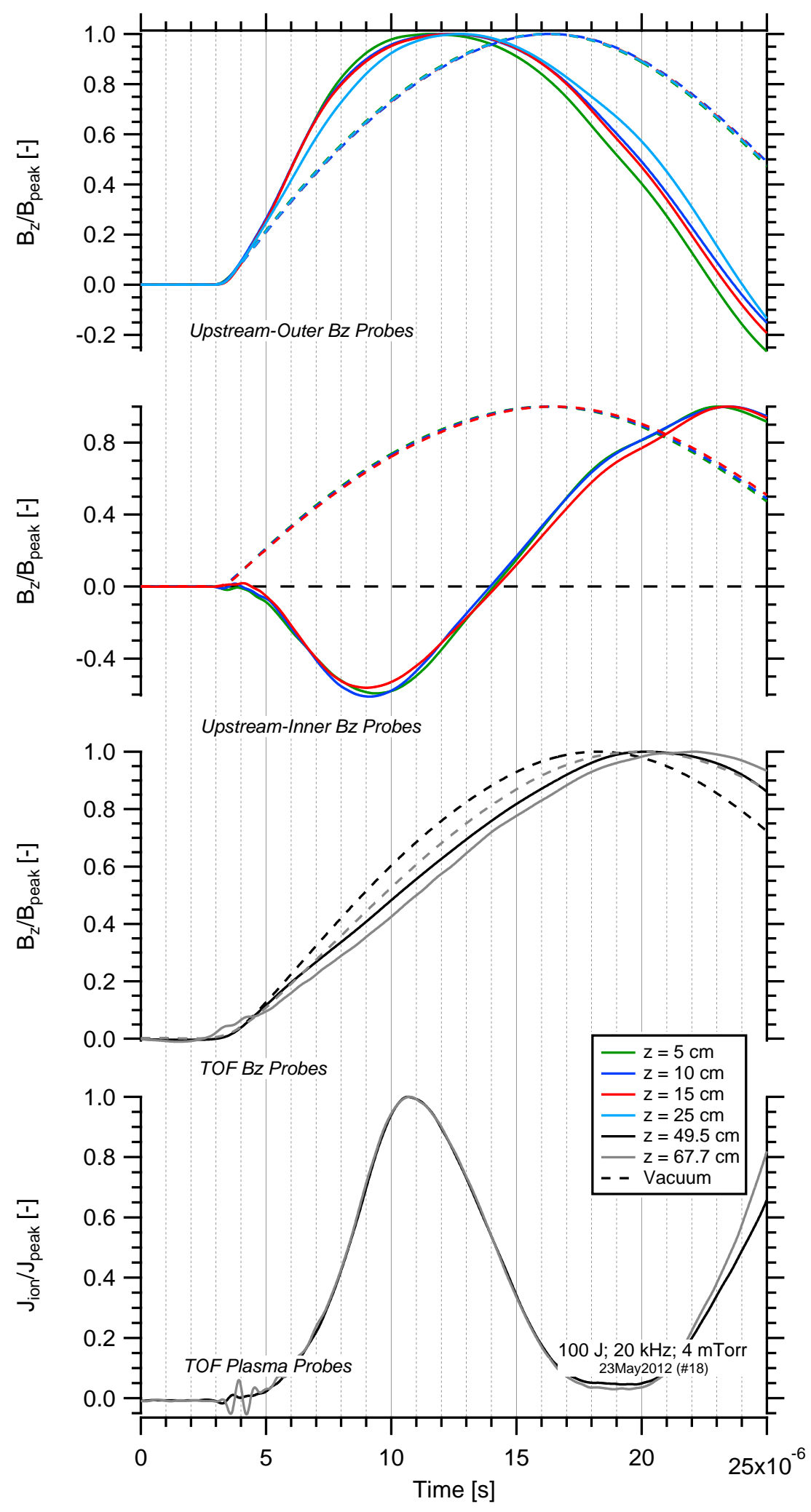

Figure 7.21: Translation results for the $20 \mathrm{kHz}$ circuit at $100 \mathrm{~J}$, with a 4 mTorr fill pressure. 

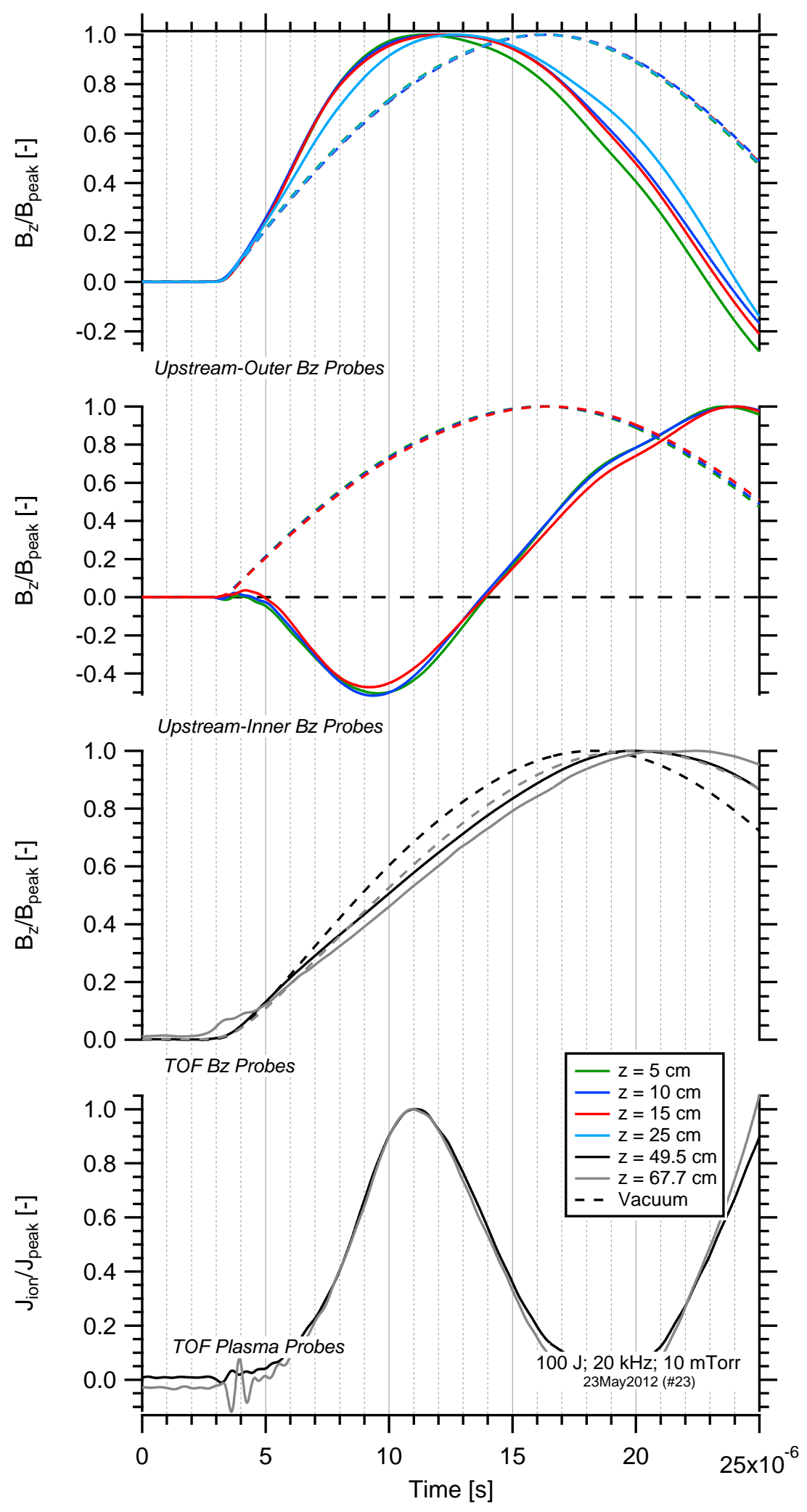

Figure 7.22: Translation results for the $20 \mathrm{kHz}$ circuit at $100 \mathrm{~J}$, with a 10 mTorr fill pressure. 

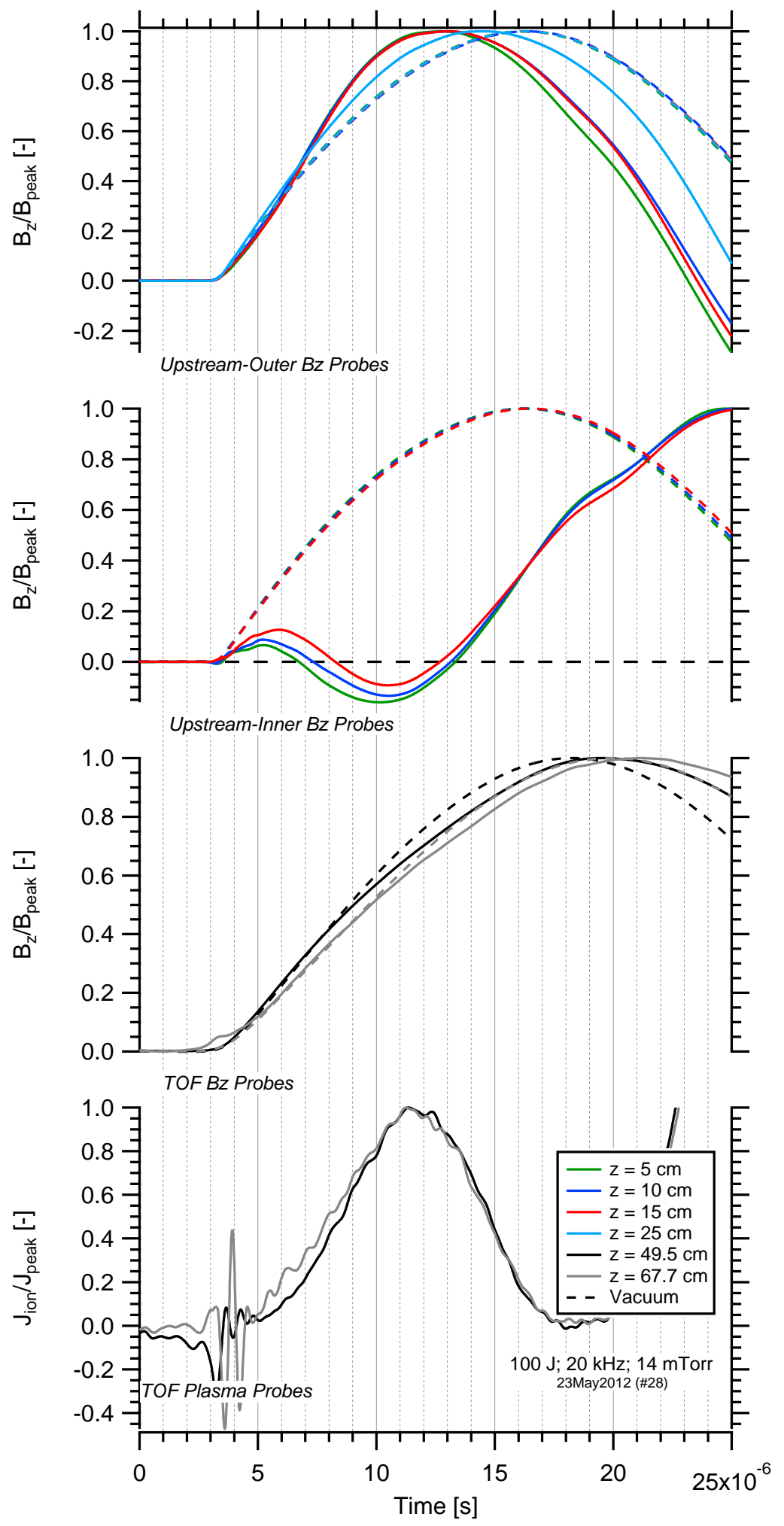

Figure 7.23: Translation results for the $20 \mathrm{kHz}$ circuit at $100 \mathrm{~J}$, with a 14 mTorr fill pressure. 

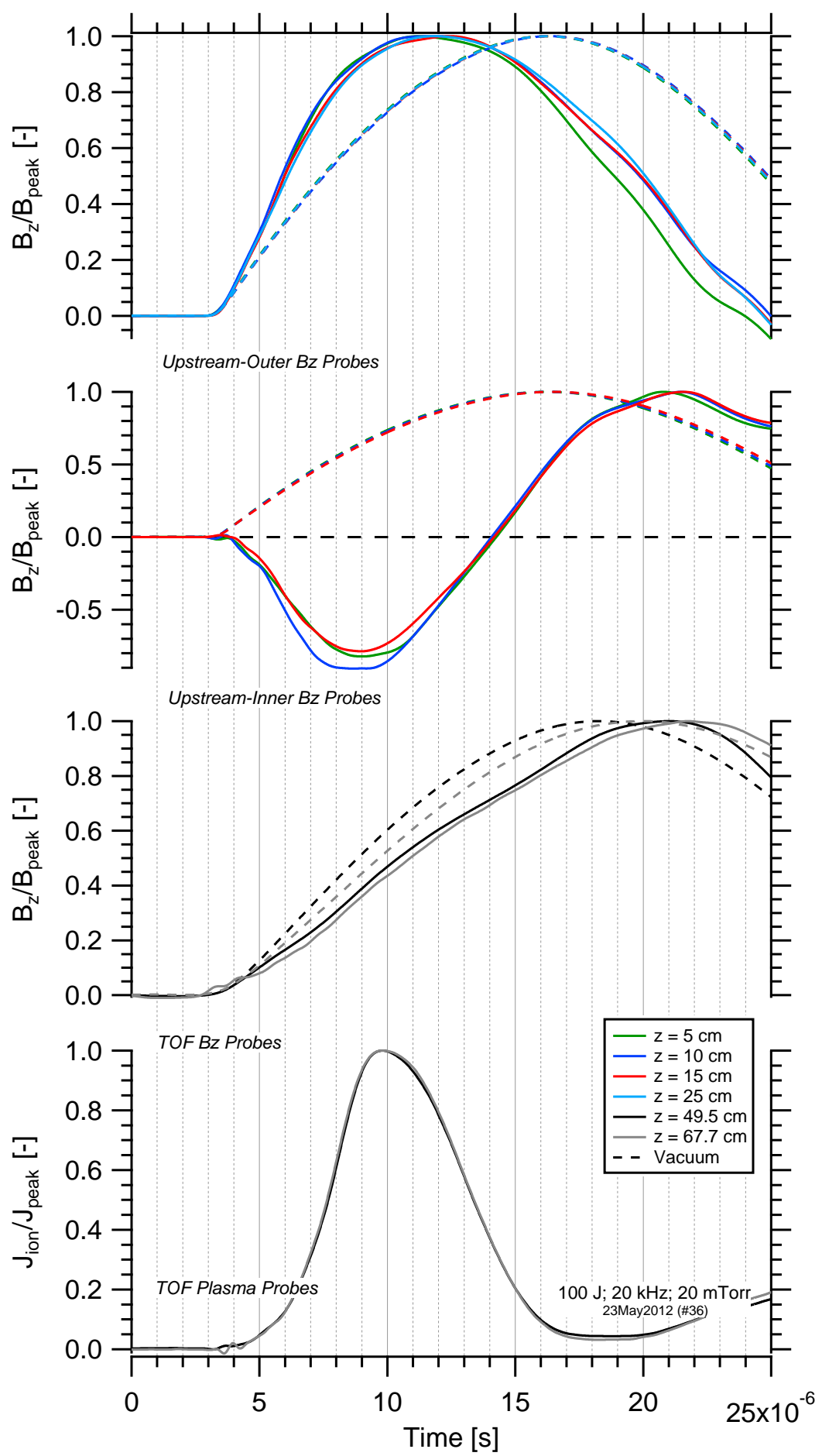

Figure 7.24: Translation results for the $20 \mathrm{kHz}$ circuit at $100 \mathrm{~J}$, with a 20 mTorr fill pressure. 

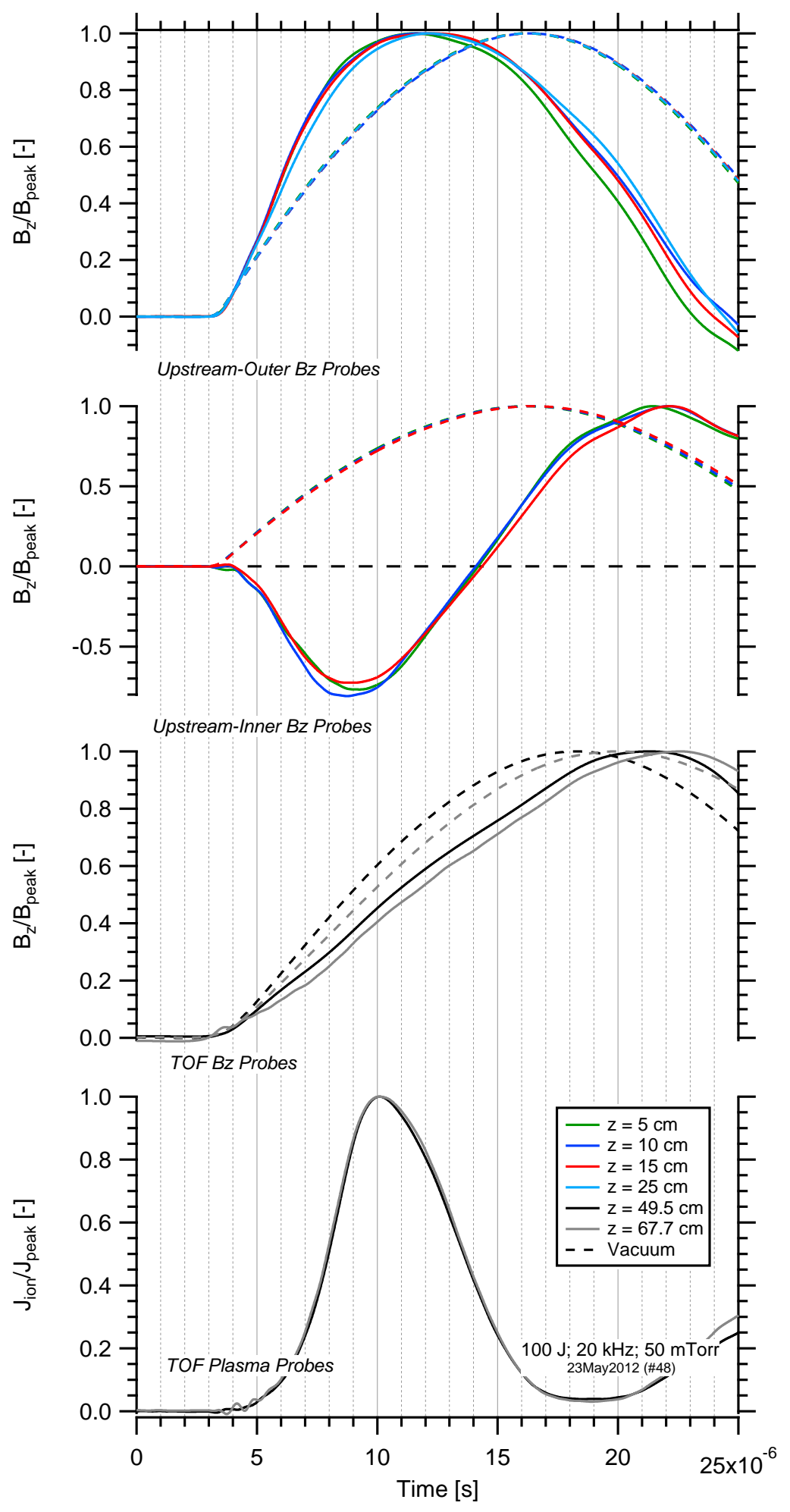

Figure 7.25: Translation results for the $20 \mathrm{kHz}$ circuit at $100 \mathrm{~J}$, with a 50 mTorr fill pressure. 


\subsubsection{Downstream Plasma Flux Measurements}

The appearance of plasma downstream of the channel on the ion flux probes was unexpected when the plasmoid did not translate. The source of the plasma was investigated by placing an ADLP $3.8 \mathrm{~cm}$ downstream of the coils' end to determine the plasma properties of plasma emerging directly downstream of the thruster. This information was then compared to the signal measured by the downstream probes. Data was collected from the probe for a $100 \mathrm{~J}, 20 \mathrm{kHz}$ discharge at 4 mTorr. The probe's bias voltage was varied from $0 \mathrm{~V}-32 \mathrm{~V}$ and the current through the probe was measured during each shot. The resulting data is displayed in Figure 7.26a. The probe current at $\mathrm{t}=20 \mu \mathrm{s}$ is shown in Figure $7.26 \mathrm{p}$ along with a curve-fit to the data from ADLP theory, used to estimate $n_{0}$ and $T_{e V}$

The ADLP results show that the probe current collected to the probe increased with bias voltage, saturating between $15-20 \mathrm{~V}$. The data was used to estimate the time-history of the temperature and density of the plasma at this location from $12-30 \mu$ s by fitting theoretical $\mathrm{I}-\mathrm{V}$ traces to the data sets. The results are displayed in Figure $7.27 \mathrm{a}$ and Figure $7.27 \mathrm{p}$. Thin-sheath approximations were used to calculate the ion currents and proved to be valid assumptions, indicated by $r_{p} / \lambda_{D}>45$ in Figure 7.27 . The curve-fit results to the data at several points in time are displayed in Appendix A. Uncertainty estimates in the data are shown as well, including errors in probe theory as well as in the error in measured current due to probe contaminants.

The ADLP data shows a dense, cool plasma traveling out of the coils with a Gaussian-like time history. Peak densities of $1.09 \times 10^{20} \mathrm{~m}^{-3}$ were measured, with a constant temperature 


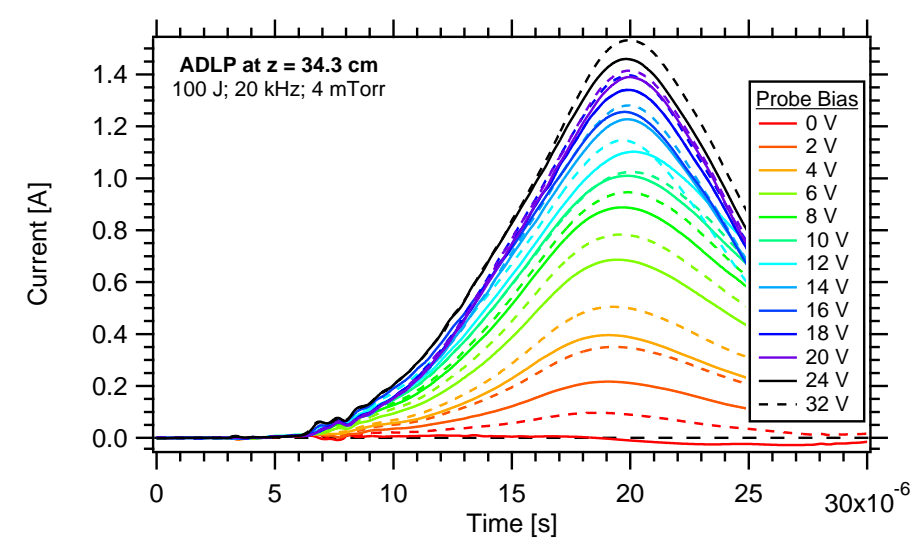

(a)

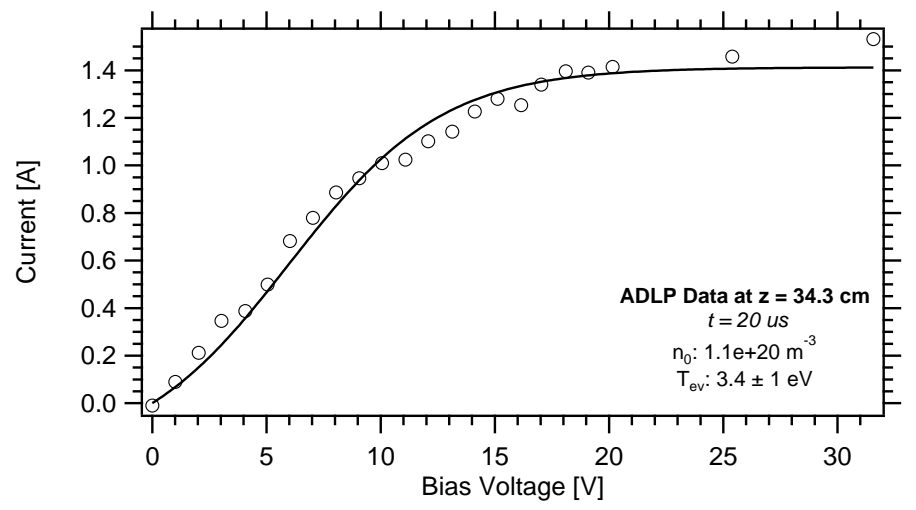

(b)

Figure 7.26: ADLP current measured $3.8 \mathrm{~cm}$ downstream of the coil exit plane for several bias voltages.

of 2-4 eV. The peak density was quite high, corresponding to an $84 \%$ ionization fraction of initial neutral fill $\left(1.29 \times 10^{20} \mathrm{~m}^{-3}\right)$. The relatively long pulse of plasma activity (FWHM of $13 \mu \mathrm{s}$ ) indicates the probe was measuring a thermal jet of plasma as opposed to coherent, high speed plasmoid. This theory is substantiated by observing the time-behavior of plasma density with respect to the magnetic field probes and downstream ion probes, shown with peak normalization in Figure 7.28.

The peak density on the ADLP appeared $10.7 \mu$ s after the inner midplane probe reached a peak reversed field and $9.2 \mu \mathrm{s}$ after the downstream probes measured their peak signal. If the ADLP was measuring a translating plasmoid at $50 \mathrm{~km} / \mathrm{s}$ or greater, the delay between the midplane magnetic probes and the ADLP peak would be $>4 \mu$ s and the 


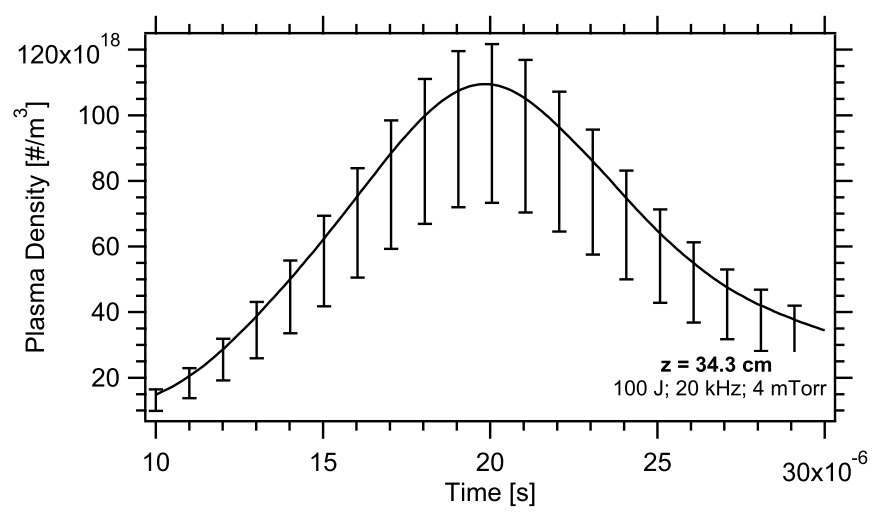

(a)

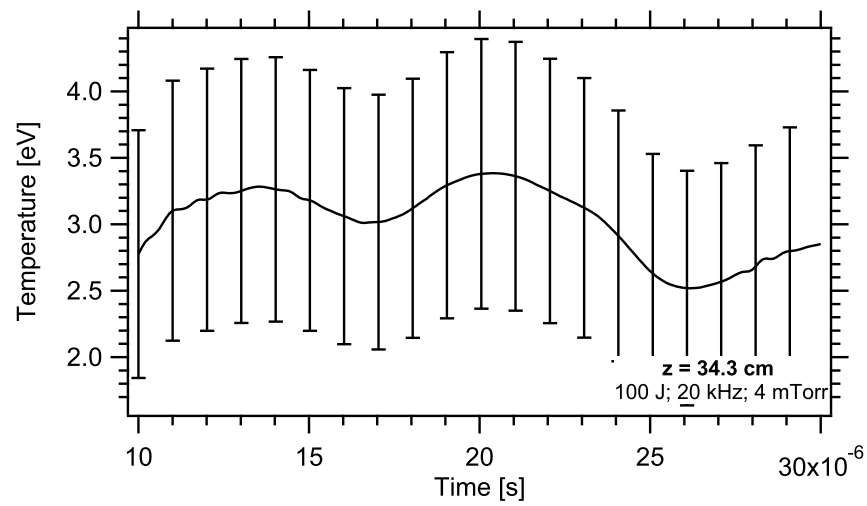

(b)

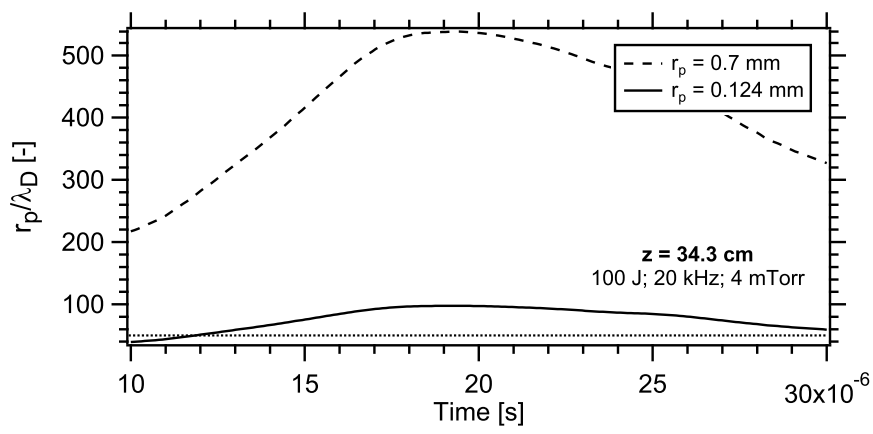

(c)

Figure 7.27: Plasma density and electron temperature measured by an ADLP at $\mathrm{z}=34.3 \mathrm{~cm}$. Figure (c) shows that thin-sheath approximations are valid for this probe for the time-interval of interest.

delay between the ADLP signal and the downstream probes would be $>6.6 \mu$ s with the downstream probes recording a peak after the ADLP. Since the ADLP signal does not correspond to a translating plasmoid, it was likely the dense plasma originally created by the AFRC released after magnetic confinement was lost. The high density of the plasma, low temperature, and long delay time support this conclusion. 

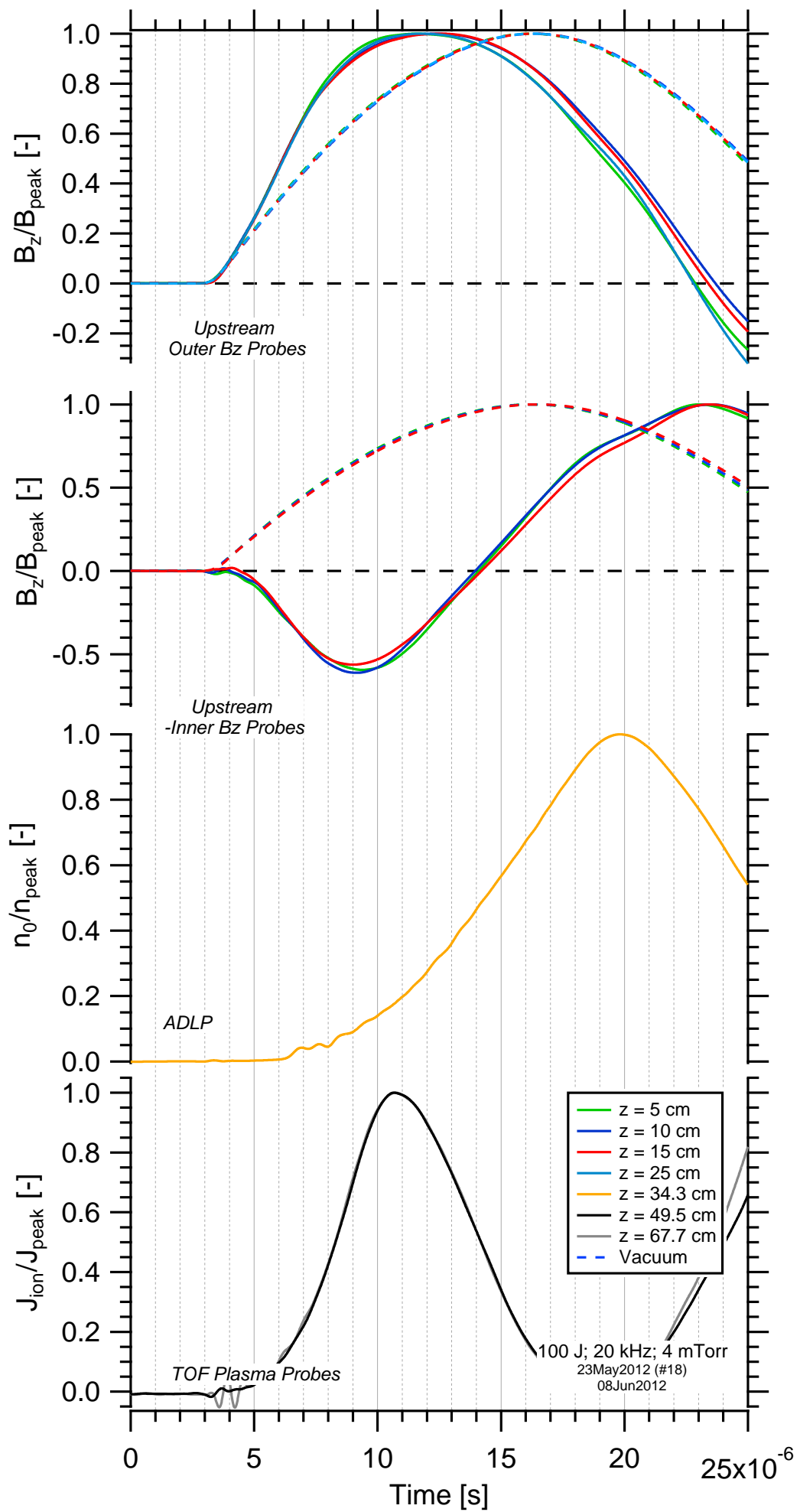

Figure 7.28: Translation results for the $100 \mathrm{~J}, 20 \mathrm{kHz}$ circuit including peak-normalized density from an ADLP. 
The dense plasma created by the AFRC emerged from the channel after the first peak on the downstream TOF plasma probes, meaning the plasma measured by the downstream probes was not part of the bulk AFRC plasma. Instead, the plasma measured by the downstream probes was likely created by one of three sources (1) hot electrons from the coil region ionizing the background gas at the probe's surface, (2) high energy plasma escaping the plasmoid's confinement, or (3) photoionization of the background gas by light emitted by the plasmoid formation. The first and third source are the most likely. The second source is highly unlikely; the delay between peaks on the downstream plasma probes are only 72 ns apart so the high energy plasma would have to be traveling in excess of $2000 \mathrm{~km} / \mathrm{s}$. This velocity is unrealistic at $1 \%$ the speed of light or $827 \mathrm{keV}$ (several orders of magntiude higher than the expected energy of $10 \mathrm{eV})$.

To determine if background ionization was responsible for the plasma measured by the probes, the mean free path for an ionization collision and an elastic collision between an electron and neutral was calculated. The mean free path $\lambda_{M}$ is the characteristic length-scale for a collision, defined as:

$$
\lambda_{M}=\frac{1}{n_{n} \sigma}
$$

where $\sigma$ is the collisional cross section and $n_{n}$ is the number density of the background neutrals. The cross section as a function of electron energy is displayed in Figure 7.29 a for ionization of argon [83] and elastic collisions with argon [84]. From this graph, it is apparent that low energy collisions (less than $15 \mathrm{eV}$ ) will simply scatter the participants and produce no ionization. When the electron energy exceeds $20 \mathrm{eV}$, ionization and elastic scattering are both just as likely. Above $100 \mathrm{eV}$, electrons are more likely to ionize the argon neutral than to scatter off of it. These results imply that only the high energy tail 


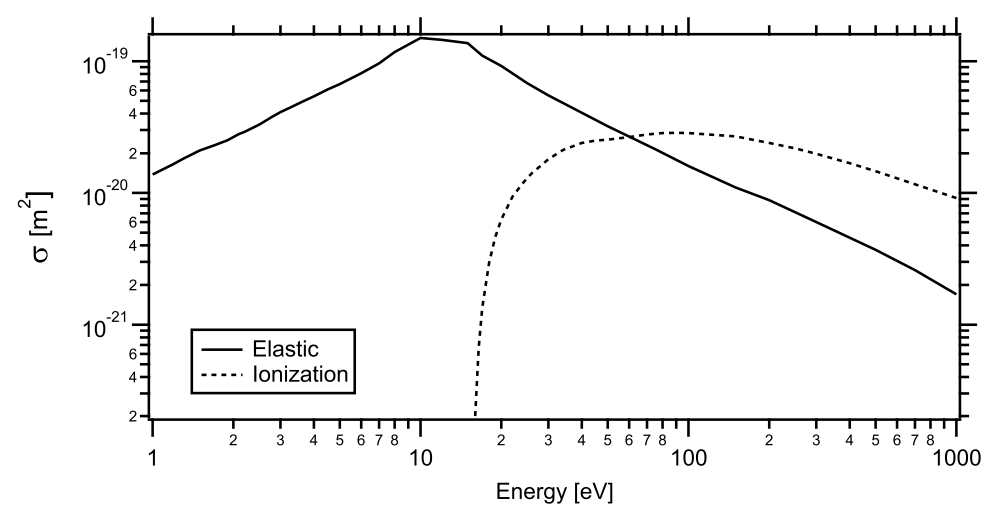

(a)

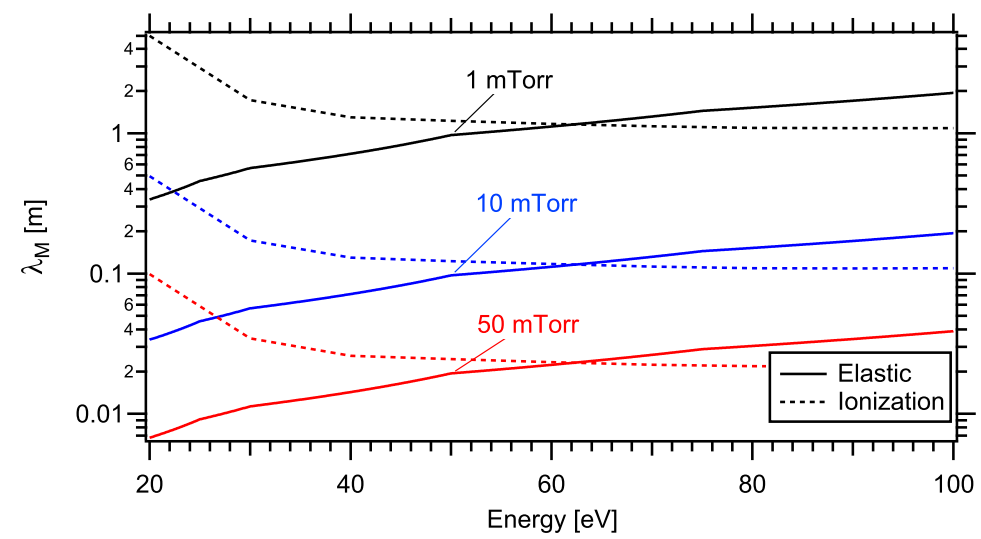

(b)

Figure 7.29: Collision cross sections for electron elastic scattering from argon and for electron ionization from argon for various electron energies (a) and mean free path for elastic and ionization scattering as a function of energy energy for various neutral fill pressures (b).

( $20 \mathrm{eV}$ and above) or a small fraction of the electron energy distribution function would be responsible for ionization of the background gas. The mean free path for the elastic and ionization collisions are displayed in Figure $7.29 \mathrm{p}$ for various fill pressures. The results in this figure show that the mean free paths for the fill pressures in this experiment are between $1 \mathrm{~cm}$ and $1 \mathrm{~m}$ for both elastic and ionzation collisions. Since the probes are located 50 $\mathrm{cm}$ downstream from the narrow end of the coil, it is possible that high energy electrons traveling from this region could ionize the background gas in front of the probes. The electrons are also just as likely to scatter off without ionization, leading to lower ionization fractions. 


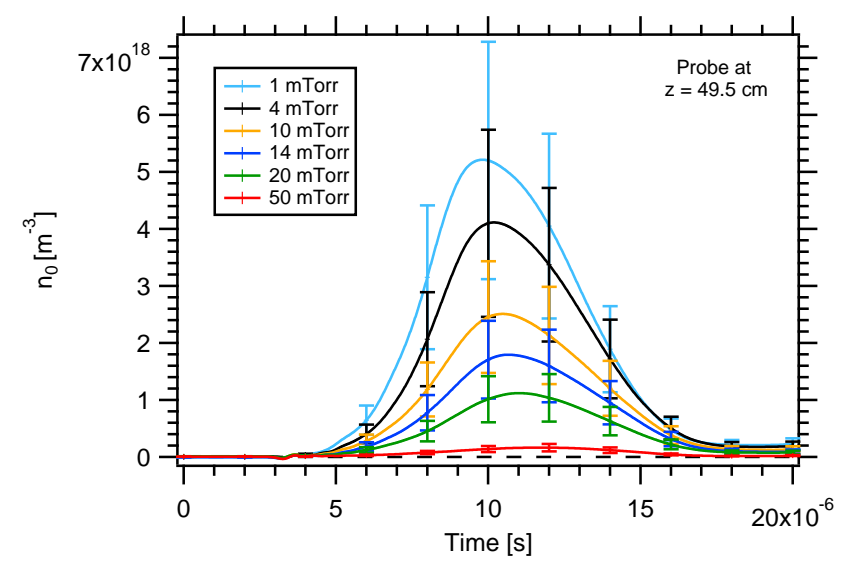

(a)

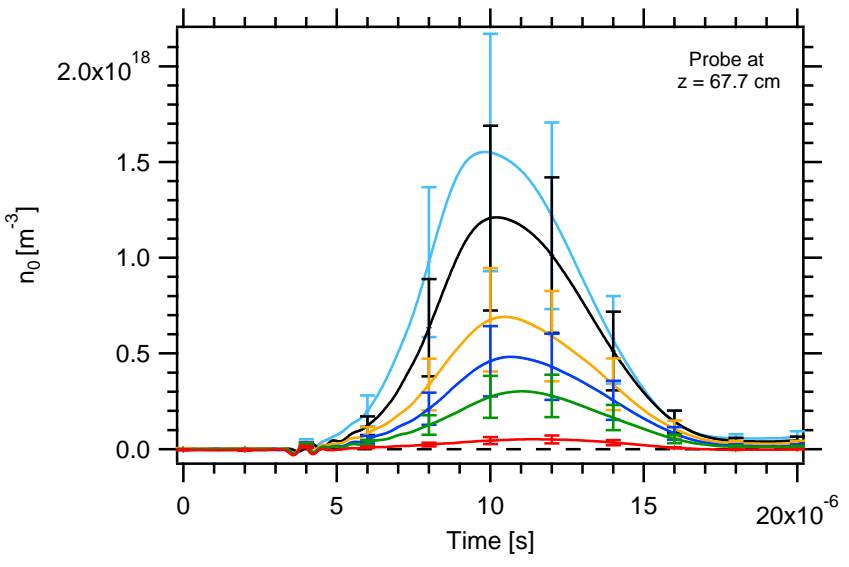

(b)

Figure 7.30: Estimated plasma density for ion saturation currents measured by downstream plasma probes at several pressures.

The mean free path decreases with density, therefore lower plasma densities should be recorded by each probe for increased fill pressures. The electrons are more likely to ionize a neutral upstream of the probes in a denser fill, leading to lower plasma densities downstream. To investigate this trend in the ion flux data, the plasma density at each probe was estimated using a fixed temperature of $1 \mathrm{eV}$ (a slight reduction from the ADLP data) and thin-sheath behavior. The results are displayed in Figure 7.30 .

The data in Figure 7.30 shows that the plasma density measured by the TOF plasma probes indeed decreases with increasing pressure. The mean free path decreases with density, meaning an electron will experience more collisions before it reaches the probes in a 
50 mTorr fill than in a 1 mTorr fill. This supports the theory that high energy electrons traveling from the coil region are responsible for ionizing the plasma measured by the downstream probes. It should be noted that the density measured by the downstream probes $\left(1 \times 10^{18} \mathrm{~m}^{-3}\right)$ is only a few percent of the neutral density $\left(1 \times 10^{20} \mathrm{~m}^{-3}\right.$ or greater $)$. This is conclusive proof that the downstream plasma probes are not measuring the accelerated AFRC plasmoid.

The high energy electrons (energies over $20 \mathrm{eV}$ ) which may have ionized the background gas were likely created during initial ionization of the AFRC by the strong inductive fields inside the coils and escaped the confinement region on open field lines. They had sufficient energy to ionize the background gas downstream of the coils, resulting in simultaneous signals on both downstream plasma probes. The density measured by the probes in the denser neutral fills was less than the density measured by the probes in less dense neutral fills because the electrons lost more energy due to more frequent collisions in the higher pressure fills. This is also why the density measured by the upstream probe is higher than the density measured by the downstream probe. These observations and the magnitude of the plasma density measured by each probe are convincing evidence that the plasma measured by the downstream probes was created by errant electrons from the discharge coils.

Another possible source for the downstream plasma could be from photoionization of the background gas. Photoionization occurs when a photon with an energy exceeding the ionization potential of the gas strikes an atom and releases an electron. For argon, this corresponds to $15.8 \mathrm{eV}$. This means that photons in the UV region of the electromagnetic spectrum will be responsible for the bulk of the photoionization. Argon has several strong emission lines in the UV spectrum from $49 \mathrm{~nm}$ to $75 \mathrm{~nm}$ [85]. These emission lines could 
excite photons in the UV region, where the photoionization cross-sections of argon are the highest [86]. However, the peak photoionization cross-sections (about 30x $10^{-21} \mathrm{~m}^{-2}$ ) are an order of magnitude smaller than the ionization cross sections shown in Figure 7.29. This implies that they are less likely to create an ionization event than electron impact collisions. Additionally, incoherant emission from the argon plasma in the discharge channel drops off as $1 / r^{2}$. This means that the probe signals measured by each probe should also decay as $1 / r^{2}$, where $r$ is their separation distance. The probes were spaced $18 \mathrm{~cm}$ apart, so the signal on the downstream probe should decay by 30 . The scaled data in Figure 6.36 show the signal decay between probes is closer to 4 . Both observations suggest that photoionization is not a likely contributor to the bulk of the plasma downstream. Further analysis on this phenomenon would require additional testing.

\subsection{Discussion of Translation Results}

The overwhelming conclusion from the translation data presented in Section 7.2 was that the plasmoid failed to translate for all conditions tested. The delays in features between probes spaced far apart appeared almost simultaneously. Additionally, signals on downstream probes appeared before signals on upstream probes. These conflicting signals do not agree with the expected profiles of a translating probe, shown in Figure 4.18 and Figure 5.39 .

While plasma activity was recorded by several plasma probes, the source of this plasma was shown to be different than a translating plasmoid. Plasma activity was measured first by the downstream probes with a peak in density observed $1 \mu$ s after the peak field reversal. However, the density of this plasma was calculated to be at least two orders of magnitude 
below the expected AFRC density and decreased with increasing pressure. The source of this plasma was likely due to ionization of the downstream neutral gas by electrons created during the initial firing of the main bank coils, as explained in Section 7.2.3 and not a translating plasmoid. After plasma activity was seen on downstream probes, a dense plasma was measured by a probe located $15 \mathrm{~cm}$ upstream of the downstream probes. While the density of plasma measured at the probe was comparable to the expected density of the AFRC, the plasma's travel across the probe lasted longer than $13 \mu \mathrm{s}$, which was much too slow for a $25 \mathrm{~cm}$ long plasmoid traveling at $100 \mathrm{~km} / \mathrm{s}$. Since plasma was seen to peak after the loss of field reversal, it was most likely the remnants of the AFRC after confinement was lost rather than a translating plasmoid.

Despite the lack of evidence supporting a translating plasmoid, an AFRC plasmoid was observed for most conditions, indicated by a magnetic field reversal on the inner wall, inductive coupling in the coil currents, and magnetic field exclusion on the outer wall. This data was presented in Section 6.4. This data also showed that the maximum lifetime of the AFRC plasmoid was much shorter than expected, with only 6-7 $\mu$ s between the start of reversal and the decay of the reversed field. The total lifetime for the plasmoid was 4-10 $\mu \mathrm{s}$. The lifetime was expected to be closer to the quarter cycle of the discharge (25 $\mu$ s for the $10 \mathrm{kHz}$ circuit; $12.5 \mu$ s for the $20 \mathrm{kHz}$ circuit). The reason for the abbreviated lifetime of the plasmoid is most likely related to its inability to translate. The source of lifetime limitation will be explored in the remainder of this dissertation. 


\subsection{Possible Failure Modes}

Several hypotheses can be made as to why the AFRC's lifetime was much shorter than expected and thus why it failed to translate. These hypotheses include a current induction limit, insufficient conductivity, and a gross instability or imbalance. Each theory is briefly explored in Sections 7.4.1, 7.4.2, and 7.4.3.

\subsubsection{Failure Mode: Current Induction Limit}

The AFRC requires an inductive drive for formation. A current is driven in the plasmoid by changing current in the coils, analogous to a magnetic transformer. Plasmoid resistance opposes this current drive through collisions. It is possible that when the rate of change of current or coil voltage falls below a certain threshold, the inductive drive is insufficient to stave off the loss of current from resistance. The plasmoid current can no longer be sustained and the plasmoid disappears. The XOCOT-T3 data for all cases was examined to see if there was some relationship between the plasmoid current lifetime and the $\mathrm{dI} / \mathrm{dt}$ of the coils (voltage). Plasmoid current lifetime is defined in this instance as the time span between when the midplane probe initially crosses through $\mathrm{B}=0$ and when the reversed field begins to decay. The decay of the reversed field indicates that the plasmoid current is no longer working to generate a reversed field. Coil voltage is quantified by the time derivative of the current, since the effective inductance (coils and plasmoid) is unknown. To provide the best representative of each case, the average waveform from each data set was used (see Section 6.4. Figure 7.31 shows the results from this scaling study, considering the outer coil and inner coil independently. The results for a $50 \mathrm{mT}$ Torr fill 

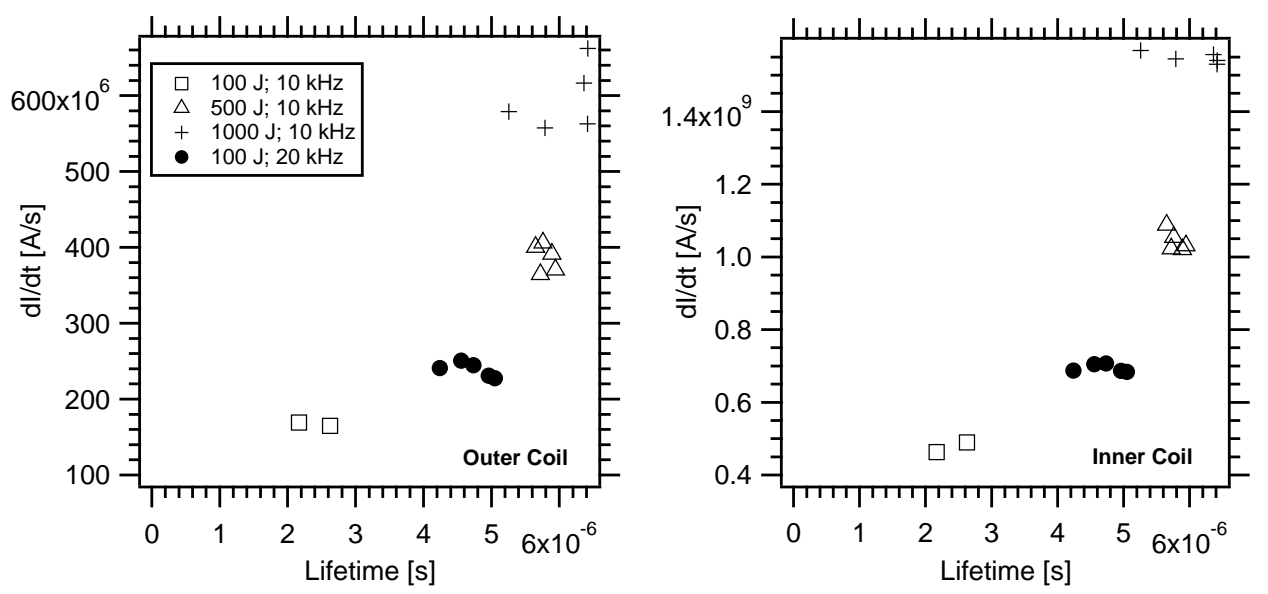

Figure 7.31: Time derivative of current compared to observed plasmoid current lifetime for all XOCOT-T3 test conditions, showing the scaling for the outer coil (left) and inner coil (right).

have been removed since all tests with 50 mTorr show a delayed and incomplete reversal.

The results from this study show that the plasma current in higher energy plasmoids disappears at a higher coil voltage (dI/dt) than their lower energy counterparts. Additionally, the plasmoid current in the $500 \mathrm{~J}$ and $1000 \mathrm{~J}$ plasmoids disappears at the same time (around $6 \mu \mathrm{s}$ ). A terminated lifetime from a current induction limit would show that all discharges would disappear at the same coil voltage and the $1 \mathrm{~kJ}$ plasmoids would be sustained several $\mu$ s past the $500 \mathrm{~J}$ plasmoids. This study does not show these trends, therefore it is likely the XOCOT-T3 plasmoid was not lifetime limited due to a drop in current induction.

\subsubsection{Failure Mode: Low Conductivity}

An AFRC plasmoid creates a bi-directional field in the annulus which excludes the outer coil field. If the conductivity is fairly low, the outer coil field can diffuse through the 
plasmoid and annihilate the reversed field. The magnetic field diffusion time through the plasmoid can be easily estimated using assumed plasma properties to determine if this is a possibility.

The 1/e timescale for magnetic field diffusion is defined by Equation 7.2 from Chen[45]:

$$
\tau_{d}=\frac{\mu_{0} L^{2}}{\eta_{\perp}}
$$

where the plasma resistivity can be estimated using the Spitzer resistivity transverse to a magnetic field [46]:

$$
\eta_{\perp}=1.29 \times 10^{4} \frac{Z \ln \Lambda}{T^{(3 / 2)}}
$$

In Equation $7.3, \mathrm{Z}$ is the ionic charge, $\ln \Lambda$ is the Coulomb logarithm, and $T$ is the electron temperature in Kelvin. The Coulomb logarithm is a function of temperature and density and it can be approximated to 10 for AFRC plasmas. The Spitzer resistivity drastically under-predicts the resistivity as discussed in Section 4.1, but will be used in this instance as a best-case scenario.

The internal temperature of an AFRC plasma can be anywhere between $10 \mathrm{eV}$ and 50 eV. Using Equation 3.22, the magnetic soakthrough time for 1-cm thick, $10 \mathrm{eV}$ plasma is $3.49 \mu \mathrm{s}$. Increasing the temperature to $20 \mathrm{eV}$ gives a soakthrough of $9.02 \mu \mathrm{s}$. A $50 \mathrm{eV}$ plasma soaks through in $32 \mu \mathrm{s}$. These estimates suggest that the plasma lifetime could be limited by soakthrough if the plasma is less than $20 \mathrm{eV}$. Internal measurements of 
temperature and magnetic field are required, however before making a definite conclusion. Internal measurements are difficult to make in FRC plasmoids as they can be highly disruptive. Before a full investigation begins, an extensive study would be required to ensure meaningful measurements can be made.

\subsubsection{Failure Mode: Instabilities and Imbalances}

AFRC plasmoids can experience global instabilities which can disrupt the confinement. They include rotational, tearing, and tilt instabilities. The instabilities can happen in any direction: radial, azimuthal, and axial. The most common instability in an AFRC has been a radial instability, as discussed in Section 3.3.5. This instability causes termination of an AFRC through a radial collapse of the plasmoid onto the inner wall.

The radial instability discussed in Section 3.3.5 is more accurately an imbalance, since it is not triggered by a small, local disturbance. The radial imbalance in an AFRC can occur when there is unequal pressure between the inner and outer radii of the plasmoid. The plasmoid will travel along the pressure gradient to the wall. This was often seen in the asynchronous coil operation for the Coaxial Slow Source experiment [50]. The parallel synchronous coil operation was designed to mitigate this issue, as derived in Section 3.3.1. However, the theory behind this operation assumes that the coil voltages on the inner and outer coil are equal. If they differ due to stray inductance, non-ideal coil coupling effects, or other imperfections, the plasmoid will slide into one of the walls. This appears to external sensors as an instability, though in nature it is the result of a pressure imbalance.

Gross radial and azimuthal instabilities of any almost any nature can be viewed from 
imaging the plasma end-on. A viewport on the far end of the XOCOT-T3 experiment provided access for fast, single frame photography of the configuration to see if radial and azimuthal instabilities are an issue. Axial instabilities are more difficult to see but may be detected in the XOCOT-T3 experiment with axial probe arrays. Since radial and azimuthal instabilities are easier to diagnose, these will be the topic of further investigation in Chapter 8 and 9.1 ,

\subsection{Summary}

The results from the XOCOT-T3 time-of-flight arrays demonstrated that despite the presence of a reversed field, the plasmoid did not translate. The field reversal lasted 4-10 $\mu \mathrm{s}$ before disappearing. The magnetic signatures on all probes have no staggered features that would suggest a translation velocity. In some instances, signatures appear on downstream probes before they appear on upstream probes. While plasma was measured downstream of the thruster, it appeared simultaneously on probes spaced $18 \mathrm{~cm}$ apart and throughout the formation sequence. These signals did not correspond to a translating AFRC plasmoid and were likely due to ionization of the background gas by stray electrons.

Several hypotheses were suggested for why the plasmoid failed to translate. One hypothesis was that the plasmoid current can only be driven when $\mathrm{dI} / \mathrm{dt}$ is above a certain threashold. This theory was ruled out for this experiment, by showing that the higher energy plasmoids disappeared at a higher $\mathrm{dI} / \mathrm{dt}$ than the lower energy plasmoids. Another theory was that the plasma was lifetime limited due to magnetic soakthrough from the outer coil. This theory was shown to be plausible as the soakthrough time for a $1 \mathrm{~cm}, 10 \mathrm{eV}$ plasma is $3.49 \mu \mathrm{s}$. Internal probing of the plasma is required to make any stronger connection between the 
XOCOT-T3's plasmoid lifetime and magnetic soakthrough. Instabilities could also be the reason for a limited lifetime and translation failure. Radial and azimuthal instabilities will be explored in Chapters 8 and 9 . 


\section{Chapter 8}

\section{Instability Study Data and Results}

The results from Chapter 7 concluded that the plasmoid failed to translate from the coils and was limited in lifetime to $4-10 \mu \mathrm{s}$. One possible theory for the limited lifetime is that the plasma experienced a disruptive instability or imbalance which terminated the configuration. This chapter discusses the additional experimental data collected from the XOCOT-T3 to explore this theory. Section 8.1 explains how Langmuir probes and fast, single frame photography were implemented to look at radial and azimuthal instabilities and imbalances. Section 8.2 presents the data from the instability and imbalance study. The results from the study are discussed in Section 8.3 .

\subsection{Instability Study Diagnostics}

Gross instabilities of a radial or azimuthal nature can be viewed by imaging the plasma end-on (along the z-axis). A fast, single frame digital camera was installed at a viewport 
opposite the discharge channel. The perspective view from the inner coil prevented the entire plasmoid from being imaged properly. To see an accurate radial plasma distribution, only an angular section of the channel could be viewed in a single frame. The camera, described in Section 5.2.4, was an intensified CCD single-frame digital camera with a minimum exposure time of $5 \mathrm{~ns}$. A $480 \mathrm{~nm}, 10 \mathrm{~nm}$ bandwidth filter was added to camera to isolate a set of Ar II emission lines.

The still images were recorded in $1 \mu \mathrm{s}$ frame widths for the $10 \mathrm{kHz}$ discharge and 500 ns frame widths for the $20 \mathrm{kHz}$ discharge. Images were collected for the first $1 / 4$ cycle only and as long as enough visible light was detectable. The camera was unable to store sequential images. Each image is from a separate shot at repeatable conditions.

The other diagnostic used in this section of research was an asymmetric double Langmuir probe. The probe and circuit is described in Section 5.2.3. The ADLP was only used in the $20 \mathrm{kHz}$ circuit, since the $10 \mathrm{kHz}$ capacitor failed before data could be collected. The probe was inserted directly into the discharge channel, at the axial midplane. The probe bias was fixed to $24 \mathrm{~V}$ for all shots. Data was collected across the entire radial cross section of the channel at one aziumuthal location, $2 \mathrm{~mm}$ apart.

\subsection{Instability Study Data}

The results from Chapter 6 show that all XOCOT-T3 discharges have the same gross behavior. The reversed field disappears, followed by the loss of the inductive load and excluded field on the outer probes. This means it was not necessary to rerun each operating point; a single setting from each circuit was sufficient to resolve the overall plasma 
behavior. Data from the $10 \mathrm{kHz}$ circuit was collected for the $500 \mathrm{~J}, 4$ mTorr discharge. Data with the $20 \mathrm{kHz}$ circuit was taken at $100 \mathrm{~J}$ with a 4 mTorr fill. Image data for both discharges is presented in Section 8.2.1. Asymmetric double Langmuir probe data is provided for the $20 \mathrm{kHz}$ circuit in Section 8.2.2

\subsubsection{Image Data}

The XOCOT-T3 plasmoid was imaged for the first quarter cycle for two different discharges. The $10 \mathrm{kHz}$ tests used a $500 \mathrm{~J} /$ pulse energy setting with a 4 mTorr static fill. The $20 \mathrm{kHz}$ tests also used 4 mTorr of gas with $100 \mathrm{~J}$ of discharge energy. Background images were captured prior to plasma shots. These images were used to establish insulator boundaries for the plasma images. The background images for each discharge is displayed in Figure 8.1. The insulator boundaries are drawn in the thick dotted lines. The thin dotted line is the entrance to the translation chamber. The camera was pointed to look along the length of the inner insulator. The red boxes indicate the areas where the edge of the inner insulator is directly orthogonal to the camera lens. This is the only region in the photographs where it will be possible to tell where the plasma is radially distributed.

Images for the $10 \mathrm{kHz}$ discharge are compiled in Figure 8.2. The $20 \mathrm{kHz}$ images are shown in Figure 8.3 . The outlines of the insulators have been drawn in each image as well as the red boxes, corresponding to the region where the radial plasma distribution can be seen. The midplane magnetic fields for each data set have been included for reference.

In the $10 \mathrm{kHz}$ data, the plasma formed off of both walls, but quickly expanded in $4 \mu \mathrm{s}$ to touch the inner wall. Shortly after this, the loss of reversal was seen on the inner magnetic 

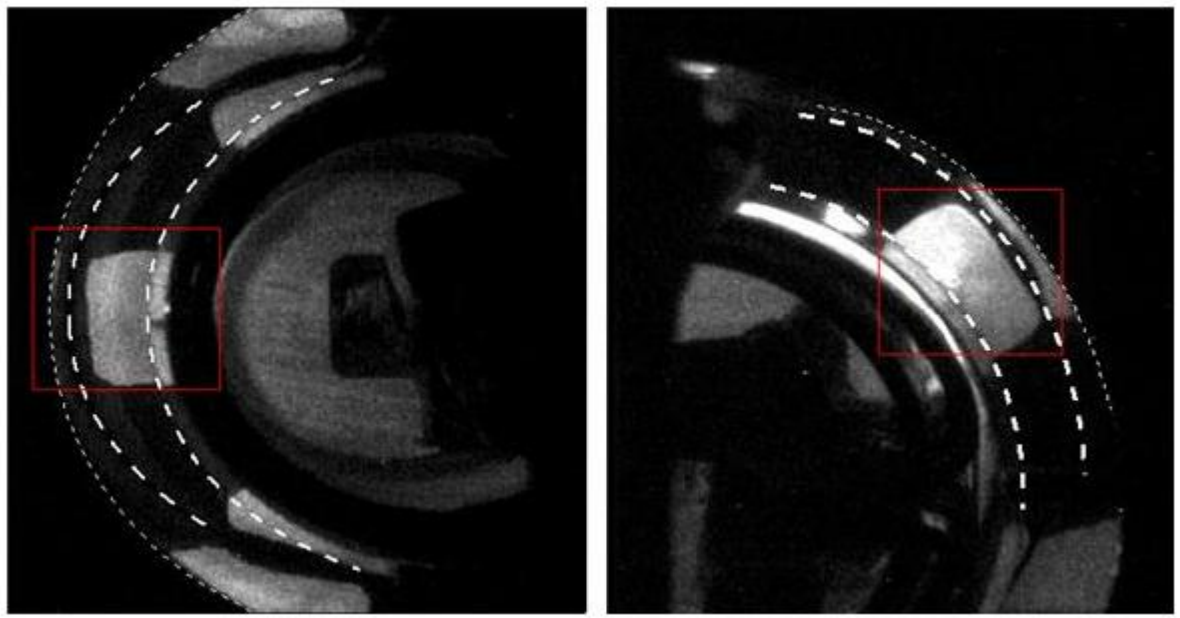

Figure 8.1: Background images for the $10 \mathrm{kHz}$ data (left) and $20 \mathrm{kHz}$ data (right), showing the location of the insulator walls and tank entrance.

field probe. A section of the plasmoid appeared to condense in the center of the channel after wall contact, but it did not correspond to a reversed field on the inner magnetic probes. The plasma extinguished fully on the inner wall at $20 \mu \mathrm{s}$, at which time the loss of excluded flux on the outer probes was noted. No azimuthal instabilities are seen within the camera view.

The $20 \mathrm{kHz}$ data was similar in behavior to the $10 \mathrm{kHz}$ circuit. The plasma formed off both walls before expanding toward the inner wall. Inner wall contact occurred around $10 \mu \mathrm{s}$, the same time the reversal began to disappear on the inner magnetic field probe. Even after wall contact, the plasma fills the entire annulus. The plasma at this point is a mixture of Argon and quartz, which is why it appears brighter to the camera. Azimuthal instabilities were not apparent in any of the photographs.

The DiCAM images are strong evidence that the plasma was radially unbalanced. Wall contact with the inner insulator appears to disrupt the field reversal, leading to shorter than expected lifetime. Langmuir probe measurements internal to the channel, presented in 

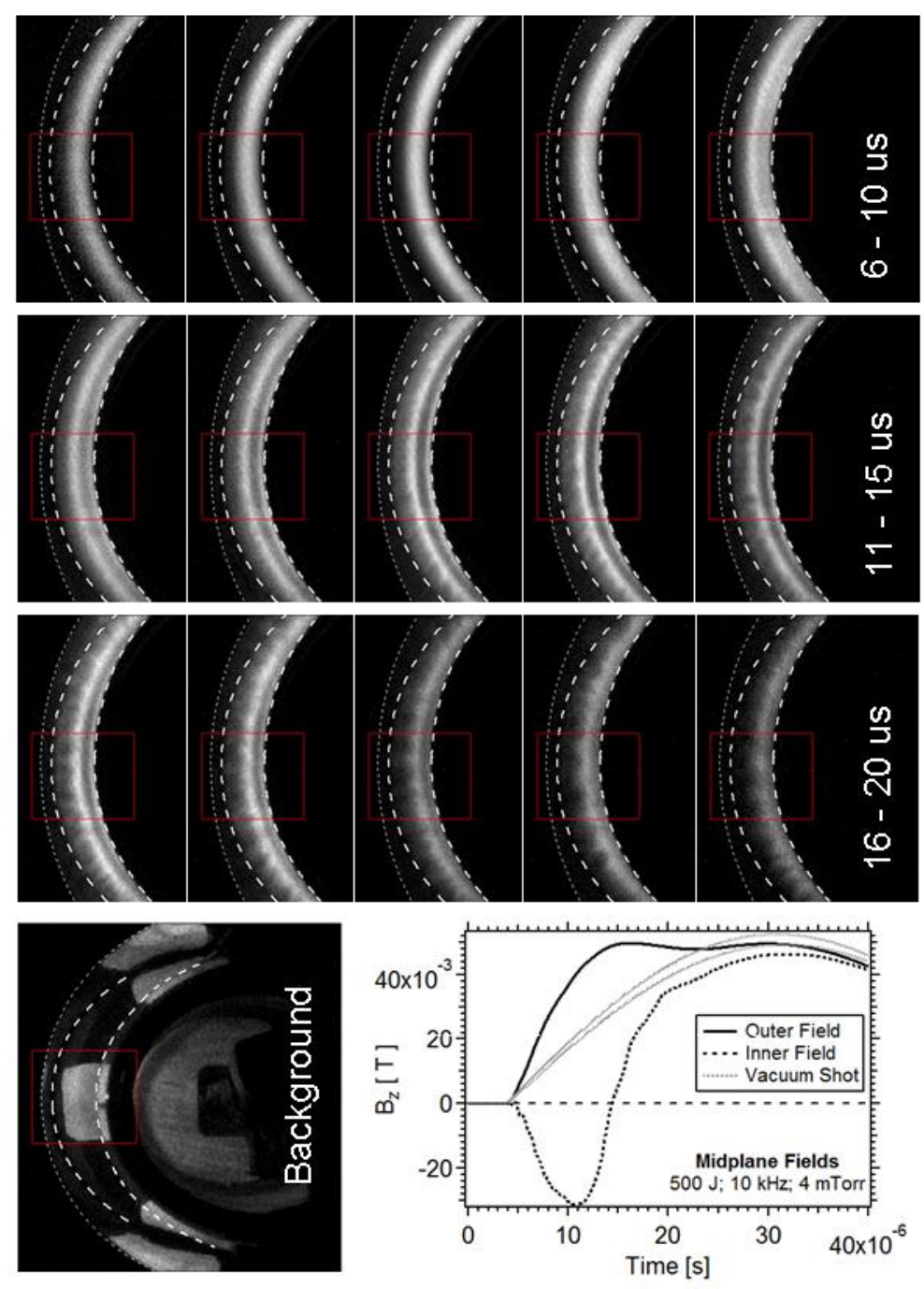

Figure 8.2: Images of the plasma from an end view for a $500 \mathrm{~J}, 4 \mathrm{mTorr}$ plasma test with the $10 \mathrm{kHz}$ circuit.

Section 8.2.2 confirm this finding. 

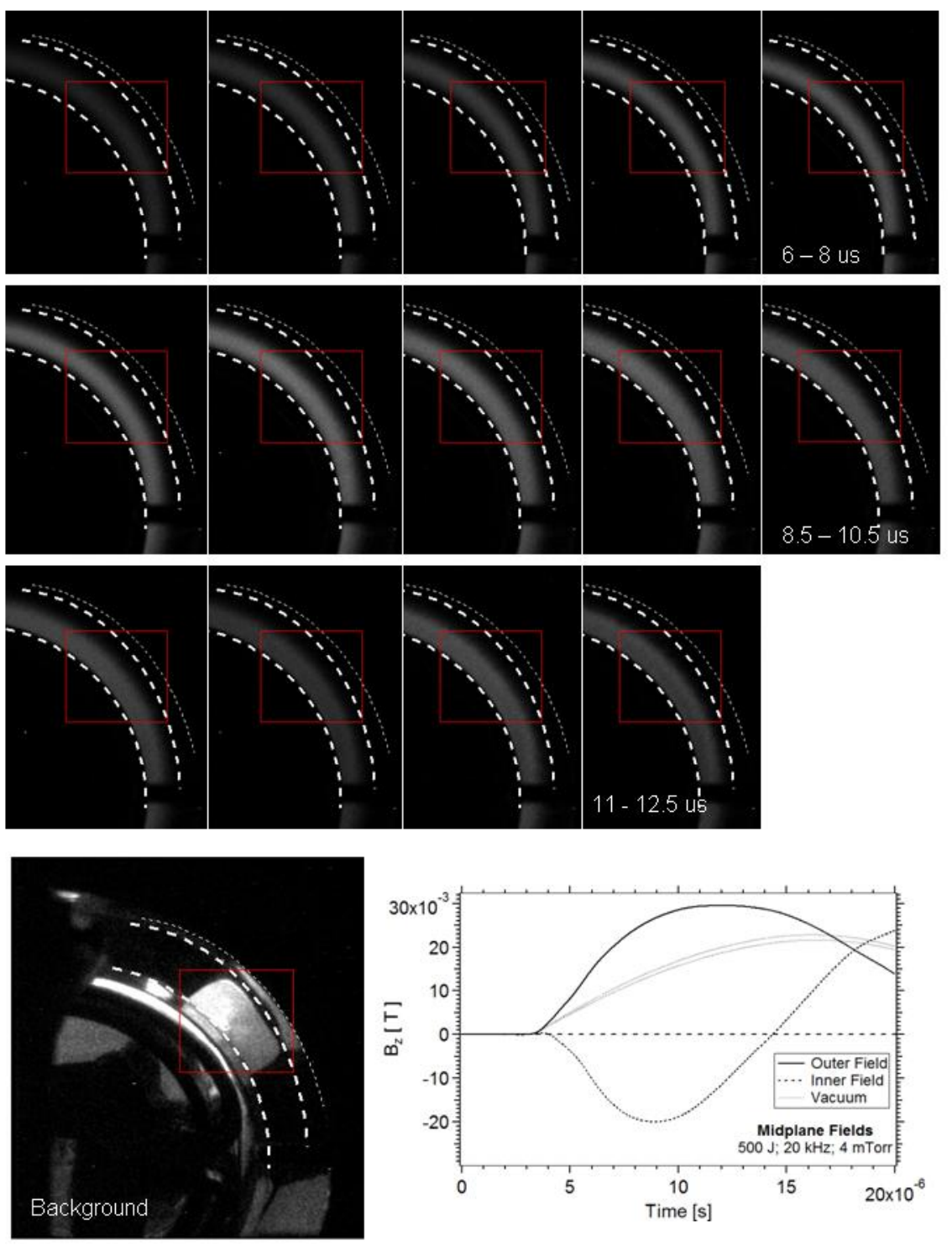

Figure 8.3: Images of the plasma from an end view for a $100 \mathrm{~J}, 4 \mathrm{mTorr}$ plasma test with the $20 \mathrm{kHz}$ circuit.

\subsubsection{Asymmetric Double Langmuir Probe Data}

An asymmetric double Langmuir probe was inserted into the discharge channel of the XOCOT-T3. The purpose of this exercise was to understand where the plasma was 
distributed spatially and temporally at the channel midplane. The probe and theory used to interpret the probe is described in Section 5.2.3. Rather than using the traditional method of sweeping the double probe to establish the I-V characteristic, the probe was fixed at a single bias.

The bias voltage was selected by building a one-sided I-V characteristic $3.8 \mathrm{~cm}$ downstream of the exit plane. The one-sided characteristic was chosen to limit the number of shots required to construct the full trace. For the one-sided characteristic, the larger electrode was biased negatively with respect to the smaller electrode to collect ions. While it would have been preferred to collect the I-V characteristic at the midplane, this was decided against to ensure the probe's longevity. FRC plasmas tend to destroy probes very quickly. Using the probe inside the channel for the $20+$ shots required to establish the voltage for saturation might have made the probe unusable for the full radial scan. The temporal data collected for each bias voltage is displayed in Figure 8.4. Each trace represents a single shot at a fixed bias voltage. The data at $20 \mu$ s was compiled to construct the I-V characteristic, also shown in Figure 8.4. Additional I-V traces from this data set are presented in Appendix A.

The data in Figure 8.4 shows that the probe saturated around 15-18 V of applied bias voltage. The plasma temperature and densities inside the channel were expected to be much greater than the plasma downstream. Higher densities increase the saturation current, while temperatures push the saturation voltage higher. Theoretical I-V characteristics are plotted in Figure 8.5. These traces assume $100 \%$ ionization of the 4-mTorr gas fill. Figure 8.5 demonstrates that a bias voltage of $24 \mathrm{~V}$ is suitable for a $5-\mathrm{eV}$ and $10-\mathrm{eV}$ plasma case. If the plasma is hotter than this, voltages in excess of $50 \mathrm{~V}$ are required. However, voltages this high can cause the probe to emit electrons and disturb the surrounding plasma. For this reason, it was decided that a conservative $24 \mathrm{~V}$ should be used for the bias. This bias will 


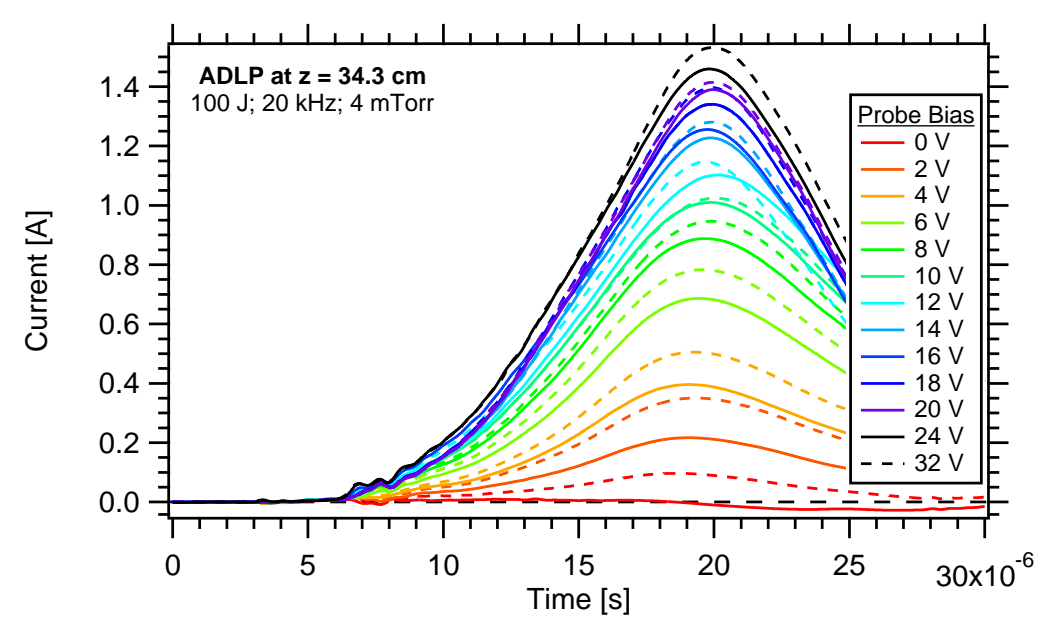

(a)

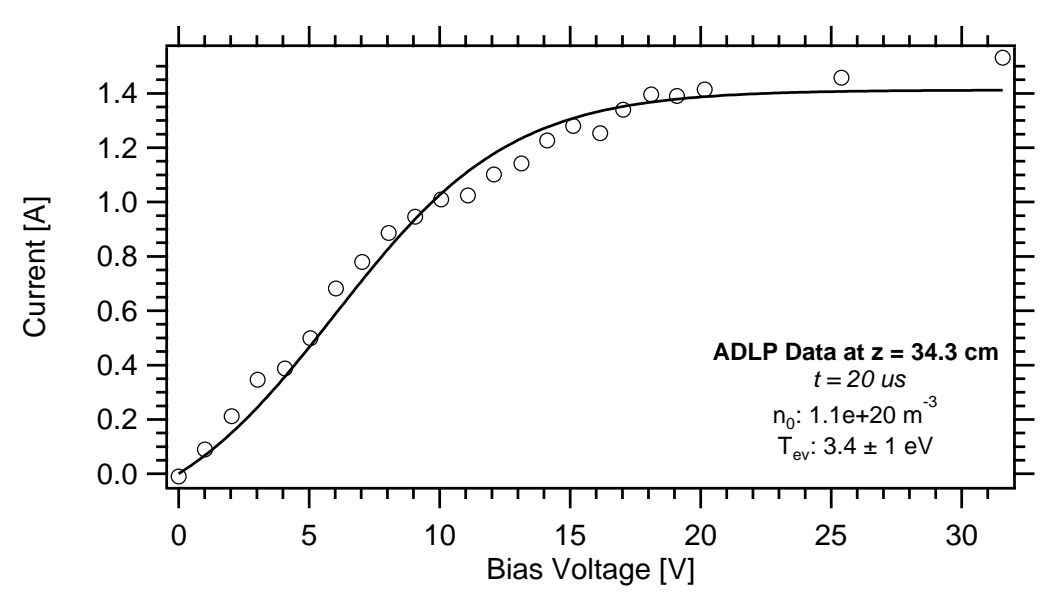

(b)

Figure 8.4: ADLP data at $\mathrm{z}=33.8 \mathrm{~cm}$, showing the temporal data at fixed bias voltages and the reconstructed I-V characteristic.

still allow the probe to be used in the qualitative radial survey and limit the effect of the probe on the plasma. Additionally, it will limit the current drawn to the probe and place less strain on the circuit electronics and current transducers.

Following selection of the bias voltage, the ADLP was inserted into the channel at the axial midplane. Data was collected at different radial locations between the insulators, shown in Figure 8.6a. The data was analyzed to get the distribution of probe current along the radius of the channel for several points in time $(6-17 \mu \mathrm{s})$. These plots are displayed in Figure 8.6p. Midplane magnetic fields are also shown for comparison in Figure 8.6c. 


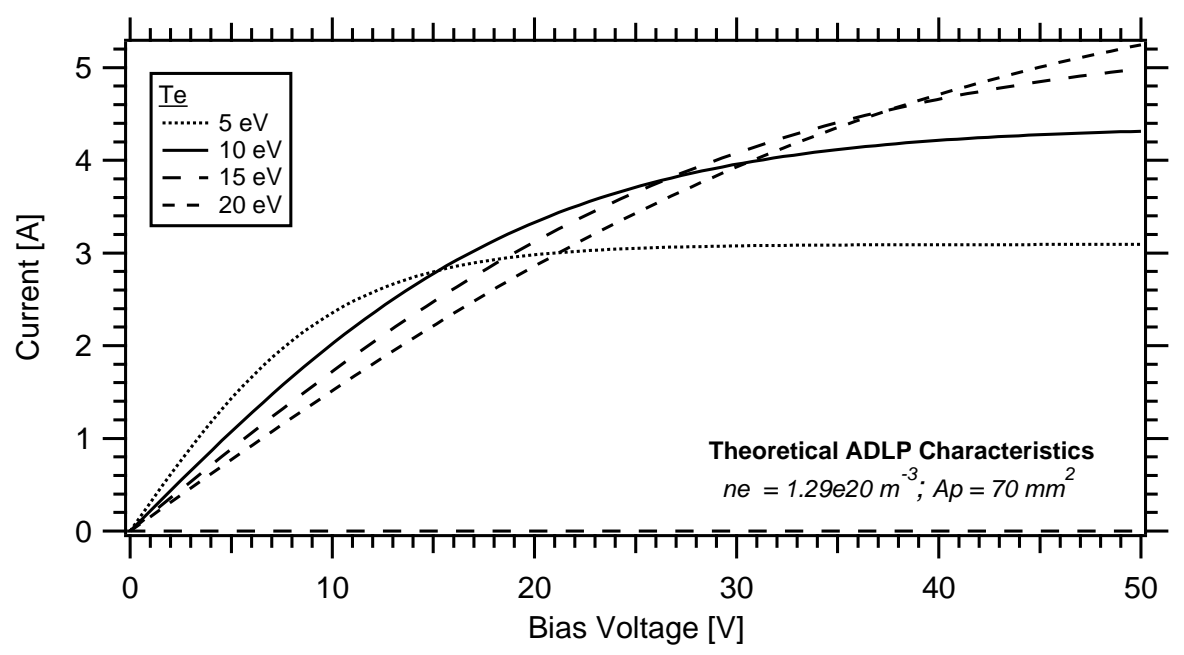

Figure 8.5: Theoretical I-V characteristics for a 4-mTorr backfill with $100 \%$ ionization and various temperatures.

The data and results in Figure 8.6 show that while plasma was detected along the entire channel width, the peak of plasma occurred near the inner insulator. Over time, the plasma near the inner wall grew and eventually the bulk of the plasma traveled to the inner wall. This trend was also seen in the images in Section 8.2.1. Wall contact with the inner insulator caused the plasmoid to lose field reversal so that the entire configuration quenched on the inner wall. What the ADLP data makes more apparent than the images was that the plasma seemed to form near the inner wall rather than at channel centerline $(\mathrm{r}=93 \mathrm{~mm})$. This suggests that the inductive plasma current was insufficient to hold itself off the inner coil.

An additional note should be made about the seemingly high level of current collected by the ADLP. The expected saturation current for this probe in a stationary, $100 \%$ ionized, $10 \mathrm{eV}$ plasma is close to $4 \mathrm{~A}$. The probe collects less than $4 \mathrm{~A}$ at the midplane, but the current collected near the inner wall exceeds $6 \mathrm{~A}$. While it is possible that the probe could be perturbing the plasma, the high level of current may come from the fact that the probe is located in a region of strong current. This increases the drift velocity of charged particles relative to the probe and increases the probe current. The current is composed primarily 

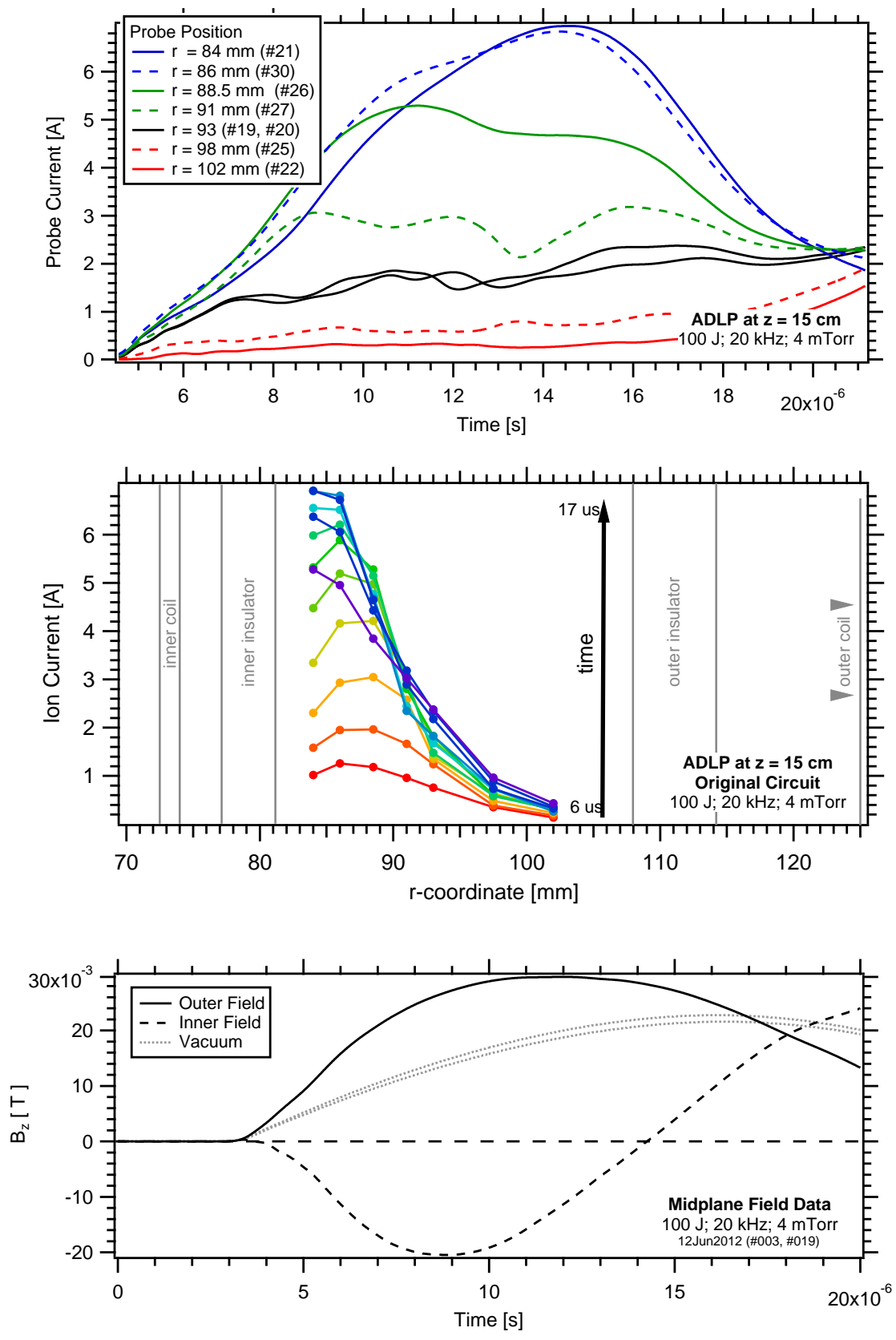

Figure 8.6: ADLP measurements at the axial midplane, including (A) the temporal probe current, (B) radial distribution of plasma current at various times, and $(\mathrm{C})$ midplane magnetic fields.

of electrons, but as discussed in Section 3.3.4 ions have been found to rotate as well.

The rotation of both species relative to the probe results in higher than expected current levels. As discussed in Section 5.2.3, the presence of a plasma current makes it difficult 
to determine plasma properties from the data. For this reason, the ADLP data at the axial midplane is reported as probe current only.

\subsection{Discussion}

The internal ADLP data and the fast, single frame images provide independent, overwhelming evidence that the plasmoid was radially unstable between the coils. Both types of data show a plasmoid that formed near the inner wall and lost field reversal just after wall contact. The plasmoid did not appear to be unstable azimuthally, up until wall contact. The radial motion therefore limited the lifetime of the plasmoid and prevented the configuration from translating out of the coils.

The most likely mechanism for the radial motion is uneven radial magnetic pressure on both sides of the configuration. Ideally, the AFRC formed in the parallel mode with synchronous coils should stay balanced between the coils. The magnetic pressure force pushing against the outer surface should be equal to the magnetic pressure force pushing against the inner surface. As was presented in Section 3.3.1, if the voltages on both coils are equal and the plasma current is twice the product of the outer coil current and the number of turns on the outer coil the pressure balance will hold. In the XOCOT-T3, it is likely that one of these conditions was not met. In Chapter 9, data will be presented to determine what circuit settings are required to maintain radial equilibrium. 


\subsection{Summary}

This chapter investigated the limited lifetime of the XOCOT-T3's annular plasmoid, using the notion that the plasmoid experienced a fatal radial or azimuthal instability or imbalance. Digital images from an intensified CCD camera were compiled from the $10 \mathrm{kHz}$ and $20 \mathrm{kHz}$ circuits at $500 \mathrm{~J}$ and $100 \mathrm{~J}$, respectively with a 4 mTorr gas fill. Using these images, it was discovered that the plasmoid was not radially centered between the coils and traveled to the inner wall about $4 \mu$ s after reversal. No azimuthal instabilities were observed. This finding was further corroborated by double probe data collected at the axial midplane for the $20 \mathrm{kHz}$ circuit. An asymmetric double Langmuir probe was inserted into the discharge channel at the axial midplane, scanning along the radius between the insulators. This data showed that the bulk of the plasma traveled to the inner wall, coinciding with the loss of the reversed field. The ADLP data also suggested that the plasma formed closest to the inner wall, which was not expected. These data sets conclude that the lifetime of the AFRC plasmoid was terminated due to a radial contact with the inner. Chapter 9 investigates the mechanism for this radial wall contact, focusing on finding what conditions are required for radial equilibrium. 


\section{Chapter 9}

\section{Radial Balance Study Data and Results}

The results from Chapter 7 concluded that the plasmoid failed to translate from the coils and was limited in lifetime to around $10 \mu \mathrm{s}$. An instability study presented in Chapter 8 determined that radial motion of the plasmoid was responsible for the short AFRC lifetime in the XOCOT-T3. This chapter conducts a study to correct for the radial motion. A brief overview on radial balance in AFRCs is given in Section 9.1. Data from circuit tuning efforts used to control the radial motion are presented in Section 9.2. Section 9.3 covers the results from this study. Section 9.4 discusses the results and how they impact future AFRC translation experiments.

\subsection{Radial Balance in AFRCs}

A radial imbalance in AFRCs arises when an unequal pressure differential occurs on the inner and outer radii of the plasmoid. The plasmoid is forced along the pressure gradient 
to the outer or inner wall. In the XOCOT-T3 experiment, the plasmoid traveled to the inner wall because of the higher magnetic field on the outer surface. Notionally, the plasmoid is supposed to remain radially balanced between the coils for the parallel synchronous operation. The theory supporting this finding was derived in Section 3.3.1. To recap, the theory stated that the plasmoid would split the annulus into equal areas assuming the voltage on the inner coil and outer coil were equal. For the XOCOT-T3, the plasma radius should be located at $r_{m}=\left(r_{o c}^{2}+r_{i c}^{2}\right) / 2$ or $r_{m}=99 \mathrm{~mm}$. Instead, it appears that the plasma sat very close to the inner coil somewhere near $r_{m}=80 \mathrm{~mm}-90 \mathrm{~mm}$.

The radial balance theory presented in Section 3.3.1 assumed that the voltages on both coils were equal at all times. In the case of no stray inductance in either the inner coil or the outer coil circuit, this is true. However, stray inductance is extremely difficult to remove in a real experiment, so the theory must be re-derived to take this into account. The new derivation is presented here.

The equations for the radial balance of an AFRC consist of 4 equations: one equation of motion for the radial position and three circuit equations for the outer coil circuit, inner coil circuit, and plasmoid circuit. The voltage due to coil resistance has been neglected since it is fairly small (10 $\mathrm{m} \Omega$ or less).

$$
\begin{gathered}
m \frac{d^{2} r_{m}}{d t^{2}}=2 \pi r_{m}\left(\frac{B_{z i}^{2}}{2 \mu_{0}}-\frac{B_{z o}^{2}}{2 \mu_{0}}\right) \\
V_{o}=\frac{d}{d t}\left(L_{o} I_{o}+M_{i o} I_{i}-M_{o p} I_{p}\right) \\
V_{i}=\frac{d}{d t}\left(L_{i} I_{i}+M_{i o} I_{o}-M_{i p} I_{p}\right) \\
0=\frac{d}{d t}\left(L_{p} I_{p}-M_{o p} I_{o}-M_{i p} I_{i}\right)-R_{p} I_{p}
\end{gathered}
$$


As was done in Section 3.3.1 and in Reference [42], only the zeroth order effects will be considered. This means that $d r^{2} / d t^{2}$ is neglected, and the radial position of the plasmoid and plasmoid current are expressed as

$$
\begin{gathered}
I_{p}=I_{p, 0}+I_{p, 1}+\ldots \\
r_{m}=r_{m, 0}+r_{m, 1}+\ldots
\end{gathered}
$$

where $I_{p, 0}$ and $r_{m, 0}$ are the zeroth order approximations, $I_{p, 1}$ and $r_{m, 1}$ are the first order approximations and so on. Using these approximations, the plasmoid current from Equation 9.1 is the same as before $I_{p, 0}=2 N_{o} I_{o}$. Invoking solenoid approximations for all inductances and observing that $V_{o}=V_{i}$, the system of equations becomes:

$$
\begin{gathered}
0=\frac{\mu_{0} N_{o}^{2} \pi r_{o c}^{2}}{l} I_{o}+L_{e o} I_{o}+\frac{\mu_{0} N_{o} N_{i} \pi r_{i c}^{2}}{l} I_{i}-\frac{2 \mu_{0} N_{o}^{2} \pi r_{m, 0}^{2}}{l} I_{o} \\
-\frac{\mu_{0} N_{i}^{2} \pi r_{i c}^{2}}{l} I_{i}-L_{e i} I_{i}-\frac{\mu_{0} N_{o} N_{i} \pi r_{i c}^{2}}{l} I_{o}+\frac{2 \mu_{0} N_{i} N_{o} \pi r_{i c}^{2}}{l} I_{o} \\
\frac{\mu_{0} N_{o} \pi r_{m, o}^{2}}{l} I_{o}-\frac{\mu_{0} N_{i} \pi r_{i c}^{2}}{l} I_{i}=-2 N_{o} \int R_{p} I_{o} d t
\end{gathered}
$$

Solving Equation 9.7 for $I_{i} / I_{o}$ and Equation 9.8 for $r_{m, 0}$ yields:

$$
\begin{gathered}
\frac{I_{i}}{I_{o}}=\frac{N_{o}^{2} r_{o c}^{2}+\frac{L_{e o} l}{\mu_{o} \pi}-2 N_{o}^{2} r_{m, 0}^{2}+N_{o} N_{i} r_{i c}^{2}}{N_{i}^{2} r_{i c}^{2}+\frac{L_{e i} l}{\mu_{0} \pi}-N_{o} N_{i} r_{i c}^{2}} \\
r_{m, 0}^{2}=\frac{N_{i} r_{i c}^{2} I_{i}}{N_{o} I_{o}}-\frac{2 l}{\mu_{0} \pi I_{o}} \int R_{p} I_{o} d t
\end{gathered}
$$

In the XOCOT-T3, the turns on the outer coil were equal to the turns on the inner coil so 
$N_{i}=N_{o}=N$. Combining Equations 9.9 and 9.10 with this information, the radius of the plasmoid to a zeroth-order approximation is

$$
r_{m, 0}^{2}=\frac{\mu_{0} \pi r_{i c}^{2}\left(N^{2} r_{o c}^{2}+\frac{L_{e o} l}{\mu_{0} \pi}+N^{2} r_{i c}^{2}\right)-\frac{2 l^{2} L_{e i}}{\mu_{0} \pi I_{o}} \int R_{p} I_{o} d t}{L_{e i} l+2 N^{2} \mu_{0} \pi r_{i c}^{2}}
$$

It's apparent from Equation 9.11 that with non-zero external parasitic inductances the plasmoid radius is no longer a simple function of the outer and inner coil radii. To study the effect of two different equation inputs, Equation 9.11 was used to predict the plasmoid's radial position in the XOCOT-T3 for the first $10 \mu \mathrm{s}$ of the $20 \mathrm{kHz}, 100 \mathrm{~J}$ condition. The results for several different plasma resistance values are displayed in Figure 9.11a. These results show that the plasmoid's position is highly sensitive to the resistance of the plasma. This matches the theory presented in Section 3.3.1 for independent coils. Resistance results in a motion of the plasmoid toward the inner insulator. Since the plasmoid current is responsible for generating the reversed field to hold the configuration off the inner wall, this makes sense. Resistance decreases the ability to drive the inductive current and lowers the plasma current. For $R_{p}=10 \mathrm{~m} \Omega$ with the XOCOT-T3 predictions, the plasmoid comes in contact with the inner insulator as the results in Figure 9.1 a indicate.

Equation 9.11 was also used to study the effect of increasing the outer coil's stray inductance $L_{e o}$. The final radial position of the plasmoid was calculated as a function of $L_{e o}$. Figure $9.1 \mathrm{p}$ displays the final radius (at $10 \mu \mathrm{s}$ ) of the plasmoid for $R_{p}=1 \mathrm{~m} \Omega$. The results show that the plasmoid's final position increases with increasing outer stray inductance $L_{e o}$. This is what is expected in experiments as well. Additional stray on the outer circuit diverts more current to the inner coil and reduces the magnetic pressure from the outer coil pushing the plasmoid toward the inner wall. It also helps minimize the voltage 


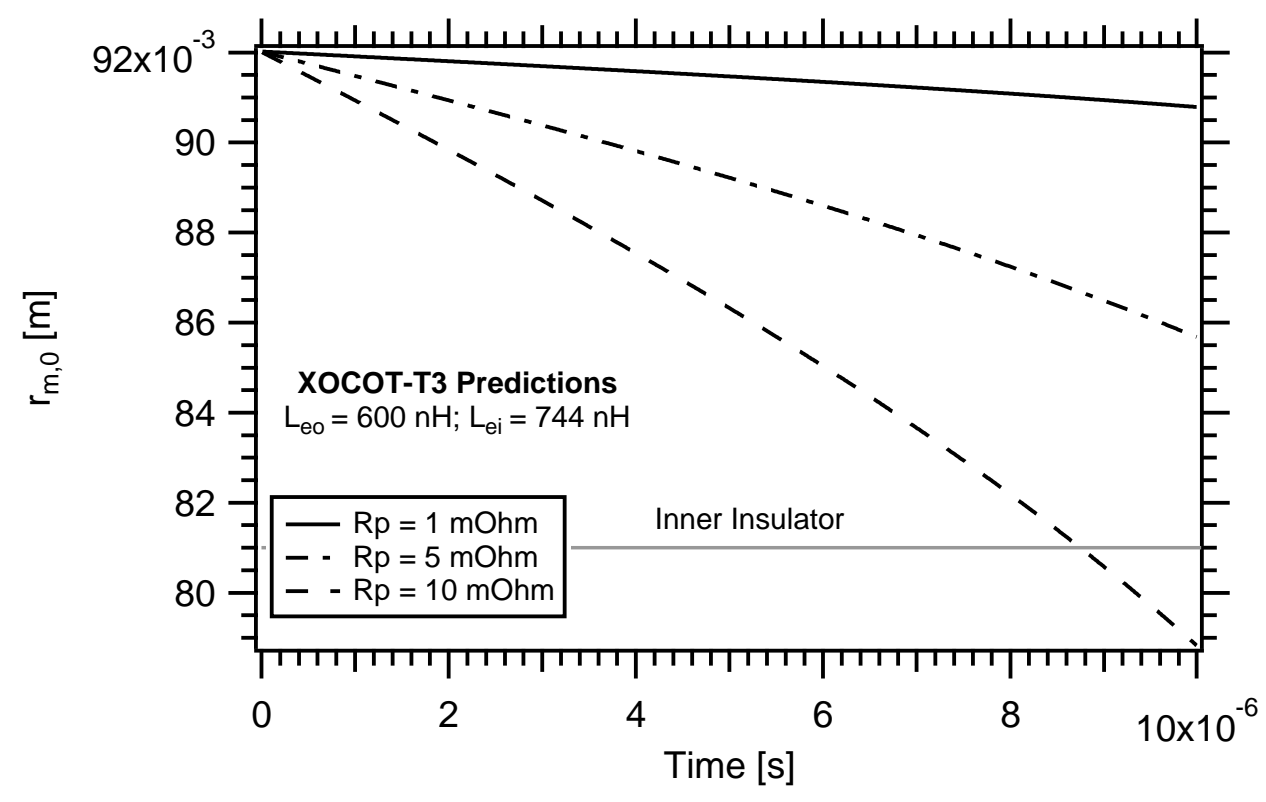

(a)

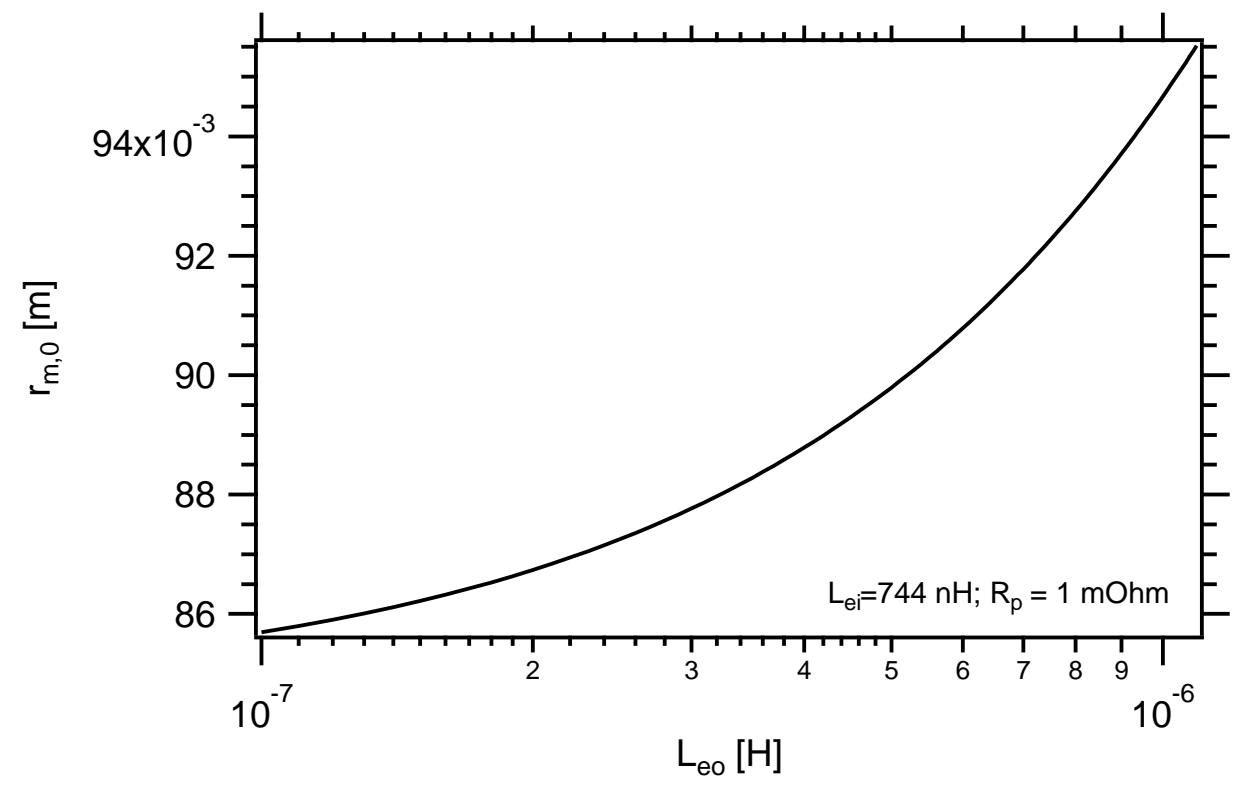

(b)

Figure 9.1: Final plasmoid radius, calculated using Equation 9.11. The radial trajectory using XOCOT-T3 circuit parameters is shown in (a) for various plasmoid resistances. The final plasmoid position as a function of outer coil stray inductance is shown in (b).

differential between the coils for increased radial balance. 


\subsection{Radial Balance Study Data}

The plasmoid's position in an AFRC experiment operating with parallel synchronous coils is theoretically a function of stray inductance on the coil circuits and plasmoid resistance, as discussed in Section 9.1. The easiest method to control the radial pressure balance in the XOCOT-T3 experiment was to add additional stray inductance to the outer coil circuit. This eased the magnetic pressure from the outer coil pushing the plasmoid toward the inner wall by diverting more current to the inner coil.

Stray inductance was added to the outer coil by increasing the loop area between the forward and return current paths. This was done for two tests. The first test added only a modest amount of stray inductance, about $185 \mathrm{nH}$ of extra inductance (or $785 \mathrm{nH}$ total). The second test increased the original amount by a factor of 1.78 from the original, using a total stray inductance of $1.072 \mu \mathrm{H}$. These versions of the circuit are referred to as Configuration \#1 and Configuration \#2, respectively. Stray inductance was added to the $20 \mathrm{kHz}$ circuit only. The capacitor for the $10 \mathrm{kHz}$ circuit failed before similar tests could be conducted.

Since tests at all pressures using the $20 \mathrm{kHz}$ circuit at $100 \mathrm{~J}$ had the same gross behavior (loss of reversed field at $14 \mu \mathrm{s}$ ), Configuration \#1 and Configuration \#2 were only tested at the $100 \mathrm{~J}, 4$ mTorr settings. Full magnetic field profiles upstream and downstream were collected from these configurations for vacuum and plasma discharges.

The vacuum data for Configuration \#1 is shown in Figure 9.2, including the coil currents and midplane fields. The data from the original version of the $20 \mathrm{kHz}$ circuit at $100 \mathrm{~J}$ is indicated in these plots by the faint dotted lines. The vacuum data from Configuration \#1 
was averaged over 6 shots. Error bars are a combination of the statistical devation (small) and the measurement uncertainty.

The vacuum currents for Configuration \#1 show the expected trends. The outer coil current is lower than the original circuit (at $2600 \mathrm{~A}$ instead of $2800 \mathrm{~A}$ ) and the inner coil current is higher (8100 A compared to 8000 A). The total circuit current drops only marginally, down 67 A. This small drop is within the shot-to-shot variation. The current ratio between the coils drops from 0.355 to 0.325 . The current ratio is defined as outer coil current divided by inner coil current. The vacuum magnetic fields change as well, when compared to the original configuration. The midplane field in the annulus drops by $2 \mathrm{mT}$, or $10 \%$ of the original field.

Following the vacuum circuit characterization with the new changes, plasma tests were conducted. A fill pressure of 4 mTorr was used and 18 shots were discharged into the neutral fill. The midplane magnetic fields from one of the shots is shown in Figure 9.3a. The magnetic field data from the coil probes and TOF array is displayed in Figure $9.3 \mathrm{~b}$ and Figure 9.3 .

The magnetic field data showed that the additional stray inductance on the outer coil circuit created a plasmoid with a higher plasmoid current. This was judged by the higher reversed midplane field on the inner wall as compared to the original configuration (see Figure 9.3 ). The field reversal also happened faster. Instead of taking a full microsecond to reverse, the field now reversed in $840 \mathrm{~ns}$. The peak reversal with the new circuit occurred $5.7 \mu \mathrm{s}$ after the switch closed, lasting less than $1 \mu$ s at the peak. The reversed field decay was much slower in the new circuit, lasting a full $\mu$ s longer. The additional stray inductance increased the configuration lifetime from $10 \mu$ to $11 \mu \mathrm{s}$. While the inner field showed marked changes, the outer field did not change appreciably for the first $3.5 \mu \mathrm{s}$, compared 
(A)

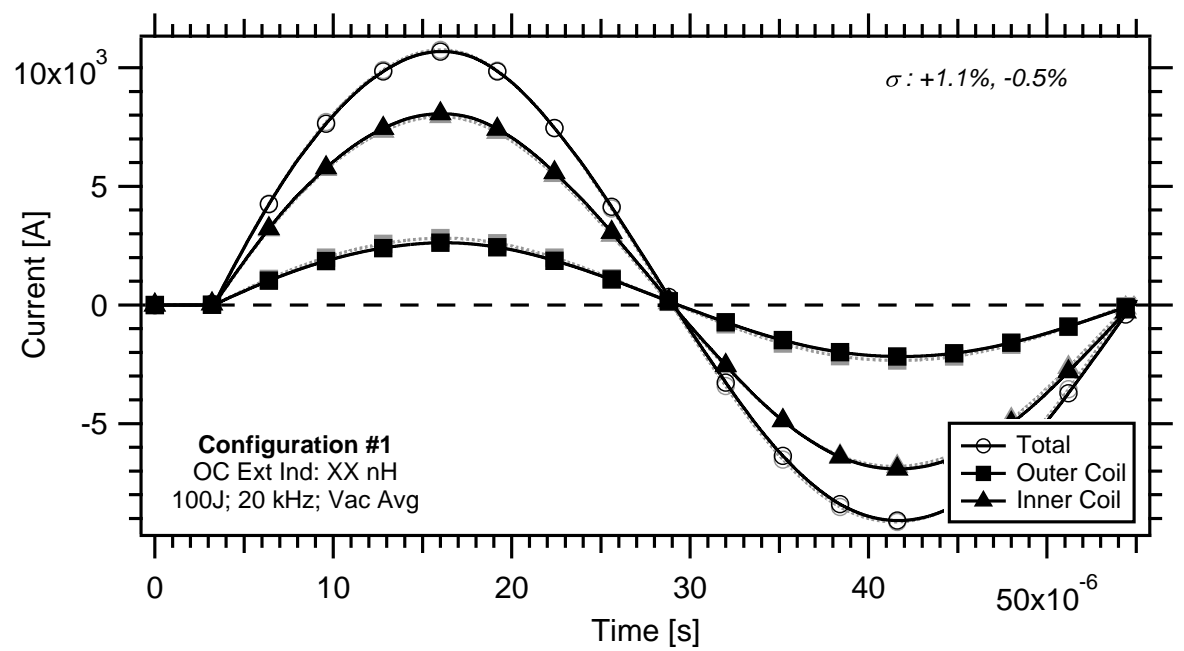

(B)
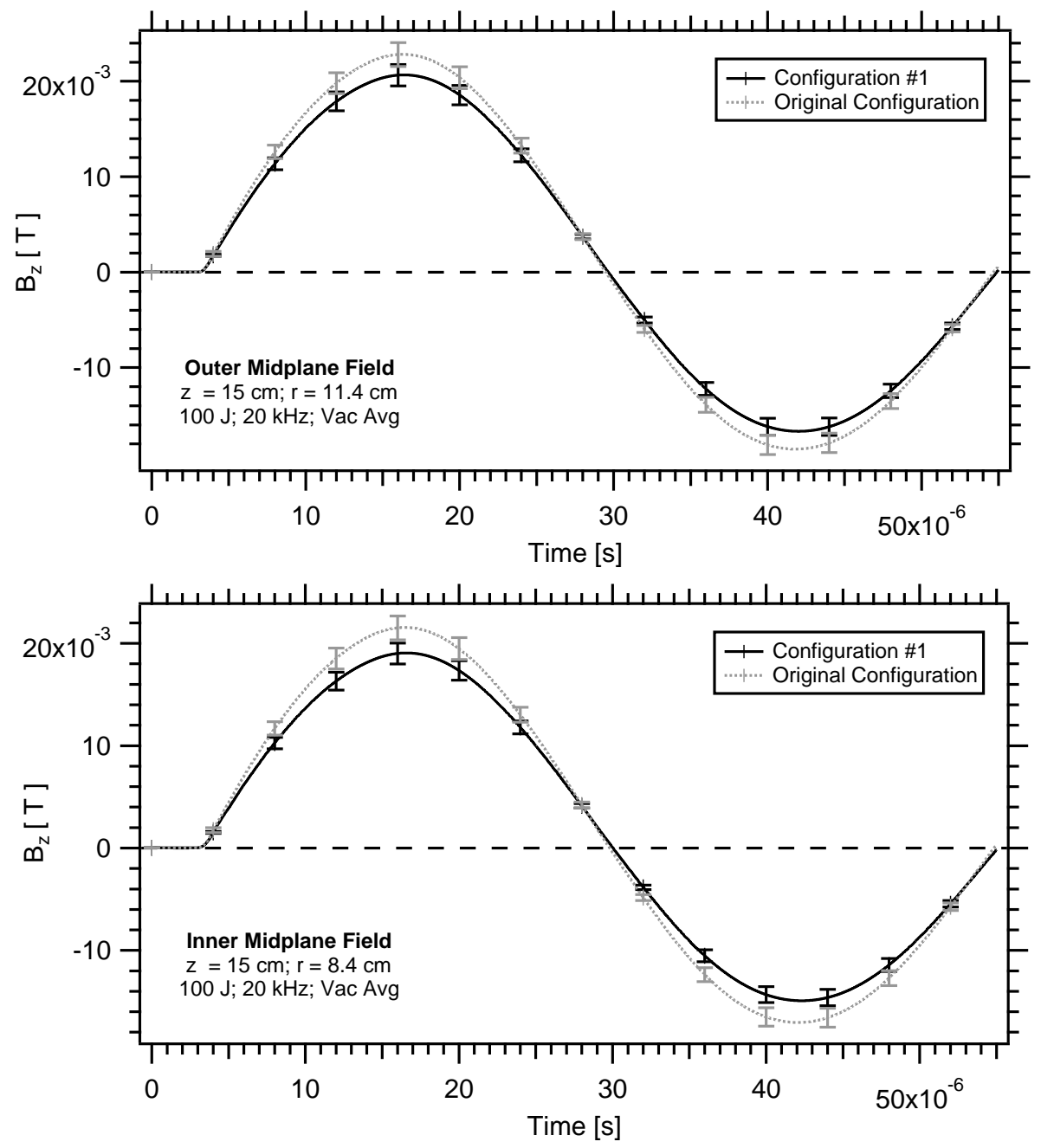

Figure 9.2: Coil currents and midplane magnetic fields for Configuration \#1. The original circuit data is shown with the faint dotted line. 
(A)

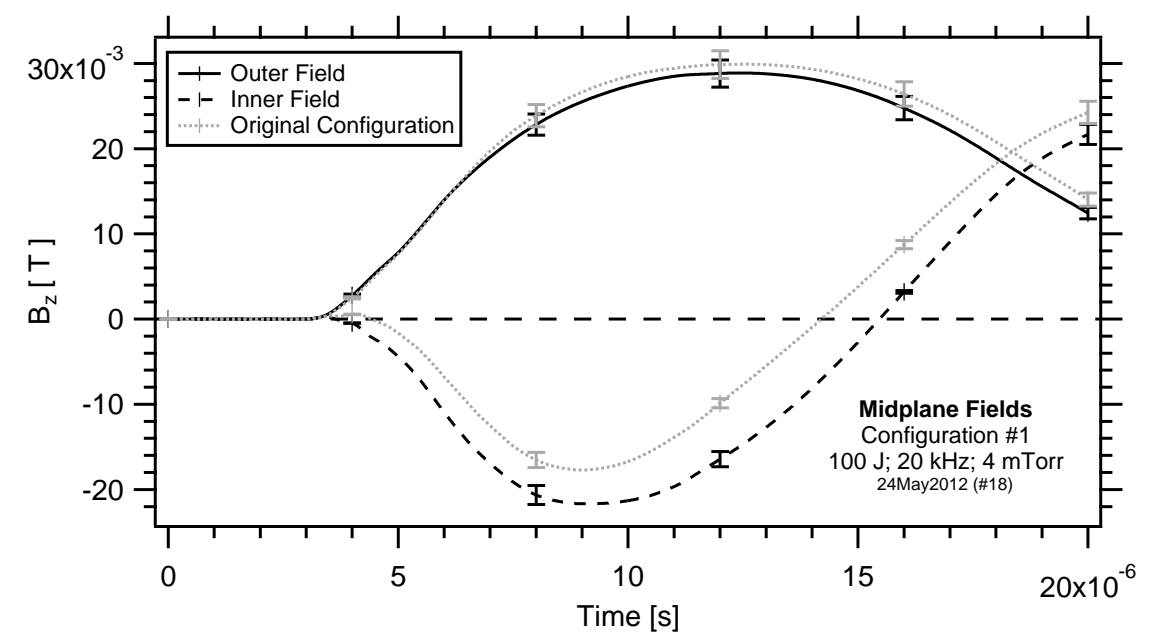

(B)
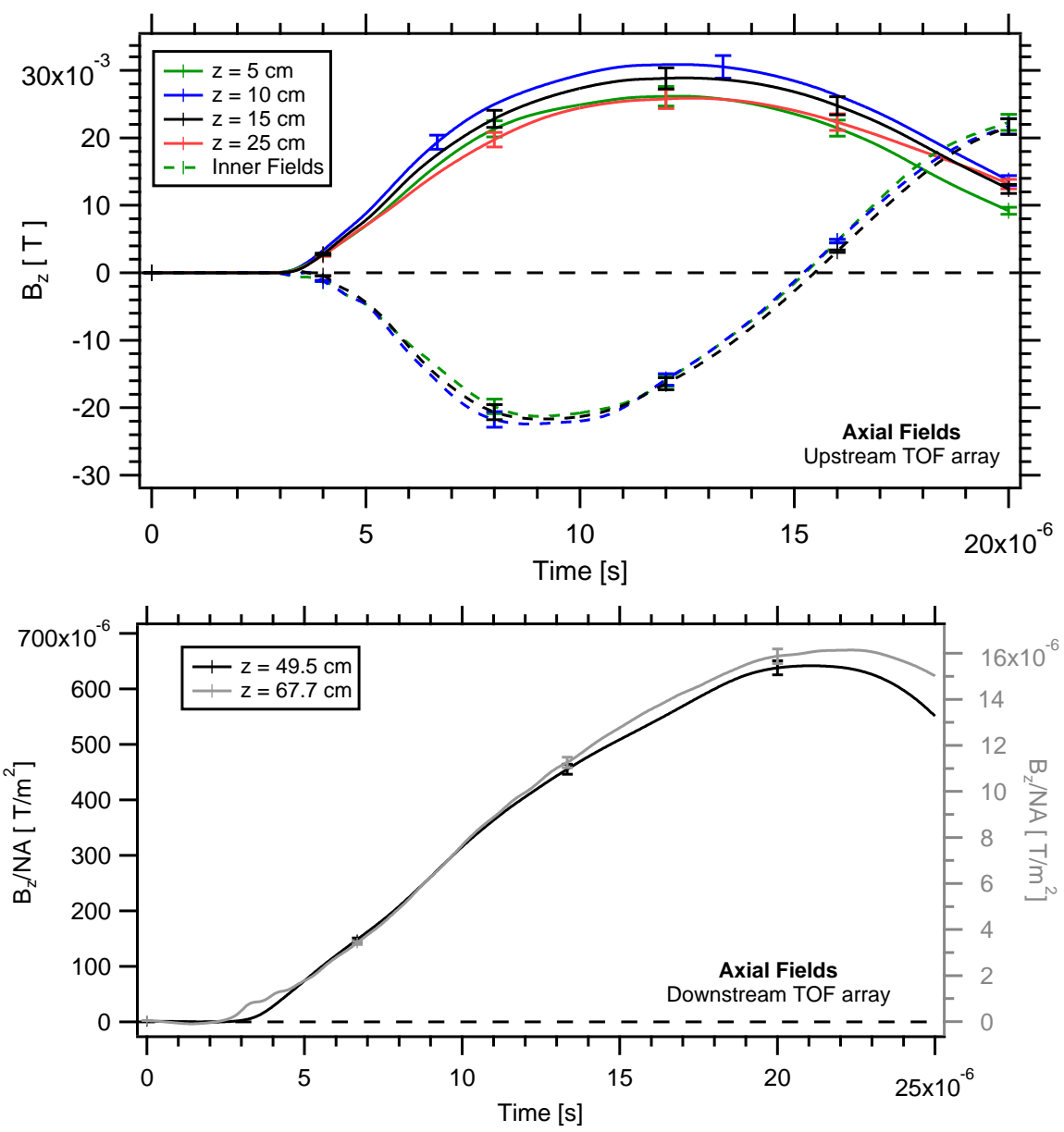

Figure 9.3: Magnetic field data for a $100 \mathrm{~J}, 4$ mTorr shot with the circuit in Configuration \#1. The data using the original circuit is indicated by the faint dotted lines. 


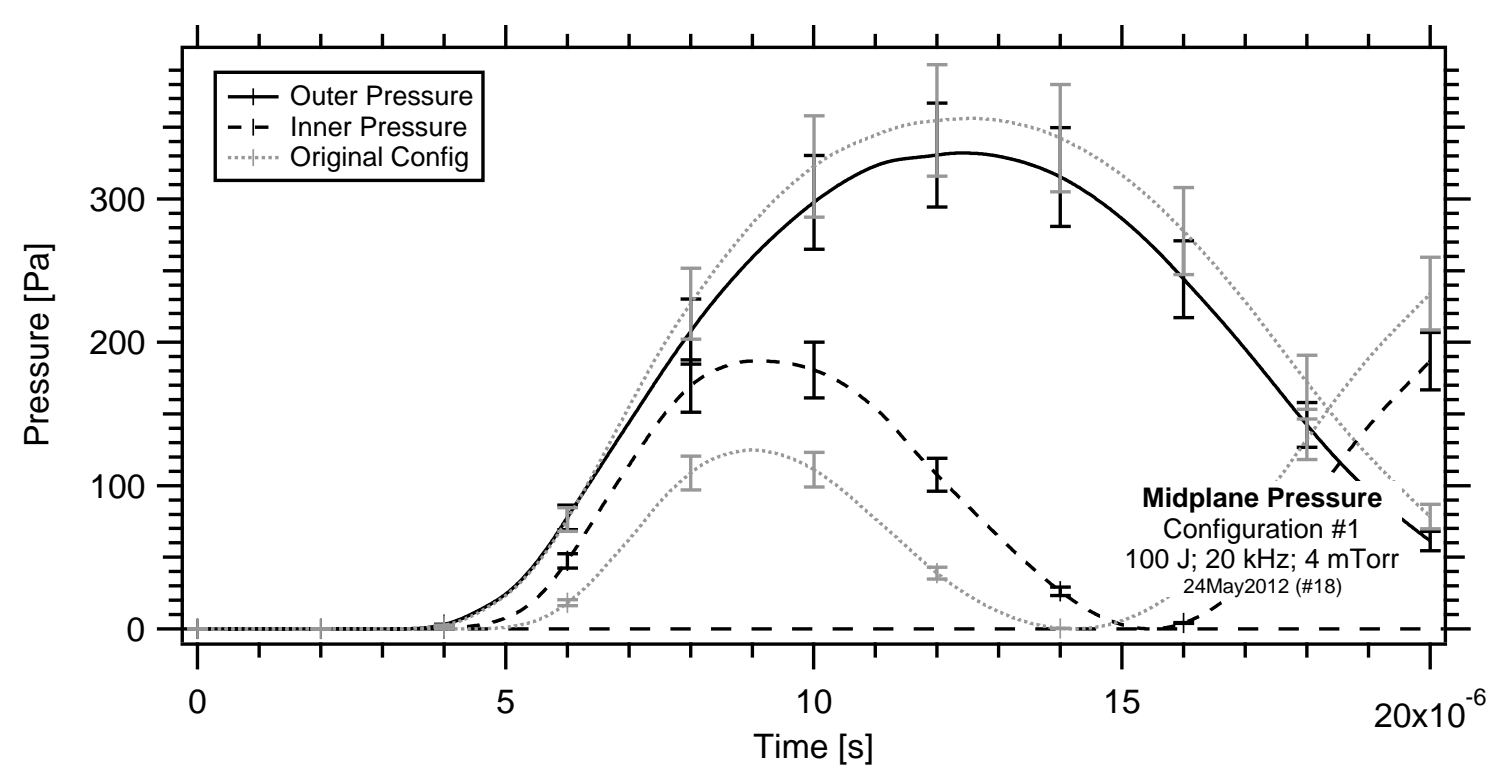

Figure 9.4: Magnetic pressure at the axial midplane for a $100 \mathrm{~J}, 4$ mTorr discharge using the $20 \mathrm{kHz}$ circuit in Configuration \#1.

to the original configuration.

The axial fields recorded by other probes along the coil and TOF array were similar in nature to the midplane probe and no evidence of translation was seen. An interesting feature that appeared in the new inner field data when compared to the original data was the extended pause near the maximum field reversal. In the original configuration, the inner field reached the maximum field reversal and then quickly disappeared. The new circuit created an extended pause, settling around the maximum for almost a full $\mu$ s before starting to disappear. This delay was too short to make considerable gains in translation, but it showed a trend in improving the lifetime.

The magnetic pressure was calculated for the axial midplane, using the data shown in Figure 9.3 a. The pressure was calculated using Equation 3.2. The results are shown in Figure 9.4. The magnetic pressure from the original circuit is shown for comparison.

The magnetic pressures data shows that a magnetic pressure differential across the plasmoid 
was still apparent, with the inner pressure lower than the outer pressure. While the pressure differential (30 Pa) was significantly less than in the original configuration, the stray inductance did not correct for the pressure differential entirely.

Images from an end-on view were recorded of the plasmoid formation, created using Configuration \#1. Still frames using a fast-shutter camera were collected from 6-11.5 $\mu \mathrm{s}$, every 500 ns. This images are available in Figure 9.5. Midplane magnetic fields are also shown in Figure 9.5 for reference.

The images for Configuration \#1 show that the plasmoid came in contact with the inner wall, but with a slight delay compared to the original circuit. The plasmoid appeared to contact the wall in Figure 9.5 at $10-10.5 \mu$ s after the switch closes. In the original circuit (consult Figure 8.3), the plasmoid hit the wall at 9-9.5 $\mu \mathrm{s}$.

Since this small amount of stray inductance added to the circuit for Configuration \#1 did not have the desired effect, additional stray inductance was added to the discharge circuit to further reduce the outer coil field. About $472 \mathrm{nH}$ of extra inductance was added to the outer coil circuit, providing a total stray inductance of $1.072 \mu \mathrm{H}$. This is referred to as Configuration \#2. This configuration was tested first at vacuum to determine how much the coil currents and fields deviate from the original circuit. The vacuum data for Configuration \#2 is shown in Figure 9.6, including the coil currents and midplane fields. The data from the original version of the $20 \mathrm{kHz}$ circuit at $100 \mathrm{~J}$ is indicated in these plots with faint dotted lines. The vacuum data from Configuration \#2 was averaged over 6 shots. Error bars are a combination of the statistical deviation (small) and the measurement uncertainty.

The vacuum currents for Configuration \#2 exhibited a marked change from the original configuration. The outer coil current was on average 454 A (or 16\%) less than the original 

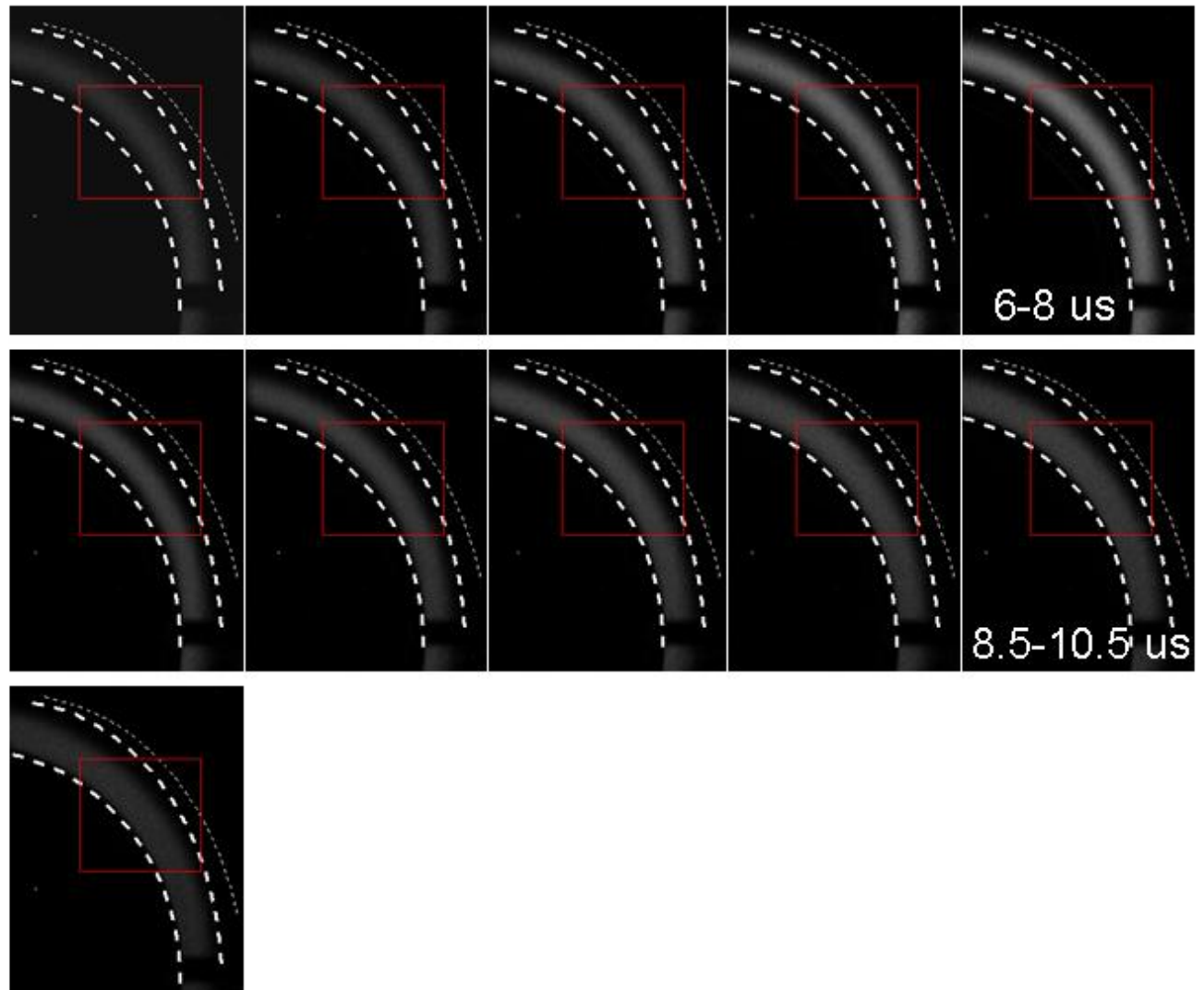

11 us
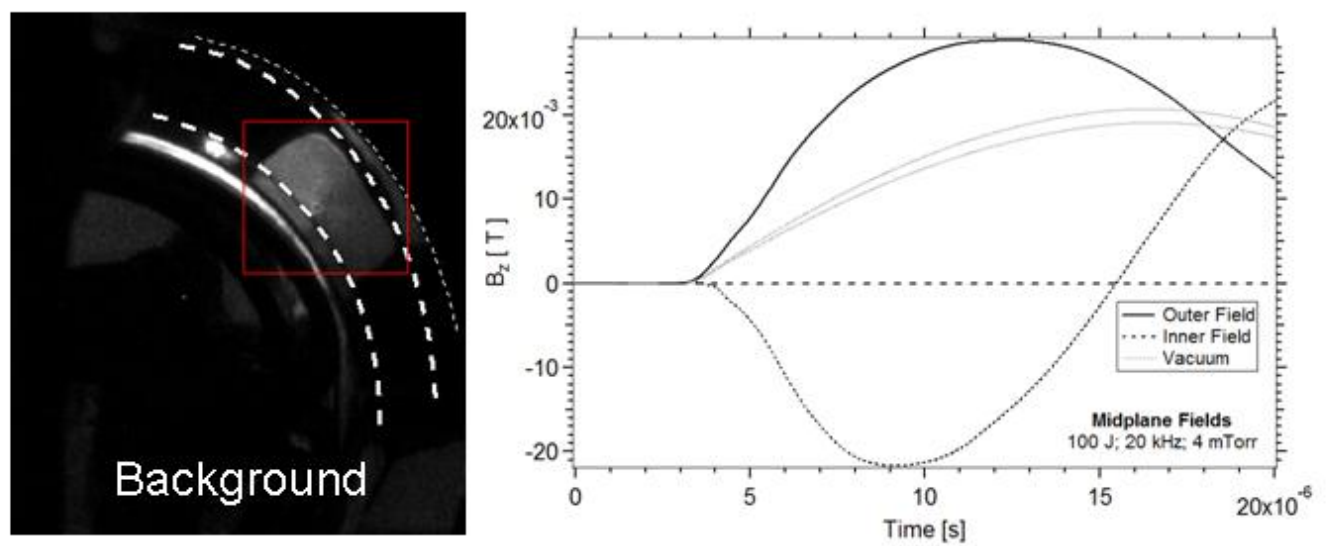

Figure 9.5: Images of plasmoid formation for a $100 \mathrm{~J}, 4$ mTorr discharge using the $20 \mathrm{kHz}$ circuit in Configuration \#1. Midplane magnetic fields are shown for reference. 
(A)

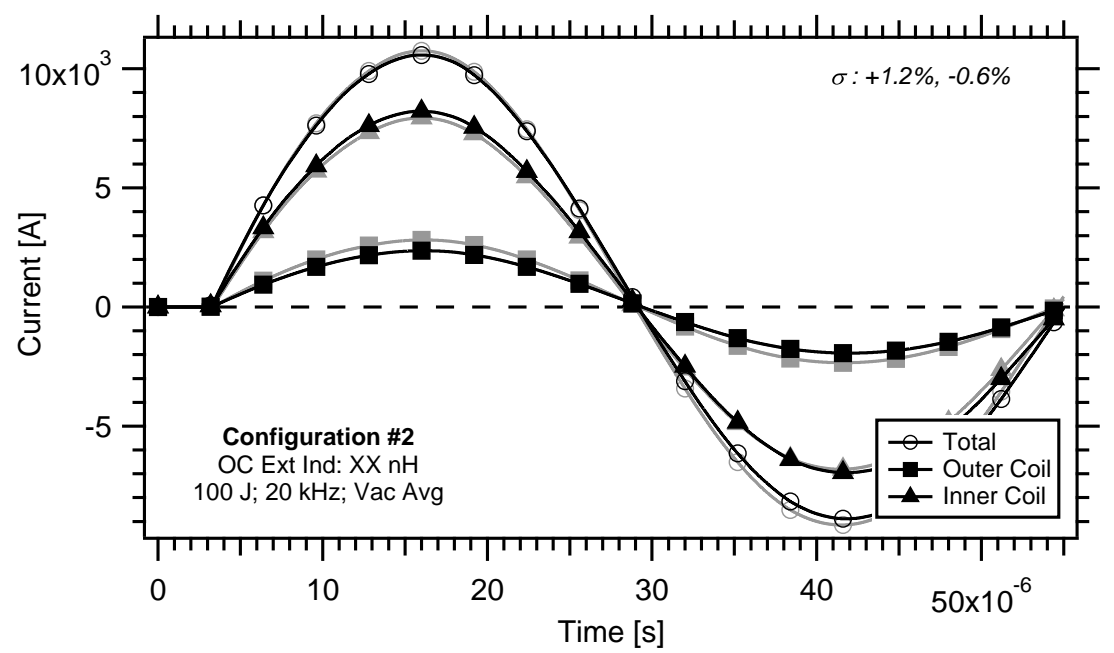

(B)
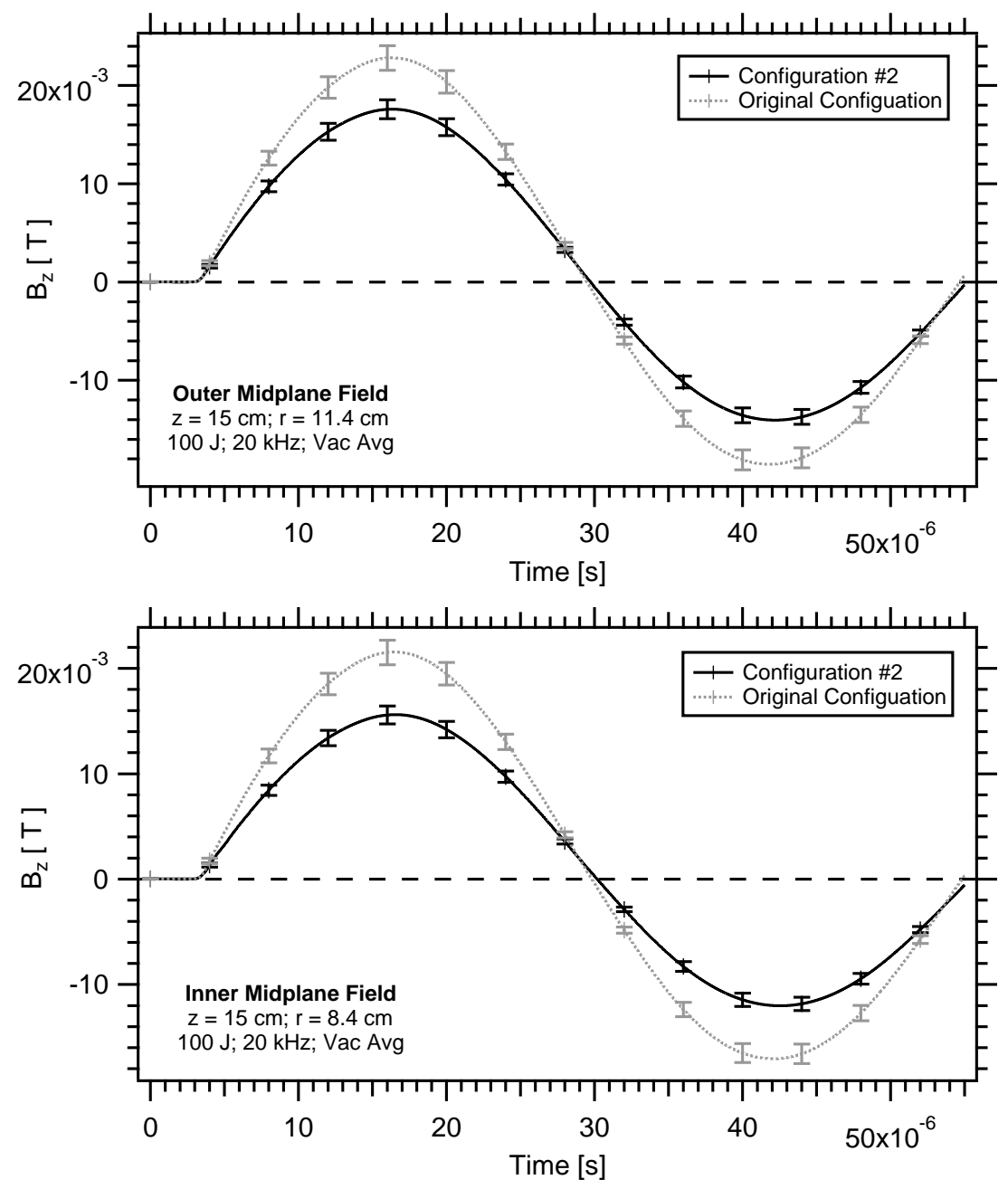

Figure 9.6: Coil currents and midplane magnetic fields for Configuration \#2. The original circuit data is shown with the faint dotted line. 
circuit. The inner coil current increased by $280 \mathrm{~A}(3 \%)$ and the total current decreased by $173 \mathrm{~A}(1.6 \%)$. The current ratio between the coils changed to 0.287 (compared to 0.355 in the original circuit). The outer vacuum field decreased by $22 \%$, about $5 \mathrm{mT}$. The magnetic field recorded by the inner probe dropped $5 \mathrm{mT}$ as well.

Plasma tests were conducted on the circuit defined as Configuration \#2, using a fill pressure of 4 mTorr and discharge energy of $100 \mathrm{~J}$. Twenty-eight test were performed with data collected from all magnetic field probes, an asymmetric double Langmuir probe at the axial midplane, and a fast-shutter, single frame camera. The midplane magnetic fields from one of the shots is shown in Figure $9.7 \mathrm{a}$. The data from the coil magnetic field probes and the TOF magnetic field probes is displayed in Figure 9.7p and Figure 9.7k.

The reversed magnetic field generated by the plasmoid in Configuration \#2 was much greater than the reversed field seen in the original circuit, about $5 \mathrm{mT}$ of additional field during the initial reversal from 4-9 $\mu \mathrm{s}$. By comparison, Configuration \#1 had a field reversal increase of about $4.4 \mathrm{mT}$. The reversal also happened sooner, in less than $630 \mathrm{~ns}$ from the switch closing. The reversal also lasted considerably longer than the original circuit. Once the peak reversed field was reached at $9.15 \mu \mathrm{s}$, the reversed field stayed constant for $2.5 \mu \mathrm{s}$ longer. The configuration lifetime with Configuration \#2 increased to $13 \mu \mathrm{s}, 3 \mu$ s longer than the original circuit.

The outer field in Configuration \#2 was less than the outer field in the original configuration. This a consequence of the lower current through the outer coil. It also helped to reduce the magnetic pressure pushing the plasmoid toward the inner wall. The axial probe signatures upstream and downstream (shown in Figure 9.7b and c) show no sign of plasmoid motion, even with the increased lifetime. This is unfortunate, but not entirely unexpected. In the 100 $\mathrm{J}$ case, there was likely little energy available for translation, especially when translation 
(A)
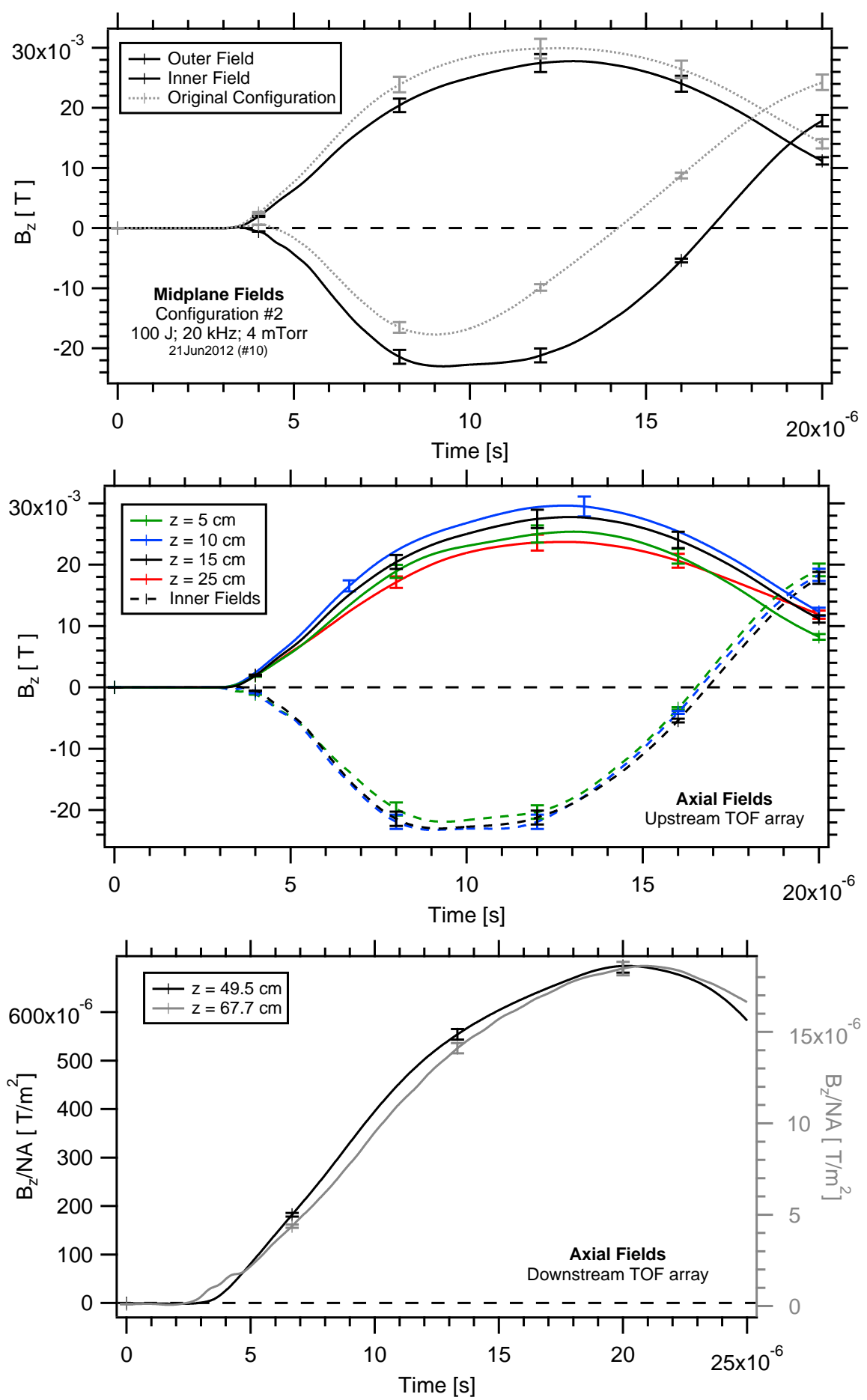

Figure 9.7: Magnetic field data for a $100 \mathrm{~J}, 4 \mathrm{mTorr}$ shot with the circuit in Configuration \#2. The data using the original circuit is indicated by the faint dotted lines. 


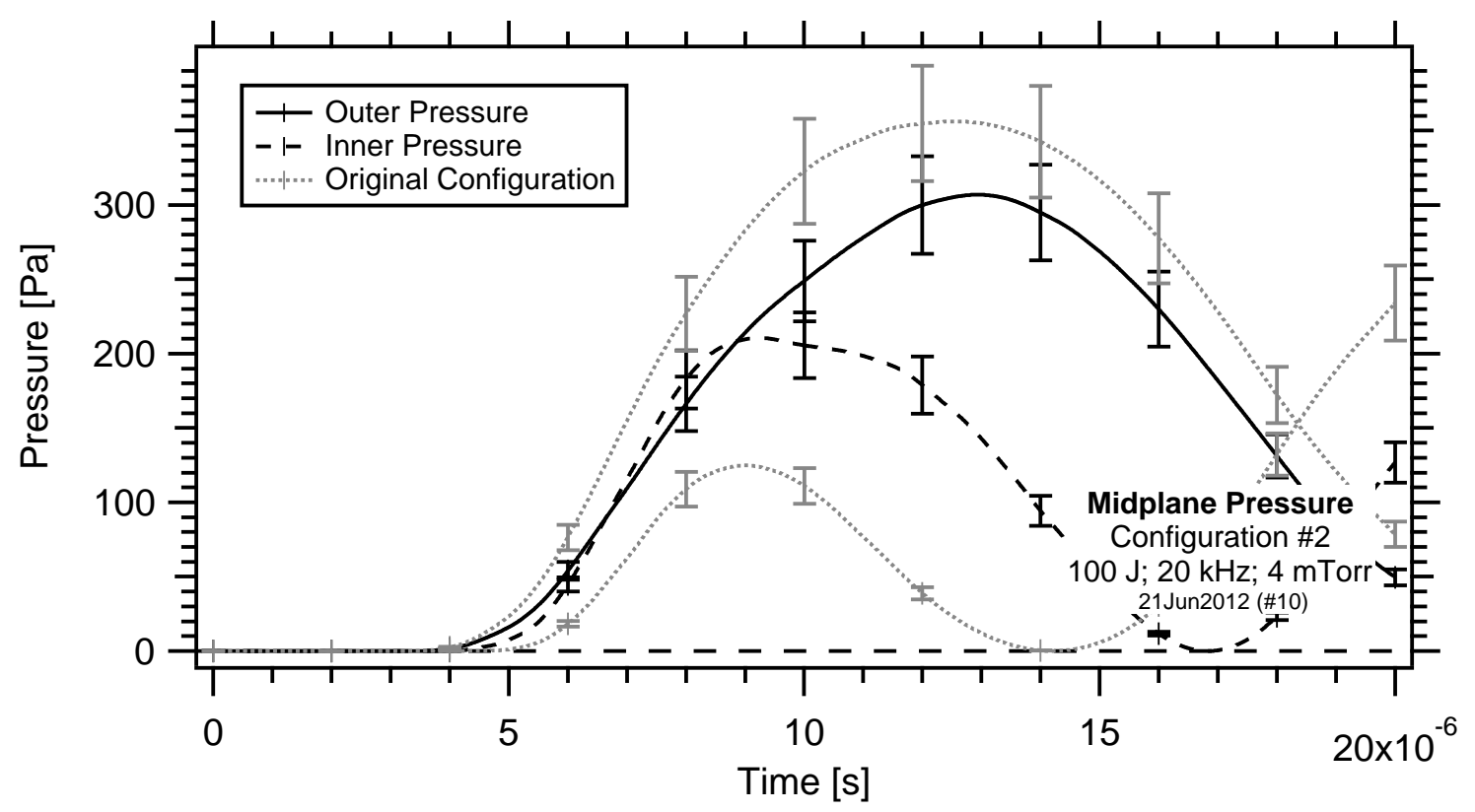

Figure 9.8: Magnetic pressure at the axial midplane for a $100 \mathrm{~J}, 4$ mTorr discharge using the $20 \mathrm{kHz}$ circuit in Configuration \#2.

must be accomplished in under $10 \mu \mathrm{s}$.

The time-history of the magnetic pressure at the axial midplane for Configuration \#2 was calculated using Equation 3.2. The results are shown in Figure 9.8. The magnetic pressure from the original circuit is also shown for comparison.

The magnetic pressures in Configuration \#2 on the inner and outer surfaces of the plasmoid were approximately equal, until $9 \mu \mathrm{s}$. The magnetic pressure on the inner wall leveled off, dropping at $12 \mu$ s when the reversed field began to decay. The outer coil current continued to climb after $9 \mu \mathrm{s}$, as the outer coil current continued to add magnetic field to the annulus.

To view the radial position of plasmoid in Configuration \#2, end-on images were collected from 6-14.5 $\mu$ s with frame widths of 500 ns. This images are presented in Figure 9.9

At the beginning of the image sequence, the plasmoid appeared fairly well centered 

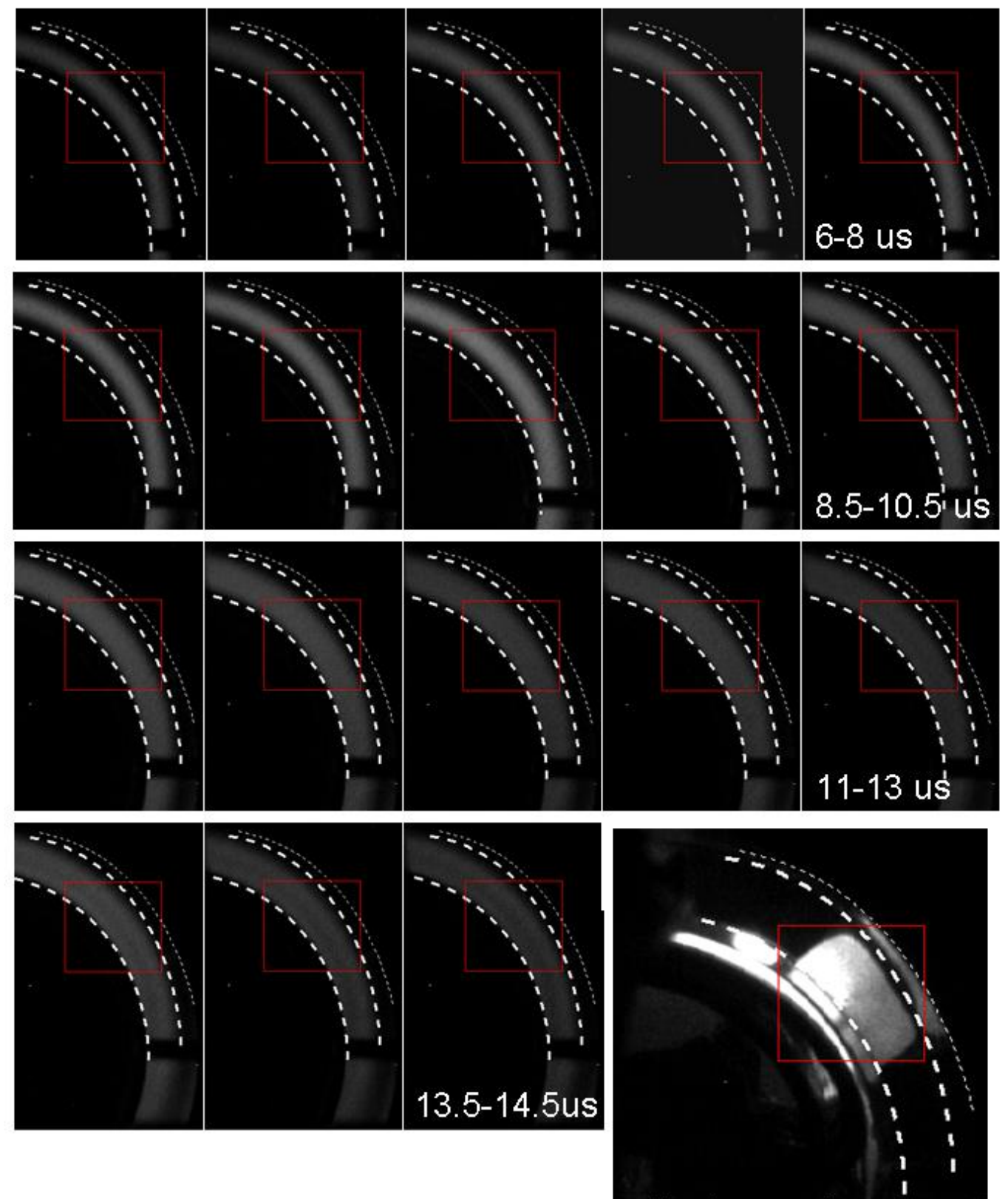

Background

Figure 9.9: Images of plasmoid formation for a $100 \mathrm{~J}, 4$ mTorr discharge using the $20 \mathrm{kHz}$ circuit in Configuration \#2. 
between the insulators. Between 9 and $9.5 \mu$ s (when the inner field reached its peak), the plasmoid began to expand radially in the channel. Inner wall contact was seen around $10.5-11 \mu \mathrm{s}$. After $11 \mu \mathrm{s}$, the plasma continued to expand though the perspective of these images makes it difficult to see if it touched the outer wall as well. Since it did not overlap the outer insulator line, it's likely that it only contacted the inner wall.

The extended lifetime of the plasmoid in Configuration \#2 warranted the use of an ADLP to determine where the bulk of the plasmoid was radially located at the coil midplane. The ADLP in Configuration \#2 was used in the same manner as previously described in Section 8.2.2. A bias voltage of $24 \mathrm{~V}$ was applied across the probe, with the larger probe tip biased to collect ions. The probe was scanned radially across the channel in $2 \mathrm{~mm}$ increments. The current collected by the probe is shown in Figure 9.10a. The radial distribution of plasma is plotted from 6-17 $\mu \mathrm{s}$ in Figure 9.10 b. Midplane magnetic fields are shown for comparison in Figure 9.10 c.

Figure 9.10 shows expected AFRC behavior. The peak current (i.e. plasma density for constant temperature and no flow velocity) was measured at the center of the channel and then tapered toward the walls. This same profile was seen in other experiments as well (consult Section 3.3.2). The plasma activity on channel centerline continues to climb until $12 \mu \mathrm{s}$, when the field reversal started to decay. Between 10 and $11 \mu \mathrm{s}$, the plasma activity near the wall starts to increase as more plasma came in contact with the wall. This likely triggered the start of the plasmoid current decay a microsecond later and loss of field reversal. The motion of the plasma appeared to be towards the inner wall, but the increase in plasma current seen in this region may have to do with plasma bombardment of the inner insulator.

While the plasmoid was more or less radially balanced in Configuration \#2, the center 
(A)
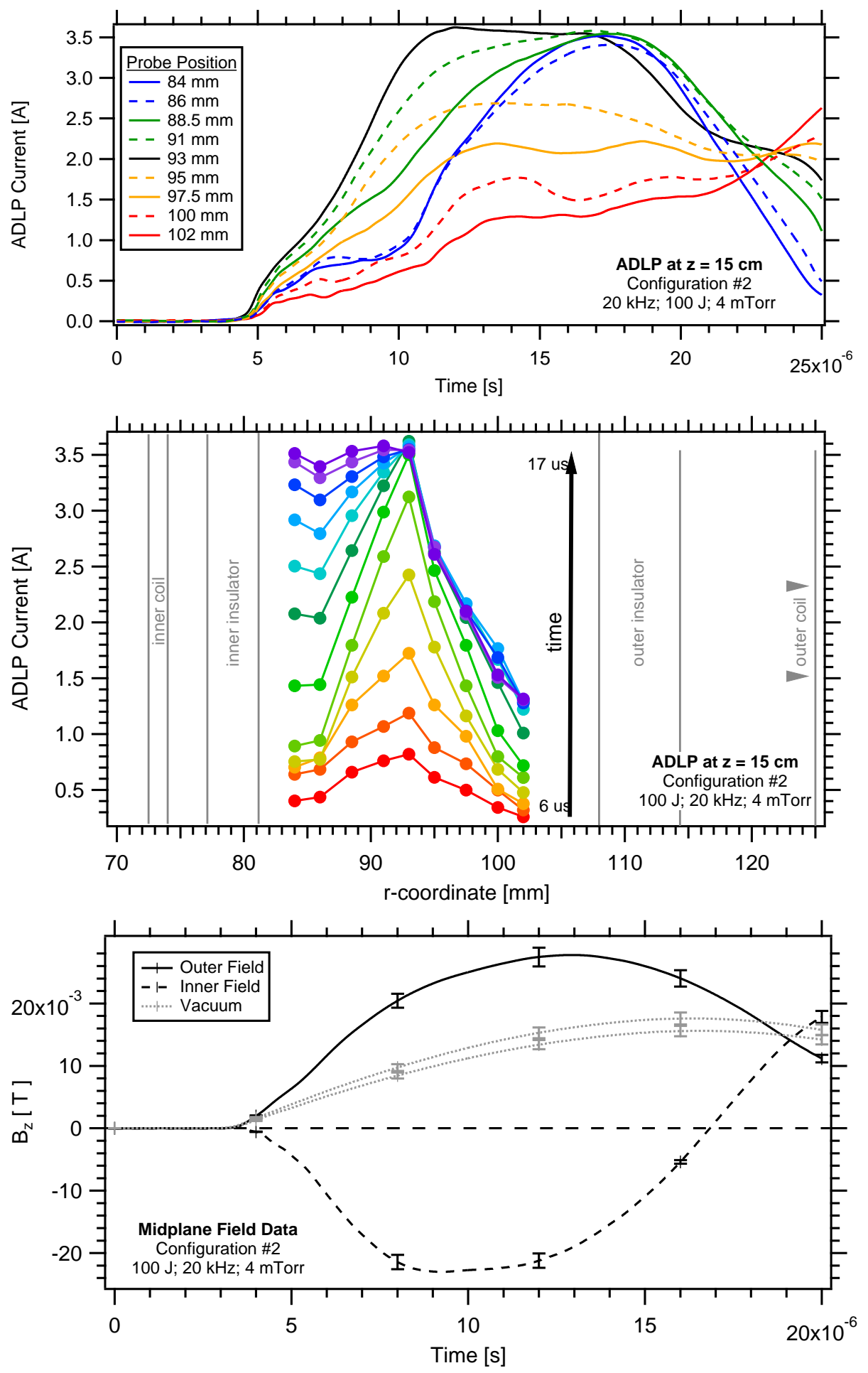

Figure 9.10: ADLP data at the axial midplane for Configuration \#2. The data collected by the probe is shown (A) along with the radial distribution along the channel (B), and midplane mangetic fields (C). 
appeared to be located at the gas center of the channel $(\mathrm{r}=93 \mathrm{~mm})$ and not the magnetic center $(\mathrm{r}=99 \mathrm{~mm})$. While this may be due to a preferential density gradient, it might also have to do with the plasma current being insufficient to push off the coil further. Additional testing with even more stray is required to find out if the equilibrium position is related to a magnetic pressure balance or a preferential gas loading.

\subsection{Radial Balance Study Results}

The radial balance study data presented in Section 9.2 demonstrated that additional stray inductance on the outer coil circuit reduced the magnetic pressure differential between the outer and inner wall. The original circuit had a pressure difference between the inner and outer wall of over $80 \mathrm{~Pa}$. When $185 \mathrm{nH}$ of stray was added to the outer coil circuit, the pressure difference dropped to $30 \mathrm{~Pa}$. The stray inductance on the outer coil was increased to $1.072 \mu \mathrm{H}$ and the pressure differential disappeared; the inner magnetic pressure was equal to the outer magnetic pressure for the first $6 \mu \mathrm{s}$.

The additional stray inductance on the outer coil circuit increased the inner coil current only slightly. An increase of 127 A (or 1.6\%) was seen in Configuration \#1. The inner coil current increased by 280 A (3\%) in Configuration \#2. The slight current increase through the inner coil did not appear to change the inner field rise-time significantly. The reversed field in all configurations stopped increasing around $9 \mu$ s with only a $300 \mathrm{~ns}$ difference between Configuration \#2 and the original circuit.

The effect of reduced pressure on the outer wall allowed the plasmoid current to reach an equilibrium state after the reversed field reached its maximum. For the case of $30 \%$ 
additional inductance in the outer coil circuit, the reversed field was noted to linger around $-0.02 \mathrm{mT}$ for $1 \mu \mathrm{s}$ before decaying toward zero. When the amount of stray inductance on the outer coil circuit was nearly doubled, an equilibrium time of $2.5 \mu$ s was seen before the field decay.

Plasmoid termination was still attributed to wall contact with both modified versions of the experiment. In Configuration \#1 (less stray), the plasmoid could be seen in images to touch the wall around $10 \mu \mathrm{s}$, at the same time the reversed field started to disappear. In Configuration \#2 (large amounts of stray), the images showed the plasma touched the wall around $11 \mu \mathrm{s}$. This also coincided with the loss of reversed field.

The mechanism for wall contact in Configuration \#1 was likely a radial imbalance, due to a pressure differential. The pressure on the outer surface of the plasmoid was always greater than the pressure on the inner surface. This drove the plasmoid into the inner wall, extinguishing it. The stray inductance correction was insufficient to reduce the outer coil field.

When this pressure differential was corrected for in Configuration \#2, the plasmoid was held off the wall after peak field reversal for a short duration of time, from 9-10 $\mu$ s. However, shortly after $10 \mu \mathrm{s}$, internal ADLP data showed the plasma activity near the inner wall jumps between 10-11 $\mu \mathrm{s}$. The peak of plasma activity stayed centered at $93 \mathrm{~mm}$, indicating that a growth in the plasmoid's midsection was responsible for this initial wall contact. It's unclear what this expansion was due to since it came after the plasmoid current (inner field) stopped climbing, but possible causes are an increase of gas pressure inside the configuration due to joule heating, poor magnetic confinement, and a radial imbalance. 


\subsection{Discussion of Radial Balance Results}

The primary purpose of the radial balance study was to determine the mechanism behind the radial plasmoid motion in the original experiment. The radial motion mechanism was traced to a pressure imbalance between the inner and outer field. When this pressure differential was corrected for by reducing the current through the outer coil, the plasmoid retained a radial balance between the insulators. The radial balance was short-lived, as an expansion in the plasmoid's minor radii resulted in wall contact with the inner insulator and eventual decay of the configuration.

The detrimental effect of the radial expansion could be corrected for by widening the space between the coils. However, this would decrease the efficiency and final position of the plasmoid, as the results in Section 4.2.3 indicate. It would also increase the energy required to accelerate the plasmoid.

The cause of radial expansion in the radially-balanced version of the XOCOT-T3 was unclear. Two possibilities include an increase of gas pressure inside the configuration due to joule heating, and lousy magnetic confinement. It also likely that the radial expansion was caused by a magnetic pressure differential, as a magnetic differential was noted at the time of wall contact. While most of the plasma appeared centered in the channel for an additional $6 \mu \mathrm{s}$, it was unclear where the plasma current was. The plasma current could have drifted through the plasmoid to the inner wall, leaving behind a dense region of plasma it had previously heated.

While the radial expansion eventually led to termination of the plasmoid, a far more serious 
concern for the plasmoid behavior is the asymptotic behavior of the plasma current. The plasmoid current in the XOCOT-T3 reached steady state after $5 \mu \mathrm{s}$, as indicated by the steady reversed field. This was approximately $1 / 2$ of the coil current rise-time. While the plasmoid current stopped increasing, the outer coil current continued to climb. If wall contact did not occur at $10 \mu \mathrm{s}$, the plasmoid would have eventually been forced into the inner wall. The asymptotic behavior in plasmoid current (or reversed field) was seen in other longer-timescale experiments as well [50], [16], [15]. In each experiment, it was seen to occur before the peak coil currents as well. The reversed-field data from CSS-U experiments [50] indicated the plasmoid current reached steady-state at 50\% of the coil current rise. The reversed-field data from the CSS-P [15] also found the plasmoid current reached a steady state at $50 \%$ of the coil current rise. In the IFRC experiment [16], the plasmoid current increased for only the first $20 \%$ of the coil current's risetime. While the immediate effect of the radial stability of the plasmoid on was unclear in the CSS-U and CSS-P due to the scale of the presented data and the effect of other instabilities, the plasmoid in the IFRC was clearly seen to move radially inward after the plasmoid current reached a steady state. The plasmoid drifted toward the inner wall, where it eventually quenched. Clear explanations for the plasma current limit were not provided.

The asymptotic plasmoid current will likely always lead to a radial collapse of the plasmoid when the coils are operated in synchronous mode. Even though the plasmoid current stops increasing, the outer coil current continues to climb and forces the plasmoid toward the inner wall. For propulsion, this means the plasmoid must be ejected at the time of peak plasmoid current which occurs well before the coil currents peak. Studies with the annular electromagnetic launcher model in Chapter 4 found that ejecting a plasmoid before the time of peak coil currents results in an efficiency loss. Peak energy efficiencies in the annular model were observed to be $55 \%$, though nominal efficiencies were close to $30 \%$. These 
efficiencies were calculated based on ejecting the plasmoid at the full quarter-cycle. If the plasmoid must be ejected well before the quarter cycle, it is unlikely that the energy efficiency will surpass $10 \%$. This observation, coupled with the high sensitivity of the plasmoid to radial imbalance, makes an AFRC-based thruster less attractive for space propulsion. 


\section{Chapter 10}

\section{Conclusion and Future Work}

\subsection{Contributions of This Work}

The objective of this research was to demonstrate AFRC translation for space propulsion using an experimental prototype, with design characteristics suitable for space propulsion. An experimental prototype was fabricated to accelerate the plasmoid using a lightweight, conical outer coil and long timescales to match the needs of a typical propulsion system. The experiment was operated at two different frequencies and tested from $100 \mathrm{~J}$ to $1 \mathrm{~kJ}$ of input energy with a static background gas of argon.

The achievement of this work has been to demonstrate that while a reversed field AFRC was formed in the experiment, it was unable to translate from the coils due to a radial collapse of the plasmoid. This collapse was linked to a magnetic pressure imbalance which forced the plasmoid toward the inner wall. Corrections made to the circuit restored the radial balance, however a radial expansion of the plasmoid's minor radius resulted in contact with the 
inner insulator. The cause of the expansion remains unclear. Three possibilities include an increase in the gas pressure inside the configuration due to joule heating, poor magnetic confinement, and a motion of the plasma current toward the inner wall.

The plasmoid's termination resulting from the radial collapse in this experiment was exacerbated by the narrow channel width. The narrow channel width left little room for plasmoid travel in the radial direction. Widening the channel would length the lifetime of the plasmoid, however it would have another consequence for the total system. The channel width used in this research was determined from numerical studies using an annular electromagnetic launcher model developed specifically for this research. The studies concluded that for the longest plasmoid trajectory at the highest efficiency, the inner coil radii should be maximized. This improves coil-plasmoid coupling and diverts more current to the outer coil to improve its effectiveness. Decreasing the radius of the inner coil would decrease the net efficiency of the system, requiring more energy to accelerate the plasmoid from the coils. Narrowing the channel width by decreasing the thickness of the insulators may also increase the plasmoid lifetime. However, the plasma in an AFRC (and FRC) is not tightly confined to the separatrix. A scrape-off layer of plasma exists just outside the separatrix boundary. Even if the main separatrix is held off the plasma wall by current repulsion, the scrape off layer can come into contact with the wall leading to massive cooling and eventual extinction of the configuration.

While future experiments may be able to detect the cause of the plasmoid's expansion, the need for these tests is called into question by two findings of this research. The first finding is that the plasmoid current's rise-time with longer timescale experiments is less than the rise-time of the coil currents. This finding was not limited to the parallel coil mode, either. In this experiment, the plasma current's rise-time was found to be half of the coil current 
rise-time. This observation is not isolated incident; data from other experiments support this finding as well. The limited rise-time of the current implies that the plasmoid will always be forced to the inner wall as the outer coil current continues to rise. To avoid a life-limiting radial collapse, the plasmoid must be ejected from the coils when the plasmoid current peaks. This leads into the second finding of this research: for peak efficiency in an AFRC thruster, the plasmoid must leave the coils at the time of the peak coil and plasma currents. If the plasmoid exhausts from the coils too quickly, energy remains in the capacitor and circuit that is unable to couple into the plasmoid. These findings combine into a net result: AFRC plasmoid thrusters are unlikely to operate with significant system efficiency.

The plasma current limit has received little attention in previous AFRC research. Some speculation can be made as to why the plasmoid current reaches an asymptote in AFRCs. Two of these theories include: plasmoid resistance and plasma drifts. Plasmoid resistance could cause the current to reach a steady state as the additional current generated by a weakening $\mathrm{dI} / \mathrm{dt}$ becomes dissipated by ohmic heating. However, the sudden onset of the plasmoid current plateau makes this theory questionable. If resistance was the source of the current drop-off, it should occur gradually not abruptly as the data suggests. Plasma drifts dominated by ions could also be a source for the current limit. Cocurrent motion of the ions has been measured in previous AFRC work on the IFRC [16], with a delayed start corresponding to when the limit in plasma current was reached. The delayed onset was explained by the authors using a rough calculation of ion relaxation times for various species. Order-of-magnitude estimates indicate that the ion plasma drifts in the IFRC were sufficient to limit the electron contribution of the current. These findings support the hypothesis that plasma drifts may be responsible for the current limit in AFRCs. Further testing and tracking of the ion motion in AFRCs is required before definitive conclusions 
can be made.

\subsection{Future Work}

While an AFRC thruster may not be an efficient source for plasmoid propulsion from an energy standpoint, the design of an AFRC is conducive to efficient propellant utilization. The narrow channel widths and relatively wide plasmoids seen in the XOCOT-T3 in end-on imaging suggest that a high fraction of propellant injected into the channel is absorbed in the plasma. The XOCOT-T3 plasmoids differ from the sheet-like configurations generated in another AFRC study so these results were not entirely expected. High propellant absorbtion into the plasma is promising for propellant efficiency as it indicates that a majority of the injected propellant can be accelerated downstream, leading to increased system efficiency.

For propellant efficiency to be an attractive benefit for AFRC propulsion, other PIPT devices with higher energy efficiency must demonstrate extremely poor propellant efficiency so that their system efficiency as a whole suffers. Recall from Section 2.1 that system efficiency is a product of energy efficiency and propellant efficiency. A combination of the low energy efficiency and high propellant efficiency in AFRCs could lead to similar or increased efficiency when compared to other PIPT devices with high energy efficiency and poor propellant efficiency. As propellant efficiency has not be studied in any of the other closed-field sources, estimates of their complete system efficiency is not available.

Testing the theory of efficient propellant utilization in AFRC thruster requires that plasmoid translation must be demonstrated at steady-state operation on an isolated thrust stand. 
These are high reaching goals for AFRC technology as plasmoid translation has yet to be demonstrated. Therefore, it is prudent to first evaluate other closed-field technologies before moving forward with additional AFRC development.

The annular electromagnetic launcher model developed for this research illuminated the sensitivity of annular electromagnetic launchers to input energy for peak efficiency. The results demonstrated that the plasmoid must travel slowly through coils to reach the end of the coils at the same time the coil and plasmoid currents peak for the highest efficiency. The end of the coils corresponded to the region of maximum mutual inductance gradient $(\mathrm{dM} / \mathrm{dz})$ which is directly proportional to the Lorentz force. These results are likely not isolated to annular geometries. They may extend to similar designs which launch a projectile through a conical inductive coil, such as the traditional conical theta-pinch. While electromagnetic launcher modeling is available in the literature for conical theta-pinch sources, lifetime considerations of the plasma were not taken into account in the work. Therefore, it is of interest to see how susceptible a design like the conical theta-pinch source is to input energy.

While electromagnetic launcher models seem to be a powerful design tool, their extension to thruster designs should be applied with caution as these models remain unverified with experimental studies. Model verification is essential before additional studies based on these models are completed. Therefore, it is of interest to build an experiment to verify the accuracy of these models. An excellent candidate for the experimental verification is the conical theta-pinch source. While bench-top experiments with conical-theta pinch sources have been completed, it is preferred to operate the thruster on an isolated thrust stand so that the true efficiency can calculated.

The future of AFRCs for propulsion technology development is questionable, stemming 
from the limit in toroidal plasma current. The current limit poses an interesting problem for plasma physics, however. One hypothesis for why the current reaches an asymptotic limit in AFRCs is that ion drifts in the plasmoid limit the electron current that can be driven by the inner coil antenna. Measuring the ion drifts and electron current with significant accuracy in a plasma is not a trivial task, especially in the dense plasma of an AFRC. However, an experiment of this nature would support or refute this current limit hypothesis. 


\section{References}

[1] B. M. Novac, I. R. Smith, M. C. Enache, and P. Senior, "Studies of a very high efficiency electromagnetic launcher," Journal of Physics D-Applied Physics, vol. 35, no. 12, pp. PII S0022-3727(02)33567-8, 2002.

[2] A. Martin and R. Eskridge, "Electrical coupling efficiency of inductive plasma accelerators," Journal of Physics D-Applied Physics, vol. 38, no. 23, pp. 4168-4179, 2005.

[3] D. E. Kirtley, J. T. Slough, M. Pfaff, and C. Pihl, "Steady operation of an electromagnetic plasmoid thruster," in Proceedings of the 8th MSS/6th LPS/5th SPS Joint Subcommittee Meeting, (Huntsville, AL), JANNAF, December 2011. Distribution A.

[4] R. G. Jahn, Physics of Electric Propulsion. Mineola, NY: Dover Publications, 1968.

[5] C. L. Dailey and R. Lovberg, "The PIT MkV Pulsed Inductive Thruster," 191155, 1993. NASA Contractor Report 191155.

[6] K. A. Polzin, "Comprehensive review of planar pulsed inductive plasma thruster research and technology," Journal of Propulsion and Power, vol. 27, no. 3, pp. 513-531.

[7] G. Fonda-Bonardi, "The operating characteristics of an electromagnetic induction plasma accelerator," in AIAA Second Propulsion Joint Specialist Conference, no. AIAA-66-567.

[8] P. J. Fimognari III, A Magnetic and Interferometric Study of a Plasma Formed in a Conical-Theta-Pinch. Doctoral dissertation, University of Alabama-Huntsville, Huntsville, AL, 2007.

[9] D. E. Kirtley, A. Pancotti, J. T. Slough, and C. Pihl, "Steady operation of an FRC thruster on Martian atmosphere and liquid water propellants," in 48th AIAA/ASME/SAE/ASEE Joint Propulsion Conference and Exhibit, no. AIAA-2012-4071, (Atlanta, GA), July 2012. 
[10] Z. Pietrzyk, G. Vlases, R. Brooks, K. Hahn, and R. Raman, "Initial results from the Coaxial Slow Source FRC device,” Nuclear Fusion, vol. 27, no. 9, pp. 1478-89, 1987.

[11] J. Phillips, "Proposal to produce large compact toroids," Report LA-8711-P, Los Alamos National Laboratory, March 1981.

[12] M. Alidieres, R. Aymar, P. Jourdan, and F. Koechlin, "Experimental study of a current sheet," Plasma Physics, vol. 10, pp. 841-850, 1968.

[13] Y. Kondoh, S. Nagao, N. Futawatari, and K. Itoh, "An experiment of double theta-pinch," Japanese Journal of Applied Physics, vol. 13, no. 6, pp. 1037-1038, 1974.

[14] G. Becker, A. Eberhagen, O. Gruber, H. Herold, J. Peiry, H. Rohr, R. Wilheim, and H. Zwicker, "The Garching belt-pinch experiments," in 5th International Conference on Plasma Physics and Controlled Nuclear Fusion, pp. 47-55, 1975.

[15] W. Pierce, R. Maqueda, R. Brooks, and R. Farengo, "Initial results from parallel coil operation of the Coaxial Slow Source field reversed configuration device," Nuclear Fusion, vol. 33, pp. 117-132, 1993.

[16] W. Harris, E. Trask, T. Roche, E. Garate, W. Heidbrink, and R. McWilliams, "Ion flow measurements and plasma current analysis in the Irvine Field Reversed Configuration," Physics of Plasmas, vol. 16, p. 112509, 2009.

[17] M. Tuszewski, "Field reversed configurations," Nuclear Fusion, vol. 28, pp. 2033-2092, 1988.

[18] M. Alidieres, R. Aymar, P. Jourdan, F. Koechlin, and S. A., "Acceleration d'un plasma dans une configuration de striction," Plasma Physics, vol. 9, pp. 73-83, 1967.

[19] D. Brown, C. W. Larson, B. Beal, and A. D. Gallimore, "Methodology and historical perspective of a hall thruster efficiency analsysis," Journal of Propulsion and Power, vol. 25, pp. 1163-1176, 2009.

[20] P. Greve, J. Haumann, H. J. Kunze, and L. K. Ullrich, "Effects of an inhomogeneous impurity distribution in a field-reversed theta-pinch," Physics of Fluids, vol. 25, no. 3, pp. 452-456, 1982.

[21] L. C. Steinhauer, "Review of field-reversed configurations," Physics of Plasmas, vol. 18, p. 07051, July 2011.

[22] T. R. Jarboe, "Review of spheromak research," Plasma Physics and Controlled Fusion, vol. 36, pp. 945-990, 1994.

[23] R. Eskridge and A. Martin, "Progress on the PT-1 prototype plasmoid thruster," Tech. Rep. 200800006053, NASA Marshall Space Flight Center, October 17-19 2007. 
[24] J. T. Slough, D. E. Kirtley, and T. Weber, "Pulsed plasmoid propulsion: The ELF thruster," in 31 st International Electric Propulsion Conference, no. IEPC-2007-265.

[25] D. E. Kirtley and J. T. Slough, "Preliminary performance measurements of the air-breathing ELF thruster," in Proceedings of the 7th MSS/5th LPS/4th SPS Joint Subcommittee Meeting, no. TP-2008-489, (Colorado Springs, CO), JANNAF, December 2010. Distribution A.

[26] G. Fonda-Bonardi, "The experimental verification of performance of an electromagnetic induction plasma thrustor," in AIAA Electric Propulsion and Plasmadynamics Conference, (Colorado Springs, CO), 1967.

[27] G. Fonda-Bonardi, "Inductive plasma accelerator development," AFAPL-TR-67-139, Air Force Propulsion Laboratory, 1968.

[28] E. Y. Choueiri and K. A. Polzin, "Faraday acceleration with radio-frequency assisted discharge," Journal of Propulsion and Power, vol. 22, no. 3, pp. 611-619, 2006.

[29] A. K. Hallock, Effect of Inductive Coil Geometry on the Operating Characteristics of a Pulsed Inductive Plasma Accelerator. Doctoral dissertation, Princeton University, Princeton, New Jersey, 2012.

[30] A. K. Hallock, E. Y. Choueiri, and K. A. Polzin, "Current sheet formation in a conical theta pinch Faraday Accelerator with Radio-frequency Assisted Discharge," in 30th International Electric Propulsion Conference, no. IEPC-2007-165.

[31] K. A. Polzin, M. F. Rose, and R. Miller, "Operational characteristics of a low-energy FARAD thruster," in 44th AIAA/ASME/SAE/ASEE Joint Propulsion Conference and Exhibit, no. AIAA-2008-5011.

[32] M. S. Feldman and E. Y. Choueiri, "Single Stage Faraday Accelerator with Radio-Frequency Assisted Discharge (SS-FARAD)," in 32nd International Electric Propulsion Conference, no. IEPC-2011-220.

[33] A. K. Hallock and K. A. Polzin, "Design of a microwave assisted discharge inductive plasma accelerator," in 46th AIAA/ASME/SAE/ASEE Joint Propulsion Conference and Exhibit, no. AIAA-2010-6527.

[34] S. J. Koelfgen, Magnetic Field and Quadruple Langmuir Probe Measurements in the Plume of the Plasmoid Thruster Experiment. Doctoral dissertation, University of Alabama in Huntsville, Huntsville, AL, 2005.

[35] K. E. Miller, J. T. Slough, and A. L. Hoffman, "An overview of the Star Thrust Experiment," in Space Technology and Applications International Forum, vol. 420, 1998. 
[36] J. T. Slough and K. E. Miller, "Flux generation and sustainment of a field reversed configuration with rotating magnetic field current drive," Physics of Plasmas, vol. 7, no. 5, pp. 1945-1950, 2000.

[37] J. T. Slough, "Propagating magnetic wave plasma accelerator (PMWAC) for deep space exploration," phase 1 final report, NASA Institute of Advanced Concepts, 2000.

[38] J. T. Slough, "Multi-megawatt propulsion based on a compact toroid thruster," in 29th International Electric Propulsion Conference, no. IEPC-2005-296.

[39] J. T. Slough and G. Votroubek, "Magnetically accelerated plasmoid (MAP) propulsion," in 42nd AIAA/ASME/SAE/ASEE Joint Propulsion Conference and Exhibit, no. AIAA-2006-4654.

[40] J. T. Slough, A. Blair, C. Pihl, and G. Votroubek, "Magnetically accelerated plasmoid (MAP) thruster - initial results and future plans," in 30th International Electric Propulsion Conference, no. IEPC-2007-16.

[41] D. E. Kirtley, Study of the Synchronous Operation of an Annular Field Reversed Configuration Device. Doctoral dissertation, University of Michigan, Ann Arbor, MI, 2008.

[42] Y. Kondoh, K. Itoh, and S. Nagao, "Motion of the double theta-pinch plasma," Journal of the Physical Society of Japan, vol. 41, no. 4, pp. 1356-1363, 1976.

[43] R. Berger, Analysis of Slow Formation of Plasmas in a Coaxial Double Theta Pinch. Doctoral dissertation, University of Washington, 1993.

[44] D. J. Rej and W. T. Armstrong, "Electron temperature measurements in the field-reversed configuration experiment FRX-C," Nuclear Fusion, vol. 24, pp. 177-182, Feb 1984.

[45] F. F. Chen, Introduction to Plasma Physics and Controlled Fusion. New York, NY: Springer, 2006.

[46] L. J. Spitzer, Physics of Fully Ionized Gases, vol. 3 of Interscience Tracts on Physics and Astronomy. New York: Interscience Publishers, Inc., 1956.

[47] M. E. Kayama, "Resistivity in the dynamic current sheath of an field reversed configuration," Physics of Plasmas, vol. 19, no. 3, pp. 032511-4, 2012.

[48] R. Farengo and R. D. Brooks, "Plasma heating and dynamics in the Coaxial Slow Source," Nuclear Fusion, vol. 32, no. 1, pp. 67-80, 1992.

[49] N. Rostoker and A. Qerushi, "Equilibrium of field reversed configurations with rotation. i. one space dimension and one type of ion," Physics of Plasmas, vol. 9, pp. 3057-3067, July 2002. 
[50] R. Raman, G. Vlases, and T. Jarboe, "Energy balance in the CSSU device," Nuclear Fusion, vol. 33, no. 11, pp. 1685-1694, 1993.

[51] Y. Kondoh, S. Okamura, S. Nagao, and M. Masuzaki, "Effects of the toroidal magnetic field on an annular plasma," Journal of the Physical Society of Japan, vol. 37, no. 1, pp. 200-205, 1974.

[52] K. Itoh, Y. Kondoh, and S. Nagao, "Toroidal plasma produced by the stabilized double-theta pinch," Nuclear Fusion, vol. 18, no. 6, pp. 769-774, 1978.

[53] R. J. Smith, Magnetic Equilibria of the Coaxial Slow Source. Doctoral dissertation, University of Washington, 1989.

[54] W. S. I. Harris, Ion Flow Measurements and Plasma Current Analysis in the Irvine Field Reversed Configuration. Doctoral dissertation, University of California-Irvine, Irvine, California, 2009.

[55] H. Gota, N. Bolte, B. Deng, G. D., V. Kiyashko, K. Knapp, R. Mendozza, M. Morehouse, T. Roche, and F. Wessel, "Two-chord interferometry using $3.39 \mu \mathrm{m}$ he-ne laser on a flux-coil-generated FRC," Review of Scientific Instruments, vol. 81, p. 10D512, 2010.

[56] R. D. Brooks and T. R. Jarboe, "Coaxial Slow Source," Final Technical Report DOE-ER-53243-5, November 1993.

[57] C. S. Niemela and L. B. King, "Numerical optimization of an annular field reversed configuration translation experiment," in 31st International Electric Propulsion Conference, no. IEPC-2009-008.

[58] C. L. Dailey and R. Lovberg, “The PIT MkV Pulsed Inductive Thruster," NASA Contractor Report 191155, July 1993.

[59] K. A. Polzin and E. Y. Choueiri, "Performance optimization criteria for pulsed inductive plasma acceleration," IEEE Transactions on Plasma Science, vol. 34, no. 3, pp. 945-953, 2006.

[60] J. T. Cassibry, "Comparison of directly and inductively coupled pulsed electromagnetic thrusters," IEEE Transactions on Plasma Science, vol. 36, no. 5, pp. 2180-2188, 2008.

[61] http://www.sagemath.org.

[62] R. Shaw, J. Booske, and M. Mccarrick, "Broadband calibration for magnetic probes for use in the Maryland spheromak," Review of Scientific Instruments, vol. 58, no. 7, pp. 1204-1210, 1987. 
[63] S. Messer, D. D. Blackwell, W. E. Amatucci, and D. N. Walker, "Broadband calibration of radio-frequency magnetic induction probes," Review of Scientific Instruments, vol. 77, no. 115104, pp. 115104-1 - 115104-7, 2006.

[64] M. P. Reilly, W. Lewis, and G. H. Miley, "Magnetic field probes for use in radio frequency plasmas," Review of Scientific Instruments, vol. 80, no. 5, pp. 053508-5, 2009.

[65] E. Everson, P. Pribyl, C. Constantin, A. Zylstra, D. Schaeffer, N. Kugland, and C. Niemann, "Design, construction, and calibration of three-axis, high-frequency magnetic probe (b-dot probe) as a diagnostic for exploding plasmas," Review of Scientific Instruments, vol. 80, no. 11, pp. 113505-8, 2009.

[66] J. Yang, J. Choi, B. Kim, N. Yoon, and S. Hwang, "A calibration method of radio frequency magnetic probe," Review of Scientific Instruments, vol. 70, no. 9, pp. 3774-3775, 1999.

[67] R. Phillips and E. Turner, "Construction and calibration techniques of high frequency magnetic probes," The Review of Scientfic Instruments, vol. 36, no. 12, pp. 1822-1825, 1965.

[68] R. L. Burden and J. D. Faires, Numerical Analysis. Belmont, CA: Thomson Brooks/Cole, 2005.

[69] G. E. Box, J. S. Hunter, and W. G. Hunter, Statistics for Experimenters. Hoboken, NJ: Wiley-Interscience, 2005.

[70] L. Schott, Electrical Probes in Plasma Diagnostics. Amsterdam: North Holland Publishing Company, 1968.

[71] I. Hutchinson, Principles of Plasma Diagnostics. Cambridge: Cambridge University Press, 2nd ed., 2002.

[72] V. Demidov, S. Ratynskaia, and K. Rypdal, "Electric probes for plasmas: The link between theory and instrument," Review of Scientific Instruments, vol. 73, no. 10, pp. 3409-3439, 2002.

[73] D. E. Koopman, "Langmuir probes and microwave measurements of the properties of streaming plasmas generated by focused laser pulses," Physics of Fluids, vol. 14, no. 8, pp. 1707-1716, 1971.

[74] P. M. Chung, L. Talbot, and K. J. Touryan, Electric Probes in Stationary and Flowing Plasmas: Theory and Application. New York, NY: Springer-Verlang, 1975.

[75] T. Sheridan, "How big is a small Langmuir probe?," Physics of Plasma, vol. 7, no. 7, pp. 3084-3088, 2000. 
[76] D. Lee and Hershkowitz, "Ion collection by planar langmuir probes: Sheridan's model and verification," Physics of Plasma, vol. 14, no. 3, pp. 033507-4, 2007.

[77] M. Tuszewski and J. A. Tobin, "The accuracy of Langmuir probe ion density measurements in low-frequency RF discharges," Plasma Sources and Science Technology, vol. 5, pp. 640-647, 1996.

[78] J. G. Laframboise, "Theory of spherical and cylindrical langmuir probes in a collisionless, maxwellian plasma at rest," University of Toronto Institute for Aerospace Studies 100, Toronto, 1966.

[79] E. Johnson and L. Malter, "A flaoting double probe method for measurements in gas discharges," Physical Review, vol. 80, pp. 58-68, 1950.

[80] J. D. Swift and M. J. R. Schwar, Electrical Probes for Plasma Diagnostics. New York, NJ: American Elsevier, 2005.

[81] A. Karamcheti and C. Steinbruchel, "Parameterization of laframboise's results for spherical and cylindrical langmuir probes," Journal of Vacuum Science and Technology A, vol. 17, pp. 3051-3056, 1999.

[82] B. Beal, L. Johnson, D. Brown, J. Blakely, and D. Bromaghim, "Improved analysis techniques for cylindrical and spherical double probes," Review of Scientific Instruments, vol. 83, pp. 073506-5, 2012.

[83] D. Rapp and P. Englander-Golden, "Total cross sections for ionizaiton and attachment in gases by electron impact. i. positive ionization," The Journal of Chemical Physics, vol. 43, no. 5, pp. 1464-1479, 1965.

[84] C. Yamabe, S. J. Buckman, and A. V. Phelps, "Measurement of free-free emission from low-energy-electron collisions with Ar," Physical Review A, vol. 27, no. 3, pp. 1345-1352, 1983.

[85] "Crc handbook of chemistry and physics."

[86] G. Marr and J. West, "Absolute photoionization cross-section tables for Helium, Neon, Argon, and Krypton in the VUV spectral regions," Atomic Data and Nuclear Data Tables, vol. 18, pp. 497-508, 1976.

[87] R. D. Milroy and J. T. Slough, "Poloidal flux loss and axial dynamics during the formation of a field-reversed configuration," Physics of Fluids, vol. 30, no. 11, pp. 3566-3576, 1987. 


\section{Appendix A}

\section{XOCOT-T3 Supplemental Data}

This section contains the additional XOCOT-T3 data used for analysis in Chapters 6, 7, 8, and 9 .

Signals from a single vacuum shot at $500 \mathrm{~J}$ using the $10 \mathrm{kHz}$ circuit is shown in Figure A.1 Unscaled coil current data and midplane b-dot signals are included. The b-dot signals have been processed to calculate the magnetic field, shown in the bottom plot of Figure A.1.

Signals from a single vacuum shot at $100 \mathrm{~J}$ using the $20 \mathrm{kHz}$ circuit is shown in Figure A.2 Unscaled coil current data and midplane b-dot signals are included. The coil current data has been scaled and filtered to calculate the total circuit current. The b-dot signals have been processed to calculate the vacuum magnetic field at the coil midplane. This plot is included at the bottom plot of Figure A.2.

Coil current, midplane magnetic field, and photometer data is shown for a single shot with the $10 \mathrm{kHz}$ circuit in Figure A.3 and with the $20 \mathrm{kHz}$ circuit in Figure A.4. A full time-history is shown, through several discharge cycles. Vacuum traces are provided for comparison.

Curve fits to ADLP data is displayed in Figure A.5 from data collected outside the coils at $\mathrm{z}=34.3 \mathrm{~cm}$. This data was used to calculate the density and temperature of the plasma emerging from the coils after magnetic confinement was lost. 

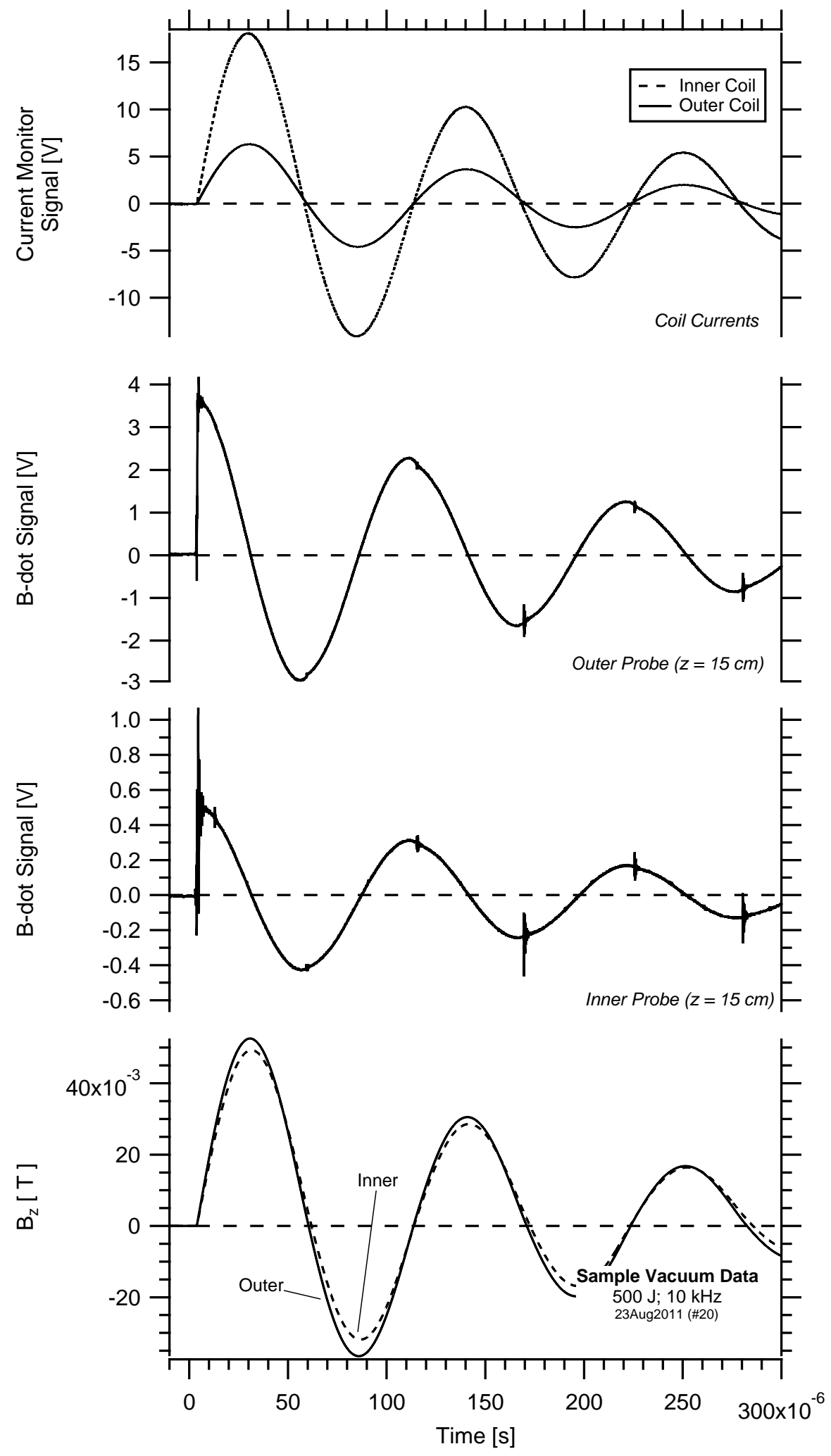

Figure A.1: Vacuum data from a shot at $500 \mathrm{~J}$ and $10 \mathrm{kHz}$. 

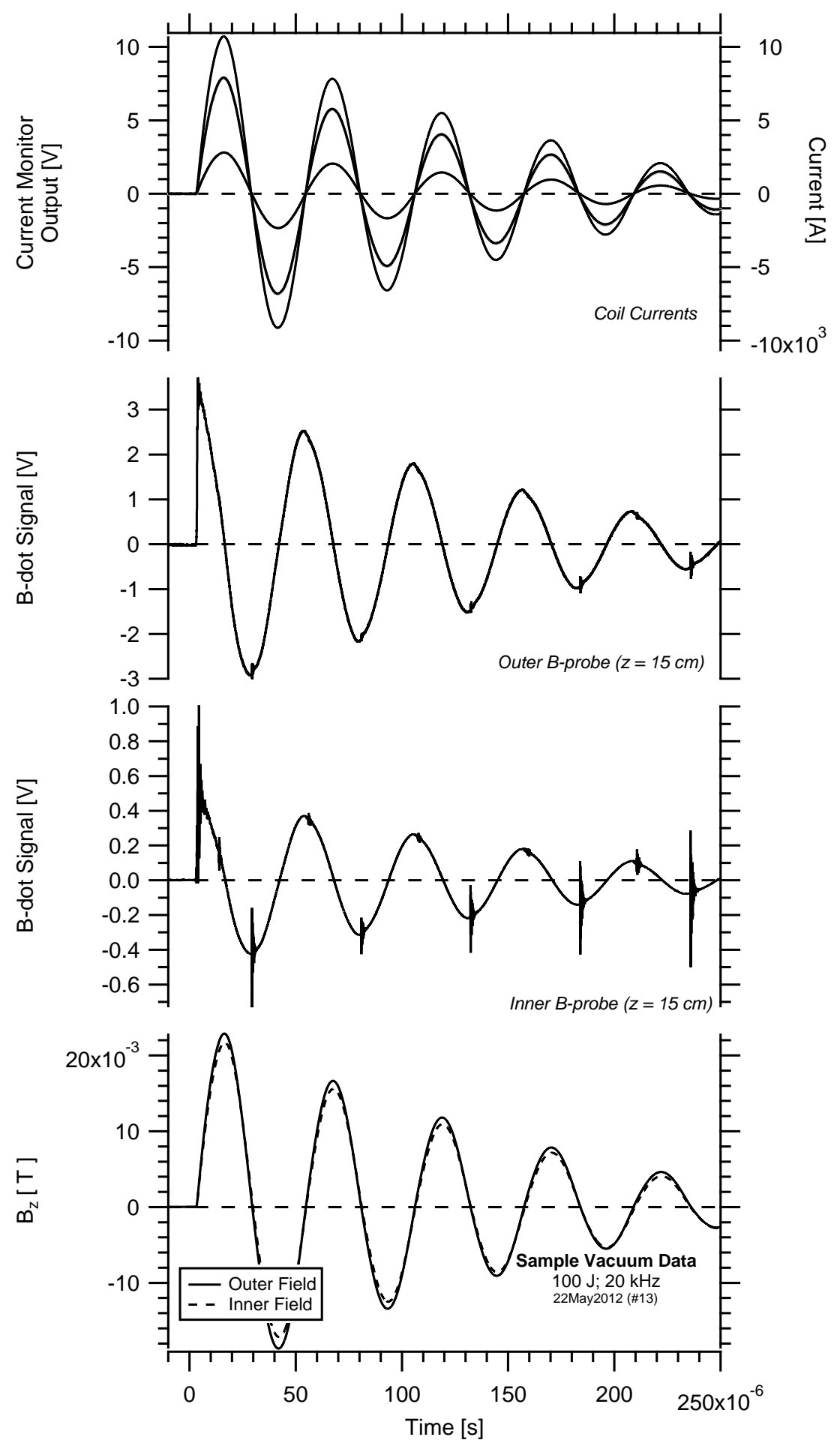

Figure A.2: Vacuum data from a shot at $100 \mathrm{~J}$ and $20 \mathrm{kHz}$. 

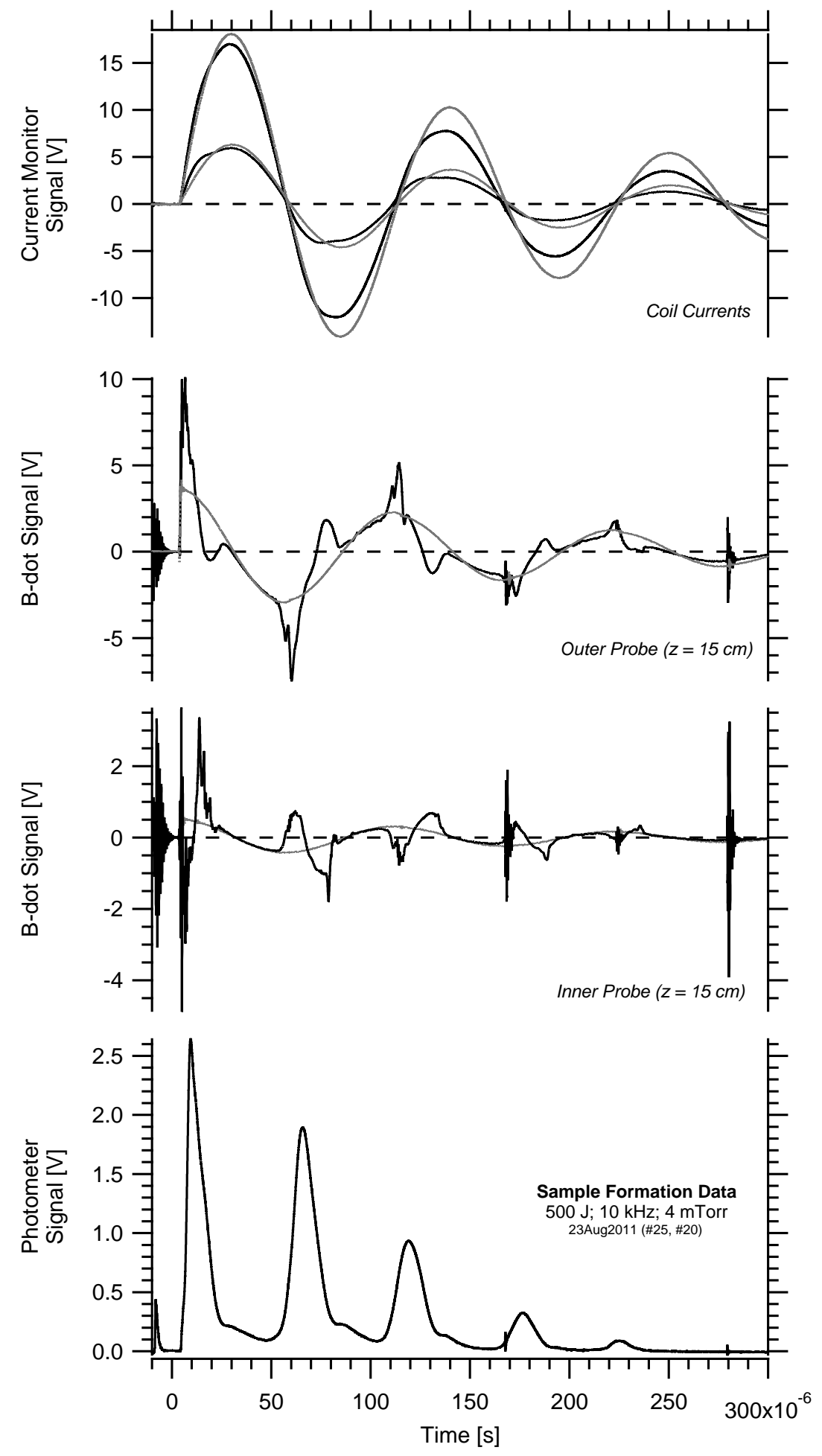

Figure A.3: Current, magnetic field, and photometer data from a shot at $500 \mathrm{~J}$ and $10 \mathrm{kHz}$, with a 4 mTorr fill of Argon. 

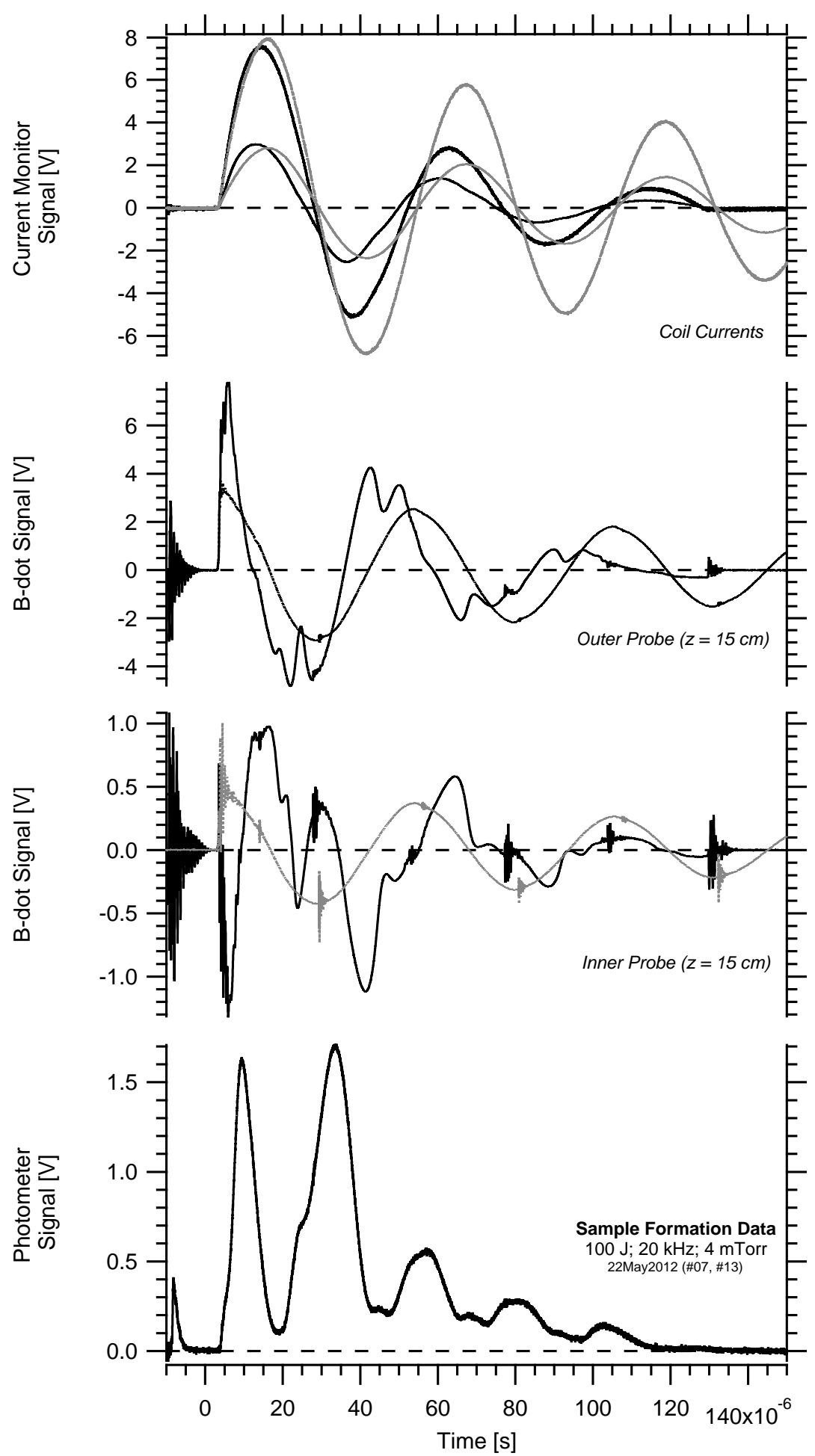

Figure A.4: Current, magnetic field, and photometer data from a shot at $100 \mathrm{~J}$ and $20 \mathrm{kHz}$, with a 4 mTorr fill of Argon. 

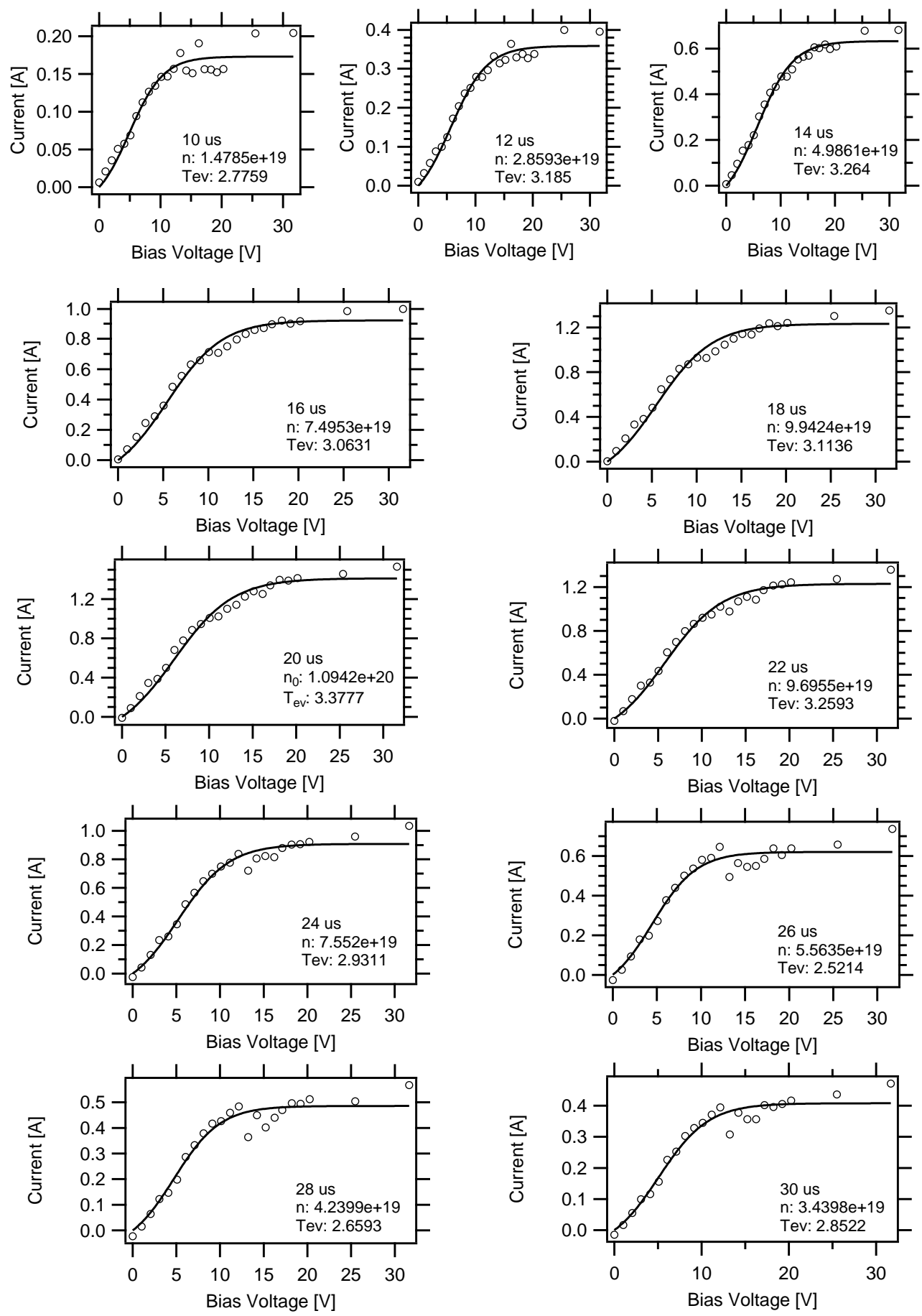

Figure A.5: Current and voltage traces from the ADLP at $\mathrm{z}=34.3 \mathrm{~cm}$. Curve fits to the data were constructed from ADLP theory. 